Recht der Informationsgesellschaft

Fabian Toros

\title{
Handlungsoptionen auf dem Weg in die Gigabit-Gesellschaft
}

Eine rechtliche Analyse von Konzessions- und Kooperationsmodellen sowie regulatorischer Entflechtungsbestimmungen 
Recht der Informationsgesellschaft

herausgegeben von

Prof. Dr. Jörg Fritzsche, Universität Regensburg, Lehrstuhl für Bürgerliches Recht, Handels- und Wirtschaftsrecht Prof. Dr. Jürgen Kühling, LL.M., Universität Regensburg, Lehrstuhl für Öffentliches Recht, Immobilienrecht, Infrastrukturrecht und Informationsrecht

Prof. Dr. Gerrit Manssen, Universität Regensburg, Lehrstuhl für Öffentliches Recht, insbesondere deutsches und europäisches Verwaltungsrecht

Prof. Dr. Robert Uerpmann-Wittzack, Maître en droit, Universität Regensburg, Lehrstuhl für Öffentliches Recht und Völkerrecht

Band 48 
Fabian Toros

\section{Handlungsoptionen auf dem Weg in die Gigabit-Gesellschaft}

Eine rechtliche Analyse von Konzessions- und Kooperationsmodellen sowie regulatorischer Entflechtungsbestimmungen 
Die Deutsche Nationalbibliothek verzeichnet diese Publikation in der Deutschen Nationalbibliografie; detaillierte bibliografische Daten sind im Internet über http://dnb.d-nb.de abrufbar.

Zugl.: Regensburg, Univ., Diss., 2020

1. Auflage 2021

(c) Fabian Toros

Publiziert von

Nomos Verlagsgesellschaft mbH \& Co. KG

Waldseestraße 3-5 | 76530 Baden-Baden

www.nomos.de

Gesamtherstellung:

Nomos Verlagsgesellschaft mbH \& Co. KG

Waldseestraße 3-5 | 76530 Baden-Baden

ISBN (Print): 978-3-8487-7959-8

ISBN (ePDF): 978-3-8487-7959-8

DOI: https://doi.org/10.5771/9783748923435

Die Bände 1 bis 33 sind im Lit-Verlag erschienen.

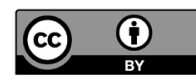

Dieses Werk ist lizensiert unter einer Creative Commons Namensnennung 4.0 International Lizenz. 
Meiner Famile 


\section{Vorwort}

Die vorliegende Monographie wurde im Sommersemester 2020 von der Fakultät für Rechtswissenschaft der Universität Regensburg als Dissertation angenommen. Gesetzgebung, Literatur und Rechtsprechung wurden bis Anfang Juli 2020 berücksichtigt. Nachträglich wurde der Regierungsentwurf zum Telekommunikationsgesetz der Bundesregierung vom 17. Dezember 2020 eingearbeitet.

Ich bedanke mich besonders bei meinem Doktorvater Professor Dr. Jürgen Kühling, LL.M. Er hat meine Dissertation umfassend betreut und mir im gesamten Bearbeitungsprozess viele wertvolle Hinweise gegeben. Die Mitarbeit an seinen vielseitigen und spannenden Projekten hat es mir ermöglicht, über die Dissertation hinaus vertiefte Kenntnisse im gesamten Regulierungsrecht zu erlangen. Insbesondere die Kontakte zu Ministerien und Behörden sowie zur Monopolkommission, die Professor Kühling mir vermittelt hat, waren für meine Forschungstätigkeit sehr hilfreich. Die Arbeit im Lehrstuhlteam hat mir große Freude bereitet und wird mir, als eine der besten Zeiten meines Lebens, stets in positiver Erinnerung bleiben.

Herrn Professor Dr. Gerrit Manssen möchte ich für die rasche Erstellung des Zweitgutachtens danken. Auf diese Weise ist es mir möglich, mit einem hochaktuellen Thema schnellstmöglich die Fachöffentlichkeit zu adressieren.

Dr. Stefan Drechsler danke ich für die umfassende und gewissenhafte Korrekturlektüre, durch die ich stets gewinnbringende und zielführende Hinweise für die Arbeit an meiner Dissertation erhielt.

Bedanken möchte ich mich auch bei den Mitgesellschaftern der schnupperkurs.media $\mathrm{GmbH}$, die mir stets den zeitlichen Freiraum gelassen haben, meine Forschung gewissenhaft betreiben zu können.

Ich möchte mich auch bei meinen Freunden bedanken, die mich auf meinem bisherigen Weg begleitet haben. Besonderer Dank gebührt den Doktoranden an der IREBS International Real Estate Business School der Universität Regensburg, die von Kollegen rasch zu Freunden geworden sind und die Promotionszeit für mich unvergesslich machen. 
Der größte Dank gilt jedoch meiner gesamten Familie, insbesondere meiner Mutter Birgit Toros und meiner Schwester Carolina Toros. Mein Vater Ahmet Toros, der in meiner Kindheit verstorben ist, bleibt unvergessen. Meiner Familie ist dieses Buch gewidmet.

Regensburg/Ratingen, Februar 2021

Fabian Toros 


\section{Inhaltsverzeichnis}

$\begin{array}{ll}\text { Kapitel 1: Einleitung } & 21\end{array}$

Kapitel 2: Die Gigabit-Gesellschaft 25

A. Die Entwicklung der Gigabit-Gesellschaft 25

I. Der Begriff der Gigabit-Gesellschaft 26

1. Der Ablauf der Infrastrukturentwicklung 26

2. Die Entstehung der „Gigabit-Gesellschaft"

II. Der Weg in die Gigabit-Gesellschaft 28

III. Zwischenergebnis 30

B. Die Anforderungen an die digitale Infrastruktur der GigabitGesellschaft 31

I. Die Qualitäts- und Kapazitätsparameter 31

1. Die Datenübertragungskapazität 32

2. Alternative Kriterien der Legaldefinition des EKEK 33

3. Relevante Kriterien ohne Veranlagung im unionalen Rechtsrahmen $\quad 34$

4. Zwischenergebnis 35

II. Die Datenübertragungstechnologien für die GigabitGesellschaft $\quad 35$

1. Glasfaser als Datenübertragungstechnologie 36

2. Alternative Datenübertragungstechnologien 37

a) Datenübertragungstechnologien auf Basis einer Kupferdoppelader 38

b) Datenübertragungstechnologien auf Basis eines Koaxialkabels 39

c) Funktechnologien $\quad 40$

III. Die optimale Infrastruktur für die Gigabit-Gesellschaft? 41

C. Der Ausbau gigabitfähiger Infrastrukturen 42

I. Statistiken zum Stand des Ausbaus gigabitfähiger Infrastrukturen

1. Die Anzahl der mit gigabitfähigen Infrastrukturen versorgten Haushalte

2. Das Verhältnis von Angebot und Nachfrage 43 
3. Der aktuelle Bandbreitenbedarf auf Basis der Verteilung der vermarkteten Bandbreiten

4. Schlussfolgerungen für die weitere Analyse

II. Risiken und Kostentreiber beim Ausbau gigabitfähiger Infrastrukturen

1. Tiefbaukosten als Kostentreiber 46

2. Die Nachfrageunsicherheit 47

3. Die Markt-, Wettbewerbs- und Regulierungsunsicherheit 50

III. Ergebnis und Schlussfolgerungen 51

D. Fazit

Kapitel 3: Exklusivität im Infrastrukturausbau am Beispiel unterschiedlicher Konzessionsmodelle

A. Begriffsbestimmung und Begriffsmerkmale der Konzession 53

I. Ursprung des Konzessionsbegriffes im nationalen Recht $\quad 54$

II. Bau- und Dienstleistungskonzessionen 54

III. Zerfaserung der Terminologie im nationalen Recht 55

IV. Ergebnis $\quad 57$

B. Exklusivität im Telekommunikationsrecht 57

I. Exklusivität bei der Vergabe von Funkfrequenzen 58

II. Exklusivität bei der Vergabe von Rufnummern 59

III. Exklusivität beim Vectoring $\quad 60$

IV. Regulatorische Behandlung neuer Netzbestandteile gemäß Art. 76 EKEK

V. Rückschlüsse und generelle Anforderungen an den Umgang mit Exklusivität im Telekommunikationsrecht $\quad 64$

C. Konzessionsmodell auf Basis der Vergabe exklusiver Wegerechte $\quad 65$

I. Aufbau und Grundstruktur 66

II. Einordnung des Konzessionsmodells auf Basis exklusiver Wegerechte

1. Bau- oder Dienstleistungskonzession

a) Einordnung des Vertragsgegenstandes als Bau- oder Dienstleistung

b) Tatbestandsvoraussetzungen einer

Dienstleistungskonzession

aa) Beschaffungsbezug im Kontext von Baukonzessionen 
bb) Eigenständige Abgrenzungskriterien für die Dienstleistungskonzession

cc) Beschaffungsbezug bei Erfüllung einer öffentlichen Aufgabe

(1) Telekommunikationsnetz der allgemeinen Versorgung

(2) Universaldienstgewährleistungsauftrag des Hoheitsträgers

(3) „e-Daseinsvorsorge“ des Staates

(4) Aufbau einer Glasfaserinfrastruktur als „öffentliche Aufgabe“

(5) Zwischenergebnis

dd) Beschaffungsvorgang nur bei einem unmittelbaren wirtschaftlichen Interesse

ee) Stellungnahme und Zwischenergebnis

c) Ergebnis

2. Einordnung in weitere Konstellationen der Begriffsverwendung

III. Allgemeine rechtliche Steuerungsvorgaben aus dem Telekommunikations(wege)recht

1. Regulierungsziele als Orientierungsmaßstab für die grundsätzliche Konzeption des Telekommunikationsrechts

a) Ziele der Regulierung im Telekommunikationsrecht

b) Umgang des Telekommunikationsrechts mit Zielkonflikten

c) Kollidierende Ziele im Konzessionsmodell auf Basis der Vergabe exklusiver Wegerechte

2. Unionsrechtliche Anforderungen an die Vergabe von

Telekommunikationswegerechten

3. Grundkonzeption der Wegerechtsvergabe im TKG

4. Novellierungserfordernisse zur Ermöglichung einer exklusiven Wegerechtsvergabe

5. Ergebnis

IV. Ausgestaltung des Konzessionsvergabeverfahrens

1. Steuerungsvorgaben des europäischen und deutschen Verfassungsrechts

a) Grundrechte

aa) Identifikation des einschlägigen Grundrechtskataloges 
bb) Vereinbarkeit mit den erwerbsbezogenen Grundrechten

(1) Unionale Gewährleistungen aus Art. 15 Abs. 1 und 16 GRCh

(2) Nationale Gewährleistungen aus Art. 12 Abs. $1 \mathrm{GG}$

cc) Vereinbarkeit mit den Gleichheitsrechten der GRCh und des GG

b) Staatsorganisationsrechtliche Vorgaben des

Art. 87f GG

aa) Zuständige Behörde für die Durchführung des Konzessionsvergabeverfahrens

(1) Kompetenzrechtliche Steuerungsvorgaben aus dem Verfassungsrecht

(2) Verfassungsrechtliche Vorgaben zur

Verwaltungsorganisation

106

(3) Ergebnis

bb) Verfahrensausgestaltung 108

cc) Ergebnis

2. Steuerungsvorgaben des Wettbewerbsrechts

a) Anwendbarkeit des Wettbewerbsrechts neben dem TKG

b) Anwendbarkeit des Wettbewerbsrechts auf staatliches Handeln

c) Anwendbarkeit des Wettbewerbsrechts aufgrund der Übertragung ausschließlicher Rechte gemäß Art. 106 AEUV

aa) Privilegiertes Unternehmen im Sinne des Art. 106 Abs. 1 AEUV

bb) Rechtsfolge der Erfüllung des Tatbestandes des Art. 106 Abs. 1 AEUV

cc) Einschränkung durch Art. 106 Abs. 2 AEUV

d) Ergebnis

3. Steuerungsvorgaben des Vergaberechts

a) Ausschluss der Anwendbarkeit der KVRL und der $\int \mathbb{S} 105 \mathrm{ff} . \mathrm{GWB}$

b) Vergaberechtliche Erleichterungen durch eine Inhouse-Vergabe

c) Steuerungsvorgaben des unionalen Primärrechts

d) Ergebnis 
4. Vereinbarkeit des Konzessionsmodells auf Basis der Vergabe exklusiver Wegerechte mit dem EU-

Beihilfenrecht

5. Folgen für die Ausgestaltung des Vergabeverfahrens

a) Vorgaben des unionalen Primärrechts für die Verfahrensausgestaltung

aa) Primärrechtliche Ver- und Gebote bei der Verfahrensausgestaltung

bb) Praktische Auswirkungen auf das Konzessionsvergabeverfahren

b) Vereinbarkeit mit den Steuerungsvorgaben des EKEK

V. Umgang mit Bestandsinfrastruktur und bereits erteilten

Wegerechten

1. Auswirkung von Bestandsinfrastruktur im Konzessionsgebiet

2. Schutzbereich des Grundrechts auf Eigentum aus Art. 17 Abs. 1 GRCh

3. Maßnahmen zum Umgang mit Bestandsinfrastruktur als Einschränkung des Eigentumsgrundrechts

4. Rechtfertigung der Einschränkungen des Eigentumsgrundrechts

a) Gesetzliche Grundlage der Eigentumsübertragungspflicht

b) Verhältnismäßigkeit der Eigentumsübertragungspflicht

aa) Legitimes Ziel und öffentliches Interesse

bb) Eignung

cc) Erforderlichkeit der Eigentumsübertragungspflicht

dd) Zahlung einer angemessenen und rechtzeitigen Entschädigung

ee) Verhältnismäßigkeit des Eigentumsentzuges im engeren Sinne

c) Zwischenergebnis

5. Eigentumsgrundrecht des Grundgesetzes (Art. 14 Abs. 1 GG)

6. Umgang mit bereits erteilten Wegerechten

7. Ergebnis

VI. Ergebnis 
D. Konzessionsmodell zum Aufbau und Betrieb staatlicher Telekommunikationsinfrastrukturen

I. Beispiele für die Ausgestaltung des Konzessionsmodells zum Aufbau und Betrieb staatlicher Infrastrukturen

1. Das griechische „Ultrafast Broadband Infrastructure Scheme“

2. Die italienische „Strategia Banda Ultralarga“ 144

3. Gemeinsamkeiten der Modelle 145

4. Klassifikation als Konzessionsmodell

II. Abgrenzung zum Konzessionsmodell auf Basis der Vergabe exklusiver Wegerechte $\quad 147$

III. Ergebnis 147

E. Konzessionsmodell auf Basis der Vergabe exklusiver Fördermittel 148

F. Ergebnis

Kapitel 4: Beschleunigung des Infrastrukturausbaus durch die Entflechtung und regulatorische Privilegierung von Wholesale-Only-Anbietern

A. Ökonomische Grundlagen der vertikalen Integration

I. Wertschöpfungsketten im Telekommunikationssektor

1. Begriffsanalyse

2. Vertikale Integration im Telekommunikationssektor

154

a) Betriebswirtschaftliche Vorteile einer vertikalen Integration

b) Gefahren für den Wettbewerb und regulatorische Handlungsoptionen

3. Wholesale-Only als betriebswirtschaftlich sinnvolles Geschäftsmodell und Beschleunigungsansatz für den Infrastrukturausbau?

4. Zwischenergebnis und Schlussfolgerungen

II. Stufenlehre zur Kategorisierung von

Entflechtungsmaßnahmen

1. Struktur des Stufensystems 161

2. Wholesale-Only-Anbieter im Stufensystem 164

III. Schlussfolgerungen für die juristische Analyse 
B. Entstehung von Wholesale-Only-Anbietern durch eine staatlich indizierte funktionelle Trennung

I. Normative Ausgestaltung der funktionellen Trennung im Telekommunikationsrecht

1. Steuerungsvorgaben des Regulierungsrechts für den Telekommunikationssektor

a) Die Grundstruktur des Art. 77 EKEK de lege lata

b) Vorgaben des $₫ 40 \mathrm{TKG}$

2. Anpassungsbedarf und -möglichkeit des Rechtsrahmens de lege ferenda?

a) Erweiterung der Entflechtungsintensität der Kompetenzgrundlage

b) Schaffung einer neuen Kompetenzgrundlage aufgrund systematischer und teleologischer Bedürfnisse?

c) Verfassungsmäßigkeit der Kompetenzgrundlage und ihrer Anwendung im Einzelfall

aa) Vereinbarkeit mit den erwerbsbezogenen Grundrechten

bb) Vereinbarkeit mit dem Grundrecht auf Eigentum

cc) Gleichbehandlung vertikal integrierter Telekommunikationsunternehmen

d) Ergebnis

II. Mögliche Aufspaltung des Unternehmens nach deutschem Kartellrecht zur Beschleunigung des Infrastrukturausbaus?

C. Freiwillige Separierung vertikal integrierter Telekommunikationsunternehmen

I. Freiwillige Aufspaltung am Beispiel des Unternehmens CETIN

II. Gesellschaftsrechtliche Vorgaben zur Aufspaltung von Unternehmen

III. Regulatorische Behandlung freiwillig getrennter Telekommunikationsunternehmen

1. Freiwillige Trennung im unionalen Rechtsrahmen

2. Umsetzung der unionalen Vorgaben im nationalen Recht

3. Anpassungsbedarf der Vorgaben de lege ferenda? 
$\begin{array}{ll}\text { IV. Zwischenergebnis } & 189\end{array}$

D. Gründung als Wholesale-Only-Unternehmen 190

I. Beispiele für ausschließlich auf der Vorleistungsebene tätige Unternehmen

1. Kommunaler Glasfaserausbau durch AB Stokab in Stockholm

2. Geschäftsmodell der Mitverlegung der deutschen GasLINE

II. Regulatorischer Umgang mit Wholesale-Only-Anbietern 193

III. Zwischenergebnis und Ausblick

E. Optimierungsvorschläge außerhalb der regulatorischen Entflechtungsbestimmungen

F. Fazit

Kapitel 5: Kooperationen im Glasfaserausbau

A. Grundlegende Erwägungen zur ökonomischen Bewertung von Kooperationen

I. Abgrenzung des Kooperationsbegriffes 204

II. Ökonomische Grundlagen 206

1. Ökonomische Auswirkungen von

Kooperationsvorhaben auf den Breitbandausbau

2. Anreize für einen kooperativen Glasfaserausbau 209

III. Schlussfolgerungen für die juristische Analyse 210

B. Der Anwendungsbereich wettbewerbsrechtlicher

Regelungsregime

I. Die Gründung der Glasfaser Nordwest als Fallbeispiel

II. Eröffnung des Anwendungsbereiches der nationalen Vorgaben zur Fusionskontrolle

1. Zusammenschluss im Sinne des GWB

a) Erfüllung eines Zusammenschlusstatbestandes 213

b) Behandlung als einheitliches Unternehmen 214

c) Zwischenergebnis 216

2. Weitere Anwendungsvoraussetzungen der Vorgaben zur Fusionskontrolle

a) Umsatzschwellenwerte 217

b) Ausnahmen 
3. Verhältnis von unionaler oder nationaler Fusionskontrolle

a) Exklusivität der Anwendungsbereiche durch das "One-Stop-Shop"-Prinzip

b) Abgrenzungskriterium des „Vollfunktionsgemeinschaftsunternehmens" im Sinne des Art. 3 Abs. 4 FKVO

c) Ergebnis

4. Ergebnis

III. Anwendbarkeit des Kartellverbotes aus $₫ 1$ GWB

1. Sperrwirkung der Vorgaben zur Fusionskontrolle?

2. Sperrwirkung des unionalen Kartellverbotes aus Art. 101 AEUV?

3. Ergebnis

C. Abgrenzung der relevanten Märkte

I. Grundlagen zur Marktabgrenzung im Wettbewerbsrecht

1. Sachliche Marktabgrenzung

2. Räumliche Marktabgrenzung

3. Möglichkeit der divergierenden Marktabgrenzung durch unterschiedliche Behörden

4. Fazit

II. Breitbandinternetzugangs- und Endkundenmärkte des Telekommunikationssektors

1. Sachliche Marktabgrenzung

a) Endkundenmarkt für festnetzbasierten Internetzugang

b) Markt für den lokal bereitgestellten Zugang zur Teilnehmeranschlussleitung

c) Markt für den zentral bereitgestellten Zugang zur Teilnehmeranschlussleitung

2. Räumliche Marktabgrenzung

a) Beschlusspraxis der Bundesnetzagentur zur räumlichen Abgrenzung der Anschlussmärkte

b) Beschlusspraxis des Bundeskartellamts zur räumlichen Abgrenzung der Anschlussmärkte

c) Abgrenzung regionaler Teilmärkte oder Auferlegung regional differenzierter Abhilfemaßnahmen auch im Regulierungsrecht?

aa) Begrenzung des räumlich-relevanten Marktes auf das Kooperationsgebiet 
bb) Möglichkeit der Auferlegung regional differenzierter regulatorischer Verpflichtungen

cc) Zwischenergebnis

3. Ergebnis und Ausblick

III. Ausschreibungsmarkt für den geförderten Breitbandausbau

IV. Zusammenfassung und Schlussfolgerungen

D. Regulatorische Rahmenbedingungen zum Schutz des Wettbewerbs bei Kooperationsvorhaben

I. Entstehung eines Gemeinschaftsunternehmens mit beträchtlicher Marktmacht

II. Beteiligung eines Unternehmens mit beträchtlicher Marktmacht am Gemeinschaftsunternehmen

1. Unternehmensbegriff des $₫ 3 \mathrm{Nr}$. 29 TKG

a) Wortlaut des $\$ 3 \mathrm{Nr} .29 \mathrm{TKG}$

b) Teleologische und unionsrechtskonforme Reduktion des $₫ 3 \mathrm{Nr} .29$ TKG?

c) Ergebnis

2. Erstreckung der telekommunikationsrechtlichen Regulierungsverfügungen auf paritätische Gemeinschaftsunternehmen

3. Novellierung der regulatorischen Rahmenbedingungen durch den EKEK

a) Regulatorische Verpflichtungszusage gemäß Art. 79 EKEK

aa) Verpflichtungszusage im Kartellrecht

bb) Öffentlich-rechtliche Verträge im Rahmen des Marktregulierungsverfahrens

cc) Verpflichtungszusage im unionalen Telekommunikationsrecht

dd) Umsetzung im Telekommunikationsgesetz

b) Privilegierungstatbestände für Kooperationsvorhaben im EKEK

aa) Regulatorische Behandlung neuer Bestandteile von Netzen mit sehr hoher Kapazität gemäß Art. 76 EKEK

bb) Kooperationsvereinbarungen im Sinne des Art. 79 Abs. 1 UAbs. 1 lit. a EKEK

cc) Novellierung des Telekommunikationsgesetzes

c) Auswirkungen des neuen Rechtsrahmens auf Kooperationsvorhaben 
d) Ergebnis

4. Optimierungsbedarf der regulatorischen

Rahmenbedingungen

a) Weitergehender normativer Anpassungsbedarf de lege ferenda

b) Änderungsbedarf im Hinblick auf die Spruchpraxis der Regulierungsbehörden

c) Zwischenergebnis

5. Ergebnis

III. Ergebnis

E. Kartellrechtliche Rahmenbedingungen zum Schutz des Wettbewerbs bei Kooperationsvorhaben

I. Steuerungsvorgaben aus dem Recht der Fusionskontrolle

1. Zusammenspiel von regulatorischen Verpflichtungen und fusionskontrollrechtlicher Wettbewerbsanalyse

2. Verpflichtungszusage im Fusionskontrollrecht

3. Zwischenergebnis und Schlussfolgerungen

II. Steuerungsvorgaben des Kartellverbots gemäß $₫ 1 \mathrm{GWB}$

1. Tatbestand des Kartellverbotes des $₫ 1 \mathrm{GWB}$

2. Freistellung vom Kartellverbot gemäß $₫ 2$ GWB

a) Ausbaukooperation und Effizienzgewinn

b) Verbraucherwohlfahrt

c) Negative Freistellungsvoraussetzungen

d) Zwischenergebnis

3. Verpflichtungszusage

4. Ergebnis und Schlussfolgerungen

Kapitel 6: Das Zusammenspiel verschiedener Handlungsoptionen auf dem Weg in die Gigabit-Gesellschaft

I. Hochkomplexe Ausgangslage

II. Zielrichtung und Anpassungsfähigkeit des

Telekommunikationsrechtsrahmens

III. Zusammenspiel von allgemeinem und sektorspezifischem Wettbewerbsrecht

IV. Keine Instrumentalisierung des allgemeinen Wettbewerbsrechts zur Beschleunigung des Glasfaserausbaus

V. Zuständigkeit und Ausstattung von Behörden 
VI. Flexibilisierung des Marktregulierungsverfahrens durch Verpflichtungszusagen

VII. Wettbewerb im Netz oder Wettbewerb um das Netz?

VIII. Keine Verpflichtung zur Umsetzung von

Geschäftsmodellen durch staatlichen Zwang

IX. Zusammenspiel der Anpassung des Rechtsregimes mit der Vergabe von Fördermitteln 


\section{Kapitel 1: Einleitung}

Die Digitalisierung von Wirtschaft und Gesellschaft ist eine der zentralen Herausforderungen der Gegenwart. Das analoge Deutschland soll zu einem „Deutschland 4.0“ werden - geprägt durch Künstliche Intelligenzen, das „Internet of Things“ und vollständig digitalisierte Prozesse. Grundvoraussetzung für das Erreichen dieses Zieles ist eine hochleistungsfähige digitale Infrastruktur. ${ }^{1}$ Klaus Steinmaurer, der Geschäftsführer der österreichischen Regulierungsbehörde, hat die Infrastruktur gar als „die Mutter der Digitalisierung “ bezeichnet. ${ }^{2}$ Vor diesem Hintergrund verwundert es nicht, dass die Bundesregierung in Deutschland eine „flächendeckende digitale Infrastruktur von Weltklasse“ ${ }^{\text {“3 }}$ schaffen möchte. Schließlich war die Verfügbarkeit einer modernen Infrastruktur schon immer Grundlage für eine prosperierende Wirtschaft, welche die Basis für den Wohlstand in der Gesellschaft ist. Die Verfügbarkeit gigabitfähiger Infrastrukturen stellt einen entscheidenden Standortfaktor für die Ansiedlung der Industrie von morgen dar. Dies betrifft nicht nur den kommunalen oder regionalen Zusammenhang, sondern auch den Wettstreit der Nationen. Die Bundesrepublik Deutschland soll nach dem Willen der Bundesregierung in dieser Entwicklung eine Vorreiterrolle einnehmen. Jedermann soll im Jahr 2025 über einen Zugang zu gigabitfähigen Infrastrukturen verfügen können. So soll der Weg in die „Gigabit-Gesellschaft“ bereitet werden.

Trotz mit hohen Summen ausgestatteter Breitbandförderprogramme der öffentlichen Hand ist derzeit nicht davon auszugehen, dass im Jahr 2025 flächendeckend gigabitfähige Infrastrukturen in Deutschland verfügbar sein werden. Es werden deshalb verschiedene Handlungsoptionen diskutiert, mit denen sich die Investitionsbedingungen verbessern lassen und der Weg in die Gigabit-Gesellschaft beschleunigt werden soll. Verbände, Ökonomen und Politiker überbieten sich mit Handlungsansätzen zur Aus-

$1 \mathrm{Zu}$ möglichen Szenarien, Nationaler IT-Gipfel, Konvergente Netze als Infrastruktur für die Gigabit-Gesellschaft, S. 2 ff.; Wernick/Fetzer/Gries u.a., Rahmenbedingungen für die Gigabitwelt 2025+, S. 17 ff.

2 https://www.wienerzeitung.at/nachrichten/wirtschaft/oesterreich/2021994-Infrastru ktur-ist-die-Mutter-der-Digitalisierung.html (zuletzt abgerufen am 1.7.2020).

3 CDU/CSU/SPD, Koalitionsvertrag 19. Legislaturperiode, Rn. 1607. 
baubeschleunigung, die vielseitige Anknüpfungspunkte zur Optimierung der Rahmenbedingungen wählen. ${ }^{4}$ Dabei werden Legislative und Exekutive mit einem komplexen ökonomischen und juristischen Spannungsfeld konfrontiert. Vor diesem Hintergrund scheint es nahezu undenkbar, sich dieser Herausforderung, geeignete Mittel und Wege zur Beschleunigung des Infrastrukturausbaus zu identifizieren, nur aus einer der genannten Perspektiven anzunähern. Dennoch kommen umfassende juristische Analysen häufig zu kurz, obwohl die tangierten rechtlichen Regelungsmaterien durch eine hohe Komplexität und Interdependenz geprägt sind. Die Modifikation einer kleinen Stellschraube in einem Rechtsregime, kann zu Friktionen in ganz anderen Regelungsmaterien führen. In der vorliegenden Untersuchung soll aus diesem Grund eine umfassende Einordnung verschiedener Handlungsoptionen erfolgen, die eine Optimierung des Rechtsrahmens zum Gegenstand haben. Nicht jede ökonomisch oder technisch sinnvolle Handlungsoption ist auch juristisch zulässig oder aufgrund der komplexen rechtlichen Anforderungen praktikabel umsetzbar. Vor diesem Hintergrund stellen sich drei zentrale Fragen, die im Rahmen der Untersuchung vertieft diskutiert werden sollen: Ist ein ökonomisch und technisch sinnvoller Handlungsansatz überhaupt rechtlich umsetzbar? Wie müsste eine konkrete Umsetzung aus rechtlicher Sicht erfolgen? Ist die Handlungsoption vor diesem Hintergrund überhaupt noch praktikabel?

Die Interdependenz von ökonomischen, technischen und juristischen Fragen im Telekommunikationssektor setzt die Klärung verschiedener Vorfragen voraus. Im folgenden zweiten Kapitel soll aus diesem Grund zunächst beleuchtet werden, in welche Phasen sich die Infrastrukturentwicklung unterscheiden lässt und was eine Gigabit-Gesellschaft auszeichnet. Entscheidend ist insbesondere der Ausbau der geeigneten Infrastruktur. Dabei können verschiedene Qualitäts- und Kapazitätsparameter, die über die reine Betrachtung der Datenübertragungsrate hinausgehen, herangezogen werden. Besonders hervorzuheben sind die Risiken und Unsicherheiten, die die Ausbaugeschwindigkeit der Unternehmen beeinflussen. Im Fo-

4 Vgl. hierzu statt vieler Kübling/Goldmedia/Enaux (Hrsg.), Rechtliche Herausforderungen bei der Schaffung von Anreizen für einen flächendeckenden Ausbau von Glasfaserinfrastrukturen; Wernick/Queder/Strube Martins u.a., Gigabitnetze für Deutschland; Wernick/Queder/Strube Martins u.a., Ansätze zur Glasfaser-ErschlieBung unterversorgter Gebiete; Deutscher Landkreistag, Flächendeckende Breitbandversorgung zu wirtschaftlichen Bedingungen sicherstellen; ZEW/Juconomy, Gutachten zur ökonomischen und rechtlichen Sinnhaftigkeit von nachfrageseitigen Förderungen im Ausbau moderner Breitbandnetze über "Voucher-Systeme". 
kus der Untersuchung stehen die Markt-, Wettbewerbs- und Regulierungsunsicherheiten.

In der weiteren Untersuchung sollen verschiedene Handlungsoptionen zur Optimierung der Bedingungen in den vorgenannten Themenkomplexen herausgearbeitet und auf ihre juristische Umsetzbarkeit hin untersucht werden. Im dritten Kapitel werden verschiedene Konzessionsmodelle vorgestellt. Hervorzuheben ist das Konzessionsmodell auf Basis der Vergabe exklusiver Wegerechte, in dem der Infrastrukturwettbewerb im Konzessionsgebiet reduziert wird und auf diese Weise die Investitionsbedingungen verbessert werden sollen. Die Umsetzbarkeit dieses Modells hängt von einer Vielzahl von Rechtsmaterien ab, die dafür unterschiedliche Steuerungswirkungen entfalten.

Das vierte Kapitel untersucht Wholesale-Only-Unternehmen, die ihre Tätigkeit auf die Errichtung und den Betrieb von Telekommunikationsinfrastrukturen beschränken. Zuweilen wird davon ausgegangen, dass die Trennung zwischen der Tätigkeit auf dem Vorleistungsmarkt und der Erbringung von Endkundendiensten einen positiven Effekt auf den Infrastrukturausbau haben kann. Es stellt sich die Frage, welche Optionen der Rechtsrahmen für die Entstehung von Wholesale-Only-Anbietern vorsieht und wie sich der unionale Rechtsrahmen in dieser Hinsicht verändert.

Ein weiterer positiver Effekt auf die Markt-, Wettbewerbs und Regulierungsunsicherheit kann von Kooperationen zwischen verschiedenen Unternehmen ausgehen, die im fünften Kapitel beleuchtet werden sollen. Von ihnen kann eine Gefahr für den Wettbewerb ausgehen, sodass - insbesondere, wenn Unternehmen mit beträchtlicher Marktmacht an der Kooperation beteiligt sind - die Anwendungsbereiche der kartellrechtlichen Vorgaben zur Fusionskontrolle, des Kartellverbots sowie des Regulierungsrechts eröffnet sind. Dies bringt die parallele Zuständigkeit von zwei Wettbewerbsbehörden mit sich. Es stellt sich die Frage, ob das Nebeneinander verschiedener Rechtsregime und Zuständigkeiten eher Vorteile oder Nachteile mit sich bringt. Durch den novellierten unionalen Rechtsrahmen werden zudem regulatorische Privilegierungsmöglichkeiten geschaffen, die einen positiven Effekt im Sinne einer Reduktion der Regulierungsunsicherheit haben könnten.

Kapitelübergreifend stellt sich dabei die Frage: Gibt es die optimale Handlungsoption für die Beschleunigung des Infrastrukturausbaus überhaupt? Oder ebnet vielmehr das Zusammenspiel verschiedener Einzelmaßnahmen den erfolgreichen Weg in die Gigabit-Gesellschaft? 


\section{Kapitel 2: Die Gigabit-Gesellschaft}

Das Telekommunikationsrecht stellt eine Materie dar, in der ökonomische und technische Fragen eine besondere Rolle spielen. Aus diesem Grund müssen vor einer vertieften juristischen Auseinandersetzung mit verschiedenen Handlungsoptionen zur Beschleunigung des Ausbaus hochleistungsfähiger Telekommunikationsinfrastrukturen zunächst einige Vorfragen geklärt werden. Hierzu soll untersucht werden, was unter dem Begriff der Gigabit-Gesellschaft zu verstehen ist und in welche Phasen sich die diesbezügliche Entwicklung unterteilen lässt (dazu A.). Aus technischer Perspektive ist zu untersuchen, welche Anforderungen die Gigabit-Gesellschaft an ihre digitale Infrastruktur stellen wird und mit welchen Übertragungstechnologien diese Anforderungen erfüllt werden können (dazu B.). Aus ökonomischer Perspektive stellt sich die Frage, wie sich der aktuelle Ausbaustand darstellt und mit welchen Risiken und Kostentreibern die Unternehmen im Rahmen von Infrastrukturausbauvorhaben konfrontiert werden (dazu C.).

\section{A. Die Entwicklung der Gigabit-Gesellschaft}

In der folgenden Untersuchung sollen angebotsseitige Handlungsoptionen auf dem Weg in die Gigabit-Gesellschaft dargestellt und aus juristischer Perspektive beleuchtet werden. Hierbei handelt es sich um Maßnahmen, die Telekommunikationsunternehmen adressieren, die gigabitfähige Infrastrukturen ausbauen. Kein vertiefter Gegenstand der Untersuchung sind hingegen solche Maßnahmen, welche die Nachfragestimulation zum Gegenstand haben. Bereits aus dem Titel dieser Untersuchung leiten sich zwei ihrer grundlegenden Prämissen ab. Zum einen ist das Ziel der darzustellenden Entwicklung die „Gigabit-Gesellschaft“ (dazu I.). Zum anderen ist der Weg in diese hoch digitalisierte Gesellschaft noch nicht abgeschlossen, sondern schreitet vielmehr stetig voran (dazu II.). 


\section{Der Begriff der Gigabit-Gesellschaft}

Um den Unterschied zwischen der „Digitalisierung der Gesellschaft“ und einer „Gigabit-Gesellschaft“ zu verdeutlichen, soll der Ablauf der Entwicklung einer Infrastruktur beleuchtet werden (dazu 1.). Anknüpfend daran lassen sich die Charakteristika der "Gigabit-Gesellschaft" herausarbeiten (dazu 2.).

\section{Der Ablauf der Infrastrukturentwicklung}

Der Weg in die Gigabit-Gesellschaft ist ein Prozess, der noch nicht abgeschlossen ist. Das Fraunhofer-Institut für offene Kommunikationssysteme FOKUS (Fraunhofer FOKUS) differenziert zwischen der Innovation, dem Wachstum und der Reife als Entwicklungsstufen von Infrastrukturen. ${ }^{5}$ Am Beginn ihrer Entwicklung steht die Innovation, während derer für einen Anwender eine Infrastruktur verfügbar wird und sich für ihn als nützlich erweist. ${ }^{6}$ Hierauf folgt die Phase des Wachstums, in der durch die Verfügbarkeit immer neuer Anwendungen ein immer größerer Nutzen durch die neue Infrastruktur erzielt werden kann. ${ }^{7}$ Hieran knüpft eine Reifephase an, in der die Gesellschaft von der Infrastruktur vollständig durchdrungen wird. In Folge dessen entsteht eine Abhängigkeit von der Infrastruktur. Zugleich steigen auch die Anforderungen an sie. ${ }^{8}$

Infrastrukturentwicklungen, die bereits die Reifephase erreicht haben, sind beispielsweise der Straßenverkehr, ${ }^{9}$ die Wasserversorgung oder die Elektrifizierung. Im Hinblick auf den Gegenstand dieser Untersuchung befinden wir uns derzeit in der Entwicklungsstufe des Wachstums, die als „Digitalisierung der Gesellschaft" ${ }^{\text {"10 }}$ bezeichnet werden kann.

5 Fraunhofer FOKUS, Netzinfrastrukturen für die Gigabitgesellschaft, S. 10, Abbildung 1.

6 Fraunhofer FOKUS, Netzinfrastrukturen für die Gigabitgesellschaft, S. 10.

7 Fraunhofer FOKUS, Netzinfrastrukturen für die Gigabitgesellschaft, S. 10.

8 Fraunhofer FOKUS, Netzinfrastrukturen für die Gigabitgesellschaft, S. 10.

9 Fraunhofer FOKUS, Netzinfrastrukturen für die Gigabitgesellschaft, S. 10.

10 Fraunhofer FOKUS, Netzinfrastrukturen für die Gigabitgesellschaft, S. 10. 


\section{Die Entstehung der „Gigabit-Gesellschaft“}

Die "Gigabit-Gesellschaft“ entsteht in Folge der „Digitalisierung der Gesellschaft". Das Bundesministerium für Verkehr und digitale Infrastruktur (BMVI) hat das Institut Fraunhofer FOKUS mit der Untersuchung wesentlicher Parameter der Strategie zum Ausbau hochleistungsfähiger digitaler Infrastrukturen und ihrer Implementierung in gesellschaftliche und wirtschaftliche Prozesse beauftragt. Im Rahmen dieser Studie entwickelte das Institut eine Definition der „Gigabit-Gesellschaft“, die das Bundesministerium für Verkehr und digitale Infrastruktur nutzt und auch dieser Untersuchung zu Grunde liegen soll.

Bei der Begriffsdefinition muss zwischen der Anwender- und der Technikperspektive differenziert werden. ${ }^{11}$ Danach ist aus der Perspektive der Anwender die Gigabit-Gesellschaft „eine fortgeschrittene Informationsgesellschaft, die vollständig von Informations- und Kommunikationstechnik durchdrungen ist, so dass die Nutzer keine technischen Beschränkungen erfahren und vernetzte Anwendungen ohne Restriktionen möglich sind." ${ }^{12}$ Dies lässt sich am Beispiel der fortschreitenden Tendenz zum automatisierten Fahren verdeutlichen, in der Kraftfahrzeuge nicht nur untereinander, sondern auch mit ihrer Umwelt (etwa mit Ampelanlagen) kommunizieren. ${ }^{13}$

Diese Anforderungen müssen durch Infrastrukturen bewältigt werden, die verschiedene Bedürfnisse miteinander in Einklang bringen. ${ }^{14}$ Aus der Perspektive der Technik ermöglichen die Netze der Gigabit-Gesellschaft „eine bedarfsgerechte Unterstützung von Anwendungen. ${ }^{15}$ Hierzu werden heterogene informations- und kommunikationstechnische Infrastrukturen umfassend und optimiert genutzt, um physische Beschränkungen überwinden zu können." ${ }^{16}$ Eine große Herausforderung stellt dabei die Interoperabilität der verschiedenen Kommunikationsprozesse dar. Letztlich

11 Fraunhofer FOKUS, Netzinfrastrukturen für die Gigabitgesellschaft, S. 11.

12 Fraunhofer FOKUS, Netzinfrastrukturen für die Gigabitgesellschaft, S. 12; im Ergebnis auch Lengsfeld, Digital Era Framework, S. 239.

13 Umfassend hierzu Sackmann, Datenschutz bei der Digitalisierung der Mobilität, S. 38 ff. m. w. N.; illustrative Animationen sind abrufbar unter: https://plattformdigitale-netze.de/fokusgruppe-aufbruch-gigabit-gesellschaft/ (zuletzt abgerufen am 1.7.2020).

14 https://www.bmvi.de/DE/Themen/Digitales/Digitale-Gesellschaft/Gigabitgesellsc haft/gigabitgesellschaft.html (zuletzt abgerufen am 1.7.2020).

15 Wernick/Fetzer/Gries u.a., Rahmenbedingungen für die Gigabitwelt 2025+, S. 32 ff.

16 Fraunhofer FOKUS, Netzinfrastrukturen für die Gigabitgesellschaft, S. 12. 
handelt es sich bei der Gigabit-Gesellschaft um eine Vision, deren tatsächliche Spezifika derzeit nur eingeschränkt vorhersehbar sind. ${ }^{17}$ Je weiter der Blick in die Zukunft geht, desto visionärer werden die Prognosen. Es handelt sich demnach in gewisser Weise um eine „Wette auf die Zukunft“. ${ }^{18}$

Die "Gigabit-Gesellschaft“ ist demnach die Zukunftsvision einer vollständig vernetzten und digitalisierten Gesellschaft. ${ }^{19}$ Dabei müssen die vielfältigen Anwenderbedürfnisse durch entsprechende Optimierung und Weiterentwicklung der Technik befriedigt werden.

\section{Der Weg in die Gigabit-Gesellschaft}

Eine Gigabit-Gesellschaft kann mithin nur auf Basis einer hochleistungsfähigen digitalen Infrastruktur entstehen. Nach einem Konzept der Netzallianz „Digitales Deutschland“, die vom Bundesministerium für Verkehr und digitale Infrastruktur geleitet wird, sollen gigabitfähige Infrastrukturen in vier Phasen ausgebaut werden. ${ }^{20}$ In der ersten Phase sollte bis zum Ende des Jahres 2018 eine flächendeckende Versorgung aller Haushalte mit einer Übertragungsrate von mindestens 50 Megabit pro Sekunde erreicht werden. Dieses Ziel deckt sich mit dem Koalitionsvertrag von CDU, CSU und SPD für die 18. Wahlperiode des Bundestags von 2013 bis 2017. ${ }^{21}$ Neubaugebiete sollten und sollen weiterhin direkt mit Glasfaseranschlüssen versorgt werden. ${ }^{22}$ Bis Mitte des Jahres 2018 wurden jedoch, wie die Bundesregierung auf eine Kleine Anfrage der FDP-Bundestagsfraktion mitteilte, lediglich 82,9 Prozent der Haushalte mit der Zielbandbreite versorgt, sodass das Ziel verfehlt wurde. ${ }^{23}$

In der zweiten Phase bis Ende des Jahres 2019 sollten alle bislang unterversorgten Gewerbegebiete mit Glasfaser versorgt werden. Neue Gewerbegebiete sollten und sollen weiterhin direkt mit Glasfaseranschlüssen ver-

17 https://plattform-digitale-netze.de/fokusgruppe-aufbruch-gigabit-gesellschaft/ (zuletzt abgerufen am 1.7.2020).

18 Kübling, WuW 2019, S. 555, 556.

19 Umfassend zur Gesellschaft der Zukunft Kollmann/Schmidt, Deutschland 4.0.

20 Die Netzallianz besteht nach dem Willen der Großen Koalition weiter fort, CDU/CSU/SPD, Koalitionsvertrag 19. Legislaturperiode, Rn. 1656 f.; Heymann/ Körner, Digitale Infrastruktur, S. 5.

21 CDU/CSU/SPD, Koalitionsvertrag 18. Legislaturperiode, S. 35.

22 BMVI, Zukunftsoffensive Gigabit-Deutschland, S. 9.

23 BT-Drs. 19/5762, S. 1. 
sorgt werden. ${ }^{24}$ Auch dieses Ziel wurde, wie sich aus der Antwort der Bundesregierung auf eine Kleine Anfrage der FDP-Bundestagsfraktion ergibt, nicht erreicht. ${ }^{25} \mathrm{Im}$ Rahmen des Koalitionsvertrages für die 19. Legislaturperiode einigte sich die Große Koalition aus CDU, CSU und SPD zudem darauf, dass insbesondere Schulen, Gewerbegebiete, soziale Einrichtungen und Krankenhäuser bis 2022 mit Glasfaser versorgt werden sollen. ${ }^{26}$

In der dritten Phase sollten bis Ende des Jahres 2020 die „Voraussetzungen für einen flächendeckenden 5G-Rollout“ geschaffen werden. ${ }^{27}$ Das Ziel ist vage formuliert, sodass sich nicht genau verifizieren lässt, ob es bis Ende des Jahres 2020 realisiert worden ist. Die 5G-Frequenzen wurden im Jahr 2019 versteigert. Allerdings sind einige der versteigerten Frequenzspektren erst im Laufe der kommenden Jahre nutzbar. Um das Ziel der Flächendeckung zu erreichen, wurde die Versteigerung mit hohen Versorgungsauflagen verknüpft. ${ }^{28}$ Diesbezüglich ist jedoch festzustellen, dass die Versorgungsauflagen auch mithilfe anderer Standardisierungen erreicht werden können und nicht zwangsläufig ein Rückgriff auf den 5G-Standard erforderlich ist. Derzeit bereitet das Bundesministerium für Verkehr und digitale Infrastruktur zudem ein Förderprogramm vor, um einen flächendeckenden Ausbau mit 5G-Infrastrukturen sicherzustellen. Dieses Programm muss jedoch noch ausgestaltet und im Anschluss im Rahmen eines EU-beihilfenrechtlichen Verfahrens von der Kommission notifiziert werden, ${ }^{29}$ sodass nicht gesichert ist, dass Fördergelder kurzfristig ausgezahlt werden können. Je nachdem wie hoch man die Anforderungen an das Ziel setzt, desto eher kommt man zu dem Schluss, dass auch dieses Ziel nicht erreicht wird.

In der vierten Phase soll bis Ende 2025 eine flächendeckende „gigabitfähige konvergente Infrastruktur in Deutschland" verfügbar sein. ${ }^{30} \mathrm{Zu}$ diesem Zeitpunkt sollen nach dem Willen der Bundesregierung alle Bürger einen „rechtlich abgesicherten Anspruch auf schnelles Internet“ geltend

24 BMVI, Zukunftsoffensive Gigabit-Deutschland, S. 9.

25 BT-Drs. 19/11357, S. 3.

26 CDU/CSU/SPD, Koalitionsvertrag 19. Legislaturperiode, Rn. 1629 f.; umfassend zu den telekommunikationspolitischen Zielen des Koalitionsvertrages Sickmann/ Neumann, Wirtschaftsdienst 2018, S. 333 ff.

27 BMVI, Zukunftsoffensive Gigabit-Deutschland, S. 9.

28 BNetzA, Beschl. v. 26. 11. 2018, Az. BK 1-17/001, Rn. $200 \mathrm{ff}$.

29 Vgl. hierzu statt vieler Kühling/Rüchardt, in: Streinz (Hrsg.), EUV/AEUV, Art. 108 AEUV, Rn. 14.

30 BMVI, Zukunftsoffensive Gigabit-Deutschland, S. 9; so auch CDU/CSU/SPD, Koalitionsvertrag 19. Legislaturperiode, Rn. $1626 \mathrm{f}$. 
machen können. ${ }^{31}$ Dieser wird derzeit ausgestaltet. ${ }^{32}$ Nach bisherigem Planungsstand soll eine Umsetzung im Rahmen des Universaldienstmechanismus erfolgen ( $\$ \$ 155 \mathrm{ff}$. TKG-RefE). ${ }^{33}$

Insgesamt lässt sich demnach feststellen, dass die Bundesregierung den Großteil ihrer Ziele im Kontext des Ausbaus digitaler Infrastrukturen verfehlt. Zuzugeben ist, dass es sich um politische Absichtserklärungen handelt, die nicht rechtsverbindlich sind. Die Konsequenzen für die Verfehlung der Ziele können lediglich in Form von Wahlergebnissen sichtbar werden. Allerdings ist für Investitionsentscheidungen in der Wirtschaft die Glaubwürdigkeit der Politik von entscheidender Bedeutung. Dies gilt insbesondere für langfristig kapitalbindende Investitionen, wie den Infrastrukturausbau. Werden politische Ziele kontinuierlich verfehlt, erzeugt dies eine Unsicherheit, die zu schlechteren Investitionsbedingungen führt und dazu beiträgt, dass sich der Ausbau weiter verzögert. Es handelt sich um einen Teufelskreis, der lediglich durch realistische Ausbauziele durchbrochen werden kann. ${ }^{34}$ Diese wiederum reduzieren Unsicherheiten, verbessern das Investitionsklima und können damit zu einer Beschleunigung des Ausbaus gigabitfähiger Infrastrukturen beitragen. Es würden sich insoweit flexiblere Ziele anbieten, die sich eher an der Entwicklung der Nachfrage orientieren und keine festen Zeiträume formulieren.

\section{Zwischenergebnis}

Die Infrastrukturentwicklung lässt sich in die Phasen der Innovation, des Wachstums und der Reife unterteilen. Der Ausbau von Telekommunikationsinfrastrukturen befindet sich derzeit in der Wachstumsphase, die auch

$31 \mathrm{CDU} / \mathrm{CSU} / \mathrm{SPD}$, Koalitionsvertrag 19. Legislaturperiode, Rn. $1661 \mathrm{f}$.

32 CDU/CSU/SPD, Koalitionsvertrag 19. Legislaturperiode, Rn. 1661 f.; hierzu umfassend und mit konkreten Ausgestaltungsvorschlägen Kühling/Toros, Rechtliche Rahmenbedingungen für die Schaffung von Anreizen für einen flächendeckenden Ausbau von Glasfaserinfrastrukturen, S. 56 ff.; hieran anknüpfend Kübling/ Toros, K\&R 2019, S. 692 ff.; Neumann, Optionen für die Ausgestaltung eines rechtlich abgesicherten Anspruchs auf schnelles Internet aus telekommunikationsrechtlicher Sicht, S. 1 ff.; Neumann/Sickmann, N\&R Beilage 1/2018, S. 1 ff.

33 Referentenentwurf des Telekommunikationsmodernisierungsgesetzes (TK-MoG), abrufbar unter: https://www.bmwi.de/Redaktion/DE/Downloads/Gesetz/telekom munikationsmodernisierungsgesetz-referentenentwurf-20201612.pdf?_blob=pub licationFile $\& v=8$ (zuletzt abgerufen am 12.1.2021).

34 Weiterführend hierzu Monopolkommission, 11. Sektorgutachten Telekommunikation (2019), Rn. $6 \mathrm{ff}$. 
als Phase der „Digitalisierung der Gesellschaft“ bezeichnet werden kann. In der Gesellschaft wird der Vorteil einer Digitalisierung immer bewusster wahrgenommen und es entstehen immer mehr digitale Applikationen. Die "Gigabit-Gesellschaft" bezeichnet die Reifephase, die sich der Digitalisierung der Gesellschaft anschließt. In ihr sind Menschen und Maschinen untereinander und miteinander vollständig vernetzt und alle Prozesse vollständig digitalisiert. Dabei ist die Gesellschaft davon abhängig, dass digitale Infrastrukturen und Dienste verfügbar sind. Vergleichbare Entwicklungen konnten etwa im Hinblick auf Mobilität und Elektrizität bereits beobachtet werden. Eine vollständige Vernetzung aus der Anwenderperspektive setzt aus der Technikperspektive eine flächendeckende, konvergente digitale Infrastruktur voraus. Diese Infrastruktur stellt damit die Basis für die weitergehende Infrastrukturentwicklung dar. Der Ausbauplan des Bundesministeriums für Verkehr und digitale Infrastruktur sieht einen schrittweisen Infrastrukturausbau in vier Phasen vor. Die Zwischenziele wurden bislang zum Großteil nicht erreicht. Mit dem Setzen realistischerer Ziele, könnten die Investitionsbedingungen verbessert werden. Bis zum Jahr 2025 soll eine flächendeckende gigabitfähige Infrastruktur vorhanden sein. Dieses Ziel soll von einem rechtlich abgesicherten Anspruch auf „schnelles Internet" flankiert werden, der derzeit ausgestaltet wird.

\section{B. Die Anforderungen an die digitale Infrastruktur der Gigabit-Gesellschaft}

Die Entstehung einer Gigabit-Gesellschaft setzt eine hochleistungsfähige digitale Infrastruktur voraus. Der Klärung bedürfen dabei die Qualitätsund Kapazitätsparameter der digitalen Infrastruktur (dazu I.) und die hierzu geeigneten Datenübertragungstechnologien (dazu II.).

\section{Die Qualitäts- und Kapazitätsparameter}

Als Orientierungspunkt für die Qualitäts- und Kapazitätsanforderungen der Gigabit-Gesellschaft kann die Legaldefinition des „Netzes mit sehr hoher Kapazität" nach Art. 2 Nr. 2 des europäischen Kodex für die elektronische Kommunikation (EKEK) ${ }^{35}$ herangezogen werden. Daraus geht her-

35 Richtlinie (EU) 2018/1972 des Europäischen Parlaments und des Rates vom 11. Dezember 2018 über den europäischen Kodex für die elektronische Kommunikation, Abl. L 321/36 v. 17.12.2018. 
vor, welche Parameter für eine hochleistungsfähige Infrastruktur insbesondere entscheidend sind. Unter einem „Netz mit sehr hoher Kapazität“ im Sinne des Art. 2 Nr. 2 EKEK ist „entweder ein elektronisches Kommunikationsnetz, das komplett aus Glasfaserkomponenten zumindest bis zum Verteilerpunkt am Ort der Nutzung besteht, oder ein elektronisches Kommunikationsnetz, das zu üblichen Spitzenlastzeiten eine ähnliche Netzleistung in Bezug auf die verfügbare Downlink- und Uplink-Bandbreite, Ausfallsicherheit, fehlerbezogene Parameter, Latenz und Latenzschwankung bieten kann [, zu verstehen]; die Netzleistung kann als vergleichbar gelten, unabhängig davon, ob der Endnutzer Schwankungen feststellt, die auf die verschiedenen inhärenten Merkmale des Mediums zurückzuführen sind, über das das Netz letztlich mit dem Netzabschlusspunkt verbunden ist.“

Der Unionsgesetzgeber verzichtet mit der Legaldefinition auf die Normierung harter Schwellenwerte wie etwa konkreter Übertragungsraten. Als Referenz für andere Übertragungstechnologien sieht er eine Glasfaserinfrastruktur vor, die möglichst nahe an den Endnutzer heranreicht. ${ }^{36}$ Diese Versorgung wird als „fibre to the home or buildung“ (FTTH/B) bezeichnet. Bevor auf einzelne Technologien eingegangen werden kann, sollen die wesentlichen Kriterien der Definition erläutert werden. Häufig wird die Diskussion hierüber auf die Datenübertragungsrate reduziert (dazu 1.). Je weiter die Entwicklung zur Gigabit-Gesellschaft jedoch voranschreitet, desto relevanter werden auch die anderen Parameter der Definition (dazu 2.). Zudem müssen bei der Identifikation der geeigneten Infrastrukturen auch solche Kriterien Berücksichtigung finden, die nicht explizit im Rechtsrahmen verankert sind (dazu 3.).

\section{Die Datenübertragungskapazität}

In der öffentlichen Diskussion erscheint die Datenübertragungskapazität einer Infrastruktur als der wichtigste Parameter. ${ }^{37}$ Die Leistungsfähigkeit eines Telekommunikationsnetzes wird insbesondere mithilfe der Band-

36 Fetzer, Impulsstudie Telekommunikationsregulierung 4.0, S. 10.

$37 \mathrm{Vgl}$. hierzu etwa https://www.sueddeutsche.de/wissen/technik-schnelles-internetwelches-tempo-fuer-welche-anwender-dpa.urn-newsml-dpa-com-20090101-180115 -99-643221 (zuletzt abgerufen am 1.7.2020); https://www.zeit.de/hamburg/2019-0 6/breitbandausbau-schnelles-internet-hamburg-versorgungsluecken-bernd-beckert (zuletzt abgerufen am 1.7.2020). 
breite bestimmt. ${ }^{38}$ Dabei wird zwischen der Breite des Frequenzbandes und der Datenübertragungskapazität differenziert. ${ }^{39}$ Für die folgende Untersuchung ist die letztere Größe relevant. Die Datenübertragungsrate wird in der Einheit Bit angegeben. ${ }^{40} \mathrm{Je}$ nach Menge der übertragenen Bit pro Zeiteinheit, erfolgt die Angabe in Kilobit (Kbit/s), Megabit (Mbit/s) oder Gigabit (Gbit/s) pro Sekunde. Die Downlink-Bandbreite bezeichnet die Geschwindigkeit, mit der ein Datenpaket heruntergeladen kann, während die Uplink-Bandbreite den umgekehrten Vorgang betrifft. Je größer die zu übertragendenden Datenmengen und die Geschwindigkeitsanforderungen sind, desto höher muss die Bandbreite sein. Die real übertragene Datenmenge wird auch als Durchsatz bezeichnet.

\section{Alternative Kriterien der Legaldefinition des EKEK}

Der unionale Gesetzgeber erwähnt in der Legaldefinition auch weitere Kriterien, die für die Bestimmung der Qualität und Kapazität einer Übertragungstechnologie herangezogen werden können. Hierzu zählen die Ausfallsicherheit, die fehlerbezogenen Parameter sowie die Latenz und ihre Schwankungen. ${ }^{41}$

Unter der Ausfallsicherheit ist die technische Verfügbarkeit eines Dienstes zu verstehen. Fällt die Telekommunikationsinfrastruktur aus, können die hierauf basierenden Dienste nicht genutzt werden. Je weiter die Digitalisierung der Gesellschaft voranschreitet und je höher der Vernetzungsgrad ist, desto abhängiger sind Wirtschaft und Gesellschaft von der technischen Verfügbarkeit von Telekommunikation. Demnach ist die Ausfallsicherheit auch für die Infrastruktur der Gigabit-Gesellschaft ein wesentlicher Qualitätsparameter.

Auch fehlerbezogene Parameter sind von entscheidender Bedeutung für die Qualität einer Übertragungstechnologie. Hierunter sind fehlerhafte Signalübertragungen - wie etwa der Verlust von Datenpaketen ${ }^{42}-$ zu fassen.

38 Bromen, in: Auer-Reinsdorff/Conrad (Hrsg.), Handbuch IT- und Datenschutzrecht, $\mathbb{S} 4$, Rn. 10.

39 Bromen, in: Auer-Reinsdorff/Conrad (Hrsg.), Handbuch IT- und Datenschutzrecht, $\mathbb{S} 4$, Rn. 10.

40 Brückner, Das globale Netz, S. 12 f.

$41 \mathrm{Zu}$ den Einzelheiten der Qualitätsmessung Keller, Breitbandkabel und Zugangsnetze, S. 439 ff.; im Überblick zu den Qualitätskriterien Nationaler IT-Gipfel, Konvergente Netze als Infrastruktur für die Gigabit-Gesellschaft, S. 5.

42 Keller, Breitbandkabel und Zugangsnetze, S. 455. 
Diese können auf unterschiedlichen Ursachen beruhen, führen aber im Ergebnis dazu, dass die Signale falsch übermittelt werden. ${ }^{43}$ Je sensibler die betreffende Signalübertragung ist und je höher die Qualitätsanforderungen sind, desto problematischer sind hohe Fehlerraten in der Datenübertragung.

Die Latenz und diesbezügliche Schwankungen sind weitere wichtige Faktoren in der Analyse der Qualität einer bestimmten Übertragungstechnologie. Die Latenz betrifft die Verzögerung zwischen dem Beginn und dem Abschluss einer Datenübertragung und wird auch als „Delay“ bezeichnet. ${ }^{44}$ Latenzschwankungen sind die Abweichungen von der Regelverzögerung und werden auch als "Jitter" bezeichnet. ${ }^{45}$ Je länger die Latenzzeiträume sind und je größer die Latenzschwankungen ausfallen, desto problematischer ist dies für Echtzeitapplikationen. Hohe Werte fallen etwa bei der Nutzung von Videotelefoniediensten auf, wo sie durch ein „Ruckeln“ der Bilder offenbar werden. Je echtzeitkritischer Dienste werden und je schädlicher Verzögerungsschwankungen sind, desto niedriger müssen Latenzen und Latenzschwankungen sein.

\section{Relevante Kriterien ohne Veranlagung im unionalen Rechtsrahmen}

Neben den explizit in Art. 2 Nr. 2 EKEK aufgezählten Vergleichskriterien, sind weitere Faktoren bei der Auswahl der Infrastruktur für die GigabitGesellschaft zu beachten. Eine Infrastruktur wird nur dann ausgebaut, wenn sie im Vergleich zu anderen Infrastrukturen wirtschaftlich im Ausbau und effizient im Betrieb ist. ${ }^{46}$ Vorteilhaft ist es deswegen, wenn für die Nutzung einer Übertragungstechnologie auf Bestandsinfrastruktur zurückgegriffen werden kann und ihre Wirtschaftlichkeit auf diese Weise schneller sichergestellt ist. Aufgrund des zunehmenden Datenverkehrs ist in der Gigabit-Gesellschaft zudem relevant, wie skalierbar eine Technologie ist, das heißt, wie viele Nutzer oder Endgeräte über die Technologie miteinander vernetzt werden können. ${ }^{47}$ Stößt eine Infrastruktur schnell an ihre Kapazitätsgrenzen, ist sie nicht für den weitergehenden Infrastrukturausbau

43 Meinel/Sack, Digitale Kommunikation, S. 129.

44 Keller, Breitbandkabel und Zugangsnetze, S. 437; Meinel/Sack, Digitale Kommunikation, S. 128.

45 Weiterführend Keller, Breitbandkabel und Zugangsnetze, S. 455; Meinel/Sack, Digitale Kommunikation, S. 129.

46 Fraunhofer FOKUS, Netzinfrastrukturen für die Gigabitgesellschaft, S. 77.

47 Fraunhofer FOKUS, Netzinfrastrukturen für die Gigabitgesellschaft, S. 77. 
geeignet. Aufgrund des hohen Innovationsgrades im Bereich der Digitalisierung ist auch die Flexibilität einer Infrastruktur relevant. ${ }^{48}$ In dieser Hinsicht ist zu beobachten, wie flexibel eine Infrastruktur im Hinblick auf sich verändernde Rahmenbedingungen oder Nutzeranforderungen angepasst werden kann.

\section{Zwischenergebnis}

Die Qualität und Leistungsfähigkeit einer Übertragungstechnologie lässt sich anhand verschiedener Kriterien ermitteln. Häufig wird die Diskussion hierüber auf die Datenübertragungskapazität einer Technologie reduziert, die auch im unionalen Rechtsrahmen als Leitkriterium verankert ist. Eine isolierte Betrachtung dieses Parameters reicht jedoch nicht aus, um die optimale Infrastruktur für die Gigabit-Gesellschaft zu identifizieren. Je höher die Qualitätsanforderungen an eine Datenübertragung werden, also je weiter der Weg zur Gigabit-Gesellschaft beschritten wird, desto stärker treten weitere Qualitätskriterien in den Mittelpunkt. Hierzu zählen Ausfallsicherheit, fehlerbezogene Parameter sowie Latenz und Latenzschwankungen, die ebenfalls im unionalen Rechtsrahmen verankert sind. Hinzutreten weitere Kriterien wie Skalierbarkeit, Wirtschaftlichkeit, Effizienz im Betrieb und Flexibilität, die bei der Auswahl der Infrastruktur für die Gigabit-Gesellschaft zu berücksichtigen sind. In Zukunft sollte die Diskussion um die geeignete Infrastruktur aus diesem Grund vielschichtiger als bisher geführt werden.

\section{Die Datenübertragungstechnologien für die Gigabit-Gesellschaft}

Anhand der genannten Kriterien lässt sich ermitteln, welche Übertragungstechnologien übergangsweise oder sogar langfristig auf dem Weg zur Gigabit-Gesellschaft genutzt werden können. Als Referenztechnologie für ein „Netz mit sehr hoher Kapazität“ sieht der unionale Gesetzgeber, wie bereits dargelegt, eine FTTB/H-Glasfaserinfrastruktur an. Auch diese Untersuchung geht davon aus, dass mithilfe dieser Übertragungstechnologie die Anforderungen der Gigabit-Gesellschaft erfüllt werden können (dazu 1.). Es stellt sich jedoch die Frage, ob diese - zumindest teilweise und

48 Fraunhofer FOKUS, Netzinfrastrukturen für die Gigabitgesellschaft, S. 77. 
temporär - auch mithilfe alternativer Datenübertragungstechnologien befriedigt werden können (dazu 2.).

\section{Glasfaser als Datenübertragungstechnologie}

Betrachtet man die Qualitäts- und Kapazitätskriterien glasfaserbasierter Telekommunikationsinfrastrukturen, ist $\mathrm{zu}$ beachten, dass sich dabei verschiedene Ausbaustufen erheblich voneinander unterscheiden. ${ }^{49}$ Abgegrenzt werden sie anhand der Frage, wie weit die Glasfaserinfrastruktur bis zum Endkunden reicht. Grundsätzlich werden alle glasfaserbasierten Infrastrukturen mit der Abkürzung „FTTX“ (fibre to the $\mathrm{x}$ ) bezeichnet, wobei der Buchstabe X flexibel ausgetauscht werden kann und anzeigt, wie weit die Glasfaserinfrastruktur ausgebaut ist. Wird etwa lediglich ein Kabelverzweiger erschlossen, der als grauer Kasten am Straßenrand steht, handelt es sich um einen FTTC-Anschluss (fibre to the curb/cabinet). ${ }^{50}$

Für die folgenden Ausführungen sind im Einklang mit dem unionalen Rechtsrahmen lediglich Teilnehmeranschlussleitungen relevant, die bis zum Haus oder der einzelnen Wohnung mithilfe der Glasfasertechnologie realisiert werden $(\mathrm{FTTB} / \mathrm{H})$. Je weiter der Glasfaserausbau in Richtung des Endkunden vorangetrieben wird, desto besser wird die Datenübertragung.

Daten werden bei der Nutzung von Telekommunikationsinfrastruktur aus Glasfaser mithilfe optischer Signale übertragen. ${ }^{51}$ Dabei können symmetrisch, also im Up- und Download zugleich, Datenübertragungsraten im Gigabit- und Terabitbereich erzielt werden. ${ }^{52}$ Auf die Einzelheiten der Qualitätskriterien kann in einer juristischen Untersuchung nicht detailliert eingegangen werden. ${ }^{53}$ Auf Basis der technischen Literatur lässt sich je-

49 Grundlegend zum technischen Aufbau eines Glasfasernetzes Keller, Breitbandkabel und Zugangsnetze, S. $237 \mathrm{ff}$.

50 Hierzu im Überblick statt vieler Kühling/Schall/Biendl, Telekommunikationsrecht, Rn. 107, Abbildung 8.

51 Fornefeld/Rokus, Technologische Grundlagen zur Breitband-Versorgung im ländlichen Raum, S. 24.

52 Bary, Kommunaler Netzausbau in der Telekommunikation, S. 15; Fornefeld/ Rokus, Technologische Grundlagen zur Breitband-Versorgung im ländlichen Raum, S. 25; Fraunhofer FOKUS, Netzinfrastrukturen für die Gigabitgesellschaft, S. 81; Reents, Ausbau und Finanzierung einer flächendeckenden Breitbandversorgung in Deutschland, S. 21.

53 Umfassend hierzu Nationaler IT-Gipfel, Konvergente Netze als Infrastruktur für die Gigabit-Gesellschaft, S.14f.; Keller, Breitbandkabel und Zugangsnetze, S. $141 \mathrm{ff}$. 
doch feststellen, dass die FTTB/H-Technologie derzeit über die höchste Leistungsfähigkeit unter den gängigen Übertragungstechnologien verfügt. ${ }^{54}$ Sie ist durch eine hohe Ausfallsicherheit geprägt und weist im Rahmen der Signalübertragung geringe fehlerbezogene Parameter ${ }^{55}$ auf. Aufgrund der Übertragung optischer Signale ergeben sich geringe Latenzen mit lediglich marginalen Schwankungen. ${ }^{56}$ Glasfaserkabel können aufgrund physikalischer Effekte Signale über lange Strecken ohne eine spürbare Leistungseinschränkung übertragen ${ }^{57}$ und werden deswegen bereits umfassend im Backbone-Netz eingesetzt, das als Hintergrundnetz fungiert und die Datenübertragung über lange Strecken ermöglicht. Die zu übertragenden Datenmengen werden bei fortschreitender Digitalisierung der Gesellschaft steigen. In dieser Hinsicht ist jedoch derzeit keine Kapazitätsgrenze für glasfaserbasierte Infrastrukturen zu erwarten. ${ }^{58} \mathrm{Je}$ individueller die Netzarchitektur für den einzelnen Teilnehmer ist, je weniger die Infrastruktur also parallel durch unterschiedliche Teilnehmer genutzt wird, ${ }^{59}$ desto leistungsfähiger ist die Infrastruktur. ${ }^{60}$

\section{Alternative Datenübertragungstechnologien}

Neben FTTB/H-Infrastrukturen können Telekommunikationsdienste auch mithilfe anderer Infrastrukturen erbracht werden. Für die Festnetzversorgung wird dabei hauptsächlich auf Kupfer- (dazu a.) oder Koaxialkabel (dazu b.) zurückgegriffen. Alternativ können die Signale auch mithilfe von Funktechnologien (dazu c.) übertragen werden.

54 Fraunhofer FOKUS, Netzinfrastrukturen für die Gigabitgesellschaft, S. 81; Wernick/Queder/Strube Martins u.a., Gigabitnetze für Deutschland, S. 24.

55 Detaillierte Werte stellen Meinel/Sack, Digitale Kommunikation, S. 118, Tabelle 3.3 zusammen.

56 Ausführlich zu den technischen Spezifika Fraunhofer FOKUS, Netzinfrastrukturen für die Gigabitgesellschaft, S. 89.

57 Anders ist dies bei Kupfertechnologien, siehe hierzu im Überblick Fraunhofer FOKUS, Netzinfrastrukturen für die Gigabitgesellschaft, S. 79, Abbildung 27.

58 Bary, Kommunaler Netzausbau in der Telekommunikation, S. 15.

59 In der Fachterminologie eine Infrastruktur bei der Nutzung durch mehrere Teilnehmer auch als „shared medium“ bezeichnet.

60 Weiterführend zu den verschiedenen Möglichkeiten der Netzarchitektur Fraunhofer FOKUS, Netzinfrastrukturen für die Gigabitgesellschaft, S. 81. 
a) Datenübertragungstechnologien auf Basis einer Kupferdoppelader

Die ursprüngliche Telekommunikationsinfrastruktur in Deutschland basiert auf einer sogenannten „Kupferdoppelader“, welche die Signale mittels elektronischer Impulse überträgt und sich insbesondere für klassische Telefondienste eignet. Mithilfe der DSL-Technologie (Digital Subscriber Line) können über diese Infrastruktur auch Daten übertragen werden. ${ }^{61}$ Deren Fortentwicklungen, die im Bereich des Vectoring eingesetzt werden, ermöglichen immer höhere Datenübertragungsraten (Kapitel 3, B. III.). Die Ertüchtigung der Bestandsinfrastruktur geht mit einem Ausbau der Glasfaserinfrastruktur einher, die immer weiter in Richtung der Teilnehmer ausgebaut wird. ${ }^{62}$ Je kürzer die Distanz ist, die mithilfe einer Kupferdoppelader bewältigt werden muss, desto höher steigt die Datenübertragungsrate, die mithilfe der Infrastruktur generiert werden kann. ${ }^{63}$ Die erzielbaren Datenübertragungsraten fallen bei kupferbasierten Technologien jedoch im Ergebnis deutlich geringer aus als bei glasfaserbasierten Infrastrukturen. ${ }^{64}$ Mithilfe der Technologie G.fast $22 \mathrm{MHz}$ können aggregierte Datenübertragungsraten von $1.500 \mathrm{Mbit} / \mathrm{s}$ erreicht werden. ${ }^{65}$ Für XG-Fast, das noch nicht verfügbar ist und für das lediglich Labortests durchgeführt wurden, werden Datenübertragungsraten von bis zu elf Gbit/s erwartet. ${ }^{66}$ Dabei können jedoch im Ergebnis nicht dieselben Qualitätskriterien erfüllt werden wie bei der Übertragungstechnologie Glasfaser. ${ }^{67} \mathrm{Zu}$ berücksichtigen ist jedoch, dass in Deutschland bereits flächendeckend Kupferdoppeladern verlegt sind. Eine Ertüchtigung der Bestandsinfrastruktur ist zu geringeren Kosten möglich als die Verlegung neuer Infrastrukturen. Solange die Bedürfnisse der Nutzer mithilfe von Bestandsinfrastrukturen befriedigt werden können, sollte aus diesem Grund auch nicht vollständig

$61 \mathrm{Zu}$ den verschiedenen DSL-basierten Übertragungstechnologien, Keller, Breitbandkabel und Zugangsnetze, S. 79.

$62 \mathrm{Zu}$ den Ausbaustufen im Überblick Kühling/Schall/Biendl, Telekommunikationsrecht, Rn. 107, Abbildung 8.

63 Fraunhofer FOKUS, Netzinfrastrukturen für die Gigabitgesellschaft, S. 79, Abbildung 27.

64 Einen Überblick über die mit den verschiedenen kupferbasierten Übertragungstechnologien erzielbaren Werte bietet, Fraunhofer FOKUS, Netzinfrastrukturen für die Gigabitgesellschaft, S. 80; Girard/Mattes/Michelsen, DIW Wochenbericht 2018, S. 532, 533.

65 Wernick/Queder/Strube Martins u.a., Gigabitnetze für Deutschland, S. 28.

66 Fraunhofer FOKUS, Netzinfrastrukturen für die Gigabitgesellschaft, S. 80.

67 Siehe hierzu im Überblick Nationaler IT-Gipfel, Konvergente Netze als Infrastruktur für die Gigabit-Gesellschaft, S. 8 f. 
von deren Optimierung abgesehen werden. Sie kann zumindest im Rahmen der „Digitalisierung der Gesellschaft“ als Übergangstechnologie genutzt werden.

\section{b) Datenübertragungstechnologien auf Basis eines Koaxialkabels}

Ursprünglich wurden Koaxialkabel, ${ }^{68}$ die auch als HFC-Kabel (hybrid fibre coax) bezeichnet werden, für die Übermittlung von Fernsehsignalen verlegt. ${ }^{69}$ Aus diesem Grund besteht in einigen Teilen Deutschlands - gerade im urbanen Bereich - bereits eine flächendeckende Infrastrukturabdeckung. Aufgrund ihres primären Einsatzes zur Übertragung von Fernsehsignalen konnten mithilfe der Technologie ursprünglich nur Signale zum Abnehmer, aber nicht wieder zurück transportiert werden. Aus diesem Grund konnten Koaxialkabel nicht als Bestandteil der Telekommunikationsinfrastruktur genutzt werden. Mittlerweile konnte die Übertragungstechnologie so modifiziert werden, dass sie auch rückkanalfähig ist. ${ }^{70}$ Stellenweise wird die Leistungsfähigkeit der Kabelnetze dadurch optimiert, dass Teile der Bestandsinfrastruktur durch Glasfaserkabel ersetzt werden.71 Mit verschiedenen Technologieupgrades kann die Übertragungsgeschwindigkeit immer weiter erhöht werden. Sie liegt derzeit bei DOCSIS 3.1 (Data Over Cable Service Interface Specification) $)^{72}$ bei bis zu $1000 \mathrm{Mbit} / \mathrm{s}$ im Download und $100 \mathrm{Mbit} / \mathrm{s}$ im Upload. ${ }^{73}$ Durch das Upgrade auf DOCSIS $3.1+{ }^{74}$ oder DOCSIS $4.0^{75}$ werden Datenübertragungsraten von bis zu zehn

68 Zum technischen Aufbau der Infrastruktur, die auf Koaxialkabel basiert umfassend, Keller, Breitbandkabel und Zugangsnetze, S. $224 \mathrm{ff}$.

69 Grundlegend zu den technischen Spezifika von Koaxialkabeln als Bestandteil der Telekommunikationsinfrastruktur, Keller, Breitbandkabel und Zugangsnetze, S. $128 \mathrm{ff}$.

70 Fraunhofer FOKUS, Netzinfrastrukturen für die Gigabitgesellschaft, S. 80.

71 Fraunhofer FOKUS, Netzinfrastrukturen für die Gigabitgesellschaft, S. 80.

$72 \mathrm{Zu}$ den technischen Spezifika von DOCSIS, Keller, Breitbandkabel und Zugangsnetze, S. $327 \mathrm{ff}$.

73 Fraunhofer FOKUS, Netzinfrastrukturen für die Gigabitgesellschaft, S. 81; Girard/Mattes/Michelsen, DIW Wochenbericht 2018, S. 532, 533; Wernick/Queder/ Strube Martins u.a., Gigabitnetze für Deutschland, S. 25.

74 Fraunhofer FOKUS, Netzinfrastrukturen für die Gigabitgesellschaft, S. 81, bezeichnet die Technologie als DOCSIS 3.1+.

75 Die Standardisierung DOCSIS 4.0 wurde durch „CableLabs“ aus den USA durchgeführt. Die Spezifika sind abrufbar unter: https://www.cablelabs.com/technologi es/docsis-4-0-technology (zuletzt abgerufen am 1.7.2020). 
Gbit/s im Download und sechs Gbit/s im Upload erreicht. Im Hinblick auf die übrigen Qualitätskriterien weisen die Glasfaserkabel insgesamt zwar bessere Werte auf, ${ }^{76}$ allerdings können die Anforderungen der Gigabit-Gesellschaft wohl bis zu einem gewissen Maß auch mithilfe von Koaxialkabeln befriedigt werden. Der Vorteil ihrer Aufrüstung liegt darin, dass die Kosten aufgrund des umfassenden Rückgriffes auf Bestandsinfrastrukturen geringer sind als beim FTTB/H-Ausbau. Werden allerdings neue Koaxialkabel verlegt, fallen ebenfalls Tiefbaukosten an, sodass die Qualitätskriterien bei der Infrastrukturauswahl eine bedeutendere Rolle einnehmen. In diesem Fall werden regelmäßig direkt Glasfaserinfrastrukturen verlegt.

\section{c) Funktechnologien}

Auch drahtlose Übertragungstechnologien wie etwa Mobilfunk oder WLAN spielen für die Gigabit-Gesellschaft eine wichtige Rolle. ${ }^{77}$ Kommunikationsprozesse und Produktionsabläufe können nur unter Rückgriff auf drahtlose Übertragungstechnologien vollständig vernetzt und digitalisiert werden. Dabei steht in den derzeitigen Planungen die Mobilfunktechnologie 5G im Mittelpunkt. Die Abkürzung 5G steht für fünfte Generation und bedeutet, dass es sich um die fünfte Standardisierung zur Weiterentwicklung der Technologie handelt. ${ }^{78}$ Die Vernetzung mithilfe satellitärer Lösungen spielt im Hinblick auf die Gigabit-Gesellschaft aufgrund der reduzierten Qualität derzeit noch keine Rolle. ${ }^{79}$ Diskutiert wird lediglich, inwieweit auf diese Technologie zurückgegriffen werden kann, um die für den Universaldienst erforderliche Basisversorgung sicherzustellen. ${ }^{80}$ Funktechnologien sind aufgrund ihrer vielfältigen Besonderheiten nicht Gegen-

$76 \mathrm{Zu}$ den technischen Details, Nationaler IT-Gipfel, Konvergente Netze als Infrastruktur für die Gigabit-Gesellschaft, S. 12; Keller, Breitbandkabel und Zugangsnetze, S. $376 \mathrm{ff}$.

77 Hierzu umfassend Fraunhofer FOKUS, Netzinfrastrukturen für die Gigabitgesellschaft, S. 82 ff.; grundlegend zu den technischen Spezifika, Keller, Breitbandkabel und Zugangsnetze, S. 191 ff.; Wernick/Queder/Strube Martins u.a., Gigabitnetze für Deutschland, S. $28 \mathrm{ff}$.

78 https:/www.fraunhofer.de/de/forschung/aktuelles-aus-der-forschung/5g-die-zuku nft-im-netz.html (zuletzt abgerufen am 1.7.2020).

79 Fraunhofer FOKUS, Netzinfrastrukturen für die Gigabitgesellschaft, S. 84 f.; zu den technischen Einzelheiten, Keller, Breitbandkabel und Zugangsnetze, S. $211 \mathrm{ff}$.

80 Umfassend zu satellitären Lösungen Goldmedia, in: Kühling/Goldmedia/Enaux (Hrsg.), Rechtliche Herausforderungen bei der Schaffung von Anreizen für einen flächendeckenden Ausbau von Glasfaserinfrastrukturen, S. 156 ff.; zur Frage der 
stand der folgenden Analyse, sodass nicht auf ihre technischen Spezifika eingegangen werden muss. Von Relevanz ist allerdings, dass die Funksignale zumeist ausgehend von Basisstationen übermittelt werden, die wiederum mit Festnetzinfrastruktur angebunden werden müssen. ${ }^{81} \mathrm{Je}$ hochwertiger diese Anbindung erfolgt, desto bessere Qualitäten können bei der Nutzung von Funktechnologien erzielt werden.

\section{Die optimale Infrastruktur für die Gigabit-Gesellschaft?}

Telekommunikationssignale können mithilfe unterschiedlicher festnetzbasierter Technologien übertragen werden. Die höchste Leistungsfähigkeit weisen FTTB/H-Technologien auf. Allerdings können auch mithilfe alternativer Übertragungstechnologien, die auf Koaxialkabeln basieren, hohe Übertragungsraten generiert werden. ${ }^{82}$ Sie können so lange genutzt werden, wie sich die übrigen Qualitätsanforderungen mit ihrer Hilfe bewältigen lassen. Hierfür entstehen im Ergebnis geringere Kosten als für die Verlegung einer neuen Infrastruktur.

Auf dem Weg in die Gigabit-Gesellschaft existiert keine optimale Infrastruktur für jede Lage und jede Situation. Vielmehr bieten alle Infrastrukturen Vor- und Nachteile, die für das konkrete Ausbaugebiet gegeneinander abgewogen werden müssen. ${ }^{83}$ Eine technologieneutrale Ausbaudiskussion fordern auch der unionale und der nationale Rechtsrahmen. ${ }^{84}$ Der Grundsatz der Technologieneutralität verbietet es, eine Übertragungstechnologie einseitig besser oder schlechter zu stellen. Je mehr die Gesellschaft jedoch zu einer Gigabit-Gesellschaft wird und je höher die Anforderungen an die Infrastruktur werden, desto weniger lassen sich die Anforderungen mit Alternativtechnologien befriedigen. Zudem sind im Rahmen der Aus-

Universaldiensterbringung mithilfe satellitärer Lösungen Kühling/Toros, Rechtliche Rahmenbedingungen für die Schaffung von Anreizen für einen flächendeckenden Ausbau von Glasfaserinfrastrukturen, S. 31 ff.; im Ergebnis auch Kühling/Toros, N\&R 2019, S. 258, 263.

81 Für den Mobilfunk besteht auch die Möglichkeit der Erschließung von Basisstationen über Richtfunk.

82 Hierzu im Überblick Wernick/Queder/Strube Martins u.a., Gigabitnetze für Deutschland, S. 36, Abbildung 3-5.

83 So im Ergebnis auch Fraunhofer FOKUS, Netzinfrastrukturen für die Gigabitgesellschaft, S. 77.

84 Siehe hierzu statt vieler Schneider, in: Fehling/Ruffert (Hrsg.), Regulierungsrecht, $\$ 8$, Rn. 9 . 
baudiskussion Pfadabhängigkeiten zu beachten, die durch die Nutzung bestimmter Technologien entstehen. Aus diesem Grund sollte die Bestandsinfrastruktur genutzt und optimiert werden. Wo dies nicht möglich ist, weil etwa bislang keine Infrastruktur besteht, sollte direkt eine zukunftsfähige Infrastruktur ausgebaut werden. Dabei sollte eine geschickte Priorisierung erfolgen. Der unionale Rechtsrahmen bietet insoweit mit dem Instrument der geografischen Erhebung, das eine umfassende Kartierung der Bestandsinfrastruktur vorsieht, ein geeignetes Instrument, um dies zu bewerkstelligen. ${ }^{85}$

\section{Der Ausbau gigabitfähiger Infrastrukturen}

Die Digitalisierung der Gesellschaft und schließlich eine Gigabit-Gesellschaft setzen den Ausbau der hierfür erforderlichen Telekommunikationsinfrastrukturen voraus. Zur weiterführenden Diskussion ist zunächst der aktuelle Ausbaustand zu ermitteln (dazu I.). Darüber hinaus sind die wesentlichen Risiken und Kostentreiber zu identifizieren, mit denen die Infrastrukturunternehmen konfrontiert werden (dazu II.).

I. Statistiken zum Stand des Ausbaus gigabitfähiger Infrastrukturen

Der aktuelle Ausbaustand kann auf Basis verschiedener Zahlenwerte betrachtet werden. Nachfolgend soll der Fokus auf den Fragen liegen, wie viele Haushalte mit gigabitfähigen Infrastrukturen versorgt sind (dazu 1.) und ob die ausgebauten Infrastrukturen tatsächlich nachgefragt werden (dazu 2.). Eine wichtige Rolle spielt darüber hinaus die aktuelle Bandbreitennachfrage (dazu 3.).

1. Die Anzahl der mit gigabitfähigen Infrastrukturen versorgten Haushalte

Bis Mitte des Jahres 2020 können nach einer Prognose der Dialog Consult GmbH (Dialog Consult) und des Verbandes der Anbieter von Telekom-

85 Umfassend hierzu Kühling/Toros, Rechtliche Rahmenbedingungen für die Schaffung von Anreizen für einen flächendeckenden Ausbau von Glasfaserinfrastrukturen, S. 107 ff.; Kühling/Toros, MMR 2020, S. 92 ff. 
munikations- und Mehrwertdiensten e. V. (VATM) bereits 51,1 Prozent der 41,9 Millionen deutschen Privathaushalte an gigabitfähige Infrastrukturen angeschlossen sein. ${ }^{86}$ Unter den Begriff der gigabitfähigen Infrastrukturen werden FTTB/H-Anschlüsse und Anschlüsse aus Koaxialkabeln, die mit DOCSIS 3.1 Technologien (B. II. 2. b.) ausgestattet sind, subsumiert. ${ }^{87}$ Etwa 16,64 Millionen Haushalte werden ausschließlich mithilfe der Übertragungstechnologie DOCSIS 3.1 versorgt. Deutlich weniger, nämlich 2,14 Millionen Haushalte, werden ausschließlich mit FTTB/H-Infrastrukturen versorgt. Insgesamt 2,61 Millionen Haushalte werden mit beiden Übertragungstechnologien parallel versorgt. ${ }^{88} \mathrm{Im}$ internationalen Vergleich belegt Deutschland damit einen der hinteren Plätze. ${ }^{89}$

\section{Das Verhältnis von Angebot und Nachfrage}

Ein wichtiger Faktor für den Ausbau ist die Nachfrage nach gigabitfähigen Infrastrukturen. ${ }^{90}$ Nur wenn die verfügbaren Infrastrukturen tatsächlich genutzt werden, können die Investitionskosten amortisiert werden. Ende des Jahres 2019 wurden nur 34,5 Prozent der verfügbaren FTTB/H-Infrastrukturen tatsächlich genutzt. Für Ende Juni 2020 wurde die Anzahl der nutzbaren Anschlüsse auf 14,7 Prozent prognostiziert. Die Nutzungsrate lag damit jedoch weiterhin lediglich bei 34,7 Prozent. ${ }^{91}$

Betrachtet man die Nutzung von HFC-Netzen, die mit DOCSIS 3.1Technologie ausgestattet sind, wurden Ende des Jahres 2019 nur 24,8 Prozent der Infrastrukturen genutzt. Für Ende Juni 2020 wurde ein Anstieg der verfügbaren Anschlüsse um 34,1 Prozent erwartet. Die Nutzungsrate sank damit leicht auf 22,8 Prozent. ${ }^{92}$ In der Zusammenschau der Nutzungsraten gigabitfähiger Glasfaser- und Kabelinfrastrukturen wurden En-

86 Dialog Consult/VATM, 2. Marktanalyse Gigabit-Anschlüsse 2020, S. 8, Abbildung 6 .

87 Dialog Consult/VATM, 2. Marktanalyse Gigabit-Anschlüsse 2020, S. 2.

$88 \mathrm{Zu}$ diesen Zahlenwerten insgesamt Dialog Consult/VATM, 2. Marktanalyse Gigabit-Anschlüsse 2020, S. 8, Abbildung 6.

89 Girard/Mattes/Michelsen, DIW Wochenbericht 2018, S. 532, S. 535, Abbildung 2.

90 DIW Econ, Ausbau von Gigabitnetzen: Wettbewerb und Regulierung, S. 15 f.

91 Dialog Consult/VATM, 2. Marktanalyse Gigabit-Anschlüsse 2020, S. 6, Abbildung 4; Böcker, BREKO Marktanalyse 19, S. 12, geht von einer Nutzungsrate von 43 Prozent aus.

92 Dialog Consult/VATM, 2. Marktanalyse Gigabit-Anschlüsse 2020, S. 5, Abbildung 3 . 
de des Jahres 2019 lediglich 27,0 Prozent der Infrastrukturen genutzt. ${ }^{93}$ Ende Juni 2020 stieg die Zahl der verfügbaren Anschlüsse nach vorherigen Schätzungen um 29,8 Prozent. Für diesen Zeitpunkt wurde zugleich ein Absinken der Nutzungsrate bis auf 25,2 Prozent prognostiziert. ${ }^{94}$ Auch in dieser Hinsicht belegt Deutschland im Vergleich zu anderen Staaten einen der hinteren Plätze. ${ }^{95}$ Es ist unsicher, ob die Glasfaserausbauvorhaben aufgrund der Auswirkungen der Corona-Pandemie in der geplanten Geschwindigkeit vorangetrieben werden können. Dies betrifft jedoch eher die Anzahl der verfügbaren Anschlüsse und deren Steigerungsrate, als die Nutzungsrate. Eine vertiefte Auseinandersetzung mit dem Zahlenmaterial soll an dieser Stelle nicht erfolgen. Es zeigt sich jedoch eindeutig, dass die Nutzung gigabitfähiger Infrastrukturen im Hinblick auf die Bezugsgröße aller deutschen Privathaushalte deutlich hinter den verfügbaren Anschlüssen zurückbleibt. ${ }^{96}$ Dies mag lokal begrenzt, etwa in Ballungsräumen, jedoch anders aussehen.

3. Der aktuelle Bandbreitenbedarf auf Basis der Verteilung der vermarkteten Bandbreiten

Als Indikator für den Bandbreitenbedarf können die vermarkteten Bandbreiten herangezogen werden. Benötigen die Kunden höhere Bandbreiten, so lässt sich dies auf Basis der abgeschlossenen Verträge ermitteln. ${ }^{97} \mathrm{Die}$ Bundesnetzagentur (BNetzA) erfasste in ihrem Jahresbericht 2019 die Verteilung der vermarkteten Bandbreiten von Festnetz-Breitbandanschlüssen. ${ }^{98}$ In dieser Statistik werden alle Übertragungstechnologien gemeinsam erfasst. Die Daten sind nicht auf gigabitfähige Infrastrukturen be-

93 Die Entwicklung der aktiven Breitbandanschlüssen für den Zeitraum zwischen 2009 und 2019 in absoluten Zahlen unabhängig von der genutzten Technologie und in Gegenüberstellung mit den aktiven DSL-Anschlüssen wird dargestellt von der BNetzA, Jahresbericht 2019, S. 48.

94 Dialog Consult/VATM, 2. Marktanalyse Gigabit-Anschlüsse 2020, S.3, Abbildung 1.

95 Girard/Mattes/Michelsen, DIW Wochenbericht 2018, S. 532, 535, Abbildung 3.

96 So auch Heymann/Körner, Digitale Infrastruktur, S. 13 f.

97 So auch Goldmedia, in: Kühling/Goldmedia/Enaux (Hrsg.), Rechtliche Herausforderungen bei der Schaffung von Anreizen für einen flächendeckenden Ausbau von Glasfaserinfrastrukturen, S. 176.

98 BNetzA, Jahresbericht 2019, S. 49. 
schränkt. Auch wenn ein Anschluss gigabitfähig ist, kann eine geringere Bandbreite als die technisch mögliche vermarktet werden.

Im Jahr 2019 wurden über 2,9 Millionen Festnetzanschlüsse mit Bandbreiten unter zehn Mbit/s zur Verfügung gestellt. Die Anzahl der vermarkteten Anschlüsse für Bandbreiten zwischen zehn bis unter $30 \mathrm{Mbit} / \mathrm{s}$ lag bei 9,6 Millionen. Mit 13,4 Millionen wurden für die meisten Anschlüsse Bandbreiten zwischen 30 bis unter $100 \mathrm{Mbit} / \mathrm{s}$ vermarktet. Für neun Millionen Anschlüsse wurden Bandbreiten zwischen $100 \mathrm{Mbit} / \mathrm{s}$ bis unter ein Gbit/s vermarktet. Die Anzahl der vermarkteten Bandbreiten mit über einem Gbit/s war mit 0,2 Millionen am geringsten. ${ }^{99}$ Der Bandbreitenbedarf der Endkunden konnte im Jahr 2019 also überwiegend ohne die Nutzung gigabitfähiger Infrastrukturen bewältigt werden. ${ }^{100}$ Dieses Ergebnis spiegelt sich in der Anzahl der aktiv genutzten gigabitfähigen Anschlüsse wider (C. I. 2.).

\section{Schlussfolgerungen für die weitere Analyse}

Auch wenn die Zahl der verfügbaren gigabitfähigen Infrastrukturen kontinuierlich ansteigt, wird der Großteil von ihnen nicht aktiv genutzt. Die Nachfrage befindet sich - bezogen auf die verfügbaren Kapazitäten - auf einem stabilen Niveau und ist sogar leicht rückläufig. Der aktuelle Bandbreitenbedarf, der sich auf Basis der vermarkteten Bandbreiten ermitteln lässt, kann auch mithilfe alternativer Infrastrukturen bewältigt werden. Es besteht somit keine große Wechselbereitschaft auf leistungsfähigere Übertragungstechnologien, zumal der Wechsel oftmals mit höheren Kosten für den Endkunden einhergeht. Hieraus folgt, dass die Nachfrage derzeit nicht zu einer Beschleunigung des Ausbaus gigabitfähiger Infrastrukturen beiträgt. Es handelt sich jedoch um eine Momentaufnahme, die nur eine eingeschränkte Steuerungswirkung auf das Verhalten der Unternehmen am Markt hat. Da Infrastrukturinvestitionen über viele Jahre amortisiert werden, ist die aktuelle Nachfrage im Vergleich zu deren Entwicklung von untergeordneter Bedeutung. Der Bandbreitenbedarf lässt sich allerdings nur schwerlich prognostizieren (C. II. 2.).

99 BNetzA, Jahresbericht 2019, S. 49.

$100 \mathrm{Zu}$ den möglichen Technologien bereits umfassend B. II. 
II. Risiken und Kostentreiber beim Ausbau gigabitfähiger Infrastrukturen

Der Ausbau gigabitfähiger Infrastrukturen bringt hohe Investitionskosten mit sich, die im Laufe der Zeit amortisiert werden müssen (dazu 1.) Hinzu treten verschiedene Risiken und Unsicherheiten, die bei der Planung von Ausbauvorhaben einkalkuliert werden müssen und die Ausbaugeschwindigkeit reduzieren können. Hierzu zählen insbesondere die Nachfrage(dazu 2.) sowie die Markt-, Wettbewerbs- und Regulierungsunsicherheit (dazu 3.).

\section{Tiefbaukosten als Kostentreiber}

Bei der Etablierung von Handlungsoptionen zur Beschleunigung des Infrastrukturausbaus ist zu berücksichtigen, dass die Tiefbaukapazitäten limitiert sind. Für andere Bereiche des Infrastrukturausbaus, insbesondere für die Energie- und Verkehrswende, wird die Expertise der gleichen Unternehmen gebraucht wie für den Ausbau gigabitfähiger Infrastrukturen. Aufgrund der hohen Nachfrage nach begrenzt verfügbaren Kapazitäten steigen die Preise. Verschiedene Studien gehen davon aus, dass etwa 50 bis 80 Prozent der Investitionskosten im Glasfaserausbau auf den Tiefbau zurückzuführen sind. ${ }^{101}$ Es wird davon ausgegangen, dass der flächendeckende Ausbau von Glasfaserinfrastrukturen etwa 45 Milliarden Euro kosten wird. ${ }^{102}$ Hierbei handelt es sich um sogenannte „sunk costs“, die unwiederbringlich verloren sind und auch bei einem Branchenausstieg nicht wieder zurückgewonnen werden können (Kapitel 4, A. I. 2. a.). Sie müssen mit einer entsprechenden Nachfrage und der Erhebung von Entgelten amortisiert werden. Dieser Aspekt führt dazu, dass die nachfolgend zu beleuchtenden Unsicherheiten umso stärker ins Gewicht fallen. Potentiale für Kostensenkungen bestehen etwa in Form der Erleichterung von Genehmigungsverfahren. ${ }^{103}$

101 Hierzu statt vieler Amendola/Pupillo, Communications \& Strategies 69 (2008), S. 85, 89; Ruble/Reichl, Intereconomis 2009, S. 30, 33; Tenbrock, Der Glasfaserausbau in Deutschland, S. 104; so auch und mit Ansätzen für eine Reduktion der Tiefbaukosten Wernick/Tenbrock/Gries u.a., Tiefbaukapazitäten als Engpass für den FTTB/H-Ausbau?, S. 11, $32 \mathrm{ff}$.

102 Gries/Plückebaum/Strube Martins, Kostentreiber für den Ausbau hochbitratiger Infrastrukturen, S. 17; IW Consult, Gigabitnetze für Deutschland, S. 9.

103 Gries/Plückebaum/Strube Martins, Kostentreiber für den Ausbau hochbitratiger Infrastrukturen, S. 50; IW Consult, Gigabitnetze für Deutschland, S. $11 \mathrm{f}$. 


\section{Die Nachfrageunsicherheit}

Für den Weg in die Gigabit-Gesellschaft ist insbesondere der Bandbreitenbedarf im Jahr 2025 relevant. Bis dahin soll nach dem Ausbauplan des Bundesministeriums für Verkehr und digitale Infrastruktur eine flächendeckende, gigabitfähige Infrastruktur verfügbar sein. Investitionen in Infrastruktur lassen sich allerdings nur dann amortisieren, wenn diese Infrastruktur nachgefragt und aktiv genutzt wird. Je niedriger die Nachfrage ist, desto weniger lassen sich die Investitionen wieder erwirtschaften. Unsicherheiten in der Entwicklung der Nachfrage können deswegen insgesamt die Geschwindigkeit des Breitbandausbaus reduzieren. Ein eigenwirtschaftlicher Ausbau erfordert das Überschreiten einer bestimmten Nachfragequote, die jedes Unternehmen individuell bestimmt und seiner Kalkulation zu Grunde legt. Wird diese Quote nicht überschritten, müssen die Lücken alternativ, etwa durch die Vergabe von Fördermitteln, geschlossen werden.

Eine Prognose der Bandbreitennutzung für eine Zeitspanne von fünf Jahren ist mit großen prognostischen Unsicherheiten belastet. So konnte vor Beginn des Jahres 2020 noch nicht vorhergesehen werden, dass der Bandbreitenbedarf in Privathaushalten aufgrund der Corona-Pandemie und der damit verbundenen vermehrten Nutzung von Homeoffice-Arbeitsplätzen stark steigen würde. ${ }^{104}$ Darüber hinaus lässt sich in einem innovationsgetriebenen Sektor wie der Digitalwirtschaft nicht antizipieren, ob eine Applikation entwickelt wird, die zu einem kurzfristigen Anstieg des Bandbreitenbedarfs führen wird. Nach dem Nielsen's Law of Internet Bandwith kann davon ausgegangen werden, dass der Bandbreitenbedarf jährlich um bis zu 50 Prozent steigt. ${ }^{105}$ Die These wurde im Jahr 1998 von Jakob Nielsen aufgestellt und lässt sich bis zum Jahr 2019 bis auf marginale Abweichungen bestätigen. ${ }^{106}$ Eine differenzierte Betrachtung der erwarteten Nutzung gigabitfähiger Anschlüsse enthält diese Analyse jedoch nicht. Eine konsolidierte Zusammenfassung der Prognosen des durchschnittlichen Bandbreitenbedarfs veröffentlichten im Jahr 2018 Andreas Neumann

104 https://www.zeit.de/digital/internet/2020-03/deutsche-internetprovider-infrastru ktur-coronavirus-homeoffice (zuletzt abgerufen am 1.7.2020).

105 Der Artikel ist abrufbar unter https://www.nngroup.com/articles/law-of-bandwi $\mathrm{dth} /$, (zuletzt abgerufen am 1.7.2020).

106 Siehe hierzu die Darstellung auf https:/www.nngroup.com/articles/law-of-band width/, (zuletzt abgerufen am 1.7.2020). 
und Jörn Sickmann. ${ }^{107}$ In dieser Untersuchung werteten die Autoren die prognostizierte Bandbreitenentwicklung aus verschiedenen Quellen aus den Jahren 2011 bis 2017 aus. ${ }^{108}$ Erwartet wurden in diesem Zeitraum Bandbreitenbedarfe für das Jahr 2025 in einem Spektrum von minimal 120 $\mathrm{Mbit} / \mathrm{s}^{109} \mathrm{bis}$ maximal $600 \mathrm{Mbit} / \mathrm{s}^{110}$ In einer dieser Studien wurde prognostiziert, dass etwa 30 Prozent der Haushalte einen Bandbreitenbedarf von mehr als einem Gbit/s haben würden. ${ }^{111} \mathrm{Im}$ Jahr 2019 wurde für das Jahr 2025 ein Bandbreitenbedarf von $505 \mathrm{Mbit} / \mathrm{s}$ im Download und 223 Mbit/s im Upload für Privatkunden und von 1,3 Gbit/s im Download und 1,1 Gbit/s im Upload für Geschäftskunden prognostiziert. ${ }^{112}$

Es lässt sich feststellen, dass in jedem Jahr der erwartete Bandbreitenbedarf für das Jahr 2025 nach oben korrigiert wurde. ${ }^{113}$ Derzeit geht aber keine der Prognosen davon aus, dass im Jahr 2025 die Mehrheit der Haushalte einen Bandbreitenbedarf im Gigabitbereich haben wird. Dies könnte dazu beitragen, dass über einen möglichst langen Zeitraum versucht wird, die Investitionen hinauszuzögern und die Nachfrage über vollständig abgeschriebene Bestandsinfrastruktur zu befriedigen, die, wie bereits dargestellt, bis zu einem gewissen Maße ertüchtigt werden kann (B. II. 2.). ${ }^{114}$

Regelmäßig fallen für die Nutzung gigabitfähiger Infrastrukturen höhere Preise an als für die Nutzung von Alternativinfrastrukturen mit niedrigeren Qualitätsstandards. ${ }^{115}$ Kann die Nachfrage auch mit Alternativinfrastrukturen zu geringeren Preisen befriedigt werden, besteht regelmäßig keine Wechselbereitschaft. ${ }^{116}$ Je höher und schneller der Bandbreitenbedarf steigt, desto niedriger sind die Nachfrageunsicherheit und das hiermit

107 Neumann/Sickmann, Stand und Perspektiven des Breitbandausbaus in Deutschland, S. 13 ff.

108 Neumann/Sickmann, Stand und Perspektiven des Breitbandausbaus in Deutschland, S. 14, Tabelle 2, mit Nachweisen für alle ausgewerteten Quellen.

109 Die Daten stammen aus der BREKO-Breitbandstudie aus dem Jahr 2012, die online nicht mehr abrufbar ist. Die Daten können demnach nur über die Sekundärquelle Neumann/Sickmann, Stand und Perspektiven des Breitbandausbaus in Deutschland, S. 14, Tabelle 2, überprüft werden.

110 BREKO, Breitbandstudie 2017, S. 15.

111 Strube Martins/Wernick/Plückebaum u.a., Die Privatkundennachfrage nach hochbitratigem Breitbandinternet im Jahr 2025, S. 21, Abbildung 3.1.

112 Böcker, BREKO Marktanalyse 19, S. 7.

113 Neumann/Sickmann, Stand und Perspektiven des Breitbandausbaus in Deutschland, S. 12, Abbildung 27.

114 Weiterführend hierzu Tenbrock, Der Glasfaserausbau in Deutschland, S. 81.

115 Tenbrock, Der Glasfaserausbau in Deutschland, S. 81.

116 Tenbrock, Der Glasfaserausbau in Deutschland, S. 81. 
verbundene Risiko, das bei Investitionsentscheidungen berücksichtigt werden muss. ${ }^{117}$

Aufgrund dieses Befundes werden verschiedene Optionen zur Stimulation der Nachfrage diskutiert. So wird erwogen, die Vermarktungsraten mithilfe sogenannter "Gigabit-Voucher" zu erhöhen. ${ }^{118}$ Hierbei handelt es sich um Kleinstbeihilfen, die den Endkunden zur Verfügung gestellt werden und als Beitrag zur Finanzierung von Anschluss- oder Vertragskosten genutzt werden können. Auch wenn die Vergabe der Voucher rechtlich möglich ist, wird kritisch diskutiert, ob sie auch ökonomisch sinnvoll ist. ${ }^{119}$ Verhaltenspsychologisch wird mithilfe eines sogenannten „Nudges“ versucht, die Kunden zu einem Verhalten zu motivieren. ${ }^{120}$ Mit dem Voucher wird ein finanzieller Anreiz in Form einer Einsparung gesetzt. Im Markt ist zu beobachten, dass verschiedene Unternehmen sehr offensiv mit dem Mittel agieren und Kunden etwa anbieten, dass keine Anschlusskosten zu zahlen sind, wenn ein Vertrag abgeschlossen wird. ${ }^{121}$ Auf diese Weise versuchen die Unternehmen sicherzustellen, dass eine hinreichende Auslastung der neuen Infrastruktur erfolgt.

Alternativ wird darüber nachgedacht, ob der Staat im Rahmen seiner Tätigkeit umfassend auf digitale Technologien - etwa in Form des e-Government - setzen sollte, um die Bandbreitennachfrage zu stimulieren. ${ }^{122}$

117 Tenbrock, Der Glasfaserausbau in Deutschland, S. 86, geht insgesamt davon aus, dass die Nachfrageunsicherheit beherrschbar sei und ein mittelhohes Risiko darstelle.

118 Hierzu umfassend Kühling/Toros, Rechtliche Rahmenbedingungen für die Schaffung von Anreizen für einen flächendeckenden Ausbau von Glasfaserinfrastrukturen, S. 82 ff.; Monopolkommission, 11. Sektorgutachten Telekommunikation (2019), Rn. 167 ff.; IW Consult, Gigabitnetze für Deutschland, S. 12 ff.; ZEW/Juconomy, Gutachten zur ökonomischen und rechtlichen Sinnhaftigkeit von nachfrageseitigen Förderungen im Ausbau moderner Breitbandnetze über "Voucher-Systeme", S. 10 ff.; deskriptiv Wissenschaftlicher Dienst, WD 5 - 3000 - 072/18, S. 2 ff.; kritisch Gerpott, Wirtschaftsdienst 2020, S. $128 \mathrm{ff}$.

119 Kritisch Gerpott, Wirtschaftsdienst 2020, S. $128 \mathrm{ff}$.

120 Vgl. hierzu statt vieler Moseley/Stoker, Resources, Conversation and Recycling 79 (2013), S. 4 ff.; IW Consult, Gigabitnetze für Deutschland, S. 12 f.; Reisch/Zhao, Behavioural Public Policy 2017, S. 190 ff.; Sunstein, Journal of Consumer Policy 37 (2014), S. $583 \mathrm{ff}$.

121 Vgl. hierzu statt vieler etwa das Vorgehen eines lokalen Anbieters in der Kommune Ratingen in Nordrhein-Westfalen, https://rp-online.de/nrw/staedte/rating en/nachdem-die-telekom-beim-internetausbau-kaum-hinterherkommt-verkabeltdie-kommitt-ganz-ratingen_aid-35570621 (zuletzt abgerufen am 1.7.2020).

122 Heuermann/Meinen/Ekango u.a., Konzepte zur Förderung von Breitbandinvestitionen im internationalen Vergleich, S. $29 \mathrm{ff}$. 
3. Die Markt-, Wettbewerbs- und Regulierungsunsicherheit

Für die Unternehmen sind bei der Investition auch Markt-, Wettbewerbsund Regulierungsunsicherheit von Relevanz. Diese Risiken sind sehr eng miteinander verzahnt. Problematisch ist für Unternehmen, dass die Entwicklung des Marktes und des Wettbewerbs nur bis zu einem gewissen Grad vorausgesehen werden kann. Ein Überbau der eigenen Infrastruktur kann nachteilig für Investitionen sein. Darüber hinaus lässt sich nicht antizipieren, ob in Zukunft noch leistungsfähigere Übertragungstechnologien entwickelt werden. ${ }^{123}$ Eine weitere Unsicherheit entsteht durch das regulatorische Regime. Gerade für Unternehmen mit beträchtlicher Marktmacht stellt sich die Frage, inwieweit sie durch Zugangsverpflichtungen dazu gezwungen werden können, anderen Unternehmen die neuen Infrastrukturen zur Verfügung zu stellen. Im Extremfall können hierdurch Investitionen entwertet werden. ${ }^{124}$ Relativierend ist einzuwenden, dass Markt-, Wettbewerbsunsicherheiten - und in regulierten Märkten auch Regulierungsunsicherheiten - für einen marktgetriebenen Ausbau typisch sind und im Sinne eines funktionsfähigen Wettbewerbs auch nicht vollständig behoben werden sollten. Vielmehr ist es üblich, dass die Risiken bei den Investitionsentscheidungen berücksichtigt werden und falls die Risiken zu hoch sind, von einer Investition Abstand genommen wird.

Es werden jedoch verschiedene Maßnahmen diskutiert, mit denen die Investitionsbedingungen verbessert und die Unsicherheiten reduziert werden können. Die Maßnahmen weisen unterschiedliche Eingriffsintensitäten auf und adressieren verschiedene der zuvor genannten Unsicherheiten. Das Konzessionsmodell auf Basis der Vergabe exklusiver Wegerechte zielt darauf $\mathrm{ab}$, in den Konzessionsgebieten den Infrastrukturwettbewerb abzuschaffen und auf diese Weise die Markt- und Wettbewerbsunsicherheit vollständig zu beseitigen. Der Wettbewerb soll von der Infrastruktur- auf die Diensteebene verlagert werden. Darüber hinaus soll ein Wettbewerb um die Konzession stattfinden (Kapitel 3, C. I.).

Ein alternativer Ansatz setzt auf Anreize zur Entflechtung vertikal integrierter Telekommunikationsunternehmen (Kapitel 4, A. I. 3.). Auf diese Weise sollen durch eine umfassende Umgestaltung des Marktes, die Diskriminierungsanreize auf der nachgelagerten Ebene reduziert und mittelbar auch die Investitionsbedingungen verbessert werden. Im Rahmen dieser

123 Weiterführend hierzu Tenbrock, Der Glasfaserausbau in Deutschland, S. $86 \mathrm{ff}$.

124 Weiterführend hierzu Tenbrock, Der Glasfaserausbau in Deutschland, S. $88 \mathrm{ff}$. 
Umgestaltung spielen auch regulatorische Privilegierungen eine entscheidende Rolle, welche die Regulierungsunsicherheiten verringern sollen.

Eine verstärkte Zusammenarbeit verschiedener Unternehmen ist Gegenstand von Kooperationsmodellen (Kapitel 5, A.). Durch die Zusammenarbeit sollen die Risiken unter den Unternehmen aufgeteilt werden. Bei all diesen Maßnahmen ist darauf zu achten, dass durch den novellierten unionalen Rechtsrahmen gemäß Art. 3 Abs. 2 EKEK zugleich die Erhöhung der Konnektivität mit hohen Bandbreiten (lit. a) als auch die Förderung des Wettbewerbs (lit. b) und des Binnenmarktes (lit. c) bei Beachtung der Interessen der Bürger (lit. d) bezweckt ist. Dieses Spannungsverhältnis tangiert alle Maßnahmen und fordert einen schonenden Ausgleich zwischen den Regulierungszielen.

\section{Ergebnis und Schlussfolgerungen}

Die Zahl der verfügbaren gigabitfähigen Anschlüsse von Endkunden steigt kontinuierlich an. Die Nachfrage nach den Infrastrukturen bleibt allerdings bei Weitem hinter dem Angebot zurück. Bis zum Jahr 2025 wird voraussichtlich keine Situation eintreten, in der die Nachfrage überwiegend mithilfe gigabitfähiger Infrastrukturen befriedigt werden kann. Hieraus resultiert eine umfassende Nachfrageunsicherheit. Kombiniert mit den hohen Investitionskosten, die für den Infrastrukturausbau erforderlich sind, und weiteren Unsicherheiten vermindert dies die Geschwindigkeit des Infrastrukturausbaus. Im Folgenden soll analysiert werden, wie sich verschiedene Handlungsoptionen auf die auf die Markt-, Wettbewerbsund Regulierungsunsicherheiten auswirken und Investitionsbedingungen gegebenenfalls verbessert werden können.

\section{Fazit}

Die fortschreitende Digitalisierung der Gesellschaft führt zur Entstehung einer Gigabit-Gesellschaft, die umfassend vernetzt ist. Das Bundesministerium für Verkehr und digitale Infrastruktur (BMVI) möchte bis zum Jahr 2025 die hierfür erforderlichen digitalen Infrastrukturen errichtet haben. Bei der Auswahl der hierfür geeigneten Infrastrukturen sind verschiedene Qualitäts- und Kapazitätskriterien zu berücksichtigen. Die Datenübertragungsrate, die mithilfe einer Infrastruktur erreicht werden kann, dominiert die derzeitige Diskussion. In der Gigabit-Gesellschaft steigen jedoch 
auch die Qualitätsanforderungen an die Übertragungstechnologien, sodass auch fehlerbezogene Parameter sowie Latenz und Latenzschwankungen zu berücksichtigen sind. Aus ökonomischer Warte spielen auch die Ausbaukosten und die Effizienz im Betrieb eine entscheidende Rolle. Auch wenn FTTB/H-Technologien als Referenzinfrastruktur herangezogen werden, um die Qualität der anderen Übertragungstechnologien zu beschreiben, handelt es sich nicht um die einzige Infrastruktur, die auf dem Weg in die Gigabit-Gesellschaft berücksichtigt werden sollte. Auch Alternativen wie Koaxialkabel, die mit DOCSIS 3.1-Übertragungstechnologien ausgestattet sind, sollten in die Strategie einbezogen werden. Problematisch ist für den Infrastrukturausbau, dass die Anzahl der verfügbaren Anschlüsse kontinuierlich steigt, jedoch bislang lediglich etwa 25 Prozent davon aktiv genutzt werden. Auch bis zum Jahr 2025 wird sich ein relevanter Anteil der Bandbreitennachfrage vermutlich mithilfe solcher Infrastrukturen befriedigen lassen, die nicht gigabitfähig sind. Hinzutreten Unsicherheiten im Hinblick auf die Entwicklung von Markt, Wettbewerb und Regulierung. In der folgenden Untersuchung sollen daher verschiedene Handlungsoptionen untersucht werden, die diese Risiken und Unsicherheiten minimieren sollen. Dabei stellt sich die Frage, ob sie rechtlich umsetzbar sind und einen entscheidenden Vorteil für die Beschleunigung des Infrastrukturausbaus mit sich bringen. 


\section{Kapitel 3: Exklusivität im Infrastrukturausbau am Beispiel unterschiedlicher Konzessionsmodelle}

Exklusivität spielt bei der Schaffung von Anreizen zur Beschleunigung des Ausbaus hochleistungsfähiger digitaler Infrastrukturen eine wichtige Rolle. Durch eine exklusive Vergabe von Gütern, Fördermitteln oder Rechten sollen die durch den Wettbewerb bedingten Unsicherheiten reduziert, auf diese Weise die Investitionsbedingungen verbessert und so Anreize für den Infrastrukturausbau in Gebieten mit Netzausbaudefizit geschaffen werden. Der Begriff der „Konzession“ wird im Kontext verschiedener Modelle und Handlungsansätze gebraucht, die Exklusivität als Ausbauanreiz nutzen. Die Modelle sind sehr heterogen. Deswegen soll im Anschluss an eine Abgrenzung des Konzessionsbegriffes und die Identifikation seiner Wesensmerkmale (dazu A.) untersucht werden, wie sich das Telekommunikationsrecht insgesamt zu einer Vergabe von Exklusivrechten verhält (dazu B.).

Drei verschiedene Exklusivitätsmodelle, die an die Vergabe von „Konzessionen" anknüpfen, sollen vertieft untersucht und miteinander verglichen werden. Besonders kontrovers diskutiert wird ein Konzept, in dem exklusive Telekommunikationswegerechte nach dem Vorbild des Energierechts vergeben werden sollen (dazu C.). Bereits umfassend genutzt werden Konzessionsmodelle zum Aufbau und Betrieb staatlicher Telekommunikationsinfrastrukturen (dazu D.). Als weitere Variante existieren Konzessionsmodelle, in denen Fördermittel lediglich exklusiv an einen Infrastrukturanbieter vergeben werden (dazu E.).

\section{A. Begriffsbestimmung und Begriffsmerkmale der Konzession}

Die Modelle und Handlungsansätze, die im Folgenden vertieft untersucht werden sollen, basieren auf der Vergabe von „Konzessionen“ durch den Staat an Infrastrukturanbieter. Um einen Vergleich zu ermöglichen, stellt sich die Frage, wie der Begriff der „Konzession“ bestimmt werden kann und welche Wesensmerkmale ihn in der deutschen Rechtswissenschaft prägen. Ausgehend von einem einheitlichen Ursprung (dazu I.) hat sich die Verwendung des Begriffes vielseitig ausdifferenziert. Neben den „Bauund Dienstleistungskonzessionen“ des förmlichen Vergaberechts (dazu II.) 
vergibt der Staat in unterschiedlichen Fallgruppen Konzessionsrechte (dazu III.). Die Begriffe und ihre Verwendung sind derart unterschiedlich, dass nicht von einem rechtsterminologisch einheitlich abgrenzbaren Konzessionsbegriff gesprochen werden kann. Dennoch ist fraglich, ob den einzelnen Begriffen verschiedene allgemeine Charakteristika zuzuordnen sind, die der nachfolgenden Betrachtung zu Grunde gelegt werden können.

\section{Ursprung des Konzessionsbegriffes im nationalen Recht}

Der Begriff „Konzession“ wird in der deutschen Rechtwissenschaft seit Beginn des 20. Jahrhunderts verwendet. ${ }^{125}$ Prägend für die frühe Entwicklungsphase war das Verständnis Otto Mayers. ${ }^{126} \mathrm{Er}$ interpretierte die „Konzession" als eine Form des Verwaltungsaktes zur Übertragung öffentlicher Hoheitsgewalt an Private, die zwingend für die Ausübung einer bestimmten Tätigkeit erforderlich war. ${ }^{127}$ Die Verleihung subjektiver Rechte ${ }^{128}$ wurde eng an das französische Institut der "concession des service public“ angelehnt. ${ }^{129}$ Dieses lässt sich am ehesten mit einer Beleihung vergleichen, durch die es Privaten gestattet wird, öffentliche Verwaltungsmaßnahmen durchzuführen. ${ }^{130}$ Ihren begrifflichen Ursprung findet die „Konzession“ also in Konstellationen, in denen Privatrechtssubjekte mit der Erbringung von Aufgaben aus dem Verantwortungsbereich des Staates beauftragt und ihnen hierfür erforderliche Hoheitsrechte übertragen wurden.

II. Bau- und Dienstleistungskonzessionen

Ausgehend von diesem Ursprung hat sich etwa der Begriff der „Bau- und Dienstleistungskonzession“ entwickelt, der jedoch nicht allein durch nationales Recht, sondern auch durch das Unionsrecht geprägt ist. Bau- und Dienstleistungskonzessionen sind dadurch gekennzeichnet, dass sich der

125 Crome, AcP 115 (1917), S. 1, 15.

126 Mayer, Deutsches Verwaltungsrecht, S. 147 ff.; Wieland, Die Konzessionsabgaben, S. $90 \mathrm{f}$.

127 Mayer, Deutsches Verwaltungsrecht, S. 147.

128 Ausführlich hierzu Wieland, Die Konzessionsabgaben, S. $98 \mathrm{ff}$.

129 Wieland, Die Konzessionsabgaben, S. 91.

130 Ausführlich zum Institut der „concession des service public“, Wieland, Die Konzessionsabgaben, S. $93 \mathrm{ff}$. 
Konzessionsnehmer gegenüber dem Konzessionsgeber vertraglich dazu verpflichtet, eine spezifische Leistung zu erbringen, und hierfür das Betriebsrisiko trägt. ${ }^{131}$ Klar konturiert wird der Begriff durch Legaldefinitionen, die im Unionsrecht in der Konzessionsvergaberichtlinie (KVRL) ${ }^{132}$ angelegt und im nationalen Recht inhaltsgleich in $\$ 105$ Abs. 1 GWB $^{133}$ enthalten sind.

Durch eine „Baukonzession“ verpflichtet sich gemäß Art. 5 Nr. 1 lit. a KVRL und $\$ 105$ Abs. 1 Nr. 1 GWB ein Privater dazu, im Auftrag des Staates eine Bauleistung zu erbringen. Eine Gegenleistung wird hierfür lediglich in Gestalt des Nutzungsrechts am entstandenen Bauwerk oder in Gestalt dieses Nutzungsrechtes zuzüglich der Zahlung eines Entgeltes gewährt. Das unternehmerische Risiko liegt beim Konzessionsnehmer. Der Begriff der „Dienstleistungskonzession“ wird hiervon gemäß Art. 5 Nr. 1 lit. b KVRL und $\$ 105$ Abs. 1 Nr. 2 GWB negativ abgegrenzt. Sie liegt dann vor, wenn der Konzessionsnehmer mit einer Dienstleistung betraut wird, die kein Bauvorhaben zum Gegenstand hat. Die Gegenleistung darf lediglich im Verwertungsrecht oder im Verwertungsrecht zuzüglich der Zahlung eines Entgeltes bestehen. Die Kriterien, auf deren Basis festzustellen ist, ob das Betriebsrisiko beim Konzessionsnehmer liegt, enthält $₫ 105$ Abs. 2 GWB. ${ }^{134}$

III. Zerfaserung der Terminologie im nationalen Recht

Neben diesem legaldefinierten und unionsrechtlich überformten Konzessionsbegriff haben sich ausgehend vom historischen Ursprung auch noch verschiedene alternative Fallgruppen herausgebildet, in denen er Verwendung findet. ${ }^{135} \mathrm{Im}$ Gewerberecht wird der Begriff der „Konzession“ gemäß

131 Nettesheim, in: Kment (Hrsg.), Konzessionen im Umwelt- und Infrastrukturrecht, S. 55, $60 \mathrm{f}$.

132 Richtlinie 2014/23/EU des Europäischen Parlaments und des Rates vom 26. Februar 2014 über die Konzessionsvergabe, ABl. L 94/1 vom 28.3.2014.

133 Die betreffenden Vorgaben des GWB werden im Rahmen der 10. GWB-Novelle nicht verändert, sodass in dem folgenden Abschnitt nicht auf dieses Gesetzgebungsverfahren eingegangen wird.

134 Friton/Stein, in: Pünder/Schellenberg (Hrsg.), Vergaberecht, $\$ 105$ GWB, Rn. 7; zur Genese der Vorschrift insbesondere Wollenschläger, in: Burgi/Dreher (Hrsg.), Beck'scher Vergaberechtskommentar, $\$ 105$ GWB, Rn. 5 ff.

$135 \mathrm{Zu}$ diesem Befund kommt bereits Stern, AöR 84 (1959), S. 137, 148; siehe ebenfalls Rennert, NZBau 2019, 411. 
$\$ 15$ Abs. 2 GewO als Synonym für eine Erlaubnis, Genehmigung oder Bewilligung gebraucht. ${ }^{136}$ Auch wenn der Staat eine Tätigkeit gestattet, die unter ein präventives Verbot mit Erlaubnisvorbehalt fällt, vergibt er entsprechende Konzessionsrechte. ${ }^{137}$

Der Staat setzt „Konzessionen“ auch als Instrument der Lenkungsverwaltung ein. So wird in der juristischen Umgangssprache beispielsweise von „Taxikonzessionen“ gesprochen. ${ }^{138}$ Nur Taxiunternehmern mit entsprechender Genehmigung ist es gestattet, ihre Dienste in einer Kommune anzubieten. ${ }^{139}$ Einen normativen Anklang im Personenbeförderungsgesetz hat der Begriff der „Konzession“ in diesem Kontext jedoch nicht gefunden. ${ }^{140}$ Es besteht ein enger Konnex zur Konzession als Synonym für eine Erlaubnis.

Eng verwandt mit den beiden vorgenannten Konstellationen ist die Nutzung des Begriffes im Kontext der Gewährung von Sondernutzungsrechten. Auch hier wird das betreffende Recht als „Konzession“ bezeichnet. So können Gaststätten an Autobahnen nur mit einer entsprechenden Konzession gemäß $₫ 15$ Abs. 3 FStrG betrieben werden. ${ }^{141}$ In diese Kategorie fällt aber auch die Vergabe von Wegerechten zur Verlegung und zum Betrieb von Infrastruktur. ${ }^{142}$ Ein typischer Fall einer Konzession im Rahmen der Gewährung von Sondernutzungsrechten ist die Vergabe besonderer Wegerechte im Sinne des $₫ 46$ Abs. 2 EnWG. ${ }^{143}$

Der Begriff „Konzession“ wird auch als Ausgestaltungsoption für Public Private Partnerships (PPP) verwendet. Hierunter sind Konstellationen zu verstehen, in denen die Gewährleistungsverpflichtung des Staates gegenüber Dritten auf den Konzessionsnehmer übertragen wird. ${ }^{144}$ Dabei wird

136 Rossi/Pfahl, in: Kment (Hrsg.), Konzessionen im Umwelt- und Infrastrukturrecht, S. 1, 7 ff., sprechen insoweit von einer ,zulassenden Konzession“; Storr, in: Kluth/Müller/Peilert (Hrsg.), Festschrift für Rolf Stober, S. 417, 419 f.

137 In diesem Sinne unter Verweis auf die wasserrechtliche Bewilligung gemäß $₫ 8$ Abs. 1 WHG, Koenig, Die öffentlich-rechtliche Verteilungslenkung, S. 105.

138 Der Begriff wird auch von der Rspr. verwendet, BVerfG, Beschl. v. 4.10.1989, Az. 1 BvL 6/83, NJW 1990, S. 1352; OLG Hamm, Urt. v. 13.6.2002, Az. 18 U 207/01, BeckRS 2002, 7016; auch in der Literatur ist der Begriff häufig verwendet, Dietlein, GewArch 1999, S. 89; Scheidler, GewArch 2011, S. 417.

139 Storr, in: Kluth/Müller/Peilert (Hrsg.), Festschrift für Rolf Stober, S. 417, 420 f.

140 Das PBefG spricht vielmehr von Genehmigungen, vgl. hierzu etwa $\$ 2$ PBefG.

141 Storr, in: Kluth/Müller/Peilert (Hrsg.), Festschrift für Rolf Stober, S. 417, 421.

142 Storr, in: Kluth/Müller/Peilert (Hrsg.), Festschrift für Rolf Stober, S. 417, $421 \mathrm{f}$.

143 Zur Abgrenzung zwischen einfachen und besonderen Wegerechten nachfolgend C. III. 4.

144 Storr, in: Kluth/Müller/Peilert (Hrsg.), Festschrift für Rolf Stober, S. 417, $424 \mathrm{f}$. 
zwar häufig auf Bau- und Dienstleistungskonzessionen zurückgegriffen, die Ausgestaltung ist jedoch nicht auf diese beiden Varianten beschränkt. Vielmehr ist es auch denkbar, dass „Konzessionen“ vergeben werden, wenn keine Bau- oder Dienstleistungen Vertragsgegenstand sind.

Der Begriff der „Konzession“ wird also in verschiedenen, sehr heterogenen Fallgruppen verwendet. Gemeinsam haben diese einen vertikalen Bezug zwischen Hoheitsträger und Privaten. Im Übrigen bestehen jedoch viele Unterschiede. Mal wird der Staat lediglich als „Genehmigungsbehörde" tätig und gestattet Tätigkeiten im Kontext von Verboten mit Erlaubnisvorbehalt, mal überträgt er seine originären Aufgaben samt Hoheitsrechten auf Dritte.

\section{Ergebnis}

Eine einheitliche Definition des Begriffes „Konzession“ ist nicht möglich. Die verschiedenen Konzessionsbegriffe verfügen über einen einheitlichen Ursprung und sind dem französischen Verwaltungsrecht entlehnt. Klar konturiert sind die Bau- und Dienstleistungskonzession, die im nationalen und unionalen Recht legaldefiniert sind. Im Übrigen wird der Begriff der „Konzession“ jedoch sehr unterschiedlich verwendet. Aus diesem Grund ist eine einheitliche Definition bisher nicht gelungen. Vielmehr muss der Begriff im jeweiligen Kontext autonom ausgelegt und abgegrenzt werden. ${ }^{145}$ In der folgenden Analyse soll der Begriff deswegen jeweils mit einem konkretisierenden Beiwort verwendet werden, der die Charakteristika des jeweiligen Kontextes hervorhebt. Eine Aussage, wonach ein bestimmtes Modell kein Konzessionsmodell darstellen könne, weil das Charakteristikum einer bestimmten Begriffsverwendung nicht erfüllt sei, kann vor diesem Hintergrund nicht überzeugen (D. I. 4.). Letztlich handelt es sich um einen so vielseitigen Begriff, dass zugleich alles und nichts hierunter subsumiert werden kann.

\section{B. Exklusivität im Telekommunikationsrecht}

In allen nachfolgend näher zu untersuchenden Konzessionsmodellen werden Rechte, Fördermittel oder sonstige Privilegierungen exklusiv an einen

145 So auch Stern, AöR 84 (1959), S. 137, 149; Storr, in: Kluth/Müller/Peilert (Hrsg.), Festschrift für Rolf Stober, S. 417, 430 f. 
Petenten vergeben. Das sektorspezifische Telekommunikationsrecht gestattet eine exklusive Rechtevergabe aus unterschiedlichen technischen und ökonomischen Gründen. ${ }^{146}$ Exemplarisch sollen die Gründe für und die Ausgestaltung exklusiver Rechtevergaben an dem Umgang mit Funkfrequenzen (dazu I.) sowie Rufnummern (dazu II.) untersucht werden. $\mathrm{Zu}$ dem sollen die regulierungsrechtliche Praxis zur Ermöglichung der Nutzung der Übertragungstechnologie Vectoring (dazu III.) und die regulatorische Behandlung neuer Netzbestandteile gemäß Art. 76 EKEK (dazu IV.) dargestellt werden. Aus der Zusammenschau der vorgenannten Konstellationen sollen Rückschlüsse auf die Zulässigkeit von und die Anforderungen an eine exklusive Rechtevergabe im Telekommunikationsrecht gezogen werden (dazu V.).

\section{Exklusivität bei der Vergabe von Funkfrequenzen}

Theoretisch existiert ein unlimitiertes Frequenzspektrum. Die Nutzung von Frequenzen durch einen Anwender führt nicht dazu, dass andere Anwender nicht auf die gleiche Frequenz zugreifen könnten. Allerdings resultieren aus der Funktion von Funkfrequenzen als integralem Bestandteil des Mobilfunknetzes hohe Qualitätsanforderungen, die insbesondere eine störungsfreie Signalübertragung erforderlich machen. ${ }^{147}$ Die parallele Verwendung einer Funkfrequenz durch verschiedene Nutzer kann dazu führen, dass sich Funkwellen gegenseitig überlagern. Diese sogenannten „Interferenzen“ führen zu erheblichen Qualitätseinbußen bei der Signalübertragung. ${ }^{148}$ Dieses Problem intensiviert sich dadurch, dass Frequenzen auch über die Landesgrenzen hinaus reichen. Aus diesem Grund ist eine internationale Koordination erforderlich, um Interferenzen zu vermeiden. ${ }^{149}$ Hinzu treten weitere technische Gründe, welche die Anzahl der

146 Kühling/Toros, Rechtliche Rahmenbedingungen für die Schaffung von Anreizen für einen flächendeckenden Ausbau von Glasfaserinfrastrukturen, S. 102.

147 Neumann/Koch, Telekommunikationsrecht, S. 341; vgl. zu den technisch-physikalischen Grundlagen der Nutzung von Frequenzen, Naab, Die Versteigerung knapper Ressourcen durch den Staat, S. 35 ff.

148 Bumke, Frequenzvergabe nach dem Telekommunikationsgesetz, S. 49; Kühling/ Schall/Biendl, Telekommunikationsrecht, Rn. 569; Meister, Das telekommunikationsrechtliche Frequenzplanungsrecht im System des allgemeinen Planungsrechts, S. 32; Neumann/Koch, Telekommunikationsrecht, S. 341.

149 Bumke, Frequenzvergabe nach dem Telekommunikationsgesetz, S. 39 ff.; Naab, Die Versteigerung knapper Ressourcen durch den Staat, S. 79 ff. 
nutzbaren Funkfrequenzen weiter reduzieren. Je höher die Frequenz etwa ist, desto komplexer wird ihre Nutzung als Bestandteil des Mobilfunknetzes. ${ }^{150}$ Das Zusammenspiel dieser technischen Aspekte führt dazu, dass ein faktisch unbegrenzt verfügbares Gut durch die Nutzung als Bestandteil der Telekommunikationsinfrastruktur tatsächlich knapp ist. ${ }^{151}$ Die Frequenzen müssen exklusiv an einzelne Anbieter vergeben werden, um die Qualitätsanforderungen zu erfüllen.

Mit verschiedenen Maßnahmen versucht der Staat, die negativen Auswirkungen der exklusiven Rechtevergabe auf den Wettbewerb so gering wie möglich zu halten. Hierzu zählt, dass die Frequenzen in einem Versteigerungsverfahren vergeben werden, in dem der Anbieter mit dem höchsten Gebot den Zuschlag erhält. Um neben der Sicherung des Wettbewerbs, auch die Verfolgung zusätzlicher Interessen sicherzustellen, wird der Verwaltungsakt durch Nebenbestimmungen ergänzt. Hierbei handelt es sich um Versorgungsauflagen, mit denen beispielsweise eine flächendeckende Mobilfunkversorgung sichergestellt werden soll. ${ }^{152}$

\section{Exklusivität bei der Vergabe von Rufnummern}

Ähnlich ist die Situation bei der Vergabe von Rufnummern. Zwar lassen sich Zahlen faktisch unbegrenzt kombinieren und auf diese Weise Rufnummern konfigurieren. Rufnummern müssen aus Praktikabilitätsgründen jedoch stets einprägsam und merkbar sein. ${ }^{153}$ Dies schränkt die Anzahl der nutzbaren Zahlenkombinationen erheblich ein. Der Effekt wird dadurch verstärkt, dass in einer globalisierten Welt eine internationale Terminierung von Sprachkommunikation möglich sein muss. Hierzu wurde ein internationaler Regelungsrahmen etabliert, der unter anderem die maximale Rufnummernlänge und spezifische Standards wie international einheitliche Ländervorwahlen vorschreibt. ${ }^{154}$

150 Meister, Das telekommunikationsrechtliche Frequenzplanungsrecht im System des allgemeinen Planungsrechts, S. 30 ff.; Neumann/Koch, Telekommunikationsrecht, S. 341.

$151 \mathrm{Naab}$, Die Versteigerung knapper Ressourcen durch den Staat, S. $36 \mathrm{ff}$.

152 Die BNetzA hat von diesem Mittel im Rahmen der aktuellen 5G-Frequenzversteigerung umfassenden Gebrauch gemacht, BNetzA, Entsch. v. 14.5.2018, Az. BK 1-17/001; sowie BNetzA, Entsch. v. 26.11.2018, Az. BK 1-17/001.

153 Neumann/Koch, Telekommunikationsrecht, S. 342.

154 Damit sind die Regelungen zwar nicht rechtlich, aber faktisch verbindlich, vgl. hierzu Neumann/Koch, Telekommunikationsrecht, S. 342. 
Technische Vorbedingungen limitieren die Anzahl der tatsächlich nutzbaren Rufnummern weiter. So ist eine Mehrfachvergabe von Rufnummern nicht möglich. Sie hätte zur Folge, dass es dem Zufall überlassen würde, bei welchem Teilnehmer der Anruf terminiert würde. ${ }^{155}$ Ein solches Risiko wäre mit den Qualitätsanforderungen an eine sichere Telekommunikationsinfrastruktur nicht zu vereinbaren. Rufnummern sind jedoch nicht derart knapp, dass der Staat einen Wettbewerb um sie im Sinne einer Versteigerung durchführt. Vielmehr wird der Staat in Form einer Verteilungsverwaltung aktiv und stellt die Einhaltung der Standards und Regeln durch die Telekommunikationsunternehmen sicher, damit die begrenzten Nummerierungsressourcen effizient eingesetzt werden.

\section{Exklusivität beim Vectoring}

Die Signalübertragungstechnologie Vectoring setzt einen exklusiven $\mathrm{Zu}$ griff auf die gesamte Teilnehmeranschlussleitung bis hin zum Endnutzer voraus, auf die normalerweise ein entbündelter Zugang gewährt wird. ${ }^{156}$

Unter Vectoring ist nach der Definition der International Telecommunication Union (ITU) aus dem Jahr 2010 ein Signalübertragungsverfahren zu verstehen, das die durch ein Kabel übertragenen Signale so koordiniert, dass das Übersprechen unterbunden und auf diese Weise die Übertragungsqualität verbessert werden kann. ${ }^{157}$ Die beim Vectoring genutzte Übertragungstechnologie heißt „Very High Speed Digital Subscriber Line 2“ (VDSL2). ${ }^{158}$ Verbreitet ist auch die Übertragungstechnologie Super-

155 Neumann/Koch, Telekommunikationsrecht, S. 341.

156 Die diesbezüglichen Regulierungsentscheidungen wurden in der Literatur zuweilen kritisch diskutiert, Dietlein/Brandenberg, IR 2005, S. 208 ff.; Dietlein/Brandenberg, IR 2005, S. 245 ff.; Kirchner, CR 2013, S. 85 ff.; Monopolkommission, 9. Sektorgutachten Telekommunikation (2015), Rn. 28 ff.; Monopolkommission, 10. Sektorgutachten Telekommunikation (2017), Rn. 59 ff.; Sörries, CR 2015, S. $162 \mathrm{ff}$.

157 Die Definition der ITU in englischer Sprache in ITU-T stellt sinngemäß die gleichen Anforderungen auf, Recommendation G.993.5 (01/2015), Self-FEXT cancellation (vectoring) for use with VDSL2 transceivers, S. 1, abrufbar unter: https: //www.itu.int/rec/T-REC-G.993.5-201902-I/en (zuletzt abgerufen am 1.7.2020); Bromen, in: Auer-Reinsdorff/Conrad (Hrsg.), Handbuch IT- und Datenschutzrecht, $₫ 4$, Rn. 18.

158 Der Übertragungsstandard hat sich weiterentwickelt, sodass mittlerweile eine Standardisierung für VDSL2 vorliegt, ITU-T, Recommendation G.993.5 (01/2015), Self-FEXT cancellation (vectoring) for use with VDSL2 transceivers, 
vectoring, durch die aufgrund der Nutzung höherer Frequenzen noch höhere Übertragungsgeschwindigkeiten erzielt werden können. ${ }^{159}$ Es handelt sich um die Nachfolgetechnologien der „Asymmetric Digital Subscriber Line“ (ADSL). Bei ADSL wurden Frequenzen von bis zu 2,2 Mhz verwendet und es konnten Übertragungsraten von 16 bis $18 \mathrm{Mbit} / \mathrm{s}$ im Up- und Download erreicht werden. Der „Digital Subscriber Line Access Multiplexer" (DSLAM) wurde am Hauptverteiler installiert und fungierte dort als Verbindungsglied zwischen Teilnehmeranschlussleitung und Konzentratornetz. VDSL und seine Nachfolgetechnologien setzen technisch ein hybrides Netz voraus, sodass bis zum Kabelverzweiger Glasfaser verlegt und der Teilnehmeranschluss mit Kupfer realisiert wird. ${ }^{160}$ Der DSLAM befindet sich nicht mehr im Hauptverteiler, sondern wird in einem Multifunktionsgehäuse direkt am Kabelverzweiger installiert. ${ }^{161}$ Es werden Frequenzen oberhalb von 2,2 Mhz genutzt und Bandbreiten von bis zu $200 \mathrm{Mbit} / \mathrm{s}$ im Up- und Download realisiert. Die Technologie wird durch das Verlegen der Abnahmestelle in die Nähe des Nutzers immer weiter fortentwickelt und beschleunigt. ${ }^{162}$

Mit Vectoring wird die Störung der Signalübertragung durch das sogenannte „Übersprechen“ unterbunden. Die Problematik entsteht dadurch, dass die Leitungen stets in Bündeln zu mehreren Kupferkabeln verlegt werden, sodass es zum Überspringen von Leitungsimpulsen auf die benachbarten Leitungen kommt. Hierdurch werden Übertragungsqualität und -geschwindigkeit erheblich beeinträchtigt. Die Vectoring-Technologie antizipiert etwaige Störungen und sendet entsprechende Gegenstörsignale aus. ${ }^{163}$ Damit die Technologie eingesetzt werden kann, wird ein Zugriff auf alle Teilnehmeranschlussleitungen im jeweiligen Kabelbündel benötigt. ${ }^{164}$ Nur so kann das Signal über die richtige Teilnehmeranschlusslei-

abrufbar unter: https://www.itu.int/rec/T-REC-G.993.5-201902-I/en (zuletzt abgerufen am 1.7.2020).

159 https:/www.golem.de/news/250-mbit-s-super-vectoring-der-telekom-hat-nur-250 -000-kunden-2005-148485.html (zuletzt abgerufen am 1.7.2020).

160 Insoweit wird auch von einem hybriden Netzaufbau gesprochen, Mertz/Pollakowski, xDSL \& Access Networks, S. 189.

$161 \mathrm{Zu}$ den technischen Einzelheiten, Keller, Breitbandkabel und Zugangsnetze, S. $412 \mathrm{ff}$.

162 Bromen, in: Auer-Reinsdorff/Conrad (Hrsg.), Handbuch IT- und Datenschutzrecht, $\mathbb{\$} 4$, Rn. 19.

163 https://breitbandbuero.de/wp-content/uploads/2017/11/171006_BBB_Infoblatt_ Vectoring.pdf (zuletzt abgerufen am 1.7.2020).

164 https://breitbandbuero.de/wp-content/uploads/2017/11/171006_BBB_Infoblatt_ Vectoring.pdf (zuletzt abgerufen am 1.7.2020). 
tung geleitet und können die übrigen Leitungen mit Störsignalen beschaltet werden. Die Technik kann deshalb nur dann sinnvoll eingesetzt werden, wenn lediglich ein Infrastrukturanbieter Zugriff auf die Teilnehmeranschlussleitungen an dem jeweiligen Einsatzpunkt der Technologie hat. Ein exklusiver Zugang ist mithin eine technische Notwendigkeit. ${ }^{165}$ Aus diesem Grund hat die Bundesnetzagentur (BNetzA) bestehende Regulierungsverfügungen ${ }^{166}$ abgeändert, um die Nutzung des Vectorings im Nahbereich ${ }^{167}$ und im Außenbereich ${ }^{168}$ zu ermöglichen. Der Zugangsanspruch auf die Teilnehmeranschlussleitung wird durch den Anspruch auf den $\mathrm{Zu}-$ gang zu einem virtuellen Ersatzprodukt ersetzt. Mit der Signalübertragungstechnologie Vectoring werden unter weitgehender Nutzung bereits existenter Infrastruktur die verfügbaren Bandbreiten erheblich erhöht (Kapitel 2, B. II. 2. a.).

IV. Regulatorische Behandlung neuer Netzbestandteile gemäß Art. 76 EKEK

Der Europäische Kodex für die elektronische Kommunikation ermöglicht gemäß Art.76 EKEK eine privilegierte regulatorische Behandlung neuer Netzbestandteile, sofern Kooperationsvereinbarungen zwischen Unternehmen mit beträchtlicher Marktmacht im Sinne des Art. 67 EKEK und anderen Unternehmen geschlossen werden (Kapitel 5, D. II. 3. b. aa.). Auf diese Weise sollen Unsicherheiten im Hinblick auf die Marktentwicklung ausgeglichen werden, die dadurch entstehen, dass die Prognosen hinsichtlich der zukünftigen Nutzung von Datenübertragungsraten ungenau sind (Ka-

165 BVerwG, Urt. v. 21.9.2018, Az. 6 C 50.16, BVerwGE 163, S. 136, Rn. 3.

166 BNetzA, Beschl. v. 22.3.2011, Az. BK 3g-09/85.

167 BNetzA, Beschl. v. 21.3.2011, Az. BK 3d-12/131; bestätigt durch VG Köln, Urt. v. 17.3.2017, Az. VG 9 K 8589/16, N\&R 2017, S. 187 ff. und BVerwG, Urt. v. 21.9.2018, Az. 6 C 8.17, BVerwGE 163, S.136. Der Nahbereich umfasst Anschlüsse, bei denen zwischen Kabelverzweiger und Betriebsstelle nicht mehr als 550 Meter liegen; hierzu umfassend Offenbächer, Die Regulierung des Vectoring, S. $284 \mathrm{ff}$.

168 BNetzA, Beschl. v. 1.9.2016, BK 3g-15/004; bestätigt durch VG Köln, Urt. v.

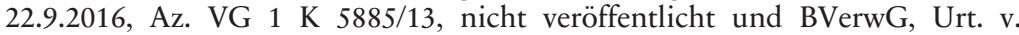
21.9.2018, Az. 6 C 50.16, BVerwGE 163, S.136. Der Außenbereich erfasst alle Anschlüsse, die außerhalb des Nahbereichs liegen; hierzu umfassend Offenbächer, Die Regulierung des Vectoring, S. $307 \mathrm{ff}$. 
pitel 2, C. II. 2.). ${ }^{169}$ Diese Unsicherheit wird in der Kalkulation von Investitionen mit einem Risikoaufschlag berücksichtigt. Dabei ist festzustellen, dass ein hoher Risikoaufschlag dazu beiträgt, dass eine Investition insgesamt als unrentabel zu klassifizieren ist. ${ }^{170} \mathrm{Je}$ mehr Unternehmen sich an einer Kooperation beteiligen, desto eher können die Lasten umgelegt werden. ${ }^{171}$ Problematisch ist jedoch, dass durch Kooperationen die Anbieterkonzentration vermindert und der Wettbewerb geschwächt werden kann. ${ }^{172} \mathrm{Um}$ positive und negative Aspekte in einen schonenden Ausgleich zu bringen, hat der EU-Gesetzgeber eine Privilegierung in Form von Regulierungserleichterungen unter Abgabe von Verpflichtungszusagen ermöglicht. Um von der Privilegierung profitieren zu können, muss das marktmächtige Unternehmen eine Verpflichtungszusage gemäß Art.79 Abs. 1 EKEK abgeben und die Netze mit sehr hoher Kapazität ${ }^{173}$ für kooperative Investitionen öffnen (Art. 76 Abs. 1 UAbs. 1 EKEK). Die Verpflichtungszusage wird auf Basis der Kriterien des Art. 76 Abs. 1 UAbs. 2 EKEK überprüft (Kapitel 5, D. II. 3. b. aa.).

Im Kern soll so ein Gleichgewicht zwischen Investitionsanreizen und Wettbewerb sichergestellt werden. Wenn die Voraussetzungen erfüllt sind, wird die Verpflichtungszusage für bindend erklärt und es werden keine zusätzlichen Verpflichtungen auferlegt (Art. 79 Abs. 2 UAbs. 1 EKEK). Die Zugangsverpflichtungen aus der Regulierungsverfügung werden also wie beim Vectoring durch eine freiwillige Selbstverpflichtung ersetzt (B. III.). ${ }^{174}$ Diese Regulierungserleichterung betrifft jedoch nur die Netzbestandteile von Netzen mit sehr hoher Kapazität. Die Ausnahme wird durch die Möglichkeit zur Auferlegung von Regulierungsverpflichtungen in Sonderfällen gemäß Art. 76 Abs. 1 UAbs. 2 und 3 EKEK relativiert und unterliegt gemäß Art. 76 Abs. 3 EKEK einem strengen Monitoring. Die Exklusivität unterscheidet sich von den vorgenannten Fällen (B. I.-III.) da-

169 Kühling, WuW 2019, S. 555, 556, spricht insoweit von einer „Wette auf die Zukunft".

170 Sickmann/Neumann, K\&R 2018, S. 92, 93. Dies betrifft insbesondere auch Gebiete, in denen ein Infrastrukturwettbewerb unter diesen Voraussetzungen ineffizient ist und wird auch in Erwägungsgrund 198 des EKEK herausgestellt.

171 Sickmann/Neumann, K\&R 2018, S. 92, 93. Hierdurch kann dazu beigetragen werden, dass Investitionen auch für kleine Unternehmen finanzierbar sind, welche die Risiken nicht alleine hätten tragen können. Dies wird auch in Erwägungsgrund 198 des EKEK herausgestellt.

172 Sickmann/Neumann, K\&R 2018, S. 92, 93 f.

173 Eine Legaldefinition für das Netz mit sehr hoher Kapazität findet sich in Art. 2 Nr. 2 EKEK.

174 Umfassend hierzu Kapitel 5, D. II. 3. a. bb. 
durch, dass sie technisch nicht notwendig ist und einen Investitionsanreiz setzen soll, und bildet damit einen Ausnahmefall im Telekommunikationsrecht.

V. Rückschlüsse und generelle Anforderungen an den Umgang mit Exklusivität im Telekommunikationsrecht

Das nationale und das unionale Telekommunikationsrecht lassen eine exklusive Vergabe von Rechten grundsätzlich nur restriktiv zu. ${ }^{175}$ Die exklusive Vergabe von Funkfrequenzen und Rufnummern ist aus technischen Gründen zwingend erforderlich. Es findet eine staatliche Verteilungsverwaltung statt, die im Rahmen der Frequenzvergabe als Versteigerungsverfahren ausgestaltet ist. Die Zulassung von exklusiven Zugriffsrechten auf Infrastruktur ist auch im Kontext der Übertragungstechnologie Vectoring technisch notwendig. Der Wettbewerb wird durch einen Anspruch auf ein Ersatzzugangsprodukt ausreichend geschützt, sodass die negativen Folgen hinreichend ausgeglichen werden. Die regulatorische Behandlung von Kooperationen im Hinblick auf Bestandteile von Netzen mit hoher Kapazität ist hingegen eine Ausnahme, da der exklusive Zugriff auf die Infrastruktur nicht technisch notwendig ist. Vielmehr sollen lediglich die Investitionsrisiken vermindert werden. Eine freiwillige Selbstverpflichtung zur $\mathrm{Zu}-$ gangsgewährung stellt sicher, dass der Wettbewerb möglichst wenig belastet wird. Der Grundsatz, dass Exklusivität im Telekommunikationsrecht lediglich aus technischen Gründen gestattet wird, wird hierdurch aufgeweicht. Sobald exklusive Rechte gewährt werden, wird durch Kompensationsmechanismen jedoch stets ein ausreichender Wettbewerb sichergestellt. Dies haben alle exklusiven Rechte gemeinsam.

175 Kühling/Toros, Rechtliche Rahmenbedingungen für die Schaffung von Anreizen für einen flächendeckenden Ausbau von Glasfaserinfrastrukturen, S. 102 f., die ihren Fokus jedoch auf die Verfügbarkeit von Gütern legen und nicht explizit auf die Übertragungstechnologie „Vectoring“ und die Regulierungserleichterungen gemäß Art. 76 EKEK eingehen. 


\section{Konzessionsmodell auf Basis der Vergabe exklusiver Wegerechte}

Das Konzessionsmodell auf Basis der Vergabe exklusiver Wegerechte ist ein Konzept zur Beschleunigung des Ausbaus von Glasfaserinfrastrukturen in Gebieten mit Netzausbaudefizit. Hierbei handelt es sich um Gebiete, in denen der Glasfaserausbau nicht im ausreichenden Maße eigenwirtschaftlich vorangetrieben wird. Das Konzessionsmodell wird besonders prominent vom Deutschen Landkreistag vertreten, der es als Teil eines Strategiepapiers im Jahr 2017 der Öffentlichkeit vorgestellt hat. ${ }^{176}$ Dieser Ausgestaltungsvorschlag soll als Referenzmodell für die nachfolgende Untersuchung dienen. ${ }^{177}$ Es handelt sich um einen Teil der Gesamtstrategie, mit deren Hilfe eine flächendeckende Versorgung mit Glasfaserinfrastrukturen zu erschwinglichen Preisen realisiert werden soll. ${ }^{178}$ Kernkritikpunkte des Deutschen Landkreistages, die mithilfe des Modells beseitigt werden sollen, sind fehlende Anreize für Investitionen in den Glasfaserausbau in Randlagen und die mangelnde Verbindlichkeit von Ausbauzusagen der Infrastrukturunternehmen im Markterkundungsverfahren, die verschiedene Folgeprobleme mit sich brächten. ${ }^{179}$

Basis für die Untersuchung sollen eine Analyse von Aufbau und Grundstruktur des Modells (dazu I.) sowie eine Einordnung in die zuvor identifizierten Kategorien zur Verwendung des Konzessionsbegriffes in der deutschen Rechtswissenschaft sein (dazu II.). ${ }^{180}$ Im Anschluss daran soll die Vereinbarkeit des Konzeptes mit dem geltenden unionalen und nationalen

176 Deutscher Landkreistag, Flächendeckende Breitbandversorgung zu wirtschaftlichen Bedingungen sicherstellen; Kübling/Toros, Rechtliche Rahmenbedingungen für die Schaffung von Anreizen für einen flächendeckenden Ausbau von Glasfaserinfrastrukturen, S. 97 ff.; Wernick/Queder/Strube Martins u.a., Ansätze zur Glasfaser-Erschließung unterversorgter Gebiete, S. 47 ff.; Wernick/Queder/ Strube Martins u.a., Gigabitnetze für Deutschland, S. $113 \mathrm{ff}$.; kritisch Kühling, N\&R 2019, S. 1; ebenfalls kritisch Monopolkommission, 10. Sektorgutachten Telekommunikation (2017), Rn. $201 \mathrm{ff}$.

177 Dieselbe Vorgehensweise liegt der Analyse von Kühling/Toros, Rechtliche Rahmenbedingungen für die Schaffung von Anreizen für einen flächendeckenden Ausbau von Glasfaserinfrastrukturen, S. 95 zugrunde.

178 Deutscher Landkreistag, Flächendeckende Breitbandversorgung zu wirtschaftlichen Bedingungen sicherstellen, S. $1 \mathrm{ff}$.

179 Deutscher Landkreistag, Flächendeckende Breitbandversorgung zu wirtschaftlichen Bedingungen sicherstellen, S. 2; Kühling/Toros, Rechtliche Rahmenbedingungen für die Schaffung von Anreizen für einen flächendeckenden Ausbau von Glasfaserinfrastrukturen, S. 97.

180 Die einzelnen Begriffsverwendungen werden dargestellt unter A. 
Recht aus verschiedenen Blickwinkeln untersucht werden. So ist etwa fraglich, welche allgemeinen Steuerungsvorgaben das Telekommunikationsrecht enthält, die bei der Umsetzung des Konzeptes Berücksichtigung finden müssten (dazu III.). Dieselbe Frage stellt sich auch im Hinblick auf andere rechtliche Rahmenbedingungen für die Ausgestaltung des Konzessionsvergabeverfahrens (dazu IV.). Um eine Umstellung des Regelungsregimes und die Funktionsfähigkeit des Modells sicherzustellen, ist zudem zu untersuchen, wie mit der Bestandsinfrastruktur im Konzessionsgebiet und bereits erteilten Wegerechten umgegangen werden müsste und welche rechtlichen Anforderungen sich hieraus ergeben (dazu V.).

\section{Aufbau und Grundstruktur}

Das Konzessionsmodell auf Basis der Vergabe exklusiver Wegerechte soll in solchen Gebieten zum Einsatz kommen, in denen ein Netzausbaudefizit besteht und deswegen in absehbarer Zeit nicht mit einem Auf- und Ausbau hochleistungsfähiger digitaler Infrastrukturen zu rechnen ist. ${ }^{181}$ Die Datengrundlage für die Gebietsabgrenzung soll in einem verbindlichen Markterkundungsverfahren gewonnen werden. Je heterogener und größer die Gebiete sind und je mehr lukrative Bestandteile ein Konzessionsgebiet hat, desto eher können die Ziele des Konzessionsmodells auf Basis der Vergabe exklusiver Wegerechte erreicht werden. ${ }^{182}$

Der Ausgestaltungsvorschlag für das Konzessionsmodell auf Basis der Vergabe exklusiver Wegerechte orientiert sich am Energierecht. Dort wird zwischen einfachen ( $\$ 46$ Abs. 1 EnWG) und qualifizierten ( $\$ 46$ Abs. 2 EnWG) Wegerechten unterschieden. ${ }^{183}$ Einfache Wegerechte dienen dem Aufbau von Versorgungsinfrastruktur, mit deren Hilfe einzelne Endnutzer angeschlossen werden. ${ }^{184}$ Die qualifizierten Wegerechte dienen hingegen der allgemeinen Versorgung und werden als „Konzessionen“ bezeich-

181 Monopolkommission, Sektorgutachten 78 (Telekommunikation 2017), Rn. 202.

182 Kühling/Toros, Rechtliche Rahmenbedingungen für die Schaffung von Anreizen für einen flächendeckenden Ausbau von Glasfaserinfrastrukturen, S. 96; Wernick/Queder/Strube Martins u.a., Ansätze zur Glasfaser-Erschließung unterversorgter Gebiete, S. 52; Wernick/Queder/Strube Martins u.a., Gigabitnetze für Deutschland, S. 113.

183 Dehenn, Die Vergabe von Wegekonzessionen nach $₫ 46$ EnWG, S. 52 ff.

184 Albrecht, in: Schneider/Theobald (Hrsg.), Recht der Energiewirtschaft, $\mathbb{9}$, Rn. 62. 
net. ${ }^{185}$ Diese Differenzierung soll nach Vorstellung des Deutschen Landkreistages einschließlich des Begriffes des „Netzes der allgemeinen Versorgung" in das Telekommunikationswegerecht übertragen werden (C. II. 1. b. cc. (1)). Die „Konzessionen“ wären danach behördlich vergebene, zeitlich begrenzte, exklusive Wegerechte zum Ausbau hochleistungsfähiger digitaler Infrastrukturen, die in den Konzessionsgebieten errichtet und betrieben werden müssen. ${ }^{186}$

Als Konzessionsvergabebehörde werden die BNetzA oder kommunale Gebietskörperschaften vorgeschlagen. ${ }^{187}$ Das exklusive Wegerecht soll mit verschiedenen Pflichten verknüpft werden. Hierzu zählt insbesondere eine Ausbau- und Betriebsverpflichtung für die neu errichtete Infrastruktur. ${ }^{188}$ Während die Betriebspflicht an andere Unternehmen delegiert werden können soll, sollen die Ausbaupflichten in einem Meilensteinplan konkretisiert werden. ${ }^{189}$

Im geltenden Regelungsregime werden Telekommunikationswegerechte an alle Petenten vergeben, welche die gesetzlichen Anforderungen erfüllen. Das vorliegende Konzessionsmodell möchte diese Vergabepraxis in den Konzessionsgebieten durch eine exklusive Rechtevergabe ablösen. Auf diese Weise soll ein Infrastrukturmonopol entstehen. Es findet ein Wettbewerb um das Konzessionsrecht statt. Im Übrigen wird der Wettbewerb von der vorgelagerten Infrastrukturebene vollständig auf die nachgelagerte Diensteebene verlagert. Die konzessionierte Infrastruktur müsste zwangsläufig von Telekommunikationsunternehmen genutzt werden, die Dienste

185 Albrecht, in: Schneider/Theobald (Hrsg.), Recht der Energiewirtschaft, $\$ 9$, Rn. 62.

186 Deutscher Landkreistag, Flächendeckende Breitbandversorgung zu wirtschaftlichen Bedingungen sicherstellen, S. 1; Kühling/Toros, Rechtliche Rahmenbedingungen für die Schaffung von Anreizen für einen flächendeckenden Ausbau von Glasfaserinfrastrukturen, S. 96; Wernick/Queder/Strube Martins u.a., Ansätze zur Glasfaser-Erschließung unterversorgter Gebiete, S. 49, die jedoch von „Lizenzen" sprechen.

187 Deutscher Landkreistag, Flächendeckende Breitbandversorgung zu wirtschaftlichen Bedingungen sicherstellen, S. 7.

188 Um einen Wholesale-Only-Betrieb des Netzes, also eine ausschließliche Erbringung von Diensten auf der Vorleistungsebene, zu ermöglichen, sollen die jeweiligen Pflichten aber auch auf Dritte übertragen werden können, Deutscher Landkreistag, Flächendeckende Breitbandversorgung zu wirtschaftlichen Bedingungen sicherstellen, S. 2 und S. 7; Kühling/Toros, Rechtliche Rahmenbedingungen für die Schaffung von Anreizen für einen flächendeckenden Ausbau von Glasfaserinfrastrukturen, S. 96.

189 Wernick/Queder/Strube Martins u.a., Ansätze zur Glasfaser-Erschließung unterversorgter Gebiete, S. 49. 
im Konzessionsgebiet anbieten wollen. Durch Zugangsentgelte und die gesicherten Bedingungen inklusive eines umfassenden Überbauschutzes sollen die Investitionsrisiken minimiert und ein lukrativer Glasfaserausbau in den Konzessionsgebieten ermöglicht werden. ${ }^{190}$ Etwaige fortbestehende Finanzierungslücken können zusätzlich mit finanziellen Förderprogrammen geschlossen werden. ${ }^{191}$

Um das Ziel des Modells zu erreichen, müssten bereits erteilte Wegerechte entzogen und die Nutzung von Bestandsinfrastruktur verhindert werden. Denn obwohl in den potentiellen Konzessionsgebieten noch keine Glasfaserinfrastruktur verlegt worden ist, kann davon ausgegangen werden, dass die Telekommunikationsunternehmen in den betreffenden Gebieten eigenwirtschaftlich eine Basisversorgung zur Verfügung stellen, die auf alternative Übertragungstechnologien zurückgreift. ${ }^{192}$ Darüber hinaus werden unter dem geltenden Regelungsregime fortlaufend neue Wegerechte vergeben. Der Deutsche Landkreistag schlägt verschiedene Handlungsoptionen zum Umgang mit Bestandsinfrastruktur vor. Unternehmen, die bereits über Infrastrukturen im Konzessionsgebiet verfügen, könnten etwa nach dem Vorbild des Energierechts dazu verpflichtet werden, ihr Eigentum gegen ein angemessenes Entgelt an den Konzessionsnehmer zu übertragen. ${ }^{193}$ Alternativ könnte die Infrastruktur im Eigentum des Anbieters verbleiben und dieser zwangsweise dazu verpflichtet werden, die Infrastruktur an den Netzanbieter zu verpachten. Darüber hinaus bestünde auch die Möglichkeit, lediglich ein Ausbauverbot für alternative

190 Kritisch Monopolkommission, 10. Sektorgutachten Telekommunikation (2017), Rn. 204, mit Bedenken im Hinblick auf den Grundsatz der Technologieneutralität.

191 Es wird jedoch mit einer deutlichen Reduktion des Förderbedarfes gerechnet; Deutscher Landkreistag, Flächendeckende Breitbandversorgung zu wirtschaftlichen Bedingungen sicherstellen, S. 7.

192 Bislang wurde der Universaldienstmechanismus aus diesem Grund für den Telekommunikationssektor noch nicht aktiviert; zur Aktualisierung des Universaldienstregimes Kübling/Toros, Rechtliche Rahmenbedingungen für die Schaffung von Anreizen für einen flächendeckenden Ausbau von Glasfaserinfrastrukturen, S. 15 ff.; Kühling/Toros, N\&R 2019, S. 258 ff.

193 Eine entsprechende Regelung für das Energierecht findet sich in $\mathbb{} 46$ Abs. 2 EnWG; Deutscher Landkreistag, Flächendeckende Breitbandversorgung zu wirtschaftlichen Bedingungen sicherstellen, S. 7; Kühling/Toros, Rechtliche Rahmenbedingungen für die Schaffung von Anreizen für einen flächendeckenden Ausbau von Glasfaserinfrastrukturen, S. 96. 
Infrastrukturanbieter im Konzessionsgebiet zu verhängen. ${ }^{194}$ Bereits erteilte Wegerechte, die noch nicht zum Ausbau von Telekommunikationsinfrastrukturen genutzt worden sind, sollen widerrufen werden. ${ }^{195}$

\section{Einordnung des Konzessionsmodells auf Basis exklusiver Wegerechte}

Im Konzessionsmodell auf Basis der Vergabe exklusiver Wegerechte wird der Begriff der „Konzession“ für behördlich vergebene, zeitlich begrenzte und exklusive Wegerechte zum Ausbau von Glasfaserinfrastrukturen im Konzessionsgebiet verwendet. ${ }^{196}$ Wie bereits dargestellt, wird der Begriff der „Konzession“ in der deutschen Rechtswissenschaft zwar nicht einheitlich verwendet, es haben sich jedoch bestimmte Fallgruppen herausgebildet (A.). Bei der „Konzession“ im Sinne des vorliegenden Konzessionsmodells könnte es sich um eine Bau- oder Dienstleistungskonzession im Sinne des Vergaberechts handeln (dazu 1.). Denkbar ist auch eine Einordnung in das weite alternative Verwendungsspektrum (dazu 2.). Eine eingehende Untersuchung ist von entscheidender Bedeutung, um die maßgeblichen rechtlichen Rahmenbedingungen identifizieren zu können.

\section{Bau- oder Dienstleistungskonzession}

Würde es sich bei der „Konzession“ des vorliegenden Modells um eine Bau- oder Dienstleistungskonzession handeln, müsste sich das Konzessionsvergabeverfahren an den Vorgaben des Vergaberechts orientieren. Die Begriffe Bau- und Dienstleistungskonzession sind, wie dargelegt, in Art. 5 Nr. 1 KVRL und in $\$ 105$ Abs. 1 GWB legaldefiniert (A. II.). Im Anschluss an die Abgrenzung des Vertragsgegenstandes (dazu a.) ist zu untersuchen,

194 Deutscher Landkreistag, Flächendeckende Breitbandversorgung zu wirtschaftlichen Bedingungen sicherstellen, S. 7; Kühling/Toros, Rechtliche Rahmenbedingungen für die Schaffung von Anreizen für einen flächendeckenden Ausbau von Glasfaserinfrastrukturen, S. 97.

195 Deutscher Landkreistag, Flächendeckende Breitbandversorgung zu wirtschaftlichen Bedingungen sicherstellen, S. 7; Kühling/Toros, Rechtliche Rahmenbedingungen für die Schaffung von Anreizen für einen flächendeckenden Ausbau von Glasfaserinfrastrukturen, S. 97.

196 Deutscher Landkreistag, Flächendeckende Breitbandversorgung zu wirtschaftlichen Bedingungen sicherstellen, S. 1. 
ob die Tatbestandsvoraussetzungen des einschlägigen Konzessionsbegriffes erfüllt sind (dazu b.).

\section{a) Einordnung des Vertragsgegenstandes als Bau- oder Dienstleistung}

Für die Abgrenzung von Bau- und Dienstleistungskonzessionen ist der Gegenstand der vertraglichen Leistungsverpflichtung maßgeblich. Eine Dienstleistungskonzession kann nur vorliegen, wenn der Vertragsgegenstand keine „Bauleistung“ ist (A. II.).

Der Ausbau der Glasfaserinfrastruktur im Konzessionsgebiet könnte eine Bauleistung darstellen. Der Begriff der Bauleistung ist normativ nicht weiter konkretisiert. Allerdings enthält das GWB in $\mathbb{1 0 5}$ Abs. 3 Nr. 1 Abgrenzungskriterien für den Begriff des „Bauauftrages“. Hierunter sind demnach „Verträge über die Ausführung (...) von Bauleistungen im $\mathrm{Zu}$ sammenhang mit einer der Tätigkeiten, die in Anhang II der Richtlinie 2014/24/EU ${ }^{197}$ (...) und Anhang I der Richtlinie 2014/25/EU ${ }^{198}$ (...) genannt sind (...) “, zu verstehen. In diesen Anhängen sind verschiedene Codes für Vertragsgegenstände aufgelistet, durch die eine exakte Identifikation der vertraglichen Leistung in der gesamten EU möglich wird. ${ }^{199}$ Die Auflistung in Anhang I der Richtlinie 2014/25/EU enthält einen Code für Tiefbauleistungen zur Errichtung von Fernmeldeleitungen, ${ }^{200}$ worunter sich auch Glasfaserleitungen subsumieren lassen. Letztlich ist dies jedoch unerheblich, da die Aufzählung der Codes nicht abschließend ist. ${ }^{201}$ Aus den vorhandenen Codes lässt sich jedoch folgern, dass die unter- und oberirdische Verlegung von Infrastruktur einen „Bauauftrag“ im Sinne des Vergaberechts darstellt. Auftrag und Konzession unterscheiden sich nicht

197 Richtlinie 2014/24/EU des Europäischen Parlaments und des Rates vom 26. Februar 2014 über die öffentliche Auftragsvergabe und zur Aufhebung der Richtlinie 2004/18/EG, Abl. L 94/65 v. 28.3.2014.

198 Richtlinie 2014/25/EU des Europäischen Parlaments und des Rates vom 26. Februar 2014 über die Vergabe von Aufträgen durch Auftraggeber im Bereich der Wasser-, Energie- und Verkehrsversorgung sowie der Postdienste und zur Aufhebung der Richtlinie 2004/17/EG, Abl. L 94/243 v. 28.3.2014 i. d. F. d. Delegierten Verordnung (EU) 2019/1829 der Kommission vom 30. Oktober 2019, Abl. L 279/27 v. 31.10. 2019.

199 Rixen, in: Burgi/Dreher (Hrsg.), Beck'scher Vergaberechtskommentar, $\mathbb{1 3 0}$ GWB, Rn. $47 \mathrm{ff}$.

200 RL 2014/25/EU, Anhang I, NACE 45.21; CPV 45210000.

201 Kühling, in: Körber/Kühling (Hrsg.), Ausschreibung von Fernwärmenetzen?, S. 9, 28. 
auf der Leistungs- sondern auf der Gegenleistungsseite, sodass sich das Begriffsverständnis übertragen lässt. Da die Abgrenzungskriterien aus dem EU-Recht stammen, lassen sich diese auch auf die Abgrenzung der Bauleistung im Sinne der KVRL übertragen. Im Ergebnis handelt es sich bei der Verlegung von Glasfaserkabeln um einen Bauauftrag, sodass sie auch bei der Integration in einen Konzessionsvertrag als Bauleistung klassifiziert werden kann. Der Konzessionsvertrag soll aber auch eine Betriebspflicht für die errichtete Infrastruktur enthalten. ${ }^{202}$ Eine solche ist nicht als Bauleistung zu klassifizieren. Vielmehr handelt es sich um eine Dienstleistung. ${ }^{203}$ Der Konzessionsvertrag soll demnach sowohl eine Bau- als auch eine Dienstleistung beinhalten. Vor diesem Hintergrund stellt sich die Frage, ob die Konzession dennoch einheitlich behandelt werden kann. Das nationale Vergaberecht enthält in den $\mathbb{\$} 110$ bis $112 \mathrm{GWB}$ Vorgaben zum Umgang mit gemischten Aufträgen, die ebenfalls auf Konzessionen mit gemischten Vertragsgegenständen Anwendung finden. ${ }^{204}$ Ein zusammenhängendes Vergabeverfahrens für alle Leistungspflichten der Konzession kann durchgeführt werden, wenn die einzelnen Vertragsbestandteile in einem akzessorischen Zusammenhang stehen. Dies erfordert einen engen rechtlichen und wirtschaftlichen Zusammenhang. ${ }^{205}$ Das ökonomische Kalkül hinter dem Konzessionsmodell auf Basis der Vergabe exklusiver Wegerechte liegt darin, dass die anfänglichen Investitionskosten für den Infrastrukturausbau durch den späteren Betrieb des Netzes amortisiert werden können. Hieraus folgt ein akzessorischer Zusammenhang zwischen den beiden Vertragselementen.

In einem nächsten Schritt ist die prägende Leistungspflicht zu identifizieren. Hierzu wurde in ständiger Rechtsprechung des Europäischen Gerichtshofes die "Schwerpunkttheorie“ entwickelt, ${ }^{206}$ die nunmehr auch

202 Deutscher Landkreistag, Flächendeckende Breitbandversorgung zu wirtschaftlichen Bedingungen sicherstellen, S. 7.

203 Gleiches gilt für den Betrieb eines Energienetzes der allgemeinen Versorgung im Sinne des $₫ 46$ Abs. 2 EnWG, Michaels/Bönnighausen, ZNER 2018, S. 7, 9.

204 Friton/Stein, in: Pünder/Schellenberg (Hrsg.), Vergaberecht, $\$ 105$ GWB, Rn. 10.

205 EuGH, Urt. v. 6.5.2010, verb. Rs. C-145/08 und C-149/08 (Club Hotel Loutraki u. a.), ECLI:EU:C:2010:247, Rn. 48; Pünder/Buchholtz, in: Pünder/Schellenberg (Hrsg.), Vergaberecht, $\$ 110 \mathrm{GWB}, \mathrm{Rn} .6 \mathrm{f}$.

206 EuGH, Urt. v. 26.5.2011, Rs. C-306/08 (Kommission/Spanien), ECLI:EU:C: 2011:347, Rn. 90 f.; EuGH, Urt. v. 29.10.2009, Rs. C-536/07 (Kommission/ Deutschland), ECLI:EU:C:2009:664, Rn. 57; EuGH, Urt. v. 18.1.2007, Rs. C-220/05 (Aroux u.a.), ECLI:EU:C:2007:31, Rn. 37. 
einfachgesetzlich in $\$ 110$ Abs. 1 GWB kodifiziert ist. ${ }^{207}$ Maßgeblich für die Abgrenzung ist der Hauptgegenstand des Vertrages, wobei die Rechtsprechung auch eine flexiblere Orientierung anhand der tatsächlichen Einzelleistungen und deren Fokus in der Leistungserbringung gestattet. ${ }^{208}$ Der Wert der Einzelleistungen kann dabei einen Anhaltspunkt bieten. ${ }^{209}$ Das Oberlandesgericht München hat einen Vertrag zur Errichtung und zum Betrieb eines Breitbandnetzes als Dienstleistungskonzession klassifiziert. ${ }^{210}$ Die Bauleistungen würden im Vergleich zu der Dienstleistung lediglich Nebenleistungen darstellen. ${ }^{211}$ Es müssten lediglich bestehende Netze aufgerüstet und wenige Netze neu gebaut werden, sodass die Bauleistung im Verhältnis zur Dienstleistung eine untergeordnete Rolle spiele. ${ }^{212}$ Dies lässt sich auf die vorliegende Situation nicht einfach übertragen. Je näher die Glasfaserversorgung an den Endkunden herangelegt werden soll, desto weniger ist es möglich, bestehende Netze aufzurüsten. Aus diesem Grund müssen zusätzliche Erwägungen angestellt werden. Während im Konzessionsmodell auf Basis der Vergabe exklusiver Wegerechte initial eine Bauleistung erforderlich ist, wird im Anschluss über mehrere Jahre hinweg eine Dienstleistung erbracht. Auch wenn die Bauleistung eine größere Rolle spielt als im Fall des Oberlandesgerichtes München, wäre bei einem Konzessionsmodell auf Basis der Vergabe exklusiver Wegerechte der Betrieb der Infrastruktur dennoch prägend. Die Infrastruktur soll nicht nur errichtet, sondern auch genutzt werden können. Ansonsten ließe sich die Zielrichtung des gesamten Handlungsansatzes, der Bevölkerung in Gebieten mit Netzausbaudefizit Glasfasernetze zur Verfügung zu stellen, nicht reali-

207 Dicks, in: Kulartz/Kus/Portz u.a. (Hrsg.), Kommentar zum GWB-Vergaberecht, $\$ 105$ GWB, Rn. 8.

$208 \mathrm{EuGH}$, Urt. v. 21.2.2008, Rs. C-421/04 (Kommission/Italien), ECLI:EU:C: 2008:102, Rn. 49; Braun, in: Müller-Wrede (Hrsg.), GWB, $\mathbb{S} 105$, Rn. 44; Noch, Vergaberecht kompakt, Kapitel A, Rn. 366; Prieß/Stein, VergabeR 2014, S. 499, 502; Walz, Die Bau- und Dienstleistungskonzession im deutschen und europäischen Vergaberecht, S. 141.

209 Hüttinger, in: Burgi/Dreher (Hrsg.), Beck'scher Vergaberechtskommentar, $\mathbb{\$} 110$ GWB, Rn. 21; Pünder/Buchholtz, in: Pünder/Schellenberg (Hrsg.), Vergaberecht, $\$ 110$ GWB, Rn. 14 ff.

210 OLG München, Beschl. v. 25.3.2011, Az. Verg 4/11, NZBau 2011, S. 380, 383; OLG Karlsruhe, Beschl. v. 14.11.2014, 15 Az. Verg. 10/14, NZBau 2015, S. 506, 507, Rn. $19 \mathrm{ff}$.

211 OLG München, Beschl. v. 25.3.2011, Az. Verg 4/11, NZBau 2011, S. $380,383$.

212 OLG München, Beschl. v. 25.3.2011, Az. Verg 4/11, NZBau 2011, S. 380, 383. 
sieren. ${ }^{213}$ Die Errichtung der Infrastruktur ist nur ein notwendiger Zwischenschritt, um den Betrieb der Glasfaserinfrastruktur zu ermöglichen. Je länger der Konzessionsvertrag dauert, desto stärker prägt die Dienstleistung den Gesamtvertrag. Es handelt sich daher um den Hauptgegenstand des Vertrages, sodass er sich insgesamt an den Anforderungen für die Dienstleistungskonzession messen lassen muss.

\section{b) Tatbestandsvoraussetzungen einer Dienstleistungskonzession}

Damit es sich bei der vorliegenden Konzession um eine Dienstleistungskonzession handelt, müssten die Tatbestandsvoraussetzungen erfüllt sein.

Der Konzessionsgeber muss den Konzessionsnehmer gemäß Art. 5 Abs. 1 lit. b KVRL und $₫ 105$ Abs. 1 Nr. 2 GWB in einem entgeltlichen Vertrag mit einer Dienstleistung betrauen. ${ }^{214}$ Ein Betrauungsakt liegt in Form des Konzessionsvertrages vor. ${ }^{215}$

Das Betriebsrisiko muss gemäß Art. 5 Abs. 2 KVRL und $\$ 105$ Abs. 2 GWB beim Konzessionsnehmer liegen. Erfasst ist sowohl ein Nachfrageals auch ein Angebotsrisiko. ${ }^{216}$ Problematisch ist diesbezüglich, dass durch die Exklusivität des Wegerechts im Konzessionsgebiet ein Infrastrukturmonopol entsteht. ${ }^{217}$ Jeder Diensteanbieter ist deshalb darauf angewiesen, das Netz des Konzessionsnehmers zu nutzen, um einen Endkunden zu errei-

213 Deutscher Landkreistag, Flächendeckende Breitbandversorgung zu wirtschaftlichen Bedingungen sicherstellen, S. 1.

214 Bis auf gleiche Nuancierungen im Rahmen des Wortlautes ohne inhaltliche Auswirkungen entsprechen sich die Begriffsfassungen des Art. 5 Abs. 1 lit.b KVRL und $\$ 105$ Abs. 1 Nr. 2 GWB, sodass sie nachfolgend nicht getrennt behandeln werden soll. Zu diesem Ergebnis kommt auch Braun, in: Müller-Wrede (Hrsg.), GWB, $\mathbb{1} 105$, Rn. 37.

215 Der Begriff der Betrauung ist jedoch nicht legaldefiniert und umstritten. Festzustellen ist jedenfalls, dass der Begriff auch im Kontext der Erbringung von „Dienstleistung von allgemeinem wirtschaftlichen Interesse“ im Sinne des Art. 106 Abs. 2 AEUV verwendet wird; Ganske, in: Reidt/Stickler/Glahs (Hrsg.), Vergaberecht, $\mathbb{S} 105$ GWB, Rn. 21 ff.; Ziekow, in: Ziekow/Völlnik (Hrsg.), Vergaberecht, $\mathbb{S} 105 \mathrm{GWB}$, Rn. 11; mit ausführlicher Begründung gehen ebenfalls vom Vorliegen eines Betrauungsaktes aus Hohenstein-Bartholl/Jacob, RdE 2017, S. $454,457 \mathrm{ff}$.

216 Ziekow, in: Ziekow/Völlnik (Hrsg.), Vergaberecht, $\$ 105$ GWB, Rn. 27.

217 Donhauser/Hölzlwimmer, VergabeR 2015, S. 509, 519f.; Walz, Die Bau- und Dienstleistungskonzession im deutschen und europäischen Vergaberecht, S. $286 \mathrm{ff}$. 
chen. ${ }^{218}$ Der Europäische Gerichtshof hat festgestellt, dass ein Betriebsrisiko auch in Konstellationen angenommen werden könne, in denen das Risiko aufgrund der Gestaltung des rechtlichen Umfeldes erheblich eingeschränkt sei, solange der Vertragspartner „aber dieses eingeschränkte Risiko in vollem Umfang oder zumindest zu einem erheblichen Teil übernimmt. “219 Dabei gilt jedoch gemäß Art. 5 Abs. 2 KVRL die Grenze, dass das Risiko nicht nur vernachlässigbar sein darf. Im Kontext der Vergabe exklusiver Wegerechte wird deswegen kritisch hinterfragt, ob das Risiko ausreicht, um vom Übergang eines Betriebsrisiko auszugehen. ${ }^{220}$ Der Kritik ist entgegenzuhalten, dass auch bei der Existenz eines exklusiven Wegerechts eine Nachfrageunsicherheit verbleibt. ${ }^{221}$ Dies manifestiert sich darin, dass etwa ein Wegzug vieler Personen aus dem Konzessionsgebiet die Investitionsbedingungen erheblich verschlechtern kann. Sofern eine Nutzung alternativer Infrastrukturen nicht vollumfänglich ausgeschlossen wird, kann auch hierin ein Betriebsrisiko liegen (C. V. 1.).222 Deswegen ist davon auszugehen, dass auch bei einer Vergabe exklusiver Wegerechte ein hinreichendes Betriebsrisiko auf den Konzessionsnehmer übertragen wird.

Das Tatbestandsmerkmal der Erforderlichkeit eines Beschaffungsvorganges ist nicht in der Legaldefinition vorausgesetzt, wird aber aus dem Gesetzeszweck des Art. 1 Abs. 1 KVRL und den Erwägungsgründen 11, 15 und 16 der KVRL hergeleitet. Für Baukonzessionen hat der Europäische Gerichtshof bereits entschieden, unter welchen Voraussetzungen von einem Beschaffungsvorgang ausgegangen werden kann (dazu aa.). Eine entsprechende Entscheidung für den Bereich der Dienstleistungskonzessio-

218 Grünewald, Die (Re)Kommunalisierung in der Energieverteilung, S. 305, spricht insoweit von einer „Abnahmegarantie“ für energierechtliche Konzessionsverträge.

219 EuGH, Urt. v. 10.9.2008, Rs. C-206/08 (Eurawasser), ECLI:EU:C:2009:540, Rn. 80.

220 Die Bezirksregierung Düsseldorf (Vergabekammer), Beschl. v. 7.7.2000, Az. VK 12/2000 - L, NZBau 2001, S. 46, 47, geht davon aus, dass das bloße „Kalkulationsrisiko" nicht ausreicht; Böckel, LKV 2003, S. 393, 395; kritisch Greb/Wegner, Die Vergabe von Konzessionen im Energiebereich, Rn. 45.

221 Dieses Risiko wird auch in der energierechtlichen Literatur berücksichtigt; Grünewald, Die (Re)Kommunalisierung in der Energieverteilung, S. 306; Sauer, Das Recht der Vergabe von Strom- und Gas-Konzessionsverträgen im EnWG, S. 243.

222 Im Energiesektor tritt noch die Entgeltregulierung hinzu, die für den Erlös aus den Konzessionsrechten eine enge Grenze setzt; Grünewald, Die (Re)Kommunalisierung in der Energieverteilung, S. 305. 
nen steht jedoch noch aus, sodass die Anforderungen umstritten sind (da$\mathrm{zu}$ bb. bis dd.). ${ }^{223}$

\section{aa) Beschaffungsbezug im Kontext von Baukonzessionen}

An das Vorliegen eines Beschaffungsbezuges bei Baukonzessionen hat der Europäische Gerichtshof in der Rechtssache Müller strenge Voraussetzungen geknüpft. ${ }^{224} \mathrm{Er}$ sei nur anzunehmen, wenn ein unmittelbares wirtschaftliches Interesse des Hoheitsträgers an der Dienstleistung bestehe. ${ }^{225}$ Es „,ist eindeutig gegeben, wenn vorgesehen ist, dass der öffentliche Auftraggeber Eigentümer der Bauleistung oder des Bauwerkes wird“226, das errichtet werden soll. Auch die Entgeltlichkeit könne als Anhaltspunkt für das unmittelbare wirtschaftliche Interesse dienen, da in der Zahlung eines Entgeltes eine Gegenleistung liege. 227 Ein unmittelbares wirtschaftliches Interesse könne auch angenommen werden, „wenn (...) der öffentliche Auftraggeber über einen Rechtstitel verfügen soll, der ihm die Verfügbarkeit der Bauwerke, die Gegenstand des Vertrages sind, im Hinblick auf ihre öffentliche Zweckbestimmung sicherstellt.“228 Es könne auch „in wirtschaftlichen Vorteilen, die der öffentliche Auftraggeber aus der zukünftigen Nutzung oder Veräußerung des Bauwerks ziehen kann, in seiner finanziellen Beteiligung an der Erstellung des Bauwerks oder in den Risiken, die er im Fall eines wirtschaftlichen Fehlschlags des Bauwerks trägt,

223 Mit dem Verweis auf das Fehlen einer höchstrichterlichen Rechtsprechung OLG Düsseldorf, Beschl. v. 28.3.2012, Az. VII-Verg 37/11, NZBau 2012, S. 518, 520; Ganske, in: Reidt/Stickler/Glahs (Hrsg.), Vergaberecht, $\$ 105$ GWB, Rn. 30.

224 EuGH, Urt. v. 25.3.2010, Rs. C-451/08 (Helmut Müller), ECLI:EU:C:2010:168, Rn. 49.

225 EuGH, Urt. v. 25.3.2010, Rs. C-451/08 (Helmut Müller), ECLI:EU:C:2010:168, Rn. 49; zur Rechtsprechung vor Erlass der Entscheidung, Kühling, in: Körber/ Kühling (Hrsg.), Ausschreibung von Fernwärmenetzen?, S. 9, 21 ff.

226 EuGH, Urt. v. 25.3.2010, Rs. C-451/08 (Helmut Müller), ECLI:EU:C:2010:168, Rn. 50.

227 EuGH, Urt. v. 25.3.2010, Rs. C-451/08 (Helmut Müller), ECLI:EU:C:2010:168, Rn. 48; EuGH, Urt. v. 18.1.2007, Rs. C-220/05 (Auroux u. a.), ECLI:EU:C: 2007:31, Rn. 45; EuGH, Urt. v. 12.7.2001, Rs. C-399/98 (Ordine degli Architetti u. a.), ECLI:EU:C:2001:401, Rn. 77.

228 EuGH, Urt. v. 25.3.2010, Rs. C-451/08 (Helmut Müller), ECLI:EU:C:2010:168, Rn. 51; EuGH, Urt. v. 12.7.2001, Rs. C-399/98 (Ordine degli Architetti u. a.), ECLI:EU:C:2001:401, Rn. 67 und 71. 
bestehen. " 229 Sofern lediglich staatliche Regelungszuständigkeiten ausgeübt würden, liege hingegen kein unmittelbares wirtschaftliches Interesse vor. ${ }^{230}$ Mangels einer entsprechenden Entscheidung für Dienstleistungskonzessionen ist umstritten, wann vom Vorliegen eines Beschaffungsbezuges ausgegangen werden kann.

bb) Eigenständige Abgrenzungskriterien für die Dienstleistungskonzession

Ein Teil der Lehre und Rechtsprechung geht davon aus, dass sich die Rechtsprechung im Fall Müller auf das Kriterium des Beschaffungsbezuges bei Dienstleistungskonzessionen nur eingeschränkt oder gar nicht übertragen lasse. ${ }^{231}$ Dies sei auf den „unterschiedlichen Charakter [...] von Bauund Dienstleistungen" 232 zurückzuführen. Auch der Gesetzgeber differenziere in $\mathbb{1 0 3}$ Abs. 3 S. 2 GWB zwischen Bau- und Dienstleistungen. Für Baukonzessionen sei in der Vorschrift das unmittelbare wirtschaftliche Interesse vorgeschrieben, während bei Dienstleistungen von einer Kodifikation abgesehen worden sei. ${ }^{233}$ Erwägungsgrund 11 der KVRL verlange lediglich einen „Nutzen“ für den Konzessionsgeber. ${ }^{234}$ Die Definition der Dienstleistungskonzession gemäß Art. 5 Abs. 1 lit. b KVRL enthalte keine Anhaltspunkte dafür, dass der Staat die Leistung selbst in Anspruch nehmen müsse. ${ }^{235}$ Ein solcher Schluss könne auch nicht aus dem Erfordernis einer Gegenleistung gezogen werden. ${ }^{236}$ Vielmehr seien Dreiecksverhältnisse, in denen Konzessionsgeber und Leistungsempfänger auseinanderfal-

229 EuGH, Urt. v. 25.3.2010, Rs. C-451/08 (Helmut Müller), ECLI:EU:C:2010:168, Rn. 52; EuGH, Urt. v. 18.1.2007, Rs. C-220/05 (Auroux u. a.), ECLI:EU:C: 2007:31, Rn. 13, 17, 18 und 45.

230 Kühling, in: Körber/Kühling (Hrsg.), Ausschreibung von Fernwärmenetzen?, S. 9, 24; Grünewald, Die (Re)Kommunalisierung in der Energieverteilung, S. 307, stellt insoweit klar, dass eine bloße Rechtevergabe nicht ausreicht.

231 OLG Düsseldorf, Beschl. v. 28.3.2012, Az. VII-Verg 37/11, NZBau 2012, S. 518, 520; Donhauser/Hölzlwimmer, VergabeR 2015, S. 509, 512 f.; Friton/Stein, in: Pünder/Schellenberg (Hrsg.), Vergaberecht, $\mathbb{\$} 105$ GWB, Rn. 20.

232 Friton/Stein, in: Pünder/Schellenberg (Hrsg.), Vergaberecht, $\mathbb{S} 105$ GWB, Rn. 20.

233 Friton/Stein, in: Pünder/Schellenberg (Hrsg.), Vergaberecht, $\mathbb{S} 105$ GWB, Rn. 21.

234 Friton/Stein, in: Pünder/Schellenberg (Hrsg.), Vergaberecht, $\mathbb{\$} 105$ GWB, Rn. 21.

235 Opitz, NVwZ 2014, S. 753, 757.

236 Opitz, NVwZ 2014, S. 753, 757. 
len, prägend für die Dienstleistungskonzession. ${ }^{237}$ Die typische Konstellation einer Dienstleistungskonzession gleiche der eines Vertrages zu Gunsten Dritter im Sinne des $\$ 328$ BGB. ${ }^{238}$ Dies werde durch Erwägungsgrund 16 der KVRL deutlich. ${ }^{239}$ Die Abnahme der angebotenen Dienstleistung, die aufgrund des Definitionsmerkmals des Betriebsrisikos erforderlich ist, könne nur in solchen Konstellationen unsicher sein, in denen eine Leistung an Dritte erbracht werde. ${ }^{240}$

Während Teile der Literatur mit dieser Begründung die Übertragbarkeit der Rechtsprechung auf Dienstleistungskonzessionen vollständig ablehnen, ${ }^{241}$ versuchen andere die Rechtsprechung im Fall Müller darauf entsprechend anzuwenden. ${ }^{242}$ Dabei wird der Beschaffungsbezug sehr weit interpretiert und bereits in solchen Konstellationen angenommen, in denen der Konzessionsnehmer die Leistung gegenüber dem Dritten erbringt und gegenüber dem Konzessionsgeber eine einklagbare Verpflichtung zur Leistungserbringung besteht. ${ }^{243}$ Sobald der Konzessionsgeber die Leistungserbringung gegenüber dem Dritten einklagen könne, liege in der Leistungserbringung eine Leistungserfüllung im Vertrag mit dem Konzessionsgeber, mithin ein Beschaffungsvorgang. ${ }^{244}$ Es sei jedoch keine „öffentliche Aufgabe“ oder eine „Aufgabe der Daseins-Vorsorge“ erforderlich. ${ }^{245}$

237 Friton/Stein, in: Pünder/Schellenberg (Hrsg.), Vergaberecht, $\mathbb{\$} 105$ GWB, Rn. 22; so auch Grünewald, Die (Re)Kommunalisierung in der Energieverteilung, S. 307.

238 Friton/Stein, in: Pünder/Schellenberg (Hrsg.), Vergaberecht, $\$ 105$ GWB, Rn. 22; Ganske, in: Reidt/Stickler/Glahs (Hrsg.), Vergaberecht, $\$ 105$ GWB, Rn. 33; Opitz, NVwZ 2014, S. 753, 757.

239 Friton/Stein, in: Pünder/Schellenberg (Hrsg.), Vergaberecht, $\mathbb{} 105$ GWB, Rn. 22; Opitz hält den Erwägungsgrund für „sprachlich missglückt" und folglich für keine Argumentationsstütze, Opitz, NVwZ 2014, S. 753, 757; kritisch im Hinblick auf diese weite Interpretation des Erwägungsgrundes Grünewald, Die (Re)Kommunalisierung in der Energieverteilung, S. 319.

240 Friton/Stein, in: Pünder/Schellenberg (Hrsg.), Vergaberecht, $\mathbb{\$} 105$ GWB, Rn. 22; Opitz, NVwZ 2014, S. 753, 757.

241 Opitz, NVwZ 2014, S. 753, 757.

242 Friton/Stein, in: Pünder/Schellenberg (Hrsg.), Vergaberecht, $\$ 105$ GWB, Rn. 22; für das Erfordernis einer „einklagbare[n] Leistungspflicht“ spricht sich auch, Ganske, in: Reidt/Stickler/Glahs (Hrsg.), Vergaberecht, $\mathbb{\$} 105$ GWB, Rn. 35, mit Verweis auf Erwägungsgrund 14 der KVRL aus.

243 Friton/Stein, in: Pünder/Schellenberg (Hrsg.), Vergaberecht, $\mathbb{} 105$ GWB, Rn. 22; kritisch Grünewald, Die (Re)Kommunalisierung in der Energieverteilung, S. 319 .

244 Friton/Stein, in: Pünder/Schellenberg (Hrsg.), Vergaberecht, $\mathbb{1} 105$ GWB, Rn. 22; Weiß, NVwZ 2014, S. 1415, 1419.

245 Friton/Stein, in: Pünder/Schellenberg (Hrsg.), Vergaberecht, $\mathbb{1} 105$ GWB, Rn. 22. 
Im Konzessionsmodell auf Basis der Vergabe exklusiver Wegerechte soll eine Betriebspflicht für das Netz verankert werden. ${ }^{246}$ Hierin liegt eine Pflicht zur Leistungserbringung gegenüber einem Dritten, die für den Konzessionsgeber, je nach Ausgestaltung des Vertrages, auch einklagbar wäre. Damit wäre nach dieser Ansicht ein Beschaffungsbezug gegeben.

\section{cc) Beschaffungsbezug bei Erfüllung einer öffentlichen Aufgabe}

Eine andere Ansicht interpretiert den Beschaffungsbezug enger und geht davon aus, dass die Leistungserbringung stets der Erfüllung einer öffentlichen Aufgabe oder einer Aufgabe der Daseinsvorsorge dienen müsse. ${ }^{247}$ Der Beschaffungsbezug komme insbesondere in Art. 1 Abs. 1 KVRL, aber auch im Erwägungsgrund 16 der KVRL zum Ausdruck. ${ }^{248}$ Eine öffentliche Aufgabe sei ein wesentlicher Bestandteil eines staatlichen Beschaffungsvorganges. ${ }^{249} \mathrm{Im}$ Energiesektor gehen Teile der Literatur davon aus, dass der Staat als „Nachfrager von Infrastrukturdienstleistungen“ auftrete und aus

246 Deutscher Landkreistag, Flächendeckende Breitbandversorgung zu wirtschaftlichen Bedingungen sicherstellen, S. 7.

247 OLG München, Beschl. v. 25.3.2011, Az. Verg 4/11, NZBau 2011, S.380, 382; Die Diskussion wird auch im Energierecht geführt, Brüning, in: Byok/Jaeger (Hrsg.), Kommentar zum Vergaberecht, $\mathbb{} 105$ GWB, Rn. 19; Diemon-Wies, VergabeR 2016, S. 162, 163; Hofmann/Zimmermann, NZBau 2016, S. 71, 72; Hohenstein-Bartholl/Jacob, RdE 2017, S.454, 459; Theobald/Wolkenhauer, DÖV 2016, S. 724, 730; kritisch Grünewald, Die (Re)Kommunalisierung in der Energieverteilung, S. 308, die aber die Erwägungen hinter dem Konzept der „Daseinsvorsorge" für beachtlich hält und zu vergleichbaren Ergebnissen kommt; kritisch auch Donhauser/Hölzlwimmer, VergabeR 2015, S. 509, 511 f.; a. A. Ziekow, in: Ziekow/Völlnik (Hrsg.), Vergaberecht, $\mathbb{1 0 5}$ GWB, Rn. 11, der davon ausgeht, dass es sich nicht um ein verpflichtendes Begriffsmerkmal handelt; so auch Opitz, NVwZ 2014, S. 753, 756.

248 Hofmann/Zimmermann, NZBau 2016, S.71, 73 f.; Kühling/Seiler, EnWZ 2017, S. 99, 102; Weiß, NVwZ 2014, S. 1415, 1419; a. A. Friton/Stein, in: Pünder/Schellenberg (Hrsg.), Vergaberecht, $\$ 105$ GWB, Rn. 22; Opitz, NVwZ 2014, S. 753, 757; eher für das Vorliegen eines Beschaffungsvorganges, aber kritisch hinsichtlich der Entgeltlichkeit, Sauer, Das Recht der Vergabe von Strom- und Gas-Konzessionsverträgen im EnWG, S. $128 \mathrm{ff}$.

249 KG Berlin, Urt. v. 22.1.2015, Az. 2 U 14/14/Kart, NZBau 2015, S. 323, 324; OLG Karlsruhe, Beschl. v. 14.11.2014, Az. 15 Verg. 10/14, NZBau 2015, S. 506, 507, Rn.16ff.; OLG München, Beschl. v. 25.3.2011, Az. Verg 4/11, NZBau 2011, S. 380,382 . 
Gründen der Daseinsvorsorge hierzu verpflichtet sei. ${ }^{250}$ Aufgrund der Unterschiede zwischen Energie- und Telekommunikationssektor kann die Diskussion freilich nicht einfach auf das Konzessionsmodell auf Basis der Vergabe exklusiver Wegerechte übertragen werden. Es ist somit zunächst zu ermitteln, worin dabei die öffentliche Aufgabe zur Errichtung eines Netzes mit Glasfaserinfrastruktur in Gebieten mit Netzausbaudefizit liegen könnte. Dafür kommen verschiedene Anknüpfungspunkt in Betracht (da$\mathrm{zu}(1)$ bis (4)).

\section{(1) Telekommunikationsnetz der allgemeinen Versorgung}

Eine öffentliche Aufgabe im Sinne der hier näher beleuchteten Ansicht könnte im Aufbau und Betrieb eines Telekommunikationsnetzes der allgemeinen Versorgung im Konzessionsgebiet liegen. Der Deutsche Landkreistag schlägt in seinem Positionspapier die Einführung dieses Begriffes in das Telekommunikationswegerecht vor. ${ }^{251}$ Der Begriff ist dem Energierecht entlehnt, dort in $\$ 3 \mathrm{Nr} .17$ EnWG legaldefiniert und wird an unterschiedlichen Stellen des Gesetzes verwendet. ${ }^{252}$ Durch diese Verwendung lässt sich auf die Funktion schließen. Der Begriff wird im Zusammenhang mit den Pflichten des $₫ 18$ Abs. 1 EnWG ${ }^{253}$ für Betreiber eines Netzes der allgemeinen Versorgung und im Kontext der Grundversorgung gemäß $\$ 36$ Abs. 2 und $\$ 38$ Abs. 1 EnWG verwendet. Bei Letzterem handelt es

250 Hofmann/Zimmermann, NZBau 2016, S.71, 73 f.; Theobald/Wolkenhauer, DÖV 2016, S. 724.

251 Deutscher Landkreistag, Flächendeckende Breitbandversorgung zu wirtschaftlichen Bedingungen sicherstellen, S. 7.

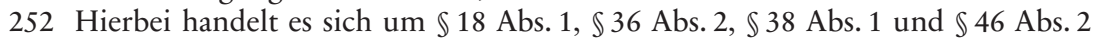
EnWG.

253 Für Betreiber eines Netzes der allgemeinen Versorgung besteht gemäß $\mathbb{} 18$ Abs. 1 EnWG die Pflicht, allgemeine Bedingungen für den Netzanschluss und die Anschlussnutzung zu veröffentlichen, im Ergebnis alle Abnehmer anzuschließen und ihnen die Energieentnahme zu gestatten; Templin, Recht der Konzessionsverträge, S. 159; Theobald, in: Theobald/Kühling (Hrsg.), Energierecht, $\mathbb{3}$ EnWG, Rn. 135; zur Abgrenzung zwischen dem Universaldienst im Sinne des Telekommunikationsrechts und der Grundversorgung im Energiesektor insbesondere Reents, Ausbau und Finanzierung einer flächendeckenden Breitbandversorgung in Deutschland, S. $88 \mathrm{ff}$. 
sich um einen Mechanismus, mit dessen Hilfe eine Basisversorgung mit Energie sichergestellt wird. 254

Die Basisversorgung mit Telekommunikationsdiensten wird hingegen über den Universaldienstmechanismus gemäß den $\$ \mathbb{S} 78 \mathrm{ff}$. TKG gewährleistet. Bei der Umsetzung des EKEK in das TKG wird es wohl zu einer Neunummerierung der Vorschriften des TKG kommen, sodass sich diese Vorgaben künftig voraussichtlich in den $\$ \$ 155 \mathrm{ff}$. TKG-RefE befinden werden. ${ }^{255}$ Dem TKG liegt ein anderer Ansatz zur Lösung einer vergleichbaren Problematik zu Grunde. Dies wird sich auch durch die Novelle nicht ändern. Eine Einführung des Begriffes im Kontext des Wegerechts ohne Anknüpfung an seine Verwendung im Energierecht würde keine Funktion erfüllen und ist deswegen nicht sinnvoll. Sollte mit dem Begriff die Einführung eines alternativen Konzeptes zur Sicherstellung der Basisversorgung im Konzessionsgebiet bezweckt werden, sind verschiedene Konstellationen zu bedenken.

Ein vollständiges Ersetzen des Universaldienstregimes des TKG durch das Konzept der allgemeinen Versorgung des Energierechts ist unionsrechtlich nicht zulässig. Die Art. 84 ff. EKEK schreiben ein Universaldienstregime vor, das zwar einen gewissen Spielraum hinsichtlich der normativen Ausgestaltung erlaubt, aber nicht das vollständige Absehen von einer Normierung gestattet, die in weiten Teilen den Vorgaben der $\$ \$ 78 \mathrm{ff}$. TKG entspricht. ${ }^{256}$ Aus diesem Grund müssten die Absicherungsmechanismen des Netzes der allgemeinen Versorgung und der Universaldienstmechanismus nebeneinanderstehen. Die Parallelexistenz von zwei Absicherungsmechanismen ist unzweckmäßig, solange sie einen identischen Gewährleistungsgehalt haben. Weicht der Gewährleistungsgehalt hingegen ab, entstünden unvermeidlich Abgrenzungsschwierigkeiten. Solche Schwierigkeiten führen zu Rechtsunsicherheiten, die vermieden werden sollten. Es be-

254 Heinlein/Weitenberg, in: Theobald/Kühling (Hrsg.), Energierecht, $\$ 36$ EnWG, Rn. 1 m. w. N.; grundlegend zur Daseinsvorsorge in der Energiewirtschaft Hermes, Staatliche Infrastrukturverantwortung, S. $94 \mathrm{ff}$.

255 Referentenentwurf des Telekommunikationsmodernisierungsgesetzes (TKMoG), abrufbar unter: https:/www.bmwi.de/Redaktion/DE/Downloads/Gesetz/ telekommunikationsmodernisierungsgesetz-referentenentwurf-20201612.pdf? blob=publicationFile $\& v=8$ (zuletzt abgerufen am 12.1.2021).

256 Im künftigen TKG werden sich die Vorgaben, wie bereits dargestellt, voraus-

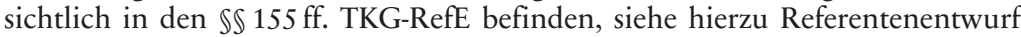
des Telekommunikationsmodernisierungsgesetzes (TK-MoG), abrufbar unter: https://www.bmwi.de/Redaktion/DE/Downloads/Gesetz/telekommunikationsm odernisierungsgesetz-referentenentwurf-20201612.pdf?_blob=publicationFile $\&$ $\mathrm{v}=8$ (zuletzt abgerufen am 12.1.2021). 
steht schlechthin kein Bedürfnis zur Etablierung eines weiteren Sicherungssystems, da mit dem Universaldienstregime ein etablierter, funktionsfähiger Mechanismus existiert. Der Aufbau und Betrieb eines Netzes der allgemeinen Versorgung kann mithin auch keine öffentliche Aufgabe im Sinne der dargelegten Ansicht darstellen.

\section{(2) Universaldienstgewährleistungsauftrag des Hoheitsträgers}

Eine öffentliche Aufgabe könnte jedoch in eben dieser Gewährleistung des Universaldienstes liegen. Hierunter versteht man die Verfügbarkeit der Dienste, die für eine wirtschaftliche und soziale Teilhabe mindestens erforderlich sind. ${ }^{257}$ Das Konzessionsmodell auf Basis der Vergabe exklusiver Wegerechte soll einen Ansatz für den Glasfaserausbau in bislang unterversorgten Gebieten darstellen. ${ }^{258}$ Bei einer Glasfaserversorgung handelt es sich jedoch, wie bereits darstellt, um eine Maximalversorgung, ${ }^{259}$ die nicht vom Universaldienst erfasst ist. Darüber hinaus ist die singuläre Ausrichtung auf die Übertragungstechnologie „Glasfaser" wegen des Grundsatzes der Technologieneutralität, der auch für die Gewährleistung des Universaldienstes gilt, problematisch. Im Ergebnis kann es sich bei der Gewährleistung des Universaldienstes aus diesen Gründen nicht um eine öffentliche Aufgabe im Sinne der vorliegend diskutierten Ansicht handeln.

„e-Daseinsvorsorge“ des Staates

Die öffentliche Aufgabe des Staates könnte in der Pflicht zur Gewährleistung einer „e-Daseinsvorsorge“ liegen. ${ }^{260}$ Der Begriff der „Daseinsvorsor-

257 Kühling/Toros, Rechtliche Rahmenbedingungen für die Schaffung von Anreizen für einen flächendeckenden Ausbau von Glasfaserinfrastrukturen, S. 15 ff.

258 Deutscher Landkreistag, Flächendeckende Breitbandversorgung zu wirtschaftlichen Bedingungen sicherstellen, S. 1.

259 Hierunter ist eine Versorgung zu verstehen, welche die Anforderungen einer Versorgung, die für die soziale und wirtschaftliche Teilhabe bei Weitem übertrifft.

260 Der Begriff wurde in einem Forschungsvorhaben des Lorenz-von-Stein-Instituts für Verwaltungswissenschaften an der Christian-Albrechts-Universität zu Kiel entwickelt, Luch/Schulz, Das Recht auf Internet als Grundlage der OnlineGrundrechte, S. 9 ff.; Luch/Schulz, in: Hill/Schliesky (Hrsg.), Herausforderung eGovernment, S. 305 ff.; Luch/Schulz, VM 2011, S. 104 ff.; Luch/Schulz, MMR 2009, 19; Schulz, in: Schliesky/Ernst/Schulz (Hrsg.), Festschrift für Edzard 
ge" wurde von Ernst Forsthoff in die Verwaltungsrechtswissenschaft eingeführt. ${ }^{261}$ Nach seinem Verständnis ist Daseinsvorsorge „alles, was von Seiten der Verwaltung geschieht, um die Allgemeinheit oder nach objektiven Merkmalen bestimmte Personenkreise in den Genuss nützlicher Leistungen zu versetzen (...).“262 Die „e-Daseinsvorsorge“ stellt einen Versuch zur Weiterentwicklung des Begriffes im Hinblick auf die Anforderungen der digitalen Gesellschaft dar. ${ }^{263}$ Den Vertretern dieser Ansicht zufolge ist unter ihr die Verantwortung des Staates zu verstehen, dass „Teile der Bevölkerung nicht von der Teilhabe an diesem neuen sozio-kulturellen Existenzminimum ausgeschlossen werden." 264 Es handele sich um ein Gegenkonzept zum Universaldienst, der nicht geeignet sei, eine zukunftsfähige Internetversorgung abzusichern. ${ }^{265}$ Mithilfe des neuen Begriffes sei eine kurzfristige und dynamische Anpassung des Gewährleistungsumfanges denkbar, die perspektivisch zumindest eine Breitbandversorgung absichern müsse. ${ }^{266} \mathrm{Im}$ geltenden nationalen Universaldienstregime wird durch den Universaldienst lediglich ein „funktionaler Internetzugang“ abgesichert und es ist umstritten, ob hierunter ein Breitbandinternetzugang zu verstehen ist. ${ }^{267}$

Bei der Umsetzung der Vorgaben der Art. $84 \mathrm{ff}$. EKEK in nationales Recht wird der Gewährleistungsumfang des Universaldienstes aktualisiert werden. ${ }^{268}$ In Zukunft wird ein „angemessener Breitbandinternetzugangs-

Schmidt-Jortzig, S. 17 ff.; Schulz, DuD 2010, S. 698 ff.; Schulz, in: Schliesky (Hrsg.), Selbstverwaltung im Staat der Informationsgesellschaft, S. $13 \mathrm{ff}$.

261 Grundlegend zur Daseinsvorsorge in der Energiewirtschaft Hermes, Staatliche Infrastrukturverantwortung, S. $94 \mathrm{ff}$.

262 Forsthoff, Lehrbuch des Verwaltungsrechts, S. 370.

$263 \mathrm{Luch} / \mathrm{Schulz}$, Das Recht auf Internet als Grundlage der Online-Grundrechte, S. 9.

$264 \mathrm{Luch} /$ Schulz, VM 2011, 104; vgl. hierzu auch Schulz, DuD 2010, S. 698, 699.

265 Schulz, DuD 2010, S.698, 701; im Ergebnis vergleichbar Holznagel/Deckers, DVB1 2009, S. 482, 488, jedoch ohne Verwendung des Begriffes der „e-Daseinsvorsorge".

$266 \mathrm{Luch} / \mathrm{Schulz}$, Das Recht auf Internet als Grundlage der Online-Grundrechte, S. 10.

267 In der Literatur wurde angenommen, dass ein „funktionaler Internetzugang“ bereits ab einer Übertragungsrate von 2 Mbit/s vorlag; Fetzer, MMR 2011, S. 707, 709; Kühling/Biendl, DÖV 2012, S. 409, 412 f.; von einer Übertragungsrate von etwa 6 Mbit/s ging Schumacher, MMR 2011, S. 711, 715 aus; im Überblick Kühling/Toros, Rechtliche Rahmenbedingungen für die Schaffung von Anreizen für einen flächendeckenden Ausbau von Glasfaserinfrastrukturen, S. 21.

268 Kübling/Toros, Rechtliche Rahmenbedingungen für die Schaffung von Anreizen für einen flächendeckenden Ausbau von Glasfaserinfrastrukturen, S. 21 ff.; Kühling/Toros, N\&R 2019, S. 258, 259. 
dienst" abgesichert werden, der sich dynamisch mit den Anforderungen der Nutzer entwickeln sollte. ${ }^{269}$ Somit wird der berechtigten Kritik der Entwickler des Konzeptes der „e-Daseinsvorsorge“ bereits über das novellierte Universaldienstregime begegnet. Die Einführung eines neuen Begriffes für die Basisversorgung mit Telekommunikationsinfrastrukturen und -diensten ist damit überflüssig. Der Begriff sollte darüber hinaus schon deshalb nicht verwendet werden, da der Universaldienstmechanismus als Gegenentwurf zur staatlichen Leistungsbereitstellung im Rahmen der „Daseinsvorsorge" entwickelt wurde. ${ }^{270}$ Hiervon losgelöst wäre eine Glasfaserversorgung ohnehin, selbst wenn man dem Begriff der "e-Daseinsvorsorge“ eine Restfunktion zuspräche, als „Luxusausstattung“271 im Sinne einer Maximalversorgung zu interpretieren und als solche nicht vom Umfang der „e-Daseinsvorsorge“ erfasst. Auch die „e-Daseinsvorsorge" lässt sich mithin nicht als öffentliche Aufgabe klassifizieren, die mit der Errichtung und dem Betrieb von Glasfaserinfrastruktur im Konzessionsgebiet verfolgt werden könnte.

\section{(4) Aufbau einer Glasfaserinfrastruktur als „öffentliche Aufgabe“}

Der Aufbau der Glasfaserinfrastruktur im Konzessionsgebiet könnte, neben den zuvor aufgeführten speziellen Konstellationen, eine nicht näher spezifizierte, allgemeine „öffentliche Aufgabe“ sein. Aus verfassungsrechtlicher Sicht wird eine solche bereits angenommen, wenn die Erfüllung der Aufgabe im öffentlichen Interesse liegt. ${ }^{272}$ Damit ist sie eng verwandt mit dem Begriff des „allgemeinen wirtschaftlichen Interesses“ aus dem EU-Beihilfenrecht, der von der Kommission ebenfalls sehr weit interpretiert wird und somit den Mitgliedstaaten einen umfassenden Spielraum bei seiner

269 Konkrete Normierungsvorschläge zur Aktualisierung des Universaldienstregimes entwickeln Kühling/Toros, Rechtliche Rahmenbedingungen für die Schaffung von Anreizen für einen flächendeckenden Ausbau von Glasfaserinfrastrukturen, S. 48 f.; siehe auch Referentenentwurf des Telekommunikationsmodernisierungsgesetzes (TK-MoG), abrufbar unter: https://www.bmwi.de/Redaktion/D E/Downloads/Gesetz/telekommunikationsmodernisierungsgesetz-referentenent wurf-20201612.pdf?__blob=publicationFile $\& v=8$ (zuletzt abgerufen am 12.1.2021).

270 Hermes, Staatliche Infrastrukturverantwortung, S. 184 ff.; Kühling, Sektorspezifische Regulierung in den Netzwirtschaften, S. 353.

$271 \mathrm{Luch} / \mathrm{Schulz}$, VM 2011, S. 104, 105.

272 Korioth, in: Maunz/Dürig (Begr.), Grundgesetz, Art. 30 GG, Rn. 14 m. w. N. 
Ausgestaltung belässt. Aus der Zusammenschau der „Strategie Europa $2020^{\text {“ }},{ }^{273}$ die ein Handlungskonzept zur umfassenden Implementierung von hochleistungsfähigen digitalen Infrastrukturen und deren Nutzung in den einzelnen Mitgliedstaaten vorsieht, und den Entscheidungen der Europäischen Kommission zur Genehmigung von Beihilfen wird offenbar, dass auch die Glasfaserversorgung bei einer entsprechenden Ausgestaltung der Beihilfe im „allgemeinen wirtschaftlichen Interesse“ liegen kann (C. IV. 2. c. aa.). Überträgt man dieses Verständnis auf den vergleichbaren Begriff der „öffentlichen Aufgabe“, kann somit die staatliche Förderung des Auf- und Ausbaus von Glasfaserinfrastrukturen im Konzessionsgebiet durchaus eine „öffentliche Aufgabe“ darstellen.

\section{(5) Zwischenergebnis}

In der Errichtung und dem Betrieb eines Netzes mit Glasfaserinfrastruktur in Gebieten mit Netzausbaudefizit liegt eine öffentliche Aufgabe. Nach der vorgenannten Ansicht wäre mithin ein staatlicher Beschaffungsvorgang zur Erfüllung dieser Aufgabe gegeben.

dd) Beschaffungsvorgang nur bei einem unmittelbaren wirtschaftlichen Interesse

Ein anderer Teil der Lehre wendet die Judikatur des Europäischen Gerichtshofes aus dem Fall Müller analog auf die Dienstleistungskonzessionen an. ${ }^{274}$ Danach könne ein Beschaffungsbezug nicht angenommen werden, „wenn lediglich für die Allgemeinheit zu erbringende Dienstleistungen Gegenstand des jeweiligen Vertrages sind." 275 Hier könnte ein unmittelbares wirtschaftliches Interesse angenommen werden, weil „der öffentliche Auftraggeber über einen Rechtstitel verfügen soll, der ihm die Verfügbarkeit der [Dienstleistung], die Gegenstand des Vertrages sind, im Hin-

273 Komm., Europe 2020, S. 11 f.; im Ergebnis auch Komm., Shaping Europe‘s Digital Future, S. 4.

274 Dicks, in: Kulartz/Kus/Portz u.a. (Hrsg.), Kommentar zum GWB-Vergaberecht, $\$ 105$ GWB, Rn. 17.

275 Kühling, in: Körber/Kühling (Hrsg.), Ausschreibung von Fernwärmenetzen?, S. 9, 37; Kübling/Seiler, EnWZ 2017, S. 99, 102. 
blick auf ihre öffentliche Zweckbestimmung sicherstellt. " ${ }^{276}$ Ein solcher Rechtstitel könnte durch die Ausbau- und die Betriebspflicht vermittelt werden, mit denen das Konzessionsrecht verbunden werden soll. 277 Diese stellen sicher, dass das Telekommunikationsnetz als Bestandteil der öffentlichen Infrastruktur genutzt werden kann. Im Ergebnis liegt damit auch nach dieser Ansicht ein unmittelbares wirtschaftliches Interesse vor.

ee) Stellungnahme und Zwischenergebnis

Auch wenn Dienstleistungs- und Baukonzession negativ voneinander abgegrenzt werden, stehen sie doch in einem engen normativen Zusammenhang. Bau- und Dienstleistungskonzessionen verfügen über keinen gänzlich unterschiedlichen Charakter. Der Unterschied liegt im konkreten Leistungsgegenstand, nicht in den übrigen Tatbestandsmerkmalen. Auch wenn der Gesetzgeber in $\mathbb{1} 103$ Abs. 3 S. 2 GWB normiert, dass für eine Bauleistung ein unmittelbares wirtschaftliches Interesse des Auftraggebers gegeben sein muss, führt dies nicht zu einer anderen Betrachtung. In $\$ 103$ Abs. 4 GWB wird normiert, dass der Dienstleistungsauftrag negativ vom Bau- und Lieferauftrag abgegrenzt werden muss. Schlüsse auf den Beschaffungsbezug im Kontext der Interpretation des $₫ 105$ GWB lassen sich hieraus jedoch nicht gewinnen. Das Tatbestandsmerkmal des „Beschaffungsbezuges" für Bau- und Dienstleistungskonzession vollständig abstrakt abzugrenzen, entbehrt aus diesem Grund einer normativen Grundlage. Genauso wenig kann es überzeugen, einen Beschaffungsbezug nur bei Vorliegen einer Aufgabe der "Daseinsvorsorge“ anzunehmen. Vielmehr ist es sinnvoll die Abgrenzungskriterien aus der Rechtsprechung des Europäischen Gerichtshofes im Fall Müller, soweit dies möglich und sinnvoll ist, auf die Dienstleistungskonzession analog anzuwenden. Im vorliegenden Fall kommen alle Ansichten zu dem Ergebnis, dass bei einem Konzessionsmodell auf Basis der Vergabe exklusiver Wegerechte ein Beschaffungsbezug gegeben wäre, sodass der Streit im Ergebnis keine Auswirkungen hat.

276 EuGH, Urt. v. 25.3.2010, Rs. C-451/08 (Helmut Müller), ECLI:EU:C:2010:168, Rn. 51; EuGH, Urt. v. 12.7.2001, Rs. C-399/98 (Ordine degli Architetti u. a.), ECLI:EU:C:2001:401, Rn. 67 und 71.

277 Deutscher Landkreistag, Flächendeckende Breitbandversorgung zu wirtschaftlichen Bedingungen sicherstellen, S. 7. 
c) Ergebnis

Der Inhalt des Konzessionsvertrages auf Basis der Vergabe eines exklusiven Wegerechtes ist eine Dienstleistungskonzession im Sinne des Vergaberechts. Ob die Regelungen der KVRL auf die Vergabe der Konzession Anwendung finden, soll an dieser Stelle offen gelassen und erst im Kontext des Rechtsrahmens für die Ausgestaltung des Konzessionsvergabeverfahrens thematisiert werden (C. IV. 3. a.). Die Konsequenzen für die Ausgestaltung des Vergabeverfahrens werden zu einem späteren Zeitpunkt zusammenhängend dargelegt (C. IV.).

\section{Einordnung in weitere Konstellationen der Begriffsverwendung}

Darüber hinaus lässt das Konzessionsmodell auf Basis der Vergabe exklusiver Wegerechte auch in andere Konstellationen einordnen, in denen der Begriff der „Konzession“ verwendet wird. So wird mit der Konzession etwa auch ein Sondernutzungsrecht vergeben (A. III.). Eine trennscharfe Abgrenzung zwischen den Konstellationen ist aber nicht nötig, da der Begriff in diversen Konstellationen verwendet wird, die in keinem Ausschließlichkeitsverhältnis zu einander stehen.

III. Allgemeine rechtliche Steuerungsvorgaben aus dem Telekommunikations(wege)recht

Das Telekommunikationswegerecht ist ein integraler Bestandteil des nationalen Telekommunikationsgesetzes. Damit ein in sich zusammenhängender und konsistenter Rechtsrahmen entsteht, muss sich das Telekommunikationswegerecht in den Gesamtrahmen einfügen und die Prinzipien berücksichtigen, die dem sektorspezifischen Regulierungsrecht zugrunde liegen. Die Regulierungsziele bilden das Grundgerüst des Regulierungsrechts für den Telekommunikationssektor. Der Gesetzgeber nutzt sie, um Leitlinien für die Rechtsanwendung und -auslegung vorzugeben. ${ }^{278}$ Sie entfalten aufgrund ihrer Stellung als einfaches Gesetzesrecht keine Bindung für den Gesetzgeber. Dennoch werden in ihnen die Grundvorstellungen des Gesetzesgebers offenbar und es besteht aufgrund des Bedürfnisses danach,

278 Neumann/Sickmann/Alkas u.a., Reformbedarf des europäischen Rechtsrahmens für elektronische Kommunikation, S. 6 m. w. N. 
den Rechtsrahmen insgesamt stimmig zu gestalten, eine faktische Bindungswirkung (dazu 1.). Darüber hinaus ist das Telekommunikationswegerecht in großen Teilen unionsrechtlich überformt, sodass die verbindlichen Steuerungsvorgaben des EKEK Berücksichtigung finden müssen (dazu 2.). Das aktuelle Telekommunikationsrecht ermöglicht derzeit noch keine exklusive Vergabe von Wegerechten (dazu 3.), sodass eine Novellierung im Rahmen dieser Steuerungsvorgaben erforderlich wäre (dazu 4.).

1. Regulierungsziele als Orientierungsmaßstab für die grundsätzliche Konzeption des Telekommunikationsrechts

Die Ziele, die der Gesetzgeber mit dem Regulierungsrecht verfolgt (dazu a.), adressieren gegenläufige Interessen (dazu b.), die miteinander in einen schonenden Ausgleich gebracht werden müssen. Dies betrifft auch das Konzessionsmodell auf Basis der Vergabe exklusiver Wegerechte, das insbesondere zum Konflikt zwischen Wettbewerbs- und Konnektivitätsziel führt (dazu c.).

\section{a) Ziele der Regulierung im Telekommunikationsrecht}

Das unionale Telekommunikationsrecht ist gemäß Art. 3 Abs. 2 EKEK auf die Verfolgung von vier Regulierungszielen ausgerichtet. Drei dieser Ziele gab es bereits in der Vorgängerrichtlinie. Danach sollen der Wettbewerb (lit. b), die Entstehung eines Binnenmarktes in der Telekommunikationswirtschaft (lit. c) sowie die Interessen der Bürgerinnen und Bürger der Europäischen Union im Rahmen der Regulierung gefördert werden (lit. d). ${ }^{279}$ Diese Ziele haben Eingang in das nationale Regulierungsrecht gefunden, wo sie in $\$ 2$ Abs. 2 TKG normiert sind. Dabei entsprechen die Ziele, die Nutzer- und Verbraucherinteressen zu wahren (Nr. 1), den Wettbewerb zu fördern (Nr.2) und den Binnenmarkt zu stärken (Nr.3), den Zielen des Art. 3 Abs. 2 lit. b bis d EKEK. ${ }^{280}$

279 Normativer Anknüpfungspunkt war Art. 8 RRL; zu den Zielen im Einzelnen und den dahinter stehenden Erwägungen Neumann/Sickmann/Alkas u.a., Reformbedarf des europäischen Rechtsrahmens für elektronische Kommunikation, S. $6 \mathrm{ff}$.

280 Ergänzt wird der Zielekanon um die Ziele der Bereitstellung des Universaldienstes (Nr.4), der Förderung des Anschluss von öffentlichen Einrichtungen (Nr.6), der effizienten und störungsfreien Frequenznutzung (Nr.7) und des 
Der Zielekanon wurde im EKEK um das Ziel erweitert, die „Konnektivität (...) des Zugangs zu und der Nutzung von Netzen (...) mit sehr hoher Kapazität“ (lit. a) zu fördern. Dieses sogenannte „Konnektivitätsziel“ impliziert einen doppelten Handlungsauftrag an die Mitgliedstaaten. Zum einen sind unter wirtschaftlichen Bedingungen Netze mit möglichst hohen Kapazitäten zu errichten. Zum anderen ist sicherzustellen, dass die Kapazitäten in allen Gebieten möglichst konvergent verteilt werden. ${ }^{281}$ Schon bevor das Konnektivitätsziel (Art. 3 Abs. 2 lit. a EKEK) in das Unionsrecht integriert wurde, hatte das nationale Regulierungsrecht über das Ziel verfügt, den Ausbau „von hochleistungsfähigen öffentlichen Telekommunikationsnetzen der nächsten Generation“ zu beschleunigen ( $\$ 2$ Abs. 2 Nr. 5 TKG). ${ }^{282}$ Es ist jedoch im Vergleich zum unionsrechtlichen Pendant weniger ambitioniert ausgestaltet und enthält kein Kapazitätsziel, ${ }^{283}$ sodass es im Rahmen der Umsetzung des EKEK in nationales Recht angepasst werden muss. ${ }^{284}$ Dies wird wohl in Gestalt des $₫ 2$ Abs. 2 Nr. 1 TKG-RefE geschehen, der die Vorgabe des EKEK mit identischem Wortlaut in nationales Recht umsetzen soll. ${ }^{285}$ Hinzu tritt die Vorgabe des $\$ 2$ Abs. 2 Nr. 3 lit. a TKG-RefE, die ebenfalls ein Konnektivitätsziel enthält. ${ }^{286}$

korrespondierenden Umganges mit Nummerierungsressourcen (Nr. 8) sowie des Schutz der öffentlichen Sicherheit (Nr. 9), die keinen Anklang im aktuellen Unionsrecht finden. Bei der Umsetzung des EKEK in nationales Recht sollte aus diesem Grund eine Streichung der Ziele evaluiert werden. Nach aktuellem Stand ist zumindest eine umfassende Neuordnung der Zielvorgaben geplant, siehe hierzu Referentenentwurf des Telekommunikationsmodernisierungsgesetzes (TK-MoG), abrufbar unter: https://www.bmwi.de/Redaktion/DE/Downloads /Gesetz/telekommunikationsmodernisierungsgesetz-referentenentwurf-2020161 2.pdf?_blob=publicationFile\&v=8 (zuletzt abgerufen am 12.1.2021).

281 Erwägungsgrund 23 des EKEK; sowie Erwägungsgrund 24 des EKEK, wonach die Kommission die Verfolgung und Verwirklichung der Ziele beobachtet und eine Rangliste der digitalen Leistungsfähigkeit aufstellt.

282 Im Rahmen der Verhandlungen zur Einführung des EKEK fand eine umfassende Debatte über die Einführung des Konnektivitätsziels statt; Neumann/Sickmann/Alkas u.a., Reformbedarf des europäischen Rechtsrahmens für elektronische Kommunikation, S. $128 \mathrm{ff}$.

283 BT-Drs. 17/5707, S. 47 f.

284 Das Konnektivitätsziel entfaltet für die BNetzA jedoch erst nach Umsetzung des EKEK Bindungswirkung, Meyer, N\&R 2019, S. 264, 265.

285 Referentenentwurf des Telekommunikationsmodernisierungsgesetzes (TKMoG), abrufbar unter: https:/www.bmwi.de/Redaktion/DE/Downloads/Gesetz/ telekommunikationsmodernisierungsgesetz-referentenentwurf-20201612.pdf? blob=publicationFile $\& v=8$ (zuletzt abgerufen am 12.1.2021).

286 Referentenentwurf des Telekommunikationsmodernisierungsgesetzes (TKMoG), abrufbar unter: https:/www.bmwi.de/Redaktion/DE/Downloads/Gesetz/ 
b) Umgang des Telekommunikationsrechts mit Zielkonflikten

Die Regulierungsziele adressieren gegenläufige Interessen. Neben individuellen unternehmerischen Interessen (beispielsweise der Konkurrenzreduktion oder Profitmaximierung) sollen auch soziale Interessen (beispielsweise die Gewährleistung des Universaldienstes) bei der Anwendung und Auslegung des Regulierungsrechts berücksichtigt werden. ${ }^{287}$ Die Ziele stehen in einem „prinzipiellen Widerspruch" 288 zueinander, wobei kein Ziel einen höheren Rang als ein anderes einnimmt (Art. 3 Abs. 2 EKEK). Aus diesem Grund hat der Normanwender die kollidierenden Interessen schonend miteinander in Ausgleich zu bringen. Ein Ziel darf nicht auf eine Art und Weise verfolgt werden, die andere Regulierungsziele unberücksichtigt lässt. Die Regulierungsziele werden durch das gesamte Gesetz konkretisiert und umgesetzt. Um einen konsistenten Rechtsrahmen zu schaffen, muss sich nicht nur der Normanwender, sondern auch der Gesetzgeber an ihnen orientieren.

c) Kollidierende Ziele im Konzessionsmodell auf Basis der Vergabe exklusiver Wegerechte

Auch das Konzessionsmodell auf Basis der Vergabe exklusiver Wegerechte adressiert verschiedene, widersprüchliche Interessenlagen. Mithilfe der Maßnahme soll insbesondere die Konnektivität der Bevölkerung erhöht werden. Hierzu werden jedoch temporäre Infrastrukturmonopole geschaffen, die in einem prinzipiellen Spannungsverhältnis zum Regulierungsziel der Wettbewerbsförderung stehen. ${ }^{289}$ Sofern das Netzausbaudefizit im Konzessionsgebiet auf eine mangelnde Nachfrage zurückzuführen ist, ist zudem fraglich, ob ein Netzausbau im Interesse der Bürger liegt. Dies lässt sich darauf zurückführen, dass die Endkundenpreise, um die Investitionen

telekommunikationsmodernisierungsgesetz-referentenentwurf-20201612.pdf? blob=publicationFile $\& v=8$ (zuletzt abgerufen am 12.1.2021).

287 Lepsius, in: Fehling/Ruffert (Hrsg.), Regulierungsrecht, S. 1055, 1079.

288 So Lepsius, in: Fehling/Ruffert (Hrsg.), Regulierungsrecht, S. 1055, 1080.

289 Wettbewerb findet lediglich im Rahmen des Verfahrens zur Konzessionsvergabe und beim Verkauf solcher Vorleistungsprodukte statt, die erforderlich sind, um den Endkunden zu erreichen; Wernick/Queder/Strube Martins u.a., Ansätze zur Glasfaser-Erschließung unterversorgter Gebiete, S. 54; Wernick/Queder/Strube Martins u.a., Gigabitnetze für Deutschland, S. 113; kritisch Monopolkommission, 10. Sektorgutachten Telekommunikation (2017), Rn. 205. 
amortisieren zu können, im Ergebnis steigen würden. Mangels entsprechender Nachfrage wären die Endkunden aller Wahrscheinlichkeit nach nicht dazu bereit, entsprechend erhöhte Preise zu zahlen. Es existiert somit ein multipolares Spannungsverhältnis. Damit sich das Konzessionsmodell auf Basis der Vergabe exklusiver Wegerechte in das Gesamtkonzept des Telekommunikationsrecht einfügt und kein normativer und systematischer Fremdkörper entsteht, dürfte die Konnektivität nicht einseitig gefördert werden. Vielmehr müsste ein schonender Ausgleich mit der Förderung des Wettbewerbs sichergestellt werden. Dies könnte durch die Integration ausreichender wettbewerblicher Elemente in das Vergabeverfahren erreicht werden, in dem auch das Interesse der Bürger umfassend berücksichtigt werden müsste.

2. Unionsrechtliche Anforderungen an die Vergabe von Telekommunikationswegerechten

Das Unionsrecht enthält in Art. 43 Abs. 1 EKEK Steuerungsvorgaben für die Vergabe von Wegerechten, die bei der Umsetzung in das TKG zu beachten sind. Die Entscheidung soll gemäß Art. 43 Abs. 1 UAbs. 1 lit. a EKEK ,auf der Grundlage einfacher, effizienter, transparenter und öffentlich zugänglicher Verfahren [erfolgen], die nichtdiskriminierend und unverzüglich angewendet werden. “290 Die Vergabeentscheidung muss von der zuständigen Behörde in der Regel innerhalb von sechs Monaten nach Antragstellung getroffen werden. ${ }^{291}$ Der Gesetzgeber geht davon aus, dass komplexe Verfahren den Wettbewerb behindern und deswegen so weit wie möglich vereinfacht werden sollten. ${ }^{292}$ Sofern die Wegerechtsvergabe an Bedingungen geknüpft wird, müssen diese gemäß Art. 43 Abs. 1 UAbs. 1 lit. b EKEK transparent und nicht diskriminierend sein. Es dürfen

290 Kühling/Toros, Rechtliche Rahmenbedingungen für die Schaffung von Anreizen für einen flächendeckenden Ausbau von Glasfaserinfrastrukturen, S. 101.

291 Ausgenommen sind gemäß Art. 43 Abs. 1 UAbs. 1 lit. a EKEK lediglich Konstellationen, in denen eine Enteignung erfolgen soll. In diesen Fällen darf der Entscheidungsprozess auch länger dauern.

292 Erwägungsgrund 104 des EKEK; der Erwägungsgrund 42 der ÄndRL 2009 war in dieser Hinsicht wortgleich; vgl. zu der hieran anknüpfenden Allgemeingenehmigung, wonach der Antrag auf Erteilung des Wegerechts stets geprüft werden muss, Kübling/Toros, Rechtliche Rahmenbedingungen für die Schaffung von Anreizen für einen flächendeckenden Ausbau von Glasfaserinfrastrukturen, S. $100 \mathrm{f}$. 
gemäß Art. 43 Abs. 1 UAbs. 2 EKEK unterschiedliche Verfahren für die Errichtung von öffentlich und nicht öffentlich zugänglichen Telekommunikationsnetzen angewandt werden. Im Ergebnis belässt das Unionsrecht einen Spielraum und charakterisiert das Verfahren lediglich, ohne Einzelheiten festzulegen. Aufgrund der Vielzahl der zu beachtenden Charakteristika ist der Spielraum jedoch nicht so groß, wie er auf den ersten Blick erscheinen mag. Dieser Eindruck manifestiert sich durch zusätzliche rechtliche Rahmenbedingungen, die den Spielraum des nationalen Gesetzgebers weiter einschränken.

Der Unionsgesetzgeber hat das Telekommunikationswegerecht seit der Rahmenrichtlinie aus dem Jahr 2002 (RRL 2002)293 kontinuierlich vereinfacht und Rahmenbedingungen zur Verfahrensbeschleunigung etabliert. So waren in Art. 11 Abs. 1 UAbs. 2 Spiegelstrich 1 RRL 2002 die Parameter der Einfachheit und Effizienz noch nicht vorgesehen. Lediglich in den Erwägungsgründen wurde erläutert, dass eine „zügige“ Vergabe von Wegerechten „für einen lauteren, wirksamen Wettbewerb“294 erforderlich sei. Der Richtliniengeber sah den Infrastrukturwettbewerb schon 2002 als essentiell für den Dienstewettbewerb an und forderte, zügig Maßnahmen zum Aufbau von Telekommunikationsinfrastrukturen zu ergreifen. 295

In der Änderungsrichtlinie aus dem Jahr 2009 (ÄndRL 2009) wurde das Wegerecht mit der Zielrichtung abgeändert, den Infrastrukturausbau weiter zu beschleunigen. ${ }^{296}$ So wurde die RRL gemäß Nr. 13 ÄndRL 2009 dahingehend ergänzt, dass Entscheidungen im Kontext der Wegerechtsvergabe innerhalb von sechs Monaten zu treffen seien. Auch in den Erwägungsgründen wurde herausgestellt, dass Wegerechte für die Errichtung von In-

293 Richtlinie 2002/21/EG des Europäischen Parlaments und des Rates vom 7.3.2002 über einen gemeinsamen Rechtsrahmen für elektronische Kommunikationsnetze und -dienste (Rahmenrichtlinie), ABl. EG L 108/33 vom 24.4.2002.

294 Erwägungsgrund 22 der RRL 2002; der Gesetzgeber verfolgt diesen Grundsatz, wie in Erwägungsgrund 103 des EKEK offenbar wird, weiterhin.

295 Erwägungsgrund 22 der RRL: „Um die Voraussetzungen für einen lauteren, wirksamen Wettbewerb zu schaffen, sollte sichergestellt werden, dass zügige, nichtdiskriminierende und transparente Verfahren zur Erteilung von Rechten für die Installation von Einrichtungen bestehen (...).“

296 Richtlinie 2009/140/EG des Europäischen Parlaments und des Rates vom 25.11.2009 zur Änderung der Richtlinie 2002/21/EG über einen gemeinsamen Rechtsrahmen für elektronische Kommunikationsnetze und -dienste, der Richtlinie 2002/19/EG über den Zugang zu elektronischen Kommunikationsnetzen und zugehörigen Einrichtungen sowie deren Zusammenschaltung und der Richtlinie 2002/20/EG über die Genehmigung elektronischer Kommunikationsnetze und -dienste, ABl. EU L 337/37 vom 18.12.2009. 
frastruktur von großer Bedeutung seien. ${ }^{297}$ Die Verfahrensparameter wurden um die Kriterien der Einfachheit und Effizienz erweitert. ${ }^{298}$

Diese Grundtendenz bleibt auch in Art. 43 EKEK ohne signifikante Änderungen erhalten. Der EU-Gesetzgeber betrachtet zeitaufwendige und kostenintensive Verfahren als Gefahr für den Wettbewerb. Grundsätzlich soll die Komplexität der Verfahren weiter reduziert werden. Dies steht einer Komplexitäts- und Kostensteigerung im Verfahren entgegen.

Im Gesetzgebungsverfahren zum EKEK in den Jahren vor 2018 wurde nicht darüber diskutiert, die entsprechenden Verfahrensparameter wieder aus dem Rechtsrahmen zu entfernen. Vielmehr wurden Rechtsrahmen und Erwägungsgründe unverändert in den EKEK übertragen. Es lassen sich demnach keine Anhaltspunkte dafür ausmachen, dass sich die Ansicht des EU-Gesetzgebers kurzfristig ändern wird. ${ }^{299}$ Demnach werden diese Maßgaben auch in Zukunft das unionale Telekommunikationswegerecht bestimmen, sodass sie der nachfolgenden Untersuchung zugrunde gelegt werden können.

\section{Grundkonzeption der Wegerechtsvergabe im TKG}

Die Grundnorm des nationalen Wegerechts ist $\$ 68$ TKG. Das Nutzungsrecht für Verkehrswege zum Aufbau von Telekommunikationslinien liegt grundsätzlich beim Bund, muss aber als Folge der Liberalisierung des Telekommunikationssektors auf private Telekommunikationsunternehmen übertragen werden. ${ }^{300}$ Betreiber oder Eigentümer eines öffentlichen Telekommunikationsnetzes oder einer öffentlichen Zwecken dienenden Telekommunikationslinie können gemäß $₫ 69$ Abs. 1 TKG beim Bund die Übertragung der Wegerechte beantragen. ${ }^{301}$ Der Bund kann die Anträge

297 Erwägungsgrund 42 der ÄndRL 2009.

298 Biendl, Rechtliche Herausforderungen beim Auf- und Ausbau von Next-Generation-Access-Networks, S. 210.

299 So auch Kühling/Toros, Rechtliche Rahmenbedingungen für die Schaffung von Anreizen für einen flächendeckenden Ausbau von Glasfaserinfrastrukturen, S. 102.

300 Hierbei handelt es sich um eine Folge des Gebotes der Privatwirtschaftlichkeit aus Art. 87f Abs. 2 S. 1 GG.

301 Zur Vergabe des Wegerechtes im Telekommunikationssektor auch Monopolkommission, 10. Sektorgutachten Telekommunikation (2017), Rn. 202. 
nur in begrenzten Ausnahmefällen ablehnen. ${ }^{302}$ Die Übertragung ist stets streng personengebunden und nicht rechtsnachfolgefähig. ${ }^{303}$ Die Antragsvoraussetzungen ergeben sich aus $₫ 69$ Abs. 2 TKG. Insbesondere müssen gemäß $\$ 69$ Abs. 2 S. 2 TKG die Voraussetzungen in der Person des Antragstellers erfüllt sein und die Übertragung der Nutzungsberechtigung darf nicht den Regulierungszielen widersprechen. Die persönlichen Voraussetzungen sind mit denen des Gewerberechts vergleichbar. ${ }^{304}$ Als Rechtsfolge ergibt sich ein unentgeltliches Nutzungsrecht, für das keine - wie auch immer geartete - Gegenleistung verlangt werden darf. ${ }^{305}$

Wird eine Telekommunikationslinie geändert oder neu gebaut, muss gemäß $₫ 68$ Abs. 3 S. 1 TKG der Träger der Wegebaulast schriftlich zustimmen. ${ }^{306}$ Werden Leitungen oberirdisch verlegt, sind gemäß $\$ 68$ Abs. 3 S. 4 TKG die Interessen des Wegebaulastträgers, die des Betreibers öffentlicher Telekommunikationsnetze und die städtebaulichen Belange gegeneinander abzuwägen. ${ }^{307}$ Zwischen einer oberirdischen und unterirdischen Leitungsverlegung besteht im Übrigen kein direktes Rangverhältnis. Lediglich bei Gesamtbaumaßnahmen soll gemäß $\$ 68$ Abs. 3 S.7 TKG auf die unterirdische Leitungsverlegung zurückgegriffen werden. Die Zustimmung kann unter den Voraussetzungen des $\$ 68$ Abs. 3 S. 8 und 9 TKG mit Nebenbestimmungen versehen werden.

Das Wegerecht des TKG erfüllt die Anforderungen des Unionsrechts, die sich aus Art. 43 Abs. 1 UAbs. 1 lit. a und b EKEK ergeben. Das Vergabeverfahren ist insbesondere einfach, effizient und schnell. Das Wegerecht wird auf Basis eines Antrages gemäß $\$ 69$ Abs. 1 TKG an alle berechtigten Interessenten vergeben. Einschränkungen und Bedingungen sind lediglich in begrenzten Ausnahmefällen zulässig. Das Telekommunikationswege-

302 Ausnahmen vom Grundsatz können sich aus dem Widmungszweck ( $\$ 68$ Abs. 1 S. 2 a. E. TKG), der Sicherheit und Ordnung ( $\$ 68$ Abs. 2 S. 1 TKG) und den Allgemeinen Technischen Bestimmungen ( $\$ 68$ Abs. 2 S. 1 TKG) ergeben.

303 Stelkens/Wabnitz, MMR 2015, S. 502.

304 Zur Begriffsbestimmung kann auf die gewerberechtliche Rechtsprechung zurückgegriffen werden; Schöttner, in: Geppert/Schütz (Hrsg.), Beck'scher TKGKommentar, $\mathbb{~ 8 , ~ R n . ~} 40 \mathrm{ff}$.

305 OVG Nordrhein-Westfalen, Urt. v. 22.5.2003, Az. 20 A 2732/01, MMR 2004, S. 53, 55.

306 Unter dem Begriff der Wegebaulast sind alle mit der Unterhaltung öffentlicher Wege zusammenhängenden Aufgaben zu verstehen. Es handelt sich um eine öffentlich-rechtliche Pflicht gegenüber der Wegeaufsichtsbehörde; Holznagel/ Enaux/Nienhaus, Telekommunikationsrecht, Rn. 571.

307 Faktisch fungiert die Vorschrift somit als Grenze des behördlichen Ermessensspielraums; Holznagel/Enaux/Nienhaus, Telekommunikationsrecht, Rn. 571. 
recht muss deswegen im Rahmen der Umsetzung des EKEK in nationales Recht insofern nicht angepasst werden. Demnach verwundert es nicht, dass sich die skizzierten Grundmechanismen im künftigen TKG nicht ändern werden. Die Vorgaben sind voraussichtlich in den $\$ \mathbb{S} 124 \mathrm{ff}$. TKGRefE enthalten. ${ }^{308}$

4. Novellierungserfordernisse zur Ermöglichung einer exklusiven Wegerechtsvergabe

Das Telekommunikationswegerecht müsste modifiziert werden, um eine exklusive Wegerechtsvergabe in Konzessionsgebieten zu ermöglichen. Vorgeschlagen wird eine Orientierung am Regelungsregime des Energiesektors. Dort wird in $\$ 46$ EnWG zwischen Wegenutzungs- und Konzessionsverträgen unterschieden, die mit einfachen oder qualifizierten Wegerechten verknüpft werden. ${ }^{309}$ Einfache Wegerechte dienen dem Anschluss einzelner Endkunden, während qualifizierte Wegerechte für den Betrieb eines Netzes der „allgemeinen Versorgung“ vergeben werden. Ein entsprechendes System sollte jedoch, wie bereits dargelegt, nicht eingeführt werden (C. II. 1. b. cc. (1)). Dennoch müsste zwischen der Vergabe von Wegerechten inner- und außerhalb eines Konzessionsgebietes differenziert werden. Nur innerhalb des Konzessionsgebietes sollen Wegerechte exklusiv vergeben werden, während der geltende Rechtsrahmen außerhalb der Konzessionsgebiete fortbestehen soll. ${ }^{310}$ Das aktuelle Wegerechtsvergabeverfahren müsste durch ein alternatives Verfahren ergänzt werden, das nur in den Konzessionsgebieten Anwendung findet.

Diese Anpassung des Rechtsrahmens müsste sich mit den unionsrechtlichen Steuerungsvorgaben aus Art. 43 Abs. 1 EKEK vereinbaren lassen. Ge-

308 Referentenentwurf des Telekommunikationsmodernisierungsgesetzes (TKMoG), abrufbar unter: https:/www.bmwi.de/Redaktion/DE/Downloads/Gesetz/ telekommunikationsmodernisierungsgesetz-referentenentwurf-20201612.pdf? blob $=$ publicationFile $\& v=8$ (zuletzt abgerufen am 12.1.2021).

309 Unterscheidungskriterium ist der Vertragsgegenstand. Während Konzessionsverträge das Verteilernetz zur allgemeinen Versorgung erfassen, sind Energieleitungen, die der Versorgung einzelner Letztverbraucher dienen, unter den Wegenutzungsvertrag zu subsumieren; Kühling/Rasbach/Busch, Energierecht, Kap. 8, Rn. 3.; Kühling/Toros, Rechtliche Rahmenbedingungen für die Schaffung von Anreizen für einen flächendeckenden Ausbau von Glasfaserinfrastrukturen, S. 103; Templin, Recht der Konzessionsverträge, S. $125 \mathrm{ff}$.

310 Deutscher Landkreistag, Flächendeckende Breitbandversorgung zu wirtschaftlichen Bedingungen sicherstellen, S. 7. 
mäß Art. 43 Abs. 1 UAbs. 2 EKEK dürfen sich, wie bereits dargelegt, die Verfahren für die Vergabe von Wegerechten für öffentliche und nicht öffentliche Telekommunikationsnetze unterscheiden. Beide Verfahren müssen sich an den Parametern aus Art. 43 Abs. 1 UAbs. 1 lit. a und b EKEK orientieren. Der Wortlaut erlaubt lediglich eine Etablierung von zwei Verfahren. Innerhalb der jeweiligen Gattung muss der Entscheidung jedoch ein einheitliches Verfahren zugrunde gelegt werden. Dies lässt sich mit den Kriterien der Einfachheit und Effizienz begründen. In einem konsistenten und verlässlichen Rechtsrahmen können Investitionen leichter geplant werden und ohne großen juristischen Beratungsaufwand erfolgen. Der Begriff des öffentlichen elektronischen Kommunikationsnetzes ist in Art. 2 Nr. 8 EKEK legaldefiniert. Die Legaldefinition wird im Rahmen der TKG-Novelle voraussichtlich nicht abgeändert, aber künftig in $\$ 3 \mathrm{Nr} .42$ TKG-RefE normiert sein. ${ }^{311}$ Sie knüpft daran an, dass das Netz „die Übertragung von Informationen zwischen Netzabschlusspunkten“ und die Nutzung öffentlich zugänglicher Telekommunikationsdienste ermöglicht. Dies ist sowohl inner- als auch außerhalb des Konzessionsgebietes bezweckt. Es handelt sich in beiden Konstellationen um ein öffentliches elektronisches Kommunikationsnetz, für die der Gesetzgeber die Einführung unterschiedlicher Wegerechtsvergabeverfahren nicht gestattet. Dies wäre also nicht mit Art. 43 Abs. 1 UAbs. 2 EKEK zu vereinbaren. Selbst wenn man dies zuließe, müsste das Verfahren jedenfalls die Kriterien des Art. 43 Abs. 1 UAbs. 1 lit. a EKEK erfüllen.

\section{Ergebnis}

Das unionale Telekommunikationsrecht enthält Steuerungsvorgaben zur Ausgestaltung des nationalen Wegerechtsvergabeverfahrens. Wegerechte müssen gemäß Art. 43 Abs. 1 UAbs. 1 lit. a EKEK „auf der Grundlage einfacher, effizienter, transparenter und öffentlich zugänglicher Verfahren“ vergeben werden. Das aktuelle Wegerecht in den $\int S 68 \mathrm{ff}$. TKG ist unionsrechtskonform ausgestaltet. Dasselbe gilt für die künftigen Vorgaben der SS 124 ff. TKG-RefE, da sie in den hier maßgeblichen Aspekten unverän-

311 Referentenentwurf des Telekommunikationsmodernisierungsgesetzes (TKMoG), abrufbar unter: https:/www.bmwi.de/Redaktion/DE/Downloads/Gesetz/ telekommunikationsmodernisierungsgesetz-referentenentwurf-20201612.pdf? blob=publicationFile $\& v=8$ (zuletzt abgerufen am 12.1.2021). 
dert bleiben. ${ }^{312}$ Der Rechtsrahmen unterscheidet jedoch nicht zwischen einfachen und exklusiven Wegerechten. Eine solche Differenzierung müsste eingeführt werden, um eine einfache Vergabe von Wegerechten außerhalb und eine Vergabe exklusiver Wegerechte innerhalb von Konzessionsgebieten zu ermöglichen. Die entsprechende Anpassung müsste sich auch mit dem Unionsrecht vereinbaren lassen. Dieses lässt gemäß Art. 43 Abs. 1 UAbs. 2 EKEK zwar die Einführung unterschiedlicher Verfahren für die Vergabe von Wegerechten für die Errichtung öffentlicher und nicht öffentlicher Telekommunikationsnetze zu. Allerdings sollen sowohl inner- als auch außerhalb des Konzessionsgebietes öffentliche Telekommunikationsnetze errichtet werden. Die Entwicklung zweier unterschiedlicher Wegerechtsvergaberegimes innerhalb einer Gattung lässt sich mit dem Wortlaut und dem Telos des Art. 43 Abs. 1 UAbs. 2 EKEK jedoch nicht vereinbaren, sodass sie unionsrechtlich unzulässig ist.

\section{Ausgestaltung des Konzessionsvergabeverfahrens}

Wie bereits dargestellt, müssen Telekommunikationswegerechte gemäß Art. 43 Abs. 1 UAbs. 1 lit. a EKEK „auf der Grundlage einfacher, effizienter, transparenter und öffentlich zugänglicher Verfahren" vergeben werden. Durch die Vergabe exklusiver Wegerechte entsteht ein Infrastrukturmonopol. Aufgrund dieses sensiblen Eingriffes in den Wettbewerb müsste sich das Verfahren zur Auswahl des Konzessionsnehmers an verschiedenen zusätzlichen rechtlichen Maßstäben messen lassen, die das Ziel haben, die negativen Auswirkungen der Beschränkung des Wettbewerbs möglichst gering zu halten. Steuerungsvorgaben ergeben sich insofern insbesondere aus dem Verfassungs- (dazu 1.), Wettbewerbs- (dazu 2.), dem Vergabe- (dazu 3.) sowie dem EU-Beihilfenrecht (dazu 4.), die bei der Ausgestaltung des Konzessionsvergabeverfahrens Berücksichtigung finden müssten (dazu 5.).

312 Referentenentwurf des Telekommunikationsmodernisierungsgesetzes (TKMoG), abrufbar unter: https:/www.bmwi.de/Redaktion/DE/Downloads/Gesetz/ telekommunikationsmodernisierungsgesetz-referentenentwurf-20201612.pdf? blob=publicationFile\&v=8 (zuletzt abgerufen am 12.1.2021). 
1. Steuerungsvorgaben des europäischen und deutschen Verfassungsrechts

Der Gesetzgeber hat im Rahmen der Normsetzung stets das nationale Verfassungsrecht zu berücksichtigen und ist zudem an das unionale Primärrecht gebunden, das ebenfalls als Verfassungsrecht klassifiziert werden kann. Steuerungsvorgaben für die Ausgestaltung des Konzessionsvergabeverfahrens ergeben sich insbesondere aus den Grundrechten (dazu a.) sowie aus Art. 87f Abs. 2 S. 1 GG (dazu b.).

\section{a) Grundrechte}

Das Konzessionsmodell auf Basis der Vergabe exklusiver Wegerechte müsste sich mit den Grundrechten vereinbaren lassen. Grundrechtsschutz wird auf völkerrechtlicher, supranationaler und nationaler Ebene gewährt. Grundrechtskataloge enthalten die Europäische Menschenrechtskonvention (EMRK) ${ }^{313}$ auf Ebene des Völkerrechts, die Europäische Grundrechtecharta $(\mathrm{GRCh})^{314}$ auf unionaler und das GG auf nationaler Ebene. Aus diesem Grund ist zunächst fraglich, an welchen dieser Gewährleistungen sich das Konzessionsmodell auf Basis der Vergabe exklusiver Wegerechte messen lassen müsste (dazu aa.). Sodann ist zu prüfen, ob seine Einführung einen ungerechtfertigten Eingriff in die erwerbsbezogenen Grundrechte (dazu bb.) der unterlegenen Bewerber im Konzessionsvergabeverfahren darstellt und welche Anforderungen an die Verfahrensausgestaltung sich aus den Gleichheitsrechten ergeben (dazu cc.). Hiervon losgelöst ist gesondert zu untersuchen, inwiefern der Umgang mit der Bestandsinfrastruktur im Hinblick auf die Eigentumspositionen problematisch sein könnte (dazu vertieft C. V.).

313 Europarat, Vertrag-Nr. 005, Konvention zum Schutze der Menschenrechte und Grundfreiheiten, SEV Nr. 005, gezeichnet am 4.11.1950 in Rom, in Kraft getreten am 3.9.1953, BGBl. 1952, II, S. 685, in der Fassung der Bekanntmachung v. 22.10.2010, BGBl. 2010, II S. 1198, zuletzt geändert durch 15. EMRK-Protokoll v. 24.6.2013, BGBl. 2014. II S. 1034.

314 Charta der Grundrechte der Europäischen Union, ABl. 2007/C 303/01 v. 14.12.2007. 
aa) Identifikation des einschlägigen Grundrechtskataloges

Das Telekommunikationswegerecht ist unionsrechtlich vorstrukturiert und mittels eines nationalen Umsetzungsgesetzes in deutsches Recht zu transformieren. Als Beurteilungsmaßstab für die Überprüfung der Grundrechtskompatibilität kommen damit einerseits das GG und andererseits die GRCh in Betracht.

Die Rechtsprechung des Bundesverfassungsgerichtes zur Zuständigkeit und hinsichtlich des Beurteilungsmaßstabes für die Prüfung von Grundrechtsverletzungen im Mehrebenensystem hat sich parallel zum europäischen Integrationsprozess entwickelt. Während es im Jahr 1974 mangels eines unionsrechtlichen Schutzstandards noch von seiner eigenen Zuständigkeit zur Prüfung der Grundrechtskompatibilität von Gemeinschaftsrechtsakten ausgegangen war, ${ }^{315}$ entwickelte es seine Rechtsprechung in verschiedenen Entscheidungen dahin, dass auch auf gemeinschaftlicher Ebene von einem ausreichenden Grundrechtsschutz auszugehen ist. ${ }^{316}$ Umstritten waren bis zuletzt jedoch die Zuständigkeit und der Beurteilungsmaßstab für die Prüfung der Vereinbarkeit unional determinierter nationaler Rechtsakte mit den Grundrechten.

Die Grundrechtecharta entfaltet ihre Bindungswirkung gemäß Art. 51 Abs. 1 S. 1 GRCh für Mitgliedstaaten ausschließlich „bei der Durchführung des Rechts der Union“. Der Europäische Gerichtshof interpretiert den Durchführungsbegriff des Art. 51 Abs. 1 S. 1 GRCh in seiner Leitent-

315 BVerfG, Beschl. v. 29.5.1974, Az. BvL 52/71 (Solange I), BVerfGE 37, S. 271, $280 \mathrm{f}$.; Diese Entscheidung ist jedoch vor dem Hintergrund kritisch zu hinterfragen, dass sich der EuGH im Vorfeld bereits etwa in den Entscheidungen EuGH, Urt. v. 12.11.1969, Rs. 29/69 (Stauder), ECLI:EU:C:1969:57, Rn. 7 und EuGH, Urt. 17.12.1970, Rs. 11/70 (Internationale Handelsgesellschaft), ECLI:EU:C: 1970:114, Rn. 3 f. entschieden hat, dass es sich bei Grundrechten um „allgemeine Rechtsgrundsätze“ des Unionsrechts handele. Er thematisiert in seiner Entscheidung EuGH, Urt. v. 14.5.1974, Rs. 4/73 (Nold), ECLI:EU:C:1974:51, Rn. 12 ff. zudem ausführlich die Grundrechte zum Schutz von Eigentum und freier Berufsausübung; hierzu weiterführend Toros/Weiß, ZJS 2020, S. 100.

316 BVerfG, Beschl. v. 22.10.1986, Az. 2 BvR 197/83 (Solange II), BVerfGE 73, S. 339, 378 ff., stellt fest, dass der Grundrechtsschutz auf Gemeinschaftsebene in ausreichendem Maß sichergestellt sei. Diese Einschätzung bestätigten BVerfG, Urt. v. 12.10.1993, Az. 2 BvR 2134/92 und 2159/92 (Maastricht), BVerfGE 89, S. 155, 175, und BVerfG, Beschl. v. 7.6.2000, Az. 2 BvL 1/97 (Bananenmarkt-Verordnung), BVerfGE 102, S. 147, 164 ff.; zusammenfassend zur historischen Entwicklung Toros/Weiß, ZJS 2020, S. 100, 100 ff.; Edenharter, DÖV, S. 349, 350 f.; Kämmerer/Kotzur, NVwZ 2020, S. 177, 178. 
scheidung Åkerberg Fransson weit. ${ }^{317}$ Es seien „keine Fallgestaltungen denkbar, die vom Unionsrecht erfasst werden, ohne dass diese [Unions-]Grundrechte anwendbar werden." 318 Sachverhalte ohne jeglichen Bezug zum Unionsrecht sollten jedoch nicht anhand der GRCh überprüft werden. ${ }^{319}$ Es müsse „einen hinreichenden Zusammenhang von einem gewissen Grad [geben], der darüber hinaus geht, dass die fraglichen Sachbereiche benachbart sind oder der eine von ihnen mittelbare Auswirkungen auf den anderen haben kann. “320 Eine parallele Anwendung sei ausgeschlossen, wenn den Mitgliedstaaten kein Umsetzungsspielraum verbleibe. ${ }^{321}$ In anderen Fällen komme es zu einer doppelten Grundrechtsbindung unter besonderer Berücksichtigung der unionsrechtlichen Vorgaben. ${ }^{322}$

Das Bundesverfassungsgericht vertrat zum Zeitpunkt dieser Entscheidung des Europäischen Gerichtshofes eine strenge Separationsthese und

317 EuGH, Urt. v. 26.2.2013, Rs. C-617/10 (Åkerberg Fransson), ECLI:EU:C: 2013:280, Rn. 21 ff., verweist umfassend auf seine ständige Rechtsprechung und bewertet die zitierte Entscheidung somit selbst nicht als Neuerung, sondern als Fortsetzung des bisherigen Vorgehens, siehe dazu Obler, NVwZ 2013, S. 1433, 1435; Thym, NVwZ 2013, S. 889, 890; Thym, DÖV 2014, 941.

318 EuGH, Urt. v. 26.2.2013, Rs. C-617/10 (Åkerberg Fransson), ECLI:EU:C: 2013:280, Rn. 21.

319 EuGH, Beschl. v. 16.1.2014, Rs. C-332/13 (Weigl), ECLI:EU:C:2014:31, Rn. 13 ff.; EuGH, Beschl. v. 6.6.2013, Rs.C-14/13 (Cholakova), ECLI:EU:C: 2013:374, Rn. 28 ff.; EuGH, Beschl. v. 8.5.2013, Rs. C-73/13 (T), ECLI:EU:C: 2013:299, Rn. 11 ff.; EuGH, Beschl. v. 14.3.2013, Rs. C-555/12 (Loreti, u. a.), ECLI:EU:C:2013:174, Rn. 16f.; EuGH, Beschl. v. 28.11.2013, Rs. C-258/13 (Sociedade Agrícola e Imobiliária da Quinta de S.Paio Lda), ECLI:EU:C:2013:810, Rn. 18 ff.; Edenharter, Grundrechtsschutz in föderalen Mehrebenensystemen, S. 886 f.; Thym, DÖV 2014, S. 941, 943.

320 EuGH, Urt. v. 6.3.2014, Rs. C-206/13 (Siragusa), ECLI:EU:C:2014:126, Rn. 24; EuGH, Urt. v. 10.7.2014, Rs. C-198/13 (Julian Hernández u. a.), ECLI:EU:C: 2014:2055, Rn.34; EuGH, Beschl. v. 24.9.2019, Rs. C-467/19 PPU (QR), ECLI: EU:C:2019:776, Rn. 38 ff.; Thym, DÖV 2014, S.941, 944; Ward, in: Peers/ Hervey/Kenner u.a. (Hrsg.), The EU Charter of Fundamental Rights, Art. 51 GRCh, Rn. 51.50.

321 EuGH, Urt. v. 26.2.2013, Rs. C-399/01 (Melloni), ECLI:EU:C:2013:107, Rn. 59; Thym, NVwZ 2013, S. 889, $891 \mathrm{f}$.

322 EuGH, Urt. v. 26.2.2013, Rs.C-617/10 (Åkerberg Fransson), ECLI:EU:C: 2013:280, Rn. 29; EuGH, Urt. v. 26.2.2013, Rs. C-399/01 (Melloni), ECLI:EU:C: 2013:107, Rn. 60; Obler, NVwZ 2013, S. 1433, 1437; Thym, NVwZ 2013, S. 889, 892; Thym, DÖV 2014, S. $941,942$. 
teilte dementsprechend die Prüfkompetenzen und Beurteilungsmaßstäbe streng zwischen den Gerichten auf. ${ }^{323}$

In den Entscheidungen Recht auf Vergessen $I^{324}$ und Recht auf Vergessen $I^{325}$ hat das Bundesverfassungsgericht diese bisherige Rechtsprechung nun aufgegeben und seine Rolle beim Grundrechtsschutz im Mehrebenensystem neu definiert. ${ }^{326}$ Dabei differenziert es zwischen Konstellationen, in denen das Unionsrecht die mitgliedstaatliche Entscheidung vollständig determiniert (Recht auf Vergessen II) und in denen den Organen der Mitgliedstaaten ein Umsetzungsspielraum bleibt (Recht auf Vergessen I).

Bei einem Umsetzungsspielraum, also in der Konstellation der Entscheidung Recht auf Vergessen I, überprüft das Bundesverfassungsgericht die Grundrechtskompatibilität anhand der nationalen Grundrechte. Es erkennt jedoch die Möglichkeit einer doppelten Grundrechtsbindung an und verwirft damit die zuvor vertretene strenge Separationsthese. ${ }^{327}$ Grundsätzlich gelten die unionalen Grundrechte als über die nationalen Grundrechte mitgewährleistet. ${ }^{328}$ Nur in Fällen eines höheren Schutzstandards des Unionsrechts, greift das Bundesverfassungsgericht auf den Beurteilungsmaßstab der GRCh zurück. ${ }^{329}$ Dies ist bei einer vollständigen Determinierung, also in einer Konstellation die der Entscheidung Recht auf Vergessen II zugrunde liegt, anders. In diesem Fall erfolgt eine Beurteilung

323 Aus diesem Grund fiel die Reaktion des BVerfG entsprechend kritisch aus, vgl. hierzu unmittelbar im Anschluss an die EuGH-Entscheidung, BVerfG, Urt. v. 24.4.2013, Az. 1 BvR 1215/07 (Antiterrordatei), BVerfGE 133, S. 277, 316; BVerfG, Pressemitteilung v. 24.4.2013, Nr. 2, abrufbar unter: https://www.bunde sverfassungsgericht.de/SharedDocs/Pressemitteilungen/DE/2013/bvg13-031.htm 1. (zuletzt abgerufen am 1.7.2020); Thym, NVwZ 2013, S. 889, 890; Toros/Weiß, ZJS 2020, S. 100, $101 \mathrm{f}$.

324 BVerfG, Beschl. v. 6.11.2019, Az. 1 BvR 16/13 (Recht auf Vergessen I), NJW 2020, S. 300.

325 BVerfG, Beschl. v. 6.11.2019, Az. 1 BvR 276/17 (Recht auf Vergessen II), NJW 2020, S. 314.

326 Kühling, NJW 2020, S. 275 ff., spricht insoweit treffend von einer „Revolution“.

327 Toros/Weiß, ZJS 2020, S. 100, 104 ff.; Streinz, DuD 2020, S. 353, 358, mit Verweis auf die wenigen Fälle, in denen es tatsächlich zu einer solchen Doppelbindung kommt.

328 BVerfG, Beschl. v. 6.11.2019, Az. 1 BvR 16/13 (Recht auf Vergessen I), NJW 2020, S. 300, 303, Rn. 55.

329 Hierzu entwickelt das BVerfG umfassende Abgrenzungskriterien, BVerfG, Beschl. v. 6.11.2019, Az. 1 BvR 16/13 (Recht auf Vergessen I), NJW 2020, S. 300, 304, Rn. 66 ff. 
anhand der $\mathrm{GRCh}^{330}$ und das Bundesverfassungsgericht legt, soweit erforderlich, dem Europäischen Gerichtshof gemäß Art. 267 AEUV vor. ${ }^{331}$

Entscheidend für die Identifikation des Beurteilungsmaßstabes ist mithin der Determinierungsgrad des deutschen Rechts durch das unionale Telekommunikationswegerecht. ${ }^{332}$ Art. 43 EKEK strukturiert das Rechtsregime zur Vergabe von Wegerechten umfassend vor (C. III. 2.). Die Vorschrift charakterisiert das Verfahrens mit Adjektiven (bspw. einfach, effizient und transparent) und normiert damit Kriterien, an denen sich der nationale Gesetzgeber bei der Umsetzung ins nationale Telekommunikationsrecht orientieren muss. Eine Vollharmonisierung wird damit jedoch nicht angestrebt. Dies würde wie beispielsweise in Art.101 EKEK ausdrücklich im Rechtsakt festgelegt. ${ }^{333}$ Auch wenn das Telekommunikationswegerecht einen hohen Determinierungsgrad aufweist, gibt es dennoch einen Umsetzungsspielraum, der eine vollständige Determinierung ausschließt. Die vorliegende Konstellation entspricht mithin der Ausgangslage der Entscheidung Recht auf Vergessen I.

Im Ergebnis würden sich sowohl der Europäische Gerichtshof als auch das Bundesverfassungsgericht für zuständig für eine Überprüfung der Grundrechtskompatibilität halten, ohne jedoch das jeweils andere Gericht als unzuständig zu klassifizieren. Sie würden jeweils ihren eigenen Grundrechtskatalog als Beurteilungsmaßstab heranziehen, jedoch die Möglichkeit einer doppelten Grundrechtsbindung anerkennen. Entscheidend ist mithin, welches Gericht die Grundrechtskonformität im Einzelfall prüft. Es ist denkbar, dass dies durch den Europäischen Gerichtshof, etwa im Wege eines Vorabentscheidungsverfahrens gemäß Art. 267 AEUV, geschieht oder im Rahmen einer Verfassungsbeschwerde durch das Bundesverfassungsgericht. Möglich und wahrscheinlich ist damit eine Prüfung durch beide Gerichte, sodass der nationale Gesetzgeber eine Kompatibilität mit beiden Grundrechtskatalogen sicherstellen sollte. Nachfolgend sollen die Unionsgrundrechte wegen ihres vom Bundesverfassungsgericht anerkannten maßgeblichen Schutzniveaus vorgezogen geprüft werden. Im An-

330 BVerfG, Beschl. v. 6.11.2019, Az. 1 BvR 267/17 (Recht auf Vergessen II), NJW 2020, S. 314, 316, Rn. 42.

331 BVerfG, Beschl. v. 6.11.2019, Az. 1 BvR 267/17 (Recht auf Vergessen II), NJW 2020, S. 314, 320, Rn. 68 ff.; Toros/Weiß, ZJS 2020, S. 100, 107 f.; Klein, DÖV 2020, S. 341, 344.

$332 \mathrm{Zu}$ den Herausforderungen bei der Abgrenzung des Determinierungsgrades von Vorschriften des EKEK bereits Toros/Weiß, ZJS 2020, S. 100, 104.

333 So auch Toros/Weiß, ZJS 2020, S. 100, 104. 
schluss soll festgestellt werden, ob das Ergebnis auch bei einer Prüfung der nationalen Grundrechte identisch wäre.

bb) Vereinbarkeit mit den erwerbsbezogenen Grundrechten

Die Einführung eines Konzessionsmodell auf Basis exklusiver Wegerechte müsste sich mit den erwerbsbezogenen Grundrechten aus der GRCh vereinbaren lassen.

(1) Unionale Gewährleistungen aus Art. 15 Abs. 1 und 16 GRCh

Im Konzessionsmodell auf Basis der Vergabe exklusiver Wegerechte kann lediglich der Konzessionsnehmer als Infrastrukturanbieter im Konzessionsgebiet tätig werden. Für alle anderen Telekommunikationsinfrastrukturunternehmen könnte hierin ein ungerechtfertigter Eingriff in die erwerbsbezogenen Grundrechte liegen. Deren Schutz wird in einem Zusammenspiel aus Art. 15 Abs. 1 und 16 GRCh gewährleistet. ${ }^{334}$

Ein Eingriff in den Schutzbereich liegt vor, wenn „eine hinreichend direkte und bedeutsame Auswirkung auf die freie Berufsausübung“ 335 gegeben ist. ${ }^{336}$ Ohne ein Wegerecht ist der Auf- und Ausbau einer hochleistungsfähigen digitalen Infrastruktur im Konzessionsgebiet und damit im Ergebnis die Tätigkeit als Infrastrukturanbieter unmöglich. Demnach liegt ein Eingriff vor, der gerechtfertigt werden müsste.

Die Rechtfertigungsanforderungen für den Eingriff in Grundrechte der GRCh ergeben sich aus Art. 52 Abs. 1 GRCh. ${ }^{337}$ Mit dem Umsetzungsge-

334 Ausführlich zum Abgrenzungsproblem zwischen Art. 15 Abs. 1 und 16 GRCh, die vom EuGH jedoch in der Rechtsprechung in der Regel einheitlich und in einem Zusammenhang geprüft werden, Drechsler, Die Unionsgrundrechte unter dem Einfluss des Prozessrechts, S. 37 ff. m. w. N.

335 EuGH, Urt. v. 23.9.2004, verb. Rs. C-435/02 und C-103/03 (Springer), ECLI:EU: C:2004:552, Rn. 49.

336 Kühling, in: Pechstein/Nowak/Häde (Hrsg.), Frankfurter Kommentar EUV/GRC/AEUV, Art. 15 GRCh, Rn. 15 f.

337 Das Telekommunikationswegerecht wird als solches abschließend in Art. 43 Abs. 1 EKEK geregelt, sodass die Regelungen die Dienstleistungsfreiheit nicht direkt angewendet werden kann. Dennoch sind sie im Rahmen der Auslegung der sekundärrechtlichen Vorschriften und auch bei der Interpretation der berufsbezogenen Grundrechte aus Art. 15 und 16 GRCh zu berücksichtigen; Müller-Graff, in: Streinz (Hrsg.), EUV/AEUV, Art. 56 AEUV, Rn. 142. 
setz zum EKEK liegt eine gesetzliche Grundlage und mit der Förderung des Ausbaus hochleistungsfähiger Infrastrukturen ein legitimer Zweck vor (C. V. 4. b. aa.). Hinsichtlich der Eignung und Erforderlichkeit verbleibt dem Gesetzgeber ein umfassender Einschätzungsspielraum. ${ }^{338}$ Es ist jedenfalls nicht völlig abwegig, dass durch Schaffung von Infrastrukturmonopolen die Investitionsbedingungen nachhaltig verbessert und damit der Ausbau in den Konzessionsgebieten gefördert wird. Die Maßnahme müsste auch erforderlich sein. Es dürfte also kein relativ milderes, gleich geeignetes Mittel zur Erreichung der Ziele gegeben sein. ${ }^{339}$ Zwar existieren mit der Fördermittelvergabe, welche die wirtschaftlichen Rahmenbedingungen ebenfalls verändert, alternative Handlungsansätze. Die ökonomischen Effekte der Maßnahme setzen eine ökonometrische Analyse voraus, die nicht zum Spektrum dieser Untersuchung gehört. Aufgrund der umfassenden Einschätzungsprärogative des Gesetzgebers, ist jedoch von ihrer Erforderlichkeit auszugehen.

Die Maßnahme müsste auch verhältnismäßig im engeren Sinne sein. Die konfligierenden Interessen müssen gegeneinander abgewogen werden. Je stärker das Ziel zu gewichten ist, dem die Maßnahme dient, desto eher ist eine Einschränkung zu rechtfertigen. ${ }^{340}$ Diesbezüglich ist festzustellen, dass den Infrastrukturunternehmen nur die Tätigkeit im Konzessionsgebiet unmöglich wird. Die Konzessionsgebiete sollen auf Gebiete mit Netzausbaudefizit beschränkt werden. ${ }^{341}$ In konzessionsfreien Gebieten könnten andere Unternehmen jedoch weiterhin als Infrastrukturanbieter tätig werden. Aus diesem Grund ist davon auszugehen, dass sich der Eingriff in die Schutzbereiche der Art. 15 Abs. 1 und 16 GRCh rechtfertigen ließe.

338 Hierzu statt vieler Kahl/Schwind, EuR 2014, S. 170, 185.

339 EuGH, Urt. v. 4.10.2018, Rs. C-384/17 (Link Logistik N\&N), ECLI:EU:C: 2018:810, Rn. 40; EuGH, Urt. v. 17.4.2018, Rs. C-414/16 (Egenberger), ECLI:EU: C:2018:257, Rn. 68; EuGH, Urt. v. 11.7.1989, Rs. 265/87 (Schräder/Hauptzollamt Gronau), ECLI:EU:C:1989:303, Rn. 21.

340 Kübling, in: Pechstein/Nowak/Häde (Hrsg.), Frankfurter Kommentar EUV/GRC/AEUV, Art. 15 GRCh, Rn. 20; Jarass, Charta der Grundrechte der Europäischen Union, Art. 16, Rn. 24.

341 An dieser Stelle soll offengelassen werden, inwieweit der Umgang mit Bestandsinfrastruktur einen ungerechtfertigten Eingriff in Art. 15 Abs. 1 und 16 GRCh bzw. Art. 12 Abs. 1 GG darstellt, vgl. hierzu C. V. 
(2) Nationale Gewährleistungen aus Art. 12 Abs. 1 GG

Die Berufsfreiheit wird im Anwendungsbereich des Grundgesetzes gemäß Art. 12 Abs. 1 GG geschützt. Auch wenn die Berufsfreiheit durch Literatur und Rechtsprechung mit einer differenzierteren Dogmatik aufwarten kann, decken sich die Ergebnisse weitestgehend mit dem Unionsrecht. ${ }^{342}$

cc) Vereinbarkeit mit den Gleichheitsrechten der GRCh und des GG

Schon aus den unionsrechtlichen Vorgaben des Art. 43 Abs. 1 UAbs. 1 lit. a EKEK lässt sich herleiten, dass die Durchführung eines transparenten und nichtdiskriminierenden Konzessionsvergabeverfahrens erforderlich ist. Auch der allgemeine Gleichheitssatz, der in Art. $20 \mathrm{GRCh}$ angelegt ist, macht die Durchführung eines solchen Verfahrens erforderlich. Kernregelungsgehalt ist, wie beim grundgesetzlichen Pendant des Art. 3 Abs. 1 GG, dass wesentlich Gleiches gleich und wesentlich Ungleiches differenziert behandelt werden muss. ${ }^{343}$ Diese Anforderungen sind einfachgesetzlich in den Verfahrensparametern der Nichtdiskriminierung und Transparenz in Art. 43 Abs. 1 UAbs. 1 lit. a und lit. b EKEK konkretisiert. Solange diese Parameter eingehalten werden, ist das Verfahren auch mit den Grundrechten kompatibel. Der allgemeine Gleichheitssatz erfordert, den Konzessionsnehmer im Rahmen eines fairen und diskriminierungsfreien Auswahlverfahrens zu ermitteln, in dem alle Telekommunikationsunternehmen die gleichen Chancen haben.

b) Staatsorganisationsrechtliche Vorgaben des Art. 87f GG

Die Einführung eines Konzessionsmodells auf Basis der Vergabe exklusiver Wegerechte müsste auch mit den Steuerungsvorgaben des Art. 87f Abs. 2 S. 1 GG zu vereinbaren sein. Anders als der Wortlaut der Vorschrift nahelegt, gelten die Vorgaben nicht lediglich für die Ausgestaltung des Univer-

342 So auch Drechsler, Die Unionsgrundrechte unter dem Einfluss des Prozessrechts, S. $493 \mathrm{f}$.

343 Heselhaus, in: Pechstein/Nowak/Häde (Hrsg.), Frankfurter Kommentar EUV/GRC/ AEUV, Art. 20 GRCh, Rn. 24; zur hier tangierten Rechtsanwendungsgleichheit äußert sich etwa Lemke, in: von der Groeben/Schwarze/Hatje (Hrsg.), Europäisches Unionsrecht, Art. 20 GRCh, Rn. 9. 
saldienstes, sondern umfassend für den gesamten Telekommunikationssektor, inklusive des Infrastrukturausbaus. ${ }^{344}$ Steuerungsvorgaben ergeben sich sowohl für die Auswahl der zuständigen Behörde (dazu aa.) als auch für die konkrete Verfahrensausgestaltung (dazu bb.).

aa) Zuständige Behörde für die Durchführung des Konzessionsvergabeverfahrens

Das Unionsrecht sieht vor, dass die Wegerechte in einem Verwaltungsverfahren vergeben werden. Hinsichtlich der Behörde, die das Konzessionsvergabeverfahren durchführen soll, werden verschiedene Ausgestaltungsoptionen diskutiert. So wäre es möglich, die exklusiven Wegerechte durch die Bundesnetzagentur vergeben zu lassen. Alternativ könnten die Wegerechte auch durch kommunale Gebietskörperschaften vergeben werden. ${ }^{345}$ Das Grundgesetz verfügt in Art. 87f GG über spezifisches Verfassungsrecht für die Wirtschaftssektoren Post und Telekommunikation. Gemäß Art. 87f Abs. 2 S. 2 GG werden „Hoheitsaufgaben im Bereich des Postwesens und der Telekommunikation [...] in bundeseigener Verwaltung ausgeführt". Hieraus lassen sich Steuerungsvorgaben zur Kompetenzverteilung (da$\mathrm{zu}(1))$ und Verwaltungsorganisation (dazu (2)) herleiten, die sich auf die Auswahl der zuständigen Behörde für die Vergabe exklusiver Wegerechte einschränkend auswirken. ${ }^{346}$

344 Gersdorf, in: von Mangoldt/Klein/Starck (Begr.), GG, Art. 87f, Rn. 50; Kühling, in: Kahl/Waldhoff/Walter (Hrsg.), Bonner Kommentar zum Grundgesetz, Art. 87f, Rn. 121; Möstl, in: Maunz/Dürig (Begr.), Grundgesetz, Art. 87f GG, Rn. 35; Windthorst, in: Sachs (Hrsg.), GG, Art. 87f, Rn. 26.

345 Deutscher Landkreistag, Flächendeckende Breitbandversorgung zu wirtschaftlichen Bedingungen sicherstellen, S. $7 \mathrm{f}$.

346 Umstritten ist, ob Art. 87f Abs. 2 S. 1 GG darüber hinaus auch eine aufgabenrechtliche Dimension zukommt, Kühling, in: Kahl/Waldhoff/Walter (Hrsg.), Bonner Kommentar zum Grundgesetz, Art. 87f, Rn. 149; differenzierend Gersdorf, in: von Mangoldt/Klein/Starck (Begr.), GG, Art. 87f, Rn. 75 ff.; a. A. Möstl, in: Maunz/Dürig (Begr.), Grundgesetz, Art. 87f, Rn. 104, der kein Bedürfnis für eine Aufgabenzuweisung sieht. 
(1) Kompetenzrechtliche Steuerungsvorgaben aus dem Verfassungsrecht

Durch Art. 87f Abs. 2 S. 2 GG werden alle hoheitlichen Verwaltungsaufgaben auf den Bund übertragen. ${ }^{347}$ Es handelt sich hierbei um das Pendant zur legislativen Kompetenzzuweisung in Art. 73 Abs. 1 Nr.7 GG und Art. $87 \mathrm{f}$ Abs. 1 GG. ${ }^{348}$ Die Verwaltungskompetenzen werden auf Bundesebene zentral zusammengefasst, sodass eine Betrauung anderer Hoheitsträger mit Regulierungsaufgaben aus dem Telekommunikationssektor ausscheidet. ${ }^{349}$ Sofern sich verschiedene Kompetenztitel überlappen, kann eine Koordinierung erforderlich sein, im Rahmen der Wegerechtsvergabe beispielsweise mit den Trägern der Straßenbaulast. Die Zusammenarbeit beschränkt sich jedoch auf die Überlappungsbereiche und erfasst nicht die gesamte Verwaltung des Kompetenztitels. Die Kompetenz zur Vergabe von Telekommunikationswegerechten liegt aus diesem Grund ausschließlich beim Bund.

(2) Verfassungsrechtliche Vorgaben zur Verwaltungsorganisation

Neben dieser kompetenzrechtlichen Strukturierung im allgemeinen Sinne enthält die Vorschrift auch spezifische Vorgaben zur Verwaltungsorganisation. Der Wortlaut des Art. 87f Abs. 2 S. 2 GG schreibt obligatorisch eine bundesunmittelbare Verwaltung vor. ${ }^{350}$ Eine Übertragung der Aufgaben auf andere Hoheitsträger wird hierdurch ausgeschlossen.

Dieser Befund wird auch durch die Auslegung mithilfe anderer Methoden gestützt. Systematisch wird in Art. 86 GG zwischen bundesmittelbarer

347 Gersdorf, in: von Mangoldt/Klein/Starck (Begr.), GG, Art. 87f, Rn. 79; Kübling, in Kahl/Waldhoff/Walter (Hrsg.), Bonner Kommentar zum Grundgesetz, Art. 87f, Rn. 150; Windthorst, in: Sachs (Hrsg.), GG, Art. 87f, Rn. 32.

348 Gersdorf, in: von Mangoldt/Klein/Starck (Begr.), GG, Art. 87f, Rn. 73; Kühling, in: Kahl/Waldhoff/Walter (Hrsg.), Bonner Kommentar zum Grundgesetz, Art. 87f GG, Rn. 150.

349 Gersdorf, in: von Mangoldt/Klein/Starck (Begr.), GG, Art. 87f, Rn. 79 f.; Kübling, in: Kahl/Waldhoff/Walter (Hrsg.), Bonner Kommentar zum Grundgesetz, Art. 87f GG, Rn. 151; Windthorst, in: Sachs (Hrsg.), GG, Art. 87f, Rn. 32.

350 Ein Verwaltungsunterbau kann dabei grundsätzlich geschaffen werden, ist jedoch nicht obligatorisch und wurde bislang auch nicht etabliert; Gersdorf, in: von Mangoldt/Klein/Starck (Begr.), GG, Art. 87f, Rn. 82; Kühling, in: Kahl/ Waldhoff/Walter (Hrsg.), Bonner Kommentar zum Grundgesetz, Art. 87f, Rn. 153; Wieland, in: Dreier (Hrsg.), Grundgesetz, Band 3, Art. 87f, Rn.22; Windthorst, in: Sachs (Hrsg.), GG, Art. 87f, Rn. 33. 
und -unmittelbarer Verwaltung differenziert. Bei bundesunmittelbarer Verwaltung wird die Subdelegation der Verwaltungsaufgabe auf andere Hoheitsträger ausgeschlossen. ${ }^{351}$ Auch Art. 87f GG folgt diesem Verständnis, indem er die bundesunmittelbare Verwaltung in Art. 87f Abs. 2 S. 2 GG als Regel und die Erledigung spezifischer Aufgaben gemäß Art. 87f Abs. 3 GG als Ausnahme versteht. ${ }^{352}$ Es reicht deswegen auch nicht aus, wenn der Bund seine Kompetenzen auf einen anderen Hoheitsträger überträgt und sich selbst seinen beherrschenden Einfluss bewahrt. ${ }^{353}$ Dies gilt insbesondere für Kompetenzen im Zusammenhang mit zentralen Regulierungsaufgaben. ${ }^{354}$ Die Vergabe exklusiver Wegerechte im Rahmen eines Konzessionsmodells wäre eindeutig als solche zentrale Regulierungsaufgabe zu klassifizieren, sodass die Aufgabe nur unmittelbar durch eine Bundesbehörde ausgeübt werden könnte. Ohnehin wäre eine Übertragung neuer Verwaltungskompetenzen auf kommunale Gebietskörperschaften durch Art. 84 Abs. 1 S. 6 und Art. 85 Abs. 1 S. 2 GG unzulässig.

\section{(3) Ergebnis}

Die Verwaltungskompetenz zur Vergabe der Wegerechte liegt gemäß Art. 87f Abs. 2 S. 2 GG beim Bund. Die Hoheitsaufgabe muss in bundesunmittelbarer Verwaltung erfüllt werden. Es wäre beispielsweise denkbar, das Bundesministerium für Verkehr und digitale Infrastruktur oder die Bundesnetzagentur mit der Aufgabe zu betrauen. Bei Kompetenzüberschneidungen, etwa mit den Trägern der Straßenbaulast, wäre eine Zusammenarbeit mit anderen Hoheitsträgern zulässig. Die zentrale Regulierungsaufgabe der Vergabe von Wegerechten müsste jedoch eine bundesunmittelbare Behörde wahrnehmen, sodass eine Subdelegation an kommunale Gebietskörperschaften ausscheidet.

351 Kühling, in: Kahl/Waldhoff/Walter (Hrsg.), Bonner Kommentar zum Grundgesetz, Art. 87f, Rn. 154.

352 Kühling, in: Kahl/Waldhoff/Walter (Hrsg.), Bonner Kommentar zum Grundgesetz, Art. 87f, Rn. 154.

353 So im Ergebnis auch Gersdorf, in: von Mangoldt/Klein/Starck (Begr.), GG, Art. 87f, Rn. 82; Kübling, in: Kahl/Waldhoff/Walter (Hrsg.), Bonner Kommentar zum Grundgesetz, Art. 87f, Rn. 154; Uerpmann-Wittzack, in: von Münch (Begr.)/ Kunig (Hrsg.), Grundgesetz, Art. 87f, Rn. 23; Windthorst, in: Sachs (Hrsg.), GG, Art. 87f, Rn. 33.

354 Kühling, in: Kahl/Waldhoff/Walter (Hrsg.), Bonner Kommentar zum Grundgesetz, Art. 87f, Rn. 155. 


\section{bb) Verfahrensausgestaltung}

Neben den Aussagen zur zuständigen Behörde lassen sich aus der Vorschrift auch Anhaltspunkte für die Ausgestaltung des Verfahrens gewinnen. Der Verfassungsgeber statuiert in Art. 87f Abs. 2 S. 2 GG insbesondere ein „Verbot der Wettbewerbsverfälschung und [einen] Wettbewerbswiederherstellungsauftrag. “355 Der Gesetzgeber und die Verwaltung haben die Pflicht dazu, die erforderlichen Rahmenbedingungen dafür zu schaffen, dass sich ein funktionsfähiger und chancengleicher Wettbewerb im Telekommunikationssektor etablieren kann. ${ }^{356}$ Sofern ein staatliches Unternehmen die Rechte erwürbe, stünde dies in einem deutlichen Widerspruch zu dieser verfassungsrechtlichen Steuerungsvorgabe, der ein „Remonopolisierungverbot" entnommen werden kann. ${ }^{357}$ Wenn man die Einführung eines Konzessionsmodells auf Basis der Vergabe exklusiver Wegerechte trotzdem für zulässig hielte, müsste jedenfalls ein wettbewerbliches Konzessionsvergabeverfahren stattfinden, in dem alle Interessenten chancengleich teilnehmen könnten.

\section{cc) Ergebnis}

Aus der Verfassung ergeben sich konkrete Steuerungsvorgaben für die Ausgestaltung eines Konzessionsvergabeverfahrens. So müssten die Wegerechte durch eine bundesunmittelbare Behörde vergeben werden. Durch das Konzessionsmodell entstünde ein Infrastrukturmonopol im Konzessionsgebiet. Sofern ein staatliches Unternehmen diese Rechte innehätte, lieBe sich dies nicht mit dem staatlichen Remonopolisierungsverbot vereinbaren, das aus Art. 87f Abs. 2 S. 2 GG abgeleitet werden kann. Hielte man dies trotzdem für zulässig, müsste das Verfahren so ausgestaltet werden, dass ein chancengleicher und diskriminierungsfreier Wettbewerb um die Wegerechte stattfinden könnte.

355 Kühling, in: Kahl/Waldhoff/Walter (Hrsg.), Bonner Kommentar zum Grundgesetz, Art. 87f, Rn. 138.

356 Biendl, Rechtliche Herausforderungen beim Auf- und Ausbau von Next-Generation-Access-Networks, S. 156; Kühling, in: Kahl/Waldhoff/Walter (Hrsg.), Bonner Kommentar zum Grundgesetz, Art. 87f, Rn. 142.

357 So auch Kübling, in: Kahl/Waldhoff/Walter (Hrsg.), Bonner Kommentar zum Grundgesetz, Art. 87f, Rn. 147. 


\section{Steuerungsvorgaben des Wettbewerbsrechts}

Im Konzessionsmodell auf Basis der Vergabe exklusiver Wegerechte erhält der Konzessionsnehmer ein Ausschließlichkeitsrecht, das ihm ein Infrastrukturmonopol einräumt. Naturgemäß konfligieren Beschränkungen des Wettbewerbs stets mit dem Wettbewerbsrecht, das auf dessen Stärkung ausgerichtet ist. Demnach ergeben sich auch aus dem Wettbewerbsrecht Steuerungsvorgaben für die Verfahrensausgestaltung. Hierzu müsste das Wettbewerbsrecht neben dem TKG anwendbar sein (dazu a.) und zur Überprüfung staatlichen Handelns genutzt werden können (dazu b.). Sofern die Tatbestandsvoraussetzungen nicht erfüllt sein sollten, könnte sich die Anwendbarkeit des Wettbewerbsrechts auch aus Art. 106 AEUV ergeben (dazu c.).

\section{a) Anwendbarkeit des Wettbewerbsrechts neben dem TKG}

Das sektorspezifische Regulierungsrecht und das Wettbewerbsrecht überschneiden sich hinsichtlich ihrer Regelungsgegenstände, Ziele und Maßstäbe. ${ }^{358}$ Während das Regulierungsrecht jedoch durch eine Ex-ante-Perspektive gekennzeichnet ist und im Vorfeld gestaltend auf den Markt einwirkt, nimmt das Kartellrecht eine Ex-post-Perspektive ein und legitimiert ein repressives Vorgehen gegen Wettbewerbsverstöße. ${ }^{359}$ Parallele behördliche Zuständigkeiten und Gesetze mit unterschiedlichen Grundansätzen können zu Konflikten führen. ${ }^{360}$

Gemäß $₫ 2$ Abs. 4 TKG finden die Regelungen des GWB Anwendung, soweit das TKG nicht über abschließende Spezialregelungen verfügt. ${ }^{361}$ Dieses Zusammenspiel bleibt im künftigen TKG unverändert. ${ }^{362}$ Das genaue Verhältnis zwischen den beiden Regelungsmaterien ist jedoch umstritten. Zuweilen wird vertreten, dass die Gesetze nebeneinander Anwen-

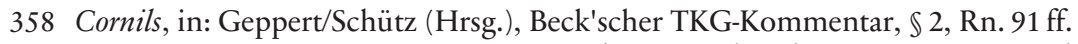

359 Neumann, N\&R 2020, S. 148, 152; Gräditz, in: Scheurle/Mayen (Hrsg.), Telekommunikationsgesetz, $\mathbb{2}$, Rn. 72.

360 Cornils, in: Geppert/Schütz (Hrsg.), Beck'scher TKG-Kommentar, $\mathbb{2}$, Rn. $91 \mathrm{ff}$.

361 Ruthig, in: Arndt/Fetzer/Scherer u.a. (Hrsg.), Telekommunikationsgesetz, $\mathbb{} 2$, Rn. 39 f.; Säcker, in: Säcker (Hrsg.), Telekommunikationsgesetz, $\$ 2, ~ R n .24$.

362 Referentenentwurf des Telekommunikationsmodernisierungsgesetzes (TKMoG), abrufbar unter: https:/www.bmwi.de/Redaktion/DE/Downloads/Gesetz/ telekommunikationsmodernisierungsgesetz-referentenentwurf-20201612.pdf? blob=publicationFile $\& v=8$ (zuletzt abgerufen am 12.1.2021). 
dung finden (Parallelitätsthese), und von anderen, dass die allgemeinen GWB-Vorschriften durch die speziellen TKG-Normen verdrängt werden (Spezialitätsthese). ${ }^{363}$ Diese Auffassungen gelangen nur dann zu unterschiedlichen Ergebnissen, wenn eine abschließende Sonderregelung im TKG vorhanden ist. Dies ist hier nicht der Fall, sodass das TKG keine Sperrwirkung gegenüber dem GWB entfaltet. Im Übrigen ist die parallele Anwendbarkeit insbesondere in solchen Konstellationen vorteilhaft, in denen sich die Regelungsmaterien gegenseitig ergänzen und Schutzlücken entgegenwirken (Kapitel 5, E. I. 1.). Würde mit der Einführung eines Konzessionsmodells auf Basis der Vergabe exklusiver Wegerechte jedoch das entsprechende Wegerechtsvergabeverfahren umfassend normiert, könnte dies unter Umständen eine Sperrwirkung entfalten.

Der vorgenannte Umstand hat jedoch nur begrenzte Auswirkungen auf die Anwendbarkeit des Wettbewerbsrechts. Gemäß $\$ 50$ Abs. 1 GWB sind die Bundes- und Landeskartellbehörden für den Vollzug der wettbewerbsrechtlichen Vorgaben der Art. $101 \mathrm{ff}$. AEUV zuständig. In dieser Hinsicht kann $\ 2$ Abs. 4 TKG wegen des Anwendungsvorranges des Unionsrechts keine Sperrwirkung des nationalen Telekommunikationsrechts gegenüber dem unionalen Wettbewerbsrecht anordnen. ${ }^{364}$ Die Kommission geht davon aus, dass das Wettbewerbsrecht und das sektorspezifische Telekommunikationsrecht nebeneinander angewendet werden. ${ }^{365}$ Unabhängig von der Ausgestaltung der exklusiven Wegerechtsvergabe im sektorspezifischen Regulierungsrecht müssten sich die Vorgaben mithin stets am primären Wettbewerbsrecht der EU messen lassen. ${ }^{366}$

b) Anwendbarkeit des Wettbewerbsrechts auf staatliches Handeln

Die Vorgaben des nationalen und unionalen Wettbewerbsrechts richten sich an Unternehmen, sodass fraglich ist, ob auch staatliches Handeln in dessen Anwendungsbereich fällt. Weder das nationale noch das unionale Wettbewerbsrecht verfügen über eine Legaldefinition des Unternehmensbegriffes. Im Rahmen der Diskussion in Literatur und Rechtsprechung ha-

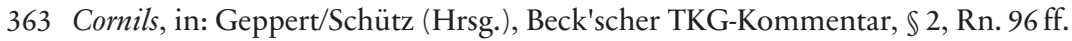

364 Cornils, in: Geppert/Schütz (Hrsg.), Beck'scher TKG-Kommentar, $\$ 2, R n .103$; Säcker, in: Säcker (Hrsg.), Telekommunikationsgesetz, $\mathbb{2}$ 2, Rn. 30.

365 Ruthig, in: Arndt/Fetzer/Scherer u.a. (Hrsg.), Telekommunikationsgesetz, $\mathbb{} 2$, Rn. 40.

366 Kling/Thomas, Kartellrecht, S. 40. 
ben sich jedoch der „institutionelle“ und der "funktionale“ Unternehmensbegriff herausgebildet, die grundsätzlich auch von Gebietskörperschaften des öffentlichen Rechts erfüllt werden können. ${ }^{367}$ Dazu müsste die Vergabe exklusiver Wegerechte zum Aufbau einer gigabitfähigen Infrastruktur jedoch insbesondere eine wirtschaftliche Tätigkeit darstellen. Eine wirtschaftliche Tätigkeit ist nach Ansicht des Europäischen Gerichtshofes ,jede Tätigkeit, die darin besteht, Güter oder Dienstleistungen auf einem bestimmten Markt anzubieten." ${ }^{368}$ Maßgeblich ist mithin, dass Waren oder Dienstleistungen entgeltlich auf einem Markt angeboten werden, wobei unerheblich ist, ob im Einzelfall Entgelte erhoben werden oder eine Gewinnerzielungsabsicht existiert. ${ }^{369}$ Sofern staatliche Institutionen ausschließlich Hoheitsgewalt ausüben, stellt dies keine wirtschaftliche Tätigkeit im Sinne des Wettbewerbsrechts dar. ${ }^{370}$ Die Wegerechte werden im Telekommunikationsrecht ohne die Verpflichtung zur Zahlung von Entgelten und Abgaben vergeben. ${ }^{371}$ Gebühren dienen dem Ausgleich der Auslagen, die der Verwaltung im Vergabeverfahren entstanden sind. ${ }^{372}$ Die derzeitigen Regelungen zur Vergabe von Wegerechten stellen aus diesem Grund keine wirtschaftliche Betätigung der Bundesnetzagentur dar. Aus dem Positionspapier des Deutschen Landkreistages lässt sich nicht folgern, dass Konzessionsabgaben erhoben werden sollen. Sofern hiervon abgesehen wird, würde die Behörde auch bei der Vergabe exklusiver Wegerechte ausschließlich hoheitlich handeln. Die Wegerechtsvergabe durch den Staat stellt mithin keine unternehmerische Tätigkeit dar, auf die das Wettbewerbsrecht direkt anwendbar wäre.

367 Emmerich/Lange, Kartellrecht, $\mathbb{3}$, Rn. 27; Emmerich, in: Immenga/Mestmäcker (Hrsg.), Wettbewerbsrecht, Band 1, Art. 101 Abs. 1 AEUV, Rn. 6 ff.

368 EuGH, Urt. v. 10.1.2006, Rs. C-222/04 (Cassa di Risparmio di Firenze u. a.), ECLI:EU:C: 2006:8, Rn. 108.

369 EuGH, Urt. v. 1.7.2008, Rs. C-49/07 (Motoe), ECLI:EU:C:2008:376, Rn. 28; Emmerich/Lange, Kartellrecht, $\mathbb{\$}$, Rn. 32 .

370 Emmerich/Lange, Kartellrecht, $\mathbb{3}$, Rn. 28.

371 Schütz, in: Geppert/Schütz (Hrsg.), Beck'scher TKG-Kommentar, $\mathbb{\$} 68$, Rn. 38.

372 Dies ergibt sich aus $\$ 142$ Abs. 1 Nr. 7 TKG, nach dem für die Übertragung von Wegerechten gemäß $\$ 69$ TKG die Zahlung einer Gebühr verlangt werden kann. Die Höhe der Gebühr berechnet sich gemäß $₫ 142$ Abs. 2 S. 2 nach der Höhe der bei der BNetzA entstandenen Auslagen. Hierdurch wird die Übertragung der Wegerechte jedoch nicht entgeltlich, Schütz, in: Geppert/Schütz (Hrsg.), Beck'scher TKG-Kommentar, $\$ 68$, Rn. 39. 
c) Anwendbarkeit des Wettbewerbsrechts aufgrund der Übertragung ausschließlicher Rechte gemäß Art. 106 AEUV

Obwohl sich der Hoheitsträger bei der Vergabe exklusiver Wegerechte im Telekommunikationssektor nicht wirtschaftlich betätigt, könnten die wettbewerblichen Vorgaben dennoch gemäß Art. 106 Abs. 1 AEUV hierauf Anwendung finden.

aa) Privilegiertes Unternehmen im Sinne des Art. 106 Abs. 1 AEUV

Dem Konzessionsnehmer müssten gemäß Art. 106 Abs. 1 Alt. 2 AEUV durch den Mitgliedstaat „, besondere oder ausschließliche Rechte“ gewährt werden. ${ }^{373}$ Ein ausschließliches Recht liegt nach der Rechtsprechung des Europäischen Gerichtshofes vor, „wenn [der Mitgliedstaat] [...] einer begrenzten Zahl von Unternehmen einen Schutz gewährt und die Fähigkeit anderer Unternehmen, die fragliche wirtschaftliche Tätigkeit im selben Gebiet zu im Wesentlichen gleichen Bedingungen auszuüben, wesentlich beeinträchtig[t] [werden] kann.“ 374

Diese Anforderungen erfüllt das Konzessionsmodell auf Basis der Vergabe exklusiver Wegerechte, da in den Konzessionsgebieten nur solche Unternehmen Telekommunikationsinfrastruktur auf- und ausbauen könnten, die über das entsprechende Wegerecht verfügen. Hinsichtlich der Gewährung des Rechts besteht umfassende Formenwahlfreiheit, sodass auch die Übertragung mittels eines Verwaltungsaktes vom Tatbestand erfasst ist. ${ }^{375}$

Eine staatliche Gewährung liegt allerdings nicht vor, wenn ein gesetzlicher Anspruch auf die Erteilung des ausschließlichen Rechts besteht und die gesetzlichen Tatbestandsvoraussetzungen erfüllt sind. Als Beispiel wird in der Literatur die Vergabe von Konzessionen angeführt. ${ }^{376}$ Der Begriff wird jedoch, wie dargelegt, nicht einheitlich verwendet und ist in jedem

373 Diese Frage stellt sich auch im Kontext der Vergabe von Energiekonzessionsverträgen, Sauer, Das Recht der Vergabe von Strom- und Gas-Konzessionsverträgen im EnWG, S. $113 \mathrm{ff}$.

374 EuGH, Urt. v. 12.12.2013, Rs. C-327/12 (Soa Nazionale Costruttori), ECLI:EU: C:2013:827, Rn. 41; EuGH, Urt. v. 25.10.2001, Rs. C-475/99 (Ambulanz Glöckner), ECLI:EU:C:2001:577, Rn. 24.

375 Klotz, in: von der Groeben/Schwarze/Hatje (Hrsg.), Europäisches Unionsrecht, Art. 106 AEUV, Rn. 21.

$376 \mathrm{Klotz}$, in: von der Groeben/Schwarze/Hatje (Hrsg.), Europäisches Unionsrecht, Art. 106 AEUV, Rn. 21. 
Kontext autonom auszulegen (A. IV.). Ein Anspruch auf Erteilung des Wegerechts existiert beim Konzessionsmodell auf Basis der Vergabe exklusiver Wegerechte ausdrücklich nicht, da das Recht ausschließlich an das Unternehmen vergeben wird, das im Konzessionsverfahren obsiegt. Im Ergebnis handelt es sich um die Gewährung eines exklusiven Rechts durch den Mitgliedstaat, sodass der Konzessionsnehmer als privilegiertes Unternehmen im Sinne des Art. 106 Abs. 1 AEUV zu klassifizieren ist.

bb) Rechtsfolge der Erfüllung des Tatbestandes des Art. 106 Abs. 1 AEUV

Gemäß Art. 106 Abs. 1 AEUV dürfen die Mitgliedstaaten, sobald sie einem Unternehmen ausschließliche Rechte gewähren, keine Maßnahmen treffen oder aufrechterhalten, die den Art. 18 und 101 bis 109 AEUV widersprechen.

cc) Einschränkung durch Art. 106 Abs. 2 AEUV

Die grundsätzliche Rechtsfolge des Art. 106 Abs. 1 AEUV könnte durch Art. 106 Abs. 2 S. 1 AEUV eingeschränkt werden. ${ }^{377}$ Danach gilt das Wettbewerbsrecht „für Unternehmen, die mit Dienstleistungen von allgemeinem wirtschaftlichem Interesse betraut sind (...), [nur] soweit die Anwendung dieser Vorschriften nicht die Erfüllung der ihnen übertragenen besonderen Aufgabe rechtlich oder tatsächlich verhindert.“

Der Konzessionsnehmer müsste mit der Erbringung der Dienstleistung betraut werden. Dies erfordert eine Übertragung der Aufgabe mittels eines Hoheitsaktes (C. IV. 1. b.) Beim Auf- und Ausbau von Telekommunikationsinfrastruktur handelt es sich um eine Dienstleistung im Sinne des Art. 106 Abs. 2 AEUV. ${ }^{378}$ Die Dienstleistung müsste von allgemeinem wirtschaftlichen Interesse sein. Das Unionsprimärrecht setzt den Begriff in Art. 14 und Art. 106 Abs. 2 S. 1 AEUV sowie im Protokoll Nr. 26 voraus, ohne ihn legal zu definieren. ${ }^{379}$ In der Rechtsprechung und der Anwen-

377 Säcker/Mohr/Wolf, Konzessionsverträge im System des europäischen und deutschen Wettbewerbsrechts, S. $73 \mathrm{f}$.

378 Kling/Thomas, Kartellrecht, S. 289 f.

379 Kommission, Leitfaden zur Anwendung der Vorschriften der Europäischen Union über staatliche Beihilfen, öffentliche Aufträge und den Binnenmarkt auf Dienstleistungen von allgemeinem wirtschaftlichem Interesse inklusive Sozialdienstleistungen v. 7.12.2010, SEC(2010) 1545 endg., S. 17. 
dungspraxis der Vorschriften wird der Begriff jedoch so verstanden, dass es sich dabei um Dienstleistungen handelt, „an denen ein allgemeines wirtschaftliches Interesse besteht, das sich von dem Interesse an anderen Tätigkeiten des Wirtschaftslebens besonders unterscheidet “" ${ }^{\text {" } 80 ~ D i e ~ M i t g l i e d s t a a-~}$ ten haben einen Spielraum bei der Auslegung des Begriffes, der einer Missbrauchskontrolle unterliegt. Die Infrastrukturanbieter bauen die Infrastruktur entgeltlich auf und aus und stellen sie den Telekommunikationsanbietern gegen Entgelt zur Verfügung, sodass es sich dabei um eine wirtschaftliche Tätigkeit handelt. ${ }^{381}$ Ein allgemeines Interesse an der Dienstleistung besteht dann, wenn nicht nur wirtschaftliche Interessen, sondern auch Interessen der Allgemeinheit verfolgt werden. Dies ist insbesondere dann der Fall, wenn die Dienstleistung auch in solchen Gebieten erbracht wird, in denen dies nicht zu wirtschaftlichen Bedingungen erfolgen kann. ${ }^{382}$ Zudem muss ein Marktversagen vorliegen. ${ }^{383}$ Das Konzessionsmodell auf Basis der Vergabe exklusiver Wegerechte soll nur in solchen Gebieten angewandt werden, in denen nicht mit einem eigenwirtschaftlichen Auf- und Ausbau der hochleistungsfähigen digitalen Infrastruktur zu rechnen ist, sodass auch diese Kriterien erfüllt sind.

Es handelt sich um eine Dienstleistung von allgemeinem wirtschaftlichen Interesse, sodass die Tatbestandsvoraussetzungen des Art. 106 Abs. 2 S. 1 AEUV erfüllt sind. Das Wettbewerbsrecht findet jedoch nur insoweit Anwendung, wie dies nicht „die Erfüllung der [den Unternehmen] übertragenen besonderen Aufgaben rechtlich oder tatsächlich verhindert. "Der Wortlaut betrifft insbesondere den Modus der Aufgabenerfüllung. Der Auswahlprozess des Konzessionsnehmers ist der Aufgabenerfüllung jedoch vorgelagert, sodass die Einschränkung des Art. 106 Abs. 2 S. 1 AEUV nicht dazu führt, dass im Rahmen des Auswahlverfahrens das Wettbewerbsrecht nicht zu beachten wäre. Die Privilegierung gilt freilich nicht unbegrenzt. Vielmehr darf gemäß Art. 106 Abs. 2 S. 2 AEUV die Entwicklung des Han-

380 Kommission, Leitfaden zur Anwendung der Vorschriften der Europäischen Union über staatliche Beihilfen, öffentliche Aufträge und den Binnenmarkt auf Dienstleistungen von allgemeinem wirtschaftlichem Interesse inklusive Sozialdienstleistungen v. 7.12.2010, SEC(2010) 1545 endg., S. 17.

381 Zum Erfordernis der wirtschaftlichen Tätigkeit Klett/Otter, in: Ministerium für Wirtschaft, Arbeit und Wohnungsbau Baden-Württemberg (Hrsg.), DAWI und Infrastrukturfinanzierung, S. 21.

382 Klett/Otter, in: Ministerium für Wirtschaft, Arbeit und Wohnungsbau BadenWürttemberg (Hrsg.), DAWI und Infrastrukturfinanzierung, S. 22.

$383 \mathrm{Klett} / \mathrm{Otter}$, in: Ministerium für Wirtschaft, Arbeit und Wohnungsbau BadenWürttemberg (Hrsg.), DAWI und Infrastrukturfinanzierung, S. 22. 
delsverkehrs durch sie nicht beeinträchtigt werden. Im Ergebnis ist eine Einschränkung lediglich in denjenigen Fällen zulässig, in denen weniger einschneidende Maßnahmen in Betracht kommen. ${ }^{384}$ Hierzu ist eine Prüfung im Einzelfall erforderlich. Diese soll jedoch erst in der Zusammenschau mit den übrigen Verfahrensanforderungen erfolgen, die sich aus anderen Rechtsmaterien ergeben können (C. IV. 3. c.).

\section{d) Ergebnis}

Das Konzessionsvergabeverfahren muss aufgrund des Art. 106 Abs. 1 AEUV in den Grenzen des Art. 106 Abs. 2 AEUV nach den Vorgaben des Wettbewerbsrechts strukturiert werden. Dieses Erfordernis könnte sich auch aus anderen rechtlichen Materien ergeben, sodass die sich hieraus ergebenden Kriterien erst zu einem späteren Zeitpunkt konkret erörtert werden sollen (C. IV. 3. c.).

\section{Steuerungsvorgaben des Vergaberechts}

Das Vergaberecht enthält Vorgaben für die Vergabe öffentlicher Aufträge und Konzessionen an private oder öffentliche Unternehmen.

\section{a) Ausschluss der Anwendbarkeit der KVRL und der $\$ \mathbb{S} 105 \mathrm{ff}$. GWB}

Der Inhalt des Konzessionsvertrages, also der Aufbau und Betrieb eines glasfaserbasierten Telekommunikationsnetzes im Konzessionsgebiet, stellt eine Dienstleistungskonzession im Sinne des Vergaberechts dar (C. II. 1.). Dies führt jedoch nicht zwangsläufig dazu, dass die Regelungen der KVRL bzw. der $\int \mathbb{S} 105$ ff. GWB auch auf das Auswahlverfahren hinsichtlich des zu betrauenden Konzessionsnehmers Anwendung finden. ${ }^{385}$ Viel-

384 Der gleiche Befund ergibt sich auch im Energierecht; Säcker/Mohr/Wolf, Konzessionsverträge im System des europäischen und deutschen Wettbewerbsrechts, S. 74.

385 Eine a. A. vertritt vermutlich das OLG Düsseldorf, Beschl. v. 15.7.2015, Az. VI-2 Kart 1/15 (V), BeckRS 2016, 2800, Rn. 55 ff., das sich mit Rechtsfragen rund um die Vorwirkung der KVRL auseinandersetzt, ohne zu prüfen, ob die Vorschriften auf die Vergabe von energierechtlichen Konzessionen überhaupt Anwendung finden. In der Revisionsentscheidung BGH, Beschl. v. 26.1.2016, Az. KVZ 
mehr ist es auch möglich, dass es sich um eine Dienstleistungskonzession handelt, die nicht unter die KVRL zu subsumieren ist. Dies hat Auswirkungen auf die Ausgestaltung des Verfahrens zur Konzessionsvergabe und wird aus diesem Grund abstrakt von der Frage der Klassifikation als Dienstleistungskonzession an dieser Stelle thematisiert (C. II. 1.). Die Möglichkeit, dass bestimmte Konzessionen nicht von der KVRL erfasst sind, ergibt sich mittelbar aus den positiv normierten Ausschlussgründen der KVRL. Dort heißt es in Art. 11 Abs. 1 KVRL, dass die Regelungen nicht auf Konzessionen Anwendung finden, „die hauptsächlich dazu dienen, dem öffentlichen Auftraggeber die Bereitstellung oder den Betrieb öffentlicher Kommunikationsnetze oder die Bereitstellung eines oder mehrerer elektronischer Kommunikationsdienste für die Öffentlichkeit zu ermöglichen." Erfasst sind somit solche Konstellationen, in denen der öffentliche Auftraggeber auf Basis der übertragenen Rechte selbst als Netzbetreiber oder Diensteanbieter tätig werden möchte. Nicht erfasst ist hingegen die Vergabe der Rechte an Dritte, die nicht den Betrieb eines eigenen Netzes oder das Angebot eigener Dienste durch den öffentlichen Auftraggeber zur Folge hat. Letzteres wäre im Rahmen des Konzessionsmodells auf Basis der Vergabe exklusiver Wegerechte jedoch der Fall. Ein positiv normierter Ausschlussgrund ist für die Anwendbarkeit der KVRL somit nicht einschlägig.

Dennoch könnten die Regelungen der KVRL und der $\mathbb{S} 105 \mathrm{ff}$. GWB auf das Auswahlverfahren, auf dessen Basis der Konzessionsnehmer mit der Erbringung der Dienstleistung betraut wird, nicht anwendbar sein. In der Literatur wird zwischen dem Treffen der Vergabeentscheidung und dem Verrichten einer Dienstleistung unterschieden. Während die Tätigkeit des Konzessionsnehmers im Rahmen des Konzessionsvertrages in den Anwendungsbereich der Regelungen der KVRL und der $\$ \mathbb{S} 105 \mathrm{ff}$. GWB falle, sei die Entscheidung der handelnden Behörde nicht erfasst. ${ }^{386}$ Es handele sich bloß um „eine für einen längeren Zeitraum (Dauer des Konzessionsvertrages) geltende Vergabeentscheidung mit anschließendem Vertragsschluss. “387 Diese Differenzierung zwischen Vergabeentscheidung und Vertrag, mag allerdings nicht zu überzeugen. Bei einer vollständig sepa-

41/15, BeckRS 2016, 5644 wird die Anwendbarkeit der Richtlinie jedoch nicht mehr thematisiert; Theobald/Wolkenhauer, DÖV 2016, S. 724, 730, nutzen das Urteil dennoch zur Stützung ihrer These, dass die Richtlinie auf die Konzessionsvergabe Anwendung finden müsse.

386 Grünewald, Die (Re)Kommunalisierung in der Energieverteilung, S. $315 \mathrm{f}$.

387 Grünewald, Die (Re)Kommunalisierung in der Energieverteilung, S. 315. 
rierten Betrachtung von Vergabeentscheidung und Konzessionsvertrag wird verkannt, dass die Vergabeentscheidung in einem Akzessorietätsverhältnis zum Konzessionsvertrag steht. Die Regelungen der KVRL strukturieren gerade das Verfahren vor, um eine Konzession zu vergeben.

Als weiteres Argument gegen die Anwendbarkeit der KVRL wird der Wortlaut des Erwägungsgrund 16 der KVRL und der entsprechenden Passage in der Gesetzesbegründung des GWB ${ }^{388}$ angeführt. ${ }^{389}$ In den Erwägungsgründen 11 bis 17 der KVRL hat der Unionsgesetzgeber Fallgruppen aufgezählt, die nach seinem Willen nicht in den Anwendungsbereich der Richtlinie fallen sollen. ${ }^{390}$ Erwägungsgrund 16 der KVRL lautet: „Außerdem sollten Vereinbarungen über die Gewährung von Wegerechten hinsichtlich der Nutzung öffentlicher Liegenschaften für die Bereitstellung oder den Betrieb fester Leitungen oder Netze, über die eine Dienstleistung für die Allgemeinheit erbracht werden soll, ebenfalls nicht als Konzession im Sinne dieser Richtlinie gelten, sofern derartige Vereinbarungen weder eine Lieferverpflichtung auferlegen, noch den Erwerb von Dienstleistungen durch den öffentlichen Auftraggeber oder den Auftraggeber für sich selbst oder für Endnutzer vorsehen."

Bei dem Konzessionsmodell auf Basis der Vergabe exklusiver Wegerechte handelt es sich um ein Wegerecht, das die Kriterien des ersten Teiles des Erwägungsgrundes erfüllt. Am Ende des Erwägungsgrundes sind Ausschlussgründe benannt. Im Rahmen des Konzessionsmodells auf Basis der Vergabe exklusiver Wegerechte dürfte demnach keine Lieferverpflichtung begründet werden. ${ }^{391}$ Betrachtet man das Energierecht, sind dort der Betrieb des Netzes und der Vertrieb der Energie durch Entflechtungsvorgaben strikt voneinander getrennt (Kapitel 4, A. II. 2.). Zwar existiert im Telekommunikationssektor kein entsprechend starkes Entflechtungsregime, allerdings sind auch hier der Betrieb des Telekommunikationsnetzes und

388 BT-Drs. 18/6281, S. 76.

389 Grünewald, Die (Re)Kommunalisierung in der Energieverteilung, S. 316 f. Die Autorin argumentiert lediglich mit Erwägungsgrund 16 der KVRL. Dies lässt sich jedoch darauf zurückführen, dass das Gesetz erst nach Schluss der Bearbeitung veröffentlicht worden ist; Weiß, Entscheidungsspielräume bei der Konzessionierung nach $₫ 46$ EnWG, S. 8; im Überblick Lau, in: Lau (Hrsg.), Vergabe von Energienetzen, S. 9, 11.

390 Grünewald, Die (Re)Kommunalisierung in der Energieverteilung, S. 319.

391 Die Frage, ob ein Konzessionsmodell auf Basis der Vergabe exklusiver Wegerechte eine Lieferverpflichtung statuiert, stellt sich auch im Energierecht; Becker/Dicks, ZNER 2014, S. 425; Grünewald, Die (Re)Kommunalisierung in der Energieverteilung, S. 316 f.; Kment/Vorwalter, EnWZ 2015, S.387, 393; Mohr, RdE 2016, S. 269, 276. 
das Angebot von Telekommunikationsdiensten an den Endkunden abstrakt voneinander zu betrachten. Dieser Umstand wird dadurch verstärkt, dass im Rahmen des Konzessionsmodells alle Diensteanbieter Zugang zur Infrastruktur im Konzessionsgebiet erhalten sollen. ${ }^{392}$ Der Betrieb des Netzes stellt damit nicht die "Belieferung“ des Endkunden dar. ${ }^{393}$ Vielmehr findet diese erst auf der nächsten Wertschöpfungsstufe der Telekommunikationswirtschaft, also im Angebot der Dienste an den Endkunden statt. ${ }^{394}$ Erst in dem Vertrag zwischen Endkunden und Diensteanbieter wird eine Verpflichtung zur Erbringung des Dienstes begründet. Demnach liegt keine Lieferverpflichtung im Sinne des Erwägungsgrundes 16 der KVRL vor. Der Wortlaut des Erwägungsgrundes „weder ... noch“ stellt klar, dass keines der beiden Kriterien erfüllt sein darf, die am Ende des Satzes aufgelistet sind. Sobald eines der Kriterien erfüllt ist, soll das Rechtsregimes nach dem Willen des unionalen und nationalen Gesetzgebers keine Anwendung finden. Die Kriterien des Ausschlussgrundes sind also erfüllt. ${ }^{395}$

Nun ist es aber nicht möglich, dass der Gesetzgeber den Anwendungsbereich einer Richtlinie bzw. eines Gesetzes auf Basis der Erwägungsgründe bzw. der Gesetzesbegründung festlegt. ${ }^{396}$ Die Argumentation mithilfe des Erwägungsgrundes 16 der KVRL wird stellenweise folgerichtig mit der Begründung abgelehnt, dass Erwägungsgründe und Gesetzesbegründungen

392 Dies lässt sich daraus schließen, dass die Konzessionsverträge „ein ausschließliches Recht zur Errichtung eines entsprechenden Netzes, nicht aber zur Versorgung der Letztverbraucher" enthalten sollen, Deutscher Landkreistag, Flächendeckende Breitbandversorgung zu wirtschaftlichen Bedingungen sicherstellen, S. 7.

393 So auch Donhauser/Hölzlwimmer, VergabeR 2015, S. 509, 518 f.; Grünewald, Die (Re)Kommunalisierung in der Energieverteilung, S. $316 \mathrm{f}$; Kment/Vorwalter, EnWZ 2015, S. 387, 393.

394 Dies lässt sich insbesondere darauf zurückführen, dass sich in der Entwicklung des Telekommunikationsrechts eine stärkere Differenzierung zwischen Infrastruktur- und Dienstebene herausgebildet hat, Kühling, Sektorspezifische Regulierung in den Netzwirtschaften, S. 71.

395 So auch Grünewald, Die (Re)Kommunalisierung in der Energieverteilung, S. 316 f.; Schwab/Giesemann, VergabeR 2014, S. 351, 366; a. A. Brück von Oertzen/ Kreggenfeld, EWerK 2016, S. 12, 14, die davon ausgehen, dass durch den Erwägungsgrund verdeutlicht werden soll, dass Situationen in denen lediglich Recht vergeben werden, nicht vom Vergaberecht erfasst werden sollen.

396 A. A. Goldbrunner, VergabeR 2016, S. 365, 369, welche die Kriterien des Erwägungsgrundes 16 der KVRL zu den Tatbestandsmerkmalen einer Dienstleistungskonzession zählt und aufgrund der Einschlägigkeit des Ausschlussgrundes davon ausgeht, dass es sich bei exklusiven Wegerechten i. S. d. \$46 EnWG nicht unter den Konzessionsbegriff zu subsumieren sind. 
allein der Auslegung dienen und kein anwendbares Recht darstellen. ${ }^{397}$ Richtigerweise entfalten sie lediglich Indizwirkung. ${ }^{398}$

Im vorliegenden Fall ergibt sich jedoch die Sondersituation, dass das Tatbestandsmerkmal des Beschaffungsbezuges in der Definition des Art. 5 Abs. 1 lit. b KVRL bzw. $\$ 105$ Abs. 1 Nr. 2 GWB nicht explizit aufgeführt wird. In der Literatur wird das Merkmal durch die Zusammenschau des Gesetzeszweckes des Art. 1 Abs. 1 KVLR und der Erwägungsgründe 11, 15 und 16 der KVRL hergeleitet. ${ }^{399}$ Sowohl der Definitionsbestandteil als solches, als auch die Einschränkung desselbigen erfolgen mithin in den Erwägungsgründen der KVRL. Der Gesetzgeber stellt in den Erwägungsgründen in einem einheitlichen Zusammenhang dar, dass das Kriterium des Beschaffungsbezuges erfüllt sein muss und stellt danach Situationen vor, in denen dies nicht der Fall ist.

Die Erweiterung der Definition um das Kriterium des Beschaffungsbezuges, als zentrales Merkmal des Vergaberechtes ist sinnvoll und schlüssig. Selbst die Gegenansicht, die den Ausschluss der Anwendbarkeit der KVRL für unzulässig erachtet, leitet den Definitionsbestandteil aus den Erwägungsgründen her (C. II. 1. b. bb.). Systematisch wäre es vorzuziehen gewesen, wenn der Gesetzgeber das Merkmal in die Legaldefinition integriert hätte und die Erwägungsgründe entsprechend ebenfalls normativ umgesetzt hätte. Argumentiert man nun, dass die Definition auf Basis der Erwägungsgründe zu erweitern ist, gestattet aber nicht zugleich eine Einschränkung des betreffenden Merkmals in dem gleichen Zusammenhang, wird mit zweierlei Maß gemessen. Überzeugender ist es den Erwägungs-

397 Donhauser/Hölzlwimmer, VergabeR 2015, S. 509, 516 f.; Ganske, in: Reidt/Stickler/Glahs (Hrsg.), Vergaberecht, $\mathbb{\$} 105$ GWB, Rn. 36 weist darauf hin, dass die Erwägungsgründe lediglich einen „Indizcharakter“ besitzen und zusätzlich eine Begründung auf Basis der Normen erfolgen müsse; Hofmann/Zimmermann, NZBau 2016, S. 71, 73 f.; Michaels/Bönnighausen, ZNER 2018, S. 7, 10; vgl. auch Probst, Auswahlkriterien bei der Vergabe von energiewirtschaftlichen Konzessionsverträgen, S. $78 \mathrm{f}$.

398 Dies ergibt sich aus der ständigen Rechtsprechung des EuGH, EuGH, Urt. v. 2.4.2009, Rs. C-134/08 (Tyson Parketthandel), ECLI:EU:C:2009:229, Rn. 16; EuGH, Urt. v. 24.11.2005, Rs. C-136/04 (Deutsches Milch-Kontor), ECLI:EU:C: 2005:716, Rn. 32 mit Verweis auf vorangegangene Judikate; Brück von Oertzen/ Kreggenfeld, EWerK 2016, S. 12, 14; Ganske, in: Reidt/Stickler/Glahs (Hrsg.), Vergaberecht, $\mathbb{S} 105$ GWB, Rn. 45; Hofmann/Zimmermann, NZBau 2016, S. 71, 73; Hohenstein-Bartholl/Jacob, RdE 2017, S. 454, 459.

399 Dicks, in: Kulartz/Kus/Portz u.a. (Hrsg.), Kommentar zum GWB-Vergaberecht, $\$ 105$ GWB, Rn. 11 ff.; Grünewald, Die (Re)Kommunalisierung in der Energieverteilung, S. 319. 
grund 16 der KVRL als Argument dafür zuzulassen, dass die Vergabe des Konzessionsrechts, das dem vorliegenden Modell zugrunde legen würde, nicht vom Anwendungsbereich der Vorgaben der KVRL und der $\$ \$ 105 \mathrm{ff}$. GWB erfasst wäre. 400

b) Vergaberechtliche Erleichterungen durch eine Inhouse-Vergabe

Konzessionen könnten bei einer Inhouse-Vergabe unter erleichterten Bedingungen, nämlich etwa unter Verzicht auf eine öffentliche Ausschreibung, vergeben werden. Die Kriterien, die hierfür kumulativ erfüllt sein müssen, ergeben sich unionsrechtlich aus Art. 17 KVRL und aus dessen Umsetzung in $\$ 108$ GWB. Verallgemeinernd handelt es sich um Kriterien der Beteiligung, Kontrolle und Wesentlichkeit des Einflusses der vergebenden Stelle auf den Konzessionär. Die Konzessionen können, wie bereits dargelegt, aus staatsorganisationsrechtlichen Gründen lediglich in bundesunmittelbarer Verwaltung vergeben werden (C. IV. 1. b. aa.). Eine Inhouse-Vergabe wäre lediglich dann denkbar, wenn die Vergabe der Rechte an den Bund oder ein bundeseigenes Unternehmen erfolgen würde. Ein diesbezüglicher Plan für den leitungsgebundenen Glasfaserausbau existiert jedoch bislang nicht. Ebenso wenig wird diskutiert, die Glasfasernetze direkt durch den Bund ausbauen zu lassen. Deswegen ist derzeit keine Inhouse-Vergabe möglich, sodass deren Folgen für die Ausgestaltung des Konzessionsvergabeverfahrens an dieser Stelle nicht diskutiert werden müssen.

c) Steuerungsvorgaben des unionalen Primärrechts

Obwohl die KVRL und der $\$ \$ 105 \mathrm{ff}$. GWB nicht anwendbar sind, würde die Konzessionsvergabe nicht in einem „rechtsfreien Raum“401 stattfinden können. ${ }^{402}$ Vielmehr leitet der Europäische Gerichtshof in seiner Rechtsprechung verschiedene Vorgaben für die Verfahrensausgestaltung aus

400 So im Ergebnis auch Grünewald, Die (Re)Kommunalisierung in der Energieverteilung, S. 319.

401 Grünewald, Die (Re)Kommunalisierung in der Energieverteilung, S. 321.

402 Grünewald, Die (Re)Kommunalisierung in der Energieverteilung, S. 321, spricht insoweit von einem „Sonderstatus“; Prieß/Marx/Hölzl, NVwZ 2011, S. 65, 68, bezeichnen die Vorgaben als „Vergaberecht light“; hierzu auch Rennert, NZBau 2019, S. 411, 414. 
dem EU-Primärrecht ab. Insoweit sind die Steuerungsvorgaben aus dem Wettbewerbs- und Vergaberecht mithin identisch. Hierbei handelt es sich insbesondere um die Niederlassungs- (Art. $49 \mathrm{ff}$. AEUV) und Dienstleistungsfreiheit (Art. $56 \mathrm{ff}$. AEUV) sowie die hieraus abgeleiteten Gebote zur Gleichbehandlung, Nichtdiskriminierung und Transparenz. ${ }^{403}$ Damit der Anwendungsbereich der Grundfreiheiten überhaupt eröffnet ist und aus ihnen Maßgaben für die Ausgestaltung des Konzessionsvergabeverfahrens abgeleitet werden können, müsste eine Binnenmarktrelevanz gegeben sein. ${ }^{404}$ Normative Abgrenzungskriterien für das Vorliegen einer Binnenmarktrelevanz existieren nicht. Vielmehr ist eine Abgrenzung anhand des Einzelfalles vorzunehmen. ${ }^{405}$ Hierbei können etwa der Wert der Konzession, die Größe und Komplexität des Gebietes sowie die Anzahl der Interessenten eine Rolle spielen. ${ }^{406}$ Maßgeblich wäre demnach insbesondere der Zuschnitt des Konzessionsgebietes. Je größer und je attraktiver die Konzessionsgebiete gefasst werden würden, desto eher könnte von einer Binnenmarktrelevanz ausgegangen werden. Im Zweifel ist jedoch davon auszugehen, dass die Binnenmarktrelevanz gegeben ist. Ziel des unionalen Gesetzgebers ist eine umfassende Liberalisierung des Telekommunikationsmarktes und eine Förderung des digitalen europäischen Binnenmarktes. ${ }^{407}$

Die Rechtsprechung fordert auf Basis der Vorgaben des unionalen Primärrechts eine transparente, nichtdiskriminierende Ausgestaltung des Verfahrens, in dem alle Teilnehmer gleichbehandelt werden, der Grundsatz der Verhältnismäßigkeit beachtet wird und ablehnende Entscheidungen

403 Dies ergibt sich aus der ständigen Rechtsprechung des EuGH, EuGH, Urt. v. 6.11.2014, Rs. C-42/13 (Cartiera dell'Adda), ECLI:EU:C:2014:2345, Rn. 47; EuGH, Urt. v. 13.4.2010, Rs. C-91/08 (Wall), ECLI:EU:C:2010:182，Rn.33; EuGH, Urt. v. 13.10.2005, Rs. C-458/03 (Parking Brixen), ECLI:EU:C:2005:605, Rn. 46 ff.; Grünewald, Die (Re)Kommunalisierung in der Energieverteilung, S. 321; Kühling/Huerkamp, in: Säcker (Hrsg.), Münchener Kommentar Europäisches und deutsches Wettbewerbsrecht - Band 3, Einleitung zum Vergaberecht, Rn. 21-29.

404 Kühling/Huerkamp, in: Säcker (Hrsg.), Münchener Kommentar Europäisches und deutsches Wettbewerbsrecht - Band 3, Einleitung zum Vergaberecht, Rn. 40.

405 Dörr, in: Burgi/Dreher (Hrsg.), Beck'scher Vergaberechtskommentar, Einleitung: Vergaberecht in Deutschland - Das Recht der öffentlichen Auftragsvergabe im Stufenbau der Rechtsordnung, Rn. 174.

406 Dörr, in: Burgi/Dreher (Hrsg.), Beck'scher Vergaberechtskommentar, Einleitung: Vergaberecht in Deutschland - Das Recht der öffentlichen Auftragsvergabe im Stufenbau der Rechtsordnung, Rn. 174; Grünewald, Die (Re)Kommunalisierung in der Energieverteilung, S. 322.

407 Erwägungsgrund 3 des EKEK. 
begründet werden. ${ }^{408}$ Konkret bedeutet dies für das Transparenzgebot, dass bereits vor dem Verfahren alle Informationen zur Konzession veröffentlicht werden müssen. ${ }^{409}$ Auf diese Weise soll jeder Mitbewerber die Möglichkeit erhalten, zumindest sein Interesse am Erhalt der Konzession zu bekunden. ${ }^{410}$ Ein angemessener Grad an Öffentlichkeit und Transparenz soll sicherstellen, dass die Möglichkeit einer Nachprüfung der vorgenannten Verfahrensanforderungen sichergestellt ist. ${ }^{411}$ Das Diskriminierungsverbot und der Gleichbehandlungsgrundsatz knüpfen hieran an und verlangen, dass etwaige Entscheidungskriterien im Vorfeld kommuniziert werden müssen. ${ }^{412}$ Die Entscheidungen müssen begründet werden und es muss einen ausreichenden Rechtsschutz geben. Alle Unternehmen müssen im gesamten Verfahren gleichbehandelt werden. ${ }^{413}$ Das Verfahren muss zudem für jedes interessierte Unternehmen zugänglich sein. ${ }^{414}$ Die Kriterien führen dazu, dass zwischen den Verfahrensschritten ausreichend Zeit für eine Wahrnehmung in der Öffentlichkeit und eine Erwägung des Einlegens von Rechtsschritten erforderlich ist.

\section{d) Ergebnis}

Das sekundärrechtlich in der KVRL normierte und einfachgesetzlich in den $\mathbb{S} 105 \mathrm{ff}$. GWB umgesetzte Vergaberecht findet auf die Vergabe der Dienstleistungskonzession, die dem Konzessionsmodell zugrunde liegt, keine Anwendung. Der Europäische Gerichtshof leitet für die Vergabe von Dienstleistungskonzessionen, die nicht in den Anwendungsbereich spezieller Regelungsregime fallen, Verfahrensausgestaltungserfordernisse aus dem unionalen Primärrecht ab. Damit decken sich die Anforderungen des

408 EuGH, Urt. v. 6.11.2014, Rs. C-42/13 (Cartiera dell'Adda), ECLI:EU:C: 2014:2345, Rn. 47; EuGH, Urt. v. 13.4.2010, Rs. C-91/08 (Wall), ECLI:EU:C: 2010:182, Rn. 33; EuGH, Urt. v. 7.12.2000, Rs. C-324/98 (Teleaustria), ECLI:EU: C:2000:669, Rn. 59 ff.; Böckel, LKV 2003, S. 393, $396 \mathrm{ff.;} \mathrm{Grünewald,} \mathrm{Die}$ (Re)Kommunalisierung in der Energieverteilung, S. 323.

409 Braun, NZBau 2011, S. 400, 402; Braun, EuZW 2012, S. 451, 452; Grünewald, Die (Re)Kommunalisierung in der Energieverteilung, S. 323.

410 EuGH, Urt. v. 21.7.2005, Rs. C-231/03 (Coname), ECLI:EU:C:2005:487, Rn. 21.

411 EuGH, Urt. v. 13.4.2014, Rs. C-91/08 (Wall), ECLI:EU:C:2010:182, Rn. 36.

412 Grünewald, Die (Re)Kommunalisierung in der Energieverteilung, S. 325.

413 Grünewald, Die (Re)Kommunalisierung in der Energieverteilung, S. 326; Burgi, NZBau 2005, S. 610, 615.

414 Grünewald, Die (Re)Kommunalisierung in der Energieverteilung, S. 326; Böckel, LKV 2003, S. 393, 397. 
Wettbewerbs- und Vergaberechts inhaltlich. Hierbei sind insbesondere die Erfordernisse der Gleichbehandlung, Nichtdiskriminierung und Transparenz hervorzuheben. Das Vergabeverfahren würde nicht in einem rechtsfreien Raum stattfinden, sondern müsste sich an diesen Vorgaben messen lassen. Die konkreten Folgen für die Verfahrensgestaltung sollen zu einem späteren Zeitpunkt gebündelt beleuchtet werden (C. IV. 5.).

4. Vereinbarkeit des Konzessionsmodells auf Basis der Vergabe exklusiver Wegerechte mit dem EU-Beihilfenrecht

Das Konzessionsmodell auf Basis der Vergabe exklusiver Wegerechte soll Förderprogramme nicht ersetzen, sondern vielmehr flankieren. ${ }^{415}$ Der Glasfaserausbau wird zukünftig auf Bundesebene insbesondere mithilfe des „Graue-Flecken-Förderprogrammes“ der Bundesregierung gefördert. ${ }^{416}$ Bei der Notifizierung der Beihilfe wird die Kommission die wirtschaftlichen Bedingungen und die Ausgangslage in Deutschland berücksichtigen. Hierzu gehört unter anderem die Art und Weise der Wegerechtsvergabe in

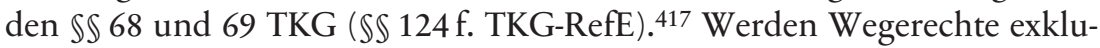
siv vergeben, entstehen Infrastrukturmonopole. Hierdurch werden die Bedingungen, unter denen die Beihilfe genehmigt wurde, wesentlich verändert. ${ }^{418}$ Dies macht eine erneute Notifizierung der Beihilfe erforderlich. ${ }^{419}$

415 Deutscher Landkreistag, Flächendeckende Breitbandversorgung zu wirtschaftlichen Bedingungen sicherstellen, S. 7.

416 Entwurf über eine Rahmenregelung der Bundesrepublik Deutschland zur Unterstützung des flächendeckenden Aufbaus von Gigabitnetzen in „grauen Flecken" vom 22.5.2019, abrufbar unter https:/www.bmvi.de/SharedDocs/DE/Anl age/DG/breitbandfoerderung-gigabit-rahmenregelung.pdf?_blob=publicationF ile (zuletzt abgerufen am 1.7.2020); zur Entwurfsfassung des Förderprogrammes Kühling/Toros, Rechtliche Rahmenbedingungen für die Schaffung von Anreizen für einen flächendeckenden Ausbau von Glasfaserinfrastrukturen, S. $11 \mathrm{ff}$.

417 Referentenentwurf des Telekommunikationsmodernisierungsgesetzes (TKMoG), abrufbar unter: https:/www.bmwi.de/Redaktion/DE/Downloads/Gesetz/ telekommunikationsmodernisierungsgesetz-referentenentwurf-20201612.pdf? blob=publicationFile\&v=8 (zuletzt abgerufen am 12.1.2021).

418 Monopolkommission, 10. Sektorgutachten Telekommunikation (2017), Rn. 206.

419 Kühling/Rüchardt, in: Streinz (Hrsg.), EUV/AEUV, Art. 108 AEUV, Rn. 14; Monopolkommission, 10. Sektorgutachten Telekommunikation (2017), Rn. 206. 
Die Anforderungen an die Notifizierung ergeben sich dabei insbesondere aus den Breitbandleitlinien der Kommission. ${ }^{420}$ Diese sehen vor, dass die negativen Auswirkungen der Beihilfe auf den Wettbewerb auf das absolute Minimum beschränkt werden müssen. ${ }^{421}$ Hierbei ist insbesondere zu prüfen, ob ein wettbewerbliches Auswahlverfahren (lit. c) durchgeführt und das Transparenzgebot beachtet wurde (lit. j). Darüber hinaus ist auch eine Beachtung des Grundsatzes der Technologieneutralität sicherzustellen (lit. e). Sofern die Anforderungen nicht eingehalten werden, kann die Kommission die Beihilfe eingehend prüfen und letztlich auch nicht genehmigen. 422

In der Diskussion über die Genehmigungsfähigkeit von Beihilfen im Zusammenhang mit dem Konzessionsmodell auf Basis der Vergabe exklusiver Wegerechte werden auch mildere Alternativmaßnahmen angeführt. So wird etwa festgestellt, dass alternative Konzepte wie Wohlverhaltensvereinbarungen oder alternative Gebietsausschreibungen weniger negative Auswirkungen auf den Wettbewerb hätten als das Konzessionsmodell auf Basis der Vergabe exklusiver Wegerechte. ${ }^{423}$ Aus den Breitbandleitlinien geht hervor, dass die Kommission den Mitgliedstaaten einen umfassenden Handlungsspielraum zubilligt. Die negativen Auswirkungen auf den Wettbewerb werden insbesondere durch die Ausgestaltung des Verfahrens begrenzt. Dieses ist im Ergebnis hinreichend wettbewerbsschützend auszugestalten. Insoweit ist davon auszugehen, dass die Anforderungen des EUPrimärrechts, die soeben dargestellt worden sind, entsprechend auch für eine Sicherstellung der Kompatibilität mit dem EU-Beihilfenrecht gelten.

420 Leitlinien der EU für die Anwendung der Vorschriften über staatliche Beihilfen im Zusammenhang mit dem schnellen Breitbandausbau, Mitteilung 2013/C 25/01, ABl. C 25/01 v. 26.1.2013; zu den übrigen Anforderungen aus dem EUBeihilfenrecht Kühling/Toros, Rechtliche Rahmenbedingungen für die Schaffung von Anreizen für einen flächendeckenden Ausbau von Glasfaserinfrastrukturen, S. $68 \mathrm{ff}$.

421 Mitteilung 2013/C 25/01, Rn. 78; Monopolkommission, 10. Sektorgutachten Telekommunikation (2017), Rn. 206.

422 Mitteilung 2013/C 25/01, Rn. 78.

423 Monopolkommission, 10. Sektorgutachten Telekommunikation (2017), Rn. 205. 
5. Folgen für die Ausgestaltung des Vergabeverfahrens

Aus den allgemeinen Anforderungen des Verfassungsrechts und ihren speziellen Ausprägungen im Wettbewerbs-, Vergabe- und EU-Beihilfenrecht ergibt sich das Bedürfnis, das Verfahren zur Vergabe exklusiver Wegerechte im Konzessionsmodell an den Anforderungen des unionalen Primärrechts auszurichten (dazu a). ${ }^{424}$ Die hieraus resultierende Verfahrensausgestaltung müsste mit den Steuerungsvorgaben des Art. 43 Abs. 1 UAbs. 1 EKEK zu vereinbaren sein (dazu b.).

a) Vorgaben des unionalen Primärrechts für die Verfahrensausgestaltung

Die vorgenannten Rechtsmaterien statuieren zur Sicherung des Wettbewerbs verschiedene Ver- sowie Gebote und konkretisieren hiermit die Wettbewerbsvorstellungen des Unionsrechts, die einen funktionsfähigen Binnenmarkt voraussetzen (dazu aa.). Dies hat praktische Auswirkungen auf die Ausgestaltung des Verfahrens (dazu bb.).

aa) Primärrechtliche Ver- und Gebote bei der Verfahrensausgestaltung

Die Rechtsquellen statuieren verschiedene Ver- und Gebote, um den Wettbewerb möglichst umfassend zu schützen. Stets ist der Grundsatz der Verhältnismäßigkeit zu beachten.

In einem Konzessionsmodell auf Basis der Vergabe exklusiver Wegerechte findet kein Wettbewerb im Netz statt. Lediglich ein Unternehmen kann im Konzessionsgebiet als Infrastrukturanbieter tätig sein. Die Situation ist vergleichbar mit dem Energierecht, in dem ebenfalls lediglich ein Unternehmen die Konzessionsrechte im jeweiligen Konzessionsgebiet innehat. Um die negativen Folgen der Monopolisierung der Infrastruktur im Konzessionsgebiet möglichst gering zu halten, wird in einem regelmäßigen Turnus ein „Wettbewerb um das Netz“ ausgetragen. ${ }^{425}$ Ein vergleichbarer

424 Ausführlich zu den Verfahrensanforderungen an eine Konzessionsvergabe im Energierecht Sauer, Das Recht der Vergabe von Strom- und Gas-Konzessionsverträgen im EnWG, S. 673 ff.; Säcker/Mohr/Wolf, Konzessionsverträge im System des europäischen und deutschen Wettbewerbsrechts, S. $75 \mathrm{ff}$.

425 Umfassend Säcker/Mohr/Wolf, Konzessionsverträge im System des europäischen und deutschen Wettbewerbsrechts, S. $123 \mathrm{ff}$. 
Mechanismus wäre zum Schutz des Wettbewerbs im Telekommunikationssektor ebenfalls erforderlich, sofern das vorliegende Konzessionsmodell etabliert würde. Dies entspricht dem generellen Umgang mit Exklusivität im Telekommunikationssektor, wonach negative Folgen für den Wettbewerb durch ein Substitut ausgeglichen werden müssen (B. I.-III.). Ein solches Substitut könnte ein „Wettbewerb um das Netz“ sein. Wie bereits dargestellt, ist das Verfahren zur Vergabe exklusiver Wegerechte unter anderem an den Maßgaben des unionalen Primärrechts auszurichten. Relevant sind diesbezüglich insbesondere das Transparenzgebot und das Diskriminierungsverbot.

\section{bb) Praktische Auswirkungen auf das Konzessionsvergabeverfahren}

Die dargestellten rechtlichen Rahmenbedingungen hätten umfassende Auswirkungen auf die Ausgestaltung des Vergabeverfahrens in einem Konzessionsmodell auf Basis der Vergabe exklusiver Wegerechte. Der Grundsatz der Verhältnismäßigkeit gebietet, den Wettbewerb umfassend zu schützen. Konkretisiert wird dies durch die Pflicht, in regelmäßigen Abständen die Konzessionen neu zu vergeben. Ewigkeitsrechte dürften nicht entstehen. Im Rahmen dieses regelmäßigen Verfahrens sind das Gebot der Fairness, das Verbot der Diskriminierung und das hiermit zusammenhängende Transparenzgebot von großer Bedeutung. ${ }^{426}$ Die Auswirkungen von Transparenz- und Diskriminierungsverbot sind, wie bereits dargestellt, eine umfassende Beteiligung der Öffentlichkeit sowie eine Implementierung verschiedenen Erfordernisse, die zu einer Erhöhung der Nachvollziehbarkeit der Entscheidung beitragen (C. IV. 3. c.). Zudem muss stets ein ausreichender Zeitraum zur Verfügung stehen, um zu sondieren, ob gegen eine Entscheidung Rechtsmittel eingelegt werden sollen. Im Ergebnis kommt es zu einer erheblichen Komplexitätssteigerung und Verzögerung im Verfahrenslauf.

426 Umfassend Möllnitz, Die Vergabe von Konzessionsverträgen nach $\$ 46$ Abs. 2 EnWG im Spannungsfeld zwischen Wettbewerb und kommunaler Daseinsvorsorge, $\mathrm{S} .71 \mathrm{ff}$. 
b) Vereinbarkeit mit den Steuerungsvorgaben des EKEK

Die rechtlichen Rahmenbedingungen, die aus einer exklusiven Rechtsvergabe entstehen, führen somit zu Verzögerungen im Konzessionsvergabeverfahren und erfordern eine umfangreiche Planung und vorlaufende Festlegung der Vergabekriterien. ${ }^{427}$ Dies steht in einem Spannungsverhältnis zu den Steuerungsvorgaben des Art. 43 Abs. 1 UAbs. 1 lit. a EKEK, wonach das Verfahren zur Vergabe von Wegerechten effizient und einfach sein soll. Die Kommission strebt eine weitere Verfahrensbeschleunigung an und steht etwaigen Verzögerungen kritisch gegenüber (C. III. 2.). Zudem soll die Vergabeentscheidung binnen sechs Monaten nach einem entsprechenden Antrag getroffen werden (C. III. 2.).

Das Transparenzgebot und die hierdurch erforderliche Einbindung der Öffentlichkeit erfordern jedoch eine umfassende Vorplanung und ausreichende Einsichtnahme- und Reaktionsmöglichkeiten. Das Verfahren ist, wie sich an der enormen Fülle der Entscheidungen im Energiekonzessionsrecht zeigt, sehr störanfällig. ${ }^{428}$ Dies liegt insbesondere daran, dass die Exklusivität den wirtschaftlichen Wert eines Wegerechtes erheblich steigert. Dies hat zur Folge, dass gegen die Vergabeentscheidungen vermehrt Rechtsbehelfe eingelegt werden, wodurch weitere Verzögerungen entstehen.

Vergleicht man die Wegerechtsvergabe in einem System mit und ohne die Vergabe exklusiver Wegerechte, ist die Durchführung eines Verfahrens zur Vergabe von Exklusivrechten, bedingt durch das unionale Wettbewerbsrecht, deutlich komplexer und ineffizienter als ohne Exklusivität. Dem Unionsgesetzgeber war das bisherige System der Wegerechtsvergabe in den mitgliedstaatlichen Telekommunikationsgesetzen bei der Novellierung des unionalen Rechtsrahmens bekannt. Dennoch wurde auf die Notwendigkeit einer Vereinfachung des Verfahrens hingewiesen. ${ }^{429}$ Der Unionsgesetzgeber spricht dem Wettbewerb im Netz und der erleichterten Möglichkeit zur Erlangung von Wegerechten eine entscheidende Bedeutung für die Beschleunigung des Infrastrukturausbaus zu. Das Konzessi-

427 Möllnitz, Die Vergabe von Konzessionsverträgen nach $₫ 46$ Abs. 2 EnWG im Spannungsfeld zwischen Wettbewerb und kommunaler Daseinsvorsorge, S. $73 \mathrm{ff}$.

428 In jüngerer Zeit etwa, BGH, Urt. v. 28.1.2020, Az. EnZR 116/18, NZKart 2020, S. 253 ff.; OLG Celle, Urt. v. 12.9.2019, Az. 13 U 41/19 (Kart), EnWZ 2019, S. 408; KG Berlin, Urt. v. 4.4.2019, Az. 2 U 5/15 Kart (Gasversorgungsnetz Berlin), NZKart 2019, S. 383.

429 Erwägungsgrund 104 des EKEK. 
onsmodell auf Basis der Vergabe exklusiver Wegerechte stünde diesem Verständnis diametral entgegen. Aus diesem Grund ließe sich ein solches Vergabeverfahren nicht rechtskonform ausgestalten.

\section{Umgang mit Bestandsinfrastruktur und bereits erteilten Wegerechten}

Die Funktionsfähigkeit eines Konzessionsmodells auf Basis der Vergabe exklusiver Wegerechte setzt eine umfassende Lösung des Umganges mit Bestandsinfrastruktur voraus (dazu 1.). ${ }^{430}$ Die Bestandsinfrastruktur ist vom Schutz des Eigentums aus Art. 17 Abs. 1 GRCh erfasst (dazu 2.). Etwaige Handlungsoptionen stellen einen Eingriff in den Schutzbereich dar (dazu 3.) und müssten sich vor diesem Hintergrund rechtfertigen lassen (dazu 4.). Zudem müsste sich das Ergebnis auch am korrespondierenden Grundrecht aus Art. 14 Abs. 1 GG messen lassen (dazu 5.). Daneben müsste auch mit bereits erteilten Wegerechten in rechtmäßiger Weise umgegangen werden (dazu 6.).

1. Auswirkung von Bestandsinfrastruktur im Konzessionsgebiet

Das Konzessionsmodell auf Basis der Vergabe exklusiver Wegerechte soll in Gebieten mit Netzausbaudefizit umgesetzt werden, in denen nicht mit einem eigenwirtschaftlichen Ausbau hochleistungsfähiger digitaler Infrastruktur zu rechnen ist. ${ }^{431}$ Die Gebiete sind jedoch nicht völlig unversorgt, sondern verfügen lediglich nicht über einen Glasfaseranschluss. Eigenwirtschaftlich wird in Deutschland zumindest eine Basisinfrastruktur zur Verfügung gestellt, die Bandbreiten bietet, die für eine soziale und wirtschaftliche Teilhabe erforderlich sind. Eine solche Basisversorgung ist auch in den Konzessionsgebieten verfügbar (C. II. 1. b. cc. (2)). Sofern dies nicht der Fall sein sollte, könnte der Universaldienstmechanismus zur Gewähr-

430 Hierbei steht lediglich der Umgang mit bereits existenter staatlicher Infrastruktur im Mittelpunkt. Der Umgang mit der im Konzessionsmodell errichteten Infrastruktur nach Auslaufen der entsprechenden Verträge soll an dieser Stelle zunächst dahinstehen. In diesem Kontext wird etwa erwogen, die Konzessionsverträge im Anschluss an das Auslaufen des ersten Konzessionsvertrages einfach auslaufen zu lassen; Wernick/Queder/Strube Martins u.a., Ansätze zur GlasfaserErschließung unterversorgter Gebiete, S. 49.

431 Deutscher Landkreistag, Flächendeckende Breitbandversorgung zu wirtschaftlichen Bedingungen sicherstellen, S. 7. 
leistung der Basisversorgung aktiviert werden. ${ }^{432}$ Je eher auf alternative Übertragungstechnologien neben dem konzessionierten Glasfasernetz zurückgegriffen werden kann, desto weniger kann sich das ökonomische Kalkül der gesicherten Investitionsamortisation durch die Nutzung der konzessionierten Infrastruktur realisieren.

Im Energiesektor wird die Funktionsfähigkeit des Konzessionsmodells mittels einer Eigentumsübertragungspflicht für die Bestandsinfrastruktur an den neuen Konzessionsnehmer sichergestellt, die durch eine Ausgleichszahlung gemäß $₫ 46$ Abs. 2 S. 2 EnWG verhältnismäßig ausgestaltet wird. ${ }^{433}$ Dies ist deshalb möglich, da im Energiesektor aus volkswirtschaftlichen Gründen lediglich ein leitungsgebundenes Energienetz bestehen kann. ${ }^{434} \mathrm{Im}$ Telekommunikationssektor existiert eine große Netzvielfalt und es können technisch mehrere Netze parallel betrieben werden. Je abgelegener ein Gebiet jedoch ist, desto weniger ist ein paralleler Infrastrukturaufbau volkswirtschaftlich sinnvoll. Dies betrifft insbesondere die potentiellen Konzessionsgebiete. Die Versorgung im Telekommunikationssektor muss jedoch nicht zwangsläufig mithilfe einer leitungsgebundenen Infrastruktur erfolgen. Vielmehr ist auch eine satelliten- oder mobilfunkgestützte Versorgung von Haushalten denkbar, ${ }^{435}$ die auch im gesamten Bundesgebiet flächendeckend verfügbar ist. ${ }^{436}$ Hieraus resultieren große Herausforderungen für den Umgang mit Bestandsinfrastruktur. Um die Funktionsfähigkeit des Konzessionsmodells auf Basis der Vergabe exklusiver Wegerechte sicherzustellen, reicht es nicht aus, lediglich den Umgang mit leitungsgebundener Festnetzinfrastruktur zu regeln. ${ }^{437}$

432 Bislang ist es in Deutschland jedoch noch nicht zu einer Aktivierung des Universaldienstes gekommen und dies wird perspektivisch auch nicht erwartet. Zum Zusammenspiel der Absicherungsmechanismen, Kübling/Toros, Rechtliche Rahmenbedingungen für die Schaffung von Anreizen für einen flächendeckenden Ausbau von Glasfaserinfrastrukturen, S. $79 \mathrm{ff}$.

433 Hierzu umfassend Braedel, Die Überlassung von Verteilungsanlagen nach Ablauf des Konzessionsvertrages gemäß $₫ 46$ Abs. 2 S. 2 EnWG, S. 71 ff.; Papier/ Schröder, Wirtschaftlich angemessene Vergütung für Netzanlagen, S. $27 \mathrm{ff}$.

434 Es liegt ein natürliches Monopol vor, Knieps, Netzökonomie, S. 109 f.

435 Kühling/Toros, Rechtliche Rahmenbedingungen für die Schaffung von Anreizen für einen flächendeckenden Ausbau von Glasfaserinfrastrukturen, S. 104.

436 Goldmedia, in: Kühling/Goldmedia/Enaux (Hrsg.), Rechtliche Herausforderungen bei der Schaffung von Anreizen für einen flächendeckenden Ausbau von Glasfaserinfrastrukturen, S. $176 \mathrm{ff}$.

437 Zur Praktikabilität dieses Ansatzes auch Kühling/Toros, Rechtliche Rahmenbedingungen für die Schaffung von Anreizen für einen flächendeckenden Ausbau von Glasfaserinfrastrukturen, S. 104. 
Diese Ausgangslage lässt an der technischen und ökonomischen Praktikabilität des Modells zweifeln. Technisch ist es kaum möglich, dass die Konzessionsgebiete nicht mit diesen alternativen Infrastrukturen versorgt werden, um die Funktionsfähigkeit eines Konzessionsmodells auf Basis der Vergabe exklusiver Wegerechte zu erreichen. Es müsste sichergestellt werden, dass die Signale von Mobilfunk und Satelliten im Konzessionsgebiet nicht verfügbar sind. Aus ökonomischer Sicht ist fraglich, wie weitgehend die Alternativversorgung für die Funktionsfähigkeit des Modells ausgeschlossen werden müsste. Diese Frage kann an dieser Stelle nicht abschließend beantwortet werden. Damit die ökonomischen Grunderwägungen zum Tragen kommen, die bei der Einführung eines Konzessionsmodells auf Basis der Vergabe exklusiver Wegerechte die Investitionsbedingungen verbessern würden, müsste jedoch im Ergebnis die Alternativversorgung des Konzessionsgebietes mit Telekommunikationsinfrastruktur möglichst umfassend vermieden werden. Je besser das Konzessionsmodell auf Basis der Vergabe exklusiver Wegerechte funktionieren soll, desto mehr Übertragungstechnologien müssen bei der Schaffung von Regelungen zum Umgang mit Bestandsinfrastruktur einbezogen werden.

\section{Schutzbereich des Grundrechts auf Eigentum aus Art. 17 Abs. 1 GRCh}

Der Europäische Gerichtshof versteht unter Eigentum „vermögenswerte Rechte, aus denen sich im Hinblick auf die Rechtsordnung eine gesicherte Rechtsposition ergibt, die eine selbstständige Ausübung dieser Rechte durch und zugunsten ihres Inhabers ermöglicht. “438 Aus diesem Grund unterfallen die Inhaber von Bestandsinfrastruktur im Konzessionsgebiet, unabhängig von der konkreten Übertragungstechnologie, dem Schutz des Eigentumsgrundrechts aus Art. 17 Abs. 1 GRCh. ${ }^{439}$

438 EuGH, Urt. v. 22.1.2013, Rs. C-283/11 (Sky Österreich/Österreichischer Rundfunk), ECLI:EU:C:2013:28, Rn. 34; Michl, Unionsgrundrechte aus der Hand des Gesetzgebers, S. 89.

439 Geschützt werden sowohl natürliche als auch juristische Personen, EuGH, Urt. v. 21.5.2019, Rs. C-235/17 (Europäische Kommission/Ungarn), ECLI:EU:C: 2019:432, Rn. 67; EuGH, Urt. v. 22.1.2013, Rs. C-283/11 (Sky Österreich/Österreichischer Rundfunk), ECLI:EU:C:2013:28, Rn. 31 ff.; Kühling, in: Pechstein/ Nowak/Häde (Hrsg.), Frankfurter Kommentar EUV/GRC/AEUV, Art. 17 GRCh, Rn. 6. 
3. Maßnahmen zum Umgang mit Bestandsinfrastruktur als Einschränkung des Eigentumsgrundrechts

Der Deutsche Landkreistag schlägt in seinem Positionspapier verschiedene Maßnahmen zum Umgang mit Bestandsinfrastruktur im Konzessionsgebiet vor. So könne analog zu $₫ 46$ Abs. 2 S. 2 EnWG eine Eigentumsübertragungspflicht vom bisherigen Infrastruktureigentümer auf den Konzessionsnehmer statuiert und diese mit einer Entschädigungszahlung verhältnismäßig ausgestaltet werden. Als Alternativen werden eine Zwangsverpachtung und ein Ausbauverbot vorgeschlagen. ${ }^{440}$

Fraglich ist, ob diese Maßnahmen den Schutzbereich des Art. 17 Abs. 1 GRCh einschränken. ${ }^{441}$ In dessen Wortlaut wird explizit zwischen einem Eigentumsentzug (S.2) und Nutzungsregelungen unterschieden (S. 3). ${ }^{442}$ Die Pflicht zur Übertragung des Eigentums wäre als für Art. 17 Abs. 1 S. 2 GRCh ausreichender, dauerhafter Eigentumsentzug ${ }^{443}$ einzuordnen. Darüber hinaus könnten die alternativen Handlungsoptionen, die vom Deutschen Landkreistag vorgeschlagen werden, eine Nutzungsbeeinträchtigung im Sinne des Art. 17 Abs. 1 S. 3 GRCh darstellen. Darunter sind diejenigen Regelungen zu verstehen, welche die Ausübung der Rechtsstellung als Eigentümer beeinträchtigen. ${ }^{444}$ Dabei legt die Rechtsprechung ein weites Begriffsverständnis zu Grunde. 445

Der Eigentümer hat herkömmlich die Möglichkeit dazu, seine Rechtsposition umfassend zu nutzen und frei über das Eigentum zu verfügen. ${ }^{446}$

440 Deutscher Landkreistag, Flächendeckende Breitbandversorgung zu wirtschaftlichen Bedingungen sicherstellen, S. 7.

441 Zur Vereinbarkeit mit der Berufsfreiheit, die hier nicht untersucht wird, Möllnitz, Die Vergabe von Konzessionsverträgen nach $\$ 46$ Abs. 2 EnWG im Spannungsfeld zwischen Wettbewerb und kommunaler Daseinsvorsorge, S. $19 \mathrm{f}$.

442 Kühling, in: Pechstein/Nowak/Häde (Hrsg.), Frankfurter Kommentar EUV/GRC/AEUV, Art. 17 GRCh, Rn. 21.

443 EuGH, Urt. v. 21.5.2019, Rs. C-235/17 (Europäische Kommission/Ungarn), ECLI:EU:C:2019:432, Rn. 67; Calliess, in: Calliess/Ruffert (Hrsg.), EUV/AEUV, Art. 17 GRCh, Rn. 15 f.; Jarass, Charta der Grundrechte der Europäischen Union, Art. 17, Rn. 18; Wollenschläger, in: von der Groeben/Schwarze/Hatje (Hrsg.), Europäisches Unionsrecht, Art. 17 GRCh, Rn. 24.

444 Kühling, in: Pechstein/Nowak/Häde (Hrsg.), Frankfurter Kommentar EUV/GRC/AEUV, Art. 17 GRCh, Rn. 24.

445 Wollenschläger, in: von der Groeben/Schwarze/Hatje (Hrsg.), Europäisches Unionsrecht, Art. 17 GRCh, Rn. 20 ff. m. w. N.

446 Ein solches Begriffsverständnis kommt etwa in der einfachgesetzlichen Ausgestaltung des Eigentums in $₫ 903$ S. 1 BGB zum Ausdruck. 
Durch eine Pflicht zur Verpachtung der Bestandsinfrastruktur wird dieses Recht weitgehend entkernt, sodass auch eine Verpachtungsverpflichtung als Einschränkung des Art. 17 GRCh zu klassifizieren wäre.

Ein Ausbauverbot muss hingegen differenziert betrachtet werden. Eigentum und Nutzungsbefugnis der Bestandsinfrastruktur bleiben in diesem Fall beim Infrastrukturanbieter. Die bestehenden Rechtspositionen werden folglich nicht eingeschränkt. Es wird vielmehr lediglich der Erwerb zusätzlicher Rechtspositionen vereitelt. Während Art. 17 Abs. 1 GRCh den Schutz bereits erworbener Rechtspositionen bezweckt, ist der Erwerb zusätzlicher Rechtspositionen vom Schutzbereich der Art. 15 und 16 GRCh erfasst. Ein Ausbauverbot stellt mithin keinen Eingriff in den Schutzbereich des Art. 17 Abs. 1 S. 1 GRCh, sondern in die erwerbsbezogenen Grundrechte dar. Als mildere Maßnahme im Vergleich zu einem Eigentumsentzug oder einer Zwangsverpachtung soll die Maßnahme im Rahmen der Erforderlichkeitsprüfung dargestellt werden (C. V. 4. b. cc.).

\section{Rechtfertigung der Einschränkungen des Eigentumsgrundrechts}

Die Einschränkungen des Schutzbereiches müssten gerechtfertigt werden. Die vorgeschlagenen Handlungsoptionen zum Umgang mit Bestandsinfrastruktur stellen unterschiedlich schwere Eingriffe in den Schutzbereich des Art. 17 Abs. 1 GRCh dar. Als schwerster Eingriff ist der vollständige Entzug der Rechtsposition, durch eine Pflicht zur Eigentumsübertragung zu klassifizieren. Die Zwangsverpachtung und das Ausbauverbot stellen im Vergleich dazu mildere Maßnahmen dar, da zumindest das Eigentum an der Infrastruktur beim Telekommunikationsunternehmen bleibt. Aus diesem Grund soll der nachfolgenden Analyse die Eigentumsübertragungspflicht zu Grunde gelegt werden. Die Rechtfertigungsvoraussetzungen für den Entzug des Eigentums ergeben sich aus Art. 17 Abs. 1 S. 2 GRCh und aus Art. 52 Abs. 1 S. 1 und S. 2 GRCh.

a) Gesetzliche Grundlage der Eigentumsübertragungspflicht

Der Entzug des Eigentums müsste sich gemäß Art. 17 Abs. 1 S. 2 GRCh auf eine gesetzliche Grundlage stützen lassen. ${ }^{447}$ Die Steuerungsvorgaben zur

447 Kühling, in: Pechstein/Nowak/Häde (Hrsg.), Frankfurter Kommentar EUV/GRC/AEUV, Art. 17 GRCh, Rn. 26. 
Strukturierung der Wegerechtsvergabe im Telekommunikationssektor ergeben sich aus Art. 43 Abs. 1 UAbs. 1 lit. a EKEK. Die Richtlinie muss effektiv in nationales Recht umgesetzt werden. ${ }^{448}$ Diese effektive Umsetzung führt dazu, dass ohnehin eine einfachgesetzliche Umsetzungsvorschrift etabliert werden muss. Diese Norm könnte auch als gesetzliche Grundlage einer Eigentumsübertragungspflicht dienen.

\section{b) Verhältnismäßigkeit der Eigentumsübertragungspflicht}

Die Eigentumsübertragungspflicht müsste auch verhältnismäßig sein. Neben den allgemeinen Voraussetzungen der Verhältnismäßigkeitsprüfung aus Art. 52 Abs. 1 S. 2 GRCh, sind dabei insbesondere die besonderen Voraussetzungen des Art. 17 Abs. 1 S. 2 GRCh zu berücksichtigen.

\section{aa) Legitimes Ziel und öffentliches Interesse}

Grundsätzlich muss jede Einschränkung einer Gewährleistung der GRCh gemäß Art. 52 Abs. 1 S. 2 GRCh ein legitimes Ziel verfolgen, das dem Gemeinwohl entspricht. Aus Art. 17 Abs. 1 S. 2 GRCh erwächst zusätzlich das Erfordernis, dass das Ziel der Maßnahme im öffentlichen Interesse liegen muss. ${ }^{449}$ Hierdurch wird sichergestellt, dass der Zweck des Eigentumsentzuges nicht allein in der Verfolgung der Interessen Privater liegt. ${ }^{450} \mathrm{Zu}$ den Zielen der Europäischen Union gehört gemäß Art. 3 Abs. 1 EUV die Sicherung einer wettbewerbsfähigen sozialen Marktwirtschaft. Der Europäische Rat hat in der strategischen und politischen Agenda der EU festgelegt, dass der digitale Wandel aktiv mitgestaltet werden soll. „Dazu muss die EU an allen Aspekten der digitalen Revolution und der künstlichen Intelligenz arbeiten: Infrastruktur, Konnektivität, Dienstleistungen, Daten, Regulie-

448 Zur ständigen Rspr. EuGH, Urt. v. 20.10.2005, Rs. C-6/04 (Kommission/Vereinigtes Königreich), ECLI:EU:C:2005:626, Rn.21; EuGH, Urt. v. 7.1.2004, Rs. C-58/02 (Kommission/Spanien), ECLI:EU:C:2004:9, Rn. 26 jeweils m. w. N.; Ruffert, in: Calliess/Ruffert (Hrsg.), EUV/AEUV, Art. 288 AEUV, Rn. 32; Ehricke, EuZW 2004, S. 359 ff.

449 Kübling, in: Pechstein/Nowak/Häde (Hrsg.), Frankfurter Kommentar EUV/GRC/AEUV, Art. 17 GRCh, Rn. 27.

450 Jarass, Charta der Grundrechte der Europäischen Union, Art. 17, Rn. 27. 
rung und Investitionen." ${ }^{451}$ Die Einführung eines Konzessionsmodells und die damit einhergehende exklusive Wegerechtsvergabe zielen letztlich darauf ab, die Investitionsbedingungen in Gebieten, in denen perspektivisch nicht mit einem Auf- und Ausbau gigabitfähiger Infrastrukturen zu rechnen ist, zu verbessern. Das Hauptziel liegt also wie beim EKEK in der „Schaffung von Anreizen für Investitionen in Hochgeschwindigkeitsbreitbandnetze". 452

Der Eingriff in das Grundrecht auf Eigentum erfolgt damit nicht einseitig zu Gunsten des Konzessionsnehmers. Vielmehr handelt es sich um eine gezielte Handlungsoption, die Gebiete mit Netzausbaudefizit zum Gegenstand hat und letztlich dazu beitragen soll, eine digitale Kluft zwischen ländlichen und städtischen Gebieten zu verhindern. Hierbei handelt es sich um ein legitimes Ziel, das im öffentlichen Interesse liegt. ${ }^{453}$

\section{bb) Eignung}

Die Maßnahme müsste gemäß Art. 52 Abs. 1 S. 2 GRCh auch zur Erreichung des vorgenannten Zieles geeignet sein. Die Eigentumsübertragungspflicht an der Bestandsinfrastruktur stellt die Funktionsfähigkeit eines Konzessionsmodells auf Basis der Vergabe exklusiver Wegerechte sicher. Je mehr Bestandsinfrastruktur im Konzessionsgebiet verbleibt, desto schlechter können die ökonomischen Ziele des Modells erreicht werden. Durch die Verbesserung der Investitionsbedingungen wird mittelbar dazu beigetragen, dass der Ausbau hochleistungsfähiger digitaler Infrastruktur beschleunigt wird. Die Maßnahme fördert also das Ziel. ${ }^{454}$

451 Europäischer Rat, Schlussfolgerung v. 20.6.2019, EUCO 9/19, S. 8, abrufbar unter: https://www.consilium.europa.eu/media/39942/20-21-euco-final-conclusions -de.pdf (zuletzt abgerufen am 1.7.2020).

452 Erwägungsgrund 3 des EKEK.

453 So auch Wernick/Queder/Strube Martins u.a., Ansätze zur Glasfaser-Erschließung unterversorgter Gebiete, S. 50.

454 Monopolkommission, 10. Sektorgutachten Telekommunikation (2017), Rn. 207; vgl. hierzu auch Wernick/Queder/Strube Martins u.a., Ansätze zur Glasfaser-Erschließung unterversorgter Gebiete, S. 50. 
cc) Erforderlichkeit der Eigentumsübertragungspflicht

Die Eigentumsübertragungspflicht müsste auch erforderlich sein, um das Ziel zu erreichen. Es darf mithin kein relativ milderes, gleich geeignetes Mittel zur Erreichung des Zieles existieren. ${ }^{455}$ Vorliegend werden vom Deutschen Landkreistag die Verpachtungspflicht und ein Ausbauverbot als relativ mildere Maßnahmen vorgeschlagen. 456

Das Ausbauverbot ist als relativ mildeste Maßnahme unter den dargestellten Handlungsoptionen zu klassifizieren. Es ist allerdings nicht genauso gut wie eine Eigentumsübertragungspflicht dazu geeignet, die Funktionsfähigkeit des Konzessionsmodells auf Basis der Vergabe exklusiver Wegerechte zu ermöglichen. Ein Ausbauverbot würde sich nämlich nicht auf die Bestandsinfrastruktur auswirken. Vielmehr würde lediglich ihre Erweiterung verhindert. $\mathrm{Ob}$ diese überhaupt noch möglich wäre, ist bereits aufgrund der Vergabe exklusiver Wegerechte fraglich.

Alternativ wird die Verpflichtung zur Verpachtung der Bestandsinfrastruktur im Konzessionsgebiet vorgeschlagen. Wesentliche Vertragspflichten eines Pachtvertrages sind in $\$ 581$ BGB normiert. ${ }^{457}$ Danach erhält der Pächter im Gegenzug für die Zahlung eines Pachtzinses ein Gebrauchsrecht und ein Recht dazu, die Früchte der Sache zu ziehen. Neben der Zahlung eines Pachtzinses ist der Pächter auch gemäß $\$ 582$ BGB dazu verpflichtet, die Sache in Stand zu halten. ${ }^{458}$ Betrachtet man die Umsetzung des Konzessionsmodells im Energierecht, ist dort in $\$ 46$ Abs. 2 S. 2 EnWG eine Eigentumsübertragungspflicht statuiert. Alternativ besteht gemäß $\$ 46$ Abs. 2 S. 3 EnWG die Möglichkeit dazu, die Infrastruktur mittels eines Pachtvertrages freiwillig zu überlassen. Der Gesetzgeber geht davon aus, dass der Vorteil der Eigentumsübertragungspflicht darin besteht, dass Eigentum und Wegerechte in einer Hand zusammenfallen.459 Der staatlichen Zwangsübertragung kann durch eine Verpachtung entgangen werden. Gleich geeignet ist das Mittel jedoch nicht.

455 Pache, in: Pechstein/Nowak/Häde (Hrsg.), Frankfurter Kommentar EUV/GRC/ AEUV, Art. 52 GRCh, Rn. 27.

456 Deutscher Landkreistag, Flächendeckende Breitbandversorgung zu wirtschaftlichen Bedingungen sicherstellen, S. 7.

457 Harke, in: Säcker/Rixecker/Oetker u.a. (Hrsg.), Münchener Kommentar zum Bürgerlichen Gesetzbuch, $\mathbb{5} 581$ BGB, Rn. 2 ff.

458 Harke, in: Säcker/Rixecker/Oetker u.a. (Hrsg.), Münchener Kommentar zum

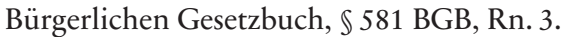

459 BT-Drs. 17/6072, S. 88. 
Im Konzessionsgebiet soll eine Netzausbaupflicht bestehen. ${ }^{460}$ Entstehen im Konzessionsgebiet also neue Gebäude, müssen diese vom Konzessionsnehmer mit Glasfaser erschlossen werden. Findet nun im Zweifel im Zeitlauf ein kontinuierlicher Wechsel von Konzessionsnehmern statt und werden diese lediglich verpflichtet ihr Netzeigentum an den Konzessionsnehmer zu verpachten, führt dies zu einer extremen Zerklüftung der Infrastruktur des Konzessionsgebietes. Im Konzessionsgebiet wäre mithin eine Infrastruktur mit sehr vielen Eigentümern gegeben. ${ }^{461}$ Aufgrund der groBen Technologievielfalt im Telekommunikationssektor stellt sich das Problem zudem in deutlich höheren Umfang als im Energierecht, wo lediglich eine leitungsgebundene Energieübertragung erfolgen kann. Im Telekommunikationssektor wären also Verträge mit den Eigentümern von allen Übertragungstechnologien abzuschließen, die das Konzessionsgebiet mit Telekommunikationsinfrastruktur erschließen. Dies würde nicht nur einen immensen bürokratischen Aufwand, sondern auch hohe Transaktionskosten bedingen. Das Mittel ist mithin nicht gleich geeignet, um die Funktionsfähigkeit des Konzessionsmodells auf Basis der Vergabe exklusiver Wegerechte sicherzustellen.

dd) Zahlung einer angemessenen und rechtzeitigen Entschädigung

Die Rechtfertigung eines Eigentumsentzuges setzt gemäß Art. 17 Abs. 1 S. 2 GRCh zudem die Zahlung einer angemessenen Entschädigung voraus. Diesbezüglich schlägt der Deutsche Landkreistag eine Regelung im Sinne des $₫ 46$ Abs. 2 S. 2 EnWG vor. ${ }^{462}$ In dieser Vorschrift ist die Eigentumsübertragungspflicht an die Zahlung einer wirtschaftlich angemessenen Vergütung gekoppelt. Sofern eine vergleichbare Regelung etabliert würde, könnte das Kriterium erfüllt werden.

460 Deutscher Landkreistag, Flächendeckende Breitbandversorgung zu wirtschaftlichen Bedingungen sicherstellen, S. 7.

461 Eine vergleichbare Situation wäre auch bei einer entsprechenden Regelung im Energierecht gegeben, Theobald, in: Theobald/Kühling (Hrsg.), Energierecht, \$46 EnWG, Rn. 58.

462 Deutscher Landkreistag, Flächendeckende Breitbandversorgung zu wirtschaftlichen Bedingungen sicherstellen, S. 7. 
ee) Verhältnismäßigkeit des Eigentumsentzuges im engeren Sinne

Daneben muss die Maßnahme auch verhältnismäßig im engeren Sinne sein. Die widerstreitenden Interessen müssen schonend miteinander in Ausgleich gebracht werden. ${ }^{463}$ Hierbei handelt es sich um das Recht auf Eigentum einerseits und das öffentliche Interesse am Aus- und Aufbau einer flächendeckenden, hochleistungsfähigen digitalen Infrastruktur andererseits. 464

Aufgrund der Vielfalt unterschiedlicher Übertragungstechnologien kann das wirtschaftliche Kalkül hinter dem Konzessionsmodell auf Basis der Vergabe exklusiver Wegerechte nur dann erreicht werden, wenn ein möglichst großer Teil der Bestandsinfrastruktur in den Eigentumsentzug integriert würde (C. V. 1.). ${ }^{465}$ Das Problem entsteht insbesondere aus der einseitigen Ausrichtung des Modells auf die Versorgung mit Glasfaserinfrastrukturen. ${ }^{466} \mathrm{Je}$ weniger Infrastrukturen in die Maßnahme integriert werden, desto ineffektiver wäre der Handlungsansatz. Dabei besteht jedoch die technische Schwierigkeit, dass die Versorgung der Konzessionsgebiete mittels satelliten- oder mobilfunkgestützter Netzanbindungen nicht vollständig verhindert werden können. Dies gilt umso mehr, je kleiner die betreffenden Gebiete abgegrenzt werden. So strahlen etwa Satelliten große Flächen Westeuropas aus und können die betreffenden Regionen bereits mit hohen Bandbreiten versorgen. 467 Je effektiver der Handlungsansatz mithin ausgestaltet würde, desto eher wäre er unverhältnismäßig. Wird er jedoch nicht effektiv ausgestaltet, so bringt er keinen Mehrwert mit sich und der Grundrechtseingriff wäre nicht zu rechtfertigen. Hierbei handelt es sich um einen diffizilen Balanceakt, der eine verhältnismäßige Ausgestaltung unwahrscheinlich macht.

463 Pache, in: Pechstein/Nowak/Häde (Hrsg.), Frankfurter Kommentar EUV/GRC/ AEUV, Art. 52 GRCh, Rn. 28.

464 Wernick/Queder/Strube Martins u.a., Ansätze zur Glasfaser-Erschließung unterversorgter Gebiete, S. 50, gehen im Rahmen ihrer Prüfung zur Vereinbarkeit des Konzessionsmodells auf Basis der Vergabe exklusiver Wegerechte nicht auf den Umgang mit Bestandsinfrastruktur ein.

465 Ebenfalls kritisch Kühling/Toros, Rechtliche Rahmenbedingungen für die Schaffung von Anreizen für einen flächendeckenden Ausbau von Glasfaserinfrastrukturen, S. 104.

466 Monopolkommission, 10. Sektorgutachten Telekommunikation (2017), Rn. 204.

467 Goldmedia, in: Kühling/Goldmedia/Enaux (Hrsg.), Rechtliche Herausforderungen bei der Schaffung von Anreizen für einen flächendeckenden Ausbau von Glasfaserinfrastrukturen, S. $176 \mathrm{ff}$. 
c) Zwischenergebnis

Zusammenfassend lässt sich feststellen, dass der Entzug des Eigentums an jeglicher Bestandsinfrastruktur im Konzessionsgebiet vor dem Hintergrund des Art. 17 Abs. 1 GRCh nicht mehr zu rechtfertigen wäre. ${ }^{468}$ Eine Beschränkung auf leitungsgebundene Festnetzinfrastruktur wäre zwar weniger eingriffsintensiv, aber nicht in gleicher Weise dazu geeignet, die Funktionsfähigkeit des Konzessionsmodells auf Basis der Vergabe exklusiver Wegerechte sicherzustellen. Vielmehr wäre in diesem Fall eine Basisversorgung als Ausweichprodukt vorhanden, welche die Nutzung der Glasfaserinfrastruktur bei einer gravierenden Preissteigerung unwahrscheinlich macht. Der entscheidende Unterschied zwischen dem Energieund Telekommunikationssektor ist die Netzvielfalt, die dieses Konzessionsmodell unpraktikabel macht und seine Vereinbarkeit im Telekommunikationssektor mit Art. 17 Abs. 1 S. 2 GRCh ausschließt.

\section{Eigentumsgrundrecht des Grundgesetzes (Art. 14 Abs. 1 GG)}

Auch wenn die Terminologie durch die Differenzierung zwischen Inhaltsund Schrankenbestimmungen und Eigentumsentzug bei Art. 14 Abs. 1 GG abweicht, wäre der Eingriff in dessen Schutzbereich aus den gleichen Gründen nicht zu rechtfertigen.

\section{Umgang mit bereits erteilten Wegerechten}

Darüber hinaus stellt sich auch die Frage, ob sich der Entzug bereits erteilter Wegerechte, um das neue Modell zu etablieren, verfassungsrechtlich rechtfertigen ließe. ${ }^{469}$ Ein solcher Widerruf bereits erteilter Genehmigungen könnte zunächst als ein ungerechtfertigter Eingriff in die Berufsfreiheit aus Art. 15 Abs. 1 GRCh und die unternehmerische Freiheit aus Art. 16 GRCh klassifiziert werden. Für den Widerruf einer wegerechtlichen Genehmigung existiert in $\$ 49 \mathrm{VwVfG}$ (Bund) eine Rechtsgrundlage, die eine umfassende Interessenberücksichtigung vorsieht. Darüber hinaus

468 a. A. Wernick/Queder/Strube Martins u.a., Ansätze zur Glasfaser-Erschließung unterversorgter Gebiete, S. 50.

469 Deutscher Landkreistag, Flächendeckende Breitbandversorgung zu wirtschaftlichen Bedingungen sicherstellen, S. 7. 
gilt auch die Argumentation, die bereits im Rahmen der generellen Vereinbarkeit des Konzessionsrechts mit den Voraussetzungen der Art. 15 Abs. 1 und 16 GRCh dargestellt wurde (C. IV. 1. a. bb. (1)).

Fraglich ist, ob der Widerruf der bereits erteilten Wegerechte nicht einen ungerechtfertigten Eingriff in das Eigentumsgrundrecht aus Art. 17 Abs. 1 S. 1 GRCh darstellt. Zwar handelt es sich bei Genehmigungen nicht um Eigentumspositionen im Sinne des Art.17 Abs. 1 GRCh, dennoch kann das hieraus resultierende Vertrauen schutzwürdig sein, sobald bereits Investitionen getätigt wurden. ${ }^{470}$ Sind noch keine Investitionen erfolgt, wird noch nicht in den Schutzbereich des Art. 17 Abs. 1 GRCh eingegriffen. Sobald erste Investitionen im Sinne von Bauvorhaben auf Basis der entsprechenden wegerechtlichen Erlaubnis getätigt wurden, müssten diese ausreichend kompensiert werden. Wurde auf Basis der Genehmigung bereits Infrastruktur aufgebaut, handelt es sich um den bereits zuvor skizzierten Umgang mit Bestandsinfrastruktur (C. V. 2.-5.).

Sofern lediglich die Wegerechte entzogen würden, ohne dass bereits Infrastruktur errichtet worden ist, müsste dennoch die Verhältnismäßigkeit für jeden Einzelfall geprüft werden. Dabei stellen sich die gleichen Probleme wie bei der Verhältnismäßigkeit des Eigentumsentzuges, sodass im Ergebnis keine Rechtfertigung möglich ist (C. V. 4. b. ee.). Der Widerruf bereits erteilter Wegerechte ließe sich nicht mit den Grundrechten aus Art. 15 Abs. 1 und 16 GRCh sowie Art. 17 Abs. 1 GRCh vereinbaren.

\section{Ergebnis}

Der Umgang mit Bestandsinfrastruktur, der zur Sicherstellung der Funktionsfähigkeit des Konzessionsmodells auf Basis der Vergabe exklusiver Wegerechte erforderlich wäre, ließe sich nicht mit den Eigentumsgrundrechten aus Art. 17 Abs. 1 GRCh und Art. 14 Abs. 1 GG vereinbaren. Dies lässt sich auf die große Netzvielfalt im Telekommunikationssektor zurückführen, sodass verschiedene Übertragungstechnologien parallel genutzt werden können, um die Endnutzer zu erreichen. Sofern die ökonomischen Grundannahmen hinter dem Konzessionsmodell auf Basis der Vergabe exklusiver Wegerechte zum Tragen kommen sollen, müssen Bestandsinfra-

470 Ein Urteil des EuGH hierzu steht noch aus, vgl. jedoch EGMR, Urt. v. 29.11.1991, Nr. 12742/87 (Pine Valley Developments Ltd. u. a./Irland), Rn.31 ff.; Jarass, Charta der Grundrechte der Europäischen Union, Art. 17, Rn. 11. 
strukturen möglichst umfassend einbezogen werden. Dies führt jedoch zu einem unverhältnismäßigen Eingriff in die Eigentumsfreiheit. Evident wird dies am Beispiel von Satelliten, die ganz Westeuropa mit Telekommunikationsinfrastruktur versorgen und infolge der Einführung des Konzessionsmodells in etwaige Handlungsansätze einbezogen werden müssten, um dessen Funktionieren in einem kleinen Konzessionsgebiet zu ermöglichen. Dies erstreckt sich neben der Eigentumsübertragungspflicht als stärkstem Eingriff auch auf die milderen Maßnahmen, die vom Deutschen Landkreistag vorgeschlagen werden.

Beim Widerruf bereits erteilter Wegerechte handelt es sich um einen Eingriff in die Art. 15 Abs. 1 und 16 GRCh, da es sich um bloße Erwerbsmöglichkeiten handelt, auf die das Eigentumsgrundrecht aus Art. 17 Abs. 1 GRCh keine Anwendung findet. Aus den vorgenannten Gründen ist dieser jedoch ebenfalls als unverhältnismäßig zu klassifizieren.

\section{Ergebnis}

Das vorliegenden Konzessionsmodell verfolgt das Ziel, mittels der Schaffung temporärer Infrastrukturmonopole die Investitionsbedingungen in den Konzessionsgebieten so zu verbessern, dass flächendeckend Glasfaserinfrastrukturen zu erschwinglichen Preisen verlegt werden. Hierzu sollen exklusive Wegerechte an Konzessionsnehmer vergeben werden, die im Gegenzug zum Aufbau und Betrieb der Glasfaserinfrastruktur im Konzessionsgebiet verpflichtet werden. Die Konzession stellt eine Dienstleistungskonzession im Sinne des Vergaberechts dar, auch wenn sie aufgrund der Einschlägigkeit spezieller Ausschlussgründe nicht von den Regelungen der KVRL und der $\$ \mathbb{S} 105 \mathrm{ff}$. GWB erfasst wird.

Das Telekommunikationswegerecht ist im Unionsrecht vorstrukturiert. Nationale Umsetzungsgesetze müssen sich an den Vorgaben des Art. 43 Abs. 1 EKEK orientieren. Hieraus ergeben sich insbesondere die Grenzen für die rechtliche Ausgestaltung. Konzessionsrechte sollen lediglich in Gebieten mit Netzausbaudefizit eingeführt werden. In den übrigen Gebieten soll das bisherige Regelungsregime weiter Anwendung finden. Es müssten mithin zwei separate Wegerechtsvergaberegime für die Errichtung öffentlicher Telekommunikationsnetze etabliert werden. Dies wäre jedoch nicht mit Art. 43 Abs. 1 UAbs. 2 EKEK zu vereinbaren.

Im Übrigen müsste sich das Verfahren an den Charakteristika des Art. 43 Abs. 1 UAbs. 1 EKEK orientieren. Besondere Relevanz haben die Kriterien der Effizienz und Einfachheit, deren Bedeutung der Unionsge- 
setzgeber in Erwägungsgrund 104 des EKEK besonders betont. Durch die Etablierung eines Infrastrukturmonopols wären aber auch die rechtlichen Rahmenbedingungen aus anderen Regelungszusammenhängen zu beachten, die einen umfassenden Wettbewerbsschutz gewährleisten. Hierbei handelt es sich insbesondere um das Verfassungs-, Wettbewerbs-, Vergabeund EU-Beihilfenrecht. Aus diesen Regelungsmaterien lässt sich herleiten, dass das Verfahren wegen Art. 87f Abs. 2 S. 2 GG zwingend von einer bundesunmittelbaren Behörde durchzuführen wäre. Im Übrigen wären in dem Verfahren insbesondere das Diskriminierungsverbot, das Gebot eines fairen Verfahrens, das Transparenzgebot und die maximale Laufzeit von Konzessionsverträgen zu beachten. Das Verfahren müsste öffentlich durchgeführt werden und es würde in der Folge zu Verzögerungen kommen. Dies stünde in einem eklatanten Spannungsverhältnis zu den Verfahrensparametern der Effizienz und Einfachheit, die in Art. 43 Abs. 1 UAbs. 1 lit. a EKEK normiert sind. Diese dürfen zwar nicht im Sinne absoluter Grenzen, sondern lediglich als relative Optimierungsgebote verstanden werden. Die Einführung eines Vergabeverfahrens, welches im diametralen Gegensatz zu diesen Parametern steht, dürfte sich jedoch nicht mehr im Umsetzungsspielraum bewegen, der vom Unionsrechtsgeber vorgesehen wurde.

Problematisch ist zudem der Umgang mit Bestandsinfrastruktur. Im Energierecht, das dem Konzessionsmodell auf Basis der Vergabe exklusiver Wegerechte als Vorbild dient, existiert aus technischen Gründen nur eine einzige, nicht duplizierbare leitungsgebundene Infrastruktur. Dies ist im Telekommunikationssektor anders, in dem unterschiedliche Übertragungstechnologien existieren. Ein Infrastrukturmonopol könnte nur funktionieren, wenn im Konzessionsgebiet keine Alternativen zur konzessionierten Infrastruktur existierten. Ein Ausbauverbot im Konzessionsgebiet würde dieses Problem nicht lösen. Die vorgeschlagenen Handlungsoptionen einer Eigentumsübertragungspflicht oder Zwangsverpachtung ließen sich jedoch vor dem Hintergrund des grundrechtlichen Eigentumsschutzes nicht rechtfertigen. Im Ergebnis lässt sich das Konzessionsmodell auf Basis der Vergabe exklusiver Wegerechte deswegen rechtlich nicht umsetzen. Es handelt sich um keine geeignete Handlungsoption zur Beschleunigung des Ausbaus von Glasfaserinfrastrukturen zu erschwinglichen Preisen. 


\section{Konzessionsmodell zum Aufbau und Betrieb staatlicher Telekommunikationsinfrastrukturen}

In verschiedenen Mitgliedstaaten der Europäischen Union werden Bauund Dienstleistungskonzessionen genutzt, um staatliche Breitbandinfrastrukturen in Gebieten mit Netzausbaudefizit aufzubauen und durch die Privatwirtschaft betreiben zu lassen. ${ }^{471}$ Diese sollen nachfolgend vorgestellt werden, um mögliche Alternativen für das Konzessionsmodell auf Basis der Vergabe exklusiver Wegerechte aufzuzeigen. Auch in Deutschland werden bereits Konzessionsmodelle genutzt, die nicht an die Vergabe exklusiver Wegerechte anknüpfen, um den Breitbandausbau in bestimm-

471 Komm., Beschl. v. 31.7.2019, SA.53135 (2019/N), Greece - Ultrafast Broadband Infrastructure Scheme, Rn. 20; Komm., Beschl. v. 7.11.2016, SA.37183 (2015/ NN), France - Plan France très haut débit, Rn. 18; Komm., Beschl. v. 30.6.2016, SA.41647 (2016/N), Italy - Strategia Banda Ultralarga, Rn. 25 ff.; Komm., Beschl. v. 26.5.2016, SA.40720 (2016/N), United Kingdom - National Broadband Scheme for the UK for 2016-2020, Rn. 40; Komm., Beschl. v. 12.12.2013, SA.37558 (2013/N), Romania - Ro-NET project, Rn. 24 ff.; Komm., Beschl. v. 4.12.2013, SA.33656 (2012/NN), Ireland - Next Generation (backhaul) Network (NGN) alongside a gas pipeline in Galway and Mayo, Rn. 18 f.; Komm., Beschl. v. 18.12.2012, SA.34199 (2012/N), Italy - Digital Plan - Super-fast broadband, Rn. 19 f.; Komm., Beschl. v. 30.11.2012, SA. 33641 (2011/N), Greece - Metropolitan Area Networks (MAN)/Fibre to the home (FTTH) Greece, Rn. 19 ff.; Komm., Beschl. v. 19.10.2011, SA.31316 (N 330/2010), France - Programme national „Très Haut Débit“-Volet B, Rn. 13; Komm., Beschl. v. 13.12.2010, SA. N 497/2010, United Kingdom - SHEFA-2 Interconnect, Rn. 19b; Komm., Beschl. v. 11.8.2010, SA. N 407/2009, Spain - Optical fibre Catalonia (Xarxa Oberta), Rn. 32 f.; Komm., Beschl. v. 30.4.2010, SA. N 646/2009, Italy - National broadband plan for rural areas in Italy, Rn. 19; Komm., Beschl. v. 30.9.2009, SA N $331 / 2008$, France - Compensation de charges pour une Délégation de Service Public (DSP) pour l'établissement et l'exploitation d'un réseau de communications électroniques à très haut débit dans le Département des Hauts-de-Seine, Rn. 31 und 56; Komm., Beschl. v. 11.3.2008, SA. N 412/2007, Italy - Aid to reduce the digital divide in Piedmont, Rn. 14 und 18; Komm., Beschl. v. 10.7.2007, SA. N 890/2006, France - Aide du Sicoval pour un réseau de très haut débit, Rn. 22; Komm., Beschl. v. 8.3.2006, Ireland - Regional Broadband Programme: Metropolitan Area Networks ("MANs"), phases II and III, Rn.23; Komm., Beschl. v. 6.4.2005, SA. N 583/2004, España - Banda ancha en zonas rurales y aisladas, Rn. 16; Komm., Beschl. v. 3.5.2005, SA. N 382/2004, France Mise en place d'une infrastructure haut débit sur le territoire de la region Limousin (DORSAL), Rn. 5 und $18 \mathrm{ff}$.; Komm., Beschl. v. 16.11.2004, SA. N 381/2004, France - Projet de réseau de télécommunication haut débit des Pyrénées-Atlantiques, Rn. 16 und 32. 
ten Regionen zu fördern. Es handelt sich um eine klassische Komponente der Förderprogramme in Form des sogenannten „Betreibermodells“. ${ }^{472}$

I. Beispiele für die Ausgestaltung des Konzessionsmodells zum Aufbau und Betrieb staatlicher Infrastrukturen

Besonders plakativ lässt sich die Funktionsweise des Modells am griechischen „Ultrafast Broadband Infrastructure Scheme“473 (dazu 1.) und der italienischen „Strategia Banda Ultralarga“"474 (dazu 2.) darstellen.

1. Das griechische „Ultrafast Broadband Infrastructure Scheme“

Mithilfe des „Ultrafast Broadband Infrastructure Scheme“ soll in Griechenland der Ausbau gigabitfähiger Infrastrukturen in weißen Flecken beschleunigt werden. ${ }^{475}$ Die weißen Flecken werden in Gebiete der Kategorie A, in denen mit einem eigenwirtschaftlichen Gigabitausbau zu rechnen ist und Gebiete der Kategorie B, in denen kein eigenwirtschaftlicher Ausbau gigabitfähiger Infrastruktur zu erwarten ist, unterteilt. ${ }^{476}$ In den Gebieten der Kategorie A werden zu Beginn des Programmes Bandbreiten von $100 \mathrm{Mbit} / \mathrm{s}$ gewährleistet, die bis zum Ende des Programmes auf $1 \mathrm{Gbit} / \mathrm{s}$ beschleunigt werden müssen. ${ }^{477}$ Die Konzession kann maximal für einen Zeitraum von 26 Jahren vergeben werden, wobei mindestens

472 Komm., Beschl. v. 22.7.2015, SA.41416 (2015/N), Deutschland - NGA-Förderregelung Baden-Württemberg, Rn. 25; Komm., Beschl. v. 21.4.2015, SA.39518 (2014/N), Deutschland - NGA-Cluster Nordhessen, Rn. 20 ff.; Komm., Beschl. v. 30.8.2012, SA.34809 (2012/N), Deutschland - NGA-Breitbandnetz Markt Reisbach, Rn. 14.

473 Komm., Beschl. v. 31.7.2019, SA.53135 (2019/N) - Greece - Ultrafast Broadband Infrastructure Scheme.

474 Komm., Beschl. v. 30.6.2016, SA.41647 (2016/N) - Italy - Strategia Banda Ultralarga.

475 Komm., Beschl. v. 31.7.2019, SA.53135 (2019/N) - Greece - Ultrafast Broadband Infrastructure Scheme, Rn. 13.

476 Die griechische Regierung geht davon aus, dass etwa $63 \%$ der Zielgebiete in die Kategorie A und $37 \%$ der Zielgebiete in die Kategorie B fallen; Komm., Beschl. v. 31.7.2019, SA.53135 (2019/N), Greece - Ultrafast Broadband Infrastructure Scheme, Rn. 14.

477 Komm., Beschl. v. 31.7.2019, SA.53135 (2019/N), Greece - Ultrafast Broadband Infrastructure Scheme, Rn. 14. 
drei Jahre auf die Bauzeit entfallen und die restliche Vertragslaufzeit für den Betrieb genutzt wird. ${ }^{478}$ Die errichtete passive Infrastruktur bleibt nach dem Ablauf des Konzessionsvertrages in staatlichem Eigentum. ${ }^{479}$ Der Konzessionsnehmer, der insbesondere die Infrastruktur auf Kosten des Staates errichtet und betreibt, wird in einem komplexen Verfahren ausgewählt, in das die Öffentlichkeit umfassend eingebunden wird. ${ }^{480}$ Gegen die Zahlung eines entsprechenden Entgeltes erhalten Telekommunikationsunternehmen Zugang zu der Infrastruktur im Konzessionsgebiet. ${ }^{481}$

\section{Die italienische „Strategia Banda Ultralarga“}

Mithilfe der „Strategia Banda Ultralarga“ soll in Italien eine hochleistungsfähige digitale Infrastruktur in „weißen Flecken“ geschaffen werden. ${ }^{482}$ Der Fokus des Programmes liegt jedoch auf der Herstellung von Breitbandanschlüssen und nicht zwingend auf dem Erreichen von Übertragungsgeschwindigkeiten im Gigabitbereich. Der Auf- und Ausbau der Infrastruktur wird vollständig mithilfe staatlicher Mittel finanziert. ${ }^{483}$ Die Infrastruktur bleibt nach Ablauf der Konzessionsverträge im Eigentum des Staates. ${ }^{484}$ Bei den Konzessionsverträgen wird zwischen einer Baukonzession, also der Errichtung der passiven Infrastruktur, und einer Dienstleistungskonzession, also dem Betrieb, der Wartung und dem Vertrieb der Infrastruktur, unterschieden. ${ }^{485}$ Es finden zwei separate Ausschreibungsverfahren statt. Es können auch mit einem einzigen Unternehmen beide Kon-

478 Komm., Beschl. v. 31.7.2019, SA.53135 (2019/N), Greece - Ultrafast Broadband Infrastructure Scheme, Rn. 19.

479 Komm., Beschl. v. 31.7.2019, SA.53135 (2019/N), Greece - Ultrafast Broadband Infrastructure Scheme, Rn. 20.

480 Komm., Beschl. v. 31.7.2019, SA.53135 (2019/N), Greece - Ultrafast Broadband Infrastructure Scheme, Rn. $27 \mathrm{ff}$.

481 Komm., Beschl. v. 31.7.2019, SA.53135 (2019/N), Greece - Ultrafast Broadband Infrastructure Scheme, Rn. $31 \mathrm{ff}$.

482 Komm., Beschl. v. 30.6.2016, SA.41647 (2016/N), Italy - Strategia Banda Ultralarga, Rn. 13.

483 Komm., Beschl. v. 30.6.2016, SA.41647 (2016/N), Italy - Strategia Banda Ultralarga, Rn. 29.

484 Komm., Beschl. v. 30.6.2016, SA.41647 (2016/N), Italy - Strategia Banda Ultralarga, Rn. 26; Wernick/Queder/Strube Martins u.a., Ansätze zur Glasfaser-Erschließung unterversorgter Gebiete, S. 47.

485 Komm., Beschl. v. 30.6.2016, SA.41647 (2016/N), Italy - Strategia Banda Ultralarga, Rn. 25. 
zessionsverträge abgeschlossen werden. ${ }^{486}$ In diesem Fall muss das Unternehmen die Tätigkeiten jedoch in separierten Unternehmenszweigen erbringen. ${ }^{487}$ Sofern beim Auf- und Ausbau des Netzes auf Bestandsinfrastruktur zurückgegriffen wird, wird das Nutzungsrecht ein Bestandteil der staatlichen Infrastruktur. ${ }^{488}$ Der Konzessionsnehmer wird in einem komplexen Auswahlprozess bestimmt, ${ }^{489}$ wobei das wirtschaftlichste Angebot gewinnt. ${ }^{490}$ Telekommunikationsunternehmen erhalten gegen Entgelt $\mathrm{Zu}$ gang zur Infrastruktur, die vom Konzessionsmodell erfasst ist. ${ }^{491}$

\section{Gemeinsamkeiten der Modelle}

Bei den betrachteten Ausgestaltungsbeispielen fallen trotz einiger Unterschiede in Detailfragen auch Gemeinsamkeiten auf. So wird die Infrastruktur jeweils durch den Staat finanziert und bleibt auch in dessen Eigentum. Der Wettbewerb wird mithilfe der Ausschreibung des Bauvorhabens und des Betriebes sichergestellt. Lediglich die Finanzierung übernimmt der Staat, sodass es sich um besondere Ausgestaltungsformen von Förderprogrammen handelt.

486 Komm., Beschl. v. 30.6.2016, SA.41647 (2016/N), Italy - Strategia Banda Ultralarga, Rn. 25; Kühling/Toros, Rechtliche Rahmenbedingungen für die Schaffung von Anreizen für einen flächendeckenden Ausbau von Glasfaserinfrastrukturen, S. 98.

487 Wernick/Queder/Strube Martins u.a., Ansätze zur Glasfaser-Erschließung unterversorgter Gebiete, S. 47.

488 Komm., Beschl. v. 30.6.2016, SA.41647 (2016/N), Italy - Strategia Banda Ultralarga, Rn. 28; Kübling/Toros, Rechtliche Rahmenbedingungen für die Schaffung von Anreizen für einen flächendeckenden Ausbau von Glasfaserinfrastrukturen, S. 98; Wernick/Queder/Strube Martins u.a., Ansätze zur Glasfaser-ErschlieBung unterversorgter Gebiete, S. 47.

489 Komm., Beschl. v. 30.6.2016, SA.41647 (2016/N), Italy - Strategia Banda Ultralarga, Rn. 34.

490 Die Kriterien werden in Komm., Beschl. v. 30.6.2016, SA.41647 (2016/N), Italy - Strategia Banda Ultralarga, Rn. 40 ff. dargestellt.

491 Komm., Beschl. v. 30.6.2016, SA.41647 (2016/N), Italy - Strategia Banda Ultralarga, Rn. $46 \mathrm{f}$. 


\section{Klassifikation als Konzessionsmodell}

Daher stellt sich die Frage, ob die Förderprogramme in Italien und Griechenland als Konzessionsmodelle bezeichnet werden können. Zuweilen werden sie in der Literatur als Lizenzmodelle bezeichnet. ${ }^{492}$ Es wird davon ausgegangen, dass es sich nicht um ein Konzessionsmodell handele, da keine exklusiven Wegerechte vergeben würden. ${ }^{493}$ Die Verwendung des Begriffes „concessions“ in den englischsprachigen Entscheidungen der Kommission zur Konformität mit dem EU-Beihilfenrecht sei missverständlich. ${ }^{494}$ Insbesondere wird gegen die Klassifikation als Konzessionsmodell eingewandt, dass ein Überbau stattfinden könne, der bei der italienischen „Strategia Banda Ultralarga“ auch zu beobachten sei. ${ }^{495}$ Nach dieser Ansicht ist eine Exklusivität, die einen Überbau der aufgebauten Infrastruktur verhindert, essentiell für eine Konzession. Ein solch enges Verständnis überzeugt jedoch vor dem Hintergrund der vielseitigen Verwendung des Konzessionsbegriffes nicht (A. I.-III.). Die Vergabe exklusiver Wegerechte prägt vielmehr lediglich den Konzessionsbegriff des Energierechts und nicht Konzessionen im Allgemeinen.

Im vorliegenden Fall lässt der Staat von privaten Unternehmen eigene Infrastruktur errichten oder betreiben. Der Staat hat an dem Aufbau und dem Betrieb seiner Infrastruktur ein unmittelbares wirtschaftliches Interesse, während das Betriebsrisiko beim Infrastrukturunternehmen liegt. Die Tatbestandsvoraussetzungen des Konzessionsbegriffes des Vergaberechts gemäß Art. 5 Abs. 1 KVRL und $\ 105$ Abs. 1 GWB sind erfüllt, sodass auch diese Methode der Ausbauförderung als Konzessionsmodell bezeichnet werden kann. Aufgrund dieser Ausrichtung soll das Modell im Folgenden als Konzessionsmodell zum Aufbau und Betrieb staatlicher Infrastruktur bezeichnet werden.

492 Wernick/Queder/Strube Martins u.a., Ansätze zur Glasfaser-Erschließung unterversorgter Gebiete, S. 47.

493 Wernick/Queder/Strube Martins u.a., Ansätze zur Glasfaser-Erschließung unterversorgter Gebiete, S. 48.

494 Wernick/Queder/Strube Martins u.a., Ansätze zur Glasfaser-Erschließung unterversorgter Gebiete, S. 48.

495 Wernick/Queder/Strube Martins u.a., Ansätze zur Glasfaser-Erschließung unterversorgter Gebiete, S. 48. 
II. Abgrenzung zum Konzessionsmodell auf Basis der Vergabe exklusiver Wegerechte

Das Konzessionsmodell auf Basis der Vergabe exklusiver Wegerechte und das vorliegende Konzessionsmodell verfolgen unterschiedliche Ansätze. Anders als beim erstgenannten Modell wird mithilfe des vorliegenden Konzessionsmodells lediglich die Förderung paralleler Infrastrukturen und nicht deren Aufbau verhindert. Es entsteht ein Ausschreibungswettbewerb um den geförderten Breitbandausbau (Kapitel 5, C. III.). Der privatwirtschaftliche Infrastrukturausbau bleibt im betreffenden Gebiet mithin möglich, auch wenn ihm fehlende Fördermittel faktisch entgegenstehen und damit innerhalb dieses Modells auch ein Wettbewerb der Infrastrukturanbieter im Konzessionsgebiet verbleibt. Insbesondere kann beim vorliegenden Fördermodell die Bestandsinfrastruktur weiterhin genutzt werden, sofern sie nicht zum Bestandteil des Konzessionsnetzes geworden ist. Das Konzessionsmodell auf Basis der Vergabe exklusiver Wegerechte ist hingegen kein Förderprogramm, sondern flankiert ein solches vielmehr. Den Modellen liegt ein unterschiedliches Konzessionsverständnis zu Grunde und sie verfolgen unterschiedliche ökonomische Ansätze, sodass sie nur eingeschränkt miteinander vergleichbar sind.

\section{Ergebnis}

Das Konzessionsmodell zum Aufbau und Betrieb staatlicher Infrastruktur unterscheidet sich grundlegend vom Konzessionsmodell auf Basis der Vergabe exklusiver Wegerechte. Es handelt sich um eine spezielle Kooperationsform zwischen Staat und Privatwirtschaft. Trotz der exklusiven Vergabe von Fördermitteln bleibt der parallele Infrastrukturausbau ohne Förderung möglich. Der Staat fungiert als Finanzierer und Eigentümer der Infrastruktur. Die wesentlichen gesetzlichen Rahmenbedingungen für den kooperativen Breitbandausbau werden später umfassend untersucht (Kapitel 5). 


\section{E. Konzessionsmodell auf Basis der Vergabe exklusiver Fördermittel}

Das portugiesische „High speed broadband Portugal“-Programm stellt ein Konzessionsmodell dar, um die digitale Kluft im Land zu verringern, und nutzt hierzu eine exklusive Vergabe von Fördermitteln. ${ }^{496}$ Auf Basis eines umfassenden Mappings und einer Abdeckungsanalyse, die durch eine Öffentlichkeitskonsultation validiert wurden, wurden 139 Gemeinden mit einem Netzausbaudefizit identifiziert. ${ }^{497}$ Mithilfe des Programmes sollte in diesen Gebieten eine Versorgungsrate von 50 Prozent mit NGA-Infrastrukturen sichergestellt werden. ${ }^{498}$ Die 139 Gemeinden wurden hierzu in fünf Gebiete unterteilt, in denen der Aufbau und Betrieb der Infrastruktur ausgeschrieben werden. ${ }^{499}$ Der Konzessionsvertrag wurde für eine Dauer von 20 Jahren an dasjenige Unternehmen vergeben, das das wirtschaftlichste Angebot eingereicht hatte. ${ }^{500}$ Dabei ist eine vertikale Integration des Unternehmens möglich, sodass es auf verschiedenen Wertschöpfungsstufen tätig werden kann. ${ }^{501}$ Andere Telekommunikationsunternehmen erhalten einen umfassenden Zugang zu der Infrastruktur..$^{502}$

Das Konzessionsmodell auf Basis der Vergabe exklusiver Fördermittel in Portugal ist zwar mit dem Konzessionsmodell verwandt, in dem staatliche

496 Komm., Beschl. v. 19.1.2011, SA.30317, Portugal - High-speed broadband Portugal, Rn. 11; zu den Erfahrungen mit diesem Modell Wernick/Queder/Strube Martins u.a., Gigabitnetze für Deutschland, S. 114.

497 Komm., Beschl. v. 19.1.2011, SA.30317, Portugal - High-speed broadband Portugal, Rn. $13 \mathrm{ff}$.

498 Komm., Beschl. v. 19.1.2011, SA.30317, Portugal - High-speed broadband Portugal, Rn. 13; Kübling/Toros, Rechtliche Rahmenbedingungen für die Schaffung von Anreizen für einen flächendeckenden Ausbau von Glasfaserinfrastrukturen, S. 99; zu den Einzelheiten Wernick/Queder/Strube Martins u.a., Gigabitnetze für Deutschland, S. 116.

499 Komm., Beschl. v. 19.1.2011, SA.30317, Portugal - High-speed broadband Portugal, Rn. 19.

500 Komm., Beschl. v. 19.1.2011, SA.30317, Portugal - High-speed broadband Portugal, Rn. 20; Kübling/Toros, Rechtliche Rahmenbedingungen für die Schaffung von Anreizen für einen flächendeckenden Ausbau von Glasfaserinfrastrukturen, S. 99.

501 Kühling/Toros, Rechtliche Rahmenbedingungen für die Schaffung von Anreizen für einen flächendeckenden Ausbau von Glasfaserinfrastrukturen, S. 99; Wernick/Queder/Strube Martins u.a., Ansätze zur Glasfaser-Erschließung unterversorgter Gebiete, S. 47.

502 Komm., Beschl. v. 19.1.2011, SA.30317, Portugal - High-speed broadband Portugal, Rn. 28 ff.; Wernick/Queder/Strube Martins u.a., Gigabitnetze für Deutschland, S. 115. 
Infrastrukturen mithilfe von Bau- und Dienstleistungskonzessionen aufgebaut werden. Allerdings steht die errichtete Infrastruktur nicht im Eigentum des Staates. Der Staat stellt die finanziellen Mittel zur Verfügung, die benötigt werden, um die Infrastruktur durch privatwirtschaftliche Unternehmen auszubauen. Das Unternehmen erhält die Investitionssicherheit durch die Stellung als Konzessionsnehmer und die Sicherheit, dass keine weiteren Fördermittel im Konzessionsgebiet vergeben werden. Im Ergebnis handelt es sich auch in dieser Hinsicht um eine öffentlich-private Kooperation. Die gesetzlichen Rahmenbedingungen für eine Ausgestaltung von Kooperationen werden später näher beleuchtet (Kapitel 5).

\section{F. Ergebnis}

Konzessionsrechte sind Grundlage für verschiedene Maßnahmen zur Erhöhung der Investitionssicherheit im Infrastrukturausbau. Dabei wird der Begriff der „Konzession“ in der deutschen Rechtswissenschaft trotz eines einheitlichen Ursprungs und der partiellen Verfügbarkeit von Legaldefinitionen nicht einheitlich verwendet. Vielmehr ist der Begriff in dem jeweiligen thematischen Zusammenhang autonom abzugrenzen und hinsichtlich seiner Wesensmerkmale zu untersuchen. Alle zuvor untersuchten Konzessionsmodelle haben gemeinsam, dass sie die Investitionsanreize mithilfe von Exklusivität erhöhen wollen. Kannte das Telekommunikationsrecht bis vor Kurzem nur die Möglichkeit, exklusive Rechte bei technischen Notwendigkeiten zu gewähren, wurde dieser Grundsatz durch den Privilegierungstatbestand in Art. 76 in Verbindung mit Art. 79 EKEK aufgeweicht. Die Verbesserung von Investitionsbedingungen kann ebenfalls, wenn auch nur unter hohen Anforderungen, eine exklusive Rechtevergabe legitimieren. Sobald eine solche stattfindet, haben aber alle Exklusivrechte gemein, dass die negativen Auswirkungen auf den Wettbewerb durch umfassende Maßnahmen ausgeglichen werden.

Das Konzessionsmodell auf Basis der Vergabe exklusiver Wegerechte ist lediglich ein Konzept, das derzeit in keinem Mitgliedstaat der Europäischen Union angewandt wird. Es ließe sich aus verschiedenen Gründen nicht rechtskonform umsetzen. Daher ist davon auszugehen, dass es in $\mathrm{Zu}-$ kunft keine weitergehende Bedeutung erlangen wird. Das Konzessionsmodell zum Aufbau und Betrieb staatlicher Telekommunikationsinfrastrukturen wird im unionalen und nationalen Recht hingegen bereits verschiedentlich umgesetzt und fügt sich in den Rechtsrahmen ein. Es handelt sich, je nach Ausgestaltung, um eine Bau- und Dienstleistungskonzessio- 
nen. Im Ergebnis fungiert der Staat als Kooperationspartner im Infrastrukturausbau. Durch die Übernahme und Aufteilung von Investitionsrisiken soll die Amortisation der Investitionen erleichtert werden. In Deutschland wird es als „Betreibermodell“ in Förderprogramme integriert.

Das Konzessionsmodell auf Basis der Vergabe exklusiver Fördermittel ist mit dem Konzessionsmodell zum Aufbau und Betrieb staatlicher Telekommunikationsinfrastrukturen verwandt. Die exklusive Fördermittelvergabe wird in den deutschen Förderprogrammen grundsätzlich umgesetzt. Hierdurch entsteht ein Ausschreibungswettbewerb im geförderten Breitbandausbau (Kapitel 5, C. III.).

Der Begriff der „Konzessionsmodelle“ ist somit ein Sammelbegriff für unterschiedliche Modelle, in denen der Staat mithilfe der Vergabe exklusiver Rechte die Investitionsbedingungen zu verbessern sucht. Solange dies innerhalb des rechtlichen Rahmens erfolgt, handelt es sich um einen praktikablen Ansatz zur Beschleunigung des Glasfaserausbaus in den betreffenden Gebieten. Der Ausbau sollte jedoch stets im schonenden Ausgleich mit dem Wettbewerb gefördert werden, um die negativen Folgen der staatlichen Marktintervention so gering wie möglich zu halten. 


\section{Kapitel 4: Beschleunigung des Infrastrukturausbaus durch die Entflechtung und regulatorische Privilegierung von Wholesale-Only-Anbietern}

Der Großteil der Unternehmen auf dem deutschen Telekommunikationsmarkt ist sowohl auf dem Vorleistungs- als auch auf dem Endkundenmarkt tätig. Diese vertikale Integration bringt in der kapitalintensiven Telekommunikationsbranche entscheidende Verbundvorteile mit sich. Ökonomische Modelle kommen zu dem Ergebnis, dass auch die Tätigkeit als Wholesale-Only-Anbieter, bei der die Unternehmen auf diese Verbundvorteile verzichten, betriebswirtschaftlich sinnvoll sein und zur Beschleunigung des Glasfaserausbaus in ländlichen Bereichen beitragen kann (dazu A.). Trotzdem sind derzeit auf dem Telekommunikationsmarkt nur wenige Wholesale-Only-Anbieter präsent. Es stellt sich die Frage, inwieweit dieser Befund auf den Rechtsrahmen zurückzuführen ist und welcher Optimierungsbedarf dort besteht.

Es sind verschiedene Szenarien denkbar, in deren Folge eine Zunahme sogenannter "Wholesale-Only-Anbieter" festzustellen wäre. ${ }^{503}$ Zum einen könnten vertikal integrierte Unternehmen entweder durch staatlichen Zwang (dazu B.) oder auf Basis einer freiwilligen Entscheidung entflochten werden (dazu C.). Alternativ könnten Telekommunikationsunternehmen direkt mit einem auf den Vorleistungsmarkt beschränkten Tätigkeitsspektrum gegründet werden (dazu D.). Bislang sind nur rudimentäre juristische Analysen über die Realisierbarkeit solcher Modelle in die Diskussion eingeflossen, sodass nachfolgend insbesondere die rechtlichen Rahmenbedingungen für die einzelnen Szenarien herausgearbeitet werden sollen. Im Fokus der Analyse soll eine Untersuchung der Rechtsvorgaben zur Entflechtung liegen. Dabei soll der Rechtsrahmen de lege lata skizziert und untersucht werden, ob sich die rudimentäre Präsenz von Wholesale-OnlyAnbietern auf dem deutschen Telekommunikationsmarkt auf ihn zurückführen lässt. Es soll insbesondere dargelegt werden, ob de lege ferenda ein Optimierungsbedarf besteht, um die Entflechtung als Instrument im Infrastrukturausbau zu nutzen. Im Anschluss daran soll untersucht werden, ob

503 Wernick/Queder/Strube Martins u.a., Ansätze zur Glasfaser-Erschließung unterversorgter Gebiete, S. 32. 
außerhalb der regulatorischen Entflechtungsbestimmungen Optimierungsmöglichkeiten für eine stärkere Durchmischung des Telekommunikationsmarktes mit vertikal integrierten Telekommunikationsunternehmen und Wholesale-Only-Anbietern bestehen (dazu E.).

\section{A. Ökonomische Grundlagen der vertikalen Integration}

Die juristische Bewertung der Verhältnismäßigkeit eingriffsintensiver Entflechtungsmaßnahmen verlangt ein Verständnis der Vor- und Nachteile einer vertikalen Integration, was eine Auseinandersetzung mit ihren ökonomischen Grundlagen erfordert. Komplexe Produktionsprozesse, wie beispielsweise die Erbringung von Endkundendiensten im Telekommunikationssektor, setzen mannigfaltige Einzelaktivitäten auf unterschiedlichen Wertschöpfungsstufen voraus. Eine vertikale Integration bringt in diesem Zusammenhang verschiedene Vorteile für das einzelne Unternehmen mit sich, die jedoch zugleich zu Risiken für den Wettbewerb führen. Allerdings kann auch eine Beschränkung des Tätigkeitsspektrums auf lediglich eine Wertschöpfungsstufe betriebswirtschaftlich sinnvoll sein (dazu I.). Vertikal integrierte Telekommunikationsunternehmen können auf verschiedene Weisen und in unterschiedlichen Intensitäten separiert werden. Martin Cave hat ein Stufenmodell entwickelt, um die Maßnahmen und ihre Auswirkungen miteinander in Verhältnis zu setzen und zu kategorisieren (dazu II.). Die Ergebnisse der ökonomischen Forschung sollen im Nachgang für die weitergehende juristische Analyse in diesem Kapitel fruchtbar gemacht werden (dazu III.).

I. Wertschöpfungsketten im Telekommunikationssektor

Auf Basis einer Analyse der Begrifflichkeiten und Zusammenhänge von Wertschöpfungsketten im Telekommunikationssektor (dazu 1.) sollen die Rahmenbedingungen untersucht werden, die eine vertikale Integration sinnvoll erscheinen lassen (dazu 2.). Zugleich soll auf ökonomische Modelle eingegangen werden, welche die Auswirkungen einer stärkeren Durchmischung des Marktes mit Wholesale-Only-Anbietern auf die Geschwindigkeit des Glasfaserausbaus zum Gegenstand haben (dazu 3.). 
1. Begriffsanalyse

Das Konzept der Wertschöpfungskette ist ein allgemeiner mikroökonomischer Ansatz. ${ }^{504}$ Danach lässt sich ein Produktionsprozess in verschiedene, aufeinander folgende Aktivitäten unterteilen. Im Vorteil sind solche Unternehmen, die einen Produktionsprozess zu geringeren Kosten und in einer höheren Qualität als die Konkurrenz durchführen können. ${ }^{505} \mathrm{Je}$ komplexer das Produkt ist, desto vielschichtiger sind die Wertschöpfungsstufen.

Der Wertschöpfungsprozess der Telekommunikationswirtschaft lässt sich in vier Stufen unterteilen, die wiederum untergliedert werden können. Hierbei handelt es sich um Tätigkeiten auf der Infrastruktur-, 506 Dienste- und Vertriebsebene. Eine weitere Wertschöpfungsstufe bildet das Management von Kunden. ${ }^{507}$

Eine allgemeinere Untergliederung, die für die nachfolgende Untersuchung maßgeblich sein soll, ist die Differenzierung zwischen Tätigkeiten auf der Vorleistungs- und auf der Diensteebene. Die Vorleistungsebene erfasst alle Tätigkeiten, die zur Erbringung von Endkundendiensten erforderlich sind. Hierbei handelt es sich insbesondere um den Aufbau, den Betrieb und die Instandhaltung von Infrastruktur. Wholesale-Only-Anbieter sind ausschließlich auf dieser Wertschöpfungsstufe tätig. ${ }^{508}$ Der Begriff leitet sich aus dem englischen Ausdruck für „Großhandel“ ab und illustriert in seiner Zusammensetzung, dass anderen Telekommunikationsunternehmen ausschließlich Vorleistungsprodukte entgeltlich zur Verfügung gestellt werden. Die Endkundenebene hingegen erfasst die Erbringung von Endkundendiensten und das hierzu gehörige Kundenmanagement. ${ }^{509}$ Un-

504 Weiterführend hierzu Porter, Wettbewerbsvorteile, S. 65 ff.

505 Harnisch, Funktionale Separierung in der Telekommunikationsindustrie, S. 13; Tenbrock, Der Glasfaserausbau in Deutschland, S. 11.

506 Mit Vorschlägen für eine weitere Subdifferenzierung im Tätigkeitsbereich Infrastruktur Tenbrock, Der Glasfaserausbau in Deutschland, S. 13; Kübling, Sektorspezifische Regulierung in den Netzwirtschaften, S. 69.

507 In der ökonomischen Literatur existieren umfassende Abhandlungen zur Abgrenzung der verschiedenen Wertschöpfungsstufen, siehe hierzu im Überblick Harnisch, Funktionale Separierung in der Telekommunikationsindustrie, S. 14; Tenbrock, Der Glasfaserausbau in Deutschland, S. 13.

508 Wernick/Queder/Strube Martins u.a., Ansätze zur Glasfaser-Erschließung unterversorgter Gebiete, S. 32.

509 Mit schematischem Überblick Schreiber, in: Geppert/Schütz (Hrsg.), Beck'scher TKG-Kommentar, $\mathbb{\$} 40$, Rn. 20; detaillierter Harnisch, Funktionale Separierung als strategisches Regulierungsinstrument auf dem europäischen Telekommuni- 
ternehmen, die ihre Tätigkeit auf diese Wertschöpfungsstufe begrenzen, werden auch als Wholebuy-Anbieter bezeichnet. Der Begriff hat seinen Ursprung ebenfalls im dargestellten englischen Vokabular und illustriert, dass die betreffenden Unternehmen alle notwendigen Produkte im Vorleistungsbereich auf Großhandelsebene einkaufen. Sobald ein Unternehmen Tätigkeiten auf beiden zentralen Wertschöpfungsstufen erbringt, wird es als vertikal integriert bezeichnet. ${ }^{510}$

\section{Vertikale Integration im Telekommunikationssektor}

Auch wenn die vertikale Integration durch die Rahmenbedingungen für eine Telekommunikationstätigkeit begünstigt (dazu a.), entstehen durch sie wettbewerbliche Risiken, denen mit regulatorischen Maßnahmen begegnet werden muss (dazu b.).

a) Betriebswirtschaftliche Vorteile einer vertikalen Integration

Die Rahmenbedingungen einer Tätigkeit im Telekommunikationssektor erhöhen aus betriebswirtschaftlicher Sicht die Attraktivität einer vertikalen Integration. ${ }^{511}$ Die ökonomische Literatur geht davon aus, dass eine vertikale Integration vor allen Dingen in Branchen mit hohem Innovationsdruck vorteilhaft sein kann, um die betriebswirtschaftlichen Risiken der Investitionen zu minimieren. ${ }^{512}$ Diese resultieren insbesondere aus den ohnehin schon hohen Kosten für den Infrastrukturausbau, zu denen etwa die Materialkosten für die leitungsgebundene Infrastruktur gehören. Diese

kationsmarkt, S. 6, der dort in Abbildung 2 die Wertschöpfungsstufen und vertikale Desintegrationsmöglichkeiten darstellt.

510 Harnisch, Funktionale Separierung in der Telekommunikationsindustrie, S. 16 f.; Neumann/Koch, Telekommunikationsrecht, S. 38.

511 Allgemein zu Branchenspezifika, die eine vertikale Integration sinnvoll erscheinen lassen, Picot/Franck, in: Hauschildt/Grün (Hrsg.), Festschrift für Eberhard Witte, S. 181, 182 ff.; Haucap/Heimeshoff/Uhde, in: Gesellschaft für öffentliche Wirtschaft (Hrsg.), Auswirkungen der Globalisierung auf die öffentlichen Banken, S. $27 \mathrm{ff}$. Konkret in Bezug auf den Telekommunikationssektor Crandall/ Eisenach/Litan, Federal Communications Law Journal 2010, S. 493, $502 \mathrm{ff}$.

512 Crandall/Eisenach/Litan, Federal Communications Law Journal 2010, S. 493, 496; zu den Folgen für den Markteintritt Neumann/Koch, Telekommunikationsrecht, S. 38. 
werden durch die sogenannten „sunk costs“ weiter angehoben. ${ }^{513}$ Hierunter sind etwa Planungs- und Tiefbaukosten zu verstehen, die im Rahmen des Infrastrukturausbaus anfallen und auch bei einem Ausstieg aus dem Markt nicht wieder liquidiert werden können. Dies erhöht das Risikoprofil von Investitionen nachhaltig und zwingt zu langfristigen Planungen.

Telekommunikationsinfrastruktur ist jedoch erforderlich, um Endkundendienste anbieten zu können. Hinter der vertikalen Integration steht aus betriebswirtschaftlicher Sicht vereinfacht folgendes Kalkül: Wird die Infrastruktur im eigenen Unternehmen vorgehalten, können die Vorleistungsprodukte regelmäßig kostengünstig genutzt und damit der Wertschöpfungsprozess preiswert abgebildet werden. In realen Ausbauszenarien besteht natürlich die Möglichkeit, dass Dritte effizienter wirtschaften und aus diesem Grund auch die Infrastruktur preiswerter zur Verfügung stellen können. Werden möglichst viele Einzelprozesse, die für das Endprodukt erforderlich sind, im selben Unternehmen erbracht, entstehen hierdurch Verbundvorteile, die in der Ökonomie als „economies of scope“ bezeichnet werden. ${ }^{514}$ Alternativ können Vorleistungsprodukte anderer Unternehmen genutzt werden.

Der Zugang zu den Infrastrukturen anderer Unternehmen wird entweder im Markt frei vereinbart oder über die Zugangsregulierung gewährleistet. Die Entgeltregulierung soll prohibitive Zugangsentgelte vermeiden. Sie schafft Anreize für den Aufbau einer eigenen Infrastruktur durch Unternehmen. Je höher der Anteil eigener Infrastruktur ist, auf die zum Erreichen des Endkunden zurückgegriffen werden kann, desto geringer fallen die zu zahlenden Entgelte aus. Die hieraus resultierenden Anreize zur Schaffung einer eigenen Infrastruktur werden als „ladder of investment“ bezeichnet. ${ }^{515}$ Dieser Ansatz führt die Grundmaxime des Gesetzgebers konsequent weiter, wonach der Infrastrukturwettbewerb die Basis für einen funktionsfähigen Dienstewettbewerb darstellt (Kapitel 3, C. III. 3.). In der Theorie folgt aus der "ladder of investment" das Bestreben der Diensteanbieter, möglichst umfassend auf eigene Infrastruktur zurückzugreifen, um die Zugangsentgelte zu reduzieren. Hierdurch soll der Infra-

513 Kühling/Schall/Biendl, Telekommunikationsrecht, Rn. 91.

514 Knieps, Netzökonomie, S. 5; Kühling/Schall/Biendl, Telekommunikationsrecht, Rn. 86.

515 Grundlegend hierzu Cave, Telecommunications Policy 30 (2006), S. 223 ff.; Kühling/Schall/Biendl, Telekommunikationsrecht, Rn. 311. 
strukturwettbewerb gefördert werden. ${ }^{516}$ In der Ausbaurealität spiegelt sich dies jedoch nur eingeschränkt wider..$^{517}$

Im Ergebnis ist also eine vertikale Integration im Telekommunikationssektor für Unternehmen insbesondere wegen der Verbundvorteile betriebswirtschaftlich sinnvoll.

b) Gefahren für den Wettbewerb und regulatorische Handlungsoptionen

Die vertikale Integration birgt jedoch - in Gestalt eines erhöhten Diskriminierungspotentials - Risiken für den Wettbewerb im Telekommunikationssektor. ${ }^{518}$ Auf der Diensteebene besteht Wettbewerb zwischen verschiedenen Anbietern. Auf der Infrastrukturebene ist dies jedoch insbesondere im Bereich der Festnetzversorgung von Endkunden anders. ${ }^{519} \mathrm{Um}$ dem Endkunden Dienste an einem festen Standort anbieten zu können, ist ein Zugang zu seiner Teilnehmeranschlussleitung erforderlich. Diese Zugänge stehen nur in einem begrenzten Umfang zur Verfügung, da nicht jeder Diensteanbieter ökonomisch sinnvoll parallele Infrastrukturen verlegen kann, um den einzelnen Endkunden zu erreichen. ${ }^{520}$ Es handelt sich um ein „natürliches Monopol“, das Gegenstand der Zugangsregulierung ist. ${ }^{521}$ Aufgrund der vertikalen Integration sind die betreffenden Telekommunikationsunternehmen zugleich auf den zuvor beschriebenen wettbewerblich und monopolistisch geprägten Wertschöpfungsstufen tätig. ${ }^{522}$ Hieraus erwächst ein vielschichtiges Missbrauchsrisiko, das sich in der Gefahr der Privilegierung des eigenen und der Diskriminierung anderer Unternehmen manifestiert.

Das Telekommunikationsrecht bietet verschiedene Möglichkeiten, um diesen Gefahren zu begegnen. Zuvorderst dienen hierzu seine ausdifferen-

516 Statt vieler Cave, Telecommunications Policy 30 (2006), S. 223, 236.

517 So bereits Kirchner, MMR 2013, S. 22, 24; differenzierend Bacache/Bourreau/ Gaudin, Review of Industrial Organization 44 (2014), S. 179, 186 ff.; kritisch Bourreau/Dogan/Manant, Telecommunications Policy 34 (2010), S. 683 ff.

$518 \mathrm{Zu}$ den Gründen für eine vertikale Desintegration im Überblick statt vieler Webb, Breaking Up is Hard to Do, S. $4 \mathrm{f}$.

519 Neumann/Koch, Telekommunikationsrecht, S. 39 f.

520 Insoweit wird in der ökonomischen Theorie auch von einer Subadditivität der Kostenfunktion gesprochen, Müller/Growitsch/Wissner, Regulierung und Investitionsanreize in der ökonomischen Theorie, S. 1.

521 Kübling, Sektorspezifische Regulierung in den Netzwirtschaften, S. 37.

522 Cave/Doyle, Network separation and investment incentives in telecommunications, S. 3, sowie S. 7 zu den verschiedenen Separierungsmöglichkeiten. 
zierten Regulierungsverpflichtungen. ${ }^{523}$ So dürfen etwa für die Gewährung des Zuganges zur Teilnehmeranschlussleitung Entgelte verlangt werden. Mithilfe der Entgeltregulierung wird verhindert, dass diese prohibitiv wirken. ${ }^{524}$ Die Quersubventionierung wettbewerblich geprägter durch monopolistisch geprägte Bereiche wird insbesondere durch Transparenzpflichten verhindert. ${ }^{525}$ Als ultima ratio steht die Verpflichtung zur funktionellen Entflechtung zur Verfügung, auf die noch intensiver eingegangen werden soll (B. I.). Die Vorgabe zur Entflechtung durch eine entsprechende regulatorische Verpflichtung ist in ihrer jetzigen Zweckrichtung ausschließlich auf den Wettbewerbsschutz ausgerichtet und soll hierzu die negativen Folgen einer vertikalen Integration eindämmen (hierzu vertieft B. I. 1.). Gleiches gilt für die Möglichkeit der freiwilligen Entflechtung (C. III.) und die regulatorische Privilegierung von Wholesale-Only-Anbietern (D. II.), die in einem einheitlichen systematischen Zusammenhang geregelt werden.

3. Wholesale-Only als betriebswirtschaftlich sinnvolles Geschäftsmodell und Beschleunigungsansatz für den Infrastrukturausbau?

Verschiedene Studien über die Anreize zur Beschleunigung des Glasfaserausbaus in der Fläche haben ökonomische Modelluntersuchungen durchgeführt, um nachzuweisen, dass auch eine Tätigkeit als Wholesale-OnlyAnbieter betriebswirtschaftlich sinnvoll sein könne und zumindest mittelbar zu einer Beschleunigung des Glasfaserausbaus beitrage. ${ }^{526}$ Während Ersteres schon allein durch die Präsenz verschiedener Wholesale-Only-Anbieter auf dem Markt deutlich wird (D. I.), ist Letzteres Gegenstand von Modelluntersuchungen.

In einer spezifisch auf Deutschland ausgerichteten Studie wurden die Haushalte in 20 Kostenklassen zu je zwei Millionen Haushalten eingeord-

523 Kühling, Sektorspezifische Regulierung in den Netzwirtschaften, S. $202 \mathrm{ff}$.

524 Kübling, Sektorspezifische Regulierung in den Netzwirtschaften, S. 83 ff.

525 Kühling, Sektorspezifische Regulierung in den Netzwirtschaften, S. 86.

526 Felten/Langer, Structurally indepent broadband infrastructure can solve perceived FTTH coverage issues, S. $10 \mathrm{ff} . ;$ Sörries, N\&R 2020, S. $161 \mathrm{ff} . ;$ Wernick/ Queder/Strube Martins u.a., Ansätze zur Glasfaser-Erschließung unterversorgter Gebiete, S. 36 ff.; Wernick/Queder/Strube Martins u.a., Gigabitnetze für Deutschland, S. $104 \mathrm{ff}$. 
net. ${ }^{527}$ Die verschiedenen Kostenklassen wurden anhand der Besiedelungsdichte voneinander abgegrenzt. Hierbei wurde die Annahme zugrunde gelegt, dass die Erschließungskosten dynamisch bei sinkender Besiedelungsdichte ansteigen würden. ${ }^{528}$ In der Folge wurden die durchschnittlichen Erschließungskosten für die jeweiligen Gebiete ermittelt und für die Berechnung der Amortisationszeiträume für Infrastrukturausbauvorhaben in den Gebieten zugrunde gelegt. ${ }^{529}$ Es handelt sich um eine sogenannte „Greenfield-Untersuchung“, die komplexe Marktzusammenhänge stark vereinfacht und unter laborähnlichen Bedingungen darstellt, indem sie für ein bestimmtes Gebiet von idealisierten Ausbaubedingungen ohne Vorsteuerung durch die tatsächlichen Gegebenheiten ausgeht. Der Name der Untersuchungsmethode leitet sich davon ab, dass der zu beobachtende Ausbau im Modell auf einer „grünen Wiese“ beginnt. In dieser stark vereinfachten Ausgangssituation seien verschiedene positive Effekte zu beobachten, die dazu beitrügen, dass für Wholesale-Only-Anbieter mit kürzeren Zeiträumen für die Amortisation von Investitionen in Infrastrukturausbauvorhaben in allen im Modell beobachteten Kostenklassen zu rechnen sei. ${ }^{530}$ Investitionen in ländlichen Regionen seien in der Folge schneller profitabel als für vertikal integrierte Unternehmen. ${ }^{531}$ Zudem sei ein geringerer Förderbedarf zu erwarten. ${ }^{532}$ Dies könne im Ergebnis zu einer Beschleunigung des Glasfaserausbaus in der Fläche beitragen. ${ }^{533}$

527 Mit einer vergleichbaren Modellrechnung, Sörries, N\&R 2020, S. 161, 165, Tabelle 1.

528 Wernick/Queder/Strube Martins u.a., Ansätze zur Glasfaser-Erschließung unterversorgter Gebiete, S. 36.

529 Wernick/Queder/Strube Martins u.a., Ansätze zur Glasfaser-Erschließung unterversorgter Gebiete, S. 36.

530 Dies wird insbesondere in der Abbildung 4-1 deutlich bei Wernick/Queder/Strube Martins u.a., Ansätze zur Glasfaser-Erschließung unterversorgter Gebiete, S. 38 .

531 Dies wird insbesondere in der Abbildung 4-2 deutlich bei Wernick/Queder/Strube Martins u.a., Ansätze zur Glasfaser-Erschließung unterversorgter Gebiete, S. 40.

532 Sörries, N\&R 2020, S. 161, 166.

533 Felten/Langer, Structurally indepent broadband infrastructure can solve perceived FTTH coverage issues, S. 15 f.; Sörries, N\&R 2020, S. 161, 166; Wernick/ Queder/Strube Martins u.a., Ansätze zur Glasfaser-Erschließung unterversorgter Gebiete, S. $41 \mathrm{f}$. 
Das Ergebnis dieser Studie ist mit der gebotenen Zurückhaltung zu betrachten, die sich aus ihrer Konstruktion als „Greenfield-Modell“ ergibt. ${ }^{534}$ Sowohl die realen Ausbaugebiete als auch die Entscheidungsfindungsprozesse der Unternehmen sind deutlich komplexer, als die Studie dies vermuten lässt. Demnach würden sich die Auswirkungen einer größeren Präsenz von Wholesale-Only-Anbietern in realen Ausbauszenarien vermutlich nicht derart evident zeigen, wie es die Modellierung auf den ersten Blick suggeriert. Die tatsächlichen Auswirkungen, auch im Vergleich mit anderen Maßnahmen zur Beschleunigung des Infrastrukturausbaus, müssten in einer ökonometrischen Analyse vertieft betrachtet werden, die hier nicht durchgeführt werden kann (hierzu auch B. I. 2. c.). Der Handlungsansatz hat jedoch keinen Einfluss auf eines der Grundprobleme des Infrastrukturausbaus auf dem Land. Dies liegt darin begründet, dass durch die geringere Siedlungsdichte im Vergleich zum urbanen Umfeld höhere Investitionskosten entstehen, die nur mithilfe weniger anschließbarer Kunden amortisiert werden können. ${ }^{535}$ Der zu untersuchende Handlungsansatz knüpft mithin nur an einer kleinen Stellschraube an, welche die weiteren Probleme des Infrastrukturausbaus im ländlichen Bereich nicht tangiert. Dennoch können auch von der Optimierung des Rechtsrahmens in Detailaspekten im Zusammenspiel mit anderen Handlungsoptionen positive Effekte für den Breitbandausbau entstehen. Wie sichtbar diese Effekte in realen Ausbauszenarien ausfallen würden, müsste jedoch in weiterführenden ökonomischen Untersuchungen dargelegt werden. Aus juristischer Sicht stellt sich die Frage, ob der Rechtsrahmen überhaupt so angepasst werden könnte, dass eine erhöhte Präsenz von Wholesale-Only-Anbietern am Markt auf diesem Wege erreichbar wäre.

\section{Zwischenergebnis und Schlussfolgerungen}

Der Wertschöpfungsprozess des Telekommunikationssektors lässt sich insbesondere in die Vorleistungs- und die Endkundenebene gliedern. Unter-

534 Dies geben auch die Verfasser der Studie, der das Modell zugrunde liegt, zu bedenken, nämlich Wernick/Queder/Strube Martins u.a., Ansätze zur Glasfaser-Erschließung unterversorgter Gebiete, S. 36; ebenso Felten/Langer, Structurally indepent broadband infrastructure can solve perceived FTTH coverage issues, S. 19.

$535 \mathrm{Zu}$ den einzelnen Kostenfaktoren Wernick, Ökonomie und Kostenstrukturen des Glasfaserausbaus, S. 5; Wernick/Gries/Bender u.a., Regionale TK-Akteure im globalen Wettbewerb, S. $15 \mathrm{f}$. 
nehmen, die auf beiden Stufen tätig sind, werden als vertikal integriert bezeichnet. Unternehmen, die ausschließlich auf Vorleistungsebene tätig sind, werden Wholesale-Only-Anbieter genannt. Betriebswirtschaftlich kann eine vertikale Integration durch die entstehenden Verbundvorteile innerhalb des Unternehmens sinnvoll sein. Zusätzlich bietet die Entgeltregulierung durch gestaffelte Zugangsentgelte weitere Anreize, eigene Infrastruktur aufzubauen. Mithilfe ökonomischer Modelle lässt sich nachweisen, dass auch die Tätigkeit als Wholesale-Only-Anbieter betriebswirtschaftlich sinnvoll sein kann. Sie illustrieren darüber hinaus, dass die Zeiträume, in denen Investitionen von Wholesale-Only-Anbietern in Infrastruktur amortisiert werden können, unter optimierten Bedingungen kürzer sein können als bei gleichartigen Investitionen vertikal integrierter Telekommunikationsunternehmen. Zudem können unter bestimmten Bedingungen auch in Regionen mit höheren Erschließungskosten Ausbauvorhaben von Wholesale-Only-Anbietern schneller profitabel sein. Hieraus wird geschlussfolgert, dass bei mehr Wholesale-Only-Anbietern auf dem Telekommunikationsmarkt mit einem beschleunigten Infrastrukturausbau in der Fläche zu rechnen sei. Diese Schlussfolgerung der Modelluntersuchung ist jedoch nur eingeschränkt aussagekräftig, da sie die komplexen Zusammenhänge der Ausbaurealität stark vereinfacht. Das Modell geht von verschiedenen Prämissen und Verhaltensweisen von Unternehmen aus, die im Markt in der Regel kumuliert auftreten. Tatsächliche Aussagen über die Auswirkungen des Handlungsansatzes in realen Ausbauszenarien lassen sich auf Basis der vorliegenden ökonomischen Modellierungen nur eingeschränkt treffen. Deswegen sind weiterführende ökonomische Analysen erforderlich, um die Effektivität des Handlungsansatzes im Zusammenspiel mit anderen Handlungsoptionen zu untersuchen.

\section{Stufenlehre zur Kategorisierung von Entflechtungsmaßnahmen}

Trotz der dargestellten betriebswirtschaftlichen Tragfähigkeit des Geschäftsmodells als Wholesale-Only-Anbieter sind nur vereinzelte Unternehmen mit dieser Ausrichtung in Deutschland aktiv. Eine größere Durchmischung des Marktes könnte durch Entflechtung bereits existierender vertikal integrierter Telekommunikationsunternehmen oder die Gründung neuer Wholesale-Only-Anbieter erreicht werden. ${ }^{536}$

536 Wernick/Queder/Strube Martins u.a., Ansätze zur Glasfaser-Erschließung unterversorgter Gebiete, S. 32. 
Damit eine sinnvolle Auseinandersetzung mit den hiermit zusammenhängenden Fragestellungen möglich ist, müssen die Entflechtungsmöglichkeiten systematisiert werden. Die Anknüpfungspunkte und Ausgestaltungsmöglichkeiten für die Separierung vertikal integrierter Telekommunikationsunternehmen sind mannigfaltig. In der juristischen Diskussion werden sie zumeist anhand des Ansatzpunktes der Entflechtung dargestellt. ${ }^{537}$ So wird etwa zwischen einer organisatorischen, eigentumsrechtlichen oder buchhalterischen Entflechtung differenziert. In der ökonomischen Literatur werden sie anhand der Intensität der Entflechtung kategorisiert, in welche die Ansatzpunkte der Entflechtung integriert werden. Die Kategorisierung basiert auf verschiedenen Publikationen des Ökonomen Martin Cave zu einem Stufensystem der Entflechtungsmöglichkeiten (dazu 1.). ${ }^{538}$ Für die folgende Diskussion kommt es nicht so sehr auf den Ansatzpunkt der Entflechtung, sondern vielmehr auf die Eingriffsintensität der Maßnahme an, sodass anhand des ökonomischen Stufenmodells untersucht werden soll, welcher Separierungsgrad erforderlich ist, um einen Wholesale-Only-Anbieter entstehen zu lassen (dazu 2.). Aus diesem Grund wird die Systematik nicht anhand der juristischen, sondern der ökonomischen Literatur diskutiert, in welche die juristischen Anknüpfungspunkte integriert werden.

\section{Struktur des Stufensystems}

Die Zuordnung einer Separierungsmaßnahme zu den einzelnen Stufen richtet sich nach der Eingriffsintensität der Maßnahme und ihrer potentiellen Wirksamkeit zur Beseitigung von Diskriminierungsanreizen. Dies lässt sich darauf zurückführen, dass die Entflechtung zumeist als Maßnahme zur Reduktion der Diskriminierung aufgrund der Tätigkeit eines Tele-

537 Vgl. hierzu statt vieler Kühling, Sektorspezifische Regulierung in den Netzwirtschaften, S. $337 \mathrm{ff}$.

538 Cave, Communications \& Strategies 64 (2006), S. 89 ff.; hierauf Bezug nehmend Crandall/Eisenach/Litan, Federal Communications Law Journal 2010, S.493, 500 f.; Harnisch, Funktionale Separierung als strategisches Regulierungsinstrument auf dem europäischen Telekommunikationsmarkt, S. 4; vgl. zudem die tabellarische Darstellung in BEREC, BoR (10) 44 Rev1, S. 5 f.; Cave/Doyle, Network separation and investment incentives in telecommunications, S.9; vgl. aber auch die Unterteilung in lediglich drei Kategorien von Olsen/Henten/Falch, Functional separation in telecommunications, S. 4; auf Basis von Cremer/de Donder/Cremer, Communications \& Strategies 68 (2008), S. 41, $50 \mathrm{ff}$. 
kommunikationsunternehmens auf verschiedenen Wertschöpfungsstufen verstanden wird. ${ }^{539} \mathrm{Je}$ intensiver die integrierte Organisationsstruktur aufgelöst wird, desto wahrscheinlicher werden die Anreize zur Diskriminierung beseitigt. ${ }^{540}$

In dieser Struktur ist die buchhalterische Entflechtung als „Grad 0“ der Separierung zu klassifizieren. Dabei werden die Umsätze für die Tätigkeit im Vorleistungs- und Endkundenbereich auf separaten buchhalterischen Konten erfasst. ${ }^{541}$ Diese Maßnahme soll Transparenz sicherstellen, um prohibitive Preise erkennen und entsprechende Maßnahmen ergreifen zu können. Sie steht damit in einem engen Konnex zur Diskriminierung aufgrund von Zugangsentgelten, kann aber nicht effektiv zur Prävention von Diskriminierung abseits der Diskriminierung mit Entgelten genutzt werden. ${ }^{542}$ Oftmals können aber auch Maßnahmen zur Bekämpfung von Diskriminierung aufgrund prohibitiver Preise oder mithilfe anderer Regulierungsmaßnahmen parallel angewandt werden. ${ }^{543}$

Als „Grad 1“ wird sodann die Gründung einer Abteilung bezeichnet, die ausschließlich für die Geschäfte des Unternehmens auf Vorleistungsebene zuständig ist. ${ }^{544}$ Die Abteilung kann auch für andere Unternehmen tätig werden. Die unternehmenseigene Diensteabteilung erhält jedoch weiterhin priorisierten Infrastrukturzugang. ${ }^{545}$

Hiervon ist die Separierung nach „Grad 2“ zu unterscheiden, in der die Geschäftsbereiche zwar faktisch in dem Unternehmen bleiben, aber virtuell voneinander getrennt werden. ${ }^{546}$ Es handelt sich um die erste Trennungsform, in der den Anbietern von Endkundendiensten aus dem eige-

539 So etwa Crandall/Eisenach/Litan, Federal Communications Law Journal 2010, S. 493, 495; zu den Diskriminierungspotentialen äußern sich Abegg/Brinkmann/ Brunekreeft u.a., Entflechtung in Netzsektoren, S. 5 ff.

540 Das BEREC stellt die Maßnahmen in einem Koordinatensystem dar, das den Schutz vor Diskriminierung und den Eingriff in die Organisationsstruktur des Telekommunikationsunternehmens in Bezug setzt, siehe BEREC, BoR (10) 44 Rev1, S. 7, Darstellung 1.

541 Hierzu ausführlich Cave, Communications \& Strategies 64 (2006), S. 89, 94.

542 Cave, Communications \& Strategies 64 (2006), S. 89, 91; hierauf Bezug nehmend BEREC, BoR (10) 44 Rev1, S. 6.

543 Cave, Communications \& Strategies 64 (2006), S. 89, 92.

544 Cave, Communications \& Strategies 64 (2006), S. 89, 94, der davon ausgeht, dass ein solches Vorgehen bei den meisten marktmächtigen Telekommunikationsunternehmen im europäischen Telekommunikationsmarkt zu beobachten ist.

545 Zur Stufung innerhalb dieses Separierungsgrades und zu Beispielen Cave, Communications \& Strategies 64 (2006), S. 89, 95.

546 Hierzu im Überblick Webb, Breaking Up is Hard to Do, S. $8 \mathrm{f}$. 
nen und aus fremden Unternehmen tatsächlich ein gleichwertiger Zugang auf der Vorleistungsebene gewährt wird. ${ }^{547}$

Die funktionelle Trennung wird als Entflechtung nach „Grad 3“ eingeordnet. ${ }^{548} \mathrm{Im}$ Unterschied zum zweiten Grad wird das Unternehmen nicht nur virtuell, sondern faktisch und physisch in unterschiedliche Wirtschaftsbereiche getrennt, die sich eigenständig mit der Tätigkeit im Vorleistungs- bzw. im Endkundenbereich beschäftigen. ${ }^{549}$ Regelmäßig werden auch selbständige Marken und ein autonomes Marketing für die unterschiedlichen Geschäftsbereiche entwickelt.

Der „Grad 4“ in der Skala der Separierungsmöglichkeiten stellt eine Intensivierung des dritten Grades dadurch dar, dass neben der Gründung eines eigenständigen Geschäftsbereiches auch eine Trennung auf Ebene der Leitung und Steuerung stattfindet. 550

In „Grad 5“ werden die Geschäftsbereiche nicht mehr lediglich innerhalb desselben Unternehmens getrennt, sondern erhalten separate Rechtspersönlichkeiten. ${ }^{551}$

Die höchste Intensität ist bei einer Separierung des „Grades 6“ festzustellen, in der das Eigentum entflochten wird. ${ }^{552}$ In diesem Fall verfügen das Unternehmen, das auf Vorleistungsebene tätig ist, und das Unternehmen, das auf Endkundenebene tätig wird, über verschiedene Eigentümer. In der Folge wird die Diskriminierung mit dem graduell stärksten Eingriff am effektivsten verhindert, da das zuvor vertikal integrierte Unternehmen vollständig separiert wird und nicht mehr zu einem Konzern gehört. ${ }^{553}$ Hierdurch entfällt jeglicher Diskriminierungsanreiz.

Die Klassifizierung orientiert sich ausschließlich an der Wirksamkeit für die Vermeidung von Zugangs- und Entgeltdiskriminierungen, während die Auswirkungen auf die Beschleunigung des Infrastrukturausbaus keine Berücksichtigung finden.

547 Cave, Communications \& Strategies 64 (2006), S. 89, 95.

548 Cave, Communications \& Strategies 64 (2006), S. 89, 95 f.

549 Zur konkreten Ausgestaltung der Organisation der funktionellen Trennung im Unternehmen Cave, Communications \& Strategies 64 (2006), S. 89, 96.

550 Cave, Communications \& Strategies 64 (2006), S. 89, $96 \mathrm{f}$.

551 Cave, Communications \& Strategies 64 (2006), S. 89, 97.

552 Cave, Communications \& Strategies 64 (2006), S. 89, 97.

$553 \mathrm{Zu}$ den Vor- und Nachteilen der einzelnen Separierungsmöglichkeiten mit umfassender Auswertung der Literatur und dem Rückgriff auf Praxisbeispiele, $C a$ ve/Doyle, Network separation and investment incentives in telecommunications, S. $11 \mathrm{ff}$. 
2. Wholesale-Only-Anbieter im Stufensystem

Die Klassifikation eines Unternehmens als Wholesale-Only-Anbieter setzt voraus, dass dessen Tätigkeit von einer Tätigkeit auf dem Endkundenmarkt ausreichend separiert ist. Denklogisch ist dies am ehesten bei einer Separierung nach dem „Grad 6“ der Stufenlehre, also bei einer eigentumsrechtlichen Entflechtung, der Fall. Diese ökonomische Betrachtung lässt sich auch mit juristischen Argumenten stützen. Der unionale Gesetzgeber knüpft die Annahme, dass ein Unternehmen ausschließlich auf der Vorleistungsebene tätig ist, in Art. 80 EKEK an strenge Voraussetzungen. So ist hierfür erforderlich, dass keine exklusiven wirtschaftlichen Beziehungen $\mathrm{zu}$ anderen Unternehmen bestehen, die Endkundendienste erbringen (hierzu ausführlich D. II.). Dies lässt sich darauf zurückführen, dass die Rechtsfolge der Erfüllung der Tatbestandsvoraussetzungen des Art. 80 Abs. 1 EKEK eine Regulierungsprivilegierung ist, die an das reduzierte Diskriminierungspotential anknüpft, das nur bei einer umfassenden Entflechtung gegeben ist.

Denkbar wäre aber auch die Entstehung eines Wholesale-Only-Anbieters mithilfe einer Entflechtung nach „Grad 5“, sofern eine ausreichende Unabhängigkeit zwischen den Tätigkeiten auf den beiden Wertschöpfungsstufen gewährleistet ist. Solche Unternehmen könnten jedoch unter Umständen, mangels Erfüllung der Tatbestandsvoraussetzungen des Art. 80 Abs. 1 EKEK, nicht von dessen regulatorischer Privilegierung profitieren. Als Ausgestaltungsbeispiel kann insoweit das Energierecht angeführt werden. Dort wurde neben der Option der eigentumsrechtlichen Entflechtung gemäß $\ 8$ EnWG auch die Ausgestaltungmöglichkeit des „Unabhängigen Systembetreibers“ im Sinne des $\$ 9$ EnWG oder des „Unabhängigen Transportnetzbetreibers“ im Sinne des $\$ 10$ EnWG geschaffen, die vergleichbar effektiv das Diskriminierungspotential reduzieren. ${ }^{554}$ Das Telekommunikationsrecht sieht entsprechende Ausgestaltungsoptionen derzeit jedoch nicht vor (B. I.). Eine Separierung nach „Grad 4“, also eine organisatorische und personelle Entflechtung reicht hingegen nicht aus, um ein Unternehmen als Wholesale-Only-Anbieter zu klassifizieren. Insoweit sind beide Geschäftsbereiche zu eng verknüpft. Dies gilt auch für geringere Entflechtungsstufen.

554 Kühling/Rasbach/Busch, Energierecht, Kap.5, Rn. 96 ff.; Rasbach, Unbundling-Regulierung in der Energiewirtschaft, S. $179 \mathrm{ff}$. 
III. Schlussfolgerungen für die juristische Analyse

Die zentralen Wertschöpfungsstufen im Telekommunikationssektor sind die Vorleistungs- und die Endkundenebene. Während sich Wholesale-Only- und Wholebuy-Anbieter auf eine der beiden Stufen beschränken, sind vertikal integrierte Telekommunikationsunternehmen auf beiden Ebenen tätig. Die vertikale Integration ist in dem von hohen initialen und langfristig gebundenen Investitionskosten geprägten Telekommunikationssektor betriebswirtschaftlich vorteilhaft. Auch die Beschränkung des Tätigkeitsbereiches auf den Vorleistungssektor kann betriebswirtschaftlich sinnvoll sein. Aus Modelluntersuchungen hierzu lässt sich ableiten, dass ein beschränkter Tätigkeitsbereich auch positive Auswirkungen auf die Geschwindigkeit des Gigabitausbaus haben könnte. Ob sich die theoretischen Erkenntnisse des Modells auch in der angenommenen Deutlichkeit in der Realität widerspiegeln würden, ist jedoch fraglich. Die Anzahl der Wholesale-Only-Anbieter auf dem deutschen Telekommunikationsmarkt ist gering. Optionen, um sie zu steigern, sollen nachfolgend näher beleuchtet werden. Das Entflechtungsregime ist derzeit auf die Förderung des Wettbewerbs ausgerichtet und müsste für den Zweck der Beschleunigung des Infrastrukturausbaus modifiziert werden. In der folgenden juristischen Analyse soll die Frage im Vordergrund stehen, ob die regulatorischen Vorgaben zur Entflechtung die Tätigkeit als Wholesale-Only-Anbieter erschweren, inwieweit sie zur Beschleunigung des Infrastrukturausbaus bereits genutzt werden können und welche Optimierungen in dieser Hinsicht erforderlich wären.

\section{B. Entstehung von Wholesale-Only-Anbietern durch eine staatlich indizierte funktionelle Trennung}

Als eine der Möglichkeiten zur vermehrten Entstehung von Wholesale-Only-Anbietern beizutragen, wird die Entflechtung vertikal integrierter Telekommunikationsunternehmen durch staatlichen Zwang angeführt. ${ }^{555}$ Das unionale und nationale Telekommunikationsrecht sehen normative Grundlagen zum Erlass von Regulierungsverfügungen vor, durch die vertikal integrierte Telekommunikationsunternehmen dazu verpflichtet werden können, sich funktionell zu trennen (dazu I.). Das Kartellrecht kennt

555 Wernick/Queder/Strube Martins u.a., Ansätze zur Glasfaser-Erschließung unterversorgter Gebiete, S. 32. 
Befugnisse, mit deren Hilfe strukturelle Abhilfemaßnahmen bei wettbewerbswidrigem Verhalten auferlegt werden können (dazu II.). Im Fokus der folgenden Analyse soll die Frage stehen, ob die Vorgaben es ermöglichen, ein Unternehmen dazu zu verpflichten, die vertikale Integration aufzulösen und zu einem Wholesale-Only-Anbieter zu werden. Darüber hinaus soll untersucht werden, ob die Vorgaben der Anpassung bedürfen und im Einzelfall angepasst werden können.

I. Normative Ausgestaltung der funktionellen Trennung im

Telekommunikationsrecht

Das unionale Telekommunikationsrecht enthält in Art. 77 EKEK die normative Grundlage für nationale Vorgaben zur Anordnung einer funktionellen Trennung. Im deutschen Telekommunikationsgesetz sind die entsprechenden Regelungen in $\$ 40$ TKG ( $\$ 31$ TKG-RefE) $)^{556}$ enthalten. Nach einer Darlegung der aktuellen rechtlichen Steuerungsvorgaben im unionalen und nationalen Regulierungsrecht (dazu 1.) sollen ein etwaiger Anpassungsbedarf und die diesbezügliche Anpassungsmöglichkeit diskutiert werden (dazu 2.).

1. Steuerungsvorgaben des Regulierungsrechts für den Telekommunikationssektor

a) Die Grundstruktur des Art. 77 EKEK de lege lata

Klarer Fokus der unionalen regulatorischen Entflechtungsvorgaben ist der Wettbewerb. Der unionale Gesetzgeber verfolgt mit der Regulierungsmaßnahme der funktionellen Trennung das Ziel, dass alle Unternehmen Vorleistungsprodukte zu gleichwertigen Bedingungen erhalten. ${ }^{557}$ Ist dies nicht mit dem üblichen Maßnahmenportfolio sicherzustellen, kann subsidiär auf die funktionelle Trennung zurückgegriffen werden. Sie ist darauf

556 Referentenentwurf des Telekommunikationsmodernisierungsgesetzes (TKMoG), abrufbar unter: https://www.bmwi.de/Redaktion/DE/Downloads/Gesetz/ telekommunikationsmodernisierungsgesetz-referentenentwurf-20201612.pdf? blob=publicationFile\&v=8 (zuletzt abgerufen am 12.1.2021).

557 Erwägungsgrund 202 des EKEK. 
ausgerichtet, die strukturellen Voraussetzungen und Anreize für Diskriminierungen zu verringern. 558

Die Maßnahme ist in ein komplexes und gestuftes Verfahren eingebettet und gemäß Art. 77 Abs. 1 UAbs. 1 EKEK als ultima ratio konzipiert. ${ }^{559}$ Die funktionelle Trennung steht gemäß Art. 77 Abs. 1 UAbs. 1 EKEK nur dann zur Verfügung, wenn die „nach den Artikeln 69 bis 74 auferlegten angemessenen Maßnahmen nicht zu einem wirksamen Wettbewerb geführt haben. " Es muss das gesamte Instrumentarium der Regulierungsverfügungen erfolglos angewandt worden sein, bevor ein Unternehmen dazu verpflichtet werden kann, seine Geschäftsbereiche funktionell zu trennen. Der Anwendungsbereich des Art. 77 Abs. 1 UAbs. 1 EKEK wird dadurch weiter eingeschränkt, dass „wichtige Wettbewerbsprobleme oder Marktversagen auf den Märkten für bestimmte Zugangsprodukte auf der Vorleistungsebene bestehen" müssen. In der Zusammenschau dieser beiden Kriterien wird deutlich, dass die Vorschrift als Maßnahme mit dem schärfsten Eingriffscharakter nur dann zum Tragen kommt, wenn tatsächlich keine andere Möglichkeit besteht, mit der Diskriminierung von Wettbewerbern umzugehen. Ausbaudefizite sind in diesen Kriterien nicht aufgenommen. Zur Beschleunigung des Glasfaserausbaus kann eine funktionelle Trennung aus diesem Grund nur dann genutzt werden, wenn neben dem Ausbaudefizit zugleich eine Diskriminierung von Wettbewerbern identifiziert wird.

Sofern eine Anordnung zur Aufteilung in verschiedene Geschäftsbereiche erfolgt, müssen die Vorleistungsprodukte gemäß Art.77 Abs. 1 UAbs. 2 EKEK allen Unternehmen zu den gleichen Konditionen zur Verfügung gestellt werden. Insoweit ist das Entflechtungsregime des Telekommunikationssektors im Vergleich zu anderen Netzwirtschaften, wie dem Energiesektor, weniger streng ausgebildet. 560

Durch einen Verweis auf Art. 68 Abs. 3 UAbs. 2 EKEK wird ein Verfahrensmechanismus implementiert, der die Hürden für die Anwendung weiter erhöht. So muss die Anwendung der Maßnahme bei der Kommission beantragt werden. ${ }^{561}$ Hierbei handelt es sich um ein Alleinstellungsmerkmal im Vergleich zu den übrigen Regulierungsmaßnahmen, die einen Antrag bei der Kommission nicht vorsehen. Im Wortlaut des Art. 68 Abs. 3

558 Erwägungsgrund 202 des EKEK.

559 Kühling, in: Ruffert (Hrsg.), Europäisches Sektorales Wirtschaftsrecht, $\mathbb{4} 4$, Rn. 128.

560 Weiterführend Schneider, in: Fehling/Ruffert (Hrsg.), Regulierungsrecht, S. 365, 384.

561 Kühling, in: Ruffert (Hrsg.), Europäisches Sektorales Wirtschaftsrecht, $\mathbb{} 4$, Rn. 128. 
UAbs. 2 EKEK wird der Ausnahmecharakter der Maßnahme dadurch hervorgehoben, dass ein Antrag lediglich „unter außergewöhnlichen Umständen" gestellt werden soll. Die strengen Anforderungen an den Antrag (Art. 77 Abs. 2 EKEK) und der hohe Konkretisierungsgrad, den er aufweisen muss (Art. 77 Abs. 3 UAbs. 1 EKEK), illustrieren ebenfalls die herausgehobene Stellung der funktionellen Trennung im Rahmen der regulatorischen Maßnahmen. Es handelt sich um keine regulatorische Standardmaßnahme. Sie darf nur dann angewandt werden, wenn hinreichende positive Effekte der Trennung im Wege einer Marktanalyse sichtbar werden, die gemäß Art. 77 Abs. 3 UAbs. 2 S. 1 EKEK im Vorfeld durchzuführen ist. ${ }^{562}$ Bei der Anwendung der Maßnahmen sollen die kollidierenden Interessen von Unternehmen und Verbrauchern Berücksichtigung finden. Sie soll nicht durchgeführt werden, wenn eine funktionelle Trennung das Verbraucherwohl gefährden und Investitionen in Netze verhindern könnte. ${ }^{563}$

Ein restriktiver Einsatz der funktionellen Entflechtung ist verfassungsrechtlich unentbehrlich, da sie einen schweren Eingriff in die Struktur eines Unternehmens darstellt, der rechtfertigungsbedürftig ist und verhältnismäßig ausgestaltet werden muss. ${ }^{564}$

Der Ausnahmecharakter des Art. 77 EKEK wird somit verschiedenen Stellen der Vorgabe deutlich. Rechtsfolge der funktionellen Trennung ist jedoch lediglich die Einrichtung separater Geschäftsbereiche. Sie ist mithin in der ökonomischen Stufenlehre als Separierung nach "Grad 3“ einzuordnen (A. II. 1.). Es entstünde durch die Anwendung der Vorgabe demnach kein Wholesale-Only-Anbieter, sondern ein vertikal integriertes Telekommunikationsunternehmen mit getrennten Geschäftsbereichen.

\section{b) Vorgaben des $\$ 40 \mathrm{TKG}$}

Die Vorschrift zur funktionellen Trennung ist in $\$ 40$ TKG umgesetzt und entspricht weitgehend den Vorgaben des Unionsrechts. ${ }^{565}$ Eine Aktualisierung der Vorschriften im Rahmen der TKG-Novelle zur Umsetzung des

562 Detailliert zur Konstruktion der Vorgabe, Kühling, in: Ruffert (Hrsg.), Europäisches Sektorales Wirtschaftsrecht, $\$ 4$, Rn. $127 \mathrm{f}$.

563 Erwägungsgrund 202 des EKEK.

564 Weiterführung zur Entflechtung und ihrer Einordnung in das Portfolio der Regulierungsmaßnahmen Kühling, Sektorspezifische Regulierung in den Netzwirtschaften, S. $337 \mathrm{ff}$.

565 Weiterführend zur Ausgestaltung der nationalen Vorschrift Klement, in: Scheurle/Mayen (Hrsg.), Telekommunikationsgesetz, $\mathbb{\$} 40 ;$ Mohr, in: Säcker (Hrsg.), Te- 
EKEK ist lediglich in Detailfragen erforderlich. Daher wird $₫ 40$ TKG - als \31 TKG-RefE - nach derzeitigem Stand weitgehend unverändert fortbestehen. ${ }^{566}$ Hinsichtlich seiner konkreten Ausgestaltung kann demnach auf die Ausführungen zum Unionsrecht verwiesen werden (B. I. 1. a.). Die funktionelle Entflechtung nach $\$ 40$ TKG kann ebenfalls ausschließlich zum Schutz des Wettbewerbs eingesetzt werden. Dies bleibt im Rahmen der TKG-Novelle unverändert. Die Förderung der Konnektivität kann allenfalls eine Nebenfolge der Maßnahme sein.

2. Anpassungsbedarf und -möglichkeit des Rechtsrahmens de lege ferenda?

Aus dem Befund, dass der Rechtsrahmen aktuell keine verpflichtende Entflechtung von Unternehmen zur Beschleunigung des Glasfaserausbaus vorsieht, könnte sich ein Änderungsbedarf de lege ferenda ergeben. Die Vorgabe zur funktionellen Entflechtung wurde durch Art. 2 Nr. 10 ÄndRL 2009/140/EG zum ersten Mal in Form des Art. 13a Zugangsrichtlinie $(Z R L)^{567}$ in den Rechtsrahmen eingeführt. Im EKEK wurden die Entflechtungsbestimmungen nicht erweitert. Vielmehr wurde im Vorfeld gar eine vollständige Abschaffung der Vorgaben diskutiert. ${ }^{568}$ Wie bereits im Zusammenhang mit der Entwicklung des Wegerechtes im unionalen Rechtsrahmen dargelegt, wird dieser Rechtsrahmen in regelmäßigen $\mathrm{Zy}$ klen überarbeitet (Kapitel 3, C. III. 2.). Perspektivisch ist zwar nicht mit einer Erweiterung der Vorgaben zu rechnen. Dennoch sollen an dieser Stelle die Anforderungen diskutiert werden, die an eine Kompetenzgrundlage zu stellen wären, die den Einsatz der Entflechtung zur Förderung der Konnektivität ermöglichen würde. Hierbei soll die Frage im Vordergrund

lekommunikationsgesetz, $\mathbb{\$} 40$; Schreiber, in: Geppert/Schütz (Hrsg.), Beck'scher TKG-Kommentar, $\$ 40$.

566 Referentenentwurf des Telekommunikationsmodernisierungsgesetzes (TKMoG), abrufbar unter: https:/www.bmwi.de/Redaktion/DE/Downloads/Gesetz/ telekommunikationsmodernisierungsgesetz-referentenentwurf-20201612.pdf? blob=publicationFile\&v=8 (zuletzt abgerufen am 12.1.2021).

567 Richtlinie 2002/19/EG des Europäischen Parlaments und des Rates vom 7.3.2002 über den Zugang zu elektronischen Kommunikationsnetzen und zugehörigen Einrichtungen sowie deren Zusammenschaltung (Zugangsrichtlinie), ABl.L 108 vom 24.4.2002, S. 7 i. d. F. d. Richtlinie 2009/140/EG vom 25.11.2009, ABl. L 337, S. 37.

568 BEREC, BoR (17) 88, S. 3. 
stehen, ob eine solche Vorschrift überhaupt in den Rechtsrahmen integriert werden kann. Dazu müsste zunächst die Eingriffsintensität der Bestandsnorm erheblich erhöht werden, um überhaupt einen Wholesale-Only-Anbieter entstehen zu lassen (dazu a.). Aus systematischen Erwägungen wäre eine neue Kompetenzgrundlage außerhalb des Marktregulierungsverfahrens erforderlich (dazu b.). Hierbei müssten die strengen verfassungsrechtlichen Anforderungen beachtet werden, die an Vorgaben zur Entflechtung vertikal integrierter Unternehmen gestellt werden (dazu c.).

a) Erweiterung der Entflechtungsintensität der Kompetenzgrundlage

Die derzeitige Kompetenzgrundlage in Art. 77 EKEK ist lediglich darauf ausgerichtet, die funktionelle Entflechtung von Unternehmen zu legitimieren. Hierbei handelt es sich um eine Separierung nach „Grad 3“ im Sinne der ökonomischen Stufenlehre (B. I. 1. a.). Durch diese entsteht jedoch kein Wholesale-Only-Anbieter, sondern lediglich ein vertikal integriertes Unternehmen mit getrennten Geschäftsbereichen. Die Entflechtungsintensität der Kompetenznorm müsste mithin deutlich erhöht werden, um den gewünschten Effekt zu erzielen. Als Vorbild hierfür könnten die regulatorischen Vorgaben des Energierechts dienen, die eine weitergehendere Entflechtung gestatten (hierzu bereits A. II. 2.). Hierbei ist jedoch zu beachten, dass diese Vorgaben ebenfalls ausschließlich darauf ausgerichtet sind, den Wettbewerb durch eine Minimierung von Diskriminierungspotentialen zu fördern. Demnach könnte ausschließlich deren Entflechtungsintensität, nicht jedoch ihre Zweckrichtung als Vorbild dienen.

b) Schaffung einer neuen Kompetenzgrundlage aufgrund systematischer und teleologischer Bedürfnisse?

Die funktionelle Entflechtung ist eine regulatorische Maßnahme, die angewandt werden kann, um Unternehmen daran zu hindern, ihre marktbeherrschende Stellung so einzusetzen, dass der Wettbewerb beeinträchtigt wird (B. I. 1.). Aus diesem Grund ist sie in das Marktregulierungsverfahren eingebunden. Eine regulatorische Verpflichtung wird in dessen Rahmen nur dann auferlegt, wenn ein Unternehmen auf einem definierten Markt über eine beherrschende Stellung verfügt. Die funktionelle Entflechtung im Besonderen ist eine Maßnahme mit herausgehobener Stellung, die nur als ultima ratio unter besonders strengen Voraussetzungen angewendet 
werden kann, wenn die übrigen Maßnahmen keine Wirkung gezeigt haben (B. I. 1. a.).

Systematisch könnte eine Erweiterung der Zweckrichtung der funktionellen Entflechtung zur Förderung der Konnektivität nicht überzeugen. Vielmehr wäre die erweiterte Kompetenzgrundlage ein systematischer Fremdkörper. Es lässt sich nicht begründen, warum die Eingriffsmaßnahmen zur Konnektivitätsförderung lediglich auf Unternehmen mit einer marktbeherrschenden Stellung angewandt werden sollten. Die ökonomischen Modelluntersuchungen, die von einem positiven Effekt von der stärkeren Marktpräsenz von Wholesale-Only-Anbietern ausgehen, formulieren eine pauschale Aussage und beschränken die Effektivität der Maßnahme nicht ausschließlich auf marktmächtige Unternehmen (A. I. 3.). Es wird allgemein festgestellt, dass die vermehrte Marktpräsenz von Wholesale-Only-Anbietern positive Effekte auf die Geschwindigkeit des Infrastrukturausbaus haben könnte. Dabei ist es irrelevant, ob die entflochtenen Unternehmen marktbeherrschend sind oder nicht.

Daher müsste eine separate Kompetenzgrundlage für die Entflechtung zur Förderung der Konnektivität außerhalb des Marktregulierungsverfahrens geschaffen werden. Hierzu müsste ein ähnlich strenges Verfahren aufgebaut werden, um den Anforderungen des Verhältnismäßigkeitsgrundsatzes zu genügen (B. I. 2. c.). Dreh- und Angelpunkt müsste die Abgrenzung geeigneter Gebiete sein, in denen sich mithilfe einer Entflechtung vertikal integrierter Unternehmen ein positiver Effekt auf den Infrastrukturausbau generieren ließe. Hierzu müsste ein vollständig neues Verfahren etabliert werden, das die Spezifika von Ausbaugebieten in den Mittelpunkt stellt. Als Anknüpfungspunkt könnte hierfür das neugeschaffene Instrument des Mapping aus Art. 22 EKEK dienen. ${ }^{569}$ Dieses soll voraussichtlich in den $\$ \$ 77 \mathrm{ff}$. TKG-RefE umgesetzt werden. ${ }^{570}$ Es ermöglicht die Erfassung von bestehender und geplanter Infrastruktur und bietet damit eine Datengrundlage, um die Infrastruktur in bestimmten Gebieten zu katalogisieren und die Ausbauperspektiven nachzuzeichnen. Der schonende Ausgleich der Interessen, die den verschiedenen Regulierungszielen zu Grunde lie-

569 Grundlegend zum Instrument des Mapping und konkreten Normierungsvorschlägen Kübling/Toros, Rechtliche Rahmenbedingungen für die Schaffung von Anreizen für einen flächendeckenden Ausbau von Glasfaserinfrastrukturen, S. 107 ff.; Kühling/Toros, MMR 2020, S. 92 ff.

570 Referentenentwurf des Telekommunikationsmodernisierungsgesetzes (TKMoG), abrufbar unter: https:/www.bmwi.de/Redaktion/DE/Downloads/Gesetz/ telekommunikationsmodernisierungsgesetz-referentenentwurf-20201612.pdf? blob=publicationFile $\& v=8$ (zuletzt abgerufen am 12.1.2021). 
gen, müsste - wie nach Art. 77 Abs. 3 UAbs. 2 S. 1 EKEK - auch im Rahmen der neuen Norm Anklang finden. ${ }^{571}$ Die Folgen einer Entflechtung für die Konnektivität, den Wettbewerb, die Interessen der Bürger und den Binnenmarkt müssten im Vorfeld - ähnlich wie nach Art. 77 Abs. 3 UAbs. 2 S. 1 EKEK - untersucht werden. Würde die Konnektivität zwar minimal gefördert, zugleich jedoch die Verbraucherinteressen erheblich geschädigt, müsste von der Maßnahme Abstand genommen werden.

c) Verfassungsmäßigkeit der Kompetenzgrundlage und ihrer Anwendung im Einzelfall

Derzeit existiert, wie bereits festgestellt, weder im unionalen noch im nationalen Telekommunikationsrecht eine Kompetenzgrundlage zur Entflechtung vertikal integrierter Telekommunikationsunternehmen zur Steigerung der Konnektivität. Auf beiden normhierarchischen Ebenen müssten mithin zunächst entsprechende Vorgaben geschaffen werden. Auch wenn der Determinierungsgrad der unionsrechtlichen Vorgaben zur Entflechtung im Marktregulierungsverfahren sehr hoch ist, ist dies für die Schaffung einer neuen Kompetenzgrundlage nicht zu prognostizieren. Damit ergibt sich eine vergleichbare Ausgangslage, wie im Rahmen der Einführung eines Konzessionsmodells auf Basis der Vergabe exklusiver Wegerechte, sodass die Kompatibilität mit dem unionalen und nationalen Grundrechtskatalog sichergestellt werden muss (Kapitel 3, C. IV. 1. a. aa.). Besonders relevant sind für die Kompetenzgrundlage zur Entflechtung von Unternehmen als Mittel zur Beschleunigung des Breitbandausbaus die erwerbsbezogenen Grundrechte (dazu aa.), das Grundrecht auf Eigentum (dazu bb.) sowie der Allgemeine Gleichheitssatz (dazu cc.).

aa) Vereinbarkeit mit den erwerbsbezogenen Grundrechten

Eine Kompetenzgrundlage zur Entflechtung vertikal integrierter Unternehmen und ihre Anwendung im Einzelfall müssten sich mit den erwerbsbezogenen Grundrechten vereinbaren lassen, die unionsrechtlich im $\mathrm{Zu}$ -

571 Siehe hierzu im Hinblick auf den entsprechenden Passus in Art. 77 EKEK bereits B. I. 1. a. sowie Erwägungsgrund 202 des EKEK. 
sammenspiel aus Art. 15 und 16 GRCh gewährleistet werden. ${ }^{572}$ In diese Grundrechte würde dadurch eingegriffen, dass den betroffenen Unternehmen eine parallele wirtschaftliche Tätigkeit auf Vorleistungs- und Endkundenebene verwehrt würde. Die Voraussetzungen einer Rechtfertigung des Eingriffes in die Grundrechte der GRCh ergeben sich aus Art. 52 Abs. 1 GRCh. ${ }^{573}$ Zunächst bedürfte es danach einer gesetzlichen Grundlage, die geschaffen werden müsste.

Mit der Beschleunigung und Förderung des Ausbaus hochleistungsfähiger Infrastrukturen würde ein legitimer Zweck verfolgt (Kapitel 3, C. V. 4. b. aa.). Hinsichtlich der Eignung und Erforderlichkeit verbleibt dem Gesetzgeber ein umfassender Einschätzungsspielraum. ${ }^{574}$ Die positiven Effekte einer stärkeren Durchmischung des Marktes mit Wholesale-Only-Anbietern auf die Geschwindigkeit des Breitbandausbaus werden derzeit lediglich auf Basis einzelner ökonomischer Modellierungen nachgewiesen (A. I. 3.). Fraglich ist, ob sich die auf Basis eines „Greenfield-Ansatzes“ gewonnen Erkenntnisse auch in realen Ausbauszenarien zeigen würden (A. I. 3.). Für eine fundierte Überprüfung der Eignung und Erforderlichkeit der Maßnahme wären weitere umfassende ökonometrische Analysen erforderlich, die auch die Effekte einer Entflechtung in solchen Gebieten untersuchen, die nicht dem Modellaufbau entsprechen. Dies wäre insbesondere für eine Bewertung der Erforderlichkeit der Entflechtung von Nöten, die einen Vergleich mit anderen Maßnahmen zur Beschleunigung des Infrastrukturausbaus erfordert. Danach dürfte es kein relativ milderes, gleich geeignetes Mittel zur Erreichung der Ziele geben. ${ }^{575}$ Die besondere Herausforderung besteht in der hohen Eingriffsintensität der Entflechtung eines Unternehmens. Zugleich stehen verschiedene Alternativmaßnahmen,

572 Zum Eingriffsbegriff EuGH, Urt. v. 23.9.2004, verb. Rs. C-435/02 und C-103/03 (Springer), ECLI:EU:C:2004:552, Rn. 49; weiterführend Kühling, in: Pechstein/ Nowak/Häde (Hrsg.), Frankfurter Kommentar EUV/GRC/AEUV, Art. 15 GRCh, Rn. $15 \mathrm{f}$.

573 An dieser Stelle soll davon ausgegangen werden, dass sich die Entflechtungsvorgaben im unionalen Rechtsrahmen abschließend geregelt werden würden und eine direkte Anwendung der Grundfreiheiten. Dennoch sind sie im Rahmen der Auslegung der sekundärrechtlichen Vorschriften und auch bei der Interpretation der berufsbezogenen Grundrechte aus Art. 15 und 16 GRCh zu berücksichtigen; Müller-Graff, in: Streinz (Hrsg.), EUV/AEUV, Art. 56 AEUV, Rn. 142.

574 Hierzu statt vieler Kahl/Schwind, EuR 2014, S. 170, 185.

575 EuGH, Urt. v. 4.10.2018, Rs. C-384/17 (Link Logistik N\&N), ECLI:EU:C: 2018:810, Rn. 40; EuGH, Urt. v. 17.4.2018, Rs. C-414/16 (Egenberger), ECLI:EU: C:2018:257, Rn.68; EuGH, Urt. v. 11.7.1989, Rs. 265/87 (Schräder/Hauptzollamt Gronau), ECLI:EU:C:1989:303, Rn. 21. 
wie etwa die Vergabe von Fördermitteln zur Verfügung, welche die Grundrechte der betroffenen Unternehmen nicht vergleichbar belasten. Die Effektivität der verschiedenen Alternativmaßnahmen lässt sich auf Basis der bisherigen ökonomischen Untersuchung jedoch an dieser Stelle nicht abschließend vergleichen. Tendenziell ist jedoch davon auszugehen, dass mit der Vergabe von Fördermitteln ein weniger eingriffsintensives und voraussichtlich sogar effektiveres Mittel zur Verfügung stünde.

Ungeachtet dessen lassen sich aus juristischer Perspektive bereits einige pauschale Aussagen über die Normkonstruktion treffen, die in jedem Fall zu beachten wären. Es böte sich an, die Erforderlichkeitsprüfung im Einzelfall bereits - wie im Rahmen der funktionellen Entflechtung gemäß Art. 77 EKEK - normativ vorzustrukturieren und die Maßnahme als ultima ratio zu konzipieren. Hierzu böte sich ein abgestuftes Maßnahmenprogramm an, an dessen Spitze die Entflechtung steht, sofern ihre Effektivität im Vergleich zu den anderen Maßnahmen hinreichend nachgewiesen wird. Die Maßnahme müsste auch verhältnismäßig im engeren Sinne sein. Die konfligierenden Interessen müssten demnach gegeneinander abgewogen werden. Dabei erscheint es sinnvoll, diese Abwägung ebenfalls normativ vorzustrukturieren und hier dem Beispiel des Art. 77 Abs. 3 UAbs. 2 S. 1 EKEK zu folgen (B. I. 1. a.).

Eine weitaus größere Herausforderung stellt es dar, dass die Anwendung der Maßnahme auch in jedem Einzelfall grundrechtskonform sein müsste. Die positiven Effekte der Entflechtung eines Unternehmens für den Breitbandausbau müssten gegen die Interessen des Unternehmens zur parallelen wirtschaftlichen Betätigung auf dem Vorleistungs- und Endkundenmarkt abgewogen werden. Problematisch wäre es in dieser Hinsicht insbesondere, wenn sich die Vorteile für den Breitbandausbau tatsächlich nur in solchen Gebieten spürbar auswirken würden, die der Modellierung gleichen und damit keinen flächendeckenden Erfolg in der komplexen Ausbaurealität versprechen. Viele Unternehmen sind nämlich nicht lediglich lokal begrenzt, sondern überregional oder gar bundesweit tätig. Positive Effekte einer vertikalen Desintegration für die Steigerung der Konnektivität in einem eng begrenzten Gebiet dürften in diesem Fall nicht ausreichen, um die Verhältnismäßigkeit einer so eingriffsintensiven Maßnahme wie einer Entflechtung sicherzustellen. Anderenfalls könnte etwa ein bundesweit tätiges Unternehmen - wie die Telekom Deutschland GmbH entflochten werden, nur um den Breitbandausbau in einem bayerischen Landkreis zu beschleunigen. Dies wäre ohne Zweifel unverhältnismäßig.

Dieser Befund gilt auch für die Gewährleistungen aus Art. 12 Abs. 1 S. 1 GG. 
bb) Vereinbarkeit mit dem Grundrecht auf Eigentum

Die Entflechtung vertikal integrierter Telekommunikationsunternehmen zur Beschleunigung des Infrastrukturausbaus müsste sich auch mit den Gewährleistungen des Art. 17 GRCh vereinbaren lassen. Eigentum sind nach Ansicht des Europäischen Gerichtshofes „vermögenswerte Rechte, aus denen sich im Hinblick auf die Rechtsordnung eine gesicherte Rechtsposition ergibt, die eine selbstständige Ausübung dieser Rechte durch und zugunsten ihres Inhabers ermöglicht. " 576 Problematisch sind solche Entflechtungen, durch die nicht nur eine unternehmensinterne Separierung - beispielsweise durch Einrichtung separater Geschäftsbereiche - gefordert wird, sondern das Unternehmen zur Veräußerung von Geschäftsanteilen verpflichtet wird. Dies wäre beispielweise bei einer eigentumsrechtlichen Entflechtung der Fall. Das Energierecht enthält entsprechende Kompetenzgrundlagen zur Förderung des Wettbewerbs (hierzu bereits A. II. 2.). Allerdings stellt sich die Frage, ob eine so umfassende und intensive Entflechtung überhaupt erforderlich wäre, um die gewünschten Effekte der Beschleunigung des Infrastrukturausbaus zu erzielen. Gegebenenfalls hätten auch weniger eingriffsintensive Entflechtungen, die eine weitgehende Unabhängigkeit der Geschäftsbereiche sicherstellen würden, bereits einen vergleichbaren Effekt. Hierzu fehlt jedoch eine ökonomische Analyse, sodass dies nicht abschließend bewertet werden kann. Aus Gründen der Erforderlichkeit müsste die relativ mildeste Ausgestaltung der Maßnahme, die zu der angestrebten Beschleunigung des Breitbandausbaus führt, gewählt werden.

Auch für die Gewährleistungen aus Art. 14 Abs. 1 GG ergeben sich keine anderen Ergebnisse.

cc) Gleichbehandlung vertikal integrierter Telekommunikationsunternehmen

Der Allgemeine Gleichheitssatz, der in Art. 20 GRCh angelegt ist, sieht wie sein grundgesetzliches Pendant in Art. 3 Abs. 1 GG - vor, dass wesentlich Gleiches gleich und wesentlich Ungleiches differenziert behandelt

576 EuGH, Urt. v. 22.1.2013, Rs. C-283/11 (Sky Österreich/Österreichischer Rundfunk), ECLI:EU:C:2013:28, Rn. 34; Michl, Unionsgrundrechte aus der Hand des Gesetzgebers, S. 89. 
werden muss. ${ }^{577}$ Die ökonomischen Modellierungen gehen davon aus, dass eine stärkere Durchmischung des Marktes mit Wholesale-Only-Anbietern positive Effekte für den Infrastrukturausbau haben könnte (A. I. 3.). Dabei wird jedoch nicht dargelegt, dass sich der gewünschte Effekt auf die Entflechtung marktmächtiger Unternehmen beschränkt. Vor diesem Hintergrund ließe es sich nicht rechtfertigen, marktmächtige Unternehmen anders zu behandeln als Unternehmen ohne beträchtliche Marktmacht. Demnach wäre die Etablierung der Kompetenzgrundlage zur Entflechtung von Unternehmen als Werkzeug zur Beschleunigung des Breitbandausbaus nicht nur aus systematischen (B. I. 2. b.), sondern auch aus primärbzw. verfassungsrechtlichen Gründen geboten. Eine Anknüpfung an eine marktbeherrschende Stellung, würde gegen den Gleichheitsgrundsatz verstoßen.

\section{dd) Zwischenergebnis}

Die Erkenntnis, dass mehr Wholesale-Only-Anbieter auf dem Telekommunikationsmarkt spürbar zur Beschleunigung des Glasfaserausbaus beitragen würden, ist keinesfalls gesichert (A. I. 3.). Eine sichtbare Beschleunigung lässt sich zwar unter optimalen Bedingungen simulieren, allerdings sind die tatsächlichen Bedingungen im Telekommunikationsmarkt deutlich komplexer und lassen sich nur schwerlich prognostizieren. Dem steht eine Maßnahme gegenüber, die der Gesetzgeber - in erheblich geringerer Eingriffsintensität - als schärftes Schwert zur Beseitigung von Wettbewerbshindernissen konzipiert hat. Nur wenn alle übrigen Regulierungsmaßnahmen fruchtlos geblieben sind, kann ein Unternehmen funktionell getrennt werden. Bei der Konstruktion der Entflechtungsbestimmung zur Förderung der Konnektivität müsste der Eingriff erheblich intensiviert werden, um einen Wholesale-Only-Anbieter entstehen zu lassen. Sowohl die Rechtsgrundlage der Entflechtung an sich als auch ihre Anwendung müssten sich am Maßstab der Berufs-, Unternehmer- und Eigentumsfreiheit aus Art. 15 und 16 sowie 17 GRCh rechtfertigen lassen. Für eine abschließende Beurteilung der Eignung und Erforderlichkeit der Maßnahme fehlen umfassende ökonomische Untersuchungen. Je niedriger die Entflechtungsintensität und je höher die Effektivität der Maßnahme wäre, desto eher ließe sich ihre Anwendung rechtfertigen. Dabei wäre eine Orientie-

577 Heselhaus, in: Pechstein/Nowak/Häde (Hrsg.), Frankfurter Kommentar EUV/GRC/AEUV, Art. 20 GRCh, Rn. 24. 
rung der normativen Ausgestaltung an Art.77 EKEK zu befürworten. Erforderlichkeit und Angemessenheit der Rechtsgrundlage ließen sich durch ihre Ausgestaltung als ultima ratio mit einer strengen Interessenabwägung sicherstellen. Aus Gründen der Gleichbehandlung müsste die Maßnahme zudem auf alle vertikal integrierten Telekommunikationsunternehmen, unabhängig von deren Marktmacht, angewandt werden können. Hinsichtlich der Möglichkeit einer grundrechtskonformen Anwendung der Vorgabe im Einzelfall, ist Skepsis geboten.

\section{d) Ergebnis}

Die Rechtsgrundlage zur funktionellen Entflechtung von Unternehmen ist konsistent auf den Wettbewerbsschutz ausgerichtet und in die Systematik des Marktregulierungsverfahrens eingebettet. Um einen Wholesale-OnlyAnbieter entstehen zu lassen, müsste ihre Eingriffsintensität zur Entflechtung vertikal integrierter Telekommunikationsunternehmen beträchtlich erhöht werden. Eine Erweiterung der Zweckrichtung der funktionellen Entflechtung auf die Steigerung der Konnektivität, würde sich nicht in den systematischen Rahmen einfügen. Vielmehr wäre eine separate Rechtsgrundlage in einem anderen Kontext zu schaffen. Diese müsste aus Gründen der Gleichbehandlung auf alle vertikal integrierten Telekommunikationsunternehmen angewandt werden können. Im Fokus dürfte deswegen nicht die Marktmacht eines Unternehmens stehen. Vielmehr sollten Gebiete mit Netzausbaudefizit identifiziert werden. Dafür könnte sich die geografische Erhebung gemäß Art. 22 EKEK eignen, die im Rahmen der Novellierung des TKG in den $\$ \$ \$ 78 \mathrm{ff}$. TKG-RefE eingeführt werden soll. 578

Bei der normativen Strukturierung müsste gewährleistet werden, dass das Ziel der Konnektivitätsförderung schonend mit den übrigen Regulierungszielen in Ausgleich gebracht wird. Ihre Vereinbarkeit mit den unionalen und nationalen Grundrechten lässt sich auf Basis der vorliegenden ökonomischen Daten nur eingeschränkt prüfen. Es lassen sich allerdings Grundsätze entwickeln, die in jedem Fall zu berücksichtigen wären. Die Verfassungsmäßigkeit ließe sich umso einfacher sicherstellen, je effektiver

578 Referentenentwurf des Telekommunikationsmodernisierungsgesetzes (TKMoG), abrufbar unter: https:/www.bmwi.de/Redaktion/DE/Downloads/Gesetz/ telekommunikationsmodernisierungsgesetz-referentenentwurf-20201612.pdf? blob=publicationFile $\& v=8$ (zuletzt abgerufen am 12.1.2021). 
die Maßnahme und je niedriger die hierfür erforderliche Entflechtungsintensität ist. Die Erforderlichkeit und Angemessenheit könnten dadurch sichergestellt werden, dass ihre Handhabung im Einzelfall bereits im Normtext vorgegeben wird. Die Verfassungsmäßigkeit des Einzelaktes ist umso schwieriger sicherzustellen, je größer der Tätigkeitsbereich des Unternehmens ist. Dies gilt insbesondere, wenn die Maßnahme nur sehr lokal begrenzt positive Effekte mit sich bringen würde. Freilich ist es insgesamt zu kritisieren, dass Regulierungsverfügungen zur Umsetzung eines bestimmten Geschäftsmodells durch staatlichen Zwang genutzt werden sollen. Dies gilt hier umso mehr, da noch nicht einmal explizit gesichert ist, ob die positiven Auswirkungen auf den Infrastrukturausbau überhaupt in einem spürbaren Maße eintreten würden. ${ }^{579}$ Vor diesem Hintergrund besteht kein Optimierungsbedarf für den ausbalancierten Rechtsrahmen. Eine Anpassungsmöglichkeit ist aus verfassungsrechtlichen Gründen aller Wahrscheinlichkeit nach ohnehin nicht gegeben.

II. Mögliche Aufspaltung des Unternehmens nach deutschem Kartellrecht zur Beschleunigung des Infrastrukturausbaus?

Während das sektorspezifische Regulierungsrecht eine Ex-ante-Perspektive einnimmt und versucht, Diskriminierungspotentiale und Marktdefizite präventiv zu beseitigen, nimmt das Kartellrecht eine Ex-post-Perspektive ein. Es ist aber ebenso darauf ausgerichtet, den Wettbewerb zu schützen und bei festgestellten Defiziten Maßnahmen zu ergreifen. Das Unionsprimärrecht enthält in Art. 101 und Art. 102 AEUV ein Kartell- und ein Missbrauchsverbot. Gemäß Art. 103 Abs. 1 AEUV können Durchführungsverordnungen erlassen werden, um der Kommission Handlungsoptionen zur Reaktion auf etwaige Verstöße zu gewähren. Relevant für den vorliegenden Fall ist insoweit Art. 7 Abs. 1 VO (EG) Nr. 1/2003, ${ }^{580}$ die der Kommission gestattet, Abhilfemaßnahmen struktureller Art bei wettbewerbsrechtswidrigem Verhalten zu ergreifen. Eine korrespondierende Kompetenz enthält $\$ 32$ Abs. 2 S. 1 GWB für das Bundeskartellamt, sofern Unternehmen

579 Ähnlich skeptisch zu den positiven Auswirkungen der Entflechtung der Deutschen Telekom AG auf den Wettbewerb bereits Kühling, Sektorspezifische Regulierung in den Netzwirtschaften, S. 343.

580 Verordnung (EG) Nr. 1/2003 des Rates vom 16.12.2002 zur Durchführung der in den Artikeln 81 und 82 des Vertrags niedergelegten Wettbewerbsregeln, ABl. L 1 vom 4.1.2003, S. 1 i. d. F. d. Verordnung (EG) Nr. 487/2009 vom 25.5.2009, ABl. L 148, S. 1. 
gegen die Vorgaben des nationalen Kartellrechts oder des Art.101 oder 102 AEUV verstoßen.

Dabei ist es jeweils erforderlich, dass der Verstoß in der Struktur des Unternehmens kausal begründet wird. ${ }^{81}$ Ein typischer Fall wäre insoweit bei einer vertikalen Integration des Unternehmens gegeben, wenn diese dazu führt, dass die marktbeherrschende Stellung wettbewerbsrechtswidrig eingesetzt wird. Die Maßnahme muss im Übrigen im konkreten Einzelfall verhältnismäßig sein. Sie muss demnach zur Abhilfe des rechtswidrigen Verhaltens geeignet und erforderlich sowie angemessen sein. Im Rahmen der Erforderlichkeitsprüfung ist zu untersuchen, ob mildere verhaltensbezogene Abhilfemaßnahmen nicht ebenfalls dazu geeignet sind, um das gesetzeswidrige Verhalten abzustellen. ${ }^{582}$ Insoweit ist die Struktur dem Telekommunikationsrecht vergleichbar (B. I. 1.), sodass die strukturellen Abhilfemaßnahmen auch im Kartellrecht lediglich als ultima ratio angewandt werden dürfen. Darüber hinaus ist eine strenge Prüfung der Angemessenheit (Verhältnismäßigkeit im engeren Sinne) erforderlich, die dieses Ergebnis weiter untermauert. Die Vorschrift enthält dabei keinen numerus clausus zulässiger Maßnahmen. Denkbar ist etwa, dass einem Unternehmen aufgegeben wird, Geschäftsbereiche zu veräußern. Kompetenzen, die der Kartellbehörde erlauben würden, ein Unternehmen - nach dem Vorbild des Energierechts - zu zerschlagen und damit eine Entflechtungsintensität auszuüben, die zur Entstehung eines Wholesale-Only-Anbieters erforderlich wäre, kennt das Kartellrecht hingegen nicht. Die Einführung solcher Kompetenznormen ist immer wieder Gegenstand der politischen Diskussion. ${ }^{583}$ Anders als in den USA ${ }^{584}$ und in Großbritannien ${ }^{585}$ wurden sie bislang jedoch nicht eingeführt. In den Staaten mit strengen Entflechtungsregimen ist die Maßnahme ausschließlich auf den Schutz und die Gewährleistung eines funktionsfähigen Wettbewerbs ausgerichtet.

Damit ergibt sich ein Bild, das weitgehend dem Befund im Telekommunikationsrecht gleicht (B. I. 1.). Strukturelle Abhilfemaßnahmen sind die ultima ratio und auf die Sicherstellung eines funktionsfähigen Wettbewerbs ausgerichtet. Tatbestandsvoraussetzung ist mithin ein Verstoß gegen Art. 101 oder Art. 102 AEUV oder das nationale Kartellrecht. In diese

581 Emmerich, in: Immenga/Mestmäcker (Hrsg.), Wettbewerbsrecht, Band 2, $\$ 32$ GWB, Rn. 34.

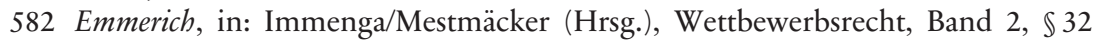
GWB, Rn. 34.

583 Nettesheim/Thomas, Entflechtung im deutschen Kartellrecht, S. $10 \mathrm{ff}$.

584 Nettesheim/Thomas, Entflechtung im deutschen Kartellrecht, S. $21 \mathrm{ff}$.

585 Nettesheim/Thomas, Entflechtung im deutschen Kartellrecht, S. $32 \mathrm{ff}$. 
Grundkonzeption würde sich das Ziel eines beschleunigten Infrastrukturausbaus in der Fläche weder systematisch noch teleologisch einfügen, da kein Zusammenhang zu den Schutzrichtungen des Kartellrechts besteht. Die Förderung des Infrastrukturausbaus ist nicht Aufgabe des Kartellrechts. Es besteht deshalb im Ergebnis kein Anpassungsbedarf de lege ferenda.

\section{Ergebnis}

Weder der unionale noch der nationale Rechtsrahmen enthalten Kompetenzen, um vertikal integrierte Telekommunikationsunternehmen mit staatlichem Zwang zu entflechten. Die funktionelle Entflechtung ist eine Maßnahme des Telekommunikationsrechts zur Bekämpfung der Diskriminierung von Zugangspetenten durch vertikal integrierte Telekommunikationsunternehmen mit signifikanter Marktmacht. Die Maßnahme ist im aktuellen Rechtsrahmen nicht auf die Beschleunigung des Infrastrukturausbaus ausgerichtet. Eine solche Funktionserweiterung ließe sich auch nicht mit dem Sinn und Zweck der Maßnahme und ihrer systematischen Stellung im Gesetz vereinbaren. Deswegen bedürfen die Vorgaben in diesem Kontext de lege ferenda keiner Anpassung. Vielmehr müsste eine neue Rechtsgrundlage geschaffen werden, die in einen anderen systematischen Kontext eingebettet werden müsste. Ohnehin ist die funktionelle Trennung als Separierung nach dem „Grad 3“ einzustufen und führt nicht dazu, dass ein Wholesale-Only-Anbieter entsteht. Durch die Maßnahme kann also lediglich die Trennung der Geschäftsbereiche auf der Vorleistungsund der Endkundenebene veranlasst werden. Dies wird sich perspektivisch nicht ändern. Die Schaffung einer separaten Rechtsgrundlage, die eine Entflechtung zur Förderung der Konnektivität gestattet, wäre zwar theoretisch möglich, im Einzelfall jedoch in der Regel nur schwerlich verhältnismäßig anwendbar. Ein positiver Effekt auf die Konnektivität, der allerdings empirisch nur rudimentär belegt ist, stünde in der Verhältnismäßigkeitsprüfung einem umfassenden Eingriff in die Eigentums- oder die Organisationsstruktur eines Unternehmens gegenüber.

Das Kartellrecht kennt ebenfalls keine normative Grundlage, welche die Kartellbehörden dazu legitimeren würde, Wholesale-Only-Anbieter durch staatlichen Zwang entstehen zu lassen. Vielmehr folgen aus den strukturellen Abhilfemaßnahmen, die im Gesetz vorgesehen sind, nur vergleichbare Kompetenzen wie im Telekommunikationsrecht. Im Kartellrecht besteht ebenfalls kein Anpassungsbedarf de lege ferenda, da die Integration von 
Maßnahmen zur Beschleunigung des Infrastrukturausbaus in den Kanon der Maßnahmen zur Abhilfe von Wettbewerbsverstößen völlig systemfremd wäre. Der Staat kann mithin nicht mittels verpflichtender Maßnahmen zur vermehrten Entstehung vertikal integrierter Telekommunikationsunternehmen beitragen. Dieses Ergebnis ist folgerichtig. Es wäre höchst bedenklich, wenn der Staat ein Geschäftsmodell für Unternehmen mit Zwang zur Erreichung eines Zieles umsetzen würde, wenn noch nicht einmal gesichert ist, ob die Maßnahme in dieser Hinsicht überhaupt hinreichend förderlich ist.

\section{Freiwillige Separierung vertikal integrierter Telekommunikations- unternehmen}

Als weitere Maßnahme, durch die Wholesale-Only-Anbieter entstehen können, wird eine freiwillige Entflechtung von zuvor vertikal integrierten Telekommunikationsunternehmen angeführt. ${ }^{586}$ Hierbei handelt es sich in der Regel um eine strategische Entscheidung, die beispielsweise vom tschechischen Telekommunikationsunternehmen CETIN a.s. getroffen wurde (dazu I.). Eine freiwillige Separierung setzt eine gesellschaftsrechtliche Trennung des Unternehmens voraus (dazu II.). Aus dem sektorspezifischen Regulierungsrecht ergeben sich insbesondere Vorgaben dazu, wie die aufgespaltenen Unternehmen aufgrund des reduzierten Missbrauchsrisikos regulatorisch zu privilegieren sind (dazu III.).

\section{Freiwillige Aufspaltung am Beispiel des Unternehmens CETIN}

In Tschechien hat sich bereits ein marktmächtiges, vertikal integriertes Telekommunikationsunternehmen aufgespalten. Infolge einer Übernahme des nationalen Unternehmens mit beträchtlicher Marktmacht durch einen Investor wurde das vormals vertikal integrierte Unternehmen aus strategischen Erwägungen im Jahr 2015 aufgespalten. ${ }^{57}$ Das Infrastrukturunternehmen tritt unter dem Namen Česká telekomunikační infrastruktura a.s. (CETIN) und der Diensteanbieter unter dem Namen O2 Czech Republic

586 Wernick/Queder/Strube Martins u.a., Ansätze zur Glasfaser-Erschließung unterversorgter Gebiete, S. 32.

587 Wernick/Queder/Strube Martins u.a., Ansätze zur Glasfaser-Erschließung unterversorgter Gebiete, S. 35 . 
a.s. am Markt auf. Mit der Aufspaltung des Unternehmens wurden verschiedene Ziele verfolgt. Durch den Fokus auf eine Wertschöpfungsstufe sollte das Unternehmen zielgerichteter und effizienter arbeiten. ${ }^{588}$ Zudem wurde durch die Separierung angestrebt, aufgrund der reduzierten Marktmacht aus der Ex-ante-Regulierung entlassen zu werden. ${ }^{589}$ Das Entlassen des Unternehmens aus der Zugangsregulierung wird zur Zeit durch die Regulierungsbehörde geprüft und auch der Wert der separierten Unternehmen ist im Vergleich jeweils deutlich gestiegen. ${ }^{590}$ Mithilfe einer gezielten und fairen Vermarktung an andere Unternehmen sollte zudem die Auslastung des Netzes erhöht werden. ${ }^{591}$ Die mögliche Beschleunigung des Ausbaus hochleistungsfähiger Infrastrukturen in Tschechien, die einen positiven Nebeneffekt der Maßnahme darstellen könnte, floss nicht explizit in die Erwägungen des Unternehmens ein. Vergleichbare Separierungsbestrebungen bestehen auf dem deutschen Telekommunikationsmarkt derzeit nicht.

\section{Gesellschaftsrechtliche Vorgaben zur Aufspaltung von Unternehmen}

Anders als bei der funktionellen Trennung erfolgt der Entschluss zur freiwilligen Aufspaltung privatautonom durch die Leitungsorgane der Gesellschaft. Die formellen Voraussetzungen hierfür ergeben sich insbesondere aus dem Gesellschaftsrecht. ${ }^{592}$ Um die Grundstrukturen einer freiwilligen

588 Godlovitch/Lemstra/Pennings u.a., Regulatory, in particular access, regimes for network investment models in Europe, S. 186; Wernick/Queder/Strube Martins u.a., Ansätze zur Glasfaser-Erschließung unterversorgter Gebiete, S. 35.

589 Godlovitch/Lemstra/Pennings u.a., Regulatory, in particular access, regimes for network investment models in Europe, S. 186; Wernick/Queder/Strube Martins u.a., Ansätze zur Glasfaser-Erschließung unterversorgter Gebiete, S. 35.

590 Dies geht aus der Zusammenschau der Überblickspräsentationen des Konzerns aus den Jahren 2017, S. 19 abrufbar unter: https://www.cetin.cz/documents/101 82/134417/2017+05+CETIN+Company+overview+FY2016.pdf/17b90788-75a9-4e 35-a808-0a4df9910fcb (zuletzt abgerufen am 1.7.2020) und aus dem Jahr 2018, S. 17 hervor, abrufbar unter: https://www.cetin.cz/documents/10182/189301/Inv estor+presentation+1H2018/0946f41a-4b11-49b1-9b3c-820bd27d9b16 (zuletzt abgerufen am 1.7.2020).

591 Godlovitch/Lemstra/Pennings u.a., Regulatory, in particular access, regimes for network investment models in Europe, S. 186; Wernick/Queder/Strube Martins u.a., Ansätze zur Glasfaser-Erschließung unterversorgter Gebiete, S. 35.

592 Hier soll lediglich auf die üblichen Konstellationen der Umwandlung eingegangen werden; siehe zu den Sonderkonstellationen für bestimmte Gesellschaftsfor- 
Entflechtungsentscheidung verstehen und ihre regulatorische Flankierung untersuchen zu können, ist eine Analyse der wesentlichen Steuerungsvorgaben für den gesellschaftsrechtlichen Ablauf einer Unternehmensaufspaltung unerlässlich.

Das Verfahren und der Ablauf der Trennung sind zivilrechtlich ausgestaltet. Das Umwandlungsgesetz (UmwG) enthält in den $\$ \mathbb{S} 123-173$ Vorgaben zur Spaltung von Unternehmen und strukturiert die diesbezüglichen Verfahren. Ist das umzuwandelnde Unternehmen eine juristische Person des öffentlichen Rechts, gelten die Sonderregelungen der $\$ \mathbb{S} 174 \mathrm{ff}$. UmwG über die Vermögensübertragung. ${ }^{593}$ Es existieren keine sektorspezifischen Regelungen für den Telekommunikationssektor, sodass die allgemeinen gesellschaftsrechtlichen Vorschriften zur Anwendung kommen. Dies ist folgerichtig, da sektorspezifische Regelungen nur für solche Konstellationen geschaffen werden müssen, in denen - für andere Branchen untypische - Besonderheiten vorliegen.

Die Spaltung einer Gesellschaft ist dadurch geprägt, dass Teile des Vermögens eines Ausgangsrechtsträgers auf den Zielrechtsträger übertragen werden. ${ }^{594}$ Das Gesetz differenziert zwischen der Aufspaltung ( $\$ 123$ Abs. 1 UmwG), der Abspaltung ( $\$ 123$ Abs. 2 UmwG) und der Ausgliederung ( $\$ 123$ Abs. 3 UmwG). 595 Eine Aufspaltung liegt gemäß $\$ 123$ Abs. 1 UmwG vor, wenn das gesamte Vermögen der Gesellschaft auf die Zielgesellschaften aufgeteilt wird, ohne dass es zu einer Abwicklung der Ausgangsgesellschaft, wohl aber zu deren Auflösung kommt. ${ }^{596}$ Dies ist bei der Abspaltung und der Ausgliederung insoweit anders, als bei ihnen die Ausgangsgesellschaft erhalten bleibt. ${ }^{597}$ Abspaltung und Ausgliederung wiederum unterscheiden sich hinsichtlich der Gegenleistung voneinander.

men und Ausgangssituationen im Überblick Leuering, in: Kraft/Redenius-Hövermann (Hrsg.), Umwandlungsrecht, S. 123, $133 \mathrm{ff}$.

593 Hierzu weiterführend Hofmann/Riethmüller, JA 2009, S. 481, 483.

594 Hofmann/Riethmüller, JA 2009, S. 481, 482.

595 Kuhlmann/Abnis, Konzern- und Umwandlungsrecht, S. 414; Leuering, in: Kraft/ Redenius-Hövermann (Hrsg.), Umwandlungsrecht, S. 123, 126; Wardenbach, in: Henssler/Strohn (Hrsg.), Gesellschaftsrecht, $\$ 123$ UmwG, Rn. 4 ff.

596 Der konkrete Ablauf einer Abspaltung wird schematisch übersichtlich dargestellt von Hofmann/Riethmüller, JA 2009, S. 481, 483; Hörtnagl, in: Schmitt/Hörtnagl/Stratz (Hrsg.), Umwandlungsgesetz, Umwandlungssteuergesetz, $\mathbb{} 123$ UmwG, Rn. 6; Leuering, in: Kraft/Redenius-Hövermann (Hrsg.), Umwandlungsrecht, S. 123, 126.

597 Hofmann/Riethmüller, JA 2009, S. 481, 483; Leuering, in: Kraft/Redenius-Hövermann (Hrsg.), Umwandlungsrecht, S. 123, 127 f.; Schwanna, in: Semler/Stengel (Hrsg.), Umwandlungsgesetz, $\mathbb{\$} 123$ UmwG, Rn. 12. 
Bei der Abspaltung erfolgt die Separierung gemäß $\$ 123$ Abs. 2 UmwG „gegen die Gewährung von Anteilen oder Mitgliedschaften dieses Rechtsträgers oder dieser Rechtsträger an die Anteilsinhaber des übertragenden Rechtsträgers“, ${ }^{9} 98$ bei der Ausgliederung „gegen Gewährung von Anteilen oder Mitgliedschaften dieses Rechtsträgers oder dieser Rechtsträger an den übertragenden Rechtsträger. " ${ }^{\text {599 }}$ Bei jeder der Separierungsvarianten können Vermögensgegenstände entweder auf eine bereits bestehende oder eine noch zu gründende Gesellschaft übertragen werden. ${ }^{600} \mathrm{Im}$ Ergebnis findet eine Gesamtrechtsnachfolge statt, auch wenn dabei nur Teile des Vermögens erfasst sind. ${ }^{601}$

Formal untergliedert das UmwG den Prozess der Aufspaltung eines Unternehmens in drei Schritte. Hierbei handelt es sich um die Vorbereitung und die Beschlussfassung über die Aufspaltung, die in deren Vollzug münden. ${ }^{602}$ In diesem Rahmen sind die gesellschaftsrechtlichen Voraussetzungen für die Separierung des Unternehmens zu erfüllen. Insbesondere werden durch die Formalia der Minderheitenschutz und die Publizität sichergestellt. ${ }^{603}$

III. Regulatorische Behandlung freiwillig getrennter Telekommunikationsunternehmen

Anders als bei der funktionellen Trennung wird die freiwillige Trennung im Telekommunikationsrecht nur rudimentär flankiert. Dies lässt sich darauf zurückführen, dass die Trennung nicht auf staatlichen Zwang, son-

598 Hörtnagl, in: Schmitt/Hörtnagl/Stratz (Hrsg.), Umwandlungsgesetz, Umwandlungssteuergesetz, $\mathbb{\$} 123 \mathrm{UmwG}$, Rn. 9.

599 In Form eines Schaubildes über die Möglichkeiten der Separierung stellen dies übersichtlich dar Hofmann/Riethmüller, JA 2009, S. 481, 483; Leuering, in: Kraft/ Redenius-Hövermann (Hrsg.), Umwandlungsrecht, S. 123, $127 \mathrm{f.}$

600 Hofmann/Riethmüller, JA 2009, S. 481, 483; Leuering, in: Kraft/Redenius-Hövermann (Hrsg.), Umwandlungsrecht, S. 123, 128.

601 Hofmann/Riethmüller, JA 2009, 481; Leuering, in: Kraft/Redenius-Hövermann (Hrsg.), Umwandlungsrecht, S. 123, 130; Wardenbach, in: Henssler/Strohn (Hrsg.), Gesellschaftsrecht, $\$ 123$ UmwG, Rn. 2.

602 Der genaue Prozess unterscheidet sich für jede der Umwandlungsarten in Detailfragen, verfügt jedoch über einen einheitlichen Grundablauf, Hofmann/ Riethmüller, JA 2009, S. 481, 483.

603 Vgl. hierzu im Einzelnen Hofmann/Riethmüller, JA 2009, S. 481, 484 f.; Kublmann/Ahnis, Konzern- und Umwandlungsrecht, S. 417 ff.; Leuering, in: Kraft/ Redenius-Hövermann (Hrsg.), Umwandlungsrecht, S. 123, 135 ff. 
dern auf eine Willensentscheidung des Unternehmens zurückzuführen ist. Demnach gibt das Telekommunikationsrecht lediglich vor, wie mit den durch die Aufspaltung entstandenen Unternehmen in regulatorischer Hinsicht umzugehen ist. Die regulatorischen Vorgaben betreffen also den Umgang mit dem Resultat einer Aufspaltung des Unternehmens, sind der autonomen Entscheidung des Unternehmens also nachgelagert. Entsprechende Regelungen sind im geltenden unionalen Rechtsrahmen in Art. 78 EKEK enthalten (dazu 1.). Die nationale Vorschrift des $\$ 41$ TKG beruht noch auf den unionalen Vorgaben des Art. 13b ZRL. Im Rahmen der Umsetzung des EKEK in deutsches Recht muss die normative Grundlage insofern aktualisiert werden (dazu 2.). Darüber hinaus ist zu untersuchen, inwieweit der Rechtsrahmen unter Umständen einer vermehrten freiwilligen Entflechtung von vertikal integrierten Telekommunikationsunternehmen entgegensteht und ob hieraus ein Anpassungsbedarf de lege ferenda resultiert (dazu 3.).

\section{Freiwillige Trennung im unionalen Rechtsrahmen}

Der Unionsgesetzgeber verfolgt bei der regulatorischen Flankierung der freiwilligen Trennung vormals vertikal integrierter Telekommunikationsunternehmen einen konservativen Ansatz. Die Vorschrift ist ausschließlich auf den Schutz des Wettbewerbs auf der Endkundenebene ausgerichtet, der auch nach der Separierung eines vertikal integrierten Unternehmens fortbestehen soll. Positiv ist festzustellen, dass der Gesetzgeber nicht aktiv auf Unternehmen einwirkt. Vielmehr führt er den Unternehmen lediglich passiv die Vorteile einer vertikalen Desintegration vor Augen. Auch nach der Trennung des Unternehmens soll der diskriminierungsfreie Zugang zum Endkunden für alle Petenten gewährleistet sein. Auf diese Weise soll verhindert werden, dass die Aufspaltung des Unternehmens dazu genutzt wird, andere Telekommunikationsunternehmen zu diskriminieren und der Regulierung missbräuchlich zu entfliehen. ${ }^{604}$ Damit ist die Zweckrichtung derjenigen mit der Rechtsfolge der Legaldefinition des $\$ 3 \mathrm{Nr} .29$ TKG vergleichbar (Kapitel 5, D. II. 1.). Die Vorgabe ist jedoch nicht auf die Förderung der Konnektivität der Bevölkerung ausgerichtet. Positive Effekte darauf sind mithin ausschließlich unbeabsichtigte Nebenfolgen, die in der Norm nicht explizit adressiert werden.

604 Erwägungsgrund 204 des EKEK. 
Dieser Befund erhärtet sich bei einer näheren Betrachtung des Art. 78 EKEK, der in seiner Struktur verschiedene Pflichten und Handlungsoptionen für unterschiedliche Regelungsadressaten statuiert. Maßgeblicher Adressat der Verpflichtungen aus Art. 78 Abs. 1 EKEK ist das marktmächtige Unternehmen (Art.67 EKEK), das seine vertikale Integration durch einen autonomen Entschluss aufheben möchte. Für alle anderen Unternehmen entfaltet die Norm keine Steuerungswirkung.

Ihre Zweckrichtung wird durch ihre Einbettung in die Struktur des Marktregulierungsverfahrens besonders unterstrichen. Der Separierungsentschluss muss der zuständigen Behörde gemäß Art. 78 Abs. 1 UAbs. 1 EKEK spätestens drei Monate vor ihrem gesellschaftsrechtlichen Vollzug gemäß Art. 78 Abs. 1 UAbs. 1 EKEK mitgeteilt werden. Ändert sich die Absicht des Unternehmens, muss die Behörde gemäß Art. 78 Abs. 1 UAbs. 2 EKEK ebenfalls informiert werden. Der Gesetzgeber interpretiert den Separierungsbegriff dabei sehr weit und subsumiert sowohl die Absicht hierunter, die Vermögenswerte am Ortsanschlussnetz auf eine eigene Rechtsperson mit einem anderen Eigentümer zu übertragen, als auch diejenige, einen getrennten Geschäftsbereich zu errichten. Dies ist zu begrüßen, da auf diese Weise eine umfassende Reaktionsfähigkeit der Regulierungsbehörden sichergestellt wird. Durch die Mitteilungspflicht wird eine behördliche Analyse und das hierauf basierende Ergreifen von Maßnahmen erst ermöglicht. Würde die Mitteilungspflicht hingegen restriktiv interpretiert, könnte die Behörde unter Umständen nur verzögert intervenieren und negative Auswirkungen auf den Wettbewerb erst mit Verzug beseitigen.

Um dem Fortbestand von Regulierungsverpflichtungen entgegenzuwirken, kann das vertikal integrierte Unternehmen vor seiner Auflösung Verpflichtungszusagen im Sinne von Art. 79 EKEK machen und auf diese Weise den diskriminierungsfreien Zugang zum Endkunden garantieren. ${ }^{605}$ Das Mittel der Verpflichtungszusage, das bereits aus dem Kartellrecht bekannt ist, wird somit auch in den Telekommunikationsrechtsrahmen eingeführt (hierzu umfassend Kapitel 5, D. II. 3. a.). Unternehmen können im neuen Rechtsrahmen durch eine Selbstverpflichtung darauf hinwirken, die Regulierungsunsicherheit zu reduzieren. Die Verpflichtungszusagen flieBen in das Regulierungsermessen der Behörde ein. Hierbei handelt es sich um eine entscheidende Modifikation des unionalen Rechtsrahmens, der

605 Erwägungsgrund 205 des EKEK; die normativen Einzelheiten ergeben sich aus Art. 79 Abs. 1 lit. c EKEK. Speziell für die vertikale Desintegration ergeben sich weitere Einzelheiten aus Art. 79 Abs. 1 UAbs. 3 EKEK; hierzu auch Kühling, in: Ruffert (Hrsg.), Europäisches Sektorales Wirtschaftsrecht, $\mathbb{\$} 4$, Rn. 137. 
im Hinblick auf die Rückführung der Regulierung zu begrüßen ist (Kapitel 5, D. II. 3. a. cc.).

Die Handlungsoptionen und -pflichten der nationalen Regulierungsbehörde ergeben sich aus Art. 78 Abs. 2 EKEK. Die Behörde muss gemäß Art. 78 Abs. 2 UAbs. 1 EKEK die Auswirkungen der Aufspaltung des Unternehmens auf bestehende Regulierungsverpflichtungen untersuchen. Hierzu nutzt sie gemäß Art. 78 Abs. 2 UAbs. 2 EKEK eine Marktanalyse und berücksichtigt etwaige Verpflichtungszusagen des Unternehmens. Die Untersuchung folgt damit dem üblichen Ablauf, der bei jeder Auferlegung von Regulierungsverpflichtungen zu beachten ist. Die Verpflichtungszusage hindert die Regulierungsbehörde nicht daran, eine Regulierungsverpflichtung aufzuerlegen. Vielmehr handelt es sich um einen Abwägungstopos, der in etwaigen Entscheidungen einbezogen werden kann. Die nationale Regulierungsbe-hörde kann auf Basis der Marktanalyse gemäß Art. 78 Abs. 2 UAbs. 4 S. 1 EKEK neue Regulierungsverpflichtungen auferlegen oder bestehende Verpflichtungen beibehalten, ändern oder aufheben. Bei der Anwendung sind jedoch die Vorgaben des Art. 80 EKEK zu beachten (D. II.). Der umfassende Rückgriff auf das neue Instrument der Verpflichtungszusagen und die Abkehr von umfassenden Regulierungsverpflichtungen können als Anreiz zur Aufspaltung von Unternehmen interpretiert werden. ${ }^{606}$ Dieser Anreiz bezieht sich jedoch exklusiv auf marktmächtige Unternehmen. Dies ist folgerichtig, da die Vorgabe in das Marktregulierungsverfahren eingebettet ist, und daher nicht zu beanstanden.

Auch Art. 78 Abs. 3 und Abs. 4 EKEK richtet sich an die nationale Regulierungsbehörde. Die besondere Rolle der Verpflichtungszusagen wird dadurch unterstrichen, dass die nationalen Regulierungsbehörden nur dann Regulierungsverpflichtungen auferlegen werden, sofern die Verpflichtungszusagen nicht ausreichen, um die Ziele des Art. 3 EKEK zu erreichen. Die Selbstregulierung der marktbeherrschenden Unternehmen wird damit gegenüber dem Regulierungszwang präferiert. Die Verpflichtungszusagen unterliegen hinsichtlich ihrer Effektivität einem intensiven Monitoring. Die Wirksamkeit wird gemäß Art. 78 Abs. 4 EKEK überwacht. Sofern gewünscht, kann die Bindungswirkung der Verpflichtungszusagen über den vereinbarten Zeitraum hinaus erstreckt werden. Hierzu ist eine erneute Erforderlichkeitsprüfung von Nöten. Die Verpflichtungszusagen erhöhen da-

606 Eine bedeutende Rolle spielt das Instrument der Verpflichtungszusage im Zusammenhang mit der Regulierung kooperativer Investitionen in die Infrastruktur. Aus diesem Grund wird an dieser Stelle erneut und vertieft auf Art. 79 EKEK zurückzukommen sein, Kapitel 5, D. II. 3. a. 
mit insgesamt die Flexibilität der Regulierung und reduzieren zugleich die Regulierungsunsicherheit. Durch eine Selbstverpflichtung kann das Unternehmen der Auferlegung umfassender regulatorischer Verpflichtungen entgegenwirken. Die positiven Auswirkungen einer umfassenden Selbstverpflichtung stellen einen Anreiz für die vertikale Desintegration dar.

Im Ergebnis wird die Behörde die Selbstverpflichtungen jedoch nur dann ausreichen lassen und für bindend erklären, wenn sie sehr umfassend die Funktion regulatorischer Verpflichtungen übernehmen. Es ist deswegen fraglich, inwieweit das Instrument einen umfassenden eigenständigen Anreiz zur freiwilligen Entflechtung darstellt. Vielmehr ist davon auszugehen, dass es ohnehin zu diesem Schritt entschlossene Unternehmen in diesem Entschluss bestätigt.

\section{Umsetzung der unionalen Vorgaben im nationalen Recht}

Die Vorgaben des $₫ 41$ TKG müssen im Rahmen der Umsetzung des EKEK in deutsches Recht umfassend überarbeitet werden. Geplant ist eine Regelung in $\$ 32$ TKG-RefE. ${ }^{607}$ Das Instrument der Verpflichtungszusage ist dem geltenden TKG noch fremd. Das Instrument soll durch $\$ 32$ Abs. 2 TKG-RefE in die Vorgaben zur freiwilligen Entflechtung implementiert werden. Weitere normative Anknüpfungspunkte für das Instrument werden in den $\$ \mathbb{S} 18$ und 19 TKG-RefE angelegt werden (Kapitel 5, D. II. 3. a. cc.). Zwar spielt die Verpflichtungszusage in Form öffentlich-rechtlicher Verträge zwischen der BNetzA und den Telekommunikationsunternehmen in der regulatorischen Praxis bereits eine Rolle, ist allerdings noch nicht normativ angelegt. ${ }^{608}$

607 Referentenentwurf des Telekommunikationsmodernisierungsgesetzes (TKMoG), abrufbar unter: https://www.bmwi.de/Redaktion/DE/Downloads/Gesetz/ telekommunikationsmodernisierungsgesetz-referentenentwurf-20201612.pdf? blob=publicationFile $\& v=8$ (zuletzt abgerufen am 12.1.2021).

608 Die BNetzA hat das Angebot zum Abschluss eines öffentlich-rechtlichen Vertrages im Vorfeld der Vectoring-II-Entscheidung umfassend zum Gegenstand ihres Regulierungsermessens gemacht, BNetzA, Beschl. v. 1.9.2016, BK 3g-15/004; bestätigt durch VG Köln, Urt. v. 22.9.2016, Az. VG 1 K 5885/13, nicht veröffentlicht und BVerwG, Urt. v. 21.9.2018, Az. 6 C 50.16, BVerwGE 163, 136. Im Vorfeld der Vectoring-II-Entscheidung der BNetzA haben sich hierzu geäußert Kühling/Bulowski, N\&R 2015, S. 262, 266 ff.; hierzu weiterführend auch Offenbächer, Die Regulierung des Vectoring, S. $313 \mathrm{ff}$. 


\section{Anpassungsbedarf der Vorgaben de lege ferenda?}

Die regulatorischen Entflechtungsvorgaben sind auf die Schaffung und Erhaltung von Wettbewerb ausgerichtet. Dies zeigt sich auch in der Konstruktion der Vorgaben zur freiwilligen Entflechtung. Für Unternehmen mit beträchtlicher Marktmacht besteht der Anreiz zur freiwilligen Entflechtung in der Aussicht, als marktmächtiges Unternehmen mit der Abgabe von Verpflichtungszusagen weniger intensive Regulierungsverpflichtungen auferlegt zu bekommen. Für Unternehmen, die nicht der Ex-anteRegulierung unterliegen, sehen die regulatorischen Vorgaben hingegen keine Anreize vor. Dies ist jedoch auch an dieser Stelle systematisch nicht möglich, da nur Unternehmen von Regulierungsprivilegierungen profitieren können, die auch reguliert werden. Anreize zur Entflechtung vertikal integrierter Telekommunikationsunternehmen ohne beträchtliche Marktmacht an dieser Stelle des Gesetzes zu implementieren, wäre systemfremd. Es sollen lediglich Anreize geschaffen werden, um eine Unternehmensstrukturierung aufzugeben, die zu Diskriminierungen tendiert. Somit besteht auch in dieser Hinsicht de lege ferenda kein Anpassungsbedarf der regulatorischen Vorgaben.

\section{Zwischenergebnis}

Vertikal integrierte Telekommunikationsunternehmen können freiwillig, etwa aus strategischen Erwägungen, beschließen, sich aufzuspalten. Ein solches Vorgehen war etwa beim tschechischen Telekommunikationsunternehmen CETIN zu beobachten.

Der Entschluss wird in diesem Zusammenhang durch die Leitungsorgane des Unternehmens getroffen. Da Telekommunikationsunternehmen regelmäßig als Gesellschaften strukturiert sind, ergeben sich die Vorgaben hierfür aus dem UmwG. Das Telekommunikationsrecht weist nur einen rudimentären Regelungsbestand auf, der darauf ausgerichtet ist, den diskriminierungsfreien Zugang und Endkundenwettbewerb auch nach der Entflechtung eines marktmächtigen Unternehmens sicherzustellen. Um dieses Ziel zu erreichen, statuiert Art. 78 EKEK wechselseitige Informations- und Handlungspflichten für Unternehmen und Behörden. Die Förderung der Konnektivität ist allenfalls unbeabsichtigte Nebenfolge. Während den Unternehmen insbesondere aufgegeben wird, die Behörden frühzeitig über die Separierungsabsichten zu informieren, müssen diese auf Basis der Informationen Maßnahmen ergreifen, um den diskriminierungsfreien $\mathrm{Zu}$ - 
gang auf der Vorleistungsebene sicherzustellen. Die Aufgabe der Regulierungsbehörde ist damit eher konservativ denn gestaltend und einflussnehmend. Durch den novellierten unionalen Rechtsrahmen wurde die Möglichkeit dazu geschaffen, durch Selbstverpflichtungen, die als Abwägungstopos in das Regulierungsermessen einbezogen werden können, die Regulierungsintensität zu reduzieren. Der eigenverantwortlichen Selbstregulierung der marktbeherrschenden Unternehmen wird auf diese Weise der Vorzug vor der verpflichtenden Regulierung durch den Staat gegeben. Durch diese Maßnahme wird die Attraktivität der freiwilligen Separierung für marktmächtige Unternehmen erhöht. Aufgrund der strengen Anforderungen an die Verpflichtungszusage beschränkt sich dieser Anreizeffekt im Wesentlichen auf solche Unternehmen, die ohnehin dazu entschlossen sind, sich zu entflechten.

\section{Gründung als Wholesale-Only-Unternehmen}

Neben der Möglichkeit, ein vertikal integriertes Unternehmen zu entflechten, kann ein Wholesale-Only-Anbieter direkt als ein solcher gegründet werden. Dies geschieht, indem bereits bei der Gründung eines Telekommunikationsunternehmens auf eine vertikale Integration verzichtet und das Tätigkeitsspektrum auf den Vorleistungsbereich fokussiert wird. In verschiedenen Mitgliedstaaten der Europäischen Union haben sich einige Unternehmen für diese strategische Ausrichtung entschieden (dazu I.). Der unionale Rechtsrahmen enthält für diese Unternehmen mit Art. 80 EKEK eine spezielle Regulierungsvorschrift für Unternehmen, die ausschließlich auf der Vorleistungsebene tätig sind, und privilegiert diese regulatorisch (dazu II.).

I. Beispiele für ausschließlich auf der Vorleistungsebene tätige Unternehmen

Um die nachfolgenden Darstellungen zu illustrieren, sollen zwei Fallbeispiele dargestellt werden. Hierbei handelt es sich um das schwedische Unternehmen AB Stokab (dazu 1.) und das deutsche Unternehmen GasLINE (dazu 2.). Beide Unternehmen konzentrieren ihr Tätigkeitsspektrum aus unterschiedlichen strategischen Erwägungen ausschließlich auf den Vorleistungsmarkt. Darüber hinaus ist auch das deutsche Kooperationsunter- 
nehmen Glasfaser Nordwest GmbH als Wholesale-Only-Anbieter tätig. ${ }^{609}$ Es soll allerdings erst im Zusammenhang mit Kooperationen zur Beschleunigung des Glasfaserausbaus vertieft betrachtet werden (Kapitel 5, B. I.).

\section{Kommunaler Glasfaserausbau durch AB Stokab in Stockholm}

In der schwedischen Hauptstadt Stockholm sind drei große Unternehmen am Ausbau der Glasfaserinfrastruktur beteiligt. ${ }^{610}$ Eines ist das Unternehmen AB Stokab, das 1994 von der Stadt Stockholm gegründet wurde und vollständig in deren Eigentum steht. ${ }^{611} \mathrm{AB}$ Stokab ist ausschließlich auf der Vorleistungsebene tätig und erbringt keine Telekommunikationsdienste gegenüber dem Endkunden. ${ }^{612}$ Die Gesellschaft ist für den Ausbau, die Wartung und den Vertrieb ihres Telekommunikationsnetzes verantwortlich, das von über 100 Diensteanbietern im Stadtgebiet genutzt wird. ${ }^{613} \mathrm{Im}$ Jahr 2017 waren 93 Prozent der Haushalte 614 und 100 Prozent der Unternehmen in Stockholm mit Glasfaseranschlüssen versorgt. ${ }^{615}$ Der Glasfaserausbau wurde bislang vollständig eigenwirtschaftlich und ohne die Inanspruchnahme von Fördermitteln finanziert. ${ }^{616}$

Im Rahmen ökonomischer Untersuchungen wurden verschiedene Komponenten für den Erfolg des Geschäftsmodells identifiziert. In Stockholm existiere ein klarer politischer Konsens darüber, dass nachhaltige Investitionen in Glasfaserinfrastruktur sozio-ökonomische Vorteile mit sich brin-

609 Sörries, N\&R 2020, S. 161, 162.

610 Zum Wettbewerb der großen Unternehmen, die sich in Stockholm am Glasfaserausbau beteiligen, ausführlich Godlovitch/Sörries/Gantumur, A tale of five cities, S. 61 ff.; eine umfassende Case-Study über das Unternehmen führen durch Godlovitch/Lemstra/Pennings u.a., Regulatory, in particular access, regimes for network investment models in Europe, S. $182 \mathrm{ff}$.

611 FTTH Council Europe, AB Stokab, S. 1; Wernick/Queder/Strube Martins u.a., Ansätze zur Glasfaser-Erschließung unterversorgter Gebiete, S. 33.

612 Godlovitch/Sörries/Gantumur, A tale of five cities, S. 13; Wernick/Queder/Strube Martins u.a., Ansätze zur Glasfaser-Erschließung unterversorgter Gebiete, S. 34.

613 FTTH Council Europe, AB Stokab, S. 1; Wernick/Queder/Strube Martins u.a., Ansätze zur Glasfaser-Erschließung unterversorgter Gebiete, S. 34.

614 Die hohe Versorgungrate für Haushalte lässt sich auch damit begründen, dass etwa 90 Prozent der Bevölkerung in Mehrfamilienhäusern wohnen, vgl. hierzu FTTH Council Europe, AB Stokab, S. 1; vgl. zum Zahlenwert Godlovitch/Sörries/ Gantumur, A tale of five cities, S. 15.

615 FTTH Council Europe, AB Stokab, S. 1.

616 FTTH Council Europe, AB Stokab, S. 2; Wernick/Queder/Strube Martins u.a., Ansätze zur Glasfaser-Erschließung unterversorgter Gebiete, S. 34. 
gen. ${ }^{617}$ Die Kommune als Eigentümerin kalkuliere mit langen Amortisierungszeiträumen für ihre Investitionen und plane langfristig, sodass ein schrittweiser und planvoller Ausbau möglich sei. ${ }^{618}$ Durch die Existenz eines umfassenden Vorleistungsangebotes werden zudem die Investitionsbedingungen für alternative Infrastrukturanbieter verschlechtert, sodass wenig Wettbewerb im Netz bestehe und die Investitionen der Stokab AB unter erleichterten Bedingungen refinanzierbar seien. ${ }^{619}$ Die hieraus folgende Marktmacht werde jedoch nicht zur Durchsetzung prohibitiver Preise genutzt. ${ }^{620}$ Das Unternehmen bekenne sich vielmehr klar zur Stellung als Wholesale-Only-Anbieter und sei bestrebt, das Netz größtmöglich auszulasten. ${ }^{621}$

Fraglich ist, ob sich diese positiven Effekte auch bei einer Umsetzung des Konzeptes in Deutschland im Glasfaserausbau in der Fläche zeigen würden. Eine einfache Übertragung der Studienergebnisse ist jedenfalls nicht möglich, da sich der Ausbau der AB Stokab auf die schwedische Hauptstadt konzentriert. Im urbanen Umfeld stellt sich ein zentrales Problem des Glasfaserausbaus in der Fläche, nämlich die Notwendigkeit zur Überbrückung großer Distanzen und die begrenzten Amortisationsmöglichkeiten wegen weniger verfügbarer Haushalte, nicht in derselben Intensität. Dennoch wird an diesem Beispiel sichtbar, dass die Tätigkeit als Wholesale-Only-Anbieter unter bestimmten Voraussetzungen ein tragfähiges Geschäftsmodell ist.

\section{Geschäftsmodell der Mitverlegung der deutschen GasLINE}

Ein Beispiel für ein deutsches Unternehmen, das ausschließlich im Vorleistungsbereich tätig ist und keine Dienstleistungen für den Endkunden erbringt, ist die GasLINE Telekommunikationsnetzgesellschaft deutscher

617 Godlovitch/Lemstra/Pennings u.a., Regulatory, in particular access, regimes for network investment models in Europe, S. 183 ff.; Wernick/Queder/Strube Martins u.a., Ansätze zur Glasfaser-Erschließung unterversorgter Gebiete, S. 34.

618 Wernick/Queder/Strube Martins u.a., Ansätze zur Glasfaser-Erschließung unterversorgter Gebiete, S. 34.

619 Wernick/Queder/Strube Martins u.a., Ansätze zur Glasfaser-Erschließung unterversorgter Gebiete, S. 34.

620 Wernick/Queder/Strube Martins u.a., Ansätze zur Glasfaser-Erschließung unterversorgter Gebiete, S. 34.

621 Wernick/Queder/Strube Martins u.a., Ansätze zur Glasfaser-Erschließung unterversorgter Gebiete, S. 34. 
Gasversorgungsunternehmen mbH \& Co. KG (GasLINE). Das Unternehmen wurde von verschiedenen Energieversorgungsunternehmen gegründet, die über ein bundesweites Gasversorgungsnetz verfügen. Gaspipelines werden etwa in einer Tiefe von einem Meter verlegt und verfügen beidseitig über einen etwa drei bis fünf Meter breiten Schutzstreifen. ${ }^{62}$ In einer Entfernung von etwa zwei Metern zur Gaspipeline verlegt GasLINE Kabelschutzrohre, in die Glasfaserkabel eingeführt werden. ${ }^{623}$ Es werden somit Tiefbauarbeiten genutzt, um mehrere Infrastrukturen parallel zu verlegen. Das Unternehmen nutzt die Leerrohre und Baustellen der teilhabenden Gasversorgungsunternehmen, um ein deutschlandweites Telekommunikationsnetz zu errichten. Hierdurch werden hohe Synergieeffekte erzielt. Die Kabel werden Diensteanbietern für ihre Tätigkeit gegenüber dem Endkunden zur Verfügung gestellt. ${ }^{624}$ Für das Unternehmen stehen also diese Synergieeffekte im Vordergrund. Die Steigerung der Konnektivität ist dabei eine Nebenfolge. Ein Glasfaserausbau ohne Zusammenhang zum Ausbau von Gaspipelines liegt nicht im ursprünglichen Unternehmensinteresse.

\section{Regulatorischer Umgang mit Wholesale-Only-Anbietern}

Der unionale Rechtrahmen enthält in Art. 80 EKEK eine Vorschrift, die Vorgaben für den regulatorischen Umgang mit Unternehmen macht, die ausschließlich auf der Vorleistungsebene tätig sind. Die Vorschrift wurde im Rahmen der Novelle des unionalen Rechtsrahmens vollständig neu konzipiert und ist demnach noch in nationales Recht umzusetzen.

Regulierungsverpflichtungen können nur solchen Unternehmen auferlegt werden, die in einem Markt über beherrschende Stellung im Sinne des Art. 67 EKEK verfügen. ${ }^{625}$ Demnach stehen vor der Auswahl der geeigneten Regulierungsverpflichtung zunächst die Marktdefinition und -analyse. Auch diese Vorschrift ist mithin ausschließlich auf den Wettbewerbsschutz ausgerichtet, nicht auf die Steigerung der Konnektivität. Der Gesetzgeber skizziert in den Erwägungsgründen zu dieser Norm, dass er davon ausgeht, dass ein umfassender Vorleistungswettbewerb auch positive

622 https://www.gasline.de/netz/lwl-backbone/ (zuletzt abgerufen am 1.7.2020).

623 https://www.gasline.de/netz/lwl-backbone/ (zuletzt abgerufen am 1.7.2020).

624 Siehe hierzu die Angaben des Unternehmens auf der eigenen Website, abrufbar unter https://www.gasline.de/netz/ (zuletzt abgerufen am 1.7.2020).

625 Kühling, in: Ruffert (Hrsg.), Europäisches Sektorales Wirtschaftsrecht, $\mathbb{} 4$, Rn. 129. 
Effekte für den Endkundenmarkt mit sich bringen kann. ${ }^{626}$ Hierdurch fügt sie sich in das zuvor skizzierte normative Umfeld ein (hierzu B. I. 1. a., C. III. 1.)

Der Unionsgesetzgeber knüpft strenge Voraussetzungen an die Feststellung, dass ein Unternehmen ausschließlich auf der Vorleistungsebene tätig ist. ${ }^{627}$ Es darf weder direkte noch indirekte Tätigkeiten auf dem Endkundenmarkt geben (Art. 80 Abs. 1 lit. a EKEK). Diese sehr enge Normierung führt dazu, dass für die Sonderregelungen nur ein sehr begrenzter Anwendungsbereich bleibt und nur eine geringe Anreizwirkung von ihr ausgeht. ${ }^{628}$ So führt etwa auch eine Beteiligung an einem Unternehmen, das Endkundendienste erbringt, dazu, dass die Tatbestandsvoraussetzungen der Vorgabe nicht erfüllt sind. Darüber hinaus darf auch keine Exklusivvereinbarung mit einem Unternehmen bestehen, das auf dem Endkundenmarkt tätig ist (Art. 80 Abs. 1 lit. b EKEK). Die strenge Interpretation einer Tätigkeit, die ausschließlich auf die Vorleistungsebene beschränkt ist, lässt sich darauf zurückführen, dass mit der Stellung als Wholesale-Only-Anbieter eine umfassende regulatorische Privilegierung einhergeht (A. III.). Diese wird wiederum mit den geringeren Diskriminierungsanreizen aufgrund der fehlenden vertikalen Integration gerechtfertigt. ${ }^{629}$ So dürfen gemäß Art. 80 Abs. 2 EKEK nur Verpflichtungen gemäß Art. 70 und Art. 73 EKEK auferlegt werden.

Fraglich ist, ob es insoweit überhaupt einer speziellen Regelung bedurft hätte. ${ }^{630}$ Die Regulierungsbehörden verfügen über ein Auswahlermessen hinsichtlich der Regulierungsverpflichtungen. Dies gestattet es ihnen, nur diejenigen Regulierungsverfügungen anzuwenden, die tatsächlich die spezifischen Gefahren für den Wettbewerb adressieren, die von einem Unternehmen ausgehen. Die Regulierungsbehörden hätten sich auch ohne eine explizite Normierung auf die aufgezählten regulatorischen Verpflichtungen beschränkt. 631

Während in Art. 70 EKEK die Nichtdiskriminierungsverpflichtung normiert ist, betrifft Art. 73 EKEK die Zugangsverpflichtung auf die Infrastruktur im Vorleistungsbereich. Der Fokus des regulatorischen Vorgehens

626 Erwägungsgrund 208 des EKEK; Sörries, N\&R 2020, S. 161, 163.

627 Kühling, in: Ruffert (Hrsg.), Europäisches Sektorales Wirtschaftsrecht, $\mathbb{S} 4$, Rn. 129; Neumann, N\&R 2018, S. 204, 209.

628 Ebenfalls kritisch Neumann, N\&R 2018, S. 204, 210.

629 Erwägungsgrund 205 des EKEK; Sörries, N\&R 2020, S. 161, 163.

630 In dieser Hinsicht ebenfalls kritisch Neumann, N\&R 2018, S. 204, 210.

631 Positiver argumentiert Sörries, N\&R 2020, S. 161, 166, der davon ausgeht, dass die Vorschrift eine „angemessene Regulierung“ ermöglicht. 
der zuständigen Behörde soll auf der Sicherstellung fairer Preise für Vorleistungsprodukte liegen. ${ }^{632}$ Hierfür sind jedoch nur Teile der regulatorischen Handlungsoptionen geeignet, sodass das Maßnahmenportfolio bereits auf normativer Ebene eingeschränkt wird. ${ }^{633}$ Das Gesetz schreibt einen erhöhten Rechtfertigungsdruck für die nationalen Regulierungsbehörden vor, um die Angemessenheit der jeweiligen Regulierungsverfügung zu begründen. Sofern die Voraussetzungen des Art. 80 Abs. 1 EKEK nicht mehr erfüllt sind, stehen gemäß Art. 80 Abs. 3 EKEK wieder alle Regulierungsverfügungen der Art. 69 bis 74 EKEK zur Verfügung. ${ }^{634}$ Dies ist folgerichtig, da in diesem Fall die Grundlage der regulatorischen Privilegierung entfällt.

Die Regulierungsbehörden führen ein regelmäßiges Monitoring durch. Zudem bestehen für die Unternehmen Mitteilungspflichten, sofern sich die für Art. 80 Abs. 1 EKEK maßgeblichen Umstände ändern. Beeinträchtigt das Unternehmen die Endkundenmärkte, können ihm im Ausnahmefall gemäß Art. 80 Abs. 4 EKEK auch die übrigen Regulierungsverfügungen auferlegt werden, auch wenn es nicht auf den betreffenden Märkten tätig ist. Hierdurch wird der Regulierungsbehörde im Einzelfall die Möglichkeit dazu eröffnet, auch strengere Maßnahmen zu ergreifen. Durch die Flexibilität des Rechtsrahmens kann die Behörde, trotz der regulatorischen Privilegierung, effektiv gegen Gefahren für den Wettbewerb vorgehen. Stets sind gemäß Art. 80 Abs. 5 EKEK die Konsultations- und Konsolidierungsverfahren aus Art. 23, 32 und 33 EKEK durchzuführen. 635

Die nationale Vorgabe in $\$ 33$ TKG-RefE ist weit kompakter konzipiert als das unionsrechtliche Pendant und wird voraussichtlich lediglich in zwei Absätze untergliedert werden. ${ }^{636}$ Dies wird mithilfe einer Konsolidierung und dem Verzicht auf deklaratorische Vorgaben des unionsrechtlichen Pendants erreicht. Die wesentlichen Inhalte der Vorgabe werden jedoch unionsrechtskonform in das nationale Recht übertragen werden.

632 Kühling, in: Ruffert (Hrsg.), Europäisches Sektorales Wirtschaftsrecht, $\mathbb{} 4$, Rn. 130.

633 Erwägungsgrund 205 des EKEK.

634 Sörries, N\&R 2020, S. 161, 163.

635 Kühling, in: Ruffert (Hrsg.), Europäisches Sektorales Wirtschaftsrecht, $\mathbb{} 4$, Rn. 131.

636 Referentenentwurf des Telekommunikationsmodernisierungsgesetzes (TKMoG), abrufbar unter: https:/www.bmwi.de/Redaktion/DE/Downloads/Gesetz/ telekommunikationsmodernisierungsgesetz-referentenentwurf-20201612.pdf? blob=publicationFile $\& v=8$ (zuletzt abgerufen am 12.1.2021). 


\section{Zwischenergebnis und Ausblick}

Unternehmen können sich von Beginn an strategisch so ausrichten, dass sie ausschließlich auf der Vorleistungsebene tätig werden. Der Unionsgesetzgeber hat in Art. 80 EKEK eine neue Norm geschaffen, um diese Unternehmen bei Bedarf ebenfalls regulieren zu können, zugleich aber dem reduzierten Missbrauchsrisiko durch eine umfassende Privilegierung gegenüber vertikal integrierten Unternehmen Rechnung zu tragen. ${ }^{637}$ Die Vorschrift ist ausschließlich auf den Schutz des Wettbewerbs ausgerichtet. Die Förderung der Konnektivität ist allenfalls eine bloße Nebenfolge.

Der Begriff des ausschließlich auf der Vorleistungsebene tätigen Unternehmens wird sehr eng interpretiert. Deswegen ist kritisch zu hinterfragen, ob von der Vorschrift überhaupt ein weitgehender Anreizeffekt ausgehen kann. Unternehmen, die in den Anwendungsbereich des Art. 80 EKEK fallen, können in der Regel nur einem reduzierten Spektrum regulatorischer Verpflichtungen unterworfen werden. Kritisch ist dazu anzumerken, dass dies auch bisher möglich gewesen wäre. Insoweit hätte es keiner neuen, aufgrund ihres Umfangs sehr komplexen, Vorschrift bedurft. Die notwendige Umsetzung in nationales Recht soll in Gestalt des $\mathbb{} 33$ TKG Ref-E erfolgen. ${ }^{638}$

Die regulatorische Privilegierung kann dazu beitragen, die Attraktivität der Gründung eines Wholesale-Only-Anbieters zu steigern. Aufgrund der hohen Anforderungen an die Entflechtungsintensität ist jedoch von einem vergleichsweise geringen Anreizeffekt auszugehen. Die Entscheidung zur Gründung als Wholesale-Only-Anbieter beruht, wie die Fallbeispiele erfolgreicher Unternehmen zeigen, in der Regel auf der strategischen Ausrichtung und dem Marktumfeld. Hierauf hat der Gesetzgeber nur begrenzte direkte Einflussmöglichkeiten. Die Norm fügt sich zwar insoweit in den skizzierten regulatorischen Rechtsrahmen ein. Da sie aber ohne einen erheblichen Mehrwert die Komplexität des Rechtsrahmens steigert, sollte der Unionsgesetzgeber erwägen, sie zu optimieren oder wieder zu entfernen.

637 Erwägungsgrund 205 des EKEK.

638 Referentenentwurf des Telekommunikationsmodernisierungsgesetzes (TKMoG), abrufbar unter: https:/www.bmwi.de/Redaktion/DE/Downloads/Gesetz/ telekommunikationsmodernisierungsgesetz-referentenentwurf-20201612.pdf? blob=publicationFile\&v=8 (zuletzt abgerufen am 12.1.2021). 


\section{E. Optimierungsvorschläge außerhalb der regulatorischen Entflechtungsbestimmungen}

Der regulatorische Rechtsrahmen für die Entflechtungsbestimmungen ist konsistent auf den Wettbewerbsschutz ausgerichtet und sollte nicht zur Förderung der Konnektivität erweitert werden.

Alternativ wurden verschiedene Anpassungsvorschläge außerhalb der regulatorischen Entflechtungsvorgaben entwickelt, um die Anzahl der Unternehmen, die sich für eine Tätigkeit als Wholesale-Only-Anbieter entscheiden, nachhaltig zu steigern.

Einer dieser Vorschläge ist die Optimierung des Förderrahmens. ${ }^{639}$ Die Kommunen können sich im Rahmen der Beantragung von Fördermitteln entweder für eine Wirtschaftlichkeitslückenförderung oder für das Betreibermodell entscheiden. Unter der Wirtschaftlichkeitslücke wird gemäß $\$ 7$ Abs. 1 des Entwurfes zum Graue-Flecken-Förderprogramm (GFFP-E) ${ }^{640}$ die „Differenz zwischen dem Barwert aller Erlöse und dem Barwert aller Kosten des Netzaufbaus und -betriebs" verstanden. Die Fördersumme entspricht der Wirtschaftlichkeitslücke. Der GFFP-E setzt den Aufbau und Betrieb von Infrastruktur gemäß $₫ 3$ Abs. 1 lit. a in ein Alternativitätsverhältnis und steht einer Fördermittelvergabe an Wholesale-Only-Anbieter nicht entgegen. ${ }^{641}$ Mithin können sich Wholesale-Only-Anbieter an Auswahlverfahren beteiligen und der Förderrechtsrahmen verhält sich hinsichtlich des Geschäftskonzeptes neutral. Beim Betreibermodell werden Fördermittel für den Aufbau einer Infrastruktur gewährt, die im Eigentum der Kommune bleibt und im Anschluss durch ein Unternehmen betrieben wird. In diesen Gebieten können Wholesale-Only-Anbieter lediglich Vorleistungsprodukte vertreiben. Betriebswirtschaftlich wäre dies jedoch nur eingeschränkt attraktiv, da das Geschäftsmodell von Wholesale-Only-Anbietern

639 Wernick/Queder/Strube Martins u.a., Ansätze zur Glasfaser-Erschließung unterversorgter Gebiete, S. 42.

640 Entwurf über eine Rahmenregelung der Bundesrepublik Deutschland zur Unterstützung des flächendeckenden Aufbaus von Gigabitnetzen in „grauen Flecken“ vom 22.5.2019, abrufbar unter https://www.bmvi.de/SharedDocs/DE/Anl age/DG/breitbandfoerderung-gigabit-rahmenregelung.pdf?_blob=publicationF ile (zuletzt abgerufen am 1.7.2020).

641 Kritisch zum vorherigen Förderrahmen Wernick/Queder/Strube Martins u.a., Ansätze zur Glasfaser-Erschließung unterversorgter Gebiete, S. 42. 
maßgeblich dadurch bestimmt wird, dass die Telekommunikationsinfrastruktur in ihrem Eigentum ist. ${ }^{642}$

Vorgeschlagen wird daher, die Wahl des Betreibermodells auf solche Konstellationen zu beschränken, in denen sich kein Wholesale-Only-Anbieter am Auswahlverfahren beteiligt, der die Infrastruktur wirtschaftlicher betreiben kann. ${ }^{643}$ Eine solche Stufung ist im derzeitigen Förderrahmen zwar nicht vorgesehen, wäre jedoch denkbar. Damit könnte ein indirekter Anreiz für ein eine vertikale Desintegration gesetzt werden. Dies wäre vor dem Hintergrund zu befürworten, dass in den betreffenden Gebieten grundsätzlich keine parallele Infrastruktur ausgebaut wird und auf diese Weise zumindest auf der Diensteebene der Wettbewerb verbessert werden könnte. Während sich die Wettbewerbsbehörden passiv verhalten sollten, darf der Fördermittelgeber den Markt seinen Vorgaben entsprechend aktiv gestalten.

Im Übrigen wird diskutiert, die derzeitige Entgeltregulierung umzugestalten und den Regulierungsansatz umfassend abzuändern. Es soll von einer Regulierung, die an Preisen orientiert wird, auf ein System gewechselt werden, das das Infrastruktureigentum in den Vordergrund stellt. Solche Modelle seien im Wasserrecht erfolgreich getestet worden. ${ }^{644}$ Diese Maßnahme adressiert allerdings wiederum insbesondere marktmächtige Unternehmen. Für den Breitbandausbau in der Fläche sind jedoch auch Unternehmen von Relevanz, die nicht über Marktmacht verfügen und lediglich regional begrenzt tätig sind. Darüber hinaus wäre eine umfassende Umstrukturierung des Rechtsrahmens erforderlich, die angesichts der in tatsächlicher Hinsicht ungewissen Effektivität der Maßnahme im Infrastrukturausbau derzeit nicht vorgenommen werden sollte. Zuvor müsste ihre Effektivität mithilfe umfassender ökonomischer Studien nachgewiesen werden, welche die Ausbaurealität besser widerspiegeln als eine Greenfield-Analyse (A. I. 3.). Darüber hinaus müssten auch die Auswirkungen auf die übrigen Regulierungsziele evaluiert werden. In jedem Fall haben positive Erfahrungen aus der Wasserwirtschaft nur eingeschränkte Aussagekraft für den Telekommunikationssektor, da die Sektoren sehr hetero-

642 Hierzu im Ergebnis auch Wernick/Queder/Strube Martins u.a., Ansätze zur Glasfaser-Erschließung unterversorgter Gebiete, S. 42.

643 Wernick/Queder/Strube Martins u.a., Ansätze zur Glasfaser-Erschließung unterversorgter Gebiete, S. 42.

644 Weiterführend hierzu Felten/Langer, Structurally indepent broadband infrastructure can solve perceived FTTH coverage issues, S. $21 \mathrm{f}$. 
gen sind und zunächst ein umfassender Vergleich der Ausgangslage erforderlich wäre. ${ }^{645}$

Rein tatsächlich müssten zudem praxisfähige Open-Access-Plattformen und technische Lösungen forciert werden, die dazu beitragen, dass eine Tätigkeit als Wholesale-Only-Anbieter überhaupt technisch möglich und sinnvoll ist. ${ }^{646}$ Durch die Vielzahl der erforderlichen Vereinbarungen mit unterschiedlichen Diensteanbietern müssen die Prozesse weiterentwickelt, vereinfacht und standardisiert werden, um das Geschäftsmodell für mehr Unternehmen tragfähig zu machen. Dieser Handlungsbedarf betrifft jedoch eher die technische Abbildbarkeit des Geschäftsmodells auf Großhandelsebene und kann nur eingeschränkt über rechtliche Vorgaben gesteuert werden.

\section{F. Fazit}

Auf dem deutschen Telekommunikationsmarkt sind nur wenige Wholesale-Only-Anbieter aktiv, obgleich betriebswirtschaftlich sowohl Argumente für eine vertikal integrierte Unternehmensorganisation als auch für eine Tätigkeit als Wholesale-Only-Anbieter sprechen. Es wird vertreten, dass eine größere Präsenz von Wholesale-Only-Anbietern auf dem deutschen Telekommunikationsmarkt den Infrastrukturausbau beschleunigen würde. Bei den zugrunde liegenden Untersuchungen handelt es sich um Modellbetrachtungen, die verschiedene Annahmen voraussetzen, die in realen Ausbauszenarien nur selten gebündelt auftreten. Insoweit wären umfassende zusätzliche ökonomische Analysen erforderlich, welche die Ausbaurealität besser widerspiegeln.

Dennoch stellt sich insbesondere die Frage, ob der regulatorische Rechtsrahmen optimiert werden muss, um die Zahl der Wholesale-OnlyAnbieter auf dem Telekommunikationsmarkt zu erhöhen. Dabei ist auffällig, dass die Vorgaben systematisch in das Marktregulierungsverfahren eingebettet sind und ausschließlich den Wettbewerbsschutz adressieren. Der Rechtsrahmen sieht keine Befugnisnormen vor, um vertikal integrierte Te-

645 Zur Übertragung von Erwägungen vom Energie- auf den Telekommunikationssektor bereits umfassend Kapitel 3, C. II. 1. b. cc. (1).

646 Gries/Plückebaum/Strube Martins, Kostentreiber für den Ausbau hochbitratiger Infrastrukturen, S. 55 f.; Wernick/Queder/Strube Martins u.a., Gigabitnetze für Deutschland, S. 105 f.; Wernick/Queder/Strube Martins u.a., Ansätze zur GlasfaserErschließung unterversorgter Gebiete, S. 45 f. 
lekommunikationsunternehmen durch staatlichen Zwang so zu entflechten, dass Wholesale-Only-Anbieter entstehen. Dies ist aber ohnehin nicht der Zweck der Norm, die als ultima ratio konzipiert ist und sicherstellen soll, dass Unternehmen ihre marktbeherrschende Stellung nicht ausnutzen, um die Konkurrenz zu diskriminieren. Einen ähnlichen Ansatz verfolgt das Kartellrecht, das ausschließlich wettbewerbswidriges Verhalten unterbinden möchte und strukturelle Abhilfemaßnahmen vorsieht, sofern die Unternehmensstruktur in einem kausalen Zusammenhang zu dem wettbewerbsrechtswidrigen Verhalten steht. Die Entflechtungsintensität und die Zweckrichtung beider Regelungsmaterien zu erweitern, um den Infrastrukturausbau zu beschleunigen - wobei die tatsächliche Wirksamkeit noch nicht einmal gesichert ist -, wäre mit der Systematik der Gesetze und ihrem Telos nicht in Einklang zu bringen. Es müsste vielmehr eine neue Rechtsgrundlage geschaffen werden, die nicht an Marktmacht anknüpft. Dabei müsste insbesondere die Identifikation von Gebieten mit Netzausbaudefizit im Vordergrund stehen. Während die Vereinbarkeit der Rechtsgrundlage mit den Grundrechten durch eine Orientierung an der Struktur des Art. 77 EKEK sichergestellt werden könnte, wäre dies bei ihrer Anwendung im Einzelfall aufgrund der hohen Eingriffsintensität deutlich komplexer. Je effektiver die Maßnahme und je weniger intensiv die Entflechtung wäre, desto eher ließe sich der Grundrechtseingriff rechtfertigen. Im Ergebnis dürfte dies jedoch eher nicht gelingen.

Die regulatorischen Vorgaben stehen einer freiwilligen Entflechtung und der Gründung eines Unternehmens als Wholesale-Only-Anbieter neutral gegenüber, honorieren aber das reduzierte Diskriminierungspotential durch regulatorische Privilegierungen. Dieses System ist konsistent und sollte nicht abgeändert werden. Außerhalb dieser Vorgaben ließe sich jedoch durch eine Optimierung der Fördermodelle die Attraktivität des Marktumfeldes für Wholesale-Only-Anbieter steigern. Die Rolle des Fördermittelgebers könnte insoweit aktiv und gestaltend sein. Wichtig ist es vor allen Dingen, Abläufe zu standardisieren und Plattformen zu etablieren, um ein attraktives Marktumfeld zu schaffen. Diese Handlungsansätze liegen jedoch außerhalb des regulatorischen Rechtsrahmens. Dieser sollte in keinem Fall mit ungewissem Ausgang für die übrigen Regulierungsziele umfassend angepasst werden, um die Konnektivität zu fördern.

Ein Allheilmittel für den Infrastrukturausbau ist eine verstärkte Marktpräsenz von Wholesale-Only-Anbietern also insgesamt nicht. Allerdings könnte eine stärkere Marktdurchmischung als Ziel in eine Gesamtstrategie zur Beschleunigung des Glasfaserausbaus einfließen. In jedem Fall handelt es sich aber lediglich um ein kleines Zahnrad im großen Getriebe potenti- 
eller Handlungsoptionen auf dem Weg in die Gigabit-Gesellschaft. Die großen Herausforderungen blieben auch dann bestehen, wenn auf dem Telekommunikationsmarkt vermehrt Wholesale-Only-Anbieter aktiv wären. Um die Wirksamkeit dieser Handlungsoption überhaupt einschätzen zu können, wären jedoch vertiefte ökonomische Untersuchungen der positiven Effekte erforderlich. 


\section{Kapitel 5: Kooperationen im Glasfaserausbau}

Die Unternehmen des Telekommunikationssektors kündigen fortlaufend neue und vielseitige Kooperationsformen zur Beschleunigung des Ausbaus von Glasfaserinfrastrukturen an. ${ }^{647}$ Auch das Bundesministerium für Verkehr und digitale Infrastruktur begrüßt solche Kooperationen grundsätzlich. ${ }^{648}$ So variabel wie die von den Unternehmen verfolgten Interessen sind auch die Möglichkeiten für die Ausgestaltung der Zusammenarbeit. Aus diesem Grund müssen im Vorfeld der juristischen Diskussion einige begriffliche und ökonomische Vorfragen zur Eingrenzung des Untersuchungsgegenstandes geklärt werden (dazu A.).

Kooperationen sind zwar grundsätzlich dazu geeignet, Investitionsszenarien für Unternehmen attraktiver zu gestalten, stellen aber zugleich eine Gefahr für den Wettbewerb in den Kooperationsgebieten dar. Das sektorspezifische Regulierungsrecht, das Recht der Fusionskontrolle und das Kartellverbot finden nebeneinander Anwendung, um diese Gefahren für den Wettbewerb möglichst gering zu halten (dazu B.). ${ }^{649}$ Dabei stellen sich die Fragen, ob jedes Instrument für sich genommen sinnvoll funktioniert und ob eine parallele Anwendbarkeit der verschiedenen Regelungsmaterien sinnvoll ist oder ob sie zu Rechtsunsicherheiten und Kompetenzstreitigkeiten zwischen den jeweils zuständigen Behörden führt.

Alle Regelungsmaterien knüpfen an eine Untersuchung der Auswirkungen des Kooperationsvorhabens auf den Wettbewerb in bestimmten Märkten an. Hierzu müssen die Märkte zunächst abgegrenzt werden (dazu C.). Im Anschluss sollen die regulatorischen (dazu D.) und die kartellrechtlichen Rahmenbedingungen (dazu E.) für die Ausgestaltung von Kooperationsvorhaben erläutert werden. Der Fokus soll auf der Frage liegen, ob der

647 Siehe hierzu etwa die Kooperationsprojekte zwischen der Deutschen Telekom und der Deutschen Glasfaser, abrufbar unter: https://www.heise.de/newsticker/ meldung/FTTH-Pilotprojekt-Telekom-und-Deutsche-Glasfaser-kooperieren-4643 866.html (zuletzt abgerufen am 1.7.2020) oder das Kooperationsprojekt zwischen Vodafone und der Deutschen Glasfaser, abrufbar unter: https://presse.deu tsche-glasfaser.de/pressreleases/vodafone-und-deutsche-glasfaser-kooperieren-beiglasfaserausbau-fuer-privatkunden-2951095 (zuletzt abgerufen am 1.7.2020).

648 BMVI, Eckpunkte Zukunftsoffensive Gigabit-Deutschland, S. 3.

649 Zur parallelen Anwendbarkeit der Regelungsmaterien aus dem TKG und dem GWB bereits Kapitel 3, C. IV. 2. a. 
Rechtsrahmen und die behördliche Beschlusspraxis die Balance zwischen der Förderung von Kooperationsvorhaben auf der einen Seite und dem Schutz des Wettbewerbs auf der anderen Seite sicherstellen. Es wird zu erörtern sein, inwieweit diesbezüglich Optimierungen erforderlich sind.

\section{A. Grundlegende Erwägungen zur ökonomischen Bewertung von Kooperationen}

Eine strukturierte Analyse rechtlicher Zusammenhänge setzt die Klärung einiger grundlegender Fragestellungen voraus, die im Rahmen der Betrachtung aller Ausgestaltungsoptionen für den kooperativen Breitbandausbau relevant sind. Es stellt sich die Frage, welche Kriterien den Begriff der „Kooperation“ bestimmen und somit zur Abgrenzung des Untersuchungsgegenstandes herangezogen werden können (dazu I.). Für die ökonomische Bewertung von Kooperationen ist von Interesse, wie sie sich auf den Breitbandausbau in der Fläche auswirken und welche Motive die Unternehmen zu einem Engagement im Rahmen des kooperativen Breitbandausbaus veranlassen (dazu II.). Die Erkenntnisse sollen für die juristischen Überlegungen in diesem Kapitel fruchtbar gemacht werden (dazu III.).

\section{Abgrenzung des Kooperationsbegriffes}

Eine wissenschaftliche Untersuchung verschiedener Kooperationsmodelle erfordert eine Abgrenzung des Untersuchungsgegenstandes. Eine besondere Herausforderung resultiert aus den vielfältigen Ausgestaltungsmöglichkeiten einer kooperativen Zusammenarbeit. ${ }^{650}$ In der Rechtswissenschaft wird der Begriff der Kooperation untechnisch verwendet, um eine mehr oder minder institutionalisierte Zusammenarbeit zwischen verschiedenen Akteuren zu illustrieren.

Einige Charakteristika sind für die Zusammenarbeit zwischen Unternehmen im Glasfaserausbau, die in diesem Kapitel dargestellt werden soll, prägend, während andere Aspekte die spezifische Ausgestaltung des konkreten Kooperationsvorhabens betreffen. ${ }^{651}$ Allgemein soll nachfolgend

650 Hierzu umfassend Baum, Morphologie der Kooperation als Grundlage für das Konzept der Zwei-Ebenen-Kooperation, S. $11 \mathrm{ff}$.

651 Weiterführend hierzu Zentes/Swoboda/Morschett, in: Zentes/Swoboda/Morschett (Hrsg.), Kooperationen, Allianzen und Netzwerke, S. 3, 5. 
unter einer Kooperation eine „nicht auf einmalige Transaktionen angelegte, meist vertraglich abgesicherte, freiwillige Verbindung [...] mit anderen rechtlich selbstständig bleibenden Unternehmen [verstanden werden], die einzelne Unternehmensaktivitäten [betrifft], um einzelwirtschaftliche Ziele besser zu erreichen als in den alternativen marktwirtschaftlichen Koordinationsformen Markt und Unternehmen."652 Die sogleich detailliert zu untersuchenden Kooperationen weisen zudem die Gemeinsamkeit auf, dass das Ziel des Ausbaus von Glasfaserinfrastruktur durch die Zusammenarbeit schneller und effizienter eigenwirtschaftlich erreicht werden soll. ${ }^{653}$

Abzugrenzen sind Kooperationen von einer Unternehmensintegration und solchen Transaktionen, die dem Investitionsvorhaben nachgelagert sind. Integrationen sind dadurch geprägt, dass die beteiligten Unternehmen ihre gesellschaftsrechtliche Selbstständigkeit verlieren. ${ }^{654}$ Transaktionen finden hingegen statt, wenn eine finanzielle Beteiligung zur Amortisation von Investitionen erst zeitlich nach dem Ausbauvorhaben, etwa in der Gestalt der Zahlung von Zugangsentgelten, erfolgt. ${ }^{655}$

652 Theurl/Schweinsberg, Neue kooperative Ökonomie, S. 4; hierauf Bezug nehmend Tenbrock/Strube Martins/Wernick u.a., Co-Invest Modelle zum Aufbau von neuen FTTB/H-Netzinfrastrukturen, S. 30; so im Ergebnis auch Carter/Elixmann/ Marcus, Unternehmensstrategische und regulatorische Aspekte von Kooperationen beim NGA-Breitbandausbau, S. 4 f.; Gerpott, in: Zentes/Swoboda/Morschett (Hrsg.), Kooperationen, Allianzen und Netzwerke, S. 1205, 1211; Gerpott, MedienWirtschaft 4/2010, S. 10, 12; Monopolkommission, 10. Sektorgutachten Telekommunikation (2017), Rn. 135; Tenbrock, Der Glasfaserausbau in Deutschland, S. 112; Tenbrock, Systematisierung und Regulierungsnotwendigkeit von Glasfaserausbaukooperationen, S. 2; in der Ökonomie wird der Begriff der "Allianz“ als Synonym genutzt, siehe etwa Zentes/Swoboda/Morschett, in: Zentes/Swoboda/ Morschett (Hrsg.), Kooperationen, Allianzen und Netzwerke, S. 3, 5.

653 Carter/Elixmann/Marcus, Unternehmensstrategische und regulatorische Aspekte von Kooperationen beim NGA-Breitbandausbau, S. $19 \mathrm{f}$.

654 Zur Abgrenzung weiterführend Carter/Elixmann/Marcus, Unternehmensstrategische und regulatorische Aspekte von Kooperationen beim NGA-Breitbandausbau, S. 5 .

655 Gerpott, MedienWirtschaft 4/2010, S. 10, 13 f.; Tenbrock, Der Glasfaserausbau in Deutschland, S. 113. 


\section{II. Ökonomische Grundlagen}

Kooperationen können auf vielfältige Weise ausgestaltet werden. ${ }^{656}$ Einen großen Stellenwert nimmt in der ökonomischen Literatur folglich die Systematisierung von Kooperationsmöglichkeiten auf Basis ihrer Auswirkungen auf die Wettbewerbsintensität ein. Abgegrenzt wird zwischen der Reichweite der Kooperation, ${ }^{657}$ ihrer technischen Ausgestaltung, ${ }^{658}$ den beteiligten Kooperationspartnern ${ }^{69}$ und ihren Beiträgen im Vorhaben. Für die juristische Untersuchung ist auch die vertragliche Grundlage einer Kooperation von entscheidender Bedeutung. ${ }^{660}$

656 Hierzu grundlegend mit umfassenden Überblick über die ökonomische Literatur, Baum, Morphologie der Kooperation als Grundlage für das Konzept der Zwei-Ebenen-Kooperation, S. $11 \mathrm{ff}$.

657 Im Überblick Gerpott, MedienWirtschaft 4/2010, S. 10, 14 ff.; zur Auswirkung der gemeinsamen Wertschöpfungsintensität Gerpott, MedienWirtschaft 4/2010, S. 10, 15; weiterführend zu den Anknüpfungspunkten auf den verschiedenen Wertschöpfungsstufen auch etwa Elixmann/Ilic/Neumann/Plückebaum, The Economics of Next Generation Access, S. 10 f., 25, 38, 40 f., 87; siehe auch BRG, CoInvestment and Commercial Offers, S. 13 f.; OECD, Developments in Fibre Technologies and Investment, S. 27; zu den Auswirkungen der Größe des Kooperationsgebietes Gerpott, MedienWirtschaft 4/2010, S. 10, 16; Tenbrock/Strube Martins/Wernick u.a., Co-Invest Modelle zum Aufbau von neuen FTTB/H-Netzinfrastrukturen, S. 31 f.; BRG, Co-Investment and Commercial Offers, S. 14.

658 Weiterführend zur Frage des komplementären oder parallelen Aufbaus von Infrastruktur und den Auswirkungen auf die Wettbewerbsintenstität, differenzierend Tenbrock, Systematisierung und Regulierungsnotwendigkeit von Glasfaserausbaukooperationen, S. 9 f.; kritisch im Hinblick auf die Wettbewerbsintensität bei einem komplementären Ausbau BKartA, Hinweise zur wettbewerbsrechtlichen Bewertung von Kooperationen beim Glasfaserausbau in Deutschland, S. 20 ff.; ähnlich Kühling/Heimeshoff/Schall, K\&R Beihefter 1/2010, S. 1, 21; a. A. Gerpott, MedienWirtschaft 4/2010, S. 10, 19.

659 Eine Differenzierungen zwischen vertikaler, horizonaler und diagonaler Kooperationsrichtung nehmen vor Tenbrock/Strube Martins/Wernick u.a., Co-Invest Modelle zum Aufbau von neuen FTTB/H-Netzinfrastrukturen, S. 31; Tenbrock, Der Glasfaserausbau in Deutschland, S. $114 \mathrm{f}$.; Morschett, in: Zentes/Swoboda/ Morschett (Hrsg.), Kooperationen, Allianzen und Netzwerke, S. 377, 392 f., differenziert lediglich zwischen vertikaler und horizontaler Kooperation. Dies lässt sich jedoch darauf zurückführen, dass der Beitrag die Spezifika von Kooperationen im Glasfaserausbau nicht berücksichtigt und allgemeiner gefasst ist.

660 Hierzu im Überblick Gerpott, MedienWirtschaft 4/2010, S. 10, 21; Tenbrock/Strube Martins/Wernick u.a., Co-Invest Modelle zum Aufbau von neuen FTTB/HNetzinfrastrukturen, S.31; Tenbrock, Der Glasfaserausbau in Deutschland, S. $115 \mathrm{f}$. 
Die nachfolgende Untersuchung soll den allgemeinen Rechtsrahmen für Kooperationsvorhaben darstellen und sich nicht auf bestimmte Ausgestaltungsoptionen beschränken. Aus diesem Grund soll auf die einzelnen Systematisierungskriterien nicht vertieft eingegangen werden. Es bleibt jedoch festzuhalten, dass Kooperationen, die eine hohe Wettbewerbsintensität belassen, auch einer geringeren wettbewerbsrechtlichen Flankierung bedürfen. Aus dem weiten Spektrum der ökonomischen Fragestellungen sind für die vorliegende Untersuchung die Effekte kooperativer Ausbauvorhaben auf den Breitbandausbau (dazu 1.) und die Motive der Unternehmen für ein Engagement in kooperativen Ausbauvorhaben relevant (dazu 2.).

1. Ökonomische Auswirkungen von Kooperationsvorhaben auf den Breitbandausbau

Die Auswirkungen kooperativer Investitionen in den Glasfaserausbau, insbesondere im Hinblick auf die Beschleunigung des Breitbandausbaus in der Fläche, waren bereits Gegenstand verschiedener ökonomischer Studien. ${ }^{661}$ Hierzu wurden Modelluntersuchungen mit unterschiedlichen Schwerpunkten durchgeführt. ${ }^{662}$ Besonders hervorzuheben sind diejenigen Studien, die sich mit den volkswirtschaftlichen und sozialen Auswirkungen vermehrter kooperativer Ausbauvorhaben beschäftigt haben. ${ }^{663}$

661 Einen Überblick bieten insoweit Abrardi/Cambini, Telecommunications Policy 2019, S. 183, 195 f.; Briglauer/Cambini/Fetzer u.a., Telecommunications Policy 2017, S. 948, 949 ff.; zum Effekt der Beschleunigung des Breitbandausbaus in der Fläche statt vieler Bourreau/Cambini/Hoernig, Cooperative Investment, Access, and Uncertainty, S. 6 ff.; Bourreau/Hoernig/Maxwell, Implementing co-investment and network sharing, S. 18 ff; Wernick/Queder/Strube Martins u.a., Gigabitnetze für Deutschland, S. $87 \mathrm{ff}$.

662 Einen Überblick über die durchgeführten Studien bieten Abrardi/Cambini, Telecommunications Policy 2019, S. 183, 196 ff.; Briglauer/Cambini/Fetzer u.a., Telecommunications Policy 2017, S. 948, 949 ff.

663 Weiterführend zu den Auswirkungen auf Investitionsverhalten und Verbraucherwohlfahrt Nitsche/Wiethaus, Industrial Organization 2010, S. 263, 266 ff.; bestätigend und ergänzend um Fragen der Auswirkungen auf soziale Aspekte und eine Analyse hinsichtlich spezifischer Kooperationsausgestaltungen Cambinil Silvestri, Information Economics and Policy 24 (2012), S. 212, 214 ff.; mit einem ähnlichen Ansatz Cambini/Silvestri, Telecommunications Policy 37 (2013), S. $861 \mathrm{ff}$. 
Nach diesen weist jede Ausgestaltungsoption Vor- und Nachteile auf, 664 die im Rahmen der konkreten Umsetzung in einen schonenden Ausgleich gebracht werden müssen. So verbessere etwa die Gründung eines Gemeinschaftsunternehmens durch die Beteiligten der Kooperation die Investitionsbedingungen, bringe aber zugleich ein erhöhtes Gefahrenpotential für den Wettbewerb mit sich. ${ }^{665}$ Diesen Risiken wird mithilfe des Rechtsrahmens begegnet. Vermehrte kooperative Ausbauvorhaben könnten jedoch diesen Studien zufolge im Ergebnis zu einer Beschleunigung des Breitbandausbaus in der Fläche beitragen. ${ }^{666}$ Neuralgisch sei jedoch die Belastung der errichteten Infrastruktur mit regulatorischen Verpflichtungen, die insbesondere bei der Zahlung geringer Zugangsentgelte die Vorteile der Kooperation wieder neutralisieren könne. ${ }^{667}$ Kritisch wird gegen den kooperativen Breitbandausbau vorgebracht, dass die hiermit einhergehende vermehrte Kommunikation zwischen den Wettbewerbern die Grundvoraussetzungen für ein kollusives Zusammenwirken schaffen und damit mittelbar zu Wohlfahrtsverlusten beitragen könnte. ${ }^{668}$

Bislang existieren allerdings nur wenige empirische Untersuchungen, die nicht nur theoretische Modellkonstellationen beleuchten, sondern die tatsächlichen Auswirkungen kooperativer Ausbauvorhaben auf den Glasfaserausbau in der Fläche. ${ }^{69}$ Eine dieser Untersuchungen hat für den französischen Markt am Beispiel von 3573 Gemeinden in einem dreijährigen Beobachtungszeitraum nachgewiesen, dass sich die auf theoretischer Grundlage modellierbaren Erkenntnisse auch in realen Ausbauszenarien zeigen. 670

664 Cambini/Silvestri, Information Economics and Policy 24 (2012), S. 212, 225 f.; umfassend hierzu mit Auswertung der ökonomischen Literatur Bourreau/Hoernig/Maxwell, Implementing co-investment and network sharing, S. $18 \mathrm{ff}$.

665 Umfassend zu den unterschiedlichen Ausgestaltungsoptionen Bourreau/Hoernig/ Maxwell, Implementing co-investment and network sharing, S. $29 \mathrm{ff}$.

666 Bourreau/Cambini/Hoernig, Cooperative Investment, Access, and Uncertainty, S. $6 \mathrm{ff}$.

667 Bourreau/Cambini/Hoernig, Cooperative Investment, Access, and Uncertainty, S. 22.

668 Bourreau/Hoernig/Maxwell, Implementing co-investment and network sharing, S. 24 f.; Krämer/Vogelsang, Review of Network Economics 2016, S. 35, 47 ff.; Neumann/Sickmann/Alkas/Koch, Reformbedarf des europäischen Rechtsrahmens für elektronische Kommunikation, S. 238; Monopolkommission, 10. Sektorgutachten Telekommunikation (2017), Rn. 138.

669 Hierzu umfassend Bourreau/Hoernig/Maxwell, Implementing co-investment and network sharing, S. $46 \mathrm{ff}$.

670 Lebourges/Liang, Estimating the impact of co-investment in fiber to the home coverage, S. $20 \mathrm{ff}$. 
An dieser Stelle müssen die Erkenntnisse der einzelnen Studien jedoch nicht im Detail nachgezeichnet werden. Festzuhalten ist jedoch, dass kooperative Investitionen den Infrastrukturausbau unter bestimmten Voraussetzungen beschleunigen können. Zugleich resultiert aus der verstärkten Zusammenarbeit auch ein Missbrauchsrisiko, dem präventiv begegnet und durch die Konstruktion der rechtlichen Vorgaben Rechnung getragen werden muss. ${ }^{671}$ Zugleich darf der Rechtsrahmen jedoch nicht dazu führen, dass die positiven Effekte von Kooperationsvorhaben vollständig aufgehoben werden.

\section{Anreize für einen kooperativen Glasfaserausbau}

Für Unternehmen können unterschiedliche Motive eine Beteiligung an Kooperationsvorhaben im Glasfaserausbau sinnvoll erscheinen lassen. ${ }^{672}$ So können die Kosten und Risiken des Ausbaus unter den Kooperationspartnern aufgeteilt werden. ${ }^{673}$ Hierdurch wird mittelbar die Finanzierung mithilfe von Eigen- oder Fremdkapital erleichtert. ${ }^{674}$ Darüber hinaus kann es für die Unternehmen attraktiv sein, die Ausbauvorhaben zu bündeln, hierdurch Synergieeffekte zu erzielen ${ }^{675}$ und einen parallelen Ausbau von Netzen zu vermeiden. ${ }^{676}$ Für die Unternehmen spricht auch die zu erwar-

$671 \mathrm{Zu}$ den Optionen der Missbrauchsprävention durch Ausgestaltung der vertraglichen Grundlagen, Bourreau/Hoernig/Maxwell, Implementing co-investment and network sharing, S. $37 \mathrm{f}$.

672 Übersichtliche grafische Darstellung bei Tenbrock/Strube Martins/Wernick u.a., Co-Invest Modelle zum Aufbau von neuen FTTB/H-Netzinfrastrukturen, S. 42, Abbildung 4-2; spezifisch für den Telekommunikationssektor Carter/Elixmann/ Marcus, Unternehmensstrategische und regulatorische Aspekte von Kooperationen beim NGA-Breitbandausbau, S. 19 f.; weiterführend mit Experteninterviews Limbach/Kübel/Zarnekow, Kooperativer Breitbandausbau in Deutschland.

673 Abrardi/Cambini, Telecommunications Policy 2019, S. 183, 195; Monopolkommission, 10. Sektorgutachten Telekommunikation (2017), Rn. 137; Tenbrock, Der Glasfaserausbau in Deutschland, S. 116; Tenbrock/Strube Martins/Wernick u.a, Co-Invest Modelle zum Aufbau von neuen FTTB/H-Netzinfrastrukturen, S. 40.

674 Tenbrock/Strube Martins/Wernick u.a., Co-Invest Modelle zum Aufbau von neuen FTTB/H-Netzinfrastrukturen, S. 40.

675 Tenbrock/Strube Martins/Wernick u.a., Co-Invest Modelle zum Aufbau von neuen FTTB/H-Netzinfrastrukturen, S. 40.

676 Weiterführend hierzu Kühling, WuW 2019, S. 555, 559; Tenbrock/Strube Martins/ Wernick u.a., Co-Invest Modelle zum Aufbau von neuen FTTB/H-Netzinfrastrukturen, S. 41. 
tende höhere Netzauslastung dafür, unter Umständen in einem kooperativen Ausbauvorhaben aktiv zu werden. Hinsichtlich der unmittelbaren und mittelbaren regulatorischen Anreize zur Beteiligung an einem Kooperationsvorhaben, ist zwischen Unternehmen mit und ohne beträchtliche Marktmacht zu differenzieren. Für marktmächtige Unternehmen könnte eine Kooperation attraktiv sein, sofern die Regulierungsbehörde sie durch regulatorische Privilegierungen belohnt (D. II. 3. b.). Solche Kooperationspartner, die nicht durch marktmachtabhängige regulatorische Verpflichtungen belastet werden, kann die Aussicht auf einen erleichterten Zugang zur Infrastruktur zur Beteiligung an kooperativen Ausbauvorhaben motivieren.

\section{Schlussfolgerungen für die juristische Analyse}

Kooperationen zur Beschleunigung des Glasfaserausbaus basieren auf langfristigen, meist vertraglich abgesicherten Vereinbarungen, die Unternehmen freiwillig eingehen, um die Bedingungen gegenüber einer Investition im freien Wettbewerb nachhaltig zu verbessern. Sie sind von nachgelagerten finanziellen Transaktionen und Unternehmensintegrationen abzugrenzen. Kooperationsvorhaben lassen sich in schier unbegrenzter Vielfalt ausgestalten und wirken sich unterschiedlich auf den Wettbewerb aus.

Dabei divergieren die betriebswirtschaftlichen Motive der Unternehmen für eine Beteiligung an Kooperationen. Durch Kooperationen lassen sich unter bestimmten Voraussetzungen Investitionsrisiken senken und Vorteile für das Allgemeinwohl erzielen. Zugleich stellt jedoch die Möglichkeit eines kollusiven Zusammenwirkens verschiedener Unternehmen, die durch Kooperationen gesteigert wird, auch eine Gefahr für die Volkswirtschaft dar. Prägendes Ziel von Wettbewerbs- und Regulierungsrecht im Umgang mit Kooperationsvorhaben ist daher die Konservierung oder gar die Steigerung der Wettbewerbsintensität in den Kooperationsgebieten sowie die Vermeidung eines solchen kollusiven Zusammenwirkens der Kooperationspartner.

Die rechtlichen Rahmenbedingungen für kooperative Glasfaserausbauvorhaben ergeben sich aus dem allgemeinen und dem sektorspezifischen Wettbewerbsrecht. Das Regulierungsrecht enthält ausschließlich Steuerungsvorgaben für Kooperationsvorhaben, an denen sich mindestens ein marktmächtiges Unternehmen beteiligt oder durch die ein marktmächtiges Unternehmen entsteht. Das allgemeine Wettbewerbsrecht erfasst hingegen auch andere Kooperationsvorhaben. Differenziert werden kann hier 
zwischen den Vorgaben zur Fusionskontrolle, die wiederum das Überschreiten spezifischer Schwellenwerte und Ausgestaltungen der Kooperation voraussetzen, und denen des allgemeinen Kartellverbots, die bei jeder Ausgestaltungsvariante auf Grundlage einer Vereinbarung zu beachten sind. Nachfolgend steht das Zusammenspiel der verschiedenen Regelungen im Vordergrund. Etwaige Detailfragen für spezifische Kooperationsausgestaltungen sollen dabei nur eine untergeordnete Rolle spielen.

\section{B. Der Anwendungsbereich wettbewerbsrechtlicher Regelungsregime}

Neben den telekommunikationsrechtlichen Vorgaben, auf die erst später eingegangen werden soll (D.), kann das allgemeine Wettbewerbsrecht Steuerungswirkungen für die Ausgestaltung von Kooperationen entfalten. Hervorzuheben sind das Recht der Fusionskontrolle (dazu II.) und das Kartellverbot (dazu III.). Veranschaulicht werden soll das Ineinandergreifen der verschiedenen Regelungsmaterien am Beispiel der Glasfaser Nordwest GmbH \& Co. KG, eines Kooperationsvorhabens, auf das alle Regelungsmaterien anwendbar sind. Die wesentlichen Eckpunkte dieses Kooperationsvorhabens sollen eingangs skizziert werden (dazu I.). Hieran anknüpfend sollen die Rahmenbedingungen für Kooperationen mit hohem Institutionalisierungsgrad untersucht werden. Dabei muss insoweit zwischen Kooperationsvorhaben, die einen hohen Institutionalisierungsgrad durch die Gründung eines Gemeinschaftsunternehmens aufweisen, und solchen mit ein geringerem Institutionalisierungsgrad differenziert werden, denen aber zumindest eine Vereinbarung im Sinne des $₫ 1 \mathrm{GWB}^{677} \mathrm{zu}$ Grunde liegt.

\section{Die Gründung der Glasfaser Nordwest als Fallbeispiel}

Die Glasfaser Nordwest GmbH \& Co. KG (Glasfaser Nordwest) ist ein Unternehmen, das von der Telekom Deutschland GmbH (Deutsche Telekom) und der EWE AG (EWE) am 8.1.2020 als Gemeinschaftsunternehmen gegründet wurde. Vor der Gründung des Unternehmens hatten sich die Bun-

677 Der Vereinbarungsbegriff wird später ausführlich thematisiert, hierzu E. II. 1. 
desnetzagentur ${ }^{678}$ und das Bundeskartellamt ${ }^{679}$ im Rahmen ihrer Kompetenzen mit den jeweiligen Fragen des regulatorischen und wettbewerbsrechtlichen Umganges mit dem Gemeinschaftsunternehmen beschäftigt. Dies macht das Unternehmen als Fallbeispiel für die nachfolgende Untersuchung besonders interessant. ${ }^{680}$ Es handelt sich um das erste Unternehmen, für das die Auswirkungen des Kooperationsvorhaben im Glasfaserausbau aus fusionskontrollrechtlicher, kartellrechtlicher und regulatorischer Perspektive durch die zuständigen Behörden beleuchtet wurden.

Die beiden Muttergesellschaften sind an der Glasfaser Nordwest paritätisch beteiligt. Das Kooperationsgebiet umfasst weite Teile des Nordwesten Deutschlands und verfügt über 460 Ortsnetze, 663 Anschlussbereiche sowie ein Ausbaupotential von 3,4 Millionen Haus- und Unternehmensstandorten. ${ }^{681}$ Das Unternehmen errichtet in diesem Gebiet als WholesaleOnly-Anbieter (Kapitel 4, A. I. 1.) aktive und passive Glasfaserinfrastruktur und vertreibt die Vorleistungsprodukte an die Muttergesellschaften und andere Telekommunikationsunternehmen. ${ }^{62}$ Unmittelbar nach seiner Gründung war das Gemeinschaftsunternehmen noch nicht Eigentümer einer eigenen Infrastruktur. Aus diesem Grund wird in der Startphase auf die Infrastruktur und Dienstleistungen der beiden Muttergesellschaften zurückgegriffen. ${ }^{683}$ Herauszustellen ist bereits an dieser Stelle, dass es sich bei der Deutschen Telekom um ein Unternehmen handelt, das auf den anschlussbezogenen Märkten im gesamten Bundesgebiet über eine beträchtliche Marktmacht verfügt (C. II. 1.). EWE hat zwar keine marktbeherrschende Stellung bei der nationalen Marktabgrenzung, ist jedoch im Kooperationsgebiet der Hauptkonkurrent der Deutschen Telekom. ${ }^{684}$ Durch die Gründung der Glasfaser Nordwest schließen sich also die beiden

678 BKartA, Beschl. v. 30.12.2019, Az. B 7-21/18; BKartA, Beschl. v. 4.12.2019, Az. B 7-21/18.

679 BNetzA, Entsch. v. 10.7.2018, Az. BK 1-18/002; BNetzA, Beschl. v. 13.7.2018, Az. BK 3-17/071.

$680 \mathrm{Zu}$ Fallbeispielen aus anderen Mitgliedstaaten der Europäischen Union umfassend Bourreau/Hoernig/Maxwell, Implementing co-investment and network sharing, S. $45 \mathrm{ff}$.

681 BKartA, Beschl. v. 30.12.2019, Az. B 7-21/18, Rn. 46, Abbildung 1.

682 BKartA, Beschl. v. 30.12.2019, Az. B 7-21/18, Rn. 45 mit Zitat aus dem Gesellschaftsvertrag, der allerdings unter die Berufs- und Geschäftsgeheimnisse fällt und deswegen nicht frei verfügbar ist.

683 Weiterführend insbesondere im Hinblick auf die Zuständigkeit zum Betrieb von aktiver oder passiver Technik BKartA, Beschl. v. 30.12.2019, Az. B 7-21/18, Rn. 48.

684 BKartA, Beschl. v. 30.12.2019, Az. B 7-21/18, Rn. 40 f. 
Hauptakteure des Infrastrukturwettbewerbes im Kooperationsgebiet zusammen.

II. Eröffnung des Anwendungsbereiches der nationalen Vorgaben zur Fusionskontrolle

Die nationalen Vorgaben zur Fusionskontrolle können angewandt werden, wenn das Kooperationsvorhaben einen der Zusammenschlusstatbestände des GWB erfüllt (dazu 1.), die Umsatzschwellen des $\$ 35$ GWB überschritten werden (dazu 2.) und die unionalen Regelungen zur Fusionskontrolle keine Anwendung finden (dazu 3.). Die Tatbestandsvoraussetzungen für die Eröffnung des Fusionskontrollrechts sind aufgrund von Verweisungen auch für das Regulierungsrecht von Relevanz, sodass sie an dieser Stelle vertieft untersucht werden sollen. ${ }^{685}$

\section{Zusammenschluss im Sinne des GWB}

Damit ein Kooperationsvorhaben als Zusammenschluss im Sinne des GWB gilt, muss einer der in $\$ 37$ Abs. 1 GWB normierten Zusammenschlusstatbestände erfüllt sein (dazu a.). Wenn zudem ein Beherrschungsverhältnis zwischen den beteiligten Unternehmen besteht, werden sie im Wettbewerbsrecht als einheitliches Unternehmen behandelt (dazu b.).

a) Erfüllung eines Zusammenschlusstatbestandes

Das Kartellrecht differenziert in dieser Hinsicht gemäß $\$ 37$ Abs. 1 GWB zwischen verschiedenen Zusammenschlusstatbeständen, die auch im Rahmen des kooperativen Glasfaserausbaus erfüllt sein müssten, um den Anwendungsbereich der Regelungsmaterie zu eröffnen. Ein Zusammenschluss kann durch Vermögens- (Nr. 1), Kontroll- (Nr. 2) oder Anteilserwerb (Nr. 3) an einem anderen Unternehmen erfolgen oder auch dadurch, dass ein erheblicher wettbewerblicher Einfluss (Nr.4) auf ein Unternehmen gewonnen wird.

$685 \mathrm{Zu}$ den Auswirkungen auf das Regulierungsrecht, D. II. 1. 
Relevant ist das Recht der Fusionskontrolle nur für solche Konstellationen, in denen die Kooperation einen hohen Institutionalisierungsgrad aufweist. Dies ist etwa bei der Gründung eines Gemeinschaftsunternehmens der Fall. Sofern jedoch lediglich eine vertragliche Vereinbarung zwischen den Kooperationspartnern geschlossen wird, die zwar zu einer verstärkten Zusammenarbeit, nicht jedoch zu einer gemeinsamen Unternehmensgründung führt, entfalten die Vorgaben keine Steuerungswirkung. Besonders interessant ist die Gründung eines paritätischen Gemeinschaftsunternehmens - wie der Glasfaser Nordwest-, das zugleich mehrere Zusammenschlusstatbestände erfüllt. Je nach konkreter Ausgestaltung ist es möglich, dass die Muttergesellschaften gemeinsam die Kontrolle an dem Gemeinschaftsunternehmen erwerben (Nr. 2). ${ }^{686}$ Hierauf ist noch detaillierter einzugehen (B. II. 1. b.). Darüber hinaus wird bei einer paritätischen Anteilsaufteilung zwischen zwei Gesellschaftern auch die Anteilsschwelle im Sinne des $₫ 37$ Abs. 1 Nr. 3 GWB überschritten. ${ }^{687}$ Danach liegt ein Zusammenschluss vor, wenn ein Unternehmen entweder 50 (lit. a) oder 25 Prozent (lit. b) der Anteile an einem anderen Unternehmen erwirbt. Bei einem paritätischen Gemeinschaftsunternehmen zweier Muttergesellschaften ist dieses Kriterium stets erfüllt, da beide Unternehmen jeweils 50 Prozent der Anteile am Gemeinschaftsunternehmen erhalten. ${ }^{688}$ Sofern sich mehr als vier Unternehmen paritätisch an der Kooperation beteiligen, sind die Voraussetzungen dieses Kriteriums aber möglicherweise nicht erfüllt. Dies hat aufgrund der Einschlägigkeit mehrere Tatbestände jedoch letztlich keine Auswirkungen.

b) Behandlung als einheitliches Unternehmen

Aus $₫ 36$ Abs. 2 GWB ergibt sich die Rechtsfolge, die an das Vorliegen eines Zusammenschlusses geknüpft wird. Verbundene Unternehmen, von

686 Spezifisch für die Glasfaser Nordwest ohne weitergehende Ausführungen festgestellt vom BKartA, Beschl. v. 30.12.2019, Az. B 7-21/18, Rn. 86; weiterführend hierzu Kling/Thomas, Kartellrecht, S. $771 \mathrm{f}$.

687 Weiterführend hierzu Crede/Franz/Peter u.a., in: Schulte (Hrsg.), Handbuch der Fusionskontrolle, Rn. $177 \mathrm{ff}$.

688 Spezifisch für die Glasfaser Nordwest festgestellt von der BNetzA, Entsch. v. 10.7.2018, Az. BK 1-18/002, S. 6; im Ergebnis auch, aber ohne weiterführende Untersuchung BKartA, Beschl. v. 30.12.2019, Az. B 7-21/18, Rn. 86; weiterführend Kling/Thomas, Kartellrecht, S. 775; kritisch Säcker, in: Säcker (Hrsg.), Telekommunikationsgesetz, $₫ 3$, Rn. 86. 
denen ein Unternehmen als abhängiges oder herrschendes Unternehmen zu klassifizieren ist, sind als einheitliches Unternehmen im Sinne des Wettbewerbsrechts zu behandeln. Das Abhängigkeits- und Beherrschungsverhältnis wird auf Basis der gesellschaftsrechtlichen Begriffsbestimmungen aus $\$ \$ 17$ und $18 \mathrm{AktG}$ festgestellt. ${ }^{69}$ Ein enger Konnex besteht zum Zusammenschluss im Wege des Kontrollerwerbs gemäß $₫ 37$ Abs. 1 Nr. 2 GWB.

Gibt es mehrere Mutterunternehmen, muss nicht jedes für sich genommen als herrschendes Unternehmen klassifiziert werden können. ${ }^{690}$ Vielmehr sieht $\$ 36$ Abs. 2 S. 2 GWB a. E. vor, dass es ausreicht, wenn die Mutterunternehmen gemeinsam einen beherrschenden Einfluss auf das Gemeinschaftsunternehmen ausüben können. ${ }^{691}$ Diese Entscheidung des Gesetzgebers kann überzeugen. Anderenfalls könnte bei der Gründung paritätischer Gemeinschaftsunternehmen kein Abhängigkeits- und Beherrschungsverhältnis angenommen werden, weil jedem Unternehmen für sich genommen der beherrschende Einfluss fehlt. In diesem Fall würde ein substantieller Bereich der Kooperationspraxis nicht von der speziell hierauf ausgerichteten Regelungsmaterie erfasst und könnte lediglich mithilfe des Kartellverbotes reguliert werden (E. II.).

Für ein gemeinsames Beherrschungsverhältnis ist zwischen den Mutterunternehmen ein „bewußtes und gewolltes Zusammenwirken mit einer gewissen Planmäßigkeit“ erforderlich. ${ }^{692}$ Vorausgesetzt wird hierfür ein "gemeinsamer Beherrschungswille" ${ }^{\prime 693}$, der sich auch in einem bloß faktischen Zusammenwirken manifestieren kann. Ob diese Kriterien erfüllt sind, muss in jedem Einzelfall geprüft werden. Aus der Gründung eines Gemeinschaftsunternehmens kann jedenfalls nicht pauschal auf einen gemeinsamen Beherrschungswillen geschlossen werden. Dies gilt auch dann,

689 Fetzer, in: Arndt/Fetzer/Scherer u.a. (Hrsg.), Telekommunikationsgesetz, $\mathbb{3}$, Rn. 112; Lünenbürger/Stamm, in: Scheurle/Mayen (Hrsg.), Telekommunikations-

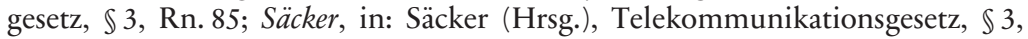
Rn. 86; Schütz, in: Geppert/Schütz (Hrsg.), Beck'scher TKG-Kommentar, $\mathbb{3}$, Rn. 90.

690 A. A. EWE/Telekom, in: BNetzA, Entsch. v. 10.7.2018, Az. BK 1-18/002, S. 23.

691 Paschke, in: Jaeger/Kokott/Pohlmann u.a. (Hrsg.), Frankfurter Kommentar zum Kartellrecht, $\mathbb{3} 6$ GWB, Rn. 103.

692 Unterrichtung des Ausschusses für Wirtschaft v. 13.6.1973, BT-Drs 7/765, S. 7.

693 Unterrichtung des Ausschusses für Wirtschaft v. 13.6.1973, BT-Drs 7/765, S. 7. 
wenn die Anteile paritätisch verteilt sind. ${ }^{694}$ Die Erweiterung des Anwendungsbereiches des Fusionskontrollrechts durch die Möglichkeit der gemeinsamen Beherrschung wird durch die Kontrolle im Einzelfall überzeugend eingeschränkt. Aufgrund der umfassenden Kasuistik entsteht so ein ausbalanciertes System, das Rechtssicherheit gewährleistet und einzelfallgerechte Lösungen ermöglicht. Ein gewichtiges Indiz für einen gemeinsamen Beherrschungswillen kann es sein, dass „gleichgerichtete Interessen eine gemeinsame Unternehmenspolitik gewährleisten“. ${ }^{695}$ Auch von der paritätischen Entsendung von Geschäftsführern kann eine Indizwirkung ausgehen. ${ }^{696}$ Regelmäßig werden die Mutterunternehmen, die ein Gemeinschaftsunternehmen zum kooperativen Ausbau von Glasfaserinfrastrukturen gründen, gleichgerichtete Interessen verfolgen. Hierdurch ist eine einheitliche Unternehmenspolitik gewährleistet. Daraus folgt ein gemeinsamer Beherrschungswille der Mutterunternehmen, sodass sie das Gemeinschaftsunternehmen gemäß $₫ 36$ Abs. 2 S. 1 GWB gemeinsam beherrschen. Die auf diese Weise verbundenen Unternehmen werden als ein einheitliches Unternehmen behandelt. ${ }^{697}$ Ansonsten würde mithilfe der Gründung eines paritätischen Gemeinschaftsunternehmens eine umfassende Umgehung der kartellrechtlichen Vorgaben ermöglicht.

\section{c) Zwischenergebnis}

Damit der Anwendungsbereich der Zusammenschlusskontrolle eröffnet ist, muss die Kooperation durch einen hohen Institutionalisierungsgrad in Form der Gründung eines Unternehmens geprägt sein. Bei Gesellschaften zum kooperativen Glasfaserausbau sind insbesondere die Zusammen-

694 Dies ergibt sich aus der ständigen Rspr., namentlich BGH, Beschl. v. 8.5.1979, Az. KVR 1/78, GRUR 1979, S. 796, 798 f.; BGH, Beschl. v. 18.11.1986, Az. KVR 9/85, NJW 1987, S.1700, 1701 f.; BGH, Beschl. v. 30.9.1986, Az. KVR 8/85, GRUR 1987, S. 307, 309; in Bezug auf $\$ 37$ Abs. 1 Nr. 2 GWB Crede/Franz/Peter u.a., in: Schulte (Hrsg.), Handbuch der Fusionskontrolle, Rn. 175.

695 BGH, Beschl. v. 8.5.1979, Az. KVR 1/78, NJW 1979, S. 2401, 2403; BGH, Beschl. v. 18.11.1986, Az. KVR 9/85, NJW 1987, S.1700, 1701; Paschke, in: Jaeger/ Kokott/Pohlmann u.a. (Hrsg.), Frankfurter Kommentar zum Kartellrecht, $\$ 36$ GWB, Rn. 107.

696 Weiterführend hierzu Thomas, in: Immenga/Mestmäcker (Hrsg.), Wettbewerbsrecht, Band 2, $\$ 36 \mathrm{GWB}, \mathrm{Rn} .777$.

697 Kablenberg, in: Loewenheim/Meessen/Riesenkampff u.a. (Hrsg.), Kartellrecht, \$36 GWB, Rn. 215 m. w. N.; Kling/Thomas, Kartellrecht, S. 765; Thomas, in: Immenga/Mestmäcker (Hrsg.), Wettbewerbsrecht, Band 2, \$36 GWB, Rn. 781. 
schlusstatbestände des Kontroll- und Anteilserwerbs, die sich aus $\$ 37$ Abs. 1 GWB ergeben, von entscheidender Bedeutung. Darüber hinaus muss auch ein Beherrschungsverhältnis im Sinne des $\$ 36$ Abs. 2 GWB vorliegen. Die Beherrschung kann bei Gemeinschaftsunternehmen gemeinsam durch die Muttergesellschaften ausgeübt werden. Ob ein Kooperationsprojekt diese Anforderungen erfüllt, ist in jedem Einzelfall separat zu überprüfen. Ein gewichtiges Indiz für einen gemeinsamen Beherrschungswillen ist die Verfolgung gleichgerichteter Interessen, die bei Unternehmensgründungen zum kooperativen Glasfaserausbau in der Regel erfüllt sein wird. Liegt ein Beherrschungsverhältnis vor, ist das Unternehmen als einheitliches Unternehmen im Sinne des gesamten GWB zu behandeln. Auf diese Weise wird effektiv eine Umgehung der speziellen kartellrechtlichen Vorgaben mithilfe der Gründung paritätischer Gemeinschaftsunternehmen unterbunden.

2. Weitere Anwendungsvoraussetzungen der Vorgaben zur Fusionskontrolle

Nicht jeder Zusammenschluss setzt eine Kontrolle der Fusion durch die Kartellbehörde voraus. Vielmehr müssen hierfür weitere Kriterien, wie das Überschreiten bestimmter Schwellenwerte, vorliegen (dazu a.). Sofern dies der Fall ist, darf keine Ausnahme erfüllt sein, die ein Absehen von einer Fusionskontrolle rechtfertigt (dazu b.).

\section{a) Umsatzschwellenwerte}

Die nationalen Vorgaben der Fusionskontrolle finden nur dann Anwendung, wenn die Schwellenwerte des $\$ 35$ Abs. 1 und Abs. 1a GWB überschritten werden. Anderenfalls müsste sich das Gemeinschaftsunternehmen lediglich an den Vorgaben des Kartellverbotes aus $₫ 1$ GWB messen lassen (E. II.).

Die Schwellenwerte sind regelmäßiger Gegenstand von GWB-Novellen. Im Rahmen der 9. GWB-Novelle wurden Systematik und Höhe der Schwellenwerte umfassend angepasst. ${ }^{698}$ Auch im Rahmen der 10. GWB-

698 BT-Drs. 18/10207, S. 70 ff.; weiterführend hierzu Jungbluth, in: Körber/Immenga (Hrsg.), Aktuelle Entwicklungen in der Fusionskontrolle zwischen Recht, Wirtschaft und Politik, S. 9, 15 f. 
Novelle sind Modifikationen dieser Vorgaben geplant. ${ }^{699}$ Maßgeblicher Referenzzeitpunkt für die Überprüfung der Schwellenwerte ist das letzte Geschäftsjahr vor dem Zusammenschluss. Gemäß $₫ 35$ Abs. 1 GWB müssen die beteiligten Unternehmen insgesamt einen Jahresumsatz von mindestens 500 Millionen Euro gemacht haben (Nr. 1). Im Inland muss eines der beteiligten Unternehmen mindestens 25 und ein anderes mindestens fünf Millionen Euro Umsatz erwirtschaftet haben (Nr. 2). Dieser Wert soll im Rahmen der 10. GWB-Novelle auf zehn Millionen Euro angehoben werden. ${ }^{700} \$ 35$ Abs. 1a GWB flexibilisiert die Schwellen, indem auch der Wert der Gegenleistung für den Zusammenschluss (Nr.3) Berücksichtigung findet. ${ }^{701}$ Die Schwellenwerte stellen sicher, dass die Fusionskontrolle nur dann erfolgt, wenn von dem Zusammenschluss tatsächlich erhebliche Auswirkungen auf den Wettbewerb ausgehen können, und tragen zur Entlastung des Bundeskartellamts bei. ${ }^{702}$

Während es folgerichtig ist, dass nur tatsächlich wettbewerbsrelevante Fusionen auch kontrolliert werden sollen, ist eine Überlastung der Behörde ein untergeordnetes Argument. Wenn mehr Fusionskontrollen für einen effektiven Wettbewerbsschutz unerlässlich sind, sollte eher eine Aufstockung des Personals als ein kontinuierliches Anheben der Schwellenwerte erwogen werden. Hierdurch entsteht nämlich die Gefahr, dass regional ausgerichtete Kooperationsvorhaben nicht mehr vom Fusionskontroll-

699 Referentenentwurf des BMWi eines Zehnten Gesetzes zur Änderung des Gesetzes gegen Wettbewerbsbeschränkungen für ein fokussiertes, proaktives und digitales Wettbewerbsrecht 4.0 (GWB-Digitalisierungsgesetz) v. 24.1.2020, S. 94 ff., abrufbar unter: https://www.bmwi.de/Redaktion/DE/Downloads/G/gw b-digitalisierungsgesetz-referentenentwurf.pdf?__blob=publicationFile\&v=10 (zuletzt abgerufen am 1.7.2020).

700 Referentenentwurf des BMWi eines Zehnten Gesetzes zur Änderung des Gesetzes gegen Wettbewerbsbeschränkungen für ein fokussiertes, proaktives und digitales Wettbewerbsrecht 4.0 (GWB-Digitalisierungsgesetz) v. 24.1.2020, S. 12, abrufbar unter: https:/www.bmwi.de/Redaktion/DE/Downloads/G/gwb-digitali sierungsgesetz-referentenentwurf.pdf?_blob=publicationFile $\& v=10$ (zuletzt abgerufen am 1.7.2020); hierzu Fülling, in: Körber/Immenga (Hrsg.), Innovation im Kartellrecht - Innovation des Kartellrechts, S. 49, 52; Kredel/Kresken, NZKart 2020, S. 2, 5; Podszun/Brauckmann, GWR 2019, S. 436.

701 BT-Drs. 18/10207, S. 72 ff.; Krueger, in: Körber/Immenga (Hrsg.), Aktuelle Entwicklungen in der Fusionskontrolle zwischen Recht, Wirtschaft und Politik, S. 79, 83; Monopolkommission, Policy Brief zur 10. GWB-Novelle, S. 6; Podszun/Brauckmann, GWR 2019, S. 436.

702 Jungbluth, in: Körber/Immenga (Hrsg.), Aktuelle Entwicklungen in der Fusionskontrolle zwischen Recht, Wirtschaft und Politik, S. 9, 15. 
recht erfasst werden. ${ }^{703}$ Dies wäre insbesondere für Glasfaserausbaukooperationen bedenklich, die - wie die Glasfaser Nordwest illustriert - regelmäBig regional ausgerichtet sind. Natürlich hat nicht jedes lokal ausgerichtete kooperative Glasfaserausbauvorhaben so gravierende Auswirkungen auf den Wettbewerb wie die Gründung der Glasfaser Nordwest, sodass nicht in jedem Fall ein Fusionskontrollverfahren erforderlich ist. Hier übernimmt das Kartellverbot eine effektive Lückenschlussfunktion (E. II.). Zu begrüßen wäre es allerdings, wenn als Reaktion auf eine Anhebung der Schwellenwerte Ausgleichsmechanismen geschaffen würden, um dem Bundeskartellamt die Möglichkeit zu geben, auch weiterhin lokale Kooperationsvorhaben einer Fusionskontrolle zu unterziehen. Hier muss die Balance zwischen effektivem Wettbewerbsschutz und der Optimierung der Rahmenbedingungen für einen kooperativen Glasfaserausbau gefunden werden.

\section{b) Ausnahmen}

Zudem darf keine der in $\$ 35$ Abs. 2 GWB aufgelisteten Ausnahmen greifen. Derzeit findet keine Fusionskontrolle statt, wenn sich gemäß $\$ 35$ Abs. 2 S. 1 GWB kleinere Kooperationspartner, die einen Jahresumsatz von unter zehn Millionen Euro erwirtschaften, zusammenschließen. Diese Ausnahme soll im Rahmen der 10. GWB-Novelle jedoch ersatzlos gestrichen werden. ${ }^{704}$ Dieser Ausnahmetatbestand war deshalb besonders, da vollständig von einer Fusionskontrolle im Sinne des $\$ 35$ Abs. 1 GWB abgesehen wurde, wenn er einschlägig war. ${ }^{705}$ Nunmehr müssen sich auch solche Kooperationen an der Fusionskontrolle messen lassen. Aufgrund des parallelen Anhebens der Schwellenwerte werden die praktischen Auswirkungen seines Wegfalls im Ergebnis wohl überschaubar sein.

703 Ebenfalls kritisch und mit Optimierungsvorschlägen Monopolkommission, Policy Brief zur 10. GWB-Novelle, S. 7; positiver Kredel/Kresken, NZKart 2020, S. 2, 5.

704 Referentenentwurf des BMWi eines Zehnten Gesetzes zur Änderung des Gesetzes gegen Wettbewerbsbeschränkungen für ein fokussiertes, proaktives und digitales Wettbewerbsrecht 4.0 (GWB-Digitalisierungsgesetz) v. 24.1.2020, S. 12, abrufbar unter: https:/www.bmwi.de/Redaktion/DE/Downloads/G/gwb-digitali sierungsgesetz-referentenentwurf.pdf?_blob=publicationFile\&v=10 (zuletzt abgerufen am 1.7.2020).

705 Thomas, in: Immenga/Mestmäcker (Hrsg.), Wettbewerbsrecht, Band 2, $\$ 35$ GWB, Rn. 87. 
Zudem werden gemäß $₫ 35$ Abs. 2 S. 2 und 3 GWB öffentliche Unternehmen und Unternehmen der Kreditwirtschaft privilegiert. Bei Kooperationen unter Beteiligung der öffentlichen Hand können diese Ausnahmetatbestände eine wichtige Rolle spielen. Diese Untersuchung beschränkt sich jedoch auf Kooperationen zwischen Telekommunikationsunternehmen, sodass sie keiner näheren Erörterung bedürfen.

\section{Verhältnis von unionaler oder nationaler Fusionskontrolle}

Die nationale und unionale Fusionskontrolle stehen in einem Exklusivitätsverhältnis zueinander. Das Zusammenspiel der Regelungsmaterien wird durch das sogenannte "One-Stop-Shop“-Prinzip geprägt (dazu a.). Entscheidend ist dabei die Frage, ob durch die Fusion ein sogenanntes „Vollfunktionsgemeinschaftsunternehmen“ entsteht (dazu b.). Relevant ist die Abgrenzung insbesondere zur Ermittlung der behördlichen Zuständigkeiten.

a) Exklusivität der Anwendungsbereiche durch das „One-Stop-Shop“Prinzip

Das „One-Stop-Shop“-Prinzip führt zur Exklusivität der Anwendungsbereiche der nationalen und unionalen Vorgaben zur Fusionskontrolle. ${ }^{706}$ Sobald der Anwendungsbereich der unionalen Fusionskontrolle eröffnet ist, sind die nationalen Regelungen nicht anwendbar. ${ }^{707}$ Dies ergibt sich unmittelbar aus Art. 23 Abs. 3 UAbs. 1 Fusionskontrollverordnung $(\mathrm{FKVO})^{708}$ und spiegelbildlich aus $\$ 35$ Abs. $3 \mathrm{GWB} .{ }^{709}$ Im Anwendungsbereich der FKVO ist gemäß Art. 23 Abs. 2 FKVO in der Regel die Kommission zuständig, während für den Vollzug des nationalen Kartellrechts die na-

706 Hierzu statt vieler Thomas, in: Immenga/Mestmäcker (Hrsg.), Wettbewerbsrecht, Band 2, $\mathbb{3 5}$ GWB, Rn. 112; Kühn, Nicht kontrollierende Minderheitsbeteiligungen in der EU-Fusionskontrolle, S. 243; im Ergebnis auch Erwägungsgrund 8 der FKVO.

707 Kühn, Nicht kontrollierende Minderheitsbeteiligungen in der EU-Fusionskontrolle, S. 243.

708 Verordnung (EG) Nr. 139/2004 des Rates vom 24.1.2004 über die Kontrolle von Unternehmenszusammenschlüssen, Abl. L 24/1 v. 29.1.2004.

709 Hierzu weiterführend Thomas, in: Immenga/Mestmäcker (Hrsg.), Wettbewerbsrecht, Band 2, $\$ 35$ GWB, Rn. $112 \mathrm{ff}$. 
tionalen Behörden zuständig sind. Ausnahmen von der vorgenannten Regel bestehen lediglich in Form der Verweisungsmöglichkeiten in Art. 4, 9 und $22 \mathrm{FKVO}$, in deren Rahmen trotz der Anwendbarkeit des unionalen Fusionskontrollrechts eine nationale Kartellbehörde zuständig sein kann.

Eine Exklusivität der Anwendungsbereiche und behördlichen Zuständigkeiten ist vor dem Hintergrund der hiermit einhergehenden erhöhten Rechtssicherheit zu begrüßen. Auf diese Weise lässt sich für die Unternehmen bereits im Vorfeld ermitteln, welche Behörde für eine Fusionskontrolle zuständig ist und bei welcher Behörde die Fusion - unter Beachtung der Fristen - anzumelden ist. Eine parallele Behördenzuständigkeit mit entsprechendem Konfliktpotential und die Gefahr einer widersprechenden behördlichen Entscheidung, lassen sich auf diese Weise effektiv bekämpfen.

b) Abgrenzungskriterium des „Vollfunktionsgemeinschaftsunternehmens" im Sinne des Art. 3 Abs. 4 FKVO

Der Dreh- und Angelpunkt für die Abgrenzung der Anwendungsbereiche der Fusionskontrollregime für den kooperativen Glasfaserbau ist die Frage, ob es sich bei dem betreffenden Gemeinschaftsunternehmen um ein „Voll-

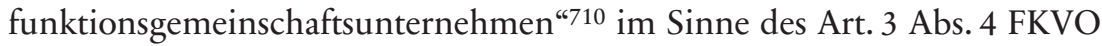
handelt. Die FKVO erfasst nur die Gründung solcher Gemeinschaftsunternehmen, die auf Dauer ,alle Funktionen einer selbstständigen wirtschaftlichen Einheit erfüll[en]“.711

Relevant ist, ob die neu gegründete Entität selbstständig am Markt auftritt und über die hierzu erforderlichen Ressourcen verfügt. ${ }^{712}$ Dafür ist das jeweilige Kooperationsprojekt umfassend zu untersuchen. Keine selbstständige wirtschaftliche Einheit ist gegeben, wenn das Gemeinschaftsunternehmen über kein eigenes Personal und keine eigenen Vermögenswerte

710 Konsolidierte Mitteilung der Kommission zu Zuständigkeitsfragen gemäß der Verordnung (EG) Nr.139/2004 des Rates über die Kontrolle von Unternehmenszusammenschlüssen, 2008/C 95/01 i. d. F. durch die Berichtigung der besagten Mitteilung 2009/C 43/09, Abl. C 43/10 v. 21.2.2009 (Mitteilung 2009/C 43/09), Ziff. 92; weiterführend zu den Abgrenzungskriterien auch Wilson, in: Dauses/Ludwigs (Hrsg.), Handbuch des EU-Wirtschaftsrechts, H. I. $\ 4$ Fusionskontrolle, Rn. $29 \mathrm{ff}$.

711 Mitteilung 2009/C 43/09, Ziff. 92.

712 Mitteilung 2009/C 43/09, Ziff. 94. 
verfügt und es sich um eine bloße Holding-Gesellschaft handelt.. ${ }^{73}$ Anders ist eine Situation zu bewerten, in der das Gemeinschaftsunternehmen neben dem Aufbau auch die Vermarktung der realisierten Infrastruktur übernimmt, sodass es am Markt aktiv präsent ist. Weitere Anhaltspunkte für eine selbstständige wirtschaftliche Einheit können aus dem Verkaufsverhalten des Gemeinschaftsunternehmens gewonnen werden. Entscheidend ist, ob ausschließlich Verkäufe an das Mutterunternehmen oder auch an Dritte erfolgen. ${ }^{714}$ Als „Dritte“ sind alle Unternehmen zu verstehen, die sich nicht an dem Gemeinschaftsunternehmen beteiligen. Die Kommission nimmt eine eigene Zuständigkeit an, wenn „wenigstens $20 \%$ des voraussichtlichen Umsatzes des Gemeinschaftsunternehmens mit Dritten erzielt werden. "715 Das Verkaufsverhalten ist für jedes Kooperationsprojekt im Einzelfall zu prüfen. Regelmäßig ist davon auszugehen, dass das Gemeinschaftsunternehmen ein großes Interesse daran hat, seine Dienstleistungen insbesondere den Muttergesellschaften feilzubieten. Die Erbringung von Dienstleistungen an Dritte wird demgegenüber nach Möglichkeit eine untergeordnete Rolle spielen. Ansonsten hätte der kooperative Glasfaserausbau keine Vorteile für die beteiligten Unternehmen, die selbst von der errichteten Infrastruktur profitieren wollen. Das Regulierungsrecht hat in dieser Hinsicht eine herausragende Bedeutung. Je umfangreicher die regulatorischen Verpflichtungen zur Gewährung des Zugangs gegenüber Dritten sind, desto eher überschreiten die Umsätze durch die Erhebung von Zugangsentgelten die 20-Prozent-Schwelle, die das Gemeinschaftsunternehmen zu einem Vollfunktionsgemeinschaftsunternehmen werden lässt.

Maßgeblicher Stichtag für die Bestimmung der Zuständigkeit ist das Datum, an dem das Kooperationsvorhaben angemeldet wird. ${ }^{716}$ Es soll davon ausgegangen werden, dass die kooperativen Gemeinschaftsunternehmen im Fokus, also zumindest zu 80 Prozent, ihre Dienstleistungen gegenüber den beteiligten Mutterunternehmen erbringen. Sollte dies nicht der Fall sein, kann im Einzelfall ein Vollfunktionsgemeinschaftsunternehmen vorliegen. Dann wären die Vorgaben der FKVO zu beachten und die Kommission wäre die zuständige Behörde. Die praktischen Auswirkungen sind jedoch gering. Die nationale Rechtsprechung orientiert sich weitgehend

713 Mitteilung 2009/C 43/09, Ziff. 94.

714 Mitteilung 2009/C 43/09, Ziff. 98.

715 Mitteilung 2009/C 43/09, Ziff. 98.

716 Mitteilung 2009/C 43/09, Ziff. 154. 
an der unionalen Rechtsprechung und Behördenpraxis. ${ }^{717}$ Im Folgenden sollen die Regelungen der nationalen Fusionskontrolle erörtert werden, die für den überwiegenden Anteil der Vorhaben von Relevanz sein werden.

Bei der Abgrenzung der behördlichen Zuständigkeiten ist das Kriterium der Marktpräsenz problematisch, das eine herausgehobene Bedeutung für den kooperativen Glasfaserausbau hat. Selbstverständlich werden die direkten Auswirkungen auf den Wettbewerb besonders offensichtlich, wenn ein Unternehmen aktiv am Markt auftritt. Eine zentrale Gefahr des kooperativen Glasfaserausbaus wird hierdurch jedoch nicht adressiert, nämlich das kollusive Zusammenwirken der beteiligten Unternehmen (A. II. 1.). Hierbei ist letztlich unerheblich, ob das Gemeinschaftsunternehmen am Markt eigenständig in großem Umfang aktiv auftritt oder nicht. Das Kooperationsunternehmen bietet in beiden Fällen die Plattform für den Informationsaustausch zwischen den beteiligten Unternehmen. Je größer die Marktmacht, der Umsatz der beteiligten Unternehmen und das Kooperationsgebiet sind, desto eher beschränken sich die hieraus resultierenden Gefahren nicht nur auf den lokalen Infrastrukturwettbewerb, sondern haben auch Auswirkungen auf den Binnenmarkt. Das unionale Fusionskontrollrecht könnte aber, wenn es sich nicht um ein Vollfunktionsgemeinschaftsunternehmen handelt, keine Anwendung finden. Daher bedarf es einer Optimierung der Abgrenzungskriterien. Die FKVO sollte auch dann Anwendung finden können, wenn kein Vollfunktionsgemeinschaftsunternehmen gegründet wird. Zugleich sollte aber auch das „One-Stop-Shop“Prinzip nicht aufgegeben werden. Eine Lösung könnte die Anwendung der FKVO auf Gemeinschaftsunternehmen mit Relevanz für den Wettbewerb im Binnenmarkt sein. Dies könnte etwa mithilfe von Schwellenwerten bestimmt werden. Die Regelung müsste jedoch hinreichend flexibel sein, um atypische Fälle weiterhin lediglich dem nationalen Fusionskontrollrecht zu unterwerfen. Hier ist eine genaue Analyse erforderlich, welche Vorgaben und welche Behörde die Wettbewerbsgefahr am effizientesten bekämpfen könnten.

\section{c) Ergebnis}

Die Anwendung der unionalen und nationalen Vorgaben zur Fusionskontrolle schließt sich gegenseitig aus. Durch die klare Abgrenzung der gesetz-

717 Kling/Thomas, Kartellrecht, S. 763 m. w. N. 
lichen Anwendungsbereiche und behördlichen Zuständigkeiten wird eine umfassende Rechtssicherheit gewährleistet. Entscheidend ist, ob im Rahmen des Kooperationsvorhabens ein Vollfunktionsgemeinschaftsunternehmen gegründet wird. Dieses Abgrenzungskriterium lässt allerdings unberücksichtigt, dass von dem Gemeinschaftsunternehmen auch Gefahren für den Wettbewerb ausgehen können, wenn das Unternehmen nicht aktiv am Markt tätig ist. Insoweit sollte das Abgrenzungskriterium optimiert werden, ohne das „One-Stop-Shop“-Prinzip aufzugeben. In der Regel ist davon auszugehen, dass das Gemeinschaftsunternehmen zwar so ausgestattet wird, dass es eine eigenständige Marktpräsenz entfalten kann. Zugleich wird die Marktpräsenz jedoch maßgeblich darauf ausgerichtet sein, die Tätigkeiten gegenüber den Muttergesellschaften zu erbringen, sodass in der Regel jedenfalls nicht mehr als 20 Prozent hiervon zur Erbringung von Tätigkeiten für Drittunternehmen aufgebracht werden. Demnach ist regelmäßig kein Vollfunktionsgemeinschaftsunternehmen gegeben, sodass sich die rechtlichen Rahmenbedingungen aus den nationalen Vorgaben zur Fusionskontrolle ergeben.

\section{Ergebnis}

Entschließen sich die Partner dazu, ihre Kooperation umfassend zu institutionalisieren, und gründen ein Gemeinschaftsunternehmen, kann der Anwendungsbereich des Fusionskontrollrechts eröffnet sein, wenn es sich beim Gemeinschaftsunternehmen um einen Zusammenschluss im Sinne des $₫ 37$ Abs. 1 GWB handelt. Besonders häufig ist dies in Form des Kontroll- oder Anteilserwerbs durch die Muttergesellschaften der Fall. Zudem ist zwischen Mutter- und Tochtergesellschaft ein Beherrschungsverhältnis erforderlich. Es ist möglich, dass dieses auch durch mehrere Muttergesellschaften gemeinschaftlich ausgeübt wird. Dies ist anhand verschiedener Indizien zu prüfen, die einen gemeinschaftlichen Beherrschungswillen manifestieren. Eine Fusionskontrolle findet nur bei einer ernstlichen Gefahr für den Wettbewerb statt, die anhand von Umsatzschwellenwerten bestimmt wird. Sowohl das unionale als auch das nationale Wettbewerbsrecht kennen eine Fusionskontrolle, die zueinander in einem Exklusivitätsverhältnis stehen. Für die Abgrenzung der Anwendungsbereiche ist es maßgeblich, ob das Gemeinschaftsunternehmen als Vollfunktionsgemeinschaftsunternehmen zu klassifizieren ist. Insoweit bedarf der unionale Rechtsrahmen noch der Optimierung. Regelmäßig kann davon ausgegangen werden, dass jedenfalls die hierfür erforderliche Verkaufsquote von zumindest 20 Pro- 
zent gegenüber Drittunternehmen nicht überschritten wird. Liegt somit kein Vollgemeinschaftsunternehmen vor, findet eine nationale Fusionskontrolle statt.

\section{Anwendbarkeit des Kartellverbotes aus $₫ 1$ GWB}

Das nationale Kartellverbot bildet für Kooperationsvereinbarungen, die nicht dem Fusionskontroll- und Regulierungsrecht unterfallen, die einzige Steuerungsvorgabe (E. II.). Als Vorfrage hierzu ist zu klären, in welchen Konstellationen die nationalen Vorgaben zur Fusionskontrolle aus $\$ 36$ GWB (dazu 1.) und das unionale Kartellverbot aus Art. 101 AEUV (dazu 2.) eine Sperrwirkung entfalten. Auf die Tatbestands- und Freistellungsvoraussetzungen soll jedoch erst im Kontext der wettbewerbsrechtlichen Steuerungsvorgaben für die Ausgestaltung von Kooperationsvorhaben eingegangen werden (E. II.). Relevant sind die vorgenannten Fragen insbesondere für die Ermittlung der behördlichen Zuständigkeit.

\section{Sperrwirkung der Vorgaben zur Fusionskontrolle?}

Eine parallele Anwendbarkeit von Fusionskontrolle und kartellrechtlicher Missbrauchskontrolle ist nicht pauschal ausgeschlossen. ${ }^{718}$ In der Beschlusspraxis des Bundeskartellamts und der Rechtsprechung wurden Abgrenzungskriterien für die Identifikation von Fusionen verwendet, die nicht zusätzlich in den Anwendungsbereich des Kartellverbotes fallen. ${ }^{719}$ In jedem Einzelfall ist zu prüfen, ob eine Fusion vorliegt, die den Ausnahmetatbestand erfüllt. ${ }^{720}$

Als „kartellrechtsneutral gilt [demnach ...] ein rein konzentratives Gemeinschaftsunternehmen, das sämtliche Funktionen eines selbstständigen Unternehmens wahrnimmt, marktbezogene Leistungen erbringt und nicht ausschließlich oder überwiegend auf einer vor- oder nachgelagerten

718 Ständige Rspr. seit BGH, Beschl. v. 1.10.1985, Az. KVR 6/84, NJW 1986, S. 1874, 1875; BGH, Beschl. v. 17.7.2018, Az. KVR 64/17, NZKart 2018, S. 541, 543, Rn. 28; a. A. Wiedemann, Gemeinschaftsunternehmen im deutschen Kartellrecht, S. 173 ff. u. 212 ff.

719 BKartA, Beschl. v. 4.12.2019, Az. B 7-21/18, Rn.23; mit Verweis auf BGH, Beschl. v. 8.5.2001, Az. KVR 12/99, BGHZ 147, S. 325, 337 f.

720 BGH, Beschl. v. 4.3.2008, Az. KVZ 55/07, Rn. 14 (zit. nach juris); BGH, Beschl. v. 8.5.2001, Az. KVR 12/99, BGHZ 147, S. 325, 336 m. w. N. 
Stufe für die Muttergesellschaft sowie nicht auf demselben Markt wie die Mütter tätig ist. “721 Die Voraussetzungen sind denen eines Vollfunktionsgemeinschaftsunternehmens sehr ähnlich (B. II. 3. b.). Wesentlicher Unterschied hierzu ist jedoch, dass keine prozentualen Schwellen definiert werden, bei deren Überschreiten die Kartellrechtsneutralität eintritt. Die Tätigkeit muss ausschließlich gegenüber den Muttergesellschaften ausgeübt werden.

Die allgemeine kartellrechtliche Definition ist nun auf das Telekommunikationsrecht zu übertragen. Dieses ist, wie bereits dargestellt, durch eine Untergliederung der Wertschöpfung in die Erbringung von Vorleistungen und Endkundendiensten geprägt (Kapitel 4, A. I. 1.). Sobald Vorleistungen auch dritten Unternehmen, die nicht an der Kooperation beteiligt sind, zur Verfügung gestellt werden, kommt eine Kartellrechtsneutralität nicht in Betracht, da die Tätigkeit nicht ausschließlich gegenüber den Mutterunternehmen ausgeübt wird. Zu den zentralen regulatorischen Verpflichtungen des Telekommunikationsrechts gehören die Zugangs- und die Entgeltverpflichtung, die einen Wettbewerb trotz der Existenz eines natürlichen Monopols ermöglichen. Grundsätzlich knüpfen die Zugangsverpflichtungen gemäß $\$ 21$ TKG an die Feststellung einer marktbeherrschenden Stellung des betreffenden Unternehmens an. Dies wird auch im künftigen Rechtsrahmen (vgl. $\$ 26$ TKG-RefE) nicht anders sein. ${ }^{722}$ Dieses Kriterium ist nicht für jedes Gemeinschaftsunternehmen erfüllt, das im Zuge einer Kooperation zum Glasfaserausbau gegründet wird. Es existieren allerdings auch marktmachtunabhängige Zugangsverpflichtungen, die allen Telekommunikationsunter-nehmen mit Endnutzerkontrolle gemäß $\mathbb{1 8}$ TKG $(\mathbb{S} 21 \mathrm{f}$. TGK-RefE) $)^{723}$ auferlegt werden können. Weitere Zugangsverpflichtungen können aus der Inanspruchnahme von Fördermitteln resultieren, die an Open-Access-Verpflichtungen geknüpft werden. Ausbauvorhaben, die vollständig auf die Gewährung des Zuganges von Dritten verzichten können,

721 BKartA, Beschl. v. 4.12.2019, Az. B 7-21/18, Rn. 23; hierzu auch BGH, Beschl. v. 8.5.2001, Az. KVR 12/99, BGHZ 147, S. 325, 336 ff. m. w. N.; OLG Düsseldorf, Beschl. v. 15.5.2019, Az. VI-W (Kart) 4/19, NZKart 2019, S. 386, 387.

722 Referentenentwurf des Telekommunikationsmodernisierungsgesetzes (TKMoG), abrufbar unter: https:/www.bmwi.de/Redaktion/DE/Downloads/Gesetz/ telekommunikationsmodernisierungsgesetz-referentenentwurf-20201612.pdf? blob=publicationFile\&v=8 (zuletzt abgerufen am 12.1.2021).

723 Referentenentwurf des Telekommunikationsmodernisierungsgesetzes (TKMoG), abrufbar unter: https://www.bmwi.de/Redaktion/DE/Downloads/Gesetz/ telekommunikationsmodernisierungsgesetz-referentenentwurf-20201612.pdf? blob=publicationFile\&v=8 (zuletzt abgerufen am 12.1.2021). 
sind eher unwahrscheinlich. Im Ergebnis sind das Recht der Fusionskontrolle und das Kartellverbot in der Regel nebeneinander anwendbar.

Die weitgehend parallele Anwendbarkeit überzeugt, ebenso wie die sehr strengen Anforderungen an eine Kartellrechtsneutralität. Das Fusionskontrollrecht ist spezifisch auf die Gefahren von Unternehmenszusammenschlüssen und der Gründung von Gemeinschaftsunternehmen ausgerichtet, während das Kartellverbot allgemein die Gefahren adressiert, die durch eine Zusammenarbeit von Unternehmen entstehen. Insoweit besteht eine Schnittmenge, die nicht problematisch ist, da sich die Vorgaben nicht widersprechen, sondern weitgehend gleichlaufen (C. II.). Ist das Unternehmen überhaupt nicht am Markt aktiv, ist es kartellrechtlich nicht relevant. Das Fusionskontrollrecht ist insoweit dazu geeignet, Gefahren eines kollusiven Zusammenwirkens überzeugend zu bannen. Würden die Vorgaben auch im Übrigen in einem strikten Exklusivitätsverhältnis zueinanderstehen, könnte dies dann zu Schutzlücken führen, wenn der Anwendungsbereich des Fusionskontrollrechts grundsätzlich eröffnet ist, aber Ausnahmetatbestände eingreifen. Durch die 10. GWB-Novelle und die mit ihrer einhergehenden Streichung des $₫ 35$ Abs. 2 S. 1 GWB (B. II. 2.) ist diese Gefahr zwar vergleichsweise gering, aber dennoch existent. Im Interesse eines möglichst umfassenden und lückenlosen Schutzes des Wettbewerbs ist die bestehende Normierung mithin vorzugswürdig und sollte beibehalten werden.

\section{Sperrwirkung des unionalen Kartellverbotes aus Art.101 AEUV?}

Sowohl das unionale (Art. 101 AEUV) als auch das nationale ( $\$ 1$ GWB) Recht enthalten ein Kartellverbot. Auch in dieser Hinsicht stellt sich wie bereits im Rahmen der Fusionskontrolle (B. II. 3. a.) die Frage, ob die unionalen oder die nationalen Vorgaben den maßgeblichen Rechtsrahmen bilden. Im Ergebnis betrifft die Frage der Abgrenzung insbesondere die behördliche Zuständigkeit, da sich Art. 101 AEUV und $\$ 1$ GWB inhaltlich in weiten Teilen decken. ${ }^{724}$ Dennoch soll an dieser Stelle eine kurze Darstellung der Abgrenzungskriterien erfolgen, um die maßgebliche behördliche Spruchpraxis zu identifizieren. Das Unionsrecht findet nur in denjenigen Fällen Anwendung, in denen spürbare Beeinträchtigungen des Han-

724 So auch Grave/Nyberg, in: Loewenheim/Meessen/Riesenkampff u.a. (Hrsg.), Kartellrecht, $\mathbb{\$} 1 \mathrm{GWB}, \mathrm{Rn} .2$. 
dels zwischen den Mitgliedstaaten durch das Vorhaben zu erwarten sind. 725

Die hohen Anforderungen, die an dieses Kriterium gestellt werden, lassen sich anhand der Entscheidung des Bundeskartellamtes zur Gründung der Glasfaser Nordwest exemplifizieren. So verfüge das Kooperationsvorhaben lediglich über eine regionale Bedeutung und etwa betroffene Märkte über einen umfassenden Ortsbezug. ${ }^{726}$ Das Kooperationsgebiet umfasse zudem weniger als zehn Prozent des Bundesgebietes. ${ }^{727}$ Die Entscheidung des Bundeskartellamts lässt sich freilich nicht pauschal auf alle Sachverhalte übertragen. Sie kann aber insgesamt überzeugen. Auch wenn der unionale Gesetzgeber das Ziel eines digitalen Binnenmarktes verfolgt, ${ }^{728}$ existiert dieser im Kontext des festnetzbasierten Infrastrukturwettbewerbs nur rudimentär. Lokal ausgerichtete Kooperationsvorhaben tangieren den Wettbewerb vor Ort und haben keine Binnenmarktrelevanz. Je größer das Kooperationsgebiet jedoch ist, desto eher kann eine solche Binnenmarktrelevanz vorliegen. Solange nicht das gesamte Bundesgebiet oder große Teile davon als Kooperationsgebiet vorgesehen wird, kann jedoch davon ausgegangen werden, dass der Handel zwischen den Mitgliedstaaten durch das jeweilige Kooperationsvorhaben nicht oder nur marginal tangiert wird. Diese Prämisse soll in der nachfolgenden Untersuchung zu Grunde gelegt werden, sodass sich die Steuerungsvorgaben ausschließlich aus dem nationalen Kartellverbot gemäß $₫ 1$ GWB ergeben. Die strenge Abgrenzung der Anwendungsbereiche und behördlichen Zuständigkeiten führt auch in diesem Kontext zu Rechtssicherheit, sodass sie sich auch positiv auf das Investitionsklima auswirkt.

\section{Ergebnis}

Für Vorhaben zum kooperativen Ausbau von Glasfaserinfrastrukturen findet in der Regel das Kartellrecht neben dem Fusionskontrollrecht Anwendung. Dies ist nur in solchen Konstellationen nicht der Fall, in denen das Vorhaben kartellrechtsneutral ist. Hierbei handelt es sich um Gemein-

725 Weiterführend hierzu Kommission, Leitlinien über den Begriff der Beeinträchtigung des zwischenstaatlichen Handels in den Artikeln 81 und 82 des Vertrages (2004/C 101/07), Abl. C 101/81 v. 27.4.2004.

726 BKartA, Beschl. v. 4.12.2019, Az. B 7-21/18, Rn. 27.

727 BKartA, Beschl. v. 4.12.2019, Az. B 7-21/18, Rn. 27.

728 Erwägungsgrund 3 des EKEK. 
schaftsunternehmen, die ausschließlich für ihre Muttergesellschaften tätig sind. Schon aufgrund der Existenz marktmachtunabhängiger Zugangsverpflichtungen gegen Entgelt ist davon auszugehen, dass dieses Kriterium im Bereich des kooperativen Glasfaserausbaus in der Regel nicht erfüllt wird. Das Kartellverbot ergibt sich aufgrund der regionalen Ausrichtung der meisten Kooperationsvorhaben aus $\mathbb{1} 1 \mathrm{GWB}$. Sofern das Vorhaben ausnahmsweise dazu geeignet ist, den Handel zwischen den Mitgliedstaaten zu beeinträchtigen, können sich die Vorgaben auch aus Art. 101 AEUV ergeben. Die daraus folgenden materiellen Unterschiede sind zwar gering, eröffnen aber die Zuständigkeit unterschiedlicher Behörden.

\section{Abgrenzung der relevanten Märkte}

Die Untersuchung der Auswirkungen von Kooperationsvorhaben auf den Wettbewerb knüpft an die Abgrenzung von Märkten an. Nur auf diese Weise lassen sich Wettbewerbskräfte und -beschränkungen zielgenau ermitteln. Sie dient als Grundlage für wettbewerbsrechtliche Konsequenzen, die von der jeweils zuständigen Vollzugsbehörde gezogen werden können. Die Marktabgrenzung folgt im gesamten Wettbewerbsrecht einer einheitlichen Systematik (dazu I.). Potentiell von kooperativen Glasfaserausbauvorhaben tangierte Märkte sollen am Beispiel der Glasfaser Nordwest veranschaulicht werden. Sowohl die zuständige Kartell- als auch die zuständige Regulierungsbehörde haben Marktabgrenzungen für die Breitbandzugangsmärkte durchgeführt. Die sachlichen Marktabgrenzungen stimmen weitgehend überein, weichen jedoch in räumlicher Hinsicht entscheidend voneinander ab (dazu II.). Die Kartellbehörden grenzen darüber hinaus weitere Märkte $a b$, die durch kooperative Glasfaserausbauvorhaben tangiert werden können, aber nicht in den Anwendungsbereich des Regulierungsrechts fallen (dazu III.). Anhand der Marktabgrenzungen lassen sich das Zusammenspiel der verschiedenen Regelungsregime des Wettbewerbsrechts illustrieren sowie Vor- und Nachteile der behördlichen Beschlusspraxis veranschaulichen.

\section{Grundlagen zur Marktabgrenzung im Wettbewerbsrecht}

Ausgangspunkt wettbewerbsrechtlicher Analysen ist stets die Frage nach der Auswirkung eines bestimmten Umstandes auf den Markt. Der Markt ist insbesondere in sachlicher (dazu 1.) und räumlicher Hinsicht (dazu 2.) 
abzugrenzen. Dabei wird sowohl auf unionaler als auch auf nationaler Ebene auf eine einheitliche Methodik zurückgegriffen, die im gesamten Wettbewerbsrecht genutzt wird und damit der Rechtssicherheit dient. Dennoch ist es nicht ausgeschlossen, dass sich die Marktabgrenzungen der Wettbewerbsbehörden unterscheiden (dazu 3.).

\section{Sachliche Marktabgrenzung}

Die Abgrenzung des sachlich relevanten Marktes erfolgt nach dem soge-

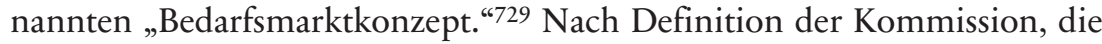
sich mit der Rechtsprechung, der Beschlusspraxis der Behörden und der Literatur deckt, erstreckt sich der sachlich relevante Markt auf „sämtliche Erzeugnisse und/oder Dienstleistungen, die von den Verbrauchern hinsichtlich ihrer Eigenschaften, Preise und ihres vorgesehenen Verwendungszwecks als austauschbar oder substituierbar angesehen werden. " ${ }^{\text {"730 }}$ Bei der sachlichen Marktabgrenzung steht also die funktionelle Austauschbarkeit aus der Sicht der Marktgegenseite im Vordergrund.

Besonders eindrücklich lassen sich diese Wettbewerbskräfte anhand der Reaktion auf eine Preiserhöhung simulieren. ${ }^{731}$ Dabei stellt sich die Frage, ob diese profitabel durchgeführt werden kann. ${ }^{732}$ Hierzu wird ermittelt, ob bei einer kleinen, aber spürbaren dauerhaften Erhöhung des Preises der Kunde zu einem Alternativprodukt wechselt. ${ }^{733}$ Dies wird mithilfe des „hypothetischen Monopolistentests“ simuliert, der sinnbildlich für seine Funktionsweise als SSNIP-Test ${ }^{734}$ abgekürzt wird. Im Rahmen des soge-

729 Dies ergibt sich aus der ständigen Rechtsprechung, namentlich BGH, Urt. v. 8.10.2019, Az. KZR 73/17, NJW 2020, S.64, 66 f., Rn. 23; BGH, Urt. v. 30.3.2011, Az. KZR 6/09, GRUR 2011, S.943, 944, Rn. 12; OLG Düsseldorf, Beschl. v. 21.10.2009, Az. VI-Kart 14/08 (V), Rn. 37 (zit. nach juris).

730 Bekanntmachung der Kommission über die Definition des relevanten Marktes im Sinne des Wettbewerbsrechts der Gemeinschaft (Bekanntmachung 97/C 372/03), Abl. C 372/5 v. 9.12.1997 über die Marktdefinition, Rn. 7; Mitteilung der Kommission über die Leitlinien zur Marktanalyse und Ermittlung beträchtlicher Marktmacht nach dem EU-Rechtsrahmen für elektronische Kommunikationsnetze und -dienste (Mitteilung 2018/C 159/01), ABl. C 159/1 v. 7.5.2018, Rn.33.

731 Bekanntmachung 97/C 372/03, Rn. 17.

732 Lenßen, Der kartellrechtlich relevante Markt, S. 106.

733 Bekanntmachung 97/C 372/03, Rn. 17.

734 SSNIP steht für „small but significant non transistory increase of price“, also eine kleine, aber signifikante und anhaltende Preiserhöhung. 
nannten „Bedarfsmarktkonzeptes" ${ }^{\text {"735 }}$ steht zwar die funktionale Austauschbarkeit im Vordergrund, wird jedoch durch weitere Aspekte im Rahmen der Untersuchung ergänzt. ${ }^{736}$ Die Substituierbarkeit des Angebotes wird bei einer vergleichbaren Auswirkung berücksichtigt. ${ }^{737}$ Von ihr ist auszugehen, wenn alternative Anbieter ihr Angebot in Reaktion auf die Preiserhöhung kurzfristig umstellen und auf dem Markt aktiv werden können. ${ }^{738}$ Eine Berücksichtigung scheidet in solchen Fällen aus, in denen eine Angebotsumstellung zwar theoretisch möglich ist, jedoch an hohe Investitionsvolumina gekoppelt wäre oder einen größeren Zeitraum in Anspruch nähme. ${ }^{739}$ Der potentielle Wettbewerb spielt im Rahmen der sachlichen Marktabgrenzung regelmäßig keine Rolle, sondern wird vielmehr im Rahmen der nachgeordneten Prüfung der Auswirkungen der Kooperation auf den Wettbewerb in die Prüfung einbezogen. ${ }^{740}$ Für die Untersuchung der Auswirkungen schreibt die Kommission keine spezifische empirische Methodik vor. ${ }^{71}$

\section{Räumliche Marktabgrenzung}

Bei der Abgrenzung des räumlichen Marktes ist zu untersuchen, über welchen geographischen Bereich sich der spezifische Markt erstreckt. ${ }^{742}$ Nach Definition der Kommission, die auch die Rechtsprechung, die Beschlusspraxis der Behörden und die Literatur zu Grunde legen, erstreckt sich der räumlich relevante Markt über „das Gebiet, in dem die beteiligten Unternehmen die relevanten Produkte oder Dienstleistungen anbieten, in dem die Wettbewerbsbedingungen hinreichend homogen sind und das sich von benachbarten Gebieten durch spürbar unterschiedliche Wettbewerbs-

735 Grundlegend zum Bedarfsmarktkonzept statt vieler Lenßen, Der kartellrechtlich relevante Markt, S. $185 \mathrm{ff}$.

736 Umfangreiche Vorgaben enthält hierzu die Bekanntmachung 97/C 372/03, Rn. $36 \mathrm{ff}$.

737 Bekanntmachung 97/C 372/03, Rn. 20.

738 Bekanntmachung 97/C 372/03, Rn. 20.

739 Bekanntmachung 97/C 372/03, Rn. 23.

740 Bekanntmachung 97/C 372/03, Rn. 24.

741 Bekanntmachung 97/C 372/03, Rn. 25 ff.; einen Überblick über empirische Untersuchungsmethoden bieten etwa Peeperkorn/Verouden, in: Faull/Nikpay (Hrsg.), The EU law of competition, S. 3, $43 \mathrm{ff}$.

742 Mitteilung 2018/C 159/01, Rn. 46. 
bedingungen unterscheidet." ${ }^{143}$ Als Abgrenzungskriterien fungieren wiederum die Wettbewerbskräfte der Substituierbarkeit von Angebot und Nachfrage sowie des potentiellen Wettbewerbs, die bereits dargestellt worden sind (C. II. 2.)..$^{74}$

3. Möglichkeit der divergierenden Marktabgrenzung durch unterschiedliche Behörden

Die Marktabgrenzung findet mithin im gesamten Wettbewerbsrecht auf Basis derselben Kriterien statt. Dabei kommen die Behörden und Gerichte jedoch nicht zwangsläufig zu denselben Ergebnissen. Die Kommission ist bestrebt, eine weitgehend einheitliche Spruchpraxis der Wettbewerbsbehörden sicherzustellen. Hierzu hat sie Bekanntmachungen, Leitlinien und Kriterien erlassen. So sollen die Behörden, „wenn sie ähnliche Fälle unter ähnlichen Umständen mit den gleichen übergeordneten Zielen untersuchen $[, \ldots]$ grundsätzlich zu ähnlichen Schlussfolgerungen kommen. ${ }^{\text {"7 }}$ (45

Bei diesen Regelungen handelt es sich um sogenanntes „soft law“, 746 das keine verbindliche Rechtsnorm, aber auch nicht ohne Bedeutung ist. ${ }^{747}$ Die Leitlinien stellen nach der Rechtsprechung der Unionsgerichte „eine Verhaltensnorm dar, die einen Hinweis auf die zu befolgende Verwaltungspraxis enthält und von der die Verwaltung im Einzelfall nicht ohne Angabe von Gründen abweichen kann, die mit dem Grundsatz der Gleichbehandlung vereinbar sind.“" ${ }^{748}$ Sie stellen faktisch ein Kompendium des

743 Bekanntmachung 97/C 372/03, Rn. 8; Stumpf, Die Abgrenzung subnationaler Märkte als regulatorischer Ansatz, S. 5.

744 Mitteilung 2018/C 159/01, Rn. 47.

745 Mitteilung 2018/C 159/01, Rn. 10.

746 Weiterführend zum Begriff des „soft law“ statt vieler Kühling, in: Ruffert (Hrsg.), Europäisches Sektorales Wirtschaftsrecht, $\mathbb{\$} 4$, Rn. 89; Müller-Graff, EuR 2012, S. 18, 18 ff.; umfassend zur Funktion des „soft law“ im Telekommunikationsrecht Bulowski, Regulierung von Internetkommunikationsdiensten, S. $224 \mathrm{ff}$.

747 Dies ergibt sich aus der ständigen Rechtsprechung des EuGH, besonders eindrücklich EuGH, Urt. v. 18.3.2010, verb. Rs. C-317/08, C-319/08 und C-320/08 (Alassini u. a.), ECLI:EU:C:2010:146, Rn. 40; EuGH, Urt. v. 24.8.2008, Rs. C-55/06 (Arcor/Deutschland), ECLI:EU:C:2008:244, Rn.94; Schwarze, EuR 2011, S.3, 5; von Graevenitz, EuZW 2013, S.169; von Graevenitz, ZRP 2019, S. 75,76 .

748 EuG, Urt. v. 8.10.2008, Rs. T-73/04 (Carbone-Lorraine/Kommission), ECLI:EU: T:2008: 416, Rn.70; mit einer vergleichbaren Aussage auch EuGH, Urt. v. 28.6.2005, verb. Rs. C-189/02 P, C-202/02 P, C-205/02 P bis C-208/02 P und 
unionalen Wettbewerbsrechts dar. ${ }^{79}$ Mit der Verwaltung in diesem Sinne ist die Kommission gemeint. Nun sind jedoch, korrespondierend mit dem normativen Anwendungsbereich, im Zusammenhang mit Kooperationen zur Beschleunigung des Glasfaserausbaus zumeist die nationalen Behörden, also die Bundesnetzagentur und das Bundeskartellamt, zuständig.

Die Bundesnetzagentur ist dazu verpflichtet, im Rahmen der Marktdefinition die Marktanalyseleitlinien gemäß $₫ 10$ Abs. 2 S. 2 TKG weitestgehend zu berücksichtigen. Dabei stellt Art. 15 Abs. 3 S. 1 RRL, auf dem die nationalen Vorgaben derzeit beruhen, klar, dass die nationalen Gegebenheiten bei der Marktanalyse zu berücksichtigen sind. Diese Berücksichtigungspflicht wird durch Art. 64 Abs. 2 UAbs. 2 EKEK beibehalten und wird sich mithin auch im Rahmen der erforderlichen TKG-Novelle nicht ändern. ${ }^{750}$ Diese faktische Bindungswirkung wird dadurch verstärkt, dass für die Marktdefinition gemäß $\$ 12$ Abs. 2 TKG ein unionales Konsultations- und Konsolidierungsverfahren durchgeführt werden muss. Dabei spielt insbesondere die Märkteempfehlung ${ }^{751}$ eine herausragende Rolle, in der die Kommission in Anhang I festlegt, welche sachlich relevanten Produktmärkte existieren. Weicht die Bundesnetzagentur von der Märkteempfehlung ab, resultiert hieraus gemäß $₫ 12$ Abs. 2 Nr. 3 S. 2 TKG ein Vetorecht der Kommission. Das nationale Recht erklärt die Marktanalyseleitlinien und die Märkteempfehlung damit zwar nicht explizit für verbindlich, allerdings wird eine faktische Verbindlichkeit durch die Hintertür konstruiert. ${ }^{752}$ An diesen Grundstrukturen werden im künftigen nationalen

C-213/02 P (Dansk Rørindustri u. a./Kommission), ECLI:EU:C:2005:408, Rn. 209; EuGH, Urt. v. 15.1.2002, Rs. C-171/00 P (Libéros/Kommission), ECLI: EU:C:2002:17, Rn. 35; weiterführend hierzu Schwarze, EuR 2011, S. 3, 8.

749 So auch BNetzA, Beschl. v. 27.8.2015, Az. BK 1-12/003, S. 67.

750 Referentenentwurf des Telekommunikationsmodernisierungsgesetzes (TKMoG), abrufbar unter: https:/www.bmwi.de/Redaktion/DE/Downloads/Gesetz/ telekommunikationsmodernisierungsgesetz-referentenentwurf-20201612.pdf? blob=publicationFile\&v=8 (zuletzt abgerufen am 12.1.2021).

751 Empfehlung der Kommission v. 9.10.2014 über relevante Produkt- und Dienstmärkte des elektronischen Kommunikationssektors, die aufgrund der Richtlinie 2002/21/EG des Europäischen Parlaments und des Rates über einen gemeinsamen Rechtsrahmen für elektronische Kommunikationsnetze und -dienste für eine Vorabregulierung in Betracht kommen (Empfehlung 2014/710/EU), Abl. L 295/79 v. 11.10.2014.

752 Zur systematischen und verfassungsrechtlichen Problematik eines solchen Vorgehens Thomas, EuR 2009, S. 423, $432 \mathrm{f}$. 
Rechtsrahmen keine Veränderungen vorgenommen. ${ }^{753}$ Das GWB enthält hingegen keine vergleichbare Regelungssystematik. Torsten Körber konstatiert jedoch auch hier „eine erhebliche faktische Autorität sowie eine Leitbildfunktion" der Kommission. ${ }^{754}$ Dies schließt jedoch unterschiedliche Marktabgrenzungen hinsichtlich vergleichbarer Produkte durch das Bundeskartellamt und die Bundesnetzagentur nicht aus. Eine solche Abweichung ist insbesondere bei der räumlichen Marktabgrenzung hinsichtlich der tangierten Märkte im kooperativen Glasfaserausbau zu beobachten (C. II. 2.).

Solange die Abweichungen lediglich in Detailfragen bestehen, ist dies im Ergebnis ohne Belang. Die Behörden sind voneinander unabhängig und sollten die Möglichkeit haben, abweichende Entscheidungen zu treffen. Problematisch wäre es hingegen, wenn die Marktabgrenzungen eklatant voneinander abwichen. Eine solche Entwicklung trüge zu einer erheblichen Rechtsunsicherheit bei und würde die Investitionsbedingungen verschlechtern. Durch die umfassend institutionalisierte gegenseitige Konsultation von Bundeskartellamt und Bundesnetzagentur in Querschnittsmaterien wird diese Gefahr jedoch ausreichend adressiert, sodass im Ergebnis kein weitergehender Handlungsbedarf besteht.

\section{Fazit}

Im Ergebnis führen die unionalen und die nationalen Wettbewerbsbehörden die Marktabgrenzung in sachlicher und räumlicher Hinsicht auf Basis derselben Kriterien durch. Dies bringt eine umfassende Rechtssicherheit mit sich. Die Marktabgrenzung der Bundesnetzagentur ist durch das Unionsrecht weitgehend determiniert. Zwar orientiert sich die nationale kartellrechtliche Beschlusspraxis auch an den Vorgaben des Unionsrechts, der Determinierungsgrad ist jedoch nicht so hoch. Deswegen können die Marktabgrenzungen von Bundesnetzagentur und Bundeskartellamt trotz der einheitlichen Methodik und der unionsrechtlichen Determinierung voneinander abweichen. Dies betrifft regelmäßig lediglich Detailfragen.

753 Referentenentwurf des Telekommunikationsmodernisierungsgesetzes (TKMoG), abrufbar unter: https:/www.bmwi.de/Redaktion/DE/Downloads/Gesetz/ telekommunikationsmodernisierungsgesetz-referentenentwurf-20201612.pdf? blob=publicationFile $\& v=8$ (zuletzt abgerufen am 12.1.2021).

754 Körber, in: Immenga/Mestmäcker (Hrsg.), Wettbewerbsrecht, Band 1, Einleitung FKVO, Rn. 77. 
Umfassenden Abweichungen wird durch gegenseitige Konsultationen in Querschnittsmaterien effektiv begegnet.

II. Breitbandinternetzugangs- und Endkundenmärkte des Telekommunikationssektors

Die Wertschöpfung im Telekommunikationssektor kann grob in die Erbringung von Vorleistungen und die Erbringung von Endkundendiensten untergliedert werden (Kapitel 4, A. I. 1.). In sachlicher Hinsicht grenzen die Bundesnetzagentur und das Bundeskartellamt die Vorleistungs- und Endkundenmärkte für den Breitbandinternetzugang (bis auf Detailfragen) einheitlich ab (dazu 1.). Während die Bundesnetzagentur jedoch räumlich von einem deutschlandweiten Markt ausgeht, nahm das Bundeskartellamt im Rahmen der Fusionskontrolle zur Gründung der Glasfaser Nordwest eine regionale Marktabgrenzung vor (dazu 2.). Fraglich ist, ob eine solche Regionalisierung auch für die regulatorische Spruchpraxis sinnvoll wäre.

\section{Sachliche Marktabgrenzung}

Bei der sachlichen Abgrenzung der Breitbandinternetzugangsmärkte ist zwischen den Endkunden- (dazu a.) und den Vorleistungsmärkten zu differenzieren. Diese sind wiederum in den lokalen (dazu b.) und den zentralen (dazu c.) Vorleistungsmarkt für den Teilnehmeranschluss zu untergliedern. Aufgrund der umfassenden Parallelen zwischen den sachlichen Marktabgrenzungen von Bundesnetzagentur und Bundeskartellamt sollen die Grundzüge der sachlichen Marktabgrenzung, die für das Verständnis der Ausführungen zur räumlichen Marktabgrenzung erforderlich sind, für beide einheitlich dargelegt werden.

\section{a) Endkundenmarkt für festnetzbasierten Internetzugang}

Der Endkundenmarkt für den festnetzbasierten Internetzugang wird von Bundesnetzagentur und Bundeskartellamt technologieneutral und unabhängig von der Übertragungskapazität abgegrenzt. Derzeit ist eine Marktabgrenzung der Bundesnetzagentur aus dem Jahr 2015 in Kraft. Im Jahr 2019 wurde ein Konsultationsverfahren für eine aktualisierte Marktabgren- 
zung und -analyse durchgeführt. ${ }^{755}$ Derzeit findet ein Notifizierungsverfahren durch die Kommission statt. ${ }^{756}$ Die neue Marktabgrenzung entfaltet erst dann ihre Bindungswirkung, wenn das Marktregulierungsverfahren vollständig abgeschlossen ist. Aus diesem Grund soll die folgende Analyse anhand der Marktabgrenzung aus dem Jahr 2015 und des Konsultationsdokuments aus dem Jahr 2019 erfolgen. Das Bundeskartellamt bezeichnet den Markt in seiner Entscheidung zur Gründung der Glasfaser Nordwest als „Massenmarkt für den festnetzbasierten Internetzugang “".757

Aus Endkundensicht ist es unerheblich, ob die Teilnehmeranschlussleitung aus Kupfer- oder aus Glasfaser besteht. ${ }^{758}$ Dies gilt sowohl für Unternehmen als auch für Privatkunden. ${ }^{759}$ Derzeit kann der Bedarf des durchschnittlichen Nachfragers mithilfe beider Technologien befriedigt werden (Kapitel 2, C. I. 3.). ${ }^{760}$ Des Weiteren findet keine Subdifferenzierung im Hinblick auf Anschlusstechnologie, Bandbreite oder Bündelprodukte statt. ${ }^{761}$ Aus diesem Grund werden beide Technologien demselben sachlichen Markt zugeordnet. ${ }^{762}$ Dies gilt jedoch nicht für die Realisierung des Anschlusses einzelner Teilnehmer mithilfe von Kabelnetzen ${ }^{763}$ oder auch nicht festnetzbasierte Möglichkeiten des Teilnehmeranschlusses. ${ }^{764}$ Hier sind die Übertragungstechnologien aus Sicht der Nachfrager, aufgrund ihrer technischen Spezifika nicht austauschbar. Die Bundesnetzagentur orientiert sich bei der Marktabgrenzung für die Vorleistungsmärkte an der

755 BNetzA, Konsultationsentwurf Markt 3a, Az. BK 1-19/001; umfassend hierzu Monopolkommission, 11. Sektorgutachten Telekommunikation (2019), Rn. $71 \mathrm{ff}$.

756 Der aktuelle Verfahrensstand ist abrufbar unter: https://circabc.europa.eu/ui/gro up/2328c58f-1fed-4402-a6cc-0f0237699dc3/library/a9ce6dbc-6dad-413d-ad5f-8b0f adf3a537? $\mathrm{p}=1 \& \mathrm{n}=10 \&$ sort=modified_DESC (zuletzt abgerufen am 1.7.2020).

757 BKartA, Beschl. v. 30.12.2019, Az. B 7-21/18, Rn. 118.

758 BNetzA, Beschl. v. 27.8.2015, Az. BK 1-12/003, S. 95.

759 BKartA, Beschl. v. 30.12.2019, Az. B 7-21/18, Rn. 122; BNetzA, Konsultationsentwurf Markt 3a, Az. BK 1-19/001, S. 55 f.

760 BNetzA, Konsultationsentwurf Markt 3a, Az. BK 1-19/001, S. 74.

761 BKartA, Beschl. v. 30.12.2019, Az. B 7-21/18, Rn. 125; BNetzA, Konsultationsentwurf Markt 3a, Az. BK 1-19/001, S. $63 \mathrm{ff}$.

762 BNetzA, Beschl. v. 27.8.2015, Az. BK 1-12/003, S. 81 ff.; BNetzA, Konsultationsentwurf Markt 3a, Az. BK 1-19/001, S. 72 ff.; Monopolkommission, 11. Sektorgutachten Telekommunikation (2019), Rn. 72 f.; Sickmann/Neumann, Deregulierung und Verbraucherwohlfahrt auf dem deutschen Telekommunikationsmarkt, S. 63.

763 BNetzA, Beschl. v. 27.8.2015, Az. BK 1-12/003, S. 99 ff.; BNetzA, Konsultationsentwurf Markt 3a, Az. BK 1-19/001, S. 75 ff.

764 BNetzA, Konsultationsentwurf Markt 3a, Az. BK 1-19/001, S. 78 ff. 
Nachfrage auf der Endkundenebene. Die Marktabgrenzung hat demnach nur einen mittelbaren Zweck. Der Endkundenmarkt wird jedoch nicht reguliert. Endkundenmärkte sollen nämlich gemäß Nr. 2 der Märkteempfehlung 2014 nur dann reguliert werden, wenn „beträchtliche und anhaltende strukturelle, rechtliche oder regulatorische Zutrittsschranken“ "(lit. a) bestehen, die Marktstruktur durch Zutrittsschranken geprägt ist, die dazu beitragen, dass langfristig kein Wettbewerb bestehen wird (lit. b), und das Wettbewerbsrecht allein nicht ausreicht, „um dem festgestellten Marktversagen angemessen entgegenzuwirken“ (lit. c). Für die Endkundenmärkte wird davon ausgegangen, dass das letztgenannte Kriterium nicht erfüllt ist. Das Kartellrecht, das auf den Vorleistungsmärkten als nicht hinreichend suffizient angesehen wird, wird im Hinblick auf die Endkundenmärkte für ausreichend erachtet. Dies kann aufgrund der umfassenden wettbewerblichen Durchdringung des Endkundenmarktes überzeugen. Hierzu tragen insbesondere die Zugangsverpflichtungen bei, die eine Tätigkeit auf dem Endkundenmarkt, auch ohne eigene Infrastruktur ermöglichen.

b) Markt für den lokal bereitgestellten Zugang zur

Teilnehmeranschlussleitung

Auf der Vorleistungsebene werden ebenfalls die Anschlusstechnologien Kupfer und Glasfaser demselben Markt zugeordnet. Hinzu treten virtuelle Ersatzprodukte, die insbesondere für die Übertragungstechnologie Vectoring von Bedeutung sind (Kapitel 3, B. III.). ${ }^{765}$ Aufgrund des verstärkten indirekten Wettbewerbsdrucks ist im Konsultationsentwurf der Märkteempfehlung der Bundesnetzagentur eine Erweiterung des Marktes 3a, der den lokal bereitgestellten Teilnehmeranschluss erfasst, um virtuell entbündelte Kabelanschlüsse geplant. ${ }^{766}$ Die Zuordnung der Anschlusstechnologien Glasfaser und Kupfer in den sachlich einheitlichen Markt ist zwar aktuell möglich, muss allerdings fortlaufend überprüft werden. Je höher der Bandbreitenbedarf in Zukunft steigt und je weniger die Bedürfnisse mithilfe eines Teilnehmeranschlusses aus Kupfer befriedigt werden können, desto eher ist von zwei separaten sachlichen Märkten für die unterschiedli-

765 Hierzu im Ergebnis BNetzA, Konsultationsentwurf Markt 3a, Az. BK 1-19/001, S. 162; BKartA, Beschl. v. 30.12.2019, Az. B 7-21/18, Rn. 193 f.

766 BNetzA, Konsultationsentwurf Markt 3a, Az. BK 1-19/001, S. 149 ff. 
chen Anschlusstechnologien auszugehen. ${ }^{767}$ Derzeit findet jedoch noch keine bandbreitenbezogene Abgrenzung von Sub-Märkten statt. ${ }^{768}$ Diesbezüglich ist eine kontinuierliche Überprüfung der Nachfrage und der Zahlungsbereitschaft der Endkunden erforderlich, ${ }^{769}$ die sich an der Regulierungsperiode orientieren kann. Danach ist gemäß Art. 67 Abs.5 lit.a EKEK in der Regel alle fünf Jahre eine Marktanalyse vorzunehmen.

\section{c) Markt für den zentral bereitgestellten Zugang zur Teilnehmer- anschlussleitung}

Dem zentralen Zugangsmarkt zur physischen Teilnehmeranschlussleitung sind die unterschiedlichen Bitstromzugangsprodukte zuzuordnen. ${ }^{770}$ Hierbei handelt es sich um Vorleistungsprodukte, die eine geringere eigene Infrastruktur voraussetzen als eine eigene lokale Teilnehmeranschlussleitung. Die Unternehmen müssen nicht jeden einzelnen Endkunden, sondern lediglich die Zugangspunkte erschließen. Eine differenzierte Unterscheidung zwischen Layer-2- und Layer-3-Bitstromzugang ist an dieser Stelle erforderlich, um die nachfolgenden Unterschiede bei der räumlichen Abgrenzung der Submärkte zu verstehen.

Die beiden Bitstromzugangsprodukte unterscheiden sich insbesondere durch den Übergabepunkt des Datenverkehrs und die Spezifika des vom Kunden begehrten Produktes. ${ }^{771}$ Der Layer-2-Bitstromzugang ist dadurch geprägt, dass die Übergabe bereits im Konzentratornetz des Unternehmens erfolgt, das den Zugang gewährt. Hierdurch rückt der Zugang in die Nähe des Endkunden, was den Aufbau einer umfassenden eigenen Infrastruktur durch den Zugangspetenten erfordert. Dieser muss die etwa 900 Zugangspunkte mit eigener Infrastruktur erschließen, um in ganz Deutschland Endkundenprodukte anbieten zu können. ${ }^{772}$ Er begehrt zudem ein Produkt, das durch eine hohe Qualität und eine geringe Beschaltung im Sinne einer rudimentären Vorkonfiguration geprägt ist. Das Bundeskartellamt

767 So im Ergebnis auch Monopolkommission, 11. Sektorgutachten Telekommunikation (2019), Rn. 102.

768 BNetzA, Konsultationsentwurf Markt 3a, Az. BK 1-19/001, S. 87 ff.

769 Zur aktuellen Datenlage bereits Kapitel 2, C. II. 2.

770 BNetzA, Beschl. v. 27.8.2015, Az. BK 1-12/003, S. 104 ff., 118.

771 Einen guten Überblick hierzu bei BNetzA, Beschl. v. 9.7.2015, Az. BK 1-14-00, S. 98, Abb. 10; Kühling/Schall/Biendl, Telekommunikationsrecht, Rn. 205, Abbildung 27.

772 BNetzA, Beschl. v. 9.7.2015, Az. BK 1-14-00, S. 97. 
rechnet den Layer-2-Bitstromzugang dem Vorleistungsmarkt für den lokalen Breitbandzugangsmarkt zu. ${ }^{773}$ Dies wird auf eine gegenseitige Substituierbarkeit mit den anderen Formen des lokalen Teilnehmeranschlusses begründet, die sich auf die hohe Anzahl der zu erschließenden Zugangspunkte zurückführen lässt. Die Bundesnetzagentur hingegen zählt den Layer-2-Bitstromzugang weiterhin zum zentral bereitgestellten Teilnehmeranschlussmarkt. Für die weitergehende Untersuchung in diesem Abschnitt kann es letztlich offenbleiben, ob eine Zuordnung zum lokalen oder zentralen Teilnehmeranschlussmarkt erfolgt. Es ist letztlich nur erheblich, dass sich der Layer-2-Bitstromzugang erheblich von seinem Pendant unterscheidet.

Beim Layer-3-Bitstromzugang erfolgt die Übergabe nämlich erst im IPKernnetz, sodass der Zugangspetent über weniger eigene Infrastruktur verfügen muss. ${ }^{774}$ Er muss lediglich 73 Übergabepunkte erschließen. ${ }^{775}$ Die Infrastruktur ist umfassend vorkonfiguriert, sodass eine individuelle Konfiguration seltener möglich ist. Die Qualitätsanforderungen des Petenten sind geringer, da der Layer-3-Bitstromzugang vornehmlich zur Erbringung von Massenprodukten genutzt wird. Entscheidend ist, dass sowohl das Bundeskartellamt als auch die Bundesnetzagentur davon ausgehen, dass sich Layer-2- und Layer-3-Bitstromzugang voneinander unterscheiden und eine sachliche Differenzierung erforderlich ist.

\section{Räumliche Marktabgrenzung}

Während die zuständigen Wettbewerbsbehörden die Märkte (bis auf Detailfragen) sachlich identisch abgrenzen, weichen sie in deren räumlichen Abgrenzung erheblich voneinander ab. Dies soll anhand der Entscheidungen der Präsidentenkammer der Bundesnetzagentur zur Abgrenzung der Anschlussmärkte und der Entscheidungen des Bundeskartellamts im Rahmen der Gründung der Glasfaser Nordwest beurteilt werden. So grenzt die Bundesnetzagentur die Zugangsmärkte in der Regel national ab (dazu a.), während das Bundeskartellamt die räumlich relevanten Märkte regional

773 BKartA, Beschl. v. 30.12.2019, Az. B 7-21/18, Rn. $192 \mathrm{ff}$.

$774 \mathrm{Zu}$ der Frage der Zuordnung von HFC-Infrastrukturen zum zentralen Teilnehmeranschlussmarkt BNetzA, Beschl. v. 9.7.2015, Az. BK 1-14-00, S. 101 ff.; weiterführend hierzu Sickmann/Neumann, Deregulierung und Verbraucherwohlfahrt auf dem deutschen Telekommunikationsmarkt, S. $64 \mathrm{f}$.

775 BNetzA, Beschl. v. 9.7.2015, Az. BK 1-14-00, S. 99. 
auf das Kooperationsgebiet beschränkt (dazu b.). Dabei ist insbesondere darauf einzugehen, ob auch die Bundesnetzagentur ihre Marktabgrenzung stärker regional ausrichten sollte, um die Auswirkungen eines Kooperationsvorhabens auf den Wettbewerb besser bewerten zu können (dazu c.).

\section{a) Beschlusspraxis der Bundesnetzagentur zur räumlichen Abgrenzung der Anschlussmärkte}

Im Rahmen der räumlichen Abgrenzung der Anschlussmärkte ist die Bundesnetzagentur damit konfrontiert, dass der Bedarf an einem Teilnehmeranschluss außerhalb eines bestimmten, räumlich sehr eng gefassten Gebietes nicht alternativ befriedigt werden kann. Es liegt eine sehr starke lokale Bindung vor. Soll ein bestimmter Endkunde erreicht werden, ist dies im lokalen Teilnehmeranschlussmarkt nur über die spezifische Teilnehmeranschlussleitung und im zentralen Teilnehmeranschlussmarkt nur über den jeweiligen Zugangspunkt möglich. ${ }^{776}$ Es kann nicht auf einen alternativen Standort zurückgegriffen werden. Das Bedarfsmarktkonzept stößt in dieser Hinsicht an seine Grenzen. ${ }^{777}$ Bei seiner konsequenten Anwendung müsste jeder Teilnehmeranschluss beziehungsweise Zugangspunkt als einzelner Markt abgegrenzt werden. Dies hätte jedoch zur Folge, dass die Auswirkungen einer Kooperation auf den Wettbewerb nicht mehr eindeutig beurteilt werden könnten. ${ }^{778}$ Eine starke Segmentierung wäre im Ergebnis unübersichtlich.

Aus diesem Grund wird auf Basis alternativer Konzepte untersucht, ${ }^{779}$ in welchen Gebieten die Wettbewerbsverhältnisse hinreichend homogen sind. ${ }^{780}$ Für den lokalen Zugangsmarkt geht die Bundesnetzagentur von einem bundesweit einheitlichen Markt aus. Dies begründet sie damit, dass die Deutsche Telekom der einzige Anbieter mit einer flächendeckenden Infrastruktur lokaler Teilnehmeranschlüsse sei ${ }^{781}$ und eine bundesweit einheitliche Preispolitik verfolge. ${ }^{782}$ Zwar setzt sich die Bundesnetzagentur

776 BNetzA, Beschl. v. 27.8.2015, Az. BK 1-12/003, S. 124.

777 BNetzA, Beschl. v. 27.8.2015, Az. BK 1-12/003, S. 124.

778 BNetzA, Beschl. v. 27.8.2015, Az. BK 1-12/003, S. 124.

779 Ausführlich zum Untersuchungsansatz der BNetzA Monopolkommission, 11. Sektorgutachten Telekommunikation (2019), Rn. $82 \mathrm{ff}$.

780 BNetzA, Beschl. v. 27.8.2015, Az. BK 1-12/003, S. 124.

781 BNetzA, Konsultationsentwurf Markt 3a, Az. BK 1-19/001, S. 191.

782 BNetzA, Beschl. v. 27.8.2015, Az. BK 1-12/003, S. 124 ff.; BNetzA, Konsultationsentwurf Markt 3a, Az. BK 1-19/001, S. 187 ff. 
auch in der neuen Märktedefinition aus dem Jahr 2019 umfassend mit Möglichkeiten zur Bildung regionaler Cluster auseinander, nimmt hiervon im Ergebnis jedoch Abstand. ${ }^{783}$ Im zentralen Zugangsmarkt differenziert die Bundesnetzagentur zwischen den beiden Submärkten für den Layer-2und den Layer-3-Bitstromzugang (C. II. 1. b.). In beiden Fällen setzt sich die Bundesnetzagentur intensiv mit der Frage auseinander, ob regionale Teilmärkte sinnvoll voneinander abgegrenzt werden können. Für den Layer-2-Bitstromzugang verneint sie dies aufgrund der homogenen Wettbewerbsverhältnisse im gesamten Bundesgebiet. Dabei stellt die Bundesnetzagentur dar, dass zwar in 20 Städten ein erhöhter Wettbewerbsdruck von lokal tätigen Infrastrukturausbauunternehmen ausgehe; dieser führe allerdings nicht zu einer solch erheblichen Heterogenität der Wettbewerbsbedingungen, dass die Märkte für den Layer-2-Bitstromzugang regional differenziert abgegrenzt werden müssten. ${ }^{784}$ Anders sei es im Layer-3-Bitstromzugangsmarkt. Dort sei von einem bundesweit einheitlichen Markt auszugehen, von dem jedoch die 20 Kommunen ${ }^{785}$ mit alternativen Netzbetreibern als sub-nationale Teilmärkte ausgenommen seien. In diesen sei der Wettbewerbsdruck so groß und eine hinreichende regionale Preisdifferenzierung festzustellen, dass regionale Märkte abgegrenzt werden könnten. ${ }^{786}$

Grundsätzlich werden die Märkte also national abgegrenzt. Eine regionale Marktabgrenzung erfolgt nur dort, wo die Deutsche Telekom mit einem erheblichen Wettbewerbsdruck konfrontiert wird. Aus juristischer Sicht lässt sich demnach festhalten, dass eine sub-nationale Marktabgrenzung im geltenden Rechtsrahmen möglich ist und bei erheblich divergierenden Wettbewerbsverhältnissen auch vorgenommen wird.

783 BNetzA, Konsultationsentwurf Markt 3a, Az. BK 1-19/001, S. $178 \mathrm{ff}$.

784 BNetzA, Beschl. v. 9.7.2015, Az. BK 1-14-00, S. 119 ff.; weiterführend zum sogenannten Patchwork-Szenario Fetzer, in: Spiecker genannt Döhmann/Krämer (Hrsg.), Network Neutrality and Open Access, S. 177, 187; Körber, in: Bechtold/ Jickeli/Rohe (Hrsg.), Festschrift zum 70. Geburtstag von Wernhard Möschel, S. 1043, $1051 \mathrm{f}$.

785 Hierbei handelt es sich um Bochum, Bottrop, Bremerhaven, Flensburg, Gelsenkirchen, Gladbeck, Herne, Karlsruhe, Kiel, Köln, Leipzig, Leverkusen, Mannheim, Osnabrück, Pforzheim, Recklinghausen, Reutlingen, Troisdorf, Tübingen und Zwickau; BNetzA, Beschl. v. 9.7.2015, Az. BK 1-14-00, S. 127, Übersicht 1.

786 BNetzA, Beschl. v. 9.7.2015, Az. BK 1-14-00, S. 127 ff. 
b) Beschlusspraxis des Bundeskartellamts zur räumlichen Abgrenzung der Anschlussmärkte

Das Bundeskartellamt hingegen begrenzt die Anschlussmärkte in ihren Entscheidungen zur Gründung der Glasfaser Nordwest räumlich auf das Kooperationsgebiet. ${ }^{787}$ Für den lokalen Zugangsmarkt wird von der Bundesnetzagentur erwogen, ob jeder Teilnehmeranschluss einen separaten Markt darstellt (C. II. 2. b.). Hiervon nimmt das Bundeskartellamt jedoch mit dem Argument Abstand, dass dies zu einer „Atomisierung“ der Märkte führe und die Auswirkungen des Kooperationsvorhabens in seiner Gesamtheit nicht mehr realistisch dargestellt werden könnten. ${ }^{788}$ Auch das Bundeskartellamt nutzt das Kriterium der Homogenität der Wettbewerbsbedingungen, um den räumlich relevanten Markt abzugrenzen. Außerhalb des Kooperationsgebietes bestehen im Fall der Glasfaser Nordwest allein mangels einer Marktpräsenz der EWE unterschiedliche Wettbewerbsbedingungen. Demnach sei es legitim, den Markt regional abzugrenzen und räumlich auf das Kooperationsgebiet zu beschränken. ${ }^{789}$

c) Abgrenzung regionaler Teilmärkte oder Auferlegung regional differenzierter Abhilfemaßnahmen auch im Regulierungsrecht?

Auch im Regulierungsrecht könnte es sinnvoll sein, die Märkte räumlich auf das Kooperationsgebiet zu begrenzen, um die Auswirkungen des Kooperationsvorhabens auf den Wettbewerb besser bewerten zu können. Hierzu besteht die Möglichkeit, einen regionalen Teilmarkt für das Kooperationsgebiet abzugrenzen (dazu aa.). Alternativ besteht die Möglichkeit regional differenzierte, regulatorische Verpflichtungen aufzuerlegen (dazu bb.). Aus Gründen der Übersichtlichkeit soll diese Option bereits an dieser Stelle beleuchtet werden.

787 BKartA, Beschl. v. 30.12.2019, Az. B 7-21/18, Rn. 215; in der Entscheidung des BKartA, Beschl. v. 4.12.2019, Az. B 7-21/18, werden die Märkte identisch abgegrenzt, sodass auf einen Nachweis verzichtet werden kann.

788 Grundlegend hierzu BKartA, Beschl. v. 30.12.2019, Az. B 7-21/18, Rn. 152 ff., 215.

789 BKartA, Beschl. v. 30.12.2019, Az. B 7-21/18, Rn. 214 
aa) Begrenzung des räumlich-relevanten Marktes auf das

Kooperationsgebiet

Die Abgrenzung subnationaler Märkte wird in der Literatur kritisch diskutiert. Gegen sie wurde in der Frühphase der Entwicklung neuer Netze hervorgebracht, dass sie den bürokratischen Aufwand für die Bundesnetzagentur als zuständige Regulierungsbehörde erheblich erhöhe und letztlich ineffizient sei. ${ }^{790}$ Hiergegen lässt sich einwenden, dass die Bundesnetzagentur im Energiebereich hunderte kleinteilige Märkte beobachtet. ${ }^{791} \mathrm{Ex}-$ pertise im Umgang mit einer Vielzahl von Marktabgrenzungen ist in der Behörde mithin vorhanden. Auch einer regional differenzierten regulatorischen Gebietsabgrenzung könnte mit vertretbarem bürokratischem Mehraufwand begegnet werden. Mithilfe der behördenintern bereits verfügbaren Erfahrung könnte sie dann effizient umgesetzt werden.

Für die Abgrenzung regional differenzierter Gebiete lässt sich das Regulierungsziel des $₫ 2$ Abs. 3 Nr. 5 TKG anführen, das eine regional differenzierte Beobachtung des Wettbewerbs im Telekommunikationssektor erfordert. ${ }^{792}$ Diese Vorgabe soll insoweit unverändert auch im künftigen nationalen Rechtsrahmen existieren. ${ }^{793}$ Die Bundesnetzagentur setzt sich im aktuellen Konsultationsentwurf für den lokalen Teilnehmeranschlussmarkt zwar mit diesem Regulierungsziel auseinander, nimmt jedoch von einer regionalen Marktabgrenzung weiterhin Abstand..$^{794}$

Subnationale Teilmärkte werden im Layer-3-Bitstrommarkt derzeit für solche Regionen abgegrenzt, in denen die Deutsche Telekom von alternativen Infrastrukturanbietern unter erheblichen Wettbewerbsdruck gesetzt wird (C. II. 2. a.). ${ }^{795}$ Dieser manifestiert sich insbesondere in den Preisen,

790 Hierzu im Rahmen der damaligen Diskussion Berger-Kögler, MMR 2008, Heft 9, S. VI, VIII; Berger-Kögler, MMR 2010, S. 303 ff.; Inderst/Kühling/Neumann/Peitz, in: Inderst/Kühling/Neumann/Peitz (Hrsg.), Der Ausbau neuer Netze in der Telekommunikation, S. 293, 304; mit dem Hinweis, dass der regulatorische Aufwand auch die Unternehmen betreffe, Neumann, Regionalisierung der Regulierung, S. 50; Ufer, N\&R 2008, S. 173, $176 \mathrm{f}$.

791 Inderst/Kübling/Neumann/Peitz, in: Inderst/Kühling/Neumann/Peitz (Hrsg.), Der Ausbau neuer Netze in der Telekommunikation, S. 293, 304.

792 Weiterführend hierzu Götz/Zenhäusern, Wirtschaftsdienst 2013, S. 260, 263.

793 Referentenentwurf des Telekommunikationsmodernisierungsgesetzes (TKMoG), abrufbar unter: https://www.bmwi.de/Redaktion/DE/Downloads/Gesetz/ telekommunikationsmodernisierungsgesetz-referentenentwurf-20201612.pdf? blob=publicationFile $\& v=8$ (zuletzt abgerufen am 12.1.2021).

794 BNetzA, Konsultationsentwurf Markt 3a, Az. BK 1-19/001, S. 198.

795 ERG, Common Position on Geographic Aspects of Market Analysis, S. 9. 
die für bestimmte Leistungen erhoben werden, und den Charakteristika der angebotenen Produkte. ${ }^{796}$ Aus auffälligen regionalen Abweichungen kann ein Wettbewerbsdruck und damit das Erfordernis einer subnationalen Marktabgrenzung gefolgert werden (C. II. 2. a.).

Beim Umgang mit kooperativen Ausbauvorhaben zur Beschleunigung des Glasfaserausbaus stellt sich jedoch die Situation gegenteilig dar. $\mathrm{Zu}$ untersuchen ist, ob sich in den betreffenden Gebieten der Wettbewerbsdruck signifikant reduziert. ${ }^{797}$ Während die Erhöhung des Wettbewerbsdrucks anhand der Preissetzungspolitik und abweichender Produktspezifika untersucht werden kann (C. II. 2. a.), sind diese Kriterien nur von eingeschränkter Aussagekraft für die Untersuchung eines gesunkenen Wettbewerbsdrucks. Gegebenenfalls ließe sich feststellen, dass im Kooperationsgebiet aufgrund des zuvor existierenden Wettbewerbsdrucks differenzierte Preise oder Produktspezifika wieder zurückgenommen werden. Eine solche Tendenz ist in Gebieten mit geringem Wettbewerbsdruck derzeit insgesamt nicht zu beobachten. Sie wird sich deswegen wohl auch nicht in solchen Konstellationen zeigen, in denen der verminderte Wettbewerbsdruck auf Kooperationsvorhaben zurückzuführen ist. Aus diesem Grund ist ein Rückgriff auf andere ökonomische Abgrenzungskriterien erforderlich. Hierzu zählen etwa die Marktzutrittsschranken und die Anzahl der Anbieter sowie ihre Marktanteile, auf deren Basis die Wettbewerbskräfte im Kooperationsgebiet überprüft werden können. ${ }^{798} \mathrm{Um}$ eine regionale Marktabgrenzung zu ermöglichen, muss sich das betreffende Gebiet von angrenzenden Regionen unterscheiden, sinnvoll mit dem Erschließungsradius der Infrastruktur in Einklang zu bringen sein und weniger als das gesamte Bundesgebiet erfassen. ${ }^{799}$ Die Homogenität der Wettbewerbsbedin-

796 Weiterführend hierzu ERG, Common Position on Geographic Aspects of Market Analysis, S. $9 \mathrm{ff}$.

797 Weiterführend hierzu Heuermann/Meinen/Ekango u.a., Konzepte zur Förderung von Breitbandinvestitionen im internationalen Vergleich, S. 25; Inderst/Kühling/ Neumann/Peitz, in: Inderst/Kühling/Neumann/Peitz (Hrsg.), Der Ausbau neuer Netze in der Telekommunikation, S. 293, $316 \mathrm{ff}$.

798 Berger-Kögler, MMR 2008, Heft 9, S. VI, VII; komplex ist die Abgrenzung der regionalen Gebiete zur Beobachtung der Wettbewerbskräfte hingegen ohne die Existenz eines klar abgegrenzten Kooperationsgebietes, weiterführend hierzu ERG, Common Position on Geographic Aspects of Market Analysis, S. 10.

799 ERG, Common Position on Geographic Aspects of Market Analysis, S. 10 f.; ausführlich hierzu Inderst/Kühling/Neumann/Peitz, in: Inderst/Kühling/Neumann/ Peitz (Hrsg.), Der Ausbau neuer Netze in der Telekommunikation, S. 293, $314 \mathrm{f}$. 
gungen in diesem Gebiet kann auf Basis der Marktanalyseleitlinien bewertet werden. 800

Exemplifizieren lassen sich die vorgenannten abstrakten Aussagen am Beispiel der Glasfaser Nordwest. Dort kooperieren mit der Deutschen Telekom und der EWE zwei Unternehmen, die im Kooperationsgebiet bislang direkte Wettbewerber im Infrastrukturausbau waren. Die EWE verfügte über eine umfassende Infrastrukturabdeckung ${ }^{801}$ und übte einen nicht unerheblichen Wettbewerbsdruck auf die Deutsche Telekom aus, auch wenn dieser bislang nicht für die Abgrenzung eines räumlichen Marktes im Kooperationsgebiet ausreichte. Durch die Bündelung der Aktivitäten zum Glasfaserausbau in der Glasfaser Nordwest reduzieren die beiden Unternehmen den bisherigen gegenseitigen Wettbewerbsdruck. Das Kooperationsgebiet ist klar definiert und abgegrenzt. Es deckt sich mit dem Gebiet, in dem die EWE und die Deutsche Telekom im Vorfeld miteinander in Konkurrenz standen. Außerhalb des Kooperationsgebietes sind keine Aktivitäten der EWE zu verzeichnen. ${ }^{802}$ Demnach wäre eine Abgrenzung des räumlich relevanten Marktes im Einklang mit dem Kooperationsgebiet überzeugend.

Für große, besonders wettbewerbssensitive Kooperationsprojekte bietet sich in Zukunft eine subnationale Marktabgrenzung an. Auf diese Weise können die Auswirkungen eines Kooperationsvorhabens auf den Wettbewerb auch aus regulatorischer Sicht effektiver bewertet werden. ${ }^{803}$ Auf dem nationalen Breitbandinternetzugangsmarkt wird ein regional ausgerichtetes Kooperationsvorhaben den Wettbewerb wohl nicht spürbar beeinträchtigen, während dies lokal anders aussehen kann. Mit einer regionalen Beobachtung der Auswirkungen könnte die Bundesnetzagentur punktgenaue und effektive Einzelfallentscheidungen treffen.

bb) Möglichkeit der Auferlegung regional differenzierter regulatorischer Verpflichtungen

Alternativ oder gar zusätzlich hierzu können regulatorische Verpflichtungen regional differenziert auferlegt werden. Diesen Schritt haben verschie-

800 ERG, Common Position on Geographic Aspects of Market Analysis, S. $13 \mathrm{ff}$.

801 BKartA, Beschl. v. 30.12.2019, Az. B 7-21/18, Rn. 40 f.

802 BKartA, Beschl. v. 30.12.2019, Az. B 7-21/18, Rn. 214.

803 Heuermann/Meinen/Ekango u.a., Konzepte zur Förderung von Breitbandinvestitionen im internationalen Vergleich, S. 22. 
dene Mitgliedstaaten der Europäischen Union bereits gewagt. ${ }^{804}$ Auf diese Weise könnte eine regional angepasste Zugangs- oder Entgeltregulierung erfolgen. Dies ist deswegen erforderlich, weil eine Zugangsregulierung stets im Zusammenhang mit der korrespondierenden Entgeltregulierung diskutiert werden muss. Gegen die Regionalisierung der Letzteren wird eingewandt, dass dies zur Folge habe, dass die bundesweit einheitliche Preispolitik nicht aufrecht erhalten bleiben könne. ${ }^{805}$ Vielmehr sei damit zu rechnen, dass eine Differenzierung bei den Vorleistungsentgelten auch eine Differenzierung der Endkundenpreise zur Folge haben würde. Über eine gestaffelte Intensität von Zugangsverpflichtungen, Begleitverpflichtungen und die Höhe der Entgelte könnte jedoch die Attraktivität für Ausbaugebiete oder Kooperationsgebiete beträchtlich gesteigert werden. ${ }^{806}$ Dabei müssten jedoch auch die vorgenannten Auswirkungen für die Tarifeinheit beachtet werden. ${ }^{807}$

Es ist zu berücksichtigen, dass derzeit noch keine ökonomischen Studien vorliegen, die untersuchen, welche Effekte die Auferlegung regionaler Abhilfemaßnahmen auf Kooperationsvorhaben haben könnte. Es handelt sich hauptsächlich um eine ökonomische Fragestellung, ob es sinnvoll ist, die Beschlusspraxis anzupassen. Eine regionale Differenzierung ist im geltenden Rechtsrahmen möglich. ${ }^{808}$ Letztlich steht in Frage, ob die Bereitschaft besteht, die nationale Preiseinheit zu Gunsten der Beschleunigung

804 Weiterführend zu konkreten Beispielen aus Großbritannien und Österreich von Weizsäcker, Regionalisierung der Regulierung im Bitstrom-Zugangsmarkt, S. 6 ff.; weiterführend auch Ehrler/Ruble/Berger, CR 2008, S. 703, 704 ff.; im Hinblick auf regionale Marktabgrenzungen in Großbritannien und Portugal Stumpf, Die Abgrenzung subnationaler Märkte als regulatorischer Ansatz, S. 13 ff.; weiterführend zur subnationalen Marktabgrenzung in verschiedenen EU-Mitgliedstaaten Heuermann/Meinen/Ekango u.a., Konzepte zur Förderung von Breitbandinvestitionen im internationalen Vergleich, S. 23 f.; Neumann, Regionalisierung der Regulierung, S. 10 ff.; Ufer, N\&R 2008, S. 173, $174 \mathrm{f}$.

805 Ehrler/Ruble/Berger, CR 2008, S. 703, 707; mit Argumenten für die Vorteile regionaler Preisdifferenzierungen Reents, Ausbau und Finanzierung einer flächendeckenden Breitbandversorgung in Deutschland, S. 132 f.; Ufer, N\&R 2008, S. $173,176$.

806 A. A. Ufer, N\&R 2008, S. 173, 176, der davon ausgeht, dass die gestaffelten Entgelte negative Auswirkungen auf den Infrastrukturausbau in der Fläche haben könnten.

807 Ehrler/Ruble/Berger, CR 2008, S. 703, 707.

808 Inderst/Kühling/Neumann/Peitz, in: Inderst/Kühling/Neumann/Peitz (Hrsg.), Der Ausbau neuer Netze in der Telekommunikation, S. 293, 321; umfassend hierzu Schöfthaler, Rechtliche Rahmenbedingungen des Breitbandausbaus, S. 157 ff. 
eines flächendeckenden Glasfaserausbaus aufzugeben. ${ }^{809}$ Dies könnte jedoch durch eine reine Anpassung der Beschlusspraxis ohne Modifikationen im Rechtsrahmen erfolgen.

\section{cc) Zwischenergebnis}

Eine Regionalisierung der Regulierung für Kooperationsvorhaben unter Beteiligung eines marktmächtigen Unternehmens bietet sich in Gebieten an, in denen der Wettbewerbsdruck für das marktmächtige Unternehmen durch die Beteiligung am Kooperationsvorhaben erheblich sinkt. Diese Feststellung kann auf Basis einer subnationalen Marktabgrenzung erfolgen, die sich mit vertretbarem bürokratischem Aufwand sicherstellen lieBe.

Zudem könnten, um einzelgerechte Lösungen zu erzielen, regional differenzierte regulatorische Verpflichtungen auferlegt werden. Auf diese Weise könnte parallel ein Wettbewerbsschutz erfolgen, ohne die wirtschaftlichen Vorteile einer Kooperation durch die Auferlegung regulatorischer Verpflichtungen vollständig zu unterminieren. Mit diesem Weg wäre jedoch eine Abkehr von einer national einheitlichen Preispolitik verbunden. Dies müsste gewollt sein. Zudem lassen sich die ökonomischen Folgen der Auferlegung regional differenzierte Abhilfemaßnahmen an dieser Stelle nicht zweifelsfrei abschätzen.

\section{Ergebnis und Ausblick}

Die Endkunden- und Vorleistungsmärkte für den Breitbandinternetzugang werden durch das Bundeskartellamt und die Bundesnetzagentur in sachlicher Hinsicht (bis auf Detailfragen) einheitlich abgegrenzt. Erhebliche Unterschiede bestehen jedoch im Hinblick auf die räumliche Marktabgrenzung. Während das Bundeskartellamt den Markt räumlich auf das Kooperationsgebiet beschränkt, geht die Bundesnetzagentur in weiten Teilen von einem nationalen Markt aus. Lediglich für den Layer-3-Bitstromzugangsmarkt grenzt sie regionale Teilmärkte ab. Bei diesen Gebieten handelt es sich um Regionen, in denen die Deutsche Telekom mit einem er-

809 Berger-Kögler, MMR 2008, Heft 9, S. VI, VIII; Inderst/Kühling/Neumann/Peitz, in: Inderst/Kühling/Neumann/Peitz (Hrsg.), Der Ausbau neuer Netze in der Telekommunikation, S. 293, 305. 
heblichen Wettbewerbsdruck durch alternative Anbieter konfrontiert wird. Dies ist im Rahmen der regulatorischen Behandlung von Kooperationsvorhaben anders. Sofern sich das marktmächtige Unternehmen an einem solchen Vorhaben beteiligt, stellt sich die Frage, ob dies den Wettbewerbsdruck in der betreffenden Region erheblich reduziert. Neben regionalen Märkten mit erhöhtem Wettbewerbsdruck für die Deutsche Telekom, sollten zukünftig auch solche mit erheblich geringerem Wettbewerbsdruck regional abgegrenzt werden. Während regionale Kooperationsvorhaben auf dem nationalen Markt vermutlich keine spürbaren Veränderungen mit sich bringen, ist dies im betreffenden Kooperationsgebiet anders. Dies kann sich negativ auf den Infrastrukturausbau auswirken. Eine auch regional differenzierte regulatorische Betrachtung eines Kooperationsvorhabens wäre aus diesem Grund zu begrüßen. Zudem könnten durch regional differenzierte Abhilfemaßnahmen einzelfallgerechte Ergebnisse erzielt werden.

\section{Ausschreibungsmarkt für den geförderten Breitbandausbau}

Neben den Breitbandinternetzugangsmärkten, die sowohl vom Regulierungsrecht als auch von der Fusionskontrolle und dem Kartellverbot erfasst sind, grenzt das Bundeskartellamt in seiner Entscheidung zur Glasfaser Nordwest zusätzlich den Ausschreibungsmarkt für den geförderten Breitbandausbau ab. ${ }^{810}$ Dabei stellt sich die Frage, ob die Abgrenzung des zusätzlichen Marktes den Wettbewerbsschutz bei Kooperationsvorhaben im Glasfaserausbau sinnvoll erweitert.

Betrachtet man die Abgrenzung von Märkten durch die Regulierungsbehörden für den Telekommunikationssektor, so fällt auf, dass das Telekommunikationsrecht monothematisch auf den Schutz des Wettbewerbs im Telekommunikationssektor ausgerichtet ist. Geschützt wird also der Wettbewerb zwischen den Telekommunikationsunternehmen in den Telekommunikationsmärkten. Das allgemeine Wettbewerbsrecht verfolgt einen breiteren Ansatz und ist auf den Schutz des Wettbewerbs in allen Märkten ausgerichtet. Dies manifestiert sich im Kontext des wettbewerbsrechtlichen Schutzes des Ausschreibungsmarktes für den geförderten Breitbandausbau. Der Schutz dieses Marktes führt letztlich zu der Frage, ob in den Fördergebieten der Schutz des Wettbewerbs der Infrastrukturanbieter (den die Bundesnetzagentur gewährleistet) durch den Wettbewerb um den In-

810 BKartA, Beschl. v. 30.12.2019, Az. B 7-21/18, Rn. 235 ff. 
frastrukturmarkt (den das Bundeskartellamt gewährleistet) erweitert wird. ${ }^{811}$ Eine vergleichbare Frage hat sich bereits bei der Diskussion um die Einführung eines Konzessionsmodells auf Basis der Vergabe exklusiver Wegerechte gestellt (Kapitel 3, C. I.). In diesem Kontext wird diskutiert, ob ein Wettbewerb im Konzessionsgebiet durch einen Wettbewerb um die Konzession ersetzt werden soll.

Nach der Idealvorstellung des Gesetzgebers belebt der Infrastrukturwettbewerb den Wettbewerb zwischen den Diensteanbietern (Kapitel 3, C. III. 2.). Vorzugswürdig wäre demnach ein möglichst umfassender Schutz des Wettbewerbs der Infrastrukturanbieter anstelle eines Wettbewerbs um den Infrastrukturmarkt. In den Fördergebieten ist bereits der Aufbau einer einzigen Infrastruktur in sogenannten „weißen Flecken“ und zumindest einer weiteren Konkurrenzinfrastruktur in sogenannten "grauen Flecken“ im Sinne der Förderterminologie nicht finanzierbar. ${ }^{812}$ Damit fehlt eine wesentliche Voraussetzung für den Wettbewerb der Infrastrukturanbieter im Fördergebiet. Ein solcher wäre nur bei einem parallelen Infrastrukturausbau mithilfe von Fördergeldern möglich. Dies wäre nicht nur ökonomisch fragwürdig, sondern darüber hinaus nicht mit dem EU-Beihilfenrecht und dem Haushaltsrecht vereinbar. Beide Rechtsmaterien fordern einen effizienten und sparsamen Einsatz von Fördergeldern. Fördergelder sollten in einem wettbewerblichen Verfahren vergeben werden. Die errichtete Infrastruktur sollte zudem möglichst vielen Diensteanbietern zur Verfügung gestellt werden, um - trotz des eingeschränkten Infrastrukturwettbewerbs einen funktionsfähigen Wettbewerb der Diensteanbieter zu ermöglichen. Insoweit ist zu begrüßen, dass die Förderrichtlinien in der Regel eine umfassende Zugangsverpflichtung für die geförderten Infrastrukturen statuieren.

Da ein Wettbewerb der Infrastrukturanbieter in den Fördergebieten demnach in der Regel nicht stattfindet, ist ein Schutz des Wettbewerbs um die Fördergelder im Fördergebiet, also des Wettbewerbs um den betreffenden Infrastrukturmarkt, sinnvoll. Was im Kontext des Konzessionsmodells auf Basis der Vergabe exklusiver Wegerechte nicht institutionalisiert werden darf (Kapitel 3, C.), ist die logische Konsequenz des Förderrahmens.

Das Bundeskartellamt grenzt einen einheitlichen sachlichen Markt ab, in den alle Programme zur Förderung des Breitbandausbaus integriert

811 Hierzu auch Kühling, WuW 2019, S. 555, 556.

812 Leitlinien der EU für die Anwendung der Vorschriften über staatliche Beihilfen im Zusammenhang mit dem schnellen Breitbandausbau (Mitteilung 2013/C 25/01), Abl. C 25/1 v. 26.1.2013, Rn. 61 ff. 
werden ${ }^{813}$ Die Förderprogramme sind hinreichend homogen, sodass sie in einen einheitlichen Markt zusammengefasst werden können. ${ }^{814}$ Dies lässt sich darauf zurückführen, dass bundesweit einheitliche Rahmenregelungen für die Ausgestaltung von Förderprogrammen gelten. ${ }^{815}$ Unerheblich sind deswegen letztlich auch die angestrebten Zielbandbreiten und die geförderten Anschlusstechnologien, da im Förderrecht der Grundsatz der Technologieneutralität gelte. ${ }^{816}$

Bei einer konsequenten Anwendung des Bedarfsmarktkonzeptes könnte freilich jede Ausschreibung im Rahmen eines Förderverfahrens als separater Markt abgegrenzt werden. Eine solche Marktabgrenzung lehnt das Bundeskartellamt auch im Kontext der Ausschreibungsmärkte für den geförderten Breitbandausbau mit dem Argument der drohenden „Atomisierung" der Märkte und der damit einhergehenden Unübersichtlichkeit der Markabgrenzung ab. ${ }^{817}$ Das Bundeskartellamt orientiert sich vielmehr daran, in welchen Gebieten sich in der Vergangenheit immer wieder dieselben Unternehmen in Ausschreibungen beworben haben und sich vermutlich auch in Zukunft bewerben werden. ${ }^{818}$ Bis auf wenige lokale Ausnahmen sei im Kooperationsgebiet insoweit eine für eine Gebietsabgrenzung hinreichende Homogenität festzustellen.

Ein fehlender Schutz für diesen angrenzenden Markt würde dazu führen, dass die Marktmacht der Deutschen Telekom im Vorleistungsbereich zunimmt, und damit auch dem Wettbewerb in den nachgelagerten Märkten schaden. Das Kartellrecht nimmt auch in dieser Hinsicht eine wichtige Rolle ein und unterstützt die Suffizienz des Rechtsrahmens im Hinblick auf den Wettbewerbsschutz beim kooperativen Glasfaserausbau. Es verhindert präventiv, dass eine Situation entsteht, die ausschließlich mithilfe des Regulierungsrechts bewältigt werden kann (E. I. 1.).

\section{Zusammenfassung und Schlussfolgerungen}

Die sachlich relevanten Endkunden- und Vorleistungsmärkte für den Breitbandinternetzugang werden von der Bundesnetzagentur und dem

813 BKartA, Beschl. v. 30.12.2019, Az. B 7-21/18, Rn. 245.

814 BKartA, Beschl. v. 30.12.2019, Az. B 7-21/18, Rn. 249.

815 BKartA, Beschl. v. 30.12.2019, Az. B 7-21/18, Rn. 249.

816 BKartA, Beschl. v. 30.12.2019, Az. B 7-21/18, Rn. 250.

817 BKartA, Beschl. v. 30.12.2019, Az. B 7-21/18, Rn. 248 und 260.

818 BKartA, Beschl. v. 30.12.2019, Az. B 7-21/18, Rn. 261. 
Bundeskartellamt im Wesentlichen gleichlaufend abgegrenzt. Dies verwundert nicht, da im gesamten Wettbewerbsrecht auf eine einheitliche Methodik zur Marktabgrenzung zurückgegriffen wird. Räumlich weichen die abgegrenzten Märkte hingegen voneinander ab. Das Bundeskartellamt betrachtet die Auswirkungen eines Kooperationsvorhabens auf den Wettbewerb im Kooperationsgebiet. Die Bundesnetzagentur hingegen grenzt die Vorleistungsmärkte grundsätzlich national ab. Subnationale Marktabgrenzungen gibt es nur für Gebiete, in denen das national marktmächtige Unternehmen, die Deutsche Telekom, von alternativen Anbietern unter erheblichen Wettbewerbsdruck gesetzt wird. In Zukunft sollten auch solche Gebiete subnational abgegrenzt werden, in denen der Wettbewerbsdruck erheblich geringer ist als in anderen Regionen. In Frage kommt dies insbesondere für Kooperationsgebiete. Zudem sollte, unter dem Vorbehalt einer ökonomischen Folgeabwägung, über die Auferlegung regionaler Abhilfemaßnahmen nachgedacht werden.

Letztlich kommen die Behörden auch im Fall paralleler Zuständigkeiten zu weitgehend einheitlichen Ergebnissen. In Fällen, in denen sie voneinander abweichen, erhöht sich der Schutzstandard. Im Ergebnis ist die Beschlusspraxis konsistent und trägt zur Rechtssicherheit bei.

Das allgemeine Wettbewerbsrecht erfasst auch solche Märkte, die nicht direkt dem Regulierungsrecht unterfallen. Hierzu ist etwa der Ausschreibungsmarkt für den geförderten Breitbandausbau zu zählen. In den Fördergebieten ist ein Wettbewerb der Infrastrukturanbieter nur sehr eingeschränkt möglich. Daher ist ein Wettbewerb um den Infrastrukturmarkt sinnvoll, der mithilfe des erweiterten Schutzes durch das Wettbewerbsrecht gewährleistet wird. Des Weiteren trägt auch das EU-Beihilfenrecht etwa in Form von „Open-Access-Verpflichtungen“ - dazu bei, dass trotz des rudimentären Wettbewerbs der Infrastrukturanbieter ein funktionsfähiger Wettbewerb in den nachgelagerten Märkten sichergestellt wird. (E. I.). Das Zusammenspiel der verschiedenen Regelungsmaterien führt zu einem umfassenden Schutz aller durch das Kooperationsvorhaben betroffenen Märkte. 
D. Regulatorische Rahmenbedingungen zum Schutz des Wettbewerbs bei Kooperationsvorhaben

Die Analyse regulatorischer Rahmenbedingungen für Kooperationsvereinbarungen zum Ausbau kooperativer Glasfaserinfrastrukturen soll auf Ausgestaltungsvarianten mit hohem Institutionalisierungsgrad durch Gründung eines Gemeinschaftsunternehmens beschränkt werden. Relevant sind hierfür zwei Optionen. Das Gemeinschaftsunternehmen selbst kann über beträchtliche Marktmacht verfügen und folglich direkter Adressat regulatorischer Verpflichtungen werden (dazu I.). Alternativ besteht die Möglichkeit der Beteiligung eines marktmächtigen Unternehmens an der Kooperation. In diesem Kontext stellt sich die Frage, ob das Gemeinschaftsunternehmen von den regulatorischen Verpflichtungen, die dem marktmächtigen Unternehmen auferlegt wurden und in Zukunft auferlegt werden, erfasst wird (dazu II.).

I. Entstehung eines Gemeinschaftsunternehmens mit beträchtlicher Marktmacht

Das Marktregulierungsverfahren des Telekommunikationsrechts ist in drei Schritte unterteilt. Regulatorische Verpflichtungen werden solchen Unternehmen auferlegt, die auf einem definierten Markt ( $\$ 10 \mathrm{TKG}$ ) über beträchtliche Marktmacht ( $\$ 11 \mathrm{TKG}$ ) verfügen. Die Rechtsfolgen der Marktanalyse ergeben sich aus $\mathbb{} 13$ TKG, in dem alle potentiellen regulatorischen Verpflichtungen enumeriert sind. Im Zuge der Novellierung des Telekommunikationsgesetzes wird sich zwar voraussichtlich die Nummerierung, nicht jedoch die skizzierte Grundsystematik der Vorgaben ändern. ${ }^{819}$ Insofern unterscheidet sich das Marktregulierungsverfahren für Gemeinschaftsunternehmen zum kooperativen Aufbau von Glasfaserinfrastrukturen nicht von dem üblichen Verfahren für andere Unternehmen des Telekommunikationssektors. Entsteht durch eine Kooperation ein Unternehmen mit beträchtlicher Marktmacht, werden ihm regulatorische Verpflichtungen auferlegt, die das Ausnutzen dieser Stellung verhindern.

819 Referentenentwurf des Telekommunikationsmodernisierungsgesetzes (TKMoG), abrufbar unter: https:/www.bmwi.de/Redaktion/DE/Downloads/Gesetz/ telekommunikationsmodernisierungsgesetz-referentenentwurf-20201612.pdf? blob=publicationFile\&v=8 (zuletzt abgerufen am 12.1.2021). 
II. Beteiligung eines Unternehmens mit beträchtlicher Marktmacht am Gemeinschaftsunternehmen

Eine andere Situation ergibt sich, wenn das Gemeinschaftsunternehmen selbst nicht über beträchtliche Marktmacht verfügt, sich allerdings ein Unternehmen an der Kooperation beteiligt, das als marktmächtig auf einem der definierten Märkte des Telekommunikationssektors zu klassifizieren ist. ${ }^{820}$ Dreh- und Angelpunkt ist hierbei die Rechtsfolge der Legaldefinition des Unternehmens aus $₫ 3$ Nr. 29 TKG (dazu 1.). Es stellt sich insbesondere die Frage, ob es für die Erstreckung der regulatorischen Verpflichtungen auf verbundene Unternehmen auf den Gründungszeitpunkt ankommt (dazu 2.). Mit dem EKEK wurde die Möglichkeit einer regulatorischen Privilegierung für Unternehmen mit beträchtlicher Marktmacht eingeführt, die eingeordnet und skizziert werden soll (dazu 3.). In diesem Zusammenhang stellt sich die Frage, ob ein weiterer Novellierungsbedarf für den regulatorischen Rechtsrahmen besteht (dazu 4.).

\section{Unternehmensbegriff des $\$ 3 \mathrm{Nr} .29 \mathrm{TKG}$}

Der Unternehmensbegriff des Telekommunikationsrechts wird in $\$ 3$ Nr. 29 TKG (und mit identischem Wortlaut in $\$ 3$ Nr. 69 TKG-RefE) ${ }^{821}$ legaldefiniert. Die Vorgabe ist von herausragender Bedeutung für die regulatorische Behandlung von Gemeinschaftsunternehmen zum kooperativen Ausbau von Glasfaserinfrastrukturen, an denen Unternehmen mit beträchtlicher Marktmacht beteiligt sind. Der Wortlaut der Norm ist zwar eindeutig (dazu a.), zur Herstellung von Einzelfallgerechtigkeit wird jedoch stellenweise eine teleologische Reduktion der Definition gefordert (dazu b.).

820 Monopolkommission, 11. Sektorgutachten Telekommunikation (2019), Rn. 122.

821 Referentenentwurf des Telekommunikationsmodernisierungsgesetzes (TKMoG), abrufbar unter: https:/www.bmwi.de/Redaktion/DE/Downloads/Gesetz/ telekommunikationsmodernisierungsgesetz-referentenentwurf-20201612.pdf? blob=publicationFile\&v=8 (zuletzt abgerufen am 12.1.2021). 
a) Wortlaut des $₫ 3 \mathrm{Nr} .29 \mathrm{TKG}$

Das Telekommunikationsrecht verzichtet auf einen vollständig autonomen Unternehmensbegriff und verweist in der Legaldefinition des $\$ 3$ Nr. 29 TKG umfassend auf die kartellrechtlichen Vorgaben zur Fusionskontrolle (B. II. 1. a.). Danach ist unter einem Unternehmen im Sinne des Telekommunikationsrechts „das Unternehmen selbst oder [die] mit ihm im Sinne des $\$ 36$ Abs. 2 und $\$ 37$ Abs. 1 und 2 des Gesetzes gegen Wettbewerbsbeschränkungen verbundenen Unternehmen“ zu verstehen. Durch die Gründung eines paritätischen Gemeinschaftsunternehmens zum kooperativen Ausbau von Glasfaserinfrastrukturen können, wie bereits dargelegt, gleich mehrere der Tatbestände des $\$ 37$ Abs. 1 GWB erfüllt werden (B. II. 1. a.). So handelt es sich regelmäßig um einen Zusammenschluss im Sinne des $₫ 37$ Abs. 1 Nr. 2 und Nr. 3 GWB (B. II. 1. a.). Das Kartellrecht behandelt die zusammengeschlossenen Unternehmen als Einheit. Diese Fiktion wird gemäß $\mathbb{3}$ Nr. 29 TKG auch auf das Telekommunikationsrecht übertragen. ${ }^{822}$ Das Gemeinschaftsunternehmen gilt als Teil der Mutterunternehmen. In der Folge wird etwa auch die Glasfaser Nordwest als Teil der Deutschen Telekom behandelt.823 Das Gemeinschaftsunternehmen ist deswegen auch von allen regulatorischen Verpflichtungen erfasst, die das Mutterunternehmen adressieren.

b) Teleologische und unionsrechtskonforme Reduktion des $₫ 3 \mathrm{Nr} .29$ TKG?

Im Rahmen des Verwaltungsverfahrens, das im Vorfeld der Gründung der Glasfaser Nordwest durch die Bundesnetzagentur durchgeführt wurde und die Frage zum Gegenstand hatte, ob das Gemeinschaftsunternehmen von den regulatorischen Verpflichtungen der Deutschen Telekom erfasst ist, wurde dieses Ergebnis des $\$ 3$ Nr. 29 TKG von den Muttergesellschaften freilich kritisiert. Die insoweit angeführten Argumente können allerdings allesamt entkräftet werden.

822 Lünenbürger/Stamm, in: Scheurle/Mayen (Hrsg.), Telekommunikationsgesetz, $\$ 3$, Rn. 87; Säcker, in: Säcker (Hrsg.), Telekommunikationsgesetz, $\mathbb{3}$ TKG, Rn. 86.

823 EWE/Telekom, in: BNetzA, Entsch. v. 10.7.2018, Az. BK 1-18/002, S. $21 \mathrm{ff}$. 
Für eine telelogische Reduktion des $₫ 3 \mathrm{Nr} .29$ TKG wird vorgebracht, dass die Rechtsfolge der Legaldefinition im Einzelfall unbillig sei. ${ }^{824}$ Zweck des $₫ 3$ Nr. 29 TKG sei es zu gewährleisten, dass die regulatorischen Verpflichtungen nicht mithilfe gesellschaftsrechtlicher Umstrukturierungen umgangen werden können. ${ }^{825}$ Man kann insoweit von der Verhinderung einer "Flucht in die gesellschaftsrechtliche Umstrukturierung" sprechen. Eine Missbrauchsgefahr bestehe aber nur in denjenigen Fällen, in denen das marktmächtige Unternehmen auf das Gemeinschaftsunternehmen einen bestimmenden Einfluss ausüben könne, der sich in einer „alleinigen Leitungsmacht ${ }^{\text {“826 }}$ manifestiere und eine Abgrenzung anhand der Kriterien der $\mathbb{S} 16,17$ Aktiengesetz (AktG) und Art. 2 Abs. 1 lit. f der Richtlinie 2004/109/EG ${ }^{827}$ erfordere. Insoweit wird auf die Fraport-Entscheidung 828 des Bundesverfassungsgerichts rekurriert. In dieser wurde eine Beherrschung erst bei einer Inhaberschaft von mehr als 50 Prozent der Geschäftsanteile angenommen. In der Entscheidung ging es um die Frage, ob ein Privatrechtssubjekt unmittelbar an Grundrechte gebunden ist, wenn der Staat Miteigentümer an der Gesellschaft ist.

Auch das Unionsrecht gebiete eine teleologische Reduktion. ${ }^{829}$ Das Sekundärrecht verfüge über keine entsprechende Definition und nehme im Rahmen des Marktregulierungsverfahrens stets ein konkretes Unternehmen in den Blick. ${ }^{830}$ Insoweit sei eine Vereinbarkeit des Unternehmensbegriffs mit dem Primärrecht erforderlich. Dieses lege in den Art. 101 und Art. 102 AEUV und den insoweit konkretisierenden Regelungen der Fusionskontrollverordnung (FKVO) den bereits dargestellten, wirtschaftlichfunktionalen Unternehmensbegriff zu Grunde (Kapitel 3, C. IV. 2. b.).

824 EWE/Telekom, in: BNetzA, Entsch. v. 10.7.2018, Az. BK 1-18/002, S. $21 \mathrm{ff}$.

825 BVerwG, Urt. v. 11.12.2013, Az. 6 C 24/12, NVwZ 2014, S. 942, 945, Rn. 23; EWE/Telekom, in: BNetzA, Entsch. v. 10.7.2018, Az. BK 1-18/002, S. 22; Lünenbürger/Stamm, in: Scheurle/Mayen (Hrsg.), Telekommunikationsgesetz, $\$ 3$, Rn. 86; Schütz, in: Geppert/Schütz (Hrsg.), Beck'scher TKG-Kommentar, $\mathbb{3}$, Rn. 89.

826 EWE/Telekom, in: BNetzA, Entsch. v. 10.7.2018, Az. BK 1-18/002, S. 23.

827 Richtlinie 2004/109/EG des Europäischen Parlaments und des Rates vom 15.12.2004 zur Harmonisierung der Transparenzanforderungen in Bezug auf Informationen über Emittenten, deren Wertpapiere zum Handel auf einem geregelten Markt zugelassen sind, und zur Änderung der Richtlinie 2001/34/EG, Abl. L 390 v. 31.12.2004, S. 38; in der Fassung der Änderung durch die Richtlinie 2013/50/EU v. 22.10.2013, Abl. L 294 v. 6.11.2013, S. 13.

828 BVerfG, Urt. v. 22.2.2011, Az. 1 BvR 699/06 (Fraport), BVerfGE 128, 226, 247.

829 EWE/Telekom, in: BNetzA, Entsch. v. 10.7.2018, Az. BK 1-18/002, S. 25.

830 EWE/Telekom, in: BNetzA, Entsch. v. 10.7.2018, Az. BK 1-18/002, S. 25. 
Dieser stellt strenge Anforderungen an den Einfluss des Mutterunternehmens auf strategische Geschäftsentscheidungen, die auch gemeinschaftlich durch zwei Muttergesellschaften ausgeübt werden können. ${ }^{831}$

Diese Argumente können jedoch nicht überzeugen. Der Befund, dass das unionale Telekommunikationsrecht keine eigene Legaldefinition des Unternehmensbegriffes enthält, ändert sich auch durch den EKEK nicht. Das Telekommunikationsrecht stellt ein sektorspezifisches, also ein spezielles Wettbewerbsrecht dar. Sofern dort keine speziellen Regelungen vorhanden sind, muss auf die allgemeinen Regelungen des Wettbewerbsrechts zurückgegriffen werden. $\$ 3 \mathrm{Nr}$. 29 TKG lässt sich mit dem allgemeinen unionalen Wettbewerbsrecht, das sich aus Art. 101 und 102 AEUV ableitet und in der FKVO spezifiziert wird, in Einklang bringen. ${ }^{832}$ Insoweit überzeugt auch die Systematik des TKG, die den Unternehmensbegriff nicht eigenständig abgrenzt, sondern auf das GWB verweist, das das deutsche allgemeine Wettbewerbsrecht enthält. Der Befund, dass die unionsrechtlichen Vorgaben jeweils ein konkretes Unternehmen in den Mittelpunkt der Marktanalyse und -definition sowie der Regulierungsverfügung stellen, entspricht der Systematik des nationalen Telekommunikationsrechts und ist auch zwingend geboten. Die Adressaten des belastenden Verwaltungsaktes sind explizit zu benennen. Diesem Erfordernis wird durch die Bezeichnung des marktmächtigen Unternehmens Genüge getan, da das Gemeinschaftsunternehmen als dessen Teil gilt. Der Gesetzgeber hat sich in $\$ 3 \mathrm{Nr} .29$ TKG dazu entschlossen, auf den gesamten $\$ 36$ Abs. 2 GWB zu verweisen. Danach ist es, wie bereits dargelegt, nicht erforderlich, dass ein einzelnes Unternehmen eine „alleinige Leitungsmacht ${ }^{\text {“833 }}$ über das Gemeinschaftsunternehmen hat. Vielmehr enthält $\$ 36$ Abs. 2 S. 2 GWB einen eigenen Zurechnungstatbestand, nach dem mehrere Mutterunternehmen gemeinsam das Tochterunternehmen beherrschen können. ${ }^{834}$ Dies kann insbesondere vor dem Hintergrund der mannigfaltigen Diskriminierungspotentiale überzeugen, die nicht zwingend eine alleinige Beherrschung des Gemeinschaftsunternehmens durch das marktmächtige

831 EuGH, Urt. v. 24.6.2015, verb. Rs. C-293/13 P und C-294/13 P (Fresh Del Monte Produce Inc./Kommission), ECLI:EU:C:2015:416, Rn.78; EuGH, Urt. v. 19.7.2012, verb. Rs. C-628/10 P und C-14/11 P (Alliance One International und Standard Commercial Tobacco/Kommission), ECLI:EU:C:2012:479, Rn. 101; BNetzA, Entsch. v. 10.7.2018, Az. BK 1-18/002, S. 4.

832 So auch BNetzA, Entsch. v. 10.7.2018, Az. BK 1-18/002, S. 3.

833 EWE/Telekom, in: BNetzA, Entsch. v. 10.7.2018, Az. BK 1-18/002, S. 23.

834 BNetzA, Entsch. v. 10.7.2018, Az. BK 1-18/002, S. 4. 
Unternehmen voraussetzen. Es besteht im Ergebnis kein Bedürfnis danach, \3 Nr. 29 TKG teleologisch zu reduzieren.

\section{c) Ergebnis}

Ein Gemeinschaftsunternehmen, das zum Zweck des kooperativen Glasfaserausbaus gegründet wird, ist in der Regel mit seinen Mutterunternehmen gemäß $\int 3$ Nr. 29 TKG verbunden. Dies bedeutet, dass die regulatorischen Verpflichtungen, die dem Mutterunternehmen auferlegt werden, auch das Gemeinschaftsunternehmen treffen. Es besteht kein Bedürfnis danach, dieses Ergebnis durch eine teleologische oder unionsrechtsrechtskonforme Reduktion des $\$ 3$ Nr. 29 TKG davon abhängig zu machen, dass das marktmächtige Unternehmen eine „alleinige Leitungsmacht“ über das Gemeinschaftsunternehmen hat. Vielmehr lässt es $₫ 36$ Abs. 2 S. 2 GWB ausreichen, dass die Mutterunternehmen gemeinsam eine Beherrschungsmacht ausüben (B. II. 1. b.). Dies überzeugt deshalb, da eine Diskriminierung nicht nur dann erfolgen kann, wenn das marktmächtige Unternehmen über die alleinige Leitungsmacht über das Gemeinschaftsunternehmen verfügt. Vielmehr ist gleichermaßen ein kollusives Zusammenwirken der Mutterunternehmen möglich.

\section{Erstreckung der telekommunikationsrechtlichen}

Regulierungsverfügungen auf paritätische Gemeinschaftsunternehmen

In ihrer Entscheidung vom 10.7.2018 hat die Bundesnetzagentur entschieden, dass die Glasfaser Nordwest nicht mit den Regulierungsverfügungen erfasst wird, die gegenüber der Deutschen Telekom erlassen wurden. ${ }^{835}$ Zwar handele es sich bei den beiden Gesellschaften um ein Unternehmen im Sinne des $\$ 3$ Nr. 29 TKG, allerdings sei die Glasfaser Nordwest zum Zeitpunkt des Marktregulierungsverfahrens, in dessen Rahmen die regulatorischen Verpflichtungen gegenüber der Deutschen Telekom erlassen worden seien, noch nicht gegründet gewesen und demnach nicht von diesen erfasst. ${ }^{836}$ Gegen dieses Ergebnis lassen sich jedoch zahlreiche Argumente anführen, sodass eine dezidierte Auseinandersetzung mit der Frage der Erheblichkeit des Gründungszeitpunktes eines Unternehmens im Hin-

835 BNetzA, Beschl. v. 13.7.2018, Az. BK 3-17/071, S. 2.

836 BNetzA, Beschl. v. 13.7.2018, Az. BK 3-17/071, S. 2. 
blick auf die Rechtsfolge des $₫ 3$ Nr. 29 TKG erforderlich ist. Bereits an dieser Stelle ist festzustellen, dass die hierdurch entstehende Lücke der regulatorischen Verpflichtungen durch eine kartellrechtliche Verpflichtungszusage geschlossen wurde (E. I. 2.).

Die Bundesnetzagentur vertritt die Auffassung, dass sich die regulatorischen Verpflichtungen nur dann auf das Tochterunternehmen erstrecken können, wenn es zum Zeitpunkt des Marktregulierungsverfahrens bereits gegründet war oder zumindest in das Verfahren einbezogen werden konnte. Aus dem Bestimmtheitsgrundsatz des $\$ 37$ Abs. 1 VwVfG folge, dass sich das Marktanalyseverfahren explizit auf das Gemeinschaftsunternehmen erstrecken müsse. ${ }^{837}$ Anderenfalls würde das Mutterunternehmen ohne beträchtliche Marktmacht verschiedener Verfahrensrechte, beispielsweise des Rechtes auf Anhörung gemäß $\$ 28$ Abs. 1 VwVfG, beraubt. ${ }^{838}$ Der Entscheidungspraxis der Kommission sei zudem zu entnehmen, dass sich eine Marktanalyse stets auf ein konkretes Unternehmen beziehen müsse, das in der Entscheidung explizit zu nennen sei. ${ }^{839}$ Hieraus wird gefolgert, dass sich die Feststellung der Marktanalyse und die Regulierungsverfügungen im Ergebnis nicht auf das neu gegründete Gemeinschaftsunternehmen erstrecken würden.

Gegen eine Erheblichkeit des Gründungszeitpunkts im Hinblick auf die Rechtsfolge des $\$ 3$ Nr. 29 TKG lassen sich jedoch vielfältige Argumente anführen. Eine Erheblichkeit des Gründungszeitpunktes ist nicht mit dem Zweck des $\$ 3$ Nr. 29 TKG zu vereinbaren. ${ }^{840}$ Die Vorgabe soll eine Flucht des marktmächtigen Unternehmens in die gesellschaftsrechtliche Umstrukturierung verhindern (D. II. 1. b.). Eine Umgehung wird allerdings erst ermöglicht, wenn die Ergebnisse des Marktregulierungsverfahrens nicht auf das neu gegründete Gemeinschaftsunternehmen erstreckt werden. ${ }^{841}$ In diesem Fall kann durch eine Auslagerung regulierter Tätigkeiten auf ein Gemeinschaftsunternehmen, das erst im Anschluss an das Marktregulierungsverfahrens mit einem dritten Unternehmen gegründet wird, die

837 BNetzA, Entsch. v. 10.7.2018, Az. BK 1-18/002, S. 10.

838 BNetzA, Entsch. v. 10.7.2018, Az. BK 1-18/002, S. 10.

839 Komm., Beschl. v. 4.8.2014, C(2014) 5698 final, S. 5; BNetzA, Entsch. v. 10.7.2018, Az. BK 1-18/002, S. 10 f.

840 So auch Vodafone, in: BNetzA, Entsch. v. 10.7.2018, Az. BK 1-18/002, Konsultationsdokument, S. 2.

841 Vodafone, in: BNetzA, Entsch. v. 10.7.2018, Az. BK 1-18/002, Konsultationsdokument, S. 2. 
Regulierung umgangen werden. ${ }^{842}$ Auf diese Weise können Unternehmen kollusiv zusammenwirken (A. II. 1.).

Die Maßgeblichkeit des Gründungszeitpunktes für das Eintreten der Rechtsfolge des $\$ 3$ Nr. 29 TKG ist nicht in dessen Wortlaut angelegt. Nimmt man dies an, wird ein neuer ungeschriebener Ausnahmebestand des $₫ 3$ Nr. 29 TKG etabliert und damit Rechtsunsicherheit geschaffen. ${ }^{843}$ Dies ist für Legaldefinitionen besonders fragwürdig, deren Funktion im Gesetzesaufbau gerade darin besteht, begriffliche Vorfragen verbindlich für den gesamten Anwendungsbereich des Gesetzes zu klären und hierdurch die Rechtssicherheit zu erhöhen. Zuzugeben ist insoweit, dass eine gefestigte Rechtsprechung und Entscheidungspraxis praeter legem nicht stets Rechtsunsicherheit begründen. Rechtssicherheit entsteht jedoch erst dann, wenn die Rechtsprechung und Entscheidungspraxis fortgeführt werden. Damit wäre zumindest temporär die Funktion der Begriffsbestimmung nicht mehr vollständig erfüllt.

Es kann zudem nicht überzeugen, dass mit einer anderslautenden Entscheidung die Verfahrensrechte des Unternehmens ohne beträchtliche Marktmacht beschnitten würden. ${ }^{844}$ Jeder Kooperationspartner ist bei Eingang des Kooperationsverhältnisses über die marktmächtige Stellung des Kooperationspartners informiert und nimmt diese bewusst im Kauf. ${ }^{845}$ Die Situation ist mit derjenigen vergleichbar, dass Anteile eines regulierten Unternehmens erworben werden. Auch dann werden die regulatorischen Verpflichtungen auf das Unternehmen erstreckt, ohne dessen Verfahrensrechte zu verletzen. ${ }^{846}$ Zudem gilt das Recht auf Anhörung aus $₫ 28$ Abs. 1 VwVfG nicht absolut. Zwar handelt es sich um eine verfassungsrechtlich fundierte Verfahrensgarantie, ${ }^{847}$ von der jedoch in bestimmten Konstellationen abgewichen werden kann. In spezifischen Fallkonstellationen er-

842 Vodafone, in: BNetzA, Entsch. v. 10.7.2018, Az. BK 1-18/002, Konsultationsdokument, S. 2; 1\&1, in: BNetzA, Entsch. v. 10.7.2018, Az. BK 1-18/002, Konsultationsdokument, S. 4.

843 So auch Vodafone, in: BNetzA, Entsch. v. 10.7.2018, Az. BK 1-18/002, Konsultationsdokument, S. 3.

844 So auch Vodafone, in: BNetzA, Entsch. v. 10.7.2018, Az. BK 1-18/002, Konsultationsdokument, S. 4.

845 1\&1, in: BNetzA, Entsch. v. 10.7.2018, Az. BK 1-18/002, Konsultationsdokument, S. 4; BKartA, in: BNetzA, Entsch. v. 10.7.2018, Az. BK 1-18/002, Stellungnahme BKartA, S. 2.

846 Vodafone, in: BNetzA, Entsch. v. 10.7.2018, Az. BK 1-18/002, Konsultationsdokument, S. 4.

847 Weiterführend zur verfassungsrechtlichen Fundierung Kallerhoff/Mayen, in:

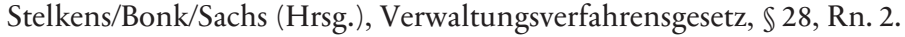


möglicht $₫ 28$ Abs. 3 VwVfG die Möglichkeit von einer Anhörung abzusehen. ${ }^{848}$ Selbst wenn diese Fallkonstellationen nicht erfüllt seien, kann eine Anhörung gemäß $₫ 45$ Abs. 1 Nr. 3, Abs. 2 VwVfG nachgeholt werden oder der Verfahrensfehler sogar gemäß $₫ 46$ VwVfG unbeachtlich sein. ${ }^{849}$ Zuzugeben ist, dass für eine Unbeachtlichkeit des in einer unterbliebenen Anhörung liegenden Verfahrensfehlers bei einer so komplexen Entscheidung wie der Auferlegung einer Regulierungsverpflichtung in der Praxis wohl kaum Raum bleibt. Darüber hinaus ist festzustellen, dass die Ausnahmen in der Praxis zunehmend restriktiv gehandhabt werden und gegen ihre extensive Handhabung verfassungsrechtliche Bedenken bestehen. ${ }^{850}$ Festzuhalten bleibt, dass der Gesetzgeber Ausnahmen von dem üblichen Verfahrenslauf gestattet. Demnach reicht ein pauschaler Rückgriff auf $\$ 28$ Abs. 1 VwVfG nicht für eine Ausnahme von der Regel des $\$ 3$ Nr. 29 TKG aus. ${ }^{851}$ Es ist vielmehr eine dezidierte Auseinandersetzung mit der Möglichkeit der Unbeachtlichkeit oder Heilung des Verfahrensfehlers in jedem konkreten Einzelfall erforderlich. Falls aber angenommen wird, dass eine pauschale Argumentation mit dem Verstoß gegen das Anhörungsrecht aus $\$ 28$ Abs. 1 VwVfG ausreicht, werden vergleichbare Konstellationen, die $\$ 3$ Nr. 29 TKG unterfallen, unterschiedlich behandelt, obwohl sich dafür kein normativer Anknüpfungspunkt findet. 852

Bei der Gründung der Glasfaser Nordwest konnte das Argument, dass die Anhörungsrechte der EWE aus $\$ 28$ Abs. 1 VwVfG durch eine Anwendung des $₫ 3$ Nr. 29 TKG umgangen würden, aus einem weiteren Grund nicht überzeugen. Konkret wurde in diesem Verfahren der Bundesnetzagentur die Erstreckung von drei Regulierungsverfügungen auf das Tochterunternehmen überprüft. ${ }^{853}$ In zwei der Verfahren war die EWE als An-

848 Vodafone, in: BNetzA, Entsch. v. 10.7.2018, Az. BK 1-18/002, Konsultationsdokument, S. 5.

849 Vodafone, in: BNetzA, Entsch. v. 10.7.2018, Az. BK 1-18/002, Konsultationsdokument, S. 5.

850 Sachs, in: Stelkens/Bonk/Sachs (Hrsg.), Verwaltungsverfahrensgesetz, $\$ 45$ VwVfG, Rn. 103 ff.

851 So auch Vodafone, in: BNetzA, Entsch. v. 10.7.2018, Az. BK 1-18/002, Konsultationsdokument, S. 5.

852 BVerwG, Urt. v. 11.12.2013, Az. 6 C 24/12, NVwZ 2014, S. 942, 943, Ls. 3; Vodafone, in: BNetzA, Entsch. v. 10.7.2018, Az. BK 1-18/002, Konsultationsdokument, S. 5.

853 Hierbei handelt es sich BNetzA, Entsch. v. 1.9.2016, Az. BK 3g-15/004; BNetzA, Entsch. v. 29.8.2013, Az. BK 3d-12/131, und BNetzA, Entsch. v. 28.10.2015, Az. BK 3h-14/114. 
tragstellerin aktiv. ${ }^{854}$ Also wurde sie in beiden Verfahren gemäß $₫ 135$ Abs. 1 TKG angehört. ${ }^{855}$ Das spezielle Anhörungsrecht für Beteiligte von Verfahren vor der Bundesnetzagentur verdrängt in seinem Anwendungsbereich die allgemeinen Vorgaben des $\$ 28$ Abs. 1 VwVfG. ${ }^{856} \$ 135$ Abs. 1 TKG ermöglicht auch die Anhörung Beteiligter, die nicht Adressat des belastenden Verwaltungsaktes sind. Dies wird gemäß $\$ 214$ Abs. 1 TKG-RefE auch künftig so sein. ${ }^{857}$ Die Anhörung der Beteiligten erfolgt nach einem einheitlichen Ablauf, sodass sich die Anhörung der Adressaten des Verwaltungsaktes und die der Antragsteller nicht unterscheiden. Aus diesem Grund kann es im konkreten Fall nicht überzeugen, wenn gerügt wird, dass keine Anhörung erfolgt sei. Diese hat stattgefunden, auch wenn die EWE vermutlich andere Argumente vortrug als bei einer neuerlichen Anhörung nach Gründung der Glasfaser Nordwest.

Auch das Bestimmtheitsgebot gebietet keine abweichende Handhabung des $₫ 3$ Nr. 29 TKG. Natürlich muss ein Verwaltungsakt im Sinne des $\$ 37$ Abs. $1 \mathrm{VwVfG}$ bestimmt sein. Es erfordert eine Benennung des Adressaten. Infolge des $\$ 3 \mathrm{Nr} .29$ TKG reicht jedoch die Benennung des marktmächtigen Mutterunternehmens aus, um dem Bestimmtheitsgebot Genüge zu tun. Das Tochterunternehmen wird nämlich als Bestandteil des Mutterunternehmens betrachtet. Wird das Mutterunternehmen benannt, ist auch das Tochterunternehmen erfasst.

Die Entscheidungspraxis der Kommission ${ }^{858}$ steht einer einheitlichen Anwendung des $\$ 3$ Nr. 29 TKG nicht entgegen, sondern gebietet vielmehr lediglich, dass das Marktanalyseverfahren sich auf ein spezifisches Unternehmen bezieht, das auch benannt werden muss. ${ }^{859}$ Insbesondere der unionale Grundsatz des effet utile spricht für eine Vereinbarkeit mit dem Unionsrecht. Die stringente Anwendung des $\$ 3$ Nr. 29 TKG stellt sicher, dass die Regulierungsverpflichtung der nationalen Regulierungsbehörde,

854 Hierbei handelt es sich um BNetzA, Entsch. v. 1.9.2016, Az. BK 3g-15/004, S. 28 und BNetzA, Entsch. v. 29.8.2013, Az. BK 3d-12/131, S. 30.

855 BNetzA, Entsch. v. 1.9.2016, Az. BK 3g-15/004; BNetzA, Entsch. v. 29.8.2013, Az. BK 3d-12/131.

856 Attendorn/Geppert, in: Geppert/Schütz (Hrsg.), Beck'scher TKG-Kommentar, $\$ 135$, Rn. 3; Bergmann, in: Scheurle/Mayen (Hrsg.), Telekommunikationsgesetz, $\mathbb{\$} 135, \mathrm{Rn} .2$.

857 Referentenentwurf des Telekommunikationsmodernisierungsgesetzes (TKMoG), abrufbar unter: https:/www.bmwi.de/Redaktion/DE/Downloads/Gesetz/ telekommunikationsmodernisierungsgesetz-referentenentwurf-20201612.pdf? blob=publicationFile\&v=8 (zuletzt abgerufen am 12.1.2021).

858 Komm., Beschl. v. 4.8.2014, C(2014) 5698 final, S. 5.

859 Komm., Beschl. v. 4.8.2014, C(2014) 5698 final, S. 5. 
die in $₫ 13$ Abs. 1 S. 1 TKG national ausgestaltet und unionsrechtlich überformt ist, auch effektiv umgesetzt werden kann. ${ }^{860}$ Eine anderweitige Regulierungspraxis weicht von der Marktanalyse ab, die im Komitologieverfahren von der Kommission bestätigt wurde, und macht aus diesem Grund einen neuerlichen Verfahrensdurchlauf erforderlich. ${ }^{861}$ Deswegen erstrecken sich die Feststellung der Marktanalyse und die Regulierungsverfügungen auch auf ein paritätisches Gemeinschaftsunternehmen, das zum Zeitpunkt des Marktregulierungsverfahrens noch nicht gegründet war. Die Spruchpraxis der BNetzA ist vor diesem Hintergrund kritisch zu hinterfragen und kann im Ergebnis nicht überzeugen.

3. Novellierung der regulatorischen Rahmenbedingungen durch den EKEK

Eine umfassende Aktualisierung haben die regulatorischen Rahmenbedingungen für den Umgang mit Kooperationen zur Beschleunigung des Glasfaserausbaus durch den EKEK erfahren. Dessen zentrales Element ist insoweit die Verpflichtungszusage, die vollständig neu in den Rechtsrahmen integriert worden ist (dazu a.). Hierbei handelt es sich um ein prägendes Instrument für verschiedene normativ vorstrukturierte Privilegierungstatbestände (dazu b.). Herauszuarbeiten ist, welche Auswirkungen der neue Rechtsrahmen auf die Kooperationspraxis haben wird (dazu c.).

\section{a) Regulatorische Verpflichtungszusage gemäß Art. 79 EKEK}

Erst im Rahmen des Trilogs zwischen Kommission, Rat und Europäischem Parlament wurde das Instrument der Verpflichtungszusage in den regulatorischen Rechtsrahmen integriert. Entlehnt ist es dem kartellrechtlichen Verwaltungsverfahren (dazu aa.). In der deutschen Regulierungspraxis wurde im Vorfeld auf das Instrument des öffentlich-rechtlichen Vertrages zurückgegriffen, das eine ähnliche Funktion erfüllte (dazu bb.). Die Vorgaben zur Einbindung der Verpflichtungszusage in das regulatorische

860 Vodafone, in: BNetzA, Entsch. v. 10.7.2018, Az. BK 1-18/002, Konsultationsdokument, S. 5.

861 Vodafone, in: BNetzA, Entsch. v. 10.7.2018, Az. BK 1-18/002, Konsultationsdokument, S. $8 \mathrm{f}$. 
Verwaltungsverfahren ergeben sich aus Art. 79 EKEK (dazu cc.), die noch vollständig in nationales Recht umzusetzen sind (dazu dd.).

aa) Verpflichtungszusage im Kartellrecht

Von dem Instrument der Verpflichtungszusage wird sowohl im unionalen als auch im nationalen Kartellrecht umfassender Gebrauch gemacht. Eine zentrale Rolle spielt es sowohl im Marktmissbrauchs- als auch im Fusionskontrollverfahren (E. I. bis II.). Normative Vorgaben für die Verpflichtungszusage im Kartellrecht finden sich in Art.9 der Verordnung (EG) Nr. 1/2003 ${ }^{862}$ und in $\$ 32 b$ GWB. ${ }^{863}$ Die Verpflichtungszusage ermöglicht eine vielfältige Flexibilisierung des kartellrechtlichen Verwaltungsverfahrens. Unternehmen können sich dadurch freiwillig zu bestimmten Verhaltensweisen verpflichten. Diese Selbstverpflichtungen können dann als Abwägungstopos in das Ermessen der Behörde einfließen. Die Verpflichtungszusagen können von den Behörden für verbindlich erklärt werden. Dabei sind sie jedoch nicht an den normierten Maßnahmenbestand oder maximale Bindungsfristen gebunden, sodass die Nutzung dieses Instrumentes das Verfahren weitgehend flexibilisiert. Auf diese Weise wird der Dialog zwischen Unternehmen und Behörde gefördert und werden einzelfallgerechte Lösungen erleichtert. Unternehmen erhalten einen Einfluss auf den Ausgang des Verwaltungsverfahrens und können den Inhalt der Verpflichtung selbst mitbestimmen.

bb) Öffentlich-rechtliche Verträge im Rahmen des Marktregulierungsverfahrens

Bereits im Vorfeld der Integration der Verpflichtungszusage in den Rechtsrahmen wurde in der deutschen Regulierungspraxis mithilfe des öffentlich-rechtlichen Vertrages ein vergleichbares Instrument genutzt. Im Rahmen der Rückführung der Regulierung zur Ermöglichung der Nutzung der Übertragungstechnologie Vectoring, die einen Zugriff auf alle Teilneh-

862 Verordnung (EG) Nr. 1/2003 vom 16.12.2002 zur Durchführung der in den Artikeln 81 und 82 des Vertrages niedergelegten Wettbewerbsregeln, ABl. L 1 v. 4.1.2003, S. 1, zuletzt geändert durch die Verordnung (EG) Nr. 487/2009 des Rates v. 25.5.2009, ABl. L 148 v. 11.6.2009, S. 1; Lettl, Kartellrecht, S. 162.

863 Weiterführend hierzu Lettl, Kartellrecht, S. 384. 
meranschlussleitungen in einem Kabelverzweiger erforderlich macht (Kapitel 3, B. III.), stellte sich die Frage, inwieweit öffentlich-rechtliche Verträge als Abwägungstopos in die Ermessenserwägungen der Regulierungsbehörde im Rahmen des Marktregulierungsverfahrens einbezogen werden können. ${ }^{864}$ Letztlich gab die Deutsche Telekom ein unwiderrufliches Angebot zum Ausbau der Infrastruktur ab, das von der Bundesnetzagentur bis zum Ablauf des Ausbauzeitraumes angenommen werden kann. ${ }^{865}$ Das Unternehmen verpflichtet sich hierdurch freiwillig zum Infrastrukturausbau. Eine tatsächlich einklagbare Verpflichtung hierzu entsteht erst mit Annahme des Angebotes. ${ }^{866}$ Das Angebot wurde im Rahmen des Regulierungsermessens der Behörde berücksichtigt und führte letztlich zu einer Abänderung der Regulierungsverfügung hinsichtlich des Zuganges zur Teilnehmeranschlussleitung. ${ }^{867}$ Letztlich ist die Funktion mit der kartellrechtlichen Verpflichtungszusage vergleichbar. Der zu Grunde liegende Rechtsgedanke wird mit dem neuen Instrument der Verpflichtungszusage nunmehr kodifiziert.

cc) Verpflichtungszusage im unionalen Telekommunikationsrecht

Die zentrale Vorschrift zur Einbindung der Verpflichtungszusage in das regulatorische Verwaltungsverfahren ist Art. 79 EKEK. Dieser muss noch in nationales Recht umgesetzt werden (hierzu sogleich in dd.). Verpflichtungszusagen können gemäß Art. 79 Abs. 1 UAbs. 1 EKEK von Unternehmen mit beträchtlicher Marktmacht gegenüber der nationalen Regulierungsbehörde abgegeben werden und Zugangsbedingungen oder Bedingungen für kooperative Investitionen beinhalten. Eine nicht abschließende Auflistung potentieller Inhalte von Verpflichtungszusagen enthält Art. 79 Abs. 1 UAbs. 1 lit. a bis c EKEK. Allgemeine Anforderungen an Form und Inhalt der Verpflichtungszusage enthält Art. 79 Abs. 1 UAbs. 2 EKEK. Auf diese Weise wird sichergestellt, dass das Angebot eine ausreichende Grundlage für die Bewertung im Sinne des Art. 79 Abs. 2 EKEK darstellt.

864 Kühling/Bulowski, Rechtsgutachten über die Zulässigkeit öffentlich-rechtlicher Verträge im Rahmen des TKG; hieran anknüpfend Kühling/Bulowski, N\&R 2015, S. 262 ff.

865 Offenbächer, Die Regulierung des Vectoring, S. 319 m. w. N.

866 Offenbächer, Die Regulierung des Vectoring, S. 316.

867 BVerwG, Urt. v. 21.9.2018, Az. 6 C 8.17, BVerwGE 163, S. 136, Rn. 6; BNetzA, Beschl. v. 1.9.2016, Az. BK 3g-15/004, S. 217, 224. 
Der Maßstab für die behördliche Überprüfung der Verpflichtungszusagen ergibt sich aus Art. 79 Abs. 2 EKEK. In der Regel erfolgt gemäß Art. 79 Abs. 2 UAbs. 1 S. 1 EKEK eine Marktprüfung inklusive einer Konsultation interessierter Dritter. Hiervon kann abgewichen werden, sofern evident eine oder mehrere Bedingungen, die an die Verpflichtungszusage gestellt werden, nicht erfüllt sind. Spezielle Äußerungsmöglichkeiten bestehen zudem gemäß Art. 79 Abs. 2 UAbs. 1 S. 2 EKEK für Ko-Investoren und Zugangsnachfrager. Diese können Änderungen an den Verpflichtungszusagen vorschlagen, welche die marktmächtigen Unternehmen berücksichtigen können, aber nicht müssen. Eine regulatorische Entlastung kann nur dann erfolgen, wenn das Angebot für eine kooperative Investition von zumindest einem Telekommunikationsunternehmen angenommen wird (D. II. 3. a. cc.). Insoweit kann die frühzeitige Konsultation der potentiellen Ko-Investoren sicherstellen, dass das marktmächtige Unternehmen gegebenenfalls Anpassungen an der Verpflichtungszusage vornehmen kann. Positiv ist zu bewerten, dass die Vorgabe auf diese Weise dialogorientiert ausgerichtet wird und dazu beiträgt, Instrumente der Selbstregulierung zu ertüchtigen.

Die speziellen Bewertungskriterien, an denen sich die Verpflichtungszusagen messen lassen müssen, sind regelmäßig im jeweiligen Regelungszusammenhang normiert. Eine Ausnahme hiervon besteht für Verpflichtungszusagen im Sinne des Art. 79 Abs. 1 UAbs. 1 lit. a EKEK, deren spezielle Bewertungskriterien mangels eigenständiger normativer Grundlage in Art. 79 Abs. 2 UAbs. 2 EKEK normiert sind. 868

Die Ausrichtung des Instrumentes auf die Förderung des Dialoges zwischen Regulierungsbehörde und Unternehmen wird insbesondere in Art. 79 Abs. 2 UAbs. 3 EKEK offenbar. In einem zwischengeschalteten Verfahrensschritt äußert sich die nationale Regulierungsbehörde dazu, ob die Verpflichtungszusage den jeweiligen Anforderungen entspricht (S. 1). Das Unternehmen kann sein Angebot anpassen (S.2). Dieser Zwischenschritt ist insbesondere deswegen vorteilhaft, weil das Instrument darauf ausgerichtet ist, die Regulierung zu flexibilisieren. Dieses Ziel kann nur mithilfe eines umfassenden Dialoges der Beteiligten erreicht werden.

Die Rechtsfolge der Verpflichtungszusage ergibt sich aus Art. 79 Abs. 3 EKEK. Danach kann die nationale Regulierungsbehörde die Verpflichtungszusage ganz oder teilweise für bindend erklären (UAbs. 1). Hiervon nicht berührt werden die speziellen Regelungen des Art. 76 Abs. 2 EKEK, auf die noch ausführlich eingegangen werden soll (D. II. 3. b. aa.).

868 Hierzu weiterführend D. II. 3. b. bb. 
Die Regulierungsbehörde muss nicht die in Art. 67 Abs. 5 EKEK vorgesehenen Bindungsfristen beachten. Verpflichtungszusagen über die regulatorische Privilegierung von Ko-Investitionen entfalten gemäß Art.79 Abs. 3 UAbs. 2 Hs. 2 EKEK eine Bindungswirkung für mindestens sieben Jahre. Hierdurch wird das Instrument noch weiter flexibilisiert. Die Ausdehnung der möglichen Bindungsfristen kann überzeugen, weil die Unternehmen sich selbst zu einem bestimmten Verhalten verpflichten. Je länger durch die Verpflichtungszusagen ein verlässlicher Regulierungsrahmen gewährleistet ist, desto stärker nimmt die Regulierungsunsicherheit ab.

Üblicherweise bleiben gemäß Art. 79 Abs. 3 UAbs. 3 EKEK die Durchführung einer Marktanalyse (Art. 67 EKEK) und die Auferlegung zusätzlicher regulatorischer Verpflichtungen (Art. 68 EKEK) unbenommen. Hierdurch wird sichergestellt, dass der regulatorische Rechtsrahmen trotz seiner Flexibilisierung nicht seine Schlagkraft einbüßt und suffizient bleibt. Von dieser Regel existiert wiederum für die regulatorische Behandlung von Ko-Investitionen gemäß Art.76 EKEK eine Ausnahme. Für die Berücksichtigung von Verpflichtungszusagen im Rahmen der Auferlegung regulatorischer Verpflichtungen enthält Art. 79 Abs. 3 UAbs. 4 EKEK spezielle Vorgaben, aus denen offenbar wird, dass die Verpflichtungszusagen umfassend in das Regulierungsermessen der nationalen Regulierungsbehörde einfließen und insbesondere in der Angemessenheitsprüfung zu berücksichtigen sind.

Die Abgabe einer Verpflichtungserklärung, also die regulatorische Selbstverpflichtung, entbindet nicht von einer Aufsicht durch die nationalen Regulierungsbehörden. Danach erfolgt gemäß Art. 79 Abs. 4 S. 1 EKEK ein umfassendes Monitoring durch die nationale Regulierungsbehörde. Falls die Verpflichtungszusage nicht eingehalten wird, kann die Regulierungsbehörde im Verfahren, das in Art. 29 EKEK vorgesehen ist, Sanktionen erlassen. Darüber hinaus kann sie unter den Voraussetzungen des Art. 79 Abs. 4 S. 3 EKEK die Situation neu bewerten.

dd) Umsetzung im Telekommunikationsgesetz

Die Vorgabe soll in $₫ 18$ TKG-RefE umgesetzt werden. ${ }^{869}$ Dabei werden jedoch verschiedene Vorgaben vorgenommen, die im unionalen Rechtsrah-

869 Referentenentwurf des Telekommunikationsmodernisierungsgesetzes (TKMoG), abrufbar unter: https:/www.bmwi.de/Redaktion/DE/Downloads/Gesetz/ 
men jeweils separat normiert sind (dazu auch D. II. 3. a. cc.), in fragwürdiger Weise verzahnt.

Auch in weiteren Detailfragen existieren Abweichungen. So sieht etwa \18 Abs. 1 TKG-RefE anders als Art. 79 EKEK vor, dass die Verpflichtungszusage im Internet zu veröffentlichen ist. Aus Gründen der Transparenz ist die weitergehende Vorgabe des Referentenentwurfes positiv zu bewerten.

Auch wenn die Vorgaben des EKEK weitaus detaillierter sind als die des Kartellrechts, zeigen sich in der Ausgestaltung im Ergebnis umfassende Parallelen.

\section{b) Privilegierungstatbestände für Kooperationsvorhaben im EKEK}

Verfügt ein Unternehmen auf einem definierten Markt über eine beträchtliche Marktmacht, sieht der Rechtsrahmen die Auferlegung regulatorischer Verpflichtungen vor. Die nationalen Regulierungsbehörden verfügen nicht über ein Entschließungsermessen, ob sie regulieren, sondern lediglich über ein Auswahlermessen hinsichtlich der regulatorischen Verpflichtung. Privilegierungstatbestände finden sich in Art.76 EKEK (dazu aa.) und Art. 79 Abs. 1 UAbs. 1 EKEK (dazu bb.). Diese sollen in den SS 16 und 17 TKG-RefE umgesetzt werden (dazu cc.).

aa) Regulatorische Behandlung neuer Bestandteile von Netzen mit sehr hoher Kapazität gemäß Art. 76 EKEK

Eine Ausnahme von der zuvor beschriebenen Systematik bildet Art.76 EKEK. Die Vorschrift sieht eine privilegierte regulatorische Behandlung von Glasfaserinfrastrukturen vor, die im Rahmen kooperativer Investitionsvorhaben unter Beteiligung eines marktmächtigen Unternehmens neu errichtet wurden. Ein unkoordiniertes Absehen von Regulierung könnte im Telekommunikationssektor, der umfassend von natürlichen Monopolen geprägt ist (Kapitel 3, B. IV.), zu erheblichen Wettbewerbsdefiziten und Beeinträchtigungen der Verbraucherinteressen führen. ${ }^{870}$ Vor diesem Hintergrund ist die normative Konstruktion zu betrachten, die erst im Tri-

telekommunikationsmodernisierungsgesetz-referentenentwurf-20201612.pdf?

blob=publicationFile $\& v=8$ (zuletzt abgerufen am 12.1.2021).

870 Weiterführend hierzu Sickmann/Neumann, Deregulierung und Verbraucherwohlfahrt auf dem deutschen Telekommunikationsmarkt, S. $96 \mathrm{ff}$.; konkret zu 
log zwischen der Kommission, dem Parlament und dem Rat der Europäischen Union ihre endgültige Gestalt erhalten hat. So war die Norm zwischen Kommission, ${ }^{871}$ Europäischem Parlament ${ }^{872}$ und Rat ${ }^{869}$ so umstritten, ${ }^{870}$ dass sie im Rahmen des Trilogverfahrens vollständig neu konzipiert wurde. ${ }^{871}$

Privilegiert $^{873}$ sind $^{874}$ gemäß ${ }^{875}$ Art. 76 Abs. 1 UAbs. 1 EKEK solche Unternehmen, deren beträchtliche Marktmacht im Marktanalyseverfahren gemäß Art. 67 EKEK festgestellt wurde. Um von der Privilegierung profitieren zu können, müssen sie Verpflichtungszusagen im Sinne des Art. 79 EKEK abgeben, die anhand der Kriterien des Art. 76 Abs. 1 UAbs. 2 EKEK zu prüfen sind. Das Ziel der Norm ist die Beschleunigung des Aufbaus von Netzen mit sehr hoher Kapazität im Sinne des Art. 2 Nr. 2 EKEK (Kapitel 3, C. III. 1. a.), wobei Art. 76 Abs. 1 UAbs. 2 EKEK davon insoweit abweicht, dass die regulatorische Privilegierung ausschließlich auf die Übertragungstechnologie Glasfaser beschränkt wird. ${ }^{876}$ Dies ist im Hinblick auf die Technologieneutralität fragwürdig, da auch alternative Infrastrukturen mit einer vergleichbaren Qualität einbezogen werden sollten. Zu denken wäre in dieser Hinsicht etwa an Koaxialkabel, die mit der Übertragungstechnologie DOCSIS 3.1 ausgestattet sind (Kapitel 2, B. II. 2. b.).

Für eine Privilegierung müssen die marktmächtigen Unternehmen den Aufbau des neuen Netzes für kooperative Investitionen öffnen. Hier ist zu klären, was unter einem „neuen Netz“ zu verstehen ist. So ist es entweder möglich, nur solche Netze zu erfassen, die nach Umsetzung des EKEK in nationales Recht errichtet werden, oder auch solche, die zwar zuvor errich-

den Auswirkungen von Kooperationsvorhaben auf die Anbieterkonzentration Sickmann/Neumann, K\&R 2018, S. 92, $92 \mathrm{f}$.

871 Kommission, COM (2016) 590 final/2, abrufbar unter: https:/ec.europa.eu/trans parency/regdoc/rep/1/2016/DE/1-2016-590-DE-F1-1.PDF (zuletzt abgerufen am 1.7.2020); hierzu weiterführend Neumann, N\&R 2016, S. 262, 270; Scherer/Heinickel, MMR 2017, S. 71, 75.

872 Europäisches Parlament, Ausschuss für Industrie, Forschung und Energie, Bericht v. 19.10.2017 - A8-0318/2017, abrufbar unter: https://www.europarl.europ a.eu/doceo/ document/A-8-2017-0318_DE.html\#title1 (zuletzt abgerufen am 1.7.2020).

873 Rat, Vorschlag v. 9.10.2017 - 12797/1/17 REV 1, abrufbar unter: http://data.cons ilium.europa.eu/doc/document/ST-12797-2017-REV-1/en/pdf (zuletzt abgerufen am 1.7.2020).

874 Umfassende Darstellung der Abweichungen bei Sickmann/Neumann, K\&R 2018, S. 92, $94 \mathrm{ff}$.

875 Neumann, N\&R 2018, S. 204, 208 f.

876 Kritisch hinsichtlich dieser Beschränkung Neumann, N\&R 2018, S. 204, 208. 
tet wurden, aber erst nach Inkrafttreten des Art. 76 EKEK für Endkundendienste in Netzen mit sehr hoher Kapazität genutzt werden können. ${ }^{877}$ Eine extensive Auslegung ist vor dem Hintergrund kritisch zu bewerten, dass die kooperative Investition von einer bloßen Zahlung für die Gewährung eines Zuganges abgegrenzt werden muss (A. I.). Daher kann jedenfalls kein bereits vollständig errichtetes Netz in den Anwendungsbereich des Art. 76 EKEK fallen. Sofern vorhandene Netze erweitert werden, stellt Art. 76 Abs. 2 UAbs. 1 EKEK zudem klar, dass lediglich die neuen Netzbestandteile von der regulatorischen Privilegierung erfasst werden. Im Ergebnis ist somit eine restriktive Interpretation des Begriffes „neues Netz“ zu favorisieren. Hinsichtlich der Kooperationsformen nennt das Gesetz zwar Beispiele wie das Miteigentum oder die Teilung des Risikos, ohne sich jedoch auf einen numerus clausus zulässiger Kooperationsformen festzulegen. ${ }^{878}$

Dass kooperative Investitionen in Glasfaserinfrastrukturen dadurch honoriert werden, dass die Regulierung partiell verringert wird, bringt verschiedene Missbrauchspotentiale mit sich, ${ }^{879}$ deren Ausnutzung mithilfe differenzierter Mechanismen verhindert wird. So werden etwaige Verpflichtungszusagen am Maßstab der Beurteilungskriterien des Art.76 Abs. 1 UAbs. 2 EKEK und des Anhangs IV des EKEK gemessen. ${ }^{880}$ Die wichtigste regulatorische Verpflichtung ist die Zugangsverpflichtung, die den Wettbewerb bei der Erbringung von Endkundendiensten trotz natürlicher Monopole ermöglicht. Bei der Kontrolle der Verpflichtungszusage wird sichergestellt, dass sowohl die Kooperationsteilnehmer als auch nicht an der Kooperation beteiligte Unternehmen (lit. a) ${ }^{881}$ einen diskriminierungsfreien Zugang zur Infrastruktur erhalten (lit. b, ii.). Auf diese Weise wird eine Remonopolisierung oder Oligopolisierung verhindert. ${ }^{82} \mathrm{Um}$ trotz der Gewährleistung des offenen Zuganges (lit. a) die Beteiligung an

877 Weiterführend zur Auslegung des Begriffes des „neuen Netzes“ Nigge/Horstmann, MMR 2018, S. 721, 724.

878 Erwägungsgrund 198; hierzu Neumann, N\&R 2018, S. 204, 209; Nigge/Horstmann, MMR 2018, S. 721, 724.

879 Umfassend hierzu Sickmann/Neumann, Deregulierung und Verbraucherwohlfahrt auf dem deutschen Telekommunikationsmarkt, S. $185 \mathrm{ff}$.

880 Weiterführend hierzu Neumann, N\&R 2018, S. 204, 209; Nigge/Horstmann, MMR 2018, S. 721, 724.

881 Zur Frage der Dauer der Zugangsberechtigung Nigge/Horstmann, MMR 2018, S. $721,724$.

$882 \mathrm{Zu}$ dieser Gefahr Sickmann/Neumann, Deregulierung und Verbraucherwohlfahrt auf dem deutschen Telekommunikationsmarkt, S. 170. 
der Kooperation zu motivieren, wird der Zugang zur vollen neuen Kapazität des Netzes nur stufenweise gewährt (lit. d). ${ }^{883}$

Eine weitere Gefahr liegt in der Ausnutzung der Verhandlungsmacht durch das marktmächtige Unternehmen bei der Ausgestaltung des Kooperationsvorhabens. ${ }^{884}$ Diese Gefahr wird in mehreren Stufen eingedämmt. So existieren hinsichtlich des Angebotes für kooperative Investitionen durch das marktmächtige Unternehmen zunächst umfassende Transparenzverpflichtungen (lit. c). ${ }^{885}$ Zudem stellen bestimmte Kriterien sicher, dass die Verhandlungsasymmetrie ausgeglichen wird (lit. d). Weitere Kriterien enthält Anhang IV des EKEK (lit. e). ${ }^{886}$

Ein zusätzliches Missbrauchspotential wird dadurch begründet, dass das Unternehmen die Angebote für kooperative Investitionen bewusst so konstruiert, dass sie nicht angenommen werden, und die regulatorische Privilegierung auf diese Weise strategisch missbraucht. ${ }^{887}$ Verhindert wird dies dadurch, dass gemäß Art. 76 Abs. 2 UAbs. 1 EKEK zumindest ein Unternehmen das Kooperationsangebot annehmen muss, bevor eine regulatorische Privilegierung erfolgt. ${ }^{888}$ Dieses Unternehmen muss ein Anbieter von Telekommunikationsnetzen oder -diensten sein. Auf diese Weise soll verhindert werden, dass das vorgenannte Kriterium durch die Wahl eines Finanzinvestors als Kooperationspartner missbraucht wird. ${ }^{889}$ Im Ergebnis wird über die Verpflichtungszusage somit sichergestellt, dass die regulatorische Privilegierung nicht als Mittel zur Umgehung regulatorischer Verpflichtungen genutzt wird. Damit das Recht unional einheitlich angewendet wird, verleiht Art. 76 Abs. 4 EKEK dem Gremium der europäischen Regulierungsbehörden (GEREK) die Kompetenz zum Erlass von Leitlinien zur einheitlichen Anwendung der Vorgaben.

Die Rechtsfolge der regulatorischen Privilegierung ergibt sich aus Art. 76 Abs. 2 EKEK. In der Regel erklärt die nationale Regulierungsbehör-

883 Weiterführend hierzu Bourreau/Hoernig/Maxwell, Implementing co-investment and network sharing, S. 42 ff.; Neumann, N\&R 2018, S. 204, 209; Nigge/Horstmann, MMR 2018, S. 721, $724 \mathrm{f}$.

884 Sickmann/Neumann, Deregulierung und Verbraucherwohlfahrt auf dem deutschen Telekommunikationsmarkt, S. 170.

885 Nigge/Horstmann, MMR 2018, S. 721, 725.

886 Hierzu weiterführend Nigge/Horstmann, MMR 2018, S. 721, 725.

887 Sickmann/Neumann, Deregulierung und Verbraucherwohlfahrt auf dem deutschen Telekommunikationsmarkt, S. 171.

888 Neumann, N\&R 2018, S. 204, 209.

$889 \mathrm{Zu}$ dieser Gefahr Sickmann/Neumann, Deregulierung und Verbraucherwohlfahrt auf dem deutschen Telekommunikationsmarkt, S. 171. 
de die Verpflichtungszusage gemäß Art. 79 Abs. 3 EKEK für bindend und erlegt keine zusätzlichen regulatorischen Verpflichtungen auf, sofern sie davon ausgeht, dass die Verpflichtungszusage die Kriterien des Art.76 Abs. 1 UAbs. 2 EKEK erfüllt, und ein Ko-Investor das Angebot des marktmächtigen Unternehmens annimmt. Die regulatorische Privilegierung erfasst ausweislich des systematischen Zusammenhanges ausschließlich Regulierungsverfügungen, die an die beträchtliche Marktmacht anknüpfen. ${ }^{890}$ Art. 76 Abs. 1 UAbs. 2 und 3 EKEK sieht hiervon jedoch Ausnahmen vor. ${ }^{891}$ Dies gilt zum einen dann, wenn bei Marktprüfungen gemäß Art. 79 Abs. 2 EKEK festgestellt wird, dass die Bedingungen des Art.76 Abs. 1 UAbs. 2 EKEK nicht erfüllt sind und die Ko-Investition für den Wettbewerb relevant ist (UAbs. 2). Als ultima ratio kann die nationale Regulierungsbehörde gemäß Art. 76 Abs. 2 UAbs. 3 EKEK auch weitere regulatorische Verpflichtungen auferlegen, sofern dies aufgrund erheblicher Wettbewerbsprobleme erforderlich ist, denen nicht auf andere Weise abgeholfen werden kann. Hieraus resultiert eine gesteigerte Darlegungslast der nationalen Regulierungsbehörden. Zuweilen wird zusätzlich die Einschränkung gemacht, dass regulatorische Verpflichtungen lediglich aufgrund solcher Aspekte auferlegt werden dürften, die zum Zeitpunkt der Verbindlichkeitserklärung der Verpflichtungszusage nicht bekannt gewesen seien. ${ }^{892}$ Damit tatsächlich sichergestellt ist, dass die Voraussetzungen der regulatorischen Privilegierung fortbestehen, sieht Art.76 Abs. 3 UAbs. 1 EKEK umfassende Überwachungsmöglichkeiten der nationalen Regulierungsbehörde vor. Darüber hinaus kann diese gemäß Art. 76 Abs. 3 UAbs. 2 EKEK im Rahmen des Verfahrens gemäß Art. 26 Abs. 1 EKEK Entscheidungen in solchen Streitigkeiten treffen, die sich auf Aspekte der Kooperationsvereinbarung beziehen, die an die Bedingungen des Art.76 Abs. 1 UAbs. 2 EKEK anknüpfen.

Entscheidender Unterschied zu den „Regulierungsferien“, die gemäß \9a TKG a. F. ${ }^{893}$ für „neue Märkte“ im Sinne des $₫ 3$ Nr. 12b TKG gewährt

890 A. A. Nigge/Horstmann, MMR 2018, S.721, 724, die auch symmetrische Zugangsverpflichtungen für erfasst halten.

891 Zu dieser Konzeption äußern sich auch Neumann, N\&R 2018, S. 204, 209; Nigge/Horstmann, MMR 2018, S. 721, 725.

892 Nigge/Horstmann, MMR 2018, S. 721, 724.

893 \$9a TKG wurde eingeführt m. W. v. 24.2.2007 durch Gesetz v. 18.2.2007, BGBl. I S. 106. 
wurden ist ${ }^{894}$ dass mithilfe der Norm vollständig von einer Regulierung abgesehen wurde. Hiervon weicht die normative Konstruktion des Art. 76 EKEK in Verbindung mit Art. 79 EKEK deutlich ab. Es wird nicht vollständig auf regulatorische Verpflichtungen verzichtet. Vielmehr wird den marktmächtigen Unternehmen lediglich offeriert, sich freiwillig zu einem bestimmten Verhalten zu verpflichten. Die Selbstverpflichtung dient als Substitut der zwangsweisen Auferlegung regulatorischer Verpflichtungen mittels eines Verwaltungsaktes. Die Verpflichtungszusagen binden die Behörde nicht, sondern dienen als zusätzlicher Abwägungstopos im Rahmen der Ermessensausübung. Denkwürdig ist vor dem Hintergrund der Entscheidung des Europäischen Gerichtshofs, in der die deutsche Regelung als unionsrechtswidrig klassifiziert wurde, ${ }^{895}$ jedoch die Vorsteuerung der behördlichen Ermessensausübung, beispielsweise hinsichtlich des Grades der Wettbewerbsbeschränkungen, die erforderlich sind, um zusätzliche regulatorische Verpflichtungen auferlegen zu können. Diese schränken das behördliche Ermessen jedoch nicht über Gebühr ein und ermöglichen die Konstruktion eines verlässlichen Rechtsrahmen, der die Investitionsbedingungen verbessert. Aufgrund dieser Unterschiede lässt sich der neue unionale Privilegierungstatbestand nicht als eine Reinkarnation der „Regulierungsferien" bezeichnen.

bb) Kooperationsvereinbarungen im Sinne des Art. 79 Abs. 1 UAbs. 1 lit. a EKEK

Von der regulatorischen Privilegierung kooperativer Investitionen in neue Glasfaserinfrastrukturen, die unter den Tatbestand des Art.76 EKEK zu subsumieren sind, sind Kooperationsvereinbarungen im Sinne des Art. 79 Abs. 1 UAbs. 1 lit. a EKEK abzugrenzen.

Dessen missverständlicher deutscher Wortlaut spricht dabei von „Kooperationsvereinbarungen in Bezug auf die Bewertung geeigneter und angemessener Verpflichtungen gemäß Artikel 68“. Dies legt nahe, dass es sich um Kooperationsvereinbarungen zwischen Behörden und Unterneh-

894 Umfassend zu der Vorgabe BT-Drs. 16/2581, S. 23; Kühling/Elbracht, Telekommunikationsrecht, Rn. 124; Dablke/Theis, CR 2007, S. 227, 227 ff.; Kühling, K\&R 2006, S. 263, 269.

895 EuGH, Urt. v. 3.12.2009, Rs. C-424/07 (Europäische Kommission/Deutschland), ECLI:EU:C:2009:749, Rn. 108; weiterführend hierzu Reents, Ausbau und Finanzierung einer flächendeckenden Breitbandversorgung in Deutschland, S. 130 ff.; Schöfthaler, Rechtliche Rahmenbedingungen des Breitbandausbaus, S. 90 ff.. 
men handele, welche die Auferlegung regulatorischer Verpflichtungen betreffen. Gemeint sind jedoch Kooperationsvereinbarungen zwischen Unternehmen, die sich auf die Auferlegung regulatorischer Verpflichtungen gemäß Art. 68 EKEK auswirken. ${ }^{896}$ Hierunter fallen etwa kommerzielle Zugangs- oder Kooperationsvereinbarungen, die keine Ko-Investitionen im Sinne des Art. 76 EKEK darstellen. Diese können im Rahmen des Regulierungsermessens im Verfahren gemäß Art. 68 EKEK berücksichtigt werden. Eindeutiger sind in dieser Hinsicht der französische und der englische Wortlaut, die diese Einbindung deutlicher herausstellen. So heißt es in der französischen Fassung „relatifs à l'évaluation d'obligation“ und in der englischen Fassung „relevant to the assessment of (...) obligations“. Es handelt sich um einen Abwägungstopos, den die Behörde im Rahmen des Regulierungsermessens berücksichtigen kann. Sofern sich das Unternehmen zu einer Verhaltensweise verpflichtet, die zusätzliche regulatorische Verpflichtungen entbehrlich macht, kann die Behörde hiervon Abstand nehmen. Das Unternehmen kann mithin darauf hinwirken, die Last regulatorischer Zwangsmaßnahmen durch eine Selbstverpflichtung zu reduzieren.

\section{cc) Novellierung des Telekommunikationsgesetzes}

Die Art. 76 und 79 EKEK sollen in $\$ 18$ TKG-RefE gebündelt in nationales Recht umgesetzt und über $\$ 19$ TKG-RefE in das Marktregulierungsverfahren eingebunden werden. ${ }^{897}$ Dies verkennt jedoch die Systematik des EKEK. Art. 79 EKEK regelt allgemeine Aspekte der Verpflichtungszusage, während die speziellen Vorgaben in der Regel separat im jeweiligen Kontext (etwa in Art. 76 EKEK) geregelt werden. Überzeugender und aufgrund der umfassenden Neusystematisierung des betreffenden Abschnittes auch praktikabler wären separate Vorschriften gewesen.

896 Nigge/Horstmann, MMR 2018, S. 721, 725.

897 Referentenentwurf des Telekommunikationsmodernisierungsgesetzes (TKMoG), abrufbar unter: https:/www.bmwi.de/Redaktion/DE/Downloads/Gesetz/ telekommunikationsmodernisierungsgesetz-referentenentwurf-20201612.pdf? blob=publicationFile\&v=8 (zuletzt abgerufen am 12.1.2021). 
c) Auswirkungen des neuen Rechtsrahmens auf Kooperationsvorhaben

Betrachtet man die Auswirkungen des novellierten unionalen Rechtsrahmens auf die regulatorischen Rahmenbedingungen für Kooperationen zum Ausbau von Glasfaserinfrastrukturen, lässt sich feststellen, dass die grundsätzlichen Steuerungsvorgaben unverändert fortbestehen und durch spezielle Privilegierungstatbestände ergänzt werden.

Der Unternehmensbegriff, der sich in $\$ 3 \mathrm{Nr} .29$ TKG manifestiert und dazu beiträgt, dass auch verbundene Unternehmen als Teil eines marktmächtigen Unternehmens verstanden werden, besteht unverändert fort. Auch weiterhin enthält das Unionsrecht keine Regelung hierzu, steht der Legaldefinition des nationalen Rechts jedoch auch nicht entgegen. Grundsätzlich erfassen folglich alle Regulierungsverfügungen, die sich an das marktmächtige Unternehmen richten, zugleich auch deren als paritätische Gemeinschaftsunternehmen gegründete Tochterunternehmen.

Durch die Umsetzung des EKEK wird der Rechtsrahmen jedoch durch spezielle Privilegierungstatbestände für kooperative Investitionen (Art. 76 EKEK) und Kooperationsvereinbarungen (Art. 79 Abs. 1 UAbs. 1 lit. a EKEK) erweitert. Art. 76 Abs. 1 UAbs. 1 EKEK erfasst explizit auch die Gründung von Gemeinschaftsunternehmen unter Beteiligung eines marktmächtigen Unternehmens. Bei der Gründung eines Gemeinschaftsunternehmens handelt es sich letztlich um eine spezielle organisatorische Ausgestaltung des Erwerbes von Miteigentum durch die Kooperationspartner und einer langfristigen Risikoteilung. Diese spezielle Norm verdrängt in ihrem Anwendungsbereich die Möglichkeit von Kooperationsvereinbarungen im Sinne des Art. 79 Abs. 1 UAbs. 1 lit. a EKEK. Wäre dies nicht der Fall, könnten die speziellen Voraussetzungen für kooperative Investitionen einfach umgangen werden. Demnach ist der Anwendungsbereich des Art. 79 Abs. 1 UAbs. 1 lit. a EKEK auf alle Kooperationen beschränkt, welche die Auferlegung regulatorischer Verpflichtungen zum Gegenstand haben.

Es nicht davon auszugehen, dass von der Möglichkeit der Privilegierung umfassende Anreize für kooperative Investitionsvorhaben ausgehen. So wäre ein Kooperationsvorhaben nach dem Vorbild der Glasfaser Nordwest auch unter dem novellierten Rechtsrahmen nicht privilegierungsfähig. Dies lässt sich auf die strengen Anforderungen zurückführen, die der Rechtsrahmen an Verpflichtungszusagen stellt. Die Initiative zur kooperativen Investition in Glasfasernetze muss stets vom marktmächtigen Unternehmen in Form eines öffentlichen Angebots kooperativer Investitionen ausgehen. Dabei kann sich das Unternehmen keinen Wunschkooperati- 
onspartner auszusuchen. Vielmehr müssen sich alle Telekommunikationsunternehmen während der gesamten Laufzeit an dem Vorhaben beteiligen können. Hiermit wird einem Missbrauchspotential und der Gefahr von Monopolen und Oligopolen vorgebeugt, ohne die Attraktivität einer Kooperation vollständig zu beseitigen. Allerdings wird diese durch die strenge regulatorische Einhegung auch nicht über Gebühr gesteigert.

\section{d) Ergebnis}

Der regulatorische Rechtsrahmen für Kooperationen im Glasfaserausbau wird durch den EKEK in Detailfragen erheblich modifiziert. Die Grundstrukturen bleiben davon jedoch unberührt. So werden paritätische Gemeinschaftsunternehmen unter Beteiligung eines marktmächtigen Unternehmens auch in Zukunft reguliert. Allerdings werden ergänzend spezielle regulatorische Privilegierungstatbestände auf Basis von Verpflichtungszusagen geschaffen. Solange diese keine kooperativen Investitionsvorhaben zum Gegenstand haben, richtet sich der regulatorische Umgang mit ihnen nach Art. 79 Abs. 1 UAbs. 1 lit. a EKEK. Hierdurch wird ein neuer Abwägungstopos für die nationale Regulierungsbehörde etabliert, welche die Selbstverpflichtung der Unternehmen in ihrem Regulierungsermessen zu berücksichtigen hat. Einen speziellen Privilegierungstatbestand für kooperative Investitionen enthält Art. 76 EKEK. Allerdings sind die regulatorischen Erleichterungen an umfassende Anforderungen geknüpft, die einen Missbrauch weitgehend ausschließen sollen. Hieraus resultiert jedoch lediglich eine geringe zusätzliche Anreizwirkung.

\section{Optimierungsbedarf der regulatorischen Rahmenbedingungen}

Vor diesem Hintergrund stellt sich die Frage, inwieweit die regulatorischen Rahmenbedingungen weiter angepasst werden müssen und können, um die Attraktivität des kooperativen Glasfaserausbaus zu steigern. Entweder könnte das Regulierungsrecht novelliert (dazu a.) oder die Regulierungspraxis der Behörde optimiert werden (dazu b.). 
a) Weitergehender normativer Anpassungsbedarf de lege ferenda

Ein weitergehender Anpassungsbedarf de lege ferenda könnte vor allen Dingen aus den geringen regulatorischen Anreizen zum kooperativen Ausbau von Glasfaserinfrastrukturen folgen. In dieser Hinsicht ist jedoch eine differenzierte Betrachtung erforderlich. Die regulatorischen Rahmenbedingungen betreffen insbesondere solche Kooperationsvorhaben, an denen sich ein Unternehmen mit beträchtlicher Marktmacht beteiligt. Nur diese Unternehmen, die schon alleine die Funktionsfähigkeit des Wettbewerbs im Telekommunikationssektor gefährden, müssen die Verpflichtungszusagen mit ihren hohen inhaltlichen Anforderungen abgeben. Ein partielles Entlassen aus der Regulierung soll nicht den Dienstewettbewerb gefährden. Kollusives Handeln soll unabhängig davon, ob es eigenständig oder mit anderen Unternehmen gemeinsam erfolgt, verhindert werden. Die Funktion der Regulierungsverfügungen übernehmen Selbstverpflichtungen. Auf diese Weise soll verhindert werden, dass in Folge der regulatorischen Privilegierung Monopole oder Oligopole entstehen. Im Kern adressiert das regulatorische Rechtsregime ausschließlich solche Kooperationsvorhaben, die ein zusätzliches Missbrauchspotential entfachen können. Je umfassender die regulatorischen Anreize ausfallen, desto stärker steigt das Missbrauchspotential. Dementsprechend empfehlen sich keine zusätzlichen regulatorischen Entlastungen. ${ }^{898}$

Kooperationen ohne Beteiligung marktmächtiger Unternehmen sind nicht Gegenstand des Regulierungsrechts, solange dadurch kein marktmächtiges Unternehmen entsteht. Es bietet sich zwar an, die Rahmenbedingungen für Kooperationen zu optimieren. Hierbei spielt jedoch der regulatorische Rechtsrahmen eine untergeordnete Rolle. Vielmehr liegt es in der Verantwortung der Unternehmen, auch vor dem Hintergrund der immer weitergehenden Open-Access-Verpflichtungen, die Voraussetzungen für eine erfolgreiche Kooperation zu schaffen. Hierzu sollten Schnittstellen optimiert und technische Hemmnisse überwunden werden, damit eine Zusammenarbeit zwischen den Unternehmen erleichtert wird. Dies sind jedoch vor allem Aspekte der Standardisierung, auf die der Rechtsrahmen nur eingeschränkt einwirken kann und soll (hierzu bereits Kapitel 4, E.). Im Ergebnis besteht somit zunächst kein weitergehender normativer Anpassungsbedarf de lege ferenda.

898 Ähnlich positiv im Hinblick auf das strikte Regelungsregime Monopolkommission, 11. Sektorgutachten Telekommunikation (2019), Rn. 125. 
b) Änderungsbedarf im Hinblick auf die Spruchpraxis der Regulierungsbehörden

Auch die Spruchpraxis der Regulierungsbehörden ist ein integraler Bestandteil der regulatorischen Rahmenbedingungen. Wie bereits dargestellt, besteht durch eine Regionalisierung der Regulierung die Möglichkeit, einzelfallgerechte Lösungen für Kooperationsvorhaben zu schaffen (C. II. 2. c.). Dies könnte jedoch durch eine reine Anpassung der Beschlusspraxis ohne Modifikationen im Rechtsrahmen erfolgen. Im Vorfeld sollte jedoch, wie bereits dargestellt, eine ökonomische Folgenabwägung vorgenommen werden (C. II. 2. c. bb.).

\section{c) Zwischenergebnis}

Bei der Debatte um eine weitergehende Optimierung der regulatorischen Rahmenbedingungen für Kooperationsvorhaben ist zwischen dem Normenbestand und der behördlichen Spruchpraxis zu differenzieren. Das Rechtsregime ist konsistent und weist eine ausgewogene Balance zwischen Möglichkeiten der regulatorischen Privilegierung und Verhinderung eines kollusiven Zusammenwirkens zwischen Kooperationspartnern auf. Spielräume bestehen im Hinblick auf die Spruchpraxis der Bundesnetzagentur. Sofern regional differenzierte regulatorische Verpflichtungen auferlegt werden, kann dies die Attraktivität von Ausbau- und Kooperationsvorhaben in den betreffenden Gebieten steigern. Erkauft wird diese Attraktivitätssteigerung allerdings mit einer Aufgabe der bundesweit einheitlichen Preisstruktur (C. II. 2. c. bb.). Hierzu bedürfte es insbesondere einer umfassenden ökonomischen Folgenabwägung, die an dieser Stelle nicht erfolgen kann.

\section{Ergebnis}

Regulatorische Rahmenbedingungen sind für Kooperationsvorhaben insbesondere in denjenigen Fällen relevant, in denen einer der Partner ein Unternehmen mit beträchtlicher Marktmacht ist. In diesem Fall erstrecken sich die regulatorischen Verpflichtungen gegenüber dem marktmächtigen Unternehmen, sofern ein Zusammenschlusstatbestand im Sinne des $\$ 37$ GWB erfüllt ist, auch auf das Gemeinschaftsunternehmen. Dieses Ergebnis kann überzeugen und muss nicht durch eine teleologische Reduktion im 
Hinblick auf den Gründungszeitpunkt des Unternehmens korrigiert werden. Anderenfalls würden die Flucht in die gesellschaftsrechtliche Umstrukturierung und ein kollusives Zusammenwirken verschiedener Kooperationsunternehmen ermöglicht. Die Entscheidung der Bundesnetzagentur, die im Rahmen der Gründung der Glasfaser Nordwest dem Gründungszeitpunkt einen hohen Stellenwert eingeräumt hat und eine Erstreckung regulatorischer Verpflichtungen auf das Gemeinschaftsunternehmen nur dann annimmt, wenn dieses vor Abschluss des Marktanalyseverfahrens gegründet wurde, ist vor diesem Hintergrund abzulehnen. Die regulatorischen Rahmenbedingungen werden in Folge der Umsetzung des EKEK in nationales Recht optimiert werden. So wird die Möglichkeit zur Abgabe einer Verpflichtungserklärung geschaffen, wodurch die Unternehmen von regulatorischen Privilegierungen profitieren können. Allerdings geht von dem Instrument nur eine geringe Attraktivitätssteigerung für Kooperationsvorhaben aus. Dies lässt sich darauf zurückführen, dass der Rechtsrahmen Möglichkeiten zum Missbrauch der Privilegierung ausschließt. Die regulatorische Entlastung wird nur unter strengen Voraussetzungen gebilligt. Ein weitergehender Optimierungsbedarf des ausbalancierten Rechtsrahmens besteht nicht. Allerdings könnte die behördliche Beschlusspraxis angepasst werden. Durch eine Regionalisierung der Regulierung könnte die Attraktivität für Ausbauvorhaben insgesamt gesteigert werden. Allerdings müsste dieser Schritt ökonomisch wohlüberlegt sein. Mit einer regional ausdifferenzierten Regulierungspraxis ginge auch die national einheitliche Preisstruktur verloren. Darüber hinaus wurden die Auswirkungen einer Regionalisierung der Regulierung von Kooperationsvorhaben noch nicht ökonomisch untersucht.

\section{Ergebnis}

Im Ergebnis entfaltet das Regulierungsrecht ausschließlich in solchen Konstellationen Steuerungswirkung, in denen sich ein marktmächtiges Unternehmen an der Kooperation beteiligt oder durch die Kooperation ein marktmächtiges Unternehmen entsteht. Der Rechtsrahmen wird durch den EKEK und die dort vorgesehene Möglichkeit der regulatorischen Privilegierung von Kooperationsvorhaben optimiert. Dem dadurch ausbalancierten Rechtsregime gelingt es, Missbrauchspotentiale gering zu halten, ohne völlig auf eine Attraktivitätssteigerung zu verzichten. Die Steuerungsvorgaben können auch durch eine regional ausdifferenzierte Regulierung weiter optimiert werden. Der Rechtsrahmen würde ein solches Vorgehen 
ermöglichen. Allerdings müssten für diesen Schritt die national einheitliche Preisstruktur aufgegeben werden und eine ökonomische Folgenabwägung erfolgen.

\section{E. Kartellrechtliche Rahmenbedingungen zum Schutz des Wettbewerbs bei Kooperationsvorhaben}

Weitere rechtliche Rahmenbedingungen, die dem Schutz des Wettbewerbs bei Kooperationsvorhaben dienen, ergeben sich aus dem Kartellrecht. Der Anwendungsbereich der Regeln wurde bereits erörtert (B. II.). Nachfolgend ist zu untersuchen, welche zusätzlichen Steuerungsvorgaben sich für die Ausgestaltung von Kooperationsvorhaben aus dem Recht der Fusionskontrolle (dazu I.) und dem Kartellverbot (dazu II.) ergeben und auf welche Weise sie den regulatorischen Wettbewerbsschutz ergänzen.

\section{Steuerungsvorgaben aus dem Recht der Fusionskontrolle}

Eine Fusionskontrolle findet in solchen Konstellationen statt, in denen umsatzstarke Unternehmen mit einem hohen Institutionalisierungsgrad kooperieren (B. II. 1. bis 2.). Ist das deutsche Recht der Fusionskontrolle einschlägig, hat das Bundeskartellamt gemäß $₫ 36$ Abs. 1 S. 1 GWB die Kompetenz, einen Zusammenschluss zu untersagen, „durch den wirksamer Wettbewerb erheblich behindert würde.“ Die Frage, ob der Wettbewerb erheblich behindert wird, wird mithilfe des SIEC-Tests ${ }^{899}$ beantwortet, der im Rahmen der 8. GWB-Novelle in den Rechtsrahmen integriert wurde. ${ }^{900}$ In der Regel sind die Voraussetzungen für eine Untersagung bei einem Zusammenschluss gegeben, durch den gemäß $\$ 36$ Abs. 1 S. 1 GWB „eine marktbeherrschende Stellung begründet oder verstärkt“ wird. Der Gesetzgeber ging davon aus, dass der Wortlaut des Gesetzes eine „zweifelsfreie, flexible und damit optimale Erfassung aller potentiell wettbewerblich kritischen Fälle“901 ermöglicht. ${ }^{902}$ Bei der Analyse der Auswirkungen

899 Bei SIEC handelt es sich um die Abkürzung der englischen Formulierung „significant impediment to effective competition“, die sich als „erhebliche Behinderung wirksamen Wettbewerbs“ übersetzen lässt.

900 BT-Drs. 17/9852, S. 28.

901 BT-Drs. 17/9852, S. 28.

902 Weiterführend hierzu statt vieler Thomas, in: Immenga/Mestmäcker (Hrsg.), Wettbewerbsrecht, Band 2, $\$ 36$ GWB, Rn. $382 \mathrm{ff}$. 
des Zusammenschlusses auf den Wettbewerb sind auch die Verpflichtungszusagen gemäß $\$ 32 \mathrm{~b}$ GWB zu berücksichtigen (E. I. 2.). Diese können erheblich dazu beitragen, dass ein eventuell zu untersagender Zusammenschluss die Wettbewerbsverhältnisse nicht etwa verschlechtert, sondern unter Umständen sogar verbessert. Ausnahmsweise kann von einer Untersagung abgesehen werden, wenn der Zusammenschluss auch eine Verbesserung des Wettbewerbs mit sich bringt und diese die Nachteile überwiegt ( $\$ 36$ Abs. 1 S. 2 Nr. $1 \mathrm{GWB}$ ) oder ein Bagatellmarkt vorliegt, bei dem die Umsatzschwellen des $\$ 36$ Abs. 1 Nr. 2 GWB nicht überschritten werden. Im Rahmen der folgenden Überlegungen soll davon ausgegangen werden, dass die Ausnahmetatbestände nicht erfüllt sind. ${ }^{903}$

Betrachtet man die spezifischen Anforderungen des Rechtsrahmens an Zusammenschlüsse zum kooperativen Ausbau von Glasfaserinfrastrukturen, muss sich die Behörde mit der Frage beschäftigen, wie sich der $\mathrm{Zu}$ sammenschluss auf den Wettbewerb in den zuvor abgegrenzten Märkten auswirken wird. ${ }^{904}$ Hierbei handelt es sich um eine Prognoseentscheidung, in deren Rahmen die Entwicklung des Marktes mit und ohne den jeweiligen Zusammenschluss untersucht werden muss. ${ }^{905}$ Die Wettbewerbsanalyse soll hier nicht umfassend dargelegt werden. Vielmehr soll eine Beschränkung der Untersuchung darauf erfolgen, wie sich die regulatorischen Verpflichtungen auf die fusionskontrollrechtliche Wettbewerbsanalyse auswirken (dazu 1.) und welche Rolle die Verpflichtungszusage gemäß $₫ 32$ b GWB im Fusionskontrollrecht spielt (dazu 2.).

\section{Zusammenspiel von regulatorischen Verpflichtungen und} fusionskontrollrechtlicher Wettbewerbsanalyse

Aus fusionskontrollrechtlicher Sicht sind für die Bewertung eines Kooperationsvorhabens zur Beschleunigung des Ausbaus von Glasfaserinfrastrukturen insbesondere seine Auswirkungen auf die Vorleistungsmärkte zum zentralen und lokalen Zugang zu Breitbandinfrastrukturen, die korrespondierenden Endkundenmärkte sowie den Ausschreibungsmarkt für den ge-

903 Ein zusätzlicher Ausnahmetatbestand für Zeitungs- und Zeitschriftenmärkte ergibt sich aus $₫ 36$ Abs. 1 S. 2 Nr. 3 GWB. Da die Ausnahme evident keinen Bezug zum Ausbau von Glasfaserinfrastrukturen aufweist, soll auf ihre Erörterung verzichtet werden.

904 BKartA, Beschl. v. 30.12.2019, Az. B 7-21/18, Rn. 280.

905 BKartA, Beschl. v. 30.12.2019, Az. B 7-21/18, Rn. 280. 
förderten Breitbandausbau von Relevanz (C. III.). Regulatorische Verpflichtungen bestehen lediglich für die Vorleistungsmärkte. Diese beseitigen jedoch nicht die marktbeherrschende Stellung des jeweiligen Unternehmens. Vielmehr tragen sie lediglich dazu bei, dass der hiermit einhergehende, umfassende Verhaltensspielraum, der missbraucht werden kann, aus regulatorischer Sicht eingeschränkt wird. ${ }^{906}$ Dies führt jedoch nicht zwangsläufig dazu, dass der Verhaltensspielraum auch aus fusionskontrollrechtlicher Perspektive hinreichend eingeschränkt wird. ${ }^{907}$ Die regulatorischen Verpflichtungen sind sehr spezifisch auf bestimmte Verhaltensweisen ausgerichtet, die durch ein natürliches Monopol im Telekommunikationssektor geprägt sind. Hierbei handelt es sich um die Zugangs- und die korrespondierende Entgeltverpflichtung, die durch weitere Verpflichtungen flankiert werden können. Die regulatorischen Verpflichtungen betreffen mithin bereits existierende Telekommunikationsnetze. Kein Einfluss kann jedoch auf erst in Zukunft zu errichtende Telekommunikationsnetze genommen werden. ${ }^{908}$ Den Glasfaserausbau betrifft aber insbesondere das koordinierende Verhalten der Kooperationspartner im Vorfeld eines Infrastrukturausbauvorhabens. Durch das gemeinsame Vorgehen, die schnelle Verlagerung von Ausbauaktivitäten oder die geschickte Bewerbung auf Ausschreibungen für Fördermittel können Konkurrenten nachhaltig verdrängt werden. Auf diese Gefahren hat das Telekommunikationsrecht mit seinem numerus clausus möglicher Regulierungsmaßnahmen keine hinreichenden Antworten. Vielmehr schließt das Fusionskontrollrecht hier eine signifikante Lücke. Dieser Befund erhärtet sich vor dem Hintergrund des fusionskontrollrechtlichen Verfahrens zur Gründung der Glasfaser Nordwest. Wie bereits dargelegt, kam die Bundesnetzagentur zur Schlussfolgerung, dass das Kooperationsunternehmen nicht von den Verpflichtungen gegenüber der Deutschen Telekom erfasst sei (D. II. 2.). Das Bundeskartellamt schließt diese Lücke, die durch die Regulierungspraxis der Bundesnetzagentur entstanden ist, mithilfe einer Verpflichtungszusage, auf die noch einzugehen ist (E. I. 2.). Hierdurch entsteht die skurrile Situation, dass das Bundeskartellamt die Regulierung eines Marktes übernimmt, für den die Bundesnetzagentur davon ausgeht, dass das Kartellrecht zur Bewältigung des Marktversagens nicht ausreiche (C. II. 1. a.). Für

906 BKartA, Beschl. v. 30.12.2019, Az. B 7-21/18, Rn. 343; weiterführend zum Zusammenspiel zwischen sektorspezifischem und allgemeinem Wettbewerbsrecht, Neumann, N\&R 2020, S. 148, $151 \mathrm{ff}$.

907 BKartA, Beschl. v. 30.12.2019, Az. B 7-21/18, Rn. 345.

908 BKartA, Beschl. v. 30.12.2019, Az. B 7-21/18, Rn. 349. 
den regulatorischen Umgang mit dem kooperativen Breitbandausbau stellt sich die Situation gegenteilig dar. Wäre das Kartellrecht nicht anwendbar, würde das Gemeinschaftsunternehmen - zumindest temporär - nicht reguliert.

\section{Verpflichtungszusage im Fusionskontrollrecht}

Nachdem bereits umfassend auf allgemeine Aspekte der kartellrechtlichen Verpflichtungszusagen eingegangen wurde, sollen nachfolgend die konkrete Einbettung des Instrumentes in das kartellrechtliche Verwaltungsverfahren illustriert und der Inhalt der Verpflichtungszusage kritisch reflektiert werden (D. II. 3. a.). Verpflichtungszusagen können genutzt werden, um die wettbewerblichen Rahmenbedingungen in den durch die Fusion betroffenen Märkten zu verändern. Die Funktionsweise soll nachfolgend durch die Rolle der Verpflichtungszusage im Rahmen der Gründung der Glasfaser Nordwest exemplifiziert werden. In einem parallel eingeleitetem Kartellverwaltungsverfahren erklärte das Bundeskartellamt Verpflichtungszusagen gemäß $\$ 32$ b Abs. 1 GWB für verbindlich. ${ }^{909}$

In dem Kartellverwaltungsverfahren wurden eine Ausbau-, eine $\mathrm{Zu}$ gangs- und eine Förderzusage abgegeben. ${ }^{910}$ Diese Einzelzusagen sind nachfolgend zunächst jeweils isoliert und anschließend in ihrem Zusammenspiel mit den übrigen Verpflichtungszusagen zu bewerten. Vor Abgabe der Verpflichtungszusagen hatte das Bundeskartellamt Bedenken gegen die Fusion angemeldet und hätte, sofern die entsprechenden Verpflichtungszusagen nicht abgegeben worden wären, das Zusammenschlussvorhaben wohl gemäß $₫ 36$ Abs. 1 S. 1 GWB untersagt. ${ }^{911}$ Die Bedenken erstreckten sich auf alle Märkte, die vom Bundeskartellamt abgegrenzt wurden und durch das Kooperationsvorhaben tangiert werden. Das Bundeskartellamt stellte in seinem Beschluss klar, dass „die im Kartellverwaltungsverfahren für bindend erklärten Verpflichtungszusagen [...] als rechtliche Rahmenbedingungen des Wettbewerbs [...] zu berücksichtigen" ${ }^{\text {"912 }}$ sind. Dies bedeutet, dass die Verpflichtungszusagen genutzt werden, um die Wettbewerbskräfte im Markt so zu justieren, dass die Fusion in den betref-

909 BKartA, Beschl. v. 4.12.2019, Az. B 7-21/18.

910 BKartA, Beschl. v. 4.12.2019, Az. B 7-21/18, Anhang Verpflichtungszusage.

911 BKartA, Beschl. v. 30.12.2019, Az. B 7-21/18, Rn. 5.

912 BKartA, Beschl. v. 30.12.2019, Az. B 7-21/18, Rn. 5. 
fenden Märkten keinen negativen, sondern vielmehr einen positiven Effekt auf den Wettbewerb hat.

Konkret erfasst die Ausbauzusage etwa Mechanismen, mit denen verhindert werden soll, dass durch geschickte Verlagerung der Ausbauaktivitäten die Investitionen alternativer Anbieter vereitelt werden können. So verpflichtete sich die Glasfaser Nordwest dazu, eine initiale Ausbauliste und eine "Shortlist“ anzufertigen, auf der Ausbaugebiete genannt werden. ${ }^{913}$ Nur wenn ein Ausbauvorhaben auf der initialen Ausbauliste oder für neun Monate auf der Shortlist stand, darf mit dem Ausbau begonnen werden. Eine Ausnahme gilt lediglich für Neubaugebiete und neu ausgewiesene Gebiete. Ein weiteres Element der Ausbauzusage ist eine Pflicht zur Erschließung ländlicher und dünn besiedelter Gebiete. ${ }^{914}$

Der erste Teil der Verpflichtungszusage, der das Ausbauverhalten des Kooperationsunternehmens transparenter macht, führt nicht zu mehr Wettbewerb zwischen den Infrastrukturunternehmen im Kooperationsgebiet. Vielmehr werden lediglich das kollusive Zusammenwirken und der Missbrauch des externen Marktmachtzuwachses der Mutterunternehmen und des Tochterunternehmens verhindert. Hiermit ist ein positiver volkswirtschaftlicher Effekt verbunden, der die Bedingungen des Wettbewerbs der Infrastrukturanbieter im Kooperationsgebiet verbessert, allerdings die Marktmacht der Kooperationsunternehmen nicht substantiell verändert und insbesondere nicht deren externen Marktmachtzuwachs tangiert.

Die Ausbauverpflichtung als zweites Element der Verpflichtungszusage wirkt in diesem Kontext systemfremd. Natürlich ist eine flächendeckende Verfügbarkeit hochleistungsfähiger Telekommunikationsinfrastrukturen volkswirtschaftlich positiv zu bewerten. ${ }^{915}$ Allerdings verbessert eine Ausbauverpflichtung, die auf „weiße Flecken“ konzentriert wird, in denen bis dato kein Wettbewerb der Infrastrukturanbieter vorliegt, den Wettbewerb im Kooperationsgebiet nicht. Im Zusammenspiel mit einer Zugangsverpflichtung können sich jedoch auf den nachgelagerten Dienstemärkten die Wettbewerbsbedingungen verbessern. Verpflichtungszusagen sollten jedoch nicht instrumentalisiert werden, um solche Interessen durchzusetzen, die nicht zum Zielekanon des Wettbewerbsrechts gehören.

913 BKartA, Beschl. v. 4.12.2019, Az. B 7-21/18, Anhang Verpflichtungszusage, S. $10 \mathrm{ff}$.

914 BKartA, Beschl. v. 4.12.2019, Az. B 7-21/18, Anhang Verpflichtungszusage, S. $13 \mathrm{f}$.

915 Hierzu statt vieler Heuermann/Meinen/Ekango u.a., Konzepte zur Förderung von Breitbandinvestitionen im internationalen Vergleich, S. $6 \mathrm{ff}$; umfassend Wernick/Gries/Bender u.a., Regionale TK-Akteure im globalen Wettbewerb, S. 17 ff. 
Mithilfe der Zugangszusage wurden die Lücken geschlossen, die durch die Entscheidung der Bundesnetzagentur (D. II. 2.) entstanden waren. Neben einer Selbstverpflichtung zur Zugangsgewährung für Layer-2- und Layer-3-Bitstromprodukte ${ }^{916}$ enthielt die Zusage auch ein Vermarktungsziel für diese Produkte. ${ }^{917}$ Hierdurch wird sichergestellt, dass die Vorleistungsprodukte tatsächlich zu attraktiven Konditionen für Drittanbieter angeboten werden. Die Zugangsverpflichtung führt für sich genommen ebenfalls nicht zu einem Wettbewerb der Infrastrukturanbieter, sondern ermöglicht lediglich einen Wettbewerb auf der nachgelagerten Diensteebene. Einen weiteren positiven Effekt bringt insoweit die verpflichtende Vermarktungsquote mit sich, die dazu beiträgt, dass die Zugangsverpflichtung nicht durch die Verknüpfung des Zuganges mit prohibitiven Entgelten konterkariert wird.

Mit der Förderzusage verpflichtete sich die Glasfaser Nordwest, nicht an Ausschreibungen für den geförderten Breitbandausbau teilzunehmen. ${ }^{918}$ Diese Verpflichtungszusage ist insgesamt kritisch zu bewerten. Sofern man von einem Ausschreibungsmarkt um den geförderten Glasfaserausbau und einer wettbewerblichen Strukturierung dieses Marktes ausgeht, fördert die Selbstverpflichtung der Glasfaser Nordwest diesen Wettbewerb nicht, sondern beeinträchtigt ihn. Im Rahmen der Fördergebiete ist, wie bereits dargelegt, nicht von einem umfassenden Wettbewerb der Infrastrukturanbieter auszugehen (C. III.). Scheidet nun die Glasfaser Nordwest von Beginn an als Wettbewerber aus, führt dies zum Verlust eines entscheidenden Marktteilnehmers. Fraglich ist darüber hinaus auch, ob sich die Deutsche Telekom und EWE an den Ausschreibungen beteiligen würden. Wäre dies nicht der Fall, schieden mehrere Unternehmen aus dem Wettbewerb im Ausschreibungsmarkt für den geförderten Glasfaserausbau im Kooperationsgebiet aus. Vorzugswürdig wäre die Verpflichtungszusage dahingehend zu modifizieren, dass die Glasfaser Nordwest zwar auch am geförderten Breitbandausbau partizipieren kann, aber durch die Konstruktion der $\mathrm{Zu}-$ sage daran gehindert wird, die eigene Marktmacht wettbewerbsschädigend auszunutzen. Hauptanknüpfungspunkt ist in dieser Hinsicht zwar die Konzeption des Förderprogrammes. Verbleibende Restrisiken, könnten al-

916 BKartA, Beschl. v. 4.12.2019, Az. B 7-21/18, Anhang Verpflichtungszusage, S. $17 \mathrm{ff}$.

917 BKartA, Beschl. v. 4.12.2019, Az. B 7-21/18, Anhang Verpflichtungszusage, S. $19 \mathrm{f}$.

918 BKartA, Beschl. v. 4.12.2019, Az. B 7-21/18, Anhang Verpflichtungszusage, S. $24 \mathrm{f}$. 
lerdings mithilfe spezifischer Verpflichtungszusagen beseitigt werden. Als Vorbild könnte insoweit die Ausbauzusage dienen, die ein kollusives $\mathrm{Zu}$ sammenwirken effektiv ausschließt.

Die Verpflichtungszusage illustriert, wie vielseitig sich das Instrument im Wettbewerbsrecht einsetzen lässt. Das behördliche Handlungsinstrumentarium beschränkt sich auf die Möglichkeit der Untersagung des Zusammenschlusses gemäß $\$ 36$ Abs. 1 S. 1 GWB. Durch die Verpflichtungszusagen wird es erheblich flexibilisiert. Die Möglichkeit der Untersagung des Zusammenschlusses fungiert dann gewissermaßen als Drohkulisse. Hätten die Deutsche Telekom und EWE sich nicht zu diesen weitreichenden Handlungen selbst verpflichtet, hätte das Bundeskartellamt die Fusion untersagt. Die Wirksamkeit der Handlungspflichten für den Schutz des Wettbewerbs ist differenziert zu betrachten. Wesentliche Teile der Ausbauund Zugangszusage beseitigen nicht den Zuwachs an Marktmacht der Kooperationsunternehmen im Sinne einer Förderung des Wettbewerbs der Infrastrukturanbieter. Vielmehr wird sichergestellt, dass eine marktbeherrschende Stellung nicht ausgenutzt wird und ein suffizienter Wettbewerb auf der nachgelagerten Ebene besteht. Darüber hinaus wird mit der Selbstverpflichtung zum Infrastrukturausbau ein zusätzliches Ziel verfolgt. Dieses kann zwar im Zusammenspiel mit der korrespondierenden Zugangsverpflichtung positive volkswirtschaftliche Effekte generieren. Allerdings gehört es nicht zum Kernbereich des Aufgabenspektrums der Kartellbehörden. Kritisch ist zu hinterfragen, ob sich die Abgabe von Verpflichtungszusagen im Kartellverwaltungsverfahren dazu eignet, Druck auf Unternehmen aufzubauen und Ziele zu erreichen, die nur entfernt dem eigentlichen Ziel des Verfahrens - nämlich dem Schutz des Wettbewerbs zuzuordnen sind. Fragwürdig ist es zudem, wenn mithilfe der Verpflichtungszusagen Wettbewerber aus dem Markt gedrängt werden. Dies stärkt im Endeffekt den Wettbewerb nicht, sondern schwächt ihn signifikant. Hier wäre ein differenzierteres Vorgehen wünschenswert. Auch wenn die Grundtendenz des Bundeskartellamtes im Umgang mit der Glasfaser Nordwest positiv zu bewerten ist, bleibt im Kontext der abgegebenen Verpflichtungszusagen ein erhebliches Optimierungspotential.

\section{Zwischenergebnis und Schlussfolgerungen}

Während das Regulierungsrecht mit einem numerus clausus möglicher Maßnahmen auf spezifische Wettbewerbsprobleme zugeschnitten ist, die in Folge natürlicher Monopole entstehen, ist das Recht der Fusionskon- 
trolle flexibler. Durch dieses werden auch solche Märkte geschützt, die nicht vom Regulierungsrecht erfasst werden. Das Recht der Fusionskontrolle schützt auch angrenzende Märkte und gewährleistet dort einen Wettbewerb, wo kein funktionsfähiger Wettbewerb der Infrastrukturanbieter möglich ist. Ein flexibles Mittel hierfür sind die kartellrechtlichen Verpflichtungszusagen, durch die wettbewerbliche Rahmenbedingungen verändert werden können. Sie eignen sich nicht nur dazu, Wettbewerbsdefizite zu reduzieren, sondern können sogar so ausgestaltet werden, dass mit ihnen eine Förderung des Wettbewerbs - insbesondere in den nachgelagerten Märkten - einhergeht. In der konkreten Situation der Gründung der Glasfaser Nordwest konnten mithilfe der Verpflichtungszusagen Defizite ausgeglichen werden, die durch die behördliche Regulierungspraxis geschaffen wurden. Allerdings ist kritisch zu hinterfragen, ob es sinnvoll ist, mithilfe dieses Instrumentes Ziele zu verfolgen, die nicht zum Kernbereich des kartellrechtlichen Zielspektrums gehören. Zudem sollten keine Zusagen abgegeben werden, die zum Ausstieg von Infrastrukturunternehmen aus dem Markt führen. Vielmehr sollte - trotz der Partizipation marktmächtiger Unternehmen - ein suffizienter und funktionsfähiger Wettbewerb ermöglicht werden.

Im Ergebnis lässt sich festhalten, dass das Recht der Fusionskontrolle das Regulierungsrecht sinnvoll ergänzt und bestimmte Wettbewerbsdefizite differenziert und effizient adressiert. Die Beschlusspraxis des Bundeskartellamtes und die konkrete Ausgestaltung der Verpflichtungszusagen weisen jedoch Optimierungsbedarf auf.

\section{Steuerungsvorgaben des Kartellverbots gemäß $₫ 1 \mathrm{GWB}$}

Das Kartellverbot findet auf alle Kooperationsvorhaben - unabhängig von ihrem Institutionalisierungsgrad - Anwendung, denen eine Vereinbarung zu Grunde liegt. Es ist insbesondere für solche Konstellationen von Relevanz, in denen der Anwendungsbereich der Fusionskontrolle, beispielsweise aufgrund der Unterschreitung der Schwellenwerte, nicht eröffnet ist (B. II. 2. a.). Damit das Kartellverbot Steuerungswirkungen entfaltet, müssen im Übrigen seine Tatbestandsvoraussetzungen erfüllt sein (dazu 1.). Sofern dies der Fall ist, besteht die Möglichkeit einer Freistellung durch die Kartellbehörden (dazu 2.). Letztlich kann die Rechtsfolge des Verbots der Vereinbarung auch mithilfe von Verpflichtungserklärungen gemäß $\$ 32 \mathrm{~b}$ GWB verhindert werden (dazu 3.). 


\section{Tatbestand des Kartellverbotes des $₫ 1$ GWB}

Damit das Kartellverbot des $\mathbb{1}$ GWB überhaupt eine Wirkung entfaltet, muss eine Vereinbarung im Sinne des Tatbestandes vorliegen. ${ }^{919}$ Hiervon ist jedenfalls bei Abschluss eines Vertrages auszugehen, ${ }^{920}$ wobei sich aus dem Gesetz keine formalen Anforderungen hieran ergeben. ${ }^{921}$ Es ist sogar unbeachtlich, wenn die Parteien die Vereinbarung ohne Bindungswirkung abschließen, was für einen zivilrechtlichen Vertrag jedoch zwingend erforderlich wäre. ${ }^{922}$ Beim kooperativen Glasfaserausbau ist aufgrund der hohen Investitionsvolumina davon auszugehen, dass die Parteien einen ordnungsgemäßen Vertrag abschließen, in dem sie die Rahmenbedingungen des Kooperationsvorhabens umfassend vereinbaren, oder zumindest eine wie auch immer geartete Vereinbarung treffen. Für die Gründung eines Gemeinschaftsunternehmens muss sogar ein Joint-Venture-Vertrag geschlossen werden, der in jedem Fall eine Vereinbarung im Sinne des $\mathbb{1} 1$ GWB darstellt. ${ }^{923}$

In einem nächsten Schritt ist zu prüfen, ob mit der Vereinbarung eine spürbare Wettbewerbsbeschränkung einhergeht. ${ }^{924}$ Die hierfür erforderliche Marktabgrenzung, wird durch das Bundeskartellamt einheitlich wie bei der Fusionskontrolle durchgeführt (C. II.). Die identifizierbaren Wettbewerbsbeschränkungen entsprechen ebenfalls der Untersuchung im Rahmen der Fusionskontrolle. Die Tatbestandsvoraussetzungen decken sich insoweit (E. I.).

919 BKartA, Hinweise zur wettbewerbsrechtlichen Bewertung von Kooperationen beim Glasfaserausbau in Deutschland, S. 14.

920 Zimmer, in: Immenga/Mestmäcker (Hrsg.), Wettbewerbsrecht, Band 2, $\mathbb{1}$ GWB, Rn. 31.

921 Weiterführend hierzu Kühling, in: Leupold/Glossner (Hrsg.), Münchener Anwalts Handbuch IT-Recht, Teil 7, Rn. 253.

922 Zimmer, in: Immenga/Mestmäcker (Hrsg.), Wettbewerbsrecht, Band 2, $\mathbb{1}$ GWB, Rn. 31.

923 BKartA, Beschl. v. 4.12.2019, Az. B 7-21/18, Rn. 28.

924 Weiterführend hierzu Kühling, in: Leupold/Glossner (Hrsg.), Münchener Anwalts Handbuch IT-Recht, Teil 7, Rn. 255 ff.; vgl. auch BKartA, Hinweise zur wettbewerbsrechtlichen Bewertung von Kooperationen beim Glasfaserausbau in Deutschland, S. $22 \mathrm{f}$. 


\section{Freistellung vom Kartellverbot gemäß $\$ 2$ GWB}

Gemäß $\ 2$ GWB können bestimmte wettbewerbsbeschränkende Vereinbarungen vom Kartellverbot freigestellt werden.925 Das Gesetz knüpft die Freistellung an „zwei positive und zwei negative Voraussetzungen."926 So ist es gemäß $\ 2$ GWB auf der einen Seite erforderlich, dass die Vereinbarung ,zur Verbesserung der Warenerzeugung oder -verteilung zur Förderung des technischen oder wirtschaftlichen Fortschritts" beiträgt (dazu a.) und das Vorhaben „unter angemessener Beteiligung der Verbraucher an dem entstehenden Gewinn" erfolgt (dazu b.). Auf der anderen Seite dürfen „den beteiligten Unternehmen [keine] Beschränkungen auferlegt werden, die für die Verwirklichung dieser Ziele nicht unerlässlich sind“ ( $\$ 2$ Abs. 1 Nr. 1 GWB, dazu c.). Zudem darf mit der Vereinbarung keine Ausschaltung des Wettbewerbs ermöglicht werden ( $\$ 2$ Abs. 1 Nr. 2 GWB, dazu ebenfalls c.). Sind die Anforderungen kumulativ erfüllt, ${ }^{927}$ werden die negativen Auswirkungen auf den Wettbewerb durch positive Effekte für die Verbraucher ausgeglichen. ${ }^{928}$ Dabei ist jeweils jeder Markt für sich genommen zu überprüfen. ${ }^{929}$

\section{a) Ausbaukooperation und Effizienzgewinn}

Die erste positive Voraussetzung, die für eine kartellrechtliche Freistellung erforderlich ist, ist ein Effizienzgewinn. Auf Basis dieses Kriteriums sollen „die durch die Vereinbarung geschaffenen objektiven Vorteile und die wirtschaftliche Bedeutung der Effizienzgewinne ermittelt werden. ${ }^{\text {"930 Ins- }}$ besondere muss zwischen den objektiv nachprüfbaren Effizienzgewinnen und der Vereinbarung ein Kausalzusammenhang bestehen. ${ }^{931}$

925 Weiterführend hierzu Kommission, Leitlinien zur Anwendung von Artikel 81 Absatz 3 EG-Vertrag (Bekanntmachung 2004/C 101/08), Abl.C 101/97 v. 27.4.2004. Die Freistellung ist nur dann erforderlich, wenn die Vereinbarung zu einer Wettbewerbsbeschränkung führt, Leitlinien Freistellung Kartellverbot, Rn. 40. Die Leitlinien können an dieser Stelle als Quelle genutzt werden, da das Kartellverbot des unionalen und nationalen Wettbewerbsrechts insoweit übereinstimmen. Dies gilt auch für die folgenden Nachweise.

926 Bekanntmachung 2004/C 101/08, Rn. 34.

927 Bekanntmachung 2004/C 101/08, Rn. 42.

928 Bekanntmachung 2004/C 101/08, Rn. 34.

929 Bekanntmachung 2004/C 101/08, Rn. 43.

930 Bekanntmachung 2004/C 101/08, Rn. 50.

931 Bekanntmachung 2004/C 101/08, Rn. 53. 
Die Komplexität des Nachweises der vorgenannten Kriterien, lässt sich anhand des Beispiels der Glasfaser Nordwest exemplifizieren. Die Muttergesellschaften des Unternehmens führten verschiedene Argumente für Effizienzgewinne an. So komme es zu Einsparungen von Kosten, ${ }^{932}$ einer Teilung des Investitionsrisikos ${ }^{933}$ und einer Senkung des Auslastungsrisikos. ${ }^{934}$ Alle potentiellen Effizienzgewinne konnten jedoch nicht substantiiert nachgewiesen werden. ${ }^{935}$ Das Bundeskartellamt führte aus, dass gerade die Risiken zu einem großen Teil auf die Unsicherheiten hinsichtlich der Entwicklung der Nachfrage zurückzuführen seien. Dies könne nicht positiv durch die Kooperation beeinflusst werden. ${ }^{936}$ Auch die bloße Existenz einer Arbeitsgemeinschaft reiche für einen Effizienzgewinn noch nicht aus. ${ }^{937}$

Alternativ könnten sich die Effizienzgewinne auch aus der Vermeidung eines Überbaus ergeben. ${ }^{938}$ In der Literatur wird angeführt, dass sich Konstellationen ergeben könnten, in denen der Ausbau erst durch das Eingehen eines Kooperationsvorhabens wirtschaftlich tragfähig werde. ${ }^{939}$ Ein diesbezüglicher Kausalzusammenhang müsste jedoch hinreichend substantiiert nachgewiesen werden. Dies ist eher bei punktuellen Ausbauvorhaben als bei Kooperationsvorhaben in einem umfassenden Kooperationsgebiet denkbar. Je größer das Kooperationsgebiet ist, desto eher ist davon auszugehen, dass auch ein paralleler Ausbau durch konkurrierende Anbieter wirtschaftlich tragfähig wäre. Ein substantiierter Nachweis des Kausalzusammenhanges wird jedenfalls bei zunehmender Größe des Kooperationsgebietes schwieriger.

932 BKartA, Beschl. v. 4.12.2019, Az. B 7-21/18, Rn. 98 ff.

933 BKartA, Beschl. v. 4.12.2019, Az. B 7-21/18, Rn. $101 \mathrm{ff}$.

934 BKartA, Beschl. v. 4.12.2019, Az. B 7-21/18, Rn. $104 \mathrm{ff}$.

$935 \mathrm{Zu}$ den diesbezüglichen Anforderungen BKartA, Hinweise zur wettbewerbsrechtlichen Bewertung von Kooperationen beim Glasfaserausbau in Deutschland, S. 25.

936 BKartA, Beschl. v. 4.12.2019, Az. B 7-21/18, Rn. 102.

937 BKartA, Hinweise zur wettbewerbsrechtlichen Bewertung von Kooperationen beim Glasfaserausbau in Deutschland, S. 19.

938 Kühling, WuW 2019, S. 555, 559; Kühling/Heimeshoff/Schall, K\&R Beihefter 1/2010, S. 1, $23 \mathrm{f}$.

939 Kübling, WuW 2019, S. 555, 559. 
b) Verbraucherwohlfahrt

Nur wenn kausal auf die Vereinbarung rückführbare Effizienzgewinne substantiiert durch die Beteiligten dargelegt werden, kommt es überhaupt auf eine Förderung der Verbraucherwohlfahrt durch das Kooperationsvorhaben an. Insoweit enthält die Entscheidung zur Gründung der Glasfaser Nordwest keine Ausführungen. Würde man einen Effizienzgewinn jedoch nachweisen, wäre eine vertiefte Auseinandersetzung mit der korrespondierenden Verbraucherwohlfahrt erforderlich. ${ }^{940}$ Der Begriff des „Verbrauchers“ ist in diesem Kontext so weit zu verstehen, dass er im Sinne der „Gesamtwohlfahrt“ zu interpretieren ist. ${ }^{941}$ Von einer „angemessenen Beteiligung" ist auszugehen, wenn jedenfalls die Nachteile durch die Verminderung des Wettbewerbs ausgeglichen werden. ${ }^{942}$ Sofern Effizienzgewinne durch die Vermeidung von Überbau dargelegt werden, müssen diese an die Verbraucher weitergegeben werden, etwa durch die Senkung der Preise für Vorleistungs- oder Endkundenprodukte. ${ }^{943}$ Sofern ein komplementärer Ausbau, also ein Ausbau der Unternehmen jeweils in unterschiedlichen Regionen mit wechselseitigen Zugangsgewährungen, erfolgen würde, könnte auch die Auswahl der Kunden erweitert werden. ${ }^{944}$ Es ist in dieser Hinsicht jedoch darauf hinzuweisen, dass mit dieser Ausweitung mannigfaltige weitere Gefahren für den Wettbewerb einhergehen. ${ }^{945}$

\section{c) Negative Freistellungsvoraussetzungen}

Die Vereinbarung in ihrer Gesamtheit und alle ihre Einzelbestandteile müssen für die Effizienzgewinne unbedingt erforderlich sein. ${ }^{946}$ Insoweit besteht ein enger Zusammenhang mit ihnen (E. II. 2. a.). Ist es schon schwierig, diese substantiiert darzulegen, so ist es umso schwieriger, die Unerlässlichkeit der Wettbewerbsbeschränkung für deren Generierung darzutun.

940 Umfassend hierzu BKartA, Hinweise zur wettbewerbsrechtlichen Bewertung von Kooperationen beim Glasfaserausbau in Deutschland, S. 26; Kühling/ Heimeshoff/Schall, K\&R Beihefter 1/2010, S. 1, 24.

941 So Kübling, WuW 2019, S. 555, 559.

942 Bekanntmachung 2004/C 101/08, Rn. 85.

943 Hierzu weiterführend Kühling, WuW 2019, S. 555, 559.

944 Kübling, WuW 2019, S. 555, 559.

945 Kübling, WuW 2019, S. 555, 559.

946 Bekanntmachung 2004/C 101/08, Rn. 73. 
Der Wettbewerb in wesentlichen Teilen des Marktes darf zu guter Letzt nicht vollständig ausgeschlossen werden. ${ }^{947}$ Der Gesetzgeber stellt insoweit klar, dass die Freistellung nicht dazu führen darf, dass der Wettbewerb im jeweiligen Markt vollständig zum Erliegen kommt.

\section{d) Zwischenergebnis}

Unter strengen Voraussetzungen können Kooperationsvorhaben, die den Wettbewerb in den jeweiligen Märkten beeinträchtigen, vom Kartellverbot freigestellt sein. Die Anforderungen an die Freistellung sind jedoch nicht einfach zu erfüllen. Bereits die substantiierte Darlegung von Effizienzvorteilen, für welche die Vereinbarung unerlässlich ist, stellt die betroffenen Unternehmen vor große Herausforderungen. Im Rahmen der Gründung der Glasfaser Nordwest haben die Argumente der Senkung der Ausbaukosten sowie der Reduktion der Risiken für Investitionen und Auslastung der Infrastruktur, die in der Regel zur Rechtfertigung von Kooperationsvorhaben herangezogen werden, aus Sicht des Bundeskartellamts nicht ausgereicht. Es bleibt zu beobachten, ob die Verhinderung des Überbaus in künftigen Verfahren als Effizienzgewinn ausreicht und hinreichend substantiiert dargelegt werden kann. Dies wird eher für punktuelle Ausbauvorhaben als für große Kooperationsgebiete gelingen. Eine regionale Marktabgrenzung erhöht ebenfalls die Komplexität.

\section{Verpflichtungszusage}

Sofern es den Unternehmen nicht gelingt, die Freistellungsvoraussetzungen substantiiert darzulegen, können sie auf Verpflichtungszusagen gemäß $\$ 32$ b GWB zurückgreifen (E. I. 2.). Auf diese Weise können die drohenden Wettbewerbsbeeinträchtigungen durch Selbstverpflichtungen beseitigt werden. Die Kartellbehörde hat die Möglichkeit dazu, die Verpflichtungszusage gemäß $\$ 32 \mathrm{~b}$ Abs. 1 S. 1 GWB für bindend zu erklären und damit die Wettbewerbsbedingungen zu verändern. Hierdurch entfallen die Voraussetzungen und es muss nicht das Verbot der Vereinbarung auf Grundlage des $₫ 1 \mathrm{GWB}$ ausgesprochen werden (E. I. 2.).

947 Bekanntmachung 2004/C 101/08, Rn. 105; Kühling, WuW 2019, S. 555, 560; Kühling/Heimeshoff/Schall, K\&R Beihefter 1/2010, S. 1, 24. 


\section{Ergebnis und Schlussfolgerungen}

Neben den Voraussetzungen der Fusionskontrolle der $\$ \$ 36 \mathrm{ff}$. GWB muss sich das Kooperationsvorhaben auch mit $\mathbb{1} 1 \mathrm{GWB}$ vereinbaren lassen, sofern die Vereinbarung nicht kartellrechtsneutral ist. Die Schutzstandards entsprechen sich weitgehend. Die Prüfung des Kartellverbots ist insbesondere für solche Konstellationen von Relevanz, in denen keine Fusion im Sinne der $\$ \$ 36 \mathrm{ff}$. GWB vorliegt. Sofern die Anwendungsvoraussetzungen beider Regelungsregime zugleich eröffnet sind, laufen die Regelungen in weiten Teilen auf dieselben Schlussfolgerungen hinaus. Wenn jedoch Fusionskontrollrecht und Regulierungsrecht keine Anwendung finden, können alle Funktionen vollständig durch das Kartellverbot ausgeglichen werden. Durch die parallele Anwendbarkeit aller Regelungsregime wird mithin sichergestellt, dass der Wettbewerb in jeder potentiellen Ausgestaltungskonstellation hinreichend geschützt wird.

\section{F. Fazit und Ausblick}

Kooperationen können den Ausbau von Glasfaserinfrastrukturen auch in solchen Gebieten ermöglichen, in denen unter normalen wirtschaftlichen Bedingungen keine Ausbauvorhaben erfolgen würden. Verschiedene Risikofaktoren von Investitionen werden durch eine Zusammenarbeit erheblich reduziert, sodass der Ausbau betriebswirtschaftlich sinnvoll werden kann. Die Motivlage für Unternehmen, um sich am Ausbau zu beteiligen, ist entsprechend gemischt und unterschiedlich. Hieraus folgt, dass sich Kooperationsvorhaben sehr vielfältig ausgestalten lassen.

Das resultierende Missbrauchsrisiko wird durch das Wettbewerbsrecht adressiert. Zu beachten sind in dieser Hinsicht das Regulierungsrecht, das Recht der Fusionskontrolle und das Kartellverbot. Die Regelungsregime sind dann parallel anwendbar, wenn das Vorhaben einen hohen Institutionalisierungsgrad in Form der Gründung eines Gemeinschaftsunternehmens aufweist, sich ein marktmächtiges Unternehmen an dem Vorhaben beteiligt und eine bestimmte Umsatzschwelle überschritten wird. Entfallen einzelne dieser Voraussetzungen, sind die betreffenden Vorgaben nicht anwendbar. Aufgrund der umfassenden Parallelen der Regelungsmaterien folgt aus der Unanwendbarkeit jedoch kein Schutzdefizit. Basis für die unterschiedlichen Maßnahmen bildet eine Abgrenzung der tangierten Märkte, die jeweils nach demselben Prinzip erfolgt. Während durch das Regulierungsrecht allerdings nur Märkte aus dem Telekommunikationssektor 
geschützt werden, verfolgt das Kartellrecht einen breiteren Ansatz. So werden etwa auch Märkte beobachtet, die nur indirekt dem Telekommunikationssektor zuzuordnen sind, für den Ausbau von Glasfaserinfrastrukturen allerdings eine wichtige Rolle spielen. Hervorzuheben ist in dieser Hinsicht der Ausschreibungsmarkt für den geförderten Breitbandausbau. Die räumliche Abgrenzung der Märkte weist hingegen Unterschiede auf. Während die Bundesnetzagentur Märkte in der Regel national abgrenzt, verfolgt das Kartellrecht einen regionalen Ansatz. Wird die Kooperation auf ein bestimmtes Gebiet begrenzt, lassen sich die Auswirkungen des Vorhabens auf den Wettbewerb am besten lokal begrenzt untersuchen. Vor diesem Hintergrund empfiehlt es sich, auch im Regulierungsrecht lokale Submärkte abzugrenzen.

Das Regulierungsrecht für Kooperationsvorhaben wurde durch den EKEK umfassend aktualisiert. Grundsätzlich gilt, dass Gemeinschaftsunternehmen, die einen Zusammenschlusstatbestand des GWB erfüllen und an denen ein marktmächtiges Unternehmen beteiligt ist, gemäß $\$ 3 \mathrm{Nr} .29$ TKG von dessen regulatorischen Verpflichtungen erfasst werden. Hierbei kommt es nicht auf den Zeitpunkt der Gründung des Unternehmens an; $\$ 3$ Nr. 29 TKG ist dementsprechend nicht teleologisch zu reduzieren. Reguliert werden auch solche Vorhaben, bei denen ein marktmächtiges Unternehmen entsteht. Privilegierungsmöglichkeiten sieht Art. 76 EKEK in Verbindung mit Art. 79 EKEK durch die Abgabe von Verpflichtungszusagen vor. Der Rechtsrahmen bedarf keiner weitergehenden Aktualisierung. Er steigert die Attraktivität von Kooperationsvorhaben zumindest ein wenig, ohne die Missbrauchsrisiken zu ignorieren. Eine Optimierung könnte durch die Regionalisierung der Regulierung erfolgen, die jedoch ökonomisch sinnvoll sein müsste. Ein entscheidendes Manko ist, dass das Regulierungsrecht erst dann eingreift, wenn bereits ein Netz besteht. Nur dann können Zugangs- und Entgeltverpflichtungen ihre Wirkung entfalten. Hierdurch wird jedoch nicht der Infrastrukturausbauwettbewerb geschützt. Diese Lücke wird durch das Recht der Fusionskontrolle und das Kartellverbot geschlossen, die auch diesen Wettbewerb in den Blick nehmen. Durch die Drohkulisse, die durch die Möglichkeit der Untersagung der Kooperation entsteht, werden die Unternehmen dazu motiviert, umfassende Verpflichtungszusagen abzugeben. Diese können so eingesetzt werden, dass der Wettbewerb durch das Kooperationsvorhaben nicht gefährdet, sondern sogar gefördert wird. Dabei dürfen diese jedoch nicht für solche Ziele instrumentalisiert werden, die nicht zum Telos des Wettbewerbsrechts gehören. Sollten sowohl das Regulierungsrecht als auch das 
Fusionskontrollrecht keine Anwendung finden, kann das Kartellverbot die Funktion der beiden Rechtsregime übernehmen.

Die Regelungsmaterien greifen also wie Zahnräder ineinander und schützen den Wettbewerb auf diese Weise umfassend. Wird normalerweise davon ausgegangen, dass das Regulierungsrecht dann Anwendung findet, wenn das Kartellrecht keinen ausreichenden Schutz bietet, liegt die Situation beim Umgang mit Kooperationsvorhaben anders. Das Regulierungsrecht für sich genommen ist keine suffiziente Antwort auf die Wettbewerbsgefahren, die aus Kooperationsvorhaben erwachsen. Nur durch die Kombination der einzelnen Rechtsbereiche wird ein umfassender Schutz des Wettbewerbs sichergestellt. 


\section{Kapitel 6: Das Zusammenspiel verschiedener Handlungs- optionen auf dem Weg in die Gigabit-Gesellschaft}

Die Infrastrukturen, die für die fortschreitende Digitalisierung auf dem Weg in die Gigabit-Gesellschaft erforderlich sind, sollen nach dem Willen der Bundesregierung bis zum Jahr 2025 ausgebaut werden. Ob dieses Ziel in die Tat umgesetzt werden kann, ist fraglich, da bereits die anvisierten Zwischenziele immer wieder verfehlt wurden. In Zukunft sollten aus diesem Grund flexiblere Ziele formuliert werden, die sich stärker an der Nachfrage der Verbraucher orientieren (Kapitel 2, A. II.).

\section{Hochkomplexe Ausgangslage}

Die Nachfrage nach gigabitfähigen Infrastrukturen stagniert im Vergleich zu deren Verfügbarkeit derzeit nämlich oder ist sogar rückläufig (Kapitel 2, C. I. 2.). Zugleich sind die Investitionen in die gigabitfähige Infrastruktur jedoch durch hohe Kosten und ein beträchtliches korrespondierendes Risiko geprägt (Kapitel 2, C. II.). Risikofaktoren sind insbesondere die Wettbewerbs-, Markt- und Regulierungsunsicherheit, die sich durch die Schwierigkeiten dabei, die Nachfrage zu prognostizieren, vervielfachen (Kapitel 2, C. II. 2.). Je ländlicher ein Gebiet geprägt ist, desto höher sind die Investitionskosten. Zugleich sinkt die Anzahl der Endkundenanschlüsse, mithilfe derer die Kosten amortisiert werden können. Hierdurch fallen die Unsicherheiten umso stärker ins Gewicht und stehen einem marktgetriebenen Ausbau gigabitfähiger Infrastrukturen in diesen Regionen entgegen. Steigt die Nachfrage, nehmen die Unternehmen den damit einhergehenden Markt- und Wettbewerbsdruck sowie die drohende Regulierung eher in Kauf. Sobald Applikationen verfügbar sind, die ausschließlich mithilfe gigabitfähiger Infrastrukturen genutzt werden können, wird die Bandbreitennachfrage sprunghaft ansteigen (Kapitel 2, C. II. 2.). Da der Ausbau entsprechender Infrastrukturen langwierig ist, sollten die - dann tatsächlich erforderlichen - Infrastrukturen zu diesem Zeitpunkt jedoch bereits vorhanden sein.

Vor diesem Hintergrund wurden verschiedene Handlungsoptionen zur Beschleunigung des Ausbaus gigabitfähiger Infrastrukturen beleuchtet, mit deren Hilfe die Markt-, Wettbewerbs- und Regulierungsunsicherheit 
gesenkt werden sollen, um hierdurch das Investitionsklima nachhaltig zu verbessern (Kapitel 2, C. II. 3.). Trotz des gemeinsamen Zieles sind die Ansätze heterogen. Im Konzessionsmodell auf Basis der Vergabe exklusiver Wegerechte soll hierzu der Wettbewerb der Infrastrukturanbieter in den Konzessionsgebieten beseitigt werden (Kapitel 3, C.). Der fehlende Wettbewerb auf dem Vorleistungsmarkt soll durch einen umfassenden Wettbewerb um das Konzessionsrecht und einen Wettbewerb auf den nachgelagerten Märkten kompensiert werden. Bei der Entflechtung vertikal integrierter Telekommunikationsunternehmen steht die Frage im Vordergrund, ob das Geschäftsmodell des Wholesale-Only-Anbieters, das den Infrastrukturausbau beschleunigen könnte, mit staatlichem Zwang oder mithilfe staatlicher Anreize durchgesetzt werden sollte (Kapitel 4). Beim kooperativen Breitbandausbau steht die Aufteilung der skizzierten Risiken zwischen den beteiligten Unternehmen im Mittelpunkt des Interesses (Kapitel 5).

All diese Handlungsansätze sollen zwar in dem diffizilen ökonomischen Spannungsfeld Vorteile mit sich bringen, müssen sich jedoch auch in den rechtlichen Rahmen einfügen. Dabei besteht die besondere Herausforderung darin, dass dieser Rahmen nicht minder komplex ist als die ökonomische Ausgangssituation und insbesondere durch starke Interdependenzen geprägt ist. Eine marginale Modifikation einer Regelung führt häufig zu Friktionen in anderen Regelungsmaterien. Dies lässt sich etwa am Konzessionsmodell auf Basis der Vergabe exklusiver Wegerechte illustrieren. Im Fokus dieser Handlungsoption steht eine Anpassung der wegerechtlichen Spezialvorgaben. Letztlich ist diese Modifikation jedoch mit dem Verfassungsrecht, dem unionalen Wettbewerbsrecht und dem Vergaberecht unvereinbar (Kapitel 3, C.). Dennoch spielte die Analyse der rechtlichen Steuerungsvorgaben in der bisherigen Diskussion um die geeigneten Handlungsoptionen auf dem Weg in die Gigabit-Gesellschaft nur eine untergeordnete Rolle.

\section{Zielrichtung und Anpassungsfähigkeit des Telekommunikations- rechtsrahmens}

Die wesentlichen speziellen Steuerungsvorgaben für den Telekommunikationssektor ergeben sich aus dem unionalen und nationalen Telekommunikationsrecht. Durch die umfassende unionsrechtliche Determinierung bestehen für den nationalen Gesetzgeber lediglich marginale Umsetzungsspielräume. Somit fehlt ihm für grundlegende, aber kurzfristige Änderun- 
gen der rechtlichen Rahmenbedingungen, die für eine Erreichung der selbst gesteckten Zielvorgaben erforderlich wären, in weiten Teilen die Verbandskompetenz. Da erst im Dezember 2018 der unionale Rechtsrahmen umfassend aktualisiert wurde, ist kurzfristig nicht mit seiner weitergehenden Novellierung zu rechnen (hierzu etwa Kapitel 3, C. III. 2.). Er kann somit gewissermaßen als „statisch“ betrachtet werden. Der nationale Gesetzgeber müsste jedoch - sofern es die Umsetzung einer Maßnahme erfordert - allein deshalb kurzfristig handeln, weil mit einer Verzögerung zu rechnen ist, bis sich eine Änderung des Rechtsrahmens in der Ausbaurealität manifestiert. Der Kreativität des nationalen Gesetzgebers, wie sich das Ziel einer flächendeckenden Versorgung mit gigabitfähigen Infrastrukturen bis zum Jahr 2025 erreichen lässt, sind damit bereits durch das unionale Telekommunikationsrecht enge Grenzen gesetzt.

Das Telekommunikationsrecht wurde ursprünglich dazu geschaffen, die Liberalisierung des zunächst durch staatliche Monopole geprägten Sektors zu flankieren. ${ }^{948}$ Dabei trieb den Gesetzgeber auch die Sorge an, dass ein allzu freier Wettbewerb dazu führen könnte, dass die Interessen der Verbraucher vernachlässigt werden. Aus diesem Grund wurden die potentiell widerstreitenden Interessen in Form von Regulierungszielen dem Gesetz vorangestellt, die dessen allgemeinen Zweck konkretisieren. ${ }^{949}$ Die Förderung des Wettbewerbs muss seitdem neben den Verbraucherinteressen bei jeder Anwendung der regulatorischen Vorgaben durch die nationale Regulierungsbehörde berücksichtigt werden.

Diese Grundausrichtung prägt jedoch nicht allein das sektorspezifische Telekommunikationsrecht. Auch das Energie-, Eisenbahn- und Postrecht sind vergleichbar konstruiert. Im Kontext der Novellierung des unionalen Telekommunikationsrechts wurde dessen Zielekanon um ein Konnektivitätsziel erweitert, das im deutschen Rechtsrahmen bereits angelegt war (Kapitel 3, C. III. 1.). Damit wird die Erhöhung des Vernetzungsgrades der Bevölkerung ein Ziel, das bei jeder Entscheidung der Regulierungsbehörde Berücksichtigung finden muss. Diese Diversifizierung des Rechtsrahmens führt dazu, dass die originäre Ausrichtung des Handelns der Regulierungsbehörden immer weiter aufgefächert wird. Je mehr widerstreitende Interessen miteinander in Einklang gebracht werden müssen, desto schwächer wird im Ergebnis der Kompromiss. Während die ursprünglichen Ziele des Rechtsrahmens eng miteinander verknüpft waren, steht das neue

948 Hierzu statt vieler Kühling, in: Ruffert (Hrsg.), Europäisches Sektorales Wirtschaftsrecht, $\$ 4$, Rn. 17.

949 Schneider, in: Fehling/Ruffert (Hrsg.), Regulierungsrecht, S. 365, 369. 
Ziel in loserem Zusammenhang zu ihnen. Dies verstärkt die Komplexität des Interessenausgleichs weiter.

Nicht in Frage gestellt werden soll an dieser Stelle, dass der Ausbau gigabitfähiger Infrastrukturen - wie jeder Ausbau von Zukunftstechnologien positive volkswirtschaftliche Effekte generieren kann. Allerdings sollte nicht jedes politische Ziel in jeder regulatorischen Entscheidung berücksichtigt werden müssen. In Zukunft sollte der Zielekanon nur zurückhaltend erweitert werden. Vielmehr sollten sich Gesetzgeber, Wissenschaft und Praxis vor Augen führen, dass das Gesetz in seiner teleologischen Grundausrichtung, seinem systematischen Aufbau und den angelegten Kompetenzen ursprünglich vorrangig auf die Schaffung und den Schutz des Wettbewerbs ausgerichtet war. Werden zusätzliche Wirkungen - wie etwa ein verbesserter Kundenschutz - angestrebt, erfolgt dies in neuen systematischen Zusammenhängen, die klar von den bisherigen Regelungsmaterien abgegrenzt werden. So sind etwa die Vorgaben zum Kundenschutz sowohl im unionalen (Titel III des EKEK) als auch im nationalen Recht (Teil 3 des TKG) separat normiert und grenzen sich klar von den übrigen Vorschriften ab. Eine Erweiterung des Gesetzeszweckes muss der Gesetzgeber auch systematisch und kompetenziell abbilden (Kapitel 4, B. I. 2. b.). Dies gilt also auch für die Förderung der Konnektivität.

Eine Erweiterung der Zielrichtung bestehender Normen ist nur möglich, wenn sie sich systematisch und teleologisch in das Gesamtbild einfügt. Hierfür eignet sich nicht jede Rechtsgrundlage. Regulatorische Verpflichtungen können nicht einfach auferlegt werden, um die Konnektivität zu fördern. Dies lässt sich darauf zurückführen, dass der regulatorische Maßnahmenkatalog der $\$ \$ 13 \mathrm{ff}$. TKG systematisch und hinsichtlich seines Zwecks darauf ausgerichtet ist, einen funktionsfähigen Wettbewerb in Märkten zu ermöglichen, die durch ein natürliches Monopol geprägt sind und von marktmächtigen Unternehmen dominiert werden. Dies wird insbesondere im Hinblick auf die Kompetenznormen zur Entflechtung von Unternehmen offenbar, welche die ultima ratio des regulatorischen Maßnahmenkataloges darstellen (Kapitel 4, B. I. 2. b.). Dessen Ertüchtigung zur Förderung der Konnektivität ließe sich weder mit dem Zweck der Vorgabe vereinbaren noch systematisch in das Marktregulierungsverfahren einfügen.

Handlungsoptionen zur Beschleunigung des Ausbaus hochleistungsfähiger Telekommunikationsinfrastrukturen, die mit bestehenden regulatorischen Maßnahmen zusammenhängen, können mithin nicht einfach dadurch umgesetzt werden, dass der Anwendungsbereich dieser Bestandsnormen erweitert wird. Hierdurch würden systematische Fremdkörper entste- 
hen, die den ausbalancierten Rechtsrahmen dysfunktional machen könnten. Vielmehr müssten - sofern dies für eine Handlungsoption erforderlich ist - eigenständige Rechtsgrundlagen geschaffen werden, die teleologisch auf die Förderung des Infrastrukturausbaus ausgerichtet sind und sich in einen neuen, auf dieses Ziel ausgerichteten systematischen Kontext einfügen. So kann es insbesondere nicht überzeugen, wenn Maßnahmen zur Förderung der Konnektivität ausschließlich gegenüber marktmächtigen Unternehmen verhängt würden. Dies wäre allerdings bei einer Einbettung in das Marktregulierungsverfahren der Fall. Dabei sind für den Infrastrukturausbau in ländlichen Gebieten auch solche Unternehmen von großer Bedeutung, deren Tätigkeit lediglich lokal ausgerichtet ist. Diese verfügen auf den Telekommunikationsmärkten, die von der Bundesnetzagentur aktuell (bis auf wenige Ausnahmen) bundesweit abgegrenzt werden (Kapitel 5, C. II. 2. a.), über keine beträchtliche Marktmacht. Sie können deswegen nicht Adressat der marktmachtabhängigen Regulierungsverpflichtungen sein. Dies würde dazu führen, dass diese Maßnahmen - insbesondere dort, wo sie am nötigsten gebraucht würden - nicht gegenüber all den Marktteilnehmern angewandt werden könnten, die entscheidend für den Infrastrukturausbau in der jeweiligen Region sind. Aus diesem Grund sollten alternative Verfahren etabliert werden, um solche Gebiete zu identifizieren, in denen Maßnahmen zur Förderung der Konnektivität nötig sind. In diesen Gebieten sollten dann alle Unternehmen, die im Rahmen des Infrastrukturausbaus tätig sind, durch die Maßnahmen zu dessen Beschleunigung adressiert werden können. Bislang gibt es jedoch keine verpflichtenden Maßnahmen, die sich hierfür eignen würden. Es handelt sich vielmehr um eine hypothetische Skizzierung der systematischen Verortung von Handlungsoptionen im Gesetz, die noch nicht entwickelt wurden. Dass insbesondere eine verpflichtende Entflechtung nicht dazu gehört, soll noch vertieft beleuchtet werden (hierzu VIII.).

Bei der Identifikation der Gebiete mit Netzausbaudefizit könnte man sich an der Abgrenzung von Fördergebieten im Beihilfenrecht orientieren. Auch die geografische Erhebung gemäß Art. 22 EKEK, die noch in nationales Recht umgesetzt werden muss, kann eingesetzt werden (Kapitel 4, B. I. 2. b.). Hierbei handelt es sich um ein Kartierungssystem, mit dessen Hilfe bereits errichtete und geplante Telekommunikationsinfrastrukturen erfasst werden können. Diese Datenbasis eignet sich für verschiedene Handlungsoptionen zur Beschleunigung des Ausbaus gigabitfähiger Infrastrukturen.

Dass diese neuen Kompetenznormen am besten im Telekommunikationsgesetz geschaffen und dort in sinnvolle Verfahren eingebettet werden, 
ist nicht zwingend. Aufgrund des hohen Determinierungsgrades des unionalen Telekommunikationsrechts ist die Schaffung nationaler Kompetenzgrundlagen im Alleingang nicht möglich. Diese müssten wohl zunächst in den unionalen Rechtsrahmen integriert und anschließend im nationalen Recht umgesetzt werden. Denkbar wäre hierfür auch ein speziell auf den Ausbau gigabitfähiger Infrastrukturen ausgerichtetes Gesetz, das systematisch und teleologisch ausschließlich die Konnektivität der Bevölkerung steigern soll. In diesem Gesetz könnte ein Maßnahmenkatalog festgelegt werden, der diejenigen Handlungsoptionen zusammenfasst, die den Infrastrukturausbaus fördern sollen. Auf diese Weise könnten diese von bestehenden Instrumentarien des Telekommunikationsrechts, insbesondere vom Marktregulierungsverfahren, entkoppelt werden. Hierdurch würde ein eigenständiger, konsistenter Rechtsrahmen geschaffen, der zwar mit dem Telekommunikationsgesetz in Einklang gebracht werden müsste, aber flexibler wäre als in einem bestehenden Gesetz. Pfadabhängigkeiten, die bei der Ausgestaltung des Telekommunikationsgesetzes entstanden sind, würden den Gesetzgeber in diesem Fall weniger beschränken.

III. Zusammenspiel von allgemeinem und sektorspezifischem Wettbewerbsrecht

Das Telekommunikationsrecht bildet jedoch nicht die einzige Regelungsmaterie, an der sich alle Handlungsoptionen zur Beschleunigung des Ausbaus gigabitfähiger Infrastrukturen messen lassen müssen. Maßnahmen zur Reduktion der Markt- und Wettbewerbsunsicherheiten stehen auch in einem Spannungsverhältnis zum GWB, das das allgemeine Wettbewerbsrecht beinhaltet. Seine Vorgaben sind, sofern das Telekommunikationsgesetz keine abschließenden Spezialregelungen enthält, parallel anwendbar. Dies führt insbesondere im Kontext des Infrastrukturausbaus zu einer sinnvollen Erweiterung des Wettbewerbsschutzes (Kapitel 5, E. I. 1.). Das GWB ist nämlich auf den Schutz des Wettbewerbs in allen Märkten ausgerichtet, während das TKG spezifisch den Wettbewerbsschutz im Telekommunikationssektor adressiert. Das GWB ermöglicht somit den Schutz solcher Märkte, die nicht direkt dem Telekommunikationssektor zuzuordnen sind, aber dennoch den Ausbau gigabitfähiger Infrastrukturen tangieren. Dies zeigt sich insbesondere am Beispiel des kooperativen Glasfaserausbaus. So schützt das Kartellrecht auch den Ausschreibungsmarkt für den geförderten Breitbandausbau (Kapitel 5, C. III.). Erst das Zusammenspiel der beiden Regelungsmaterien ermöglicht einen umfassenden Wettbe- 
werbsschutz im Infrastrukturausbau. Zugleich offenbart jedoch die Einschlägigkeit der beiden Regelungsmaterien, dass den Handlungsoptionen zur Reduktion des Wettbewerbsdrucks, um den Glasfaserausbau zu beschleunigen, enge Grenzen gesetzt sind.

IV. Keine Instrumentalisierung des allgemeinen Wettbewerbsrechts zur Beschleunigung des Glasfaserausbaus

Während das Zusammenspiel des allgemeinen Wettbewerbsrechts und des Telekommunikationsrechts zu begrüßen ist, um einen umfassenden Wettbewerbsschutz sicherzustellen, sind weitere Tendenzen kritisch zu betrachten. Dazu gehört es, dass die Handlungsoptionen des GWB dazu genutzt werden, um den flächendeckenden Ausbau hochleistungsfähiger digitaler Infrastrukturen voranzutreiben. Ein solches Vorgehen des Bundeskartellamts war im Kartellverwaltungsverfahren bei der Gründung der Glasfaser Nordwest zu beobachten (Kapitel 5, E. I. 2.). Mit einer Ausbauzusage verpflichtete sich das Kooperationsunternehmen in einem Verfahren, das ausschließlich dem Schutz des Wettbewerbs dient, dazu, eine bestimmte Anzahl gigabitfähiger Teilnehmeranschlüsse im Kooperationsgebiet auszubauen. Das GWB ist jedoch nicht darauf ausgerichtet, den Ausbau gigabitfähiger Infrastrukturen zu beschleunigen. Auch wenn das Unternehmen die Verpflichtungszusage äußerlich freiwillig abgab, geschah dies nur auf Grund des behördlichen Drucks. Ohne die Abgabe weitreichender Verpflichtungszusagen wäre die Kooperation behördlich nicht genehmigt worden. Zwar ermöglicht der Ausbau der Glasfaserinfrastruktur in den betreffenden Gebieten aufgrund der Verfügbarkeit entsprechender Infrastrukturen einen Dienstewettbewerb überhaupt mit, der einen entsprechenden Bandbreitenbedarf voraussetzt. Zudem ist der Ausbau hochleistungsfähiger Technologien grundsätzlich volkswirtschaftlich positiv zu bewerten. Allerdings sollte die Genehmigung von Kooperationsvorhaben nur von solchen Verpflichtungszusagen abhängig gemacht werden, die in einem direkten Zusammenhang mit dem Telos des betreffenden Gesetzes stehen. Auch dieser fragwürdigen Vermengung verschiedener Ziele kann durch ein separates Gesetz vorgebeugt werden. 


\section{Zuständigkeit und Ausstattung von Behörden}

Alle gesetzlichen Kompetenzgrundlagen müssen von den zuständigen Behörden angewandt werden. Im hiesigen Kontext sind dies die Bundesnetzagentur und das Bundeskartellamt. Diese sind, dem Zweck der jeweiligen Gesetze folgend, kompetenziell so ausgestattet, dass sie insbesondere den Wettbewerbsschutz und den Schutz der Verbraucherinteressen bewerkstelligen können. Zudem spiegelt sich diese Ausrichtung auch im Aufbau und in der Ausstattung der Behörden wider. Die fortschreitende Diversifizierung des Zielekanons muss auch in diesem Kontext Anklang finden. Sollen die Behörden im Rahmen ihrer Tätigkeit auch den Infrastrukturausbau berücksichtigen, müssen sie hierzu über fachlich kompetentes Personal verfügen und mit entsprechenden Exekutivkompetenzen ausgestattet werden.

Hierzu könnte eine entsprechende Abteilung in einer bestehenden Behörde gegründet werden. Auf diese Weise könnte sichergestellt werden, dass Maßnahmen zur Sicherstellung eines funktionsfähigen Wettbewerbs von solchen zur Förderung des Infrastrukturausbaus klar getrennt sind und die Aufgaben nicht in fragwürdiger Weise vermengt werden. Ein Austausch der Abteilungen und der Behörden untereinander könnte in Querschnittsmaterien durch umfassende Konsultationsmechanismen gewährleistet werden. Solche existieren bereits im Verhältnis zwischen Bundesnetzagentur und Bundeskartellamt und stellen sicher, dass die Behörden trotz im Detail abweichender Entscheidungen grundsätzlich eine schlüssige und aufeinander abgestimmte Spruchkammerpraxis verfolgen.

VI. Flexibilisierung des Marktregulierungsverfahrens durch Verpflichtungszusagen

Die Integration von Verpflichtungszusagen in den Telekommunikationsrechtsrahmen ist - trotz der Kritik an der Anwendung ihres kartellrechtlichen Pendants durch das Bundeskartellamt im Einzelfall (hierzu IV.) - insgesamt positiv zu bewerten. Im Telekommunikationsrecht ist zu beobachten, dass der Rechtsrahmen immer weiter ausdifferenziert wird. Die Verfahren werden - insbesondere durch die Vielzahl der zu berücksichtigenden Interessen - immer komplexer. Ein umfassend durchstrukturiertes normatives Geflecht erhöht die Regulierungssicherheit. Behördliche Entscheidungen werden vorhersehbar und planbar. Darüber hinaus werden 
im Verfahren bereits die Interessen umfassend abgewogen und die Verhältnismäßigkeit geprüft.

Die umfassende Normierung bringt jedoch auch Nachteile mit sich. Denn je ausdifferenzierter ein Rechtsrahmen ist, desto weniger Flexibilität bleibt der zuständigen Behörde im Rahmen ihrer Entscheidungsfindung. Die Verpflichtungszusage durchbricht diese Tendenz und flexibilisiert das Verfahren (Kapitel 5, D. II. 3.). Dadurch, dass die Verpflichtungszusagen lediglich einen Einzeltopos ihrer Ermessenerwägungen darstellen, wird die Behörde in ihrer Entscheidungsfindung nicht eingeschränkt. Hierdurch fügt sich das Instrument schlüssig in den Rechtsrahmen ein. Insoweit bietet es einen Vorteil gegenüber der zuvor praktizierten Flexibilisierung des Marktregulierungsverfahrens mithilfe öffentlich-rechtlicher Verträge, die im TKG nicht explizit normiert waren.

Mit dem Instrument der Verpflichtungszusage verknüpfen sich Hoffnungen: Die Regulierung könnte, insbesondere aufgrund der Möglichkeit des umfassenden Rückgriffes auf das Instrument im Marktregulierungsverfahren gemäß Art. 79 Abs. 1 UAbs. 1 lit. a EKEK, zurückgeführt werden. Die Sicherstellung eines funktionsfähigen Wettbewerbes könnte dem Markt überantwortet werden, in den der Staat nicht mehr aktiv interveniert, sondern auf dem er passiv als Schiedsrichter sicherstellt, dass die wettbewerbsrechtlichen Spielregeln eingehalten werden. Ob die Verpflichtungszusage diese Hoffnungen erfüllen kann, bleibt abzuwarten. Dies hängt insbesondere davon $\mathrm{ab}$, wie intensiv das Instrument in künftigen Marktregulierungsverfahren eingesetzt werden wird.

\section{Wettbewerb im Netz oder Wettbewerb um das Netz?}

Die Komplexität des Zusammenspieles verschiedener Materien, die die Beschleunigung des Ausbaus hochleistungsfähiger digitaler Telekommunikationsinfrastrukturen beeinflussen, offenbart sich bei einer kritischen Reflexion eines Detailaspektes der Spruchkammerpraxis des Bundeskartellamtes im kooperativen Glasfaserausbau. Wo es keinen sinnvollen Wettbewerb der Infrastrukturanbieter gibt, stellt die Behörde einen funktionsfähigen Wettbewerb auf nachgelagerter Ebene sicher. Anstelle des Wettbewerbs der Infrastrukturanbieter wird ein Wettbewerb um den Infrastrukturmarkt durchgeführt. Dieser entsteht durch die exklusive Vergabe von Fördergeldern (Kapitel 5, C. III.). Genau dieses Kalkül liegt auch dem Kooperationsmodell auf Basis der Vergabe exklusiver Wegerechte zu Grunde, das einen Wettbewerb der Infrastrukturanbieter durch einen Wettbewerb um den 
Infrastrukturmarkt ersetzen möchte (Kapitel 3, C. IV. 5. a. aa.). In diesem Fall entstünde der Markt durch die exklusive Vergabe des Konzessionsrechtes. Der ausdifferenzierte Rechtsrahmen steht einer funktionsfähigen Institutionalisierung dieses Modells aber entgegen, die zudem impraktikabel und daher nicht realisierbar erscheint und aus volkswirtschaftlichen Gründen abzulehnen ist (Kapitel 3, C.).

In ländlichen Regionen, in denen das Konzessionsmodell auf Basis der Vergabe exklusiver Wegerechte eingeführt werden soll, gibt es einen solchen Wettbewerb um das Netz jedoch faktisch bereits. In den Fördergebieten besteht das Problem, dass sich die Infrastruktur nicht marktgetrieben und kostendeckend ausbauen lässt. Der Förderrahmen ist grundsätzlich wettbewerblich ausgerichtet, ermöglicht jedoch nur einen begrenzten Wettbewerb der Infrastrukturen, obgleich schon Unternehmen im Markt konkurrieren können. Dies lässt sich darauf zurückführen, dass das EUBeihilfenrecht und das Haushaltsrecht einer umfassenden Förderung eines parallelen Infrastrukturausbaus entgegenstehen. Der Förderrahmen ermöglicht mit dem „Graue-Flecken-Förderprogramm“ lediglich den Ausbau von zwei konkurrierenden Infrastrukturen (Kapitel 5, C. III.). ${ }^{950}$ Eine Förderung weiterer paralleler Infrastrukturen würde aber ohnehin dem Grundsatz des sparsamen Einsatzes von Haushaltsmitteln entgegenstehen. Durch umfassende Open-Access-Verpflichtungen wird trotzdem ein funktionsfähiger Wettbewerb auf der nachgelagerten Ebene sichergestellt. Aufgrund der Exklusivität in der Fördermittelvergabe entsteht ein Ausschreibungsmarkt im geförderten Breitbandausbau. Diese Folge der Konstruktion des EU-Beihilfenrechts sichert die Spruchkammerpraxis des Bundeskartellamtes zusätzlich, auch gegen Verschiebungen durch den kooperativen Breitbandausbau, ab. Das Bundeskartellamt geht mit den Folgen der Ausgestaltung des Förderregimes offensiv um und versucht, den funktionsfähigen Wettbewerb um den Markt sicherzustellen.

Die konkrete Umsetzung des Wettbewerbsschutzes durch das Bundeskartellamt in der Form, dass Unternehmen eine Teilnahme am Ausschreibungswettbewerb verwehrt wird, ist hingegen zu kritisieren (Kapitel 5, E. I. 2.). Aufgrund der abgegebenen Verpflichtungszusagen können Unternehmen, die zentral für den Breitbandausbau in der Region sind, nicht mehr in einem integralen Marktbestandteil aktiv werden. Dies ist widersprüchlich und schädigt den Wettbewerb eher, als dass es ihm nützt. Vielmehr sollte die Funktionsfähigkeit des Wettbewerbs um die Fördergelder dadurch sichergestellt werden, dass die Marktpräsenz der Unternehmen

950 So auch Kübling, WuW 2019, S. 555, 556. 
zwar ermöglicht, ein kollusives Verhalten jedoch verhindert wird. Zu den Missbrauchsgefahren zählt insbesondere ein selektives Ausbauverhalten in den Fördergebieten, in dem nur deren attraktive Bereiche ausgebaut werden. Hauptanknüpfungspunkt zur Verhinderung eines Missbrauchs wäre in dieser Hinsicht zwar das Förderprogramm, allerdings könnten Restrisiken für den Wettbewerb durch entsprechende Verpflichtungszusagen beseitigt werden. Ein Vorbild könnte insoweit die Ausbauzusage im selben Verfahren sein, die eine missbräuchliche Verlagerung der Ausbauaktivitäten verhindert (Kapitel 5, E. I. 2.). Anstelle eines pauschalen Vorgehens durch einen vollständigen Wettbewerbsausschluss müssten die konkret in den Verfahren identifizierten Missbrauchspotentiale durch einzelfallgerechte und spezifisch auf das konkrete Kooperationsgebiet ausgerichtete Maßnahmen ersetzt werden.

Grundsätzlich ist der Schutz des Wettbewerbs, um den Infrastrukturmarkt in Fördergebieten aber positiv zu bewerten, da er die Ausbaurealität wahrnimmt und einen Lösungsweg bietet. Der Wettbewerb der Infrastrukturanbieter wird in Fördergebieten durch einen Wettbewerb um die Fördergelder ersetzt.

\section{Keine Verpflichtung zur Umsetzung von Geschäftsmodellen durch staatlichen Zwang}

Kategorisch abzulehnen sind alle Handlungsoptionen, die das Wettbewerbsrecht instrumentalisieren, um Geschäftsmodelle mithilfe staatlichen Zwanges umzusetzen. Auch wenn von der Tätigkeit als Wholesale-OnlyAnbieter ein positiver Effekt für den Glasfaserausbau ausgehen kann, darf die Exekutive dieses Geschäftsmodell nicht mithilfe des Wettbewerbsrechts zwangsweise durchsetzen (Kapitel 4, B. I.). Ob tatsächlich flächendeckende positive Auswirkungen zu erwarten sind, müsste jedoch ohnehin zunächst mithilfe umfassender ökonomischer Studien nachgewiesen werden (Kapitel 4, A. I. 3.).

Es sollte keine Rechtsgrundlage dafür geschaffen werden, Unternehmen zu einer Entflechtung zu verpflichten, um den Infrastrukturausbau zu fördern, sofern eine Entflechtung nicht bereits aus wettbewerblichen Gründen geboten ist. Eine Ertüchtigung der bestehenden Norm ist aus systematischen und teleologischen Gründen nicht möglich. Die Anwendung einer neuen Rechtsgrundlage zur Entflechtung von Unternehmen zur Förderung der Konnektivität ließe sich im Einzelfall verfassungsrechtlich schwerlich rechtfertigen (Kapitel 4, B. I. 3.). 
Vielmehr sollte der Gesetzgeber sich darauf beschränken, Anreize zu schaffen, um das Geschäftsmodell des Wholesale-Only-Anbieters attraktiver zu machen. Dies ist jedoch nur dort möglich, wo sich der spezifische Anreiz in den Regelungsrahmen einfügt. Im Regulierungsrecht sind im Rahmen des Marktregulierungsverfahrens demnach nur solche Anreize denkbar, die marktmächtige Unternehmen betreffen (Kapitel 4, C. III. 3.). $\mathrm{Da}$ jedoch im Infrastrukturausbau auch Unternehmen ohne beträchtliche Marktmacht aktiv sind, die nicht mit der Spruchpraxis der Wettbewerbsschutzbehörden konfrontiert werden, können dadurch nur marginale Effekte erzielt werden. Hauptanknüpfungspunkt müssten demnach Anreize durch den Förderrahmen sein (Kapitel 4, E.). Insoweit ist es unverfänglich, wenn der Staat die Vergabe öffentlicher Gelder von Belangen abhängig macht, die volkswirtschaftlich positive Effekte mit sich bringen. Die Vorgaben können jedoch nur dann Steuerungswirkung entfalten, wenn nicht Unternehmen die Aktivität im Markt durch die behördliche Spruchpraxis untersagt wird (Kapitel 5, E. I. 2.). Auch aus dieser Perspektive ist die Verpflichtungszusage zu kritisieren, die der Glasfaser Nordwest die Beteiligung am Ausschreibungswettbewerb im geförderten Breitbandausbau verwehrt (hierzu VII.). Dies ist hier jedoch von untergeordneter Bedeutung, da die Glasfaser Nordwest ohnehin als Wholesale-Only-Anbieter tätig ist (Kapitel 5, B. I.) und deswegen keine zusätzlichen Anreize für eine Entflechtung gesetzt werden müssen.

Die Rolle des Fördermittelgebers kann auch aktiv und gestaltend sein. Hieraus ergibt sich ein differenziertes Handlungsprogramm für die Behörden. Die Wettbewerbsschutzbehörden sollten sich möglichst passiv verhalten und lediglich als Schiedsrichter einen funktionsfähigen Wettbewerb sicherstellen. Der Fördermittelgeber kann und darf hingegen aktiven Einfluss auf den Markt nehmen. Wer zahlt, bestimmt die Regeln, nach denen die Fördermittel vergeben werden.

Anders als die Auferlegung regulatorischer Verpflichtungen, denen sich das Unternehmen fügen muss, sind die Fördermittelvergaben von Freiwilligkeit geprägt. Möchte das Unternehmen bestimmte Bedingungen nicht akzeptieren, an welche die Vergabe von Fördermitteln geknüpft sind, kann es von einer Beteiligung an der Ausschreibung absehen. Diese freiwillige Verzichtsmöglichkeit besteht im Rahmen des üblichen Regulierungsrechts jedoch nicht, sodass Modifikationen des Rechtsrahmens hier eine höhere Eingriffsintensität aufweisen und sich schwerer mit den Grundrechten vereinbaren lassen.

In jedem Fall müssten zudem außerhalb des rechtlichen Rahmens die Bedingungen für eine vermehrte Marktpräsenz von Wholesale-Only-An- 
bietern geschaffen werden. Hier steht die Branche in der Pflicht, Plattformen zu etablieren und Prozesse zu standardisieren, um Unternehmen den Vertrieb ihrer Infrastruktur zu ermöglichen. Gerade wenn Unternehmen nur lokal über Infrastruktur verfügen, ist es von Vorteil, wenn nicht in jedem Einzelfall komplexe technische Abläufe und Verträge entwickelt werden müssen, die zu hohen Transaktionskosten führen. Der Rechtsrahmen steht einer solchen Prozessoptimierung nicht entgegen (Kapitel 4, E.). Die Unternehmen müssten solche Aktivitäten freiwillig und im Markt organisieren. Die nationale Regulierungsbehörden kann und darf sie hierzu nicht verpflichten.

IX. Zusammenspiel der Anpassung des Rechtsregimes mit der Vergabe von Fördermitteln

Es ergibt sich ein differenziertes Bild der Handlungsoptionen, die sich für die Beschleunigung des Infrastrukturausbaus auf dem Weg in die GigabitGesellschaft eignen. Ungeeignet sind Konzessionsmodelle auf Basis der Vergabe exklusiver Wegerechte, die sich nicht in den Rechtsrahmen einfügen. Alternative Konzessionsmodelle, die etwa die exklusive Vergabe von Fördermitteln betreffen, sind hingegen als Handlungsoptionen grundsätzlich geeignet und werden bereits großflächig angewandt. Ein differenziertes Bild zeigt sich auch bei Handlungsoptionen zur Steigerung der Marktpräsenz von Wholesale-Only-Anbietern. Eine verpflichtende Entflechtung zur Förderung der Konnektivität ist im bestehenden Rechtsrahmen nicht möglich. Eine Erweiterung der bestehenden Normen ließe einen systematischen und teleologischen Fremdkörper entstehen. Eine neue Rechtsgrundlage ließe sich im Einzelfall schwerlich grundrechtskonform anwenden. Unbedenklich sind hingegen Anreize für die Tätigkeit als WholesaleOnly-Anbieter, die nicht die bestehenden rechtlichen Vorgaben verändern, sondern den Förderrahmen oder das Vertriebsumfeld optimieren sollen.

Kooperationen eignen sich durch die Aufteilung des Risikos zwischen verschiedenen Unternehmen grundsätzlich dazu, den Glasfaserausbau zu beschleunigen. Dabei ist jedoch stets darauf zu achten, dass sich die Ausgestaltung des konkreten Kooperationsvorhabens mit dem Wettbewerbsrecht vereinbaren lässt. Der Rechtsrahmen adressiert diese Gefahren effektiv, auch wenn die Spruchpraxis der Behörden im Einzelfall noch optimierungsbedürftig ist.

Eine weitere entscheidende Handlungsoption auf dem Weg in die Gigabit-Gesellschaft ist die Vergabe von Fördermitteln. Alle näher untersuch- 
ten und für vorteilhaft befundenen Handlungsoptionen haben gemeinsam, dass sie für sich genommen keine Lösung für ein weiteres entscheidendes Problem des flächendeckenden Ausbaus gigabitfähiger Infrastrukturen bieten. Das Verhältnis von Investitionskosten und Amortisationsmöglichkeiten bleibt auch bei einer Optimierung des Rechtsrahmens im ländlichen Raum um ein Vielfaches schlechter als im urbanen Bereich. Die Handlungsansätze optimieren einen bereits umfassend ausdifferenzierten Rechtsrahmen und stoßen dabei auf die skizzierten Grenzen. Die hohen Investitionskosten werden durch die untersuchten Handlungsoptionen nicht gesenkt. Der Staat kann diese aber in Form von Fördermitteln teilweise übernehmen und somit für Unternehmen tragbar machen. Wenn der Staat die Kosten übernimmt, kann auch in völlig abgelegenen Orten gigabitfähige Infrastruktur verlegt werden. Auf diese Weise lassen sich auch hochgesteckte politische Ziele - unabhängig von der Frage, wie sinnvoll diese sind - verfolgen. Dies findet seine Grenze lediglich in den Kapazitäten der Bauunternehmen. Fördermittel können auch zur Stimulation der Nachfrage, etwa in Form von Gigabit-Vouchern, eingesetzt werden und dazu beitragen, die Nachfrageunsicherheit zu reduzieren (Kapitel 2, C. II. 2.).

Der Weg in die Gigabit-Gesellschaft lässt sich mit einer Rennstrecke vergleichen. Alle beschriebenen Handlungsoptionen sind darauf ausgerichtet, an dem Rennauto High-End-Optimierungen vorzunehmen, um ZehntelSekunden zu gewinnen. Der eigentliche Motor für den Ausbau sind jedoch die Fördergelder. Die High-End-Optimierung des Rechtsrahmens kann nur dann seine Wirkung erzielen, wenn ein optimaler Motor verbaut wurde. Ansonsten kommt es bei den erheblichen Zeitverlusten nicht auf das Einsparen von Zehntel-Sekunden an, die mithilfe der High-End-Optimierung erreicht werden. Ein suffizienter Förderrahmen ist mithin entscheidend, damit die skizzierten Handlungsoptionen überhaupt sinnvoll eingesetzt werden können. Von ihnen geht jedoch - und das haben alle Ansätze gemeinsam - für sich genommen keine eklatante Beschleunigung des Infrastrukturausbaus aus. Ein flächendeckender Ausbau gigabitfähiger Infrastrukturen und ein zügiger Weg in die Gigabit-Gesellschaft können nur dann gelingen, wenn verschiedene Handlungsoptionen gemeinsam verfolgt werden. Eine Kombination der Vergabe von Fördermitteln mit einer Optimierung des Rechtsrahmens in Detailfragen verspricht hier den größten Erfolg. 


\section{Literaturverzeichnis}

Alle Links, die in den nachfolgend bibliographischen Angaben aufgelistet sind, wurden zuletzt am 1.7.2020 abgerufen.

Abegg, Peter/Brinkmann, Michael/Brunekreeft, Gert/Götz, Georg/Krancke, Jan/Müller, Christoph/Schmidt, Claudia, Entflechtung in Netzsektoren - ein Vergleich, Bremen Energy Working Papers No. 19, Bremen 2014, abrufbar unter https://www. econstor.eu/bitstream/10419/113278/1/807078735.pdf.

Abrardi, Laura/Cambini, Carlo, Ultra-fast broadband investment and adoption - A survey, Telecommunications Policy 2019, S. 183-198.

Amendola, Giovanni Battista/Pupillo, Lorenzo Maria, The Economics of Next Generation Access Networks and Regulatory Governance: Towards Geographic Patterns of Regulation, Communications \& Strategies 69 (2008), S. 85-105.

Arndt, Hans-Wolfgang/Fetzer, Thomas/Scherer, Joachim/Graulich, Kurt (Hrsg.), Telekommunikationsgesetz - Kommentar, 2. Aufl., Berlin 2015.

Auer-Reinsdorff, Astrid/Conrad, Isabell (Hrsg.), Handbuch IT- und Datenschutzrecht, 3. Aufl., München 2019.

Bacache, Maya/Bourreau, Marc/Gaudin, Germain, Dynamic Entry and Investment in New Infrastructures: Empirical Evidence from the Fixed Broadband Industry, Review of Industrial Organization 44 (2014), S. 179-209.

Bary, Tarek-Leander, Kommunaler Netzausbau in der Telekommunikation - Nationale und europäische Rahmenbedingungen für den Infrastrukturausbau, Hamburg 2014.

Baum, Heiko, Morphologie der Kooperation als Grundlage für das Konzept der Zwei-Ebenen-Kooperation, Wiesbaden 2011.

Becker, Peter/Dicks, Heinz-Peter, Vergabe von Wegenutzungsrechten - Rechtsrahmen für Konzessionsvergaben und für Partnerschaften mit kommunalen Netzbetreibern, ZNER 2014, S. 425-430.

Berger-Kögler, Ulrike, Regionalisierung der Regulierung - erste Beispiele aus Großbritannien und Österreich - Implikationen für Deutschland, MMR 2008, Heft 9 , S. VI-VIII.

Berger-Kögler, Ulrike, Die künftige Regulierung des Bitstrommarkts - Ein Weg zur Regionalisierung, MMR 2010, S. 303-309.

Berkeley Research Group LLC (BRG), Co-Investment and Commercial Offers, London 2017, abrufbar unter: https://www.thinkbrg.com/pp/publication-667.pdf?32609.

Biendl, Michael, Rechtliche Herausforderungen beim Auf- und Ausbau von NextGeneration-Access-Networks - Telekommunikations-Infrastrukturrecht nach der TKG-Novelle 2012, Münster 2015. 
Böckel, Markus, Vergaberechtliche Behandlung von Dienstleistungskonzessionen, LKV 2003, S. 393-399.

Böcker, Jens, BREKO Marktanalyse 19 - Marktbefragung der BREKO-Netzbetreiber, Bonn 2019, abrufbar unter: https://brekoverband.de/wp-content/uploads/2019/0 8/BREKOMarktanalyse19_final.pdf.

Body of European Regulators for Electronic Communications (BEREC), BoR (10) 44 Rev1 - BEREC Guidance on functional seperation under Articles 13a and $13 \mathrm{~b}$ of the revised Access Directive and nationale experiences, Riga 2011, abrufbar unter: https://berec.europa.eu/doc/berec/bor_10_44rev1.pdf.

Body of European Regulators for Electronic Communications (BEREC), BoR (17) 88 - BEREC views on Article 77 of the draft Code, Vertically separate undertakings, Riga 2018, abrufbar unter: https://berec.europa.eu/eng/document_register/ subject_matter/berec/download/0/7034-berec-views-on-article-77-of-the-draftc_0.pd $\bar{f}$

Bourreau, Marc/Cambini, Carlo/Hoernig, Steffen, Cooperative Investment, Access, and Uncertainty, Florenz 2016, abrufbar unter https:/www.researchgate.net/ publication/321483255_Cooperative_Investment_Access_and_Uncertainty/link/ 5a26af130f7e9b71dd0c61a9/download.

Bourreau, Marc/Dogan, Pinar/Manant, Matthieu, A critical review of the "ladder of investment” approach, Telecommunications Policy 34 (2010), S. 683-696.

Bourreau, Marc/Hoernig, Steffen/Maxwell, Winston, Implementing co-investment and network sharing, Brüssel 2020, abrufbar unter: https:/www.cerre.eu/sites/cerre/ files/cerre_implementing_co-investment_and_network_sharing-26.05.2020.pdf.

Braedel, Stephanie, Die Überlassung von Verteilungsanlagen nach Ablauf des Konzessionsvertrages gemäß $\$ 46$ Abs. 2 S. 2 EnWG, Baden-Baden 2011.

Braun, Christian, Der Retter in der Not: Dienstleistungskonzession?, NZBau 2011, S. 400-402.

Braun, Christian, Dienstleistungskonzession im europäischen Wandel, EuZW 2012, S. 451-455.

Briglauer, Wolfgang/Cambini, Carlo/Fetzer, Thomas/Hüschelrath, Kai, The European Electronic Communications Code - A critical appraisal with a focus on incentivizing investment in next generation broadband networks, Telecommunications Policy 2017, S. 948-961.

Brück von Oertzen, Martin/Kreggenfeld, Lena, Die Novelle des $\$ 46$ EnWG im Spannungsfeld des Vergaberechts, EWerK 2016, S. 12-16.

Brückner, Volkmar, Das globale Netz - Wirkungsweise und Grenzen der Datenübertragung im globalen Netz, Wiesbaden 2015.

Bulowski, Stefan, Regulierung von Internetkommunikationsdiensten - Zur Anwendbarkeit des Telekommunikationsrechts auf Voice over IP, Instant Messaging und E-Mail-Dienste, Baden-Baden 2019.

Bumke, Susanne, Frequenzvergabe nach dem Telekommunikationsgesetz - Unter besonderer Berücksichtigung der Integration ökonomischer Handlungsrationalität in das Verwaltungsverfahren, Berlin 2006. 
Bundeskartellamt (BKartA), Hinweise zur wettbewerbsrechtlichen Bewertung von Kooperationen beim Glasfaserausbau in Deutschland, Bonn 2010, abrufbar unter: https://www.bundeskartellamt.de/SharedDocs/Publikation/DE/Sonstiges/W ettbewerbsrechtliche \%20Bewertung\%20von\%20Kooperationen $\% 20$ beim $\% 20 \mathrm{Gl}$ asfaserausbau.pdf?_blob=publicationFile $\& v=3$.

Bundesministerium für Verkehr und digitale Infrastruktur (BMVI), Eckpunkte Zukunftsoffensive Gigabit-Deutschland, abrufbar unter: https://www.bmvi.de/Shar edDocs/DE/Anlage/DG/eckpunkte-zukunfts-offensive.pdf?_blob=publicationFi le.

Bundesministerium für Verkehr und digitale Infrastruktur (BMVI), Zukunftsoffensive Gigabit-Deutschland - Offensive der Netzallianz zum Ausbau gigabitfähiger konvergenter Netze bis 2025, Bonn 2017, abrufbar unter: https://www.bmvi.de/ SharedDocs/DE/Publikationen/DG/netzallianz-digitales-deutschland.pdf?_blob =publicationFile.

Bundesnetzagentur (BNetzA), Jahresbericht 2019 - Netze für die digitale Welt, Bonn 2020, abrufbar unter: https://www.bundesnetzagentur.de/SharedDocs/Me diathek/Jahresberichte/JB2019.pdf?_blob=publicationFile\&v=6.

Bundesverband Breitbandkommunikation (BREKO), Breitbandstudie 2017 Marktbefragung der BREKO-Mitgliedsunternehmen, Bonn 2017, abrufbar unter: https://brekoverband.de/wp-content/uploads/2017/11/BREKO-Breitbandstu die-2017.pdf.

Burgi, Martin, Die Vergabe von Dienstleistungskonzessionen - Verfahren, Vergabekriterien, Rechtsschutz, NZBau 2005, S. 610-617.

Burgi, Martin/Dreher, Meinrad (Hrsg.), Beck'scher Vergaberechtskommentar - Gesetz gegen Wettbewerbsbeschränkungen, 3. Aufl., München 2017.

Byok, Jan/Jaeger, Wolfgang (Hrsg.), Kommentar zum Vergaberecht - Vergaberechtliche Vorschriften des GWB, 4. Aufl., Frankfurt am Main 2018.

Calliess, Christian/Ruffert, Matthias (Hrsg.), EUV/AEUV - Das Verfassungsrecht der Europäischen Union mit Europäischer Grundrechtecharta. Kommentar, 5. Aufl., München 2016.

Cambini, Carlo/Silvestri, Virginia, Technology investment and alternative regulatory regimes with demand uncertainity, Information Economics and Policy 24 (2012), S. 212-230.

Cambini, Carlo/Silvestri, Virginia, Investment sharing in broadband networks, Telecommunications Policy 37 (2013), S. 861-878.

Carter, Kenneth/Elixmann, Dieter/Marcus, Scott, Unternehmensstrategische und regulatorische Aspekte von Kooperationen beim NGA-Breitbandausbau, WIK Diskussionsbeitrag Nr. 356, Bad Honnef 2011, abrufbar unter: https://www.wik.org /uploads/media/WIK_Diskussionsbeitrag_Nr_357_02.pdf.

Cave, Martin, Encouraging infrastructure competition via the ladder of investment, Telecommunications Policy 30 (2006), S. 223-237.

Cave, Martin, Six Degrees of Separation - Operational Seperation as a Remedy in European Telecommunications Regulation, Communications \& Strategies 64 (2006), S. 89-103. 
Cave, Martin/Doyle, Chris, Network separation and investment incentives in telecommunications, Coventry 2007, abrufbar unter: https://www.researchgate.net/publication/228982666_Network_separation_and_investment_incentives_in_telecommunications/link/54103c880cf2f2b29a3fc647/download.

Christlich Demokratische Union Deutschlands (CDU)/Christlich-Soziale Union in Bayern (CSU)/Sozialdemokratische Partei Deutschlands (SPD), Koalitionsvertrag 18. Legislaturperiode, Deutschlands Zukunft gestalten, Berlin 2013, abrufbar unter: https:/www.kas.de/c/document_library/get_file?uuid=2f9f5a98-5d120987-7f12-aca250c6ea92\&groupId=252038.

Christlich Demokratische Union Deutschlands (CDU)/Christlich-Soziale Union in Bayern (CSU)/Sozialdemokratische Partei Deutschlands (SPD), Koalitionsvertrag 19. Legislaturperiode, Ein neuer Aufbruch für Europa. Eine neue Dynamik für Deutschland. Ein neuer Zusammenhalt für unser Land, Berlin 2018, abrufbar unter: https://www.bundesregierung.de/resource/blob/656734/847984/5b8bc 23590d4cb2892b31c987ad672b7/2018-03-14-koalitionsvertrag-data.pdf?downloa $\mathrm{d}=1$.

Crandall, Robert/Eisenach, Jeffrey/Litan, Robert, Vertical Separation of Telecommunications Networks - Evidence from Five Countries, Federal Communications Law Journal 2010, S. 493-539.

Cremer, Helmuth/de Donder, Philippe/Cremer, Jacques, Costs and Benefits of Vertical Divestiture, Communications \& Strategies 68 (2008), S. 41-56.

Crome, Carl, Der Konzessionsvertrag und seine Ausführung im Kriege - Ein Beitrage zur Lehre von der Unerschwinglichkeit der Leistung, AcP 115 (1917), S. 157.

Dablke, Peter/Theis, Nikolaus, „Neue Märkte“ in der Rechtsanwendung - Tatbestandsmerkmale und Europarechtskonformität des neuen \$9a TKG, CR 2007, S. 227-231.

Dauses, Manfred/Ludwigs, Markus (Hrsg.), Handbuch des EU-Wirtschaftsrechts, 49. Ergänzungslieferung, München November 2019.

Dehenn, Daniel, Die Vergabe von Wegekonzessionen nach $\$ 46$ EnWG - Kommunaler Stromnetzbetrieb zwischen Daseinsvorsorge und Wettbewerb, Lohmar 2015.

Deutscher Landkreistag, Flächendeckende Breitbandversorgung zu wirtschaftlichen Bedingungen sicherstellen, Berlin 2017, abrufbar unter: https://www.landk reistag.de/images/stories/publikationen/170620_Pospap_Breitband.pdf.

Dialog Consult GmbH (Dialog Consult)/ Verband der Anbieter von Telekommunikations- und Mehrwertdiensten e. V. (VATM), 2. Marktanalyse Gigabit-Anschlüsse 2020, Köln 2020, abrufbar unter: https:/www.vatm.de/wp-content/uplo ads/2020/04/VATM_Gigabit-Studie_290420_.pdf.

Diemon-Wies, Ingeborg, Vergabe von Konzessionen - Überblick über die neuen Regelungen im GWB-Entwurf, VergabeR 2016, S. 162-165.

Dietlein, Johannes, Konzessionsübertragung und Konzessionshandel im Taxi-Gewerbe, GewArch 1999, S. 89-95. 
Dietlein, Johannes/Brandenberg, Alexandra, Regulierung „Neuer Märkte“ im Telekommunikationsrecht am Beispiel von VDSL (Teil 2), IR 2005, S. 245-248.

Dietlein, Johannes/Brandenberg, Alexandra, Regulierung „Neuer Märkte“ im Telekommunikationsrecht am Beispiel von VDSL (Teil 1), IR 2005, S. 208-211.

DIW Econ, Ausbau von Gigabitnetzen: Wettbewerb und Regulierung, Berlin 2018, abrufbar unter: https://www.vatm.de/wp-content/uploads/2018/10/DIW_Econ_ VATM_Gigabitnetz_Wettbewerb_und_Regulierung-1.pdf.

Donhauser, Christoph/Hölzlwimmer, Jennifer, Die neue Richtlinie über die Konzessionsvergabe und ihre Auswirkungen auf die Vergabe von Wegenutzungskonzessionen nach $₫ 46$ EnWG, VergabeR 2015, S. 509-521.

Drechsler, Stefan, Die Unionsgrundrechte unter dem Einfluss des Prozessrechts Bestandsaufnahme und Perspektiven im Europäischen Grundrechtsverbund am Beispiel der EU-Wirtschaftsgrundrechte, Baden-Baden 2019.

Dreier, Horst (Hrsg.), Grundgesetz - Kommentar, Band 3, 3. Aufl., Tübingen 2018.

Edenharter, Andrea, Grundrechtsschutz in föderalen Mehrebenensystemen - Inspiration des EU-Grundrechtsschutzes durch die Grundrechtsentwicklung in Deutschland und der Schweiz sowie durch die EMRK, Tübingen 2018.

Edenharter, Andrea, Die EU-Grundrechte-Charta als Prüfungsmaßstab des Bundesverfassungsgerichts, DÖV 2020, S. 349-357.

Ehricke, Ulrich, Vermerke der Kommission zur Umsetzung von Richtlinien, EuZW 2004, S. 359-364.

Ehrler, Matthias/Ruble, Ernst-Olav/Berger, Ernst Georg, Regionalisierung der TK Regulierung: Mehr oder weniger Wettbewerb?, CR 2008, S. 703-708.

Elixmann, Dieter/Ilic, Dragan/Neumann, Karl-Heinz/Plückebaum, Thomas, The Economics of Next Generation Access - Final Report, Bad Honnef 2008, abrufbar unter:

file_2008_09_15_V1.pdf.

Emmerich, Volker/Lange, Knut Werner, Kartellrecht - Ein Studienbuch, 14. Aufl., München 2018.

Europäische Kommission (Komm.), Europe 2020 - A European strategy for smart, sustainable and inclusive growth, Brüssel 2010, abrufbar unter: https:/ec.europa .eu/eu2020/pdf/COMPLET\%20EN\%20BARROSO\%20\%20\%20007\%20-020Eur ope\%202020\%20-\%20EN\%20version.pdf.

Europäische Kommission (Komm.), Shaping Europe’s Digital Future, Brüssel 2020, abrufbar unter: https:/ec.europa.eu/info/sites/info/files/communication-shaping -europes-digital-future-feb2020_en_4.pdf.

European Regulators Group (ERG), Common Position on Geographic Aspects of Market Analysis, Brüssel 2008, abrufbar unter: https://berec.europa.eu/doc/ publications/erg_08_20_final_cp_geog_aspects_081016.pdf.

Fehling, Michael/Ruffert, Matthias (Hrsg.), Regulierungsrecht, Tübingen 2010.

Felten, Benoît/Langer, Thomas, Structurally independent broadband infrastructure can solve perceived FTTH coverage issues, La Garenne Colombes 2014, abrufbar unter: https://papers.ssrn.com/sol3/papers.cfm?abstract_id=2794850. 
Fetzer, Thomas, Breitbandinternetzugang als Universaldienst? - Rechtliche Zulässigkeit und ökonomische Angemessenheit einer Universaldienstverpflichtung, MMR 2011, S. 707-711.

Fetzer, Thomas, Open Access and Access Regulation in Increasingly Regionalized Telecommunication Markets, in: Spiecker genannt Döhmann, Indra/Krämer, Jan (Hrsg.), Network Neutrality and Open Access, Baden-Baden 2011, S. 177190.

Fetzer, Thomas, Impulsstudie Telekommunikationsregulierung 4.0, Mannheim 2017, abrufbar unter: https://www.econstor.eu/bitstream/10419/175372/1/10151 5056X.pdf.

Fornefeld, Martin/Rokus, Marius, Technologische Grundlagen zur Breitband-Versorgung im ländlichen Raum, Düsseldorf 2010, abrufbar unter: https://micus-duess eldorf.de/images/download/publikationen/MICUS_Grundlagen-Breitband-Vers orgung.pdf.

Forsthoff, Ernst, Lehrbuch des Verwaltungsrechts - Band 1, 10. Aufl. München 1973.

Fraunhofer-Institut für offene Kommunikationssysteme (Fraunhofer FOKUS), Netzinfrastrukturen für die Gigabitgesellschaft, Berlin 2016, abrufbar unter: http://publica.fraunhofer.de/eprints/urn_nbn_de_0011-n-4260110.pdf.

FTTH Council Europe, AB Stokab - Stockholm makes high-tech economy and society a reality. Council-owned AB Stokab rolls out large-scale darb fibre network across city, Brüssel 2013, abrufbar unter: https://www.ftthcouncil.eu/documents/ CaseStudies/STOKAB.pdf.

Fülling, Daniel, GWB-Digitalisierungsgesetz und Kommission Wettbewerbsrecht 4.0 als Bausteine digitaler Ordnungspolitik, in: Körber, Torsten/Immenga, Ulrich (Hrsg.), Innovation im Kartellrecht - Innovation des Kartellrechts - Referate der 2. Kölner Kartellrechtsgespräche vom 26. Juni 2019, Baden-Baden 2020, S. 49-56.

Geppert, Martin/Schütz, Raimund (Hrsg.), Beck'scher TKG-Kommentar, 4. Aufl., München 2013.

Gerpott, Torsten, Unternehmenskooperationen beim Bau und bei der Nutzung von Glasfaseranschlussnetzen - Systematisierung von Zusammenarbeitsvarianten und Analyse ihrer Wettbewerbswirkungen im deutschen Markt für Breitbandanschlüsse, MedienWirtschaft 4/2010, S. 10-24.

Gerpott, Torsten, Voucher für Glasfaseranschlüsse: Warum Bund und Länder auf sie verzichten sollten, Wirtschaftsdienst 2020, S. 128-132.

Girard, Yann/Mattes, Anselm/Michelsen, Claus, Gigabitzugang in Deutschland: im internationalen Vergleich rückständig, aber auch wenig nachgefragt, DIW Wochenbericht 2018, S. 532-541.

Godlovitch, Ilsa/Lemstra, Wolter/Pennings, Christoph/Neumann, Karl-Heinz/de Streel, Alexandre/Stronzik, Marcus/Stumpf, Ulrich/Kroon, Peter/Lucidi, Stefano/Tseveen, Gantumur/Plueckebaum, Thomas/Baldacchino, Vincent/Chailoou, Valerie/van den Peijl, Sebastiaan, Regulatory, in particular access, regimes for network investment models in Europe, Brüssel 2016, abrufbar unter: https://op.europa.eu/en/p ublication-detail/-/publication/c0da75d9-9a8c-11e6-9bca-01aa75ed71a1. 
Godlovitch, Ilsa/Sörries, Bernd/Gantumur, Tseveen, A tale of five cities - The implications of broadband business models on choice, price and quality, Bad Honnef 2017, abrufbar unter: https://www.stokab.se/Documents/Nyheter\%20bilagor/A $\% 20$ tale $\% 20$ of $\% 20$ five $\% 20$ cities.pdf.

Goldbrunner, Loni, Das neue Recht der Konzessionsvergabe, VergabeR 2016, S. 365-384.

Götz, Georg/Zenhäusern, Patrick, Investitionen und Regulierung bei schnellen Internetzugängen in Deutschland und der Schweiz, Wirtschaftsdienst 2013, S. 260 266.

von Graevenitz, Albrecht, Mitteilungen, Leitlinien, Stellungnahmen - Soft Law der EU mit Lenkungswirkung, EuZW 2013, S. 169-173.

von Graevenitz, Albrecht, Wider die Verrechtlichung von soft law der Europäischen Kommission, ZRP 2019, S. 75-78.

Greb, Klaus/Wegner, Mario, Die Vergabe von Konzessionen im Energiebereich - Ein Leitfaden für die kommunale Praxis, Kronach 2012.

von der Groeben, Hans/Schwarze, Jürgen/Hatje, Armin (Hrsg.), Europäisches Unionsrecht - Vertrag über die Europäische Union, Vertrag über die Arbeitsweise der Europäischen Union, Charta der Grundrechte der Europäischen Union, 7. Aufl., München 2015.

Gries, Christin-Isabel/Plückebaum, Thomas/Strube Martins, Sonia, Treiber für den Ausbau hochbitratiger Infrastrukturen, Bad Honnef 2016, abrufbar unter: https:/w ww.wik.org/fileadmin/Studien/2016/VATM_Hochbitratige_Infrastrukturen.pdf.

Grünewald, Stefanie, Die (Re)Kommunalisierung in der Energieverteilung - Grenzen und Möglichkeiten kommunaler wirtschaftlicher Betätigung in der Elektrizitätsverteilung - Zugleich eine kritische Auseinandersetzung mit dem energiewirtschaftlichen Konzessionsvergaberecht, Berlin 2016.

Harnisch, Christian, Funktionale Separierung als strategisches Regulierungsinstrument auf dem europäischen Telekommunikationsmarkt, Arbeitspapiere des Institutes für Genossenschaftswesen der Westfälischen Wilhelms-Universität Münster Nr. 83, Münster 2009, abrufbar unter: http://www.wiwi.uni-muenster.d e/06/forschen/veroeffentlichungen/2009/material/ap83harnisch.pdf.

Harnisch, Christian, Funktionale Separierung in der Telekommunikationsindustrie - Eine komparative Analyse alternativer Ausgestaltungsoptionen vor dem Hintergrund des technologischen Wandels, Aachen 2010.

Haucap, Justus/Heimeshoff, Ulrich/Uhde, André, Vertikale Entflechtung netzgebundener Industrien - Kosten und Nutzen aus ökonomischer Sicht, in: Gesellschaft für öffentliche Wirtschaft (Hrsg.), Auswirkungen der Globalisierung auf die öffentlichen Banken - Trennung von Betrieb und Infrastruktur, Leipzig 2007, S. 27-65.

Henssler, Martin/Strohn, Lutz (Hrsg.), Gesellschaftsrecht, 4. Aufl., München 2019.

Hermes, Georg, Staatliche Infrastrukturverantwortung - Rechtliche Grundstrukturen netzgebundener Transport- und Übertragungssysteme zwischen Daseinsvorsorge und Wettbewerbsregulierung am Beispiel der leitungsgebundenen Energieversorgung in Europa, Tübingen 1998. 
Heuermann, Arnulf/Meinen, Philipp/Ekango, Albert Njoume/Pavel, Ferdinand/Wissmann, Daniel, Konzepte zur Förderung von Breitbandinvestitionen im internationalen Vergleich, Berlin 2009, abrufbar unter: https://diw-econ.de/wp-content/ uploads/2014/01/diwkompakt_2009-052.pdf.

Heymann, Eric/Körner, Kevin, Digitale Infrastruktur - Engpässe hemmen Europa, Frankfurt am Main 2018, abrufbar unter: https://www.dbresearch.de/PROD/RP S_DE-PROD/PROD0000000000478197/Digitale_Infrastruktur\%3A_Engp\%C3\% A4sse_hemmen_Europa.PDF.

Hofmann, Heiko/Riethmüller, Tobias, Einführung in das Umwandlungsrecht, JA 2009, S. 481-488.

Hofmann, Heiko/Zimmermann, Manuel, Rechtsrahmen für die Vergabe von Wegenutzungsverträgen im Energiebereich nach der neuen Konzessionsvergaberichtlinie - Droht eine Fortsetzung der gegenwärtig bestehenden Rechtsunsicherheit?, NZBau 2016, S. 71-76.

Hohenstein-Bartholl, Christine/Jacob, Martin, Strom- und Gaskonzessionsverträge vermitteln keine Dienstleistungskonzessionen - Abhandlung aus vergabe- und energiewirtschaftlicher Sicht, RdE 2017, S. 454-462.

Holznagel, Bernd/Deckers, Sebastian, Breites Band im weiten Land - Neue Herausforderungen für die Daseinsvorsorge im föderalen Bundesstaat, DVBl. 2009, S. 482-489.

Holznagel, Bernd/Enaux, Christoph/Nienhaus, Christian, Telekommunikationsrecht, 2. Aufl., München 2006.

Ilic, Dragan/Neumann, Andreas/Plückebaum, Thomas, The Economics of Next Generation Access - Addendum, Bad Honnef 2009, abrufbar unter: https:// www.wik.org/uploads/media/ECTA_Study_Addendum_2009.pdf.

Immenga, Ulrich/Mestmäcker, Ernst-Joachim (Hrsg.), Wettbewerbsrecht, Band 1 Kommentar zum Europäischen Kartellrecht, 6. Aufl., München 2019.

Immenga, Ulrich/Mestmäcker, Ernst-Joachim (Hrsg.), Wettbewerbsrecht, Band 2 Deutsches Kartellrecht, 6. Aufl., München 2020.

Inderst, Roman/Kühling, Jürgen/Neumann, Karl-Heinz/Peitz, Martin, Nationale versus sub-nationale Marktabgrenzung und Regulierung bei NGA, in: Inderst, Roman/ Kühling, Jürgen/Neumann, Karl-Heinz/Peitz, Martin (Hrsg.), Der Ausbau neuer Netze in der Telekommunikation - Institutionelle, ökonomische und juristische Betrachtungen, Baden-Baden 2012, S. 293-324.

IW Consult GmbH (IW Consult), Der Weg zur Gigabitgesellschaft - Eine wettbewerbsorientierte Migration für eine flächendeckende Gigabitversorgung in Deutschland, Köln 2018, abrufbar unter: https://www.vatm.de/wp-content/uplo ads/2018/10/2018-08_IW-Studie_Glasfasermigration_01.pdf .

Jaeger, Wolfgang/Kokott, Juliane/Pohlmann, Petra/Schroeder, Dirk (Hrsg.), Frankfurter Kommentar zum Kartellrecht, 95. Ergänzungslieferung, Köln März 2020.

Jarass, Hans D., Charta der Grundrechte der Europäischen Union - unter Einbeziehung der vom EuGH entwickelten Grundrechte, der Grundrechtsregelungen der Verträge und der EMRK, 3. Aufl., München 2016. 
Jungbluth, Armin, Die 9. GWB-Novelle - Änderungen beim Ministerialerlaubnisverfahren und bei den Aufgreifschwellen in der Fusionskontrolle, in: Körber, Torsten/Immenga, Ulrich (Hrsg.), Aktuelle Entwicklungen in der Fusionskontrolle zwischen Recht, Wirtschaft und Politik - Referate der 1. Kölner Kartellrechtsgespräche vom 13. April 2018, Baden-Baden 2019, S. 9-16.

Kahl, Wolfang/Schwind, Manuel, Europäische Grundrechte und Grundfreiheiten Grundbausteine einer Interaktionslehre, EuR 2014, S. 170-195.

Kahl, Wolfgang/Waldhoff, Christian/Walter, Christian (Hrsg.), Bonner Kommentar zum Grundgesetz, 204. Aufl., Heidelberg Juni 2020.

Kämmerer, Jörn Axel/Kotzur, Markus, Vollendung des Grundrechtsverbunds oder Heimholung des Grundrechtsschutzes? - Die BVerfG-Beschlüsse zum „Recht auf Vergessen“ als Fanal, NVwZ 2020, S. 177-184.

Keller, Andres, Breitbandkabel und Zugangsnetze - Technische Grundlagen und Standards, 2. Aufl., Berlin 2011.

Kirchner, Christian, Regulierungsanreize für den Auf- und Ausbau von NGA-Netzen - Schlussfolgerungen aus dem Kroes-Memo vom 12.7.2012, MMR 2013, S. 22-26.

Kirchner, Christian, Regulierungsanreize für VDSL-Vectoring - Regulatorische Möglichkeiten und Grenzen einer Aufrüstung von Kupferzugängen für NGANetze, CR 2013, S. 85-89.

Klein, Eckhart, Kompetenzielle Würdigung und verfassungsprozessuale Konsequenzen der „Recht auf Vergessen“-Entscheidungen, DÖV 2020, S. 341-349.

Klett, Michael/Otter, Jan Philipp, Dienstleistungen von allgemeinem wirtschaftlichem Interesse, in: Ministerium für Wirtschaft, Arbeit und Wohnungsbau Baden-Württemberg (Hrsg.), DAWI und Infrastrukturfinanzierung - EU-Beihilfenrecht, Stuttgart 2016, abrufbar unter: https://wm.baden-wuerttemberg.de/filead $\mathrm{min} /$ redaktion/m-wm/intern/Publikationen/Wirtschaftsstandort/Leitfaden_EUBeihilfenrecht_DAWI_und_Infrastrukturfinanzierung_Band-3.pdf.

Kling, Michael/Thomas, Stefan, Kartellrecht, 2. Aufl., München 2016.

Kment, Martin/Vorwalter, Sebastian, Streitfragen der energiewirtschaftlichen Konzession, EnWZ 2015, S. 387-395.

Knieps, Günter, Netzökonomie - Grundlagen - Strategien - Wettbewerbspolitik, Wiesbaden 2007.

Koenig, Christian, Die öffentlich-rechtliche Verteilungslenkung - Grund und Grenzen einer Deregulierung am Beispiel der Vergabe von Konzessionen, Kontingenten und Genehmigungen zur unternehmerischen Nutzung öffentlich verwalteter Güter, Berlin 1994.

Kollmann, Tobias/Schmidt, Holger, Deutschland 4.0 - Wie die digitale Transformation gelingt, Wiesbaden 2016.

Körber, Torsten, Regulierung der Netzindustrien: Übergangsstadium oder Daueraufgabe des Staates? - Zum Verhältnis von Kartell- und Regulierungsrecht, in: Bechtold, Stefan/Jickeli, Joachim/Rohe, Mathias (Hrsg.), Festschrift zum 70. Geburtstag von Wernhard Möschel - Recht, Ordnung und Wettbewerb, Baden-Baden 2011, S. 1043-1056. 
Kraft, Julia/Redenius-Hövermann, Julia (Hrsg.), Umwandlungsrecht, Tübingen 2015.

Krämer, Jan/Vogelsang, Ingo, Co-Investments and Tacit Collusion in Regulated Network Industries - Experimental Evidence, Review of Network Economics 2016, S. 35-61.

Kredel, Nicolas/Kresken, Jan, Das „GWB 10.0“ - Eckpunkte des Referentenentwurfs für das GWB-Digitalisierungsgesetz, NZKart 2020, S. 2-8.

Krueger, Birgit, Die Kartellamtspraxis nach der 9. GWB-Novelle am Beispiel der Fusionskontrolle, in: Körber, Torsten/Immenga, Ulrich (Hrsg.), Aktuelle Entwicklungen in der Fusionskontrolle zwischen Recht, Wirtschaft und Politik - Referate der 1. Kölner Kartellrechtsgespräche vom 13. April 2018, Baden-Baden 2019, S. 79-88.

Kühling, Jürgen, Sektorspezifische Regulierung in den Netzwirtschaften - Typologie, Wirtschaftsverwaltungsrecht, Wirtschaftsverfassungsrecht, München 2004.

Kübling, Jürgen, \$9a TKG-E - Innovationsschutz durch Regulierungsverzicht oder Steigerung der Regulierungskomplexität?, K\&R 2006, S. 263-272.

Kühling, Jürgen, Ausschreibungspflichten beim Abschluss von Gestattungsverträgen für die Verlegung von Fernwärmeleitungen, in: Körber, Torsten/Kühling, Jürgen (Hrsg.), Ausschreibung von Fernwärmenetzen?, Baden-Baden 2016, S. 9-62.

Kühling, Jürgen, Netzwirtschaftsübergreifende Lerneffekte bei neuen Gemeinwohlherausforderungen aktivieren, N\&R 2019, S. 1.

Kühling, Jürgen, Wettbewerbliche Leitplanken auf dem Weg in die „Gigabit“-Gesellschaft, WuW 2019, S. 555-561.

Kühling, Jürgen, Das „Recht auf Vergessenwerden“ vor dem BVerfG - November(r)evolution für die Grundrechtsarchitektur im Mehrebenensystem, NJW 2020, S. 275-280.

Kühling, Jürgen/Biendl, Michael, Zulässiger Universaldienstumfang im Zeitalter des Breitbandausbaus - Europa- und verfassungsrechtliche Grenzen staatlicher Fördermodelle, DÖV 2012, S. 409-417.

Kühling, Jürgen/Bulowski, Stefan, Rechtsgutachten über die Zulässigkeit öffentlichrechtlicher Verträge im Rahmen des TKG, Bonn 2015, abrufbar unter: https://w ww.bundesnetzagentur.de/DE/Service-Funktionen/Beschlusskammern/1_GZ/B K3-GZ/2015/BK3-15-0004/Rechtsgutachten/BK3-15-0004_Rechtsgutachten_dow nload.pdf?_blob=publicationFile\&v=3.

Kübling, Jürgen/Bulowski, Stefan, Zulässigkeit und Ausgestaltungsmöglichkeiten öffentlich-rechtlicher Verträge im Rahmen des TKG, N\&R 2015, S. 262-275.

Kühling, Jürgen/Elbracht, Alexander, Telekommunikationsrecht, Heidelberg 2008.

Kühling, Jürgen/Goldmedia/Enaux, Christoph (Hrsg.), Rechtliche Herausforderungen bei der Schaffung von Anreizen für einen flächendeckenden Ausbau von Glasfaserinfrastrukturen, Bonn/Berlin 2019, abrufbar unter: https:/www.bmvi.de/Shar edDocs/DE/Anlage/DG/Digitales/rechtsgutachten-ausbauanreize-glasfaser-goldm edia-kuehling.pdf?_blob=publicationFile.

Kühling, Jürgen/Heimeshoff, Ulrich/Schall, Tobias, Künftige Regulierung moderner Breitbandinfrastrukturen, K\&R Beihefter 1/2010, S. 1-32. 
Kübling, Jürgen/Rasbach, Winfried/Busch, Claudia, Energierecht, 4. Aufl., Baden-Baden 2018.

Kühling, Jürgen/Schall, Tobias/Biendl, Michael, Telekommunikationsrecht, 2. Aufl., Heidelberg 2014.

Kühling, Jürgen/Seiler, Martin, Der Ausschluss der Inhouse-Vergabe von Energiekonzessionen nach der Novellierung von $\$ 46$ EnWG im Mehrebenensystem, EnWZ 2017, S. 99-106.

Kübling, Jürgen/Toros, Fabian, Rechtliche Rahmenbedingungen für die Schaffung von Anreizen für einen flächendeckenden Ausbau von Glasfaserinfrastrukturen, Regensburg 2019.

Kühling, Jürgen/Toros, Fabian, Universaldienst der Zukunft - Zur Novellierung der s\$ 78 ff. TKG, N\&R 2019, S. 258-263.

Kühling, Jürgen/Toros, Fabian, Der rechtlich abgesicherte Anspruch auf „schnelles Internet" - quo vadis?, K\&R 2019, S. 692-697.

Kühling, Jürgen/Toros, Fabian, Rechtliche Rahmenbedingungen für das neue Kartierungssystem beim Ausbau von TK-Infrastrukturen - Die geografische Erhebung gem. Art. 22 EKEK, MMR 2020, S. 92-96.

Kublmann, Jens/Abnis, Erik, Konzern- und Umwandlungsrecht, 4. Aufl., Heidelberg 2016.

Kühn, Hendrik, Nicht kontrollierende Minderheitsbeteiligungen in der EU-Fusionskontrolle, Baden-Baden 2017.

Kulartz, Hans-Peter/Kus, Alexander/Portz, Norbert/Prieß, Hans-Joachim (Hrsg.), Kommentar zum GWB-Vergaberecht, 4. Aufl., Düsseldorf 2016.

Lau, Niels, Vergabe von Strom- und Gasnetzen im Zeichen der Energiewende Eine Einführung, in: Lau, Niels (Hrsg.), Vergabe von Energienetzen - Konzessionsverträge - Strom, Gas, Fernwärme, Köln 2016, S. 9-12.

Lebourges, Marc/Liang, Julienne, Estimating the impact of co-investment in fiber to the home coverage, Trient 2018, abrufbar unter: https://www.econstor.eu/ bitstream/10419/184953/1/Lebourges-Liang.pdf.

Lengsfeld, Jörn, Digital Era Framework - Ein Bezugsrahmen für das Digitale Informationszeitalter, Bad Waldsee 2019, abrufbar unter: https://joernlengsfeld.com/f iles/digital-era-framework-de.pdf.

Lenßen, Markus, Der kartellrechtlich relevante Markt - Prinzip - Konzeption - Methode, Baden-Baden 2009.

Lettl, Tobias, Kartellrecht, 4. Aufl., München 2017.

Leupold, Andreas/Glossner, Silke (Hrsg.), Münchener Anwalts Handbuch IT-Recht, 3. Aufl., München 2013.

Limbach, Felix/Kübel, Hannes/Zarnekow, Rüdiger, Kooperativer Breitbandausbau in Deutschland - Eine Expertenbefragung unter Unternehmensführern und Kooperationsverantwortlichen der deutschen Telekommunikationsbranche, Berlin 2013. 
Loewenheim, Ulrich/Meessen, Karl/Riesenkampff, Alexander/Kersting, Christian/MeyerLindemann, Hans Jürgen (Hrsg.), Kartellrecht - Kommentar, 4. Aufl., München 2020.

Luch, Anika/Schulz, Sönke, Die E-Daseinsvorsorge als Grundlage der Online-Handlungsfreiheit und „Eintrittskarte“ zur Digitalen Agora, VM 2011, S. 104-112.

Luch, Anika/Schulz, Sönke, eDaseinsvorsorge - Neuorientierung des überkommenen (Rechts-)Begriffs „Daseinsvorsorge“ im Zuge technischer Entwicklungen?, MMR 2009, S. 19-24.

Luch, Anika/Schulz, Sönke, E-Daseinsvorsorge - Staatliche Schutzpflichten und Sozialstaatsprinzip im Lichte der „Virtualisierung“ des Lebens, in: Hill, Hermann/ Schliesky, Utz (Hrsg.), Herausforderung e-Government - E-Volution des Rechtsund Verwaltungssystems, Baden-Baden 2009, S. 305-336.

Luch, Anika/Schulz, Sönke, Das Recht auf Internet als Grundlage der Online-Grundrechte, Kiel 2013.

von Mangoldt, Hermann/Klein, Friedrich/Starck, Christian (Begr.), GG - Kommentar zum Grundgesetz, 7. Aufl., München 2018.

Maunz, Theodor/Dürig, Günter (Begr.), Grundgesetz - Kommentar, 90. Ergänzungslieferung, München Februar 2020.

Mayer, Otto, Deutsches Verwaltungsrecht - Band 2, Leipzig 1896.

Meinel, Christoph/Sack, Harald, Digitale Kommunikation - Vernetzen, Multimedia, Sicherheit, Berlin/Heidelberg 2009.

Meister, Johannes, Das telekommunikationsrechtliche Frequenzplanungsrecht im System des allgemeinen Planungsrechts, Baden-Baden 2004.

Mertz, Andreas/Pollakowski, Martin, xDSL \& Access Networks - Grundlagen, Technik und Einsatzaspekte von HDSL, ADSL und VDSL, München 2000.

Meyer, Matti, Vorwirkungen des Konnektivitätsziels auf das geltende TKG vor Ablauf der Umsetzungsfrist des EU-Kommunikationskodex?, N\&R 2019, S. 264268.

Michaels, Sascha/Bönnighausen, Rabea, Zu Inhalt und Folgen der Zweifel über die Anwendbarkeit des Teils 4 des GWB und der Konzessionsvergabeverordnung auf Konzessionierungsverfahren für Strom- und Gasverteilnetze, ZNER 2018, S. 7-13.

Michl, Fabian, Unionsgrundrechte aus der Hand des Gesetzgebers, Tübingen 2018.

Mohr, Jochen, Energiewirtschaftliche Konzessionsverträge und Unionsrecht, RdE 2016, S. 269-279.

Möllnitz, Christina, Die Vergabe von Konzessionsverträgen nach $₫ 46$ Abs. 2 EnWG im Spannungsfeld zwischen Wettbewerb und kommunaler Daseinsvorsorge, Tübingen 2016.

Monopolkommission, 9. Sektorgutachten Telekommunikation (2015) - Märkte im

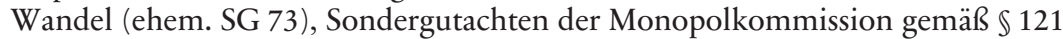
Abs. 2 TKG, Bonn 2015, abrufbar unter: https://www.monopolkommission.de/i mages/PDF/SG/s73_volltext.pdf. 
Monopolkommission, 10. Sektorgutachten Telekommunikation (2017) - Auf Wettbewerb bauen! (ehem. SG 78), Sondergutachten der Monopolkommission gemäß \121 Abs. 2 TKG, Bonn 2017, abrufbar unter: https://www.monopolkom mission.de/images/PDF/SG/s78_volltext.pdf.

Monopolkommission, 11. Sektorgutachten Telekommunikation (2019) - Staatliches Augenmaß beim Netzausbau, Sektorgutachten gemäß $\$ 121$ Abs. 2 TKG, Bonn 2019, abrufbar unter: https://www.monopolkommission.de/images/PDF/S G/11sg_telekommunikation.pdf.

Monopolkommission, Policy Brief zur 10. GWB-Novelle, 10. GWB-Novelle - Herausforderungen auf digitalen und regionalen Märkten begegnen!, Bonn 2020, abrufbar unter: https://www.monopolkommission.de/images/Policy_Brief/MK_ Policy_Brief_4.pdf.

Moseley, Alice/Stoker, Gerry, Nudging citizens? Prospects and pitfalls confronting a new heuristic, Resources, Conversation and Recycling 79 (2013), S. 4-10.

Müller, Christine/Growitsch, Christian/Wissner, Matthias, Regulierung und Investitionsanreize in der ökonomischen Theorie - IRIN Working Paper im Rahmen des Arbeitspakets: Smart Grid-gerechte Weiterentwicklung der Anreizregulierung, Bad Honnef 2010, abrufbar unter: https://www.wik.orguploads/media/WI K_Diskussionsbeitrag_Nr_349.pdf.

Müller-Graff, Peter-Christian, Das „Soft Law“ der europäischen Organisationen, EuR 2012, S. 18-34.

Müller-Wrede, Malte (Hrsg.), GWB - Vergaberecht, Kommentar, Köln 2016.

von Münch, Ingo (Begr.)/Kunig, Philip (Hrsg.), Grundgesetz - GG. Kommentar, 6. Aufl., München 2012.

Naab, Philipp, Die Versteigerung knapper Ressourcen durch den Staat - Insbesondere zur Versteigerung von Mobilfunkfrequenzen nach dem Telekommunikationsgesetz, Baden-Baden 2010.

Nationaler IT-Gipfel, Konvergente Netze als Infrastruktur für die Gigabit-Gesellschaft, 2016, abrufbar unter: https://plattform-digitale-netze.de/app/uploads/201 6/11/PF1_Gigabit_Konvergente_Netze_web_20161111.pdf.

Nettesheim, Martin, Marktdesign durch EU-Recht - Das Beispiel der Konzessionsvergabe, in: Kment, Martin (Hrsg.), Konzessionen im Umwelt- und Infrastrukturrecht, Tübingen 2016.

Nettesheim, Martin/Thomas, Stefan, Entflechtung im deutschen Kartellrecht - Wettbewerbspolitik, Verfassungsrecht, Wettbewerbsrecht, Tübingen 2011.

Neumann, Andreas, Der Kommissionsvorschlag für einen europäischen Kodex für die elektronische Kommunikation - Geplante Änderungen im Bereich der Marktregulierung, N\&R 2016, S. 262-272.

Neumann, Andreas, Das Ergebnis der Trilog-Verhandlungen zum Kodex für die elektronische Kommunikation im Bereich der Marktregulierung, N\&R 2018, S. 204-211. 
Neumann, Andreas, Optionen für die Ausgestaltung eines rechtlich abgesicherten Anspruchs auf schnelles Internet aus telekommunikationsrechtlicher Sicht, Bonn 2019, abrufbar unter: http://www.irnik.de/publikationen/gutachten/201902-01_BREKO_Gutachten_rechtlich_abgesicherter_Anspruch_auf_schnelles_Int ernet.pdf.

Neumann, Andreas, Reform des Rechtswegs im Telekommunikationsrecht, N\&R 2020, S. 148-161.

Neumann, Andreas/Koch, Alexander, Telekommunikationsrecht, 2. Aufl., Frankfurt am Main 2013.

Neumann, Andreas/Sickmann, Jörn, Schaffung eines rechtlich abgesicherten Anspruchs auf einen Zugang zum schnellen Internet - Eine Analyse der Zielvorgabe im Koalitionsvertrag zwischen CDU, CSU und SPD, N\&R-Beilage 1/2018, S. 1-12.

Neumann, Andreas/Sickmann, Jörn, Stand und Perspektiven des Breitbandausbaus in Deutschland - IRNIK-Diskussionspapier Nr.5, Bonn 2018, abrufbar unter: http://www.irnik.de/publikationen/dp/irnik_dp_005.pdf.

Neumann, Andreas/Sickmann, Jörn/Alkas, Hasan/Koch, Alexander, Reformbedarf des europäischen Rechtsrahmens für elektronische Kommunikation - Recht und Ökonomie der Telekommunikationsregulierung in Zeiten der Gigabit-Gesellschaft, Frankfurt am Main 2017.

Neumann, Karl-Heinz, Regionalisierung der Regulierung, Bad Honnef 2014, abrufbar unter: https://www.vatm.de/wp-content/uploads/2018/07/Studie_Regionalisi erung_der_Regulierung.pdf.

Nigge, Ralf/Horstmann, Niklas, Marktregulierung und Investitionsanreize im europäischen Kodex für elektronische Kommunikation - Neue Ansätze der Regulierung von Unternehmen mit beträchtlicher Marktmacht, MMR 2018, S. 721-726.

Nitsche, Rainer/Wiethaus, Lars, Access Regulation and investment in next generation networks - A ranking of regulatory regimes, Industrial Organization 2010, S. 263-272.

Noch, Rainer, Vergaberecht kompakt - Handbuch für die Praxis, 7. Aufl., Köln 2017.

Offenbächer, Philipp, Die Regulierung des Vectoring - Der entbündelte Zugang zum Teilnehmeranschluss im Kräftefeld von Wettbewerb und hochleistungsfähigen Telekommunikationsnetzen, Baden-Baden 2019.

Ohler, Christoph, Grundrechtliche Bindungen der Mitgliedstaaten nach Art. 51 GRCh, NVwZ 2013, S. 1433-1438.

Olsen, Ole/Henten, Anders/Falch, Morten, Functional separation in telecommunications - A comparative analysis of infrastructural areas, Montreal 2008, abrufbar unter: http://www.imaginar.org/taller/its2008/104.pdf.

Opitz, Marc, Die Zukunft der Dienstleistungskonzession, NVwZ 2014, S. 753-760. 
Organisation für wirtschaftliche Zusammenarbeit und Entwicklung (OECD), Developments in Fibre Technologies and Investment, OECD Digital Economy Papers No. 142, Paris 2008, abrufbar unter: https:/www.oecd-ilibrary.org/docserve r/230526125804.pdf?expires=1593246533\&id=id\&accname=guest\&checksum=D 01671B9126B31827E833C08989E6C4B.

Papier, Hans-Jürgen/Schröder, Meinhard, Wirtschaftlich angemessene Vergütung für Netzanlagen - Zur verfassungskonformen Auslegung des $\$ 46$ Abs. 2 S. 2 EnWG, Baden-Baden 2012.

Pechstein, Matthias/Nowak, Carsten/Häde, Ulrich (Hrsg.), Frankfurter Kommentar EUV/GRC/AEUV, Tübingen 2017.

Peeperkorn, Luc/Verouden, Vincent, The economics of competition, in: Faull, Jonathan/Nikpay, Ali (Hrsg.), The EU law of competition, 3. Aufl., Oxford 2014, S. 3-90.

Peers, Steve/Hervey, Tamara/Kenner, Jeff/Ward, Angela (Hrsg.), The EU Charter of Fundamental Rights - A Commentary, Oxford, Portland, Oregon 2014.

Picot, Arnold/Franck, Egon, Vertikale Integration, in: Hauschildt, Jürgen/Grün, Oskar (Hrsg.), Festschrift für Eberhard Witte - Ergebnisse empirischer betriebswirtschaftlicher Forschung, Stuttgart 1993, S. 181-219.

Podszun, Rupprecht/Brauckmann, Fabian, GWB-Digitalisierungsgesetz: Der Referentenentwurf des BMWi zur 10. GWB-Novelle, GWR 2019, S. 436-438.

Porter, Michael, Wettbewerbsvorteile - Spitzenleistungen erreichen und behaupten, 8. Aufl., Frankfurt am Main 2014.

Prieß, Hans-Joachim/Marx, Fridhelm/Hölzl, Josef, Kodifizierung des europäischen Rechtes zur Vergabe von Dienstleistungskonzessionen nicht notwendig - Überlegungen am Beispiel der europäischen Regeln für die Trinkwasserversorgung, NVwZ 2011, S. 65-72.

Prieß, Hans-Joachim/Stein, Roland, Die neue EU-Konzessionsvergaberichtlinie, VergabeR 2014, S. 499-512.

Probst, Matthias Ernst, Auswahlkriterien bei der Vergabe von energiewirtschaftlichen Konzessionsverträgen - Eine Untersuchung der europa-, verfassungs-, energie-, kartell- und kommunalrechtlichen Anforderungen an Kommunen in Konzessionsverfahren, München 2016.

Pünder, Hermann/Schellenberg, Martin (Hrsg.), Vergaberecht, 3. Aufl., Baden-Baden 2019.

Rasbach, Winfried, Unbundling-Regulierung in der Energiewirtschaft, München 2009.

Reents, Reent Ricklef, Ausbau und Finanzierung einer flächendeckenden Breitbandversorgung in Deutschland, Tübingen 2016.

Reidt, Olaf/Stickler, Thomas/Glahs, Heike (Hrsg.), Vergaberecht - Kommentar, 4. Aufl., Köln 2018.

Reisch, Lucia/Zhao, Min, Behavioural economics, consumer behaviour and consumer policy: state of the art, Behavioural Public Policy 2017, S. 190-206.

Rennert, Klaus, Konzessionen vor dem Verwaltungsgericht, NZBau 2019, S.411417. 
Rossi, Matthias/Pfahl, Sebastian, Grundstrukturen der Konzession im Umwelt- und Infrastrukturrecht, in: Kment, Martin (Hrsg.), Konzessionen im Umwelt- und Infrastrukturrecht, Tübingen 2016, S. 1-32.

Ruffert, Matthias (Hrsg.), Europäisches Sektorales Wirtschaftsrecht, 2. Aufl., BadenBaden 2020.

Ruble, Ernst-Olav/Reichl, Wolfgang, Incentives for Investments in Next Generation Access and Customer Choice: a Dichotomy?, Intereconomics 2009, S. 30-40.

Sachs, Michael (Hrsg.), GG - Grundgesetz. Kommentar, 8. Aufl., München 2018.

Säcker, Franz Jürgen (Hrsg.), Telekommunikationsgesetz - Kommentar, 3. Aufl., Frankfurt am Main 2013.

Säcker, Franz Jürgen (Hrsg.), Münchener Kommentar Europäisches und deutsches Wettbewerbsrecht - Band 3 - Vergaberecht I, 2. Aufl., München 2018.

Säcker, Franz Jürgen/Mohr, Jochen/Wolf, Maik, Konzessionsverträge im System des europäischen und deutschen Wettbewerbsrechts, Frankfurt am Main 2011.

Säcker, Franz Jürgen/Rixecker, Roland/Oetker, Hartmut/Limperg, Bettina (Hrsg.), Münchener Kommentar zum Bürgerlichen Gesetzbuch, 8. Aufl., München 2020.

Sackmann, Florian, Datenschutz bei der Digitalisierung der Mobilität - Eine sektorspezifische Analyse der Leistungsfähigkeit und des Weiterentwicklungsbedarfs der Datenschutzordnung, Baden-Baden 2020.

Sauer, Mirko, Das Recht der Vergabe von Strom- und Gas-Konzessionsverträgen im EnWG - Legitimität und Anwendung eines Wettbewerbsinstruments im Kontext des Unions- und deutschen Verfassungsrechts, Baden-Baden 2012.

Scheidler, Alfred, Die Taxikonzession - eine Sonderform der gewerberechtlichen Erlaubnis, GewArch 2011, S. 417-424.

Scherer, Joachim/Heinickel, Caroline, Ein Kodex für den digitalen Binnenmarkt Vorschlag der EU-Kommission für eine Reform des Rechts der elektronischen Kommunikation, MMR 2017, S. 71-77.

Scheurle, Klaus-Dieter/Mayen, Thomas (Hrsg.), Telekommunikationsgesetz - Kommentar, 3. Aufl., München 2018.

Schmitt, Joachim/Hörtnagl, Robert/Stratz, Rolf-Christian (Hrsg.), Umwandlungsgesetz, Umwandlungssteuergesetz, 8. Aufl., München 2018.

Schneider, Jens-Peter/Theobald, Christian (Hrsg.), Recht der Energiewirtschaft - Praxishandbuch, 4. Aufl., München 2013.

Schöfthaler, Jochen, Rechtliche Rahmenbedingungen des Breitbandausbaus, Hamburg 2018.

Schulte, Josef (Hrsg.), Handbuch der Fusionskontrolle, 3. Aufl., Köln 2020.

Schulz, Sönke, E-Daseinsvorsorge - Der staatliche und kommunale Grundversorgungsauftrag in einer technisierten Informationsgesellschaft, in: Schliesky, Utz (Hrsg.), Selbstverwaltung im Staat der Informationsgesellschaft, Kiel 2010.

Schulz, Sönke, Informations- und Kommunikationstechnologie als Grundversorgung, DuD 2010, S. 698-702. 
Schulz, Sönke, Das Grundrecht auf Achtung und Gewährung eines menschenwürdigen Existenzminimums, in: Schliesky, Utz/Ernst, Christian/Schulz, Sönke (Hrsg.), Festschrift für Edzard Schmidt-Jortzig - Die Freiheit des Menschen in Kommune, Staat und Europa, Heidelberg 2011, S. 17-46.

Schumacher, Pascal, Breitband-Universaldienst: Möglichkeiten und Grenzen deutscher Politik - Funktionales Internet endlich für alle?, MMR 2011, S. 711-715.

Schwab, Andreas/Giesemann, Amelie, Mit mehr Regeln zu mehr Rechtssicherheit? Die Überarbeitung des europäischen Vergaberechts, VergabeR 2014, S. 351-369.

Schwarze, Jürgen, Soft Law im Recht der Europäischen Union, EuR 2011, S. 3-18.

Semler, Johannes/Stengel, Arndt (Hrsg.), Umwandlungsgesetz, 4. Aufl., München 2017.

Sickmann, Jörn/Neumann, Andreas, Deregulierung und Verbraucherwohlfahrt auf dem deutschen Telekommunikationsmarkt - Studie im Auftrag des Verbraucherzentrale Bundesverband e.V., Bonn 2017, abrufbar unter: https:/www.vzbv. de/sites/default/files/downloads/2017/11/23/2017-10-04_studie_deregulierung_u nd_verbraucherwohlfahrt_tk-markt.pdf.

Sickmann, Jörn/Neumann, Andreas, „Digitalland“ Deutschland? - Telekommunikationspolitische Ziele im Koalitionsvertrag zwischen CDU, CSU und SPD, Wirtschaftsdienst 2018, S. 333-339.

Sickmann, Jörn/Neumann, Andreas, Regulierungsferien für Koinvestitionen? - Die Diskussion um Art. 74 eines künftigen europäischen Kodex für die elektronische Kommunikation, K\&R 2018, S. 92-99.

Sörries, Bernd, Die Vectoring-Technologie: Fluch oder Segen? - Die Telekommunikationspolitik am Scheideweg, CR 2015, S. 162-167.

Sörries, Bernd, Neues Geschäftsmodell, neue Regulierung? - Das Geschäftsmodell des reinen Vorleistungsanbieters und seine rechtliche und ökonomische Bedeutung, N\&R 2020, S. 161-166.

Stelkens, Ulrich/Bonk, Heinz Joachim/Sachs, Michael (Hrsg.), Verwaltungsverfahrensgesetz - Kommentar, 9. Aufl., München 2018.

Stelkens, Ulrich/Wabnitz, Miriam, Neuere Rechtsprechung der Instanzgerichte zu \$S 68 bis 75 TKG - Telegraphenwegerechtliche Traditionen in Zeiten des Breitbandausbaus, MMR 2015, S. 502-506.

Stern, Klaus, Zur Problematik des energiewirtschaftlichen Konzessionsvertrages, AöR 84 (1959), S. 137-184.

Storr, Stefan, Konfusion um die Konstruktion der Konzession, in: Kluth, Winfried/ Müller, Martin/Peilert, Andreas (Hrsg.), Festschrift für Rolf Stober - Wirtschaft - Verwaltung - Recht, Köln 2008, S. 417-432.

Streinz, Rudolf (Hrsg.), EUV/AEUV - Vertrag über die Europäische Union, Vertrag über die Arbeitsweise der Europäischen Union, Charta der Grundrechte der Europäischen Union, 3. Aufl., München 2018.

Streinz, Rudolf, „Recht auf Vergessenwerden“ zwischen Unionsrecht und Verfassungsrecht - Das Datenschutzrecht als Ansatz für Neuerungen des „Kooperationsverhältnisses“ zwischen EuGH und BVerfG, DuD 2020, S. 353-359. 
Strube Martins, Sonia/Wernick, Christian/Plückebaum, Thomas/Henseler-Unger, Iris, Die Privatkundennachfrage nach hochbitratigem Breitbandinternet im Jahr 2025, Bad Honnef 2017, abrufbar unter: https://www.wik.org/fileadmin/Studien /2017/Die_Privatkundennachfrage_nach_hochbitratigem_Breitbandinternet_im JJahr_2025_FINAL.pdf.

Stumpf, Ulrich, Die Abgrenzung subnationaler Märkte als regulatorischer Ansatz, Bad Honnef 2010, abrufbar unter: https://www.wik.org/uploads/media/WIK_Di skussionsbeitrag_Nr_334.pdf.

Sunstein, Cass, Nudging: A Very Short Guide, Journal of Consumer Policy 37 (2014), S. 583-588.

Templin, Wolf, Recht der Konzessionsverträge - Eine historische, verfassungsrechtliche, rechtstatsächliche und rechtspolitische Analyse des Konzessionsvertrages als Instrument gemeindlicher Energiepolitik, München 2009.

Tenbrock, Sebastian, Systematisierung und Regulierungsnotwendigkeit von Glasfaserausbaukooperationen - Arbeitspapiere des Instituts für Genossenschaftswesen der Westfälischen Wilhelms-Universität Münster, Nr. 108, Münster 2011, abrufbar unter: http://www.ifg-muenster.de/forschen/veroeffentlichungen/2011/m aterial/ap108_tenbrock_neu.pdf.

Tenbrock, Sebastian, Der Glasfaserausbau in Deutschland - Eine empirische Untersuchung der Ausgestaltungsformen und Kooperationsvarianten, Aachen 2013.

Tenbrock, Sebastian/Strube Martins, Sonia/Wernick, Christian/Queder, Fabian/HenselerUnger, Iris, Co-Invest Modelle zum Aufbau von neuen FTTB/H-Netzinfrastrukturen, Bad Honnef 2018, abrufbar unter: https://www.wik.org/uploads/media/WI K_Diskussionsbeitrag_Nr_430.pdf.

Theobald, Christian/Kühling, Jürgen (Hrsg.), Energierecht - Loseblattkommentar, 104. Ergänzungslieferung, München Dezember 2019.

Theobald, Christian/Wolkenhauer, Sören, Vergabe von Strom- und Gaskonzessionen - Einschätzungen zur aktuellen Novellierung des $₫ 46$ EnWG, DÖV 2016, S. 724-732.

Theurl, Theresia/Schweinsberg, Andrea, Neue kooperative Ökonomie, Tübingen 2004.

Thomas, Stefan, Die Bindungswirkung von Mitteilungen, Bekanntmachungen und Leitlinien der EG-Kommission, EuR 2009, S. 423-444.

Thym, Daniel, Die Reichweite der EU-Grundrechte-Charta - Zu viel Grundrechtsschutz?, NVwZ 2013, S. 889-896.

Thym, Daniel, Blaupausenfallen bei der Abgrenzung von Grundgesetz und Grundrechtecharta, DÖV 2014, S. 941-951.

Toros, Fabian/Weiß, Martin, Echte Kooperation?! -Wandel des Grundrechtsschutzes im Mehrebenensystem - Zu den Entscheidungen „Recht auf Vergessen I“ und „Recht auf Vergessen II“ des BVerfG, ZJS 2020, S. 100-108.

Ufer, Frederic, Regionalisierung als Ausstieg aus der Regulierung, N\&R 2008, S. 173-177. 
von Weizsäcker, Carl Christian, Regionalisierung der Regulierung im Bitstrom-Zugangsmarkt - ZEI Discussion Paper C189/2008, Bonn 2008, abrufbar unter: https://www.zei.uni-bonn.de/dateien/discussion-paper/dp_c189_von_Weizsaeck er.pdf.

Walz, Rainer, Die Bau- und Dienstleistungskonzession im deutschen und europäischen Vergaberecht - Merkmale und dogmatische Einordnung der vergaberechtlichen Konzession sowie ihre Abgrenzung zum klassischen Bau-, Lieferund Dienstleistungsauftrag, Köln 2009.

Webb, Malcolm, Breaking Up is Hard to Do - The Emergence of Functional Separation as a Regulatory Remedy, Genf 2008, abrufbar unter: https:/www.itu.int/IT U-D/treg/Events/Seminars/GSR/GSR08/discussion_papers/Malcolm_Webb_sessi on3.pdf.

Weiß, Holger, Stand und Perspektiven des Rechts der Strom- und Gaskonzessionsvergabe, NVwZ 2014, S. 1415-1420.

Weiß, Wolfgang, Entscheidungsspielräume bei der Konzessionierung nach $₫ 46$ EnWG - Eine kritische Betrachtung der jüngsten BGH-Rechtsprechung, München 2015.

Wernick, Christian, Ökonomie und Kostenstrukturen des Glasfaserausbaus, Bad Honnef 2016, abrufbar unter: https://www.wik.org/fileadmin/Studien/2016/Stu die_OEkonomie_Glasfaserausbau.pdf.

Wernick, Christian/Gries, Christin/Bender, Christian/Tenbrock, Sebastian/Strube Martins, Sonia, Regionale TK-Akteure im globalen Wettbewerb, Bad Honnef 2016, abrufbar unter: https:/www.wik.org/fileadmin/Studien/2016/Regionale_TK_Ak teure_im_globalen_Wettbewerb.pdf.

Wernick, Christian/Queder, Fabian/Strube Martins, Sonia/Gries, Christin, Ansätze zur Glasfaser-Erschließung unterversorgter Gebiete, Bad Honnef 2017, abrufbar unter: https://www.wik.org/fileadmin/Studien/2017/2017_DIHK_Studie.pdf.

Wernick, Christian/Queder, Fabian/Strube Martins, Sonia/Gries, Christin/Tenbrock, Sebastian/Bender, Christian, Gigabitnetze für Deutschland, Bad Honnef 2016, abrufbar unter: https://www.wik.org/fileadmin/Studien/2017/Gigabitnetze_Deutsc hland.pdf.

Wernick, Christian/Tenbrock, Sebastian/Gries, Christin/Henseler-Unger, Iris/Plückebaum, Thomas, Tiefbaukapazitäten als Engpass für den FTTB/H-Ausbau? - Empfehlungen zur Effizienzsteigerung und Kostensenkung für den Markt und die öffentliche Hand, Bad Honnef 2018, abrufbar unter: https://www.wik.org/filead $\mathrm{min} /$ Studien/2018/WIK-Tiefbaustudie.pdf.

Wernick, Christian/Fetzer, Thomas/Gries, Christin/Tenbrock, Sebastian/Queder, Fabian/ Henseler Unger, Iris/Strube Martins, Sonia, Rahmenbedingungen für die Gigabitwelt 2025+ (RaGiga), Bad Honnef 2018, abrufbar unter: https://www.wik.org/fil eadmin/Studien/2018/Ragiga_20180706.pdf.

Wiedemann, Gerhard, Gemeinschaftsunternehmen im deutschen Kartellrecht, Heidelberg 1981.

Wieland, Joachim, Die Konzessionsabgaben - Zur Belastung wirtschaftsverwaltungsrechtlicher Erlaubnisse mit Abgaben, Berlin 1991. 
Wissenschaftlicher Dienst, WD 5 - 3000 - 072/18 - Gigabit-Voucher als Maßnahme zur nachfrageseitigen Förderung des Breitbandausbaus, abrufbar unter: https:// www.bundestag.de/resource/blob/562382/46d1eed5f19626d0008327fb5fdbebc7/ wd-5-072-18-pdf-data.pdf.

Zentes, Joachim/Swoboda, Bernhard/Morschett, Dirk (Hrsg.), Kooperationen, Allianzen und Netzwerke - Grundlagen - Ansätze - Perspektiven, 2. Aufl., Wiesbaden 2005.

ZEW - Leibniz-Zentrum für Europäische Wirtschaftsforschung GmbH (ZEW)/ Juconomy Rechtsanwälte (Juconomy), Gutachten zur ökonomischen und rechtlichen Sinnhaftigkeit von nachfrageseitigen Förderungen im Ausbau moderner Breitbandnetze über „Voucher-Systeme“, Köln 2019, abrufbar unter: https:/ww w.vatm.de/wp-content/uploads/2019/09/ZEW_Juconomy_Gutachten_VoucherSysteme_BREKO_VATM.pdf.

Ziekow, Jan/Völlnik, Uwe-Carsten (Hrsg.), Vergaberecht - Kommentar, 3. Aufl., München 2018. 


\section{Verzeichnis nur über das Internet abrufbarer Entscheidungen}

In diesem Verzeichnis werden, um die Übersichtlichkeit der Fußnoten zu erhöhen, die Fundstellen aller Entscheidungen angegeben, die lediglich über das Internet abrufbar sind. Die Links wurden zum letzten Mal am 1.7.2020 geprüft.

\section{Bundesnetzagentur}

Bundesnetzagentur (BNetzA), Beschl. v. 21.3.2011, Az. BK 3d-12/131, abrufbar unter: http:/www.t-regs.com/wp-content/uploads/2013/11/2013-08-30_BNetzA_D E-2013-1484_Vectoring_Adopted-Measures.pdf.

Bundesnetzagentur (BNetzA), Beschl. v. 22.3.2011, Az. BK 3g-09/85, abrufbar unter: https://www.bundesnetzagentur.de/DE/Service-Funktionen/Beschlusskamm ern/1_GZ/BK3-GZ/2009/BK3-09-0085/BK3-09-0085_RegVfg.pdf?_blob=publica tionFile $\& v=3$.

Bundesnetzagentur (BNetzA), Entsch. v. 29.8.2013, Az. BK 3d-12/131, abrufbar unter: http://www.t-regs.com/wp-content/uploads/2013/11/2013-08-30_BNetzA_D E-2013-1484_Vectoring_Adopted-Measures.pdf.

Bundesnetzagentur (BNetzA), Beschl. v. 9.7.2015, Az. BK 1-14-00, abrufbar unter: https://www.bundesnetzagentur.de/SharedDocs/Downloads/DE/Sachgebiete/Tel ekommunikation/Unternehmen_Institutionen/Marktregulierung/Marktanalyse n/Festlegung_Markt3b_ME2014.pdf?_blob=publicationFile\&v=2.

Bundesnetzagentur (BNetzA), Beschl. v. 27.8.2015, Az. BK 1-12/003, abrufbar unter: https://www.bundesnetzagentur.de/SharedDocs/Downloads/DE/Sachgebiete /Telekommunikation/Unternehmen_Institutionen/Marktregulierung/Marktanal ysen/Festlegung_Markt3a_ME2014.pdf?_blob=publicationFile\&v=3.

Bundesnetzagentur (BNetzA), Entsch. v. 28.10.2015, Az. BK 3h-14/114, abrufbar unter: https://www.bundesnetzagentur.de/DE/Service-Funktionen/Beschlusska mmern/1_GZ/BK3-GZ/2014/BK3-14-0114/Anlagen/BK3-14-0114_Erg\%C3\%A4n zung_Feststellung_08_2019_download_bf.pdf?_blob=publicationFile\&v=2.

Bundesnetzagentur (BNetzA), Entsch. v. 1.9.2016, Az. BK 3g-15/004, abrufbar unter: https://www.bundesnetzagentur.de/DE/Service-Funktionen/Beschlusskamm ern/1_GZ/BK3-GZ/2015/BK3-15-0004/BK3-15-0004_Regulierungsverfuegung_d ownload_BF.pdf?_blob=publicationFile $\& v=6$.

Bundesnetzagentur (BNetzA), Entsch. v. 14.5.2018, Az. BK 1-17/001, abrufbar unter: https://www.bundesnetzagentur.de/SharedDocs/Downloads/DE/Sachgebiete /Telekommunikation/Unternehmen_Institutionen/Frequenzen/OffentlicheNetz e/Mobilfunk/DrahtloserNetzzugang/Mobilfunk2020/20180514_Entscheidungen _I_II.pdf?_blob=publicationFile\&v $=5$. 
Bundesnetzagentur (BNetzA), Entsch. v. 10.7.2018, Az. BK 1-18/002, abrufbar unter: https:/www.bundesnetzagentur.de/DE/Service-Funktionen/Beschlusskamm ern/1_GZ/BK3-GZ/2017/BK3-17-0071/BK3-17-0071_Beschl_Marktdefinitionen Marktanalysen_download_bf.pdf?_blob=publicationFile\&v=5.

Bundesnetzagentur (BNetzA), Beschl. v. 13.7.2018, Az. BK 3-17/071, abrufbar unter: https:/www.bundesnetzagentur.de/DE/Service-Funktionen/Beschlusskamm ern/1_GZ/BK3-GZ/2017/BK3-17-0071/BK3-17-0071_Beschluss_download_bf.pdf ?_blob=publicationFile\&v=3.

Bundesnetzagentur (BNetzA), Beschl. v. 26. 11. 2018, Az. BK 1-17/001, abrufbar unter: https:/www.bundesnetzagentur.de/SharedDocs/Downloads/DE/Sachgebi ete/Telekommunikation/Unternehmen_Institutionen/Frequenzen/OffentlicheN etze/Mobilfunk/DrahtloserNetzzugang/Mobilfunk2020/20181126_Entscheidung en_III_IV.pdf?_blob=publicationFile\&v=3.

Bundesnetzagentur (BNetzA), Konsultationsentwurf Markt 3a, Az. BK 1-19/001, abrufbar unter: https:/www.bundesnetzagentur.de/DE/Service-Funktionen/Besc hlusskammern/1_GZ/BK1-GZ/2019/BK1-19-0001/BK1-19-0001_Konsultationsen twurf_bf.pdf;jsessionid=318F0E205B2D9DDFE3FC2013DB76893F?_blob=publ icationFile $\& v=3$.

\section{Bundeskartellamt}

Die Entscheidungen des Bundeskartellamtes sind zwar auch in Teilen über das Online-Angebot BECK-RS abrufbar. Allerdings werden dort für diese Untersuchung entscheidende Passagen der Beschlüsse (insbes. die Anlagen) nicht abgedruckt. Aus diesem Grund wurde auf die Entscheidungen zurückgegriffen, die das Bundeskartellamt auf seiner Website veröffentlicht hat.

Bundeskartellamt (BKartA), Beschl. v. 4.12.2019, Az. B 7-21/18, abrufbar unter: https://www.bundeskartellamt.de/SharedDocs/Entscheidung/DE/Entscheidunge n/Fusionskontrolle/2019/B7-21-18.pdf;jsessionid=AE03CA3CABB0EFEEAD53D 56B4BFF668D.1_cid390?_blob=publicationFile\&v=4.

Bundeskartellamt (BKartA), Beschl. v. 30.12.2019, Az. B 7-21/18, abrufbar unter: https://www.bundeskartellamt.de/SharedDocs/Entscheidung/DE/Entscheidunge n/Fusionskontrolle/2020/B7-21-18.pdf?_blob=publicationFile\&v=2.

\section{Europäischer Gerichtshof für Menschenrechte}

Europäischer Gerichtshof für Menschenrechte (EGMR), Urt. v. 29.11.1991, Nr. 12742/87 (Pine Valley Developments Ltd. u. a./Irland), abrufbar unter: https://hudoc.echr.coe.int/eng\#\{\%22itemid\%22:[\%22001-57711\%22]\}. 


\section{Europäische Kommission}

Europäische Kommission (Komm.), Beschl. v. 16.11.2004, SA. N 381/2004, France - Projet de réseau de télécommunication haut débit des Pyrénées-Atlantiques, abrufbar unter: https://ec.europa.eu/competition/state_aid/cases/164636/164636 _509434_33_2.pdf.

Europäische Kommission (Komm.), Beschl. v. 6.4.2005, SA. N 583/2004, España Banda ancha en zonas rurales y aisladas, abrufbar unter: https://ec.europa.eu/co mpetition/state_aid/cases/197328/197328_516348_28_2.pdf.

Europäische Kommission (Komm.), Beschl. v. 3.5.2005, SA. N 382/2004, France Mise en place d'une infrastructure haut débit sur le territoire de la region Limousin (DORSAL), abrufbar unter: https://ec.europa.eu/competition/state_aid/c ases/164637/164637_509484_51_2.pdf.

Europäische Kommission (Komm.), Beschl. v. 8.3.2006, Ireland - Regional Broadband Programme: Metropolitan Area Networks ("MANs"), phases II and III, abrufbar unter: https://ec.europa.eu/competition/state_aid/cases/199656/199656_5 81678_68_2.pdf.

Europäische Kommission (Komm.), Beschl. v. 10.7.2007, SA. N 890/2006, France Aide du Sicoval pour un réseau de très haut débit, abrufbar unter: https://ec.eur opa.eu/competition/state_aid/cases/218142/218142_723454_30_2.pdf.

Europäische Kommission (Komm.), Beschl. v. 11.3.2008, SA. N 412/2007, Italy Aid to reduce the digital divide in Piedmont, abrufbar unter: https://ec.europa.e u/competition/state_aid/cases/221120/221120_811738_34_1.pdf.

Europäische Kommission (Komm.), Beschl. v. 30.9.2009, SA N 331/2008, France Compensation de charges pour une Délégation de Service Public (DSP) pour l'établissement et l'exploitation d'un réseau de communications électroniques à très haut débit dans le Département des Hauts-de-Seine, abrufbar unter: https:// ec.europa.eu/competition/state_aid/cases/226425/226425_1095475_172_2.pdf.

Europäische Kommission (Komm.), Beschl. v. 30.4.2010, SA. N 646/2009, Italy National broadband plan for rural areas in Italy, abrufbar unter: https:/ec.europ a.eu/competition/state_aid/cases/233970/233970_1114455_30_1.pdf.

Europäische Kommission (Komm.), Beschl. v. 11.8.2010, SA. N 407/2009, Spain Optical fibre Catalonia (Xarxa Oberta), abrufbar unter: https:/ec.europa.eu/com petition/state_aid/cases/232264/232264_1136486_80_2.pdf.

Europäische Kommission (Komm.), Beschl. v. 13.12.2010, SA. N 497/2010, United Kingdom - SHEFA-2 Interconnect, abrufbar unter: https:/ec.europa.eu/competi tion/state_aid/cases/238173/238173_1193590_58_2.pdf.

Europäische Kommission (Komm.), Beschl. v. 19.1.2011, SA.30317, Portugal High-speed broadband Portugal, abrufbar unter: https://ec.europa.eu/competitio n/state_aid/cases/236635/236635_1199063_71_2.pdf.

Europäische Kommission (Komm.), Beschl. v. 19.10.2011, SA.31316 (N 330/2010), France - Programme national „Très Haut Débit“-Volet B, abrufbar unter: https:/ /ec.europa.eu/competition/state_aid/cases/237100/237100_1268937_108_3.pdf. 
Europäische Kommission (Komm.), Beschl. v. 30.8.2012, SA.34809 (2012/N), Deutschland - NGA-Breitbandnetz Markt Reisbach, abrufbar unter: https://ec.e uropa.eu/competition/state_aid/cases/244707/244707_1362269_106_1.pdf.

Europäische Kommission (Komm.), Beschl. v. 30.11.2012, SA. 33641 (2011/N), Greece - Met-ropolitan Area Networks (MAN)/Fibre to the home (FTTH) Greece, abrufbar unter: https://ec.europa.eu/competition/state_aid/cases/242048/ 242048_1393123_88_1.pdf.

Europäische Kommission (Komm.), Beschl. v. 18.12.2012, SA.34199 (2012/N), Italy - Digital Plan - Super-fast broadband, abrufbar unter: https://ec.europa.eu/com petition/state_aid/cases/244965/244965_1400328_95_2.pdf.

Europäische Kommission (Komm.), Beschl. v. 4.12.2013, SA.33656 (2012/NN), Ireland - Next Generation (backhaul) Network (NGN) alongside a gas pipeline in Galway and Mayo, abrufbar unter: https:/ec.europa.eu/competition/state_aid/ca ses/243213/243213_1504550_221_2.pdf.

Europäische Kommission (Komm.), Beschl. v. 12.12.2013, SA.37558 (2013/N), Romania - Ro-NET project, abrufbar unter: https://ec.europa.eu/competition/state _aid/cases/250354/250354_1501667_115_2.pdf.

Europäische Kommission (Komm.), Beschl. v. 4.8.2014, C(2014) 5698 final, S. 5, abrufbar unter https://www.anacom.pt/streaming/CE_EN2014_1638and1639Ma rket1and2.pdf?contentId=1311812\&field=ATTACHED_FILE.

Europäische Kommission (Komm.), Beschl. v. 21.4.2015, SA.39518 (2014/N), Deutschland - NGA-Cluster Nordhessen, abrufbar unter: https://ec.europa.eu/c ompetition/state_aid/cases/257205/257205_1675759_69_2.pdf.

Europäische Kommission (Komm.), Beschl. v. 22.7.2015, SA.41416 (2015/N), Deutschland - NGA-Förderregelung Baden-Württemberg, abrufbar unter: https: //ec.europa.eu/competition/state_aid/cases/257876/257876_1719703_130_2.pdf.

Europäische Kommission (Komm.), Beschl. v. 26.5.2016, SA.40720 (2016/N), United Kingdom - National Broadband Scheme for the UK for 2016-2020, abrufbar unter: https://https://ec.europa.eu/competition/state_aid/cases/263954/263 954_1760328_135_4.pdf.

Europäische Kommission (Komm.), Beschl. v. 30.6.2016, SA.41647 (2016/N), Italy - Strategia Banda Ultralarga, abrufbar unter: https:/ec.europa.eu/competition/st ate_aid/cases/264095/264095_1764969_101_2.pdf.

Europäische Kommission (Komm.), Beschl. v. 7.11.2016, SA.37183 (2015/NN), France - Plan France très haut débit, abrufbar unter: https://ec.europa.eu/compe tition/state_aid/cases/260985/260985_1876109_165_2.pdf.

Europäische Kommission (Komm.), Beschl. v. 31.7.2019, SA.53135 (2019/N), Greece - Ultrafast Broadband Infrastructure Scheme, abrufbar unter: https:/ec.e uropa.eu/competition/state_aid/cases1/201937/278209_2093880_210_2.pdf. 